WSRC-TR--93-086

DE93 012834

\title{
FLOWTRAN-TF v1.2 Source Code (U)
}

S. E. Aleman, R. E. Cooper, G. P. Flach, L. L. Hamm, S. Lee, F. G. Smith III

Publication Date: February, 1993

Westinghouse Savannah River Company Savannah River Site

Aiken, SC 29808 
TASK NUMBER: $89-021-1$

DOCUMENT TITLE: FLOWTRAN-TF v1.2 Source Code (U)

DOCUMENT NUMBER: WSRC-TR-93-086

REVISION NUMBER: 0

\section{APPROVALS}

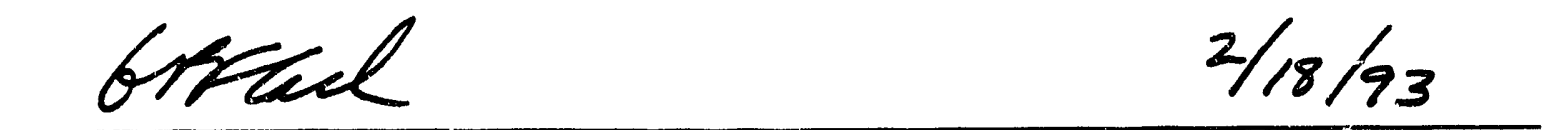

G. P. FLACH, TASK LEADER

DATE

Aavida. Gombey 2/24/9.3 D. A. CROWLEY, NES CODE DEV ELOPMENT GROUP MANAGER DATE

$\frac{\text { Q Jowett }}{\text { A. J. GARRETT, NES MANAGER }} \frac{2 / 24 / 93}{\text { DATE }}$




\section{Acknowledgements}

The FLOWTRAN-TF code development effort was initiated in early 1989 . Several members of the Code Development Group have contributed to FLOWTRAN-TF development as summarized alphabetically below:

\section{Contributor Principal Contributions}

Sebastian Aleman Axial wall drag, neutronic power distribution, splines, code architecture, documentation, coding, graphics, testing

Roger Cooper Coding, graphics

Greg Flach Program leadership, interfacial drag, axial wall drag, phase transition logic, fluid finite-differencing and solution methods, equations of state, documentation, coding, testing, benchmarking

Larry Hamm

Program concept, initiation and leadership, all aspects of physical model development and computational implementation, interfacial heat and mass transfer, code architecture, coding, testing, benchmarking

Andrea Kielpinski Interfacial heat and mass transfer coefficients, flow regime map, documentation

Larry Koffman Documentation

Si Young Lee Channel inlet void distribution, CCFL correlation, inter-subchannel mixing, interfacial heat and mass transfer coefficients, rib void model, equations of state, documentation, coding, testing, benchmarking

Andy Shadday Azimuthal wall drag, decumentation, testing

Frank Smith Solid heat conduction, neutronic power distribution, wall heat transfer, irterfacial heat and mass transfer, fluid finite differencing and solution methods, code architecture and $/ / O$, documentation, coding, testing, benchmarking

The developers are grateful to the many technical reviewers of FLOWTRAN-TF. These include Ken Moore, Garry Gose, Ken Katsma and M. Paulsen of Computer Simulation \& Analysis, Inc. and Steve Hensel and Jeff Jerrell of WSRC. The helpful suggestions of the DOE Independent Review Panel (DIRP) are also appreciated.

The developers also appreciate the support of the DOE, former WSRC Nuclear Engineering Section manager Ken Huffman and present manager Al Garrett, and former Code Development Group managers Larry Harnm and Bill Horion and current manager Dave Crowley. 
This page intentionally left blank 


\section{Table of Contents}

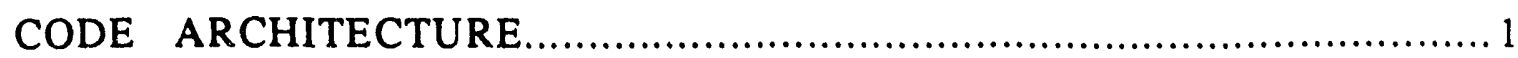

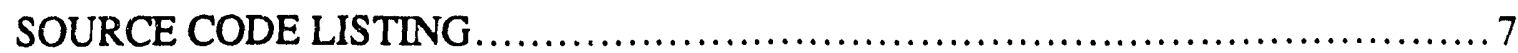

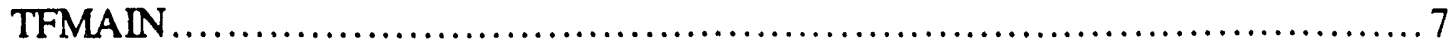

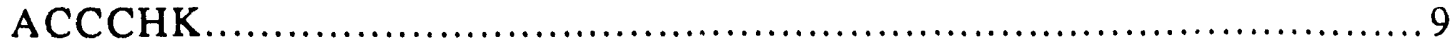

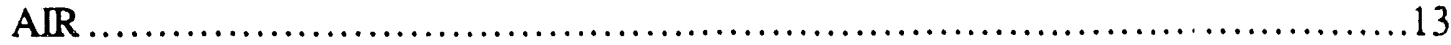

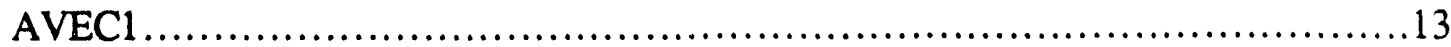

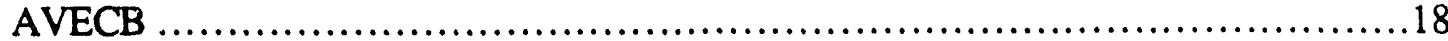

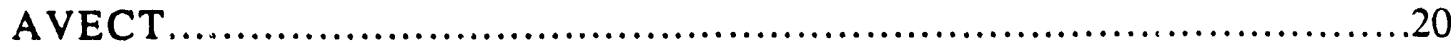

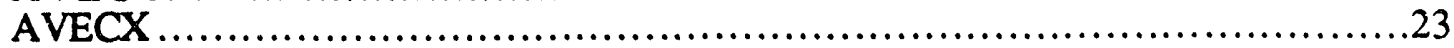

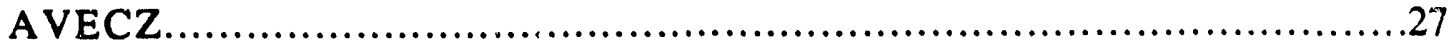

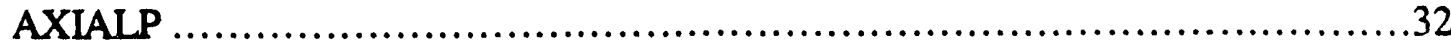

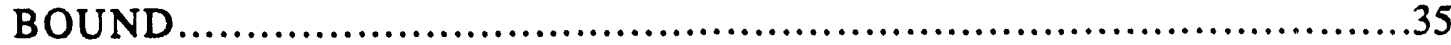

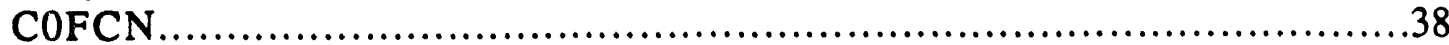

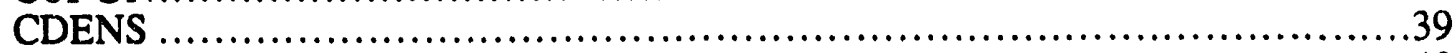

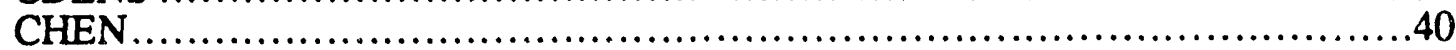

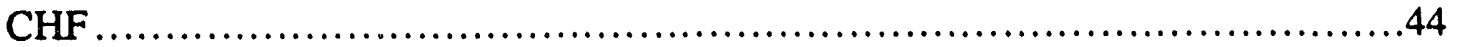

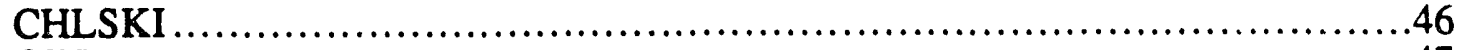

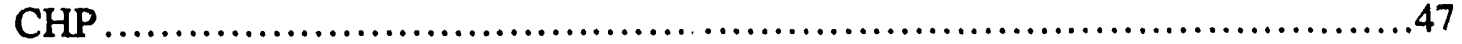

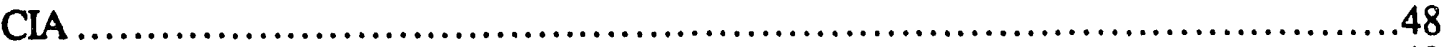

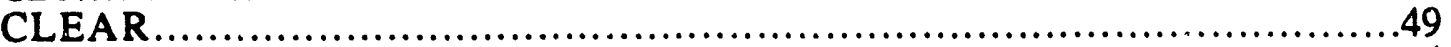

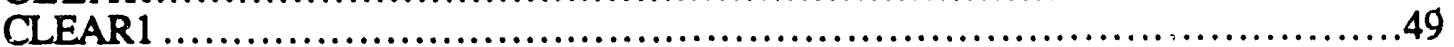

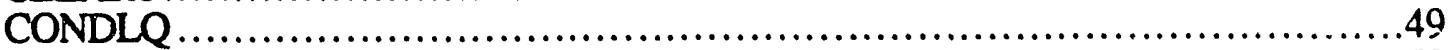

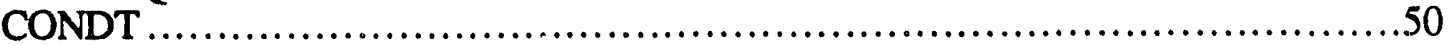

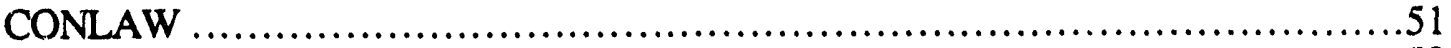

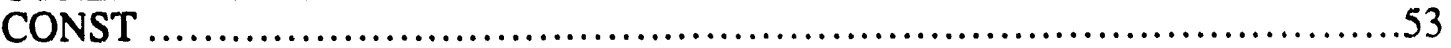

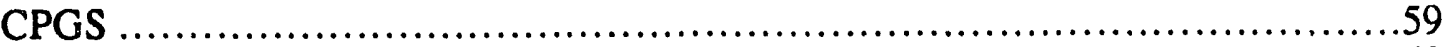

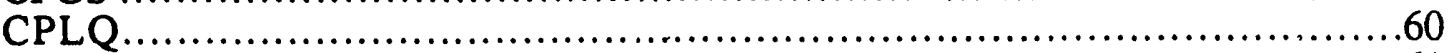

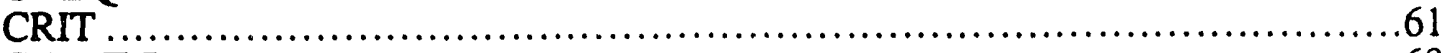

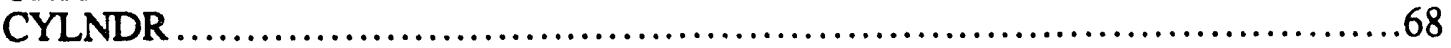

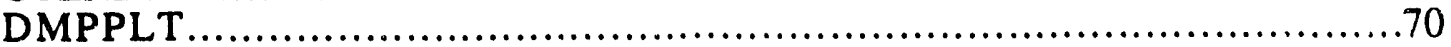

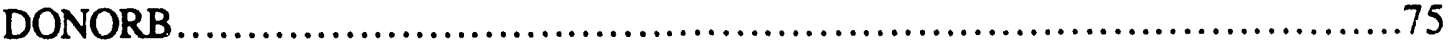

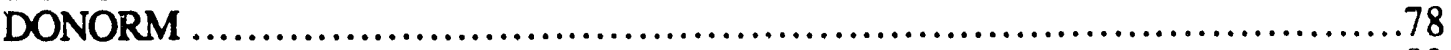

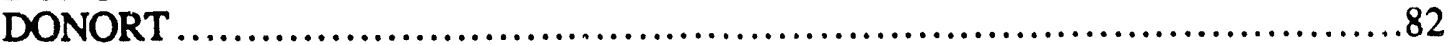

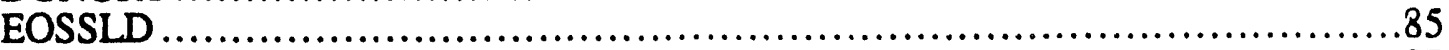

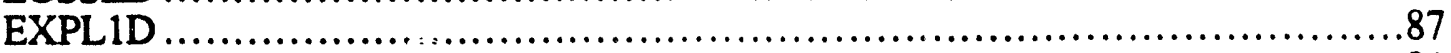

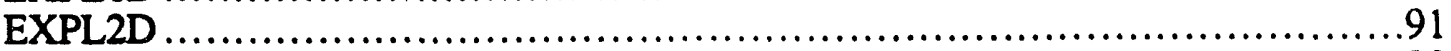

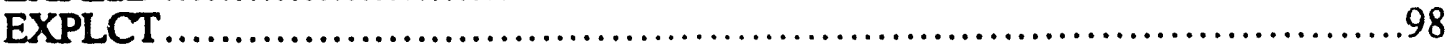

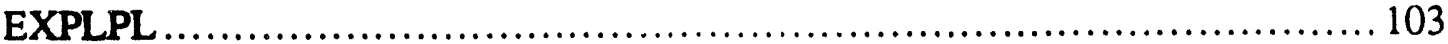

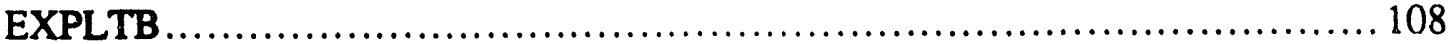

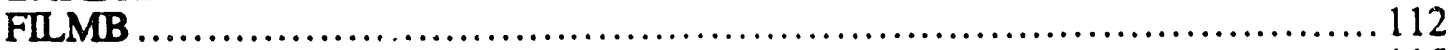

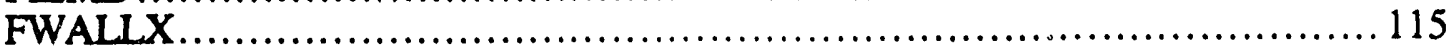

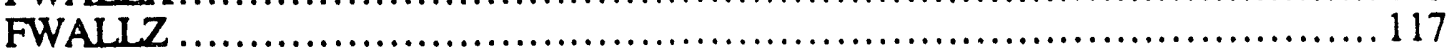

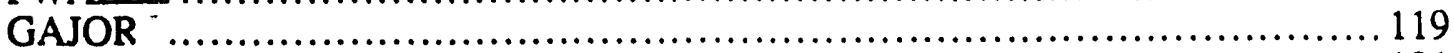

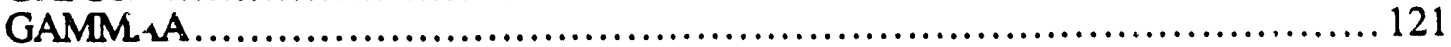

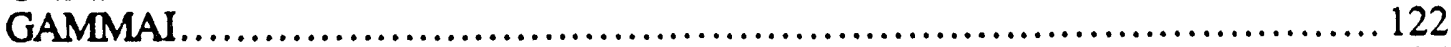

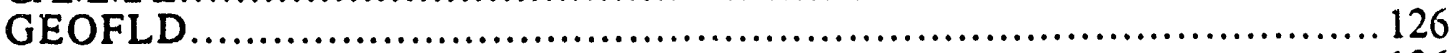

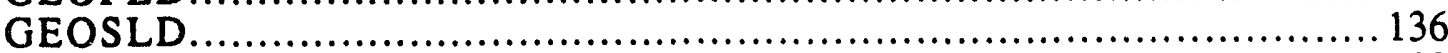

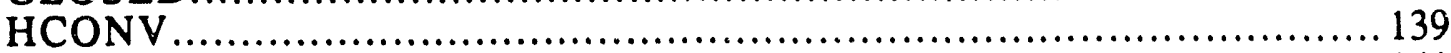

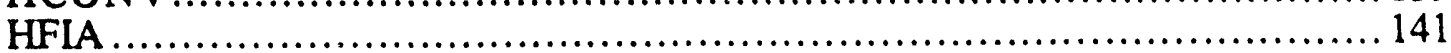




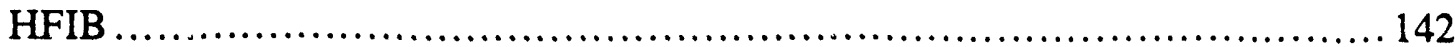

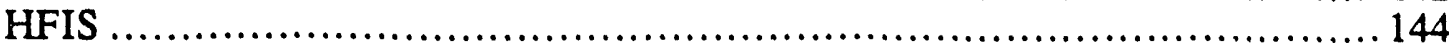

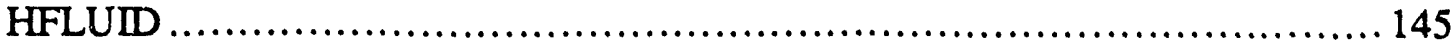

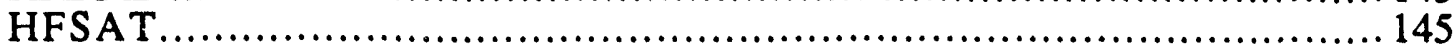

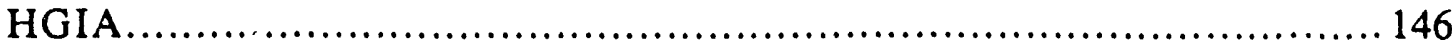

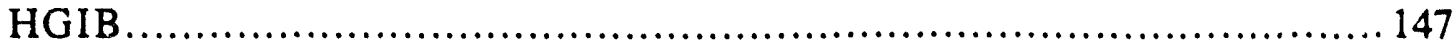

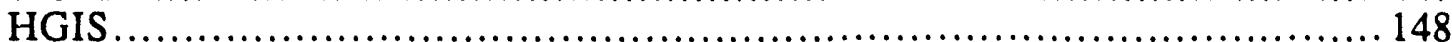

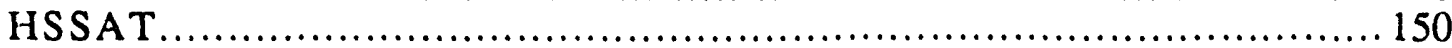

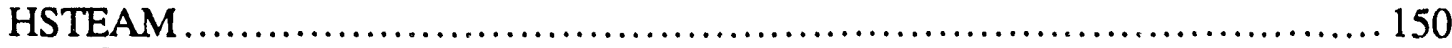

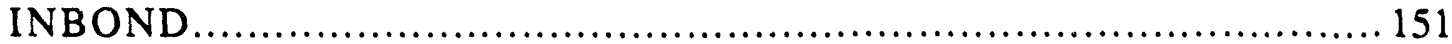

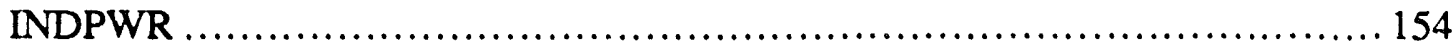

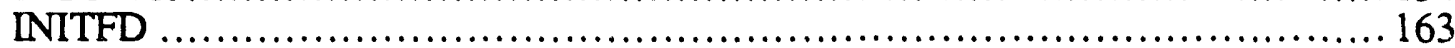

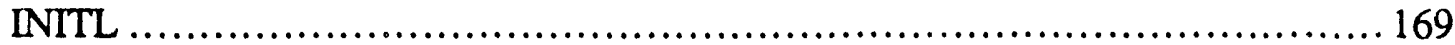

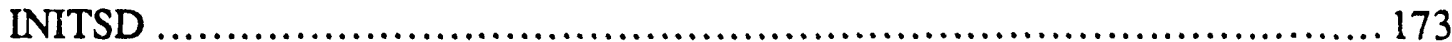

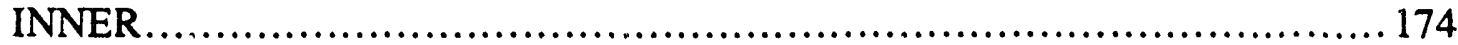

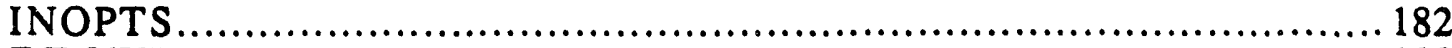

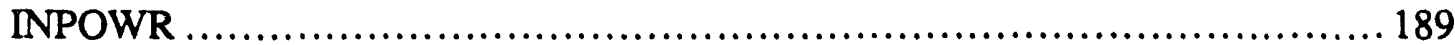

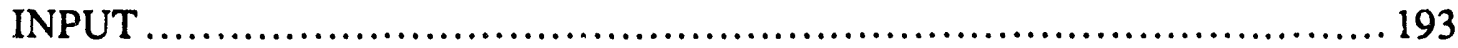

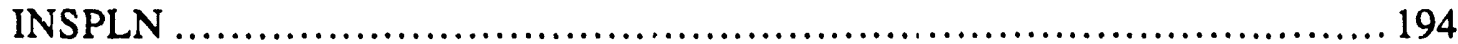

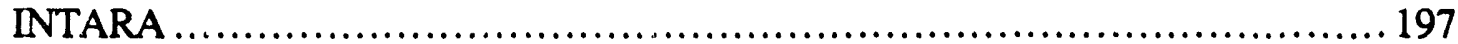

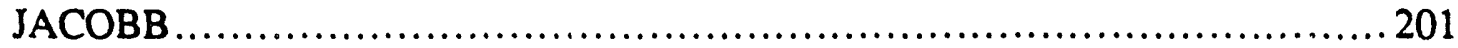

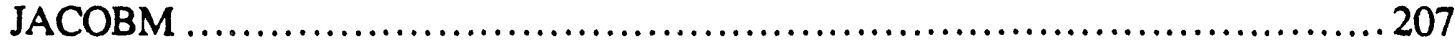

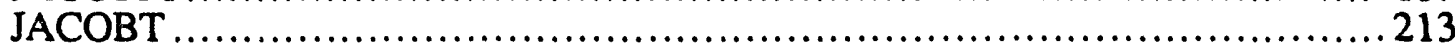

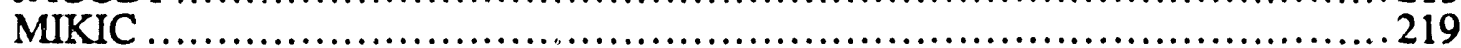

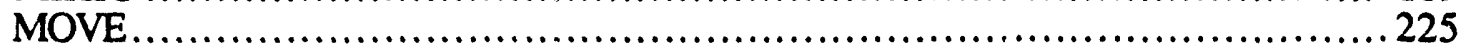

NEWTON .......................................................................... 225

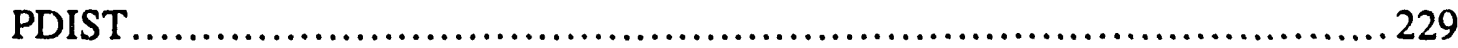

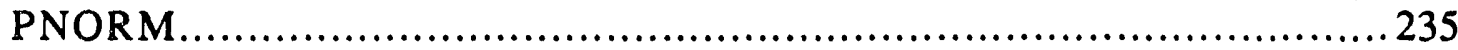

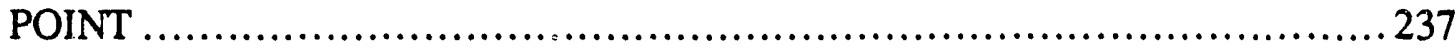

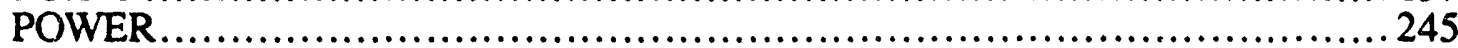

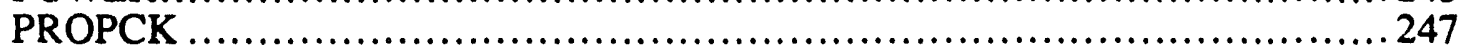

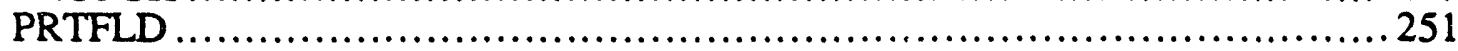

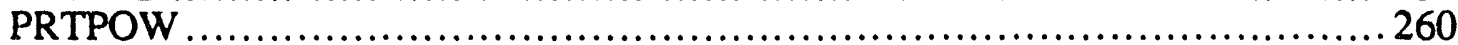

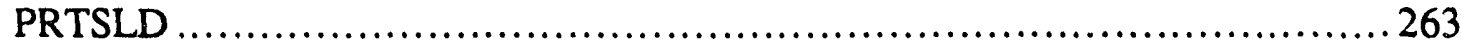

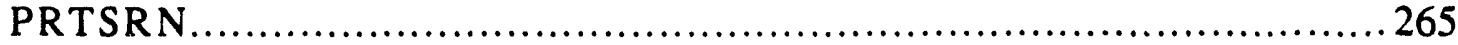

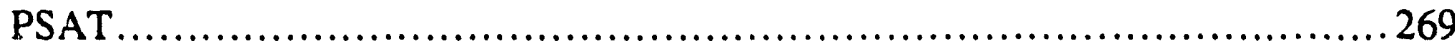

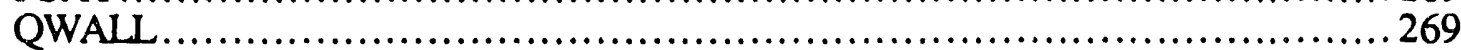

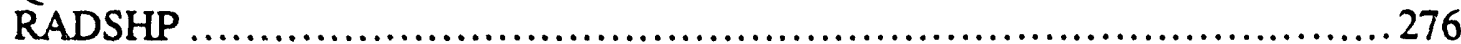

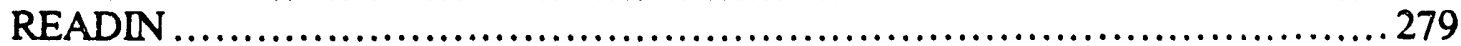

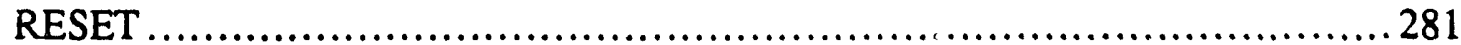

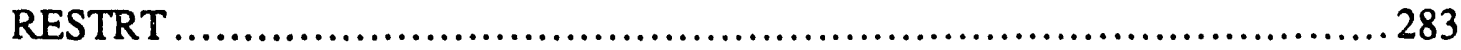

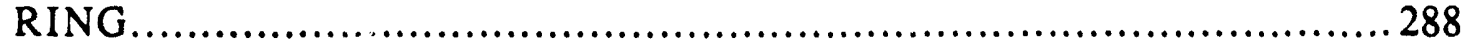

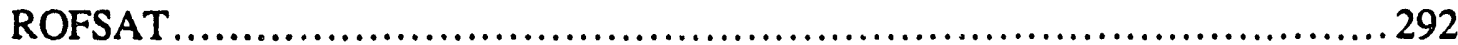

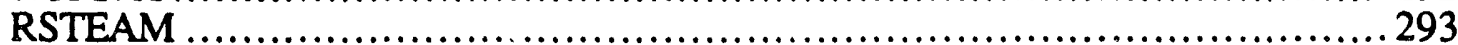

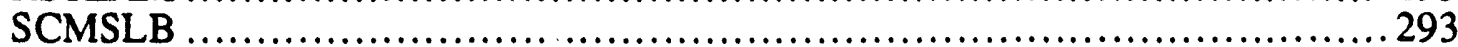

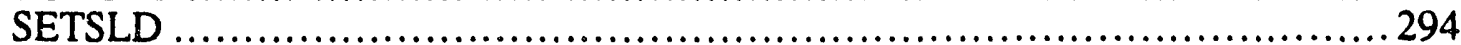

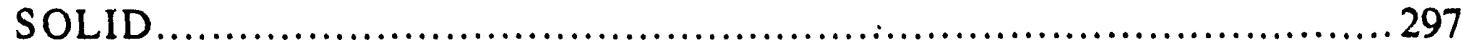

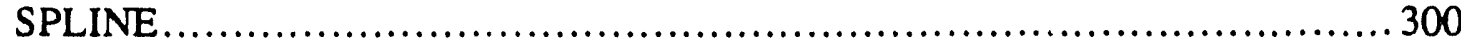

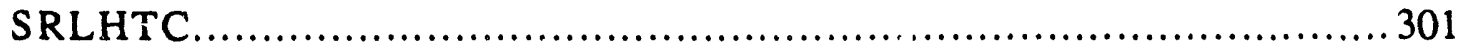

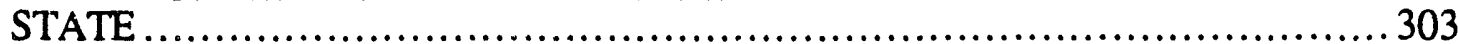

STORE

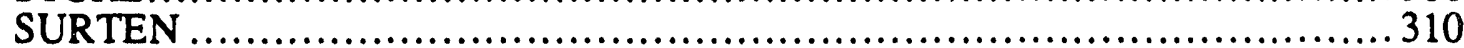

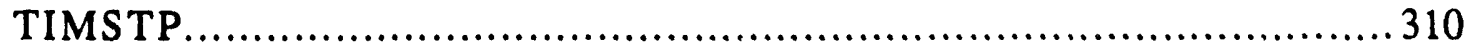

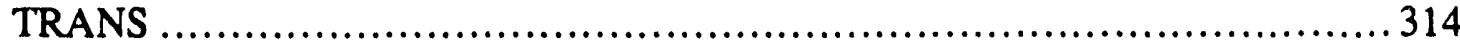




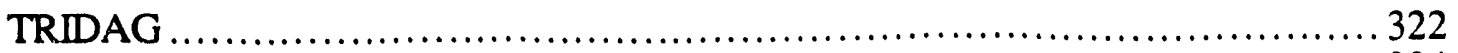

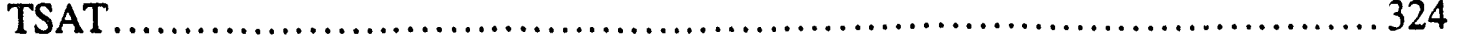

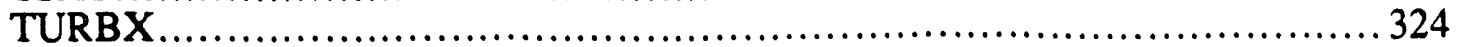

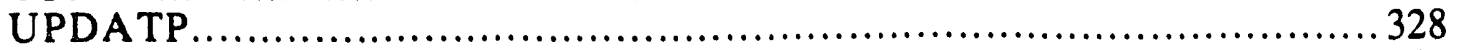

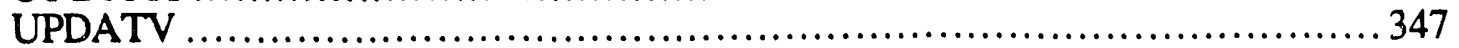

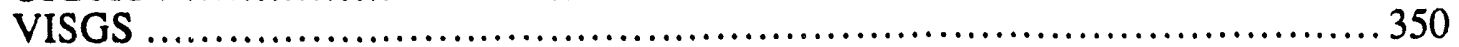

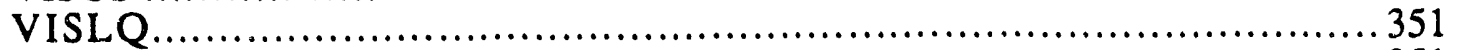

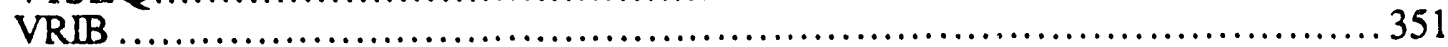

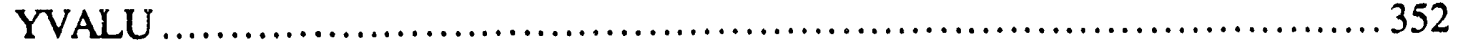

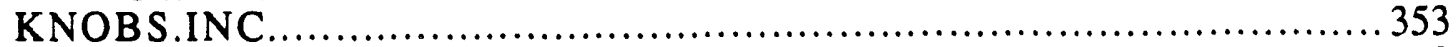

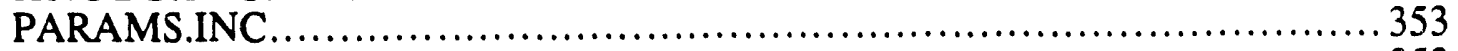

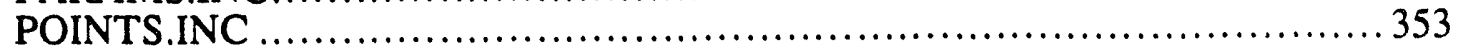


This page intentionally left blank 


\section{Code Architecture}

Figure 1 illustrates the architecture of FLOWTRAN-TF v1.2. 


\begin{tabular}{|l|ll|r|}
\hline Pg. 2 of 354 & WSRC-TR-93-086 Rev. 0 FLOWTRAN-TF v1.2 Source Listing & 2/15/93 \\
\hline
\end{tabular}

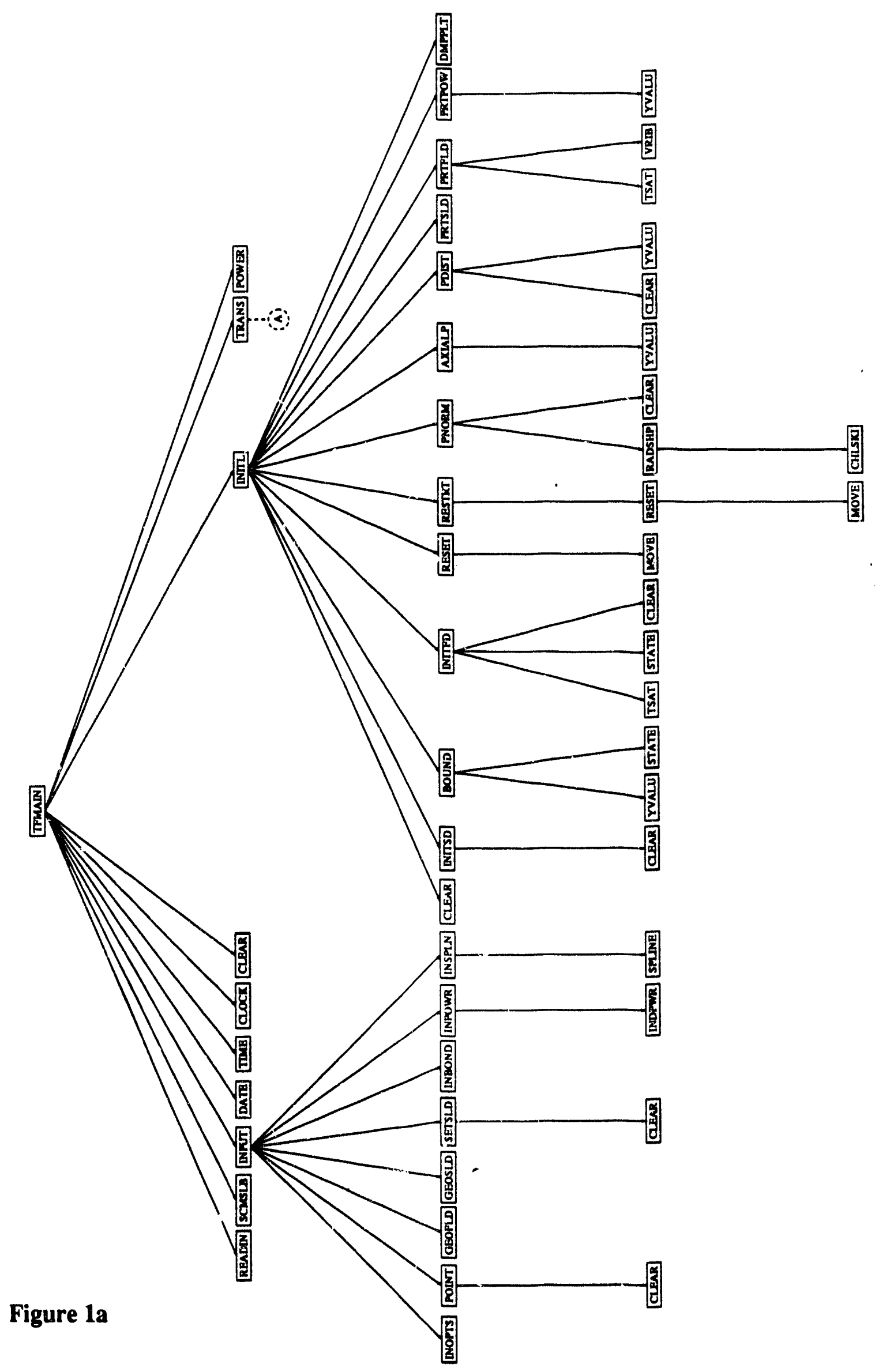




\begin{tabular}{|l|l|l|l|}
\hline $2 / 15 / 93$ & WSRC-TR-93-086 Rev.0 & FLOWTRAN-TF v1.2 Source Listing & Pg. 3 of 354 \\
\hline
\end{tabular}

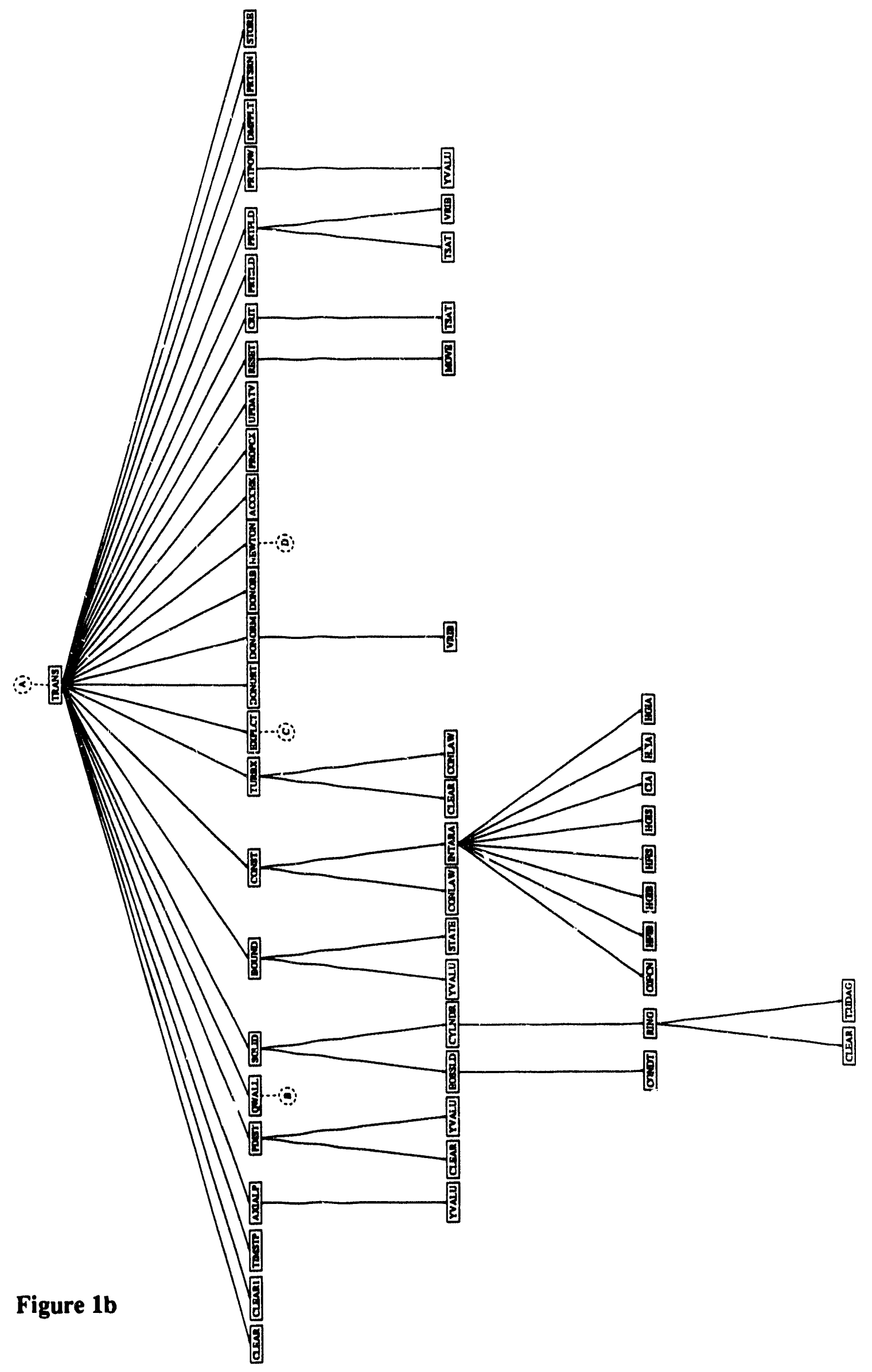




\begin{tabular}{|l|ll|r|}
\hline Pg. 4 of 354 & WSRC-TR-93.086 Rev. 0 & FLOWTRAN-TF v1.2 Source Listing & $2 / 15 / 93$ \\
\hline
\end{tabular}

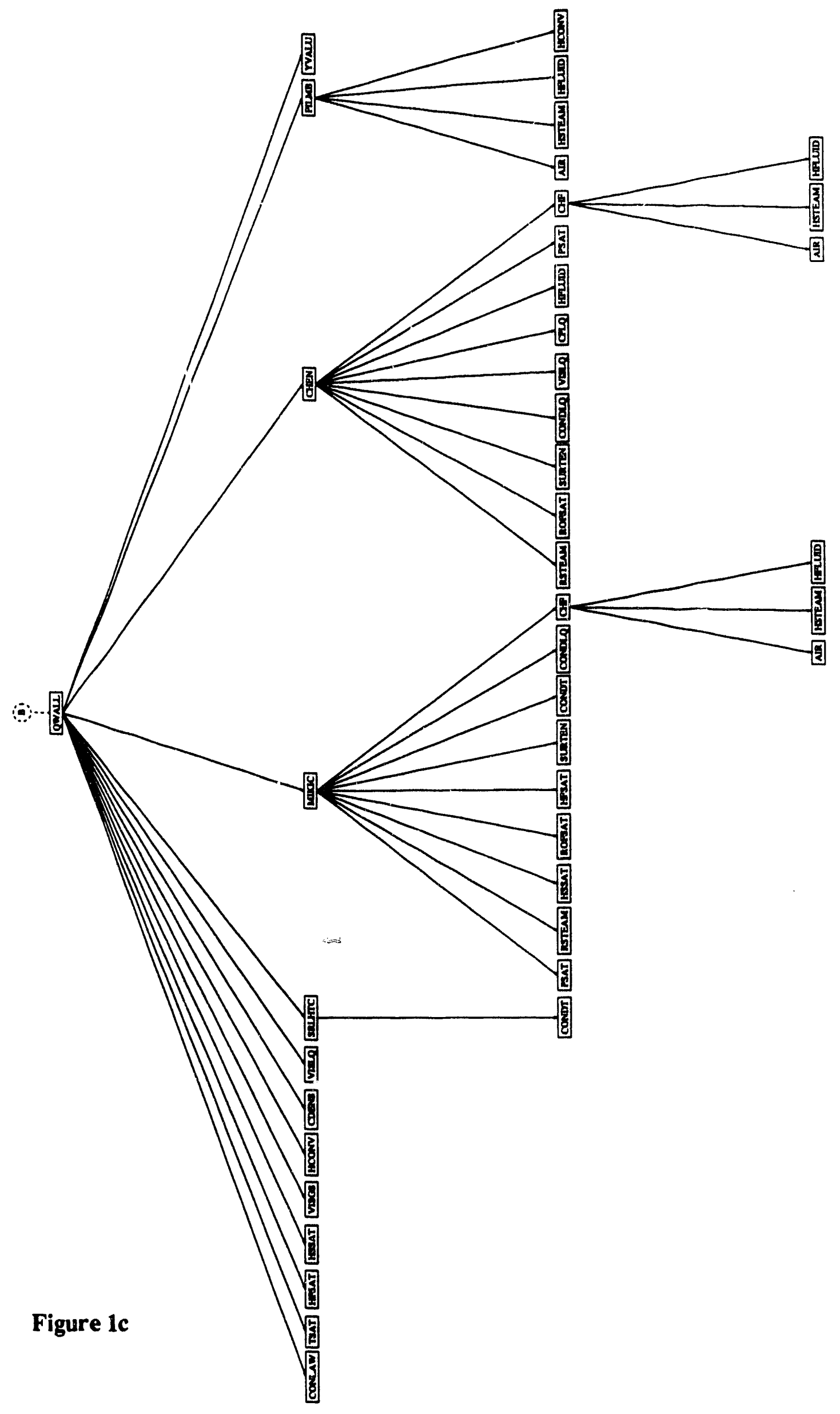




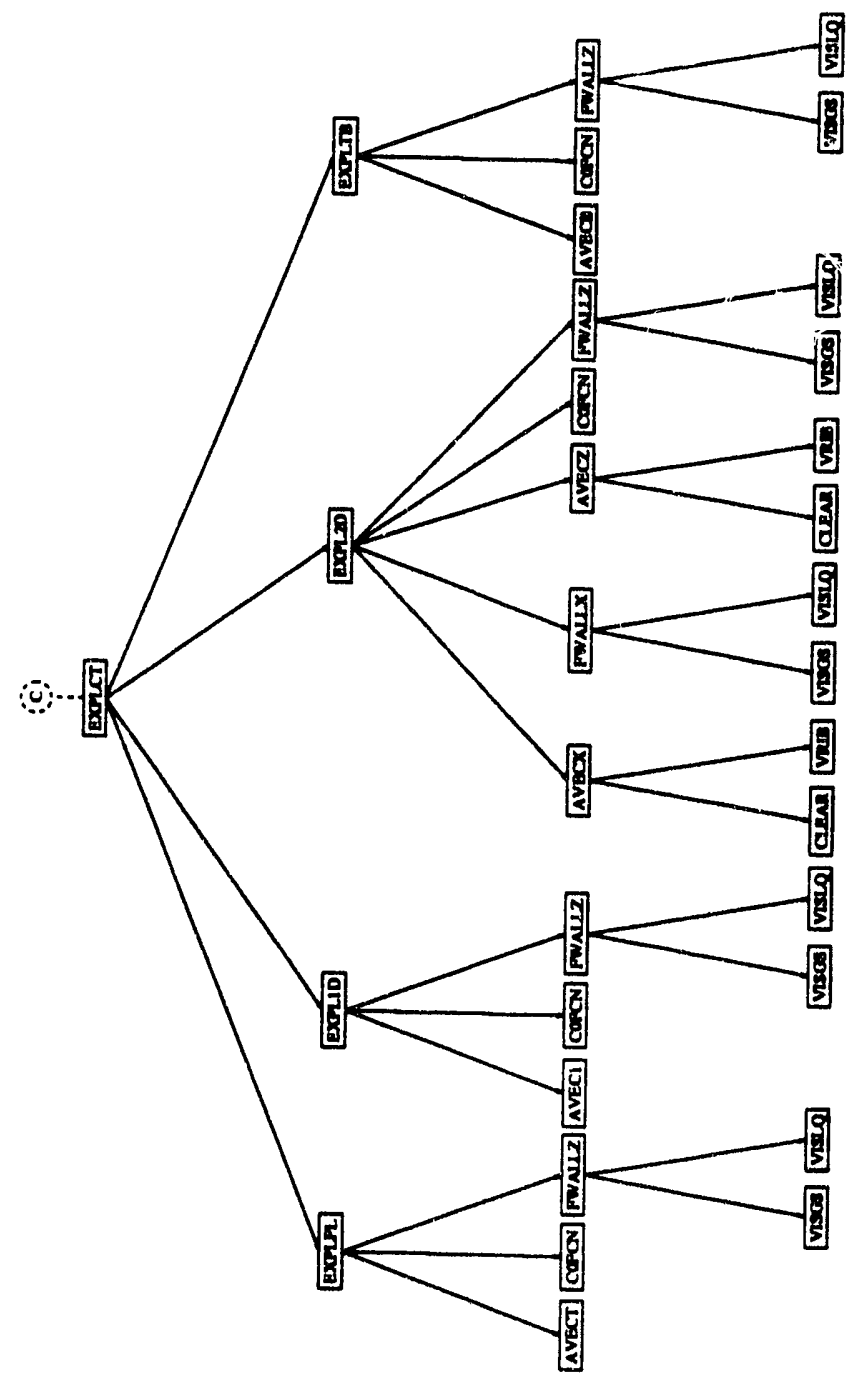

Figure 1d 
\begin{tabular}{|l|lll|r|}
\hline Pg. 6 of 354 & WSRC-TR-93-086 Rev. 0 & FLOWTRAN-TF v1.2 Source Listing & 2/15/93 \\
\hline
\end{tabular}

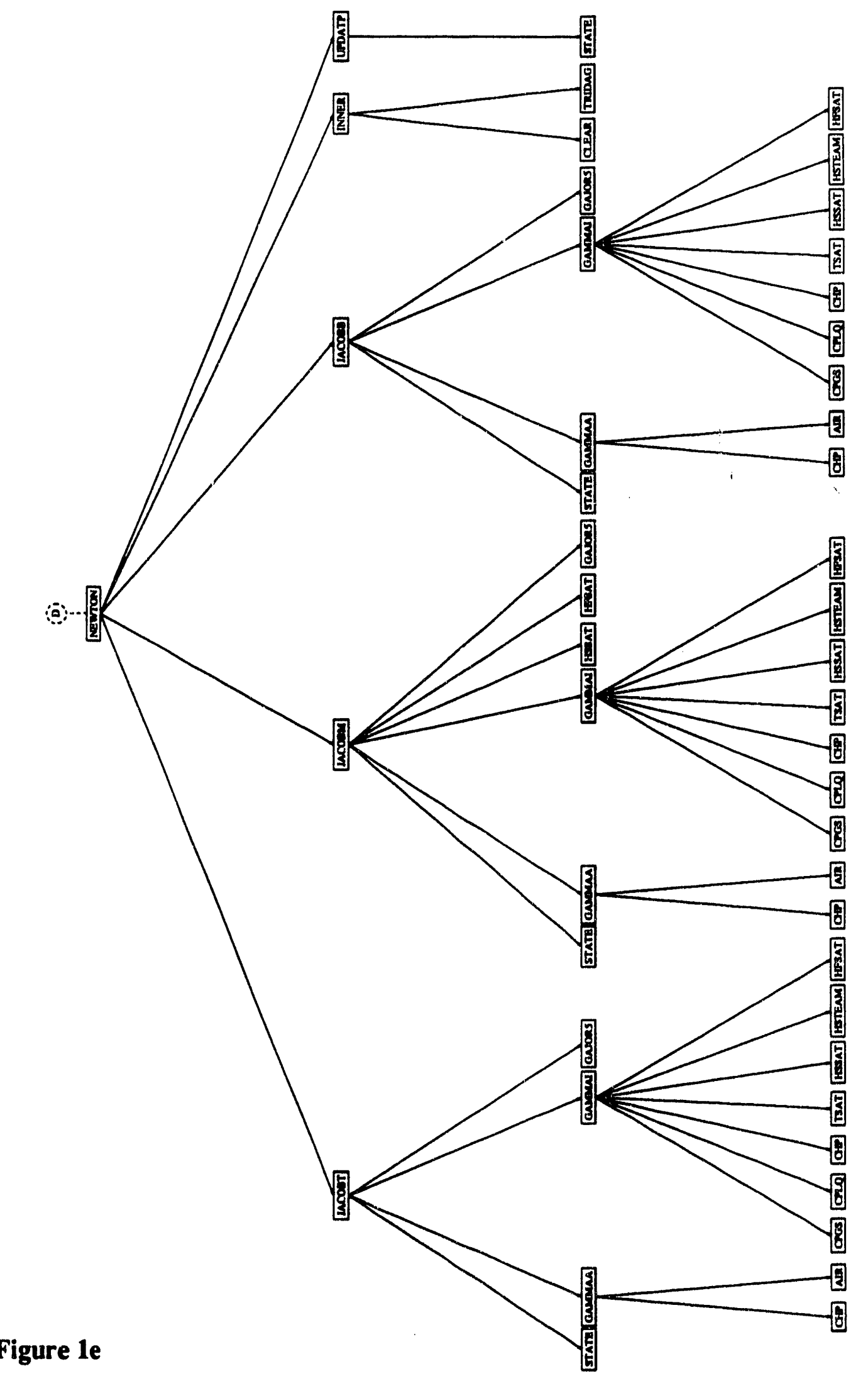




\section{Source Code Listing}

The FORTRAN coding for FLOWTRAN-TF v1.2 is listed below. The listing starts with the main program followed by subroutines in alphabetical order. Three INCLUDE files complete the listing.

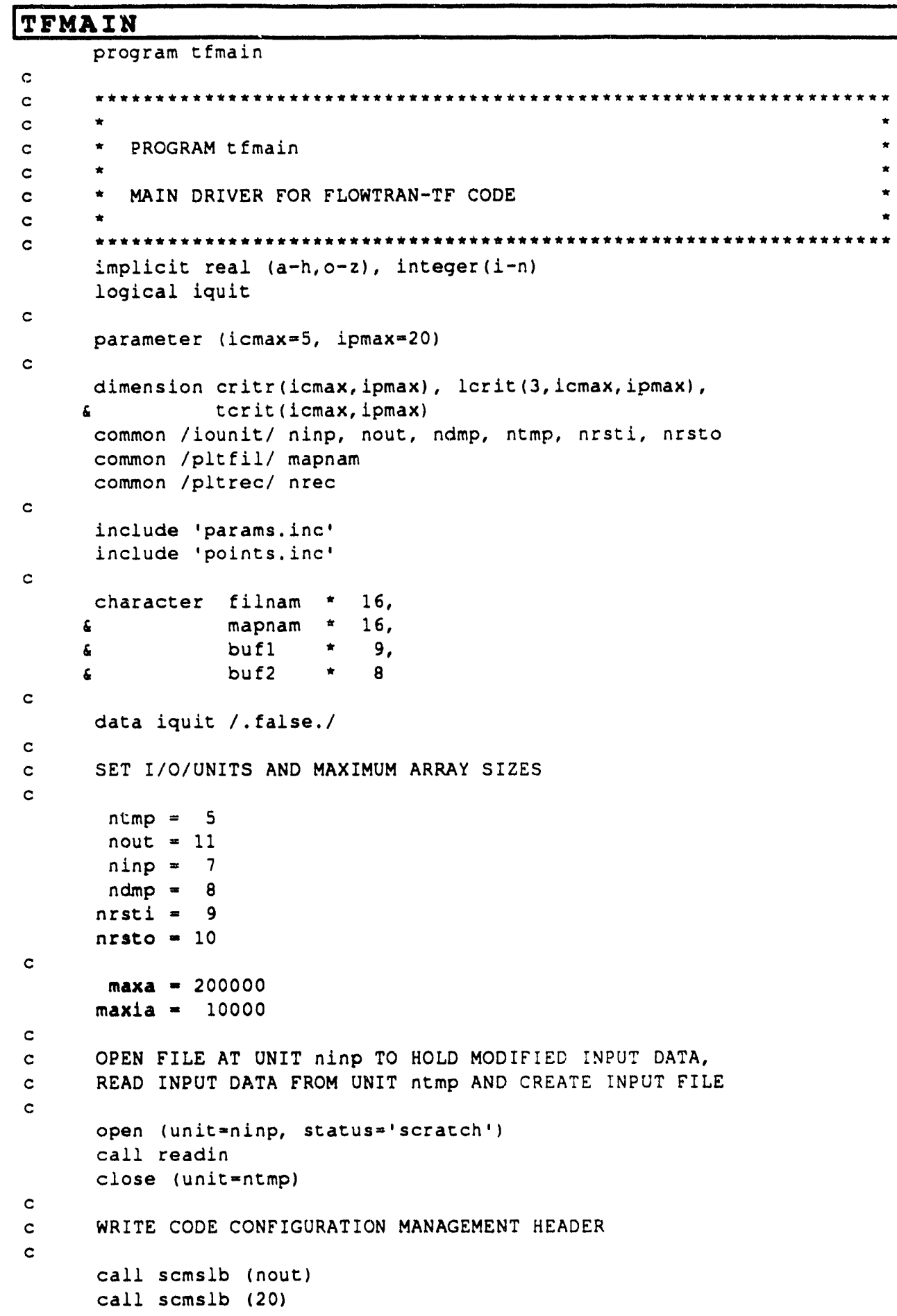




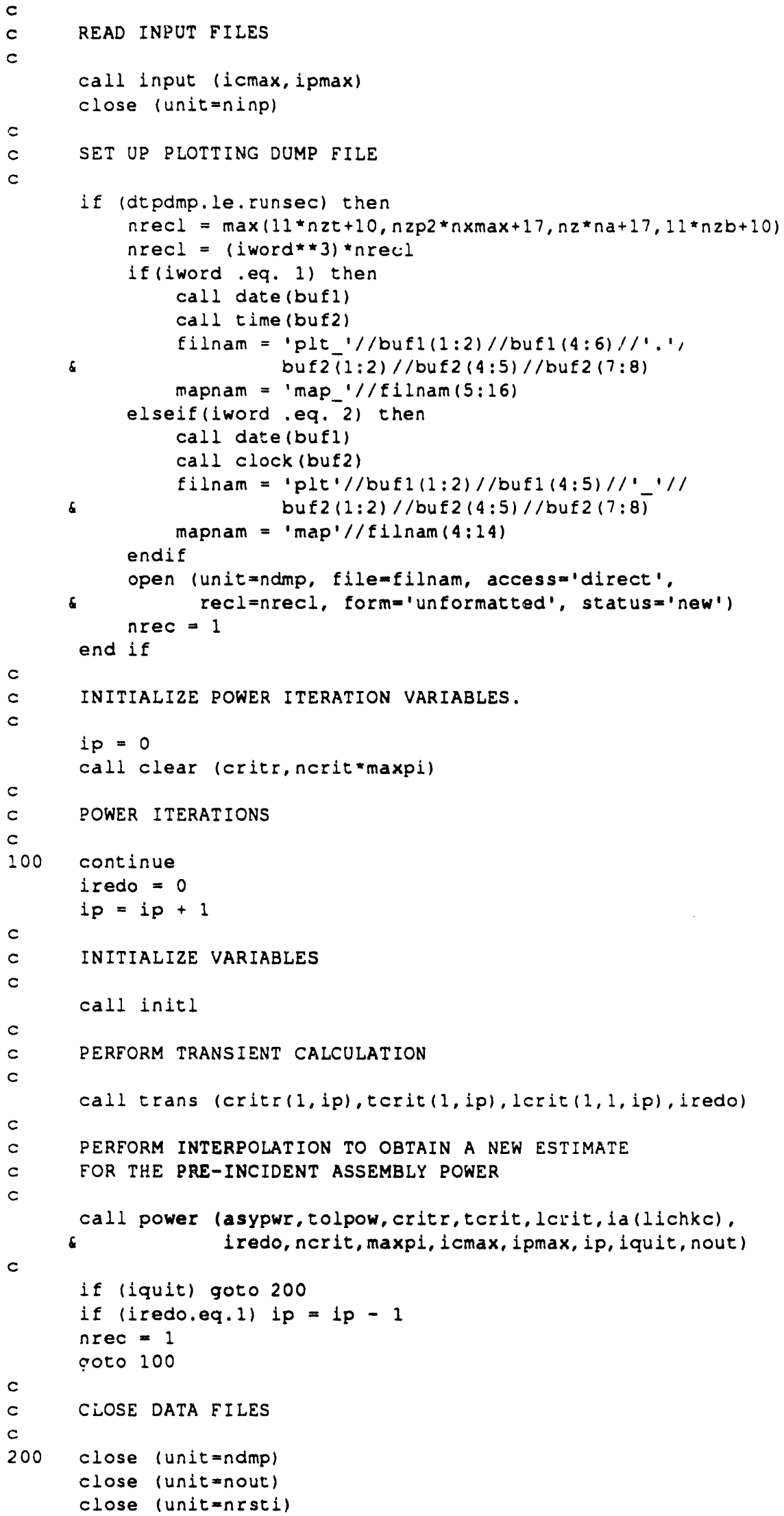



stop end

\section{ACCCHR}

subroutine accchk (pt, alpt, tgt, tft, xat, rogt, roft, hgt, hft,

\& $p$,alp , tg, tf , xa , rog, rof, hg , hf,

\& $\quad p b, a l p b, t g b, t f b, x a b, r o g b, r o f b, h g b, h f b$.

* ptn, alptn, tgtn, tftn, xatn, rogtn, roftn, hgtn, hftn,

* pn, alpn, tgn, ten, xan, rogn, rofn, hgn, hen.

- pbn, alpbn, tgbn, tfbn, xabn, rogbn, rofbn, hgbn, hfbn.

* volt, volc, volb,tsurf,tsrfn,

nsurf, na, nz, nzt, nzp1.nzp2, nxmax, nchn, nx, nzo,

ierr, istdy, tol, tolss, nstdy, delt, tsec, dif, difp, difalp, diftg, diftf, difxa, difts, delhm, steady)

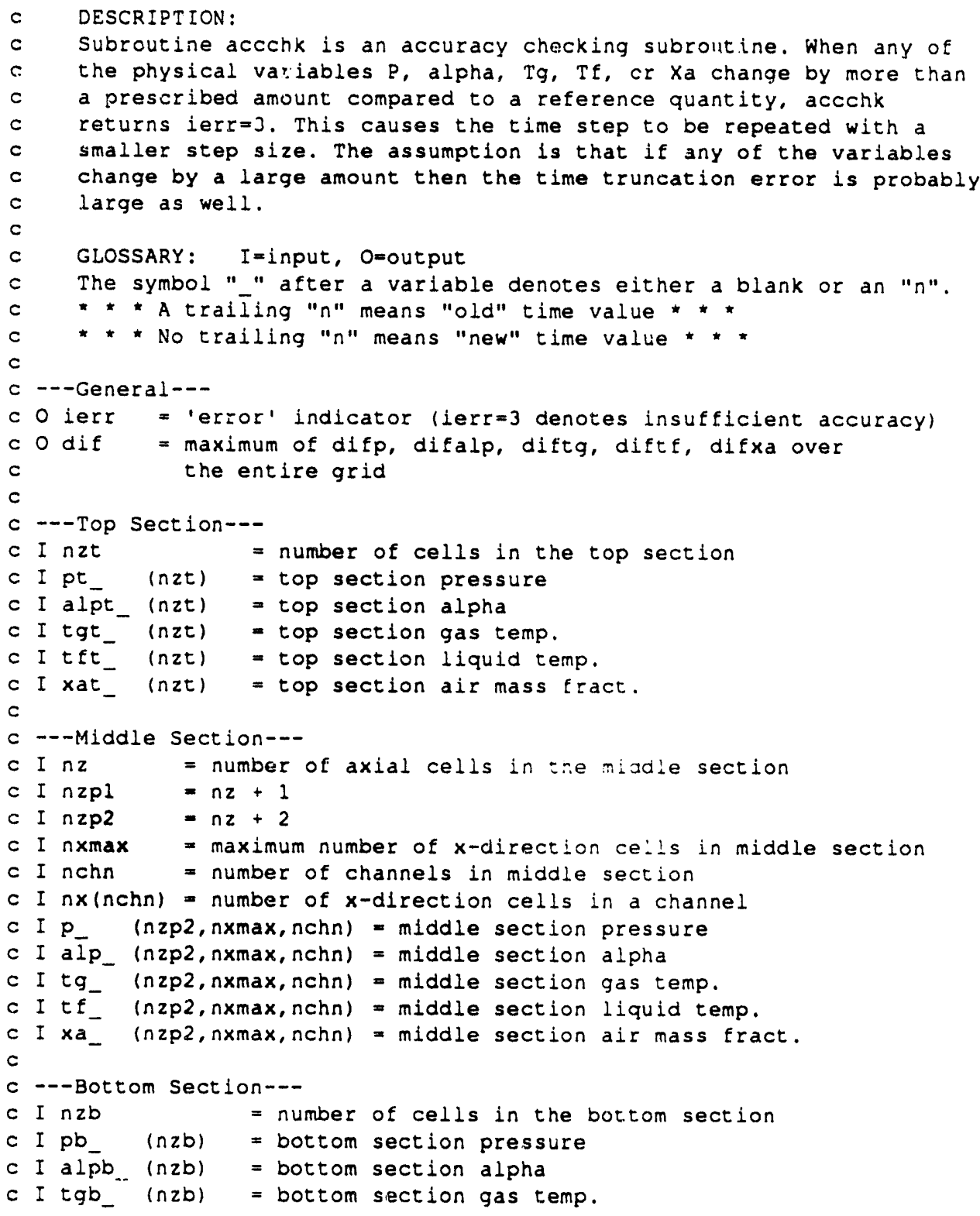




\begin{tabular}{|l|ll|r|}
\hline Pg. 10 of 354 & WSRC-TR-93-086 Rev. 0 & FLOWTRAN-TF v1.2 Source Code & $2 / 15 / 93$ \\
\hline
\end{tabular}

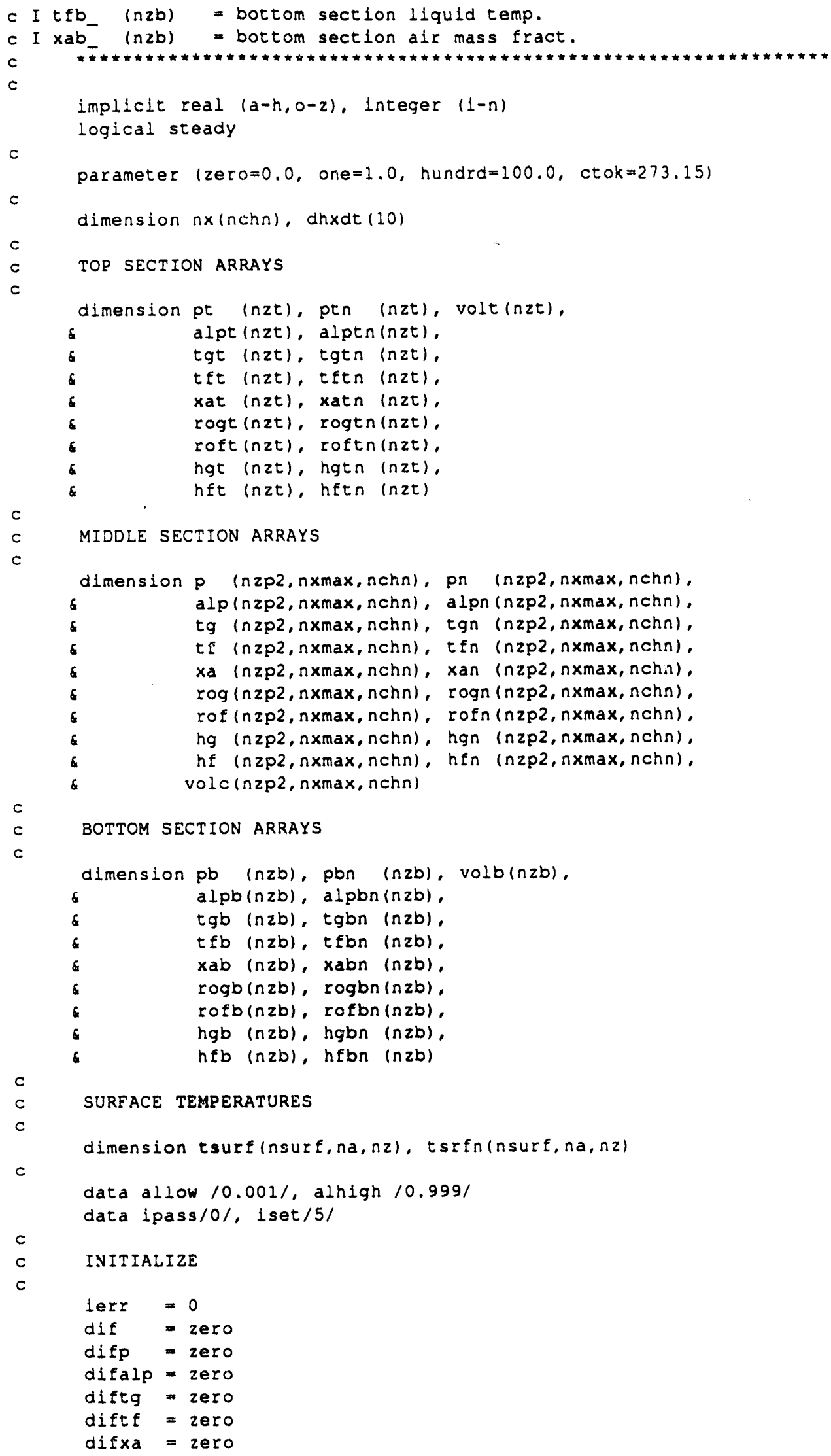



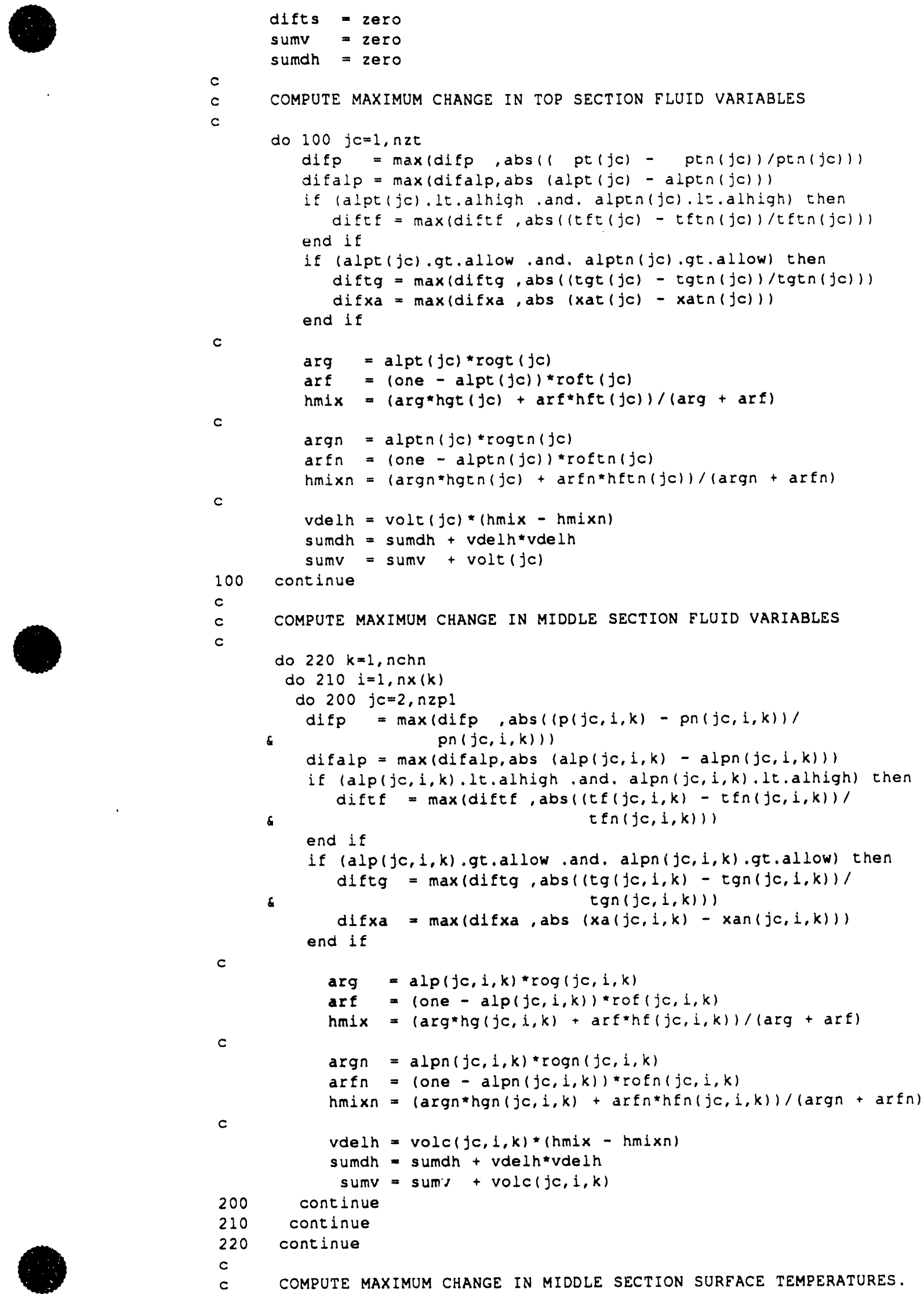


\begin{tabular}{|l|ll|r|}
\hline Pg. 12 of 354 & WSRC-TR-93-086 Rev. 0 & FLOWTRAN-TF v1.2 Source Code & $2 / 15 / 93$ \\
\hline
\end{tabular}

c

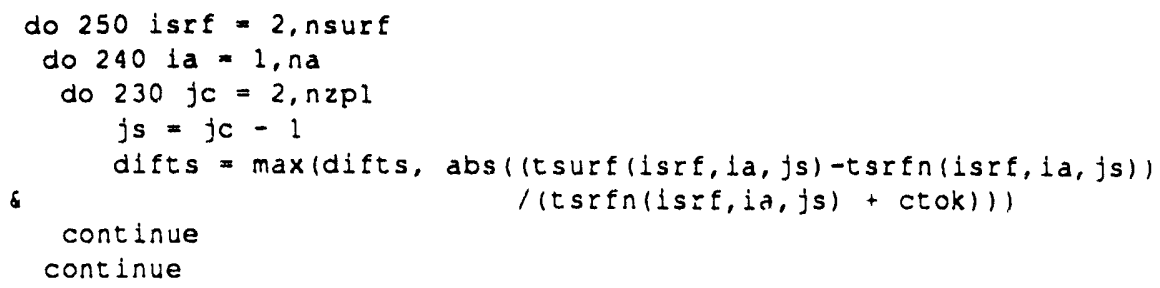



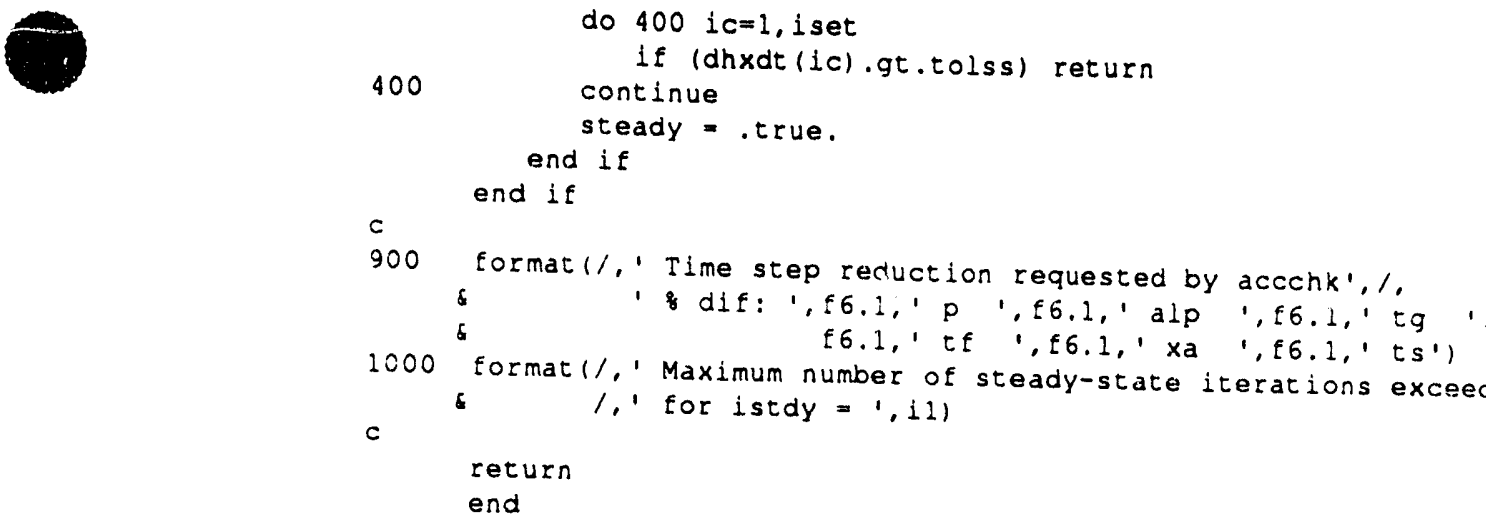

\section{AIR}

subroutine air (p,t, roa, droadp, droadt, ha, dhadp, dhadt)

INPUT:

$P=$ PARTIAL PRESSURE OF AIR, Pa

$t$ - MIXTURE GAS TEMPERATURE, $K$

\section{AVEC 1}

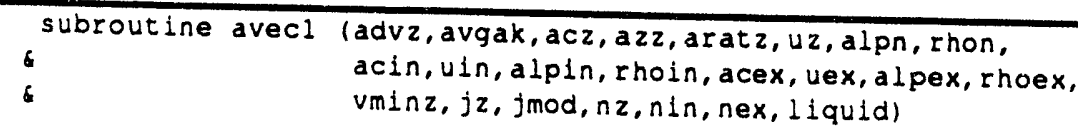




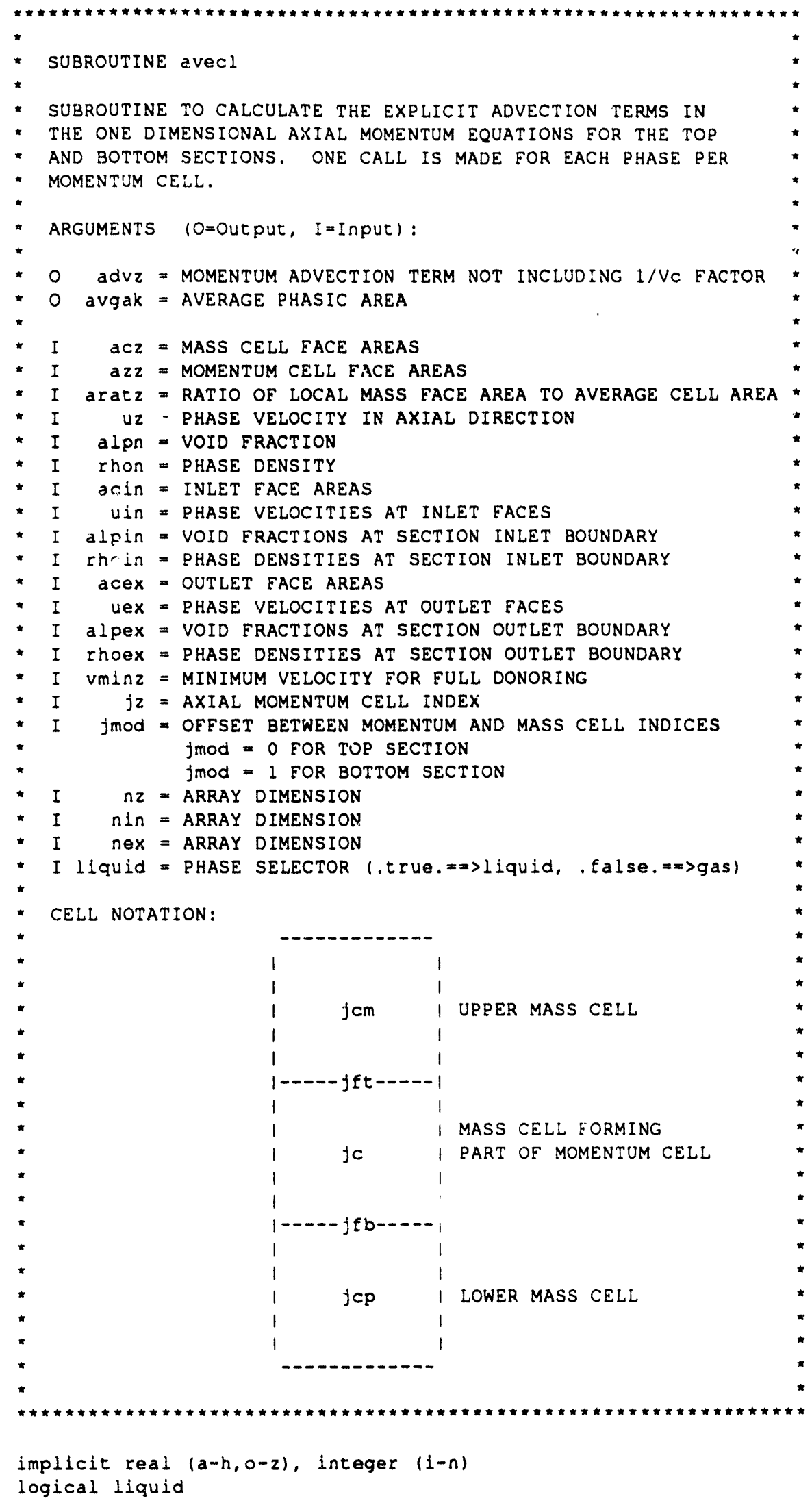


$c$

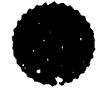

$c$

dimension $\operatorname{acz}(n z), u z(n z), \operatorname{arat} z(n z)$,

dimension $\operatorname{achn}(n i n), u \ln (n i n), \operatorname{acex}(\operatorname{nex})$, uex(nex),

\& alpin(nin), thoin(nin), alpex(nex), rhoex(nex)

C

dimension aavg:21

parameter $($ zero $=0.0$, hal $f=0.5$, one $=1.0, t w o=2.0)$

data epsalp/1.0e-4/

$\mathrm{c}$

INITIALIZATIONS

aavg $(1)=$ zero

$\operatorname{aavg}(2)=$ zero

$\operatorname{adv} z=z e r o$

signt $=$-one

$c$

LOOP OVER TWO AXIAL MASS CELLS FORMING THE MOMENTUM CELL

do 100 mcell $=1,2$

signt $=(-$ one $) *$ signt

SET LOCAL CELL POINTERS:

$j z$ = MOMENTUM CELL INDEX

$j C=$ MASS CELL INDEX

$j \mathrm{Cm}=$ MASS CELL ABOVE jC

$j C P=$ MASS CELL BELOW $j C$

fft = INDEX FOR TOP FACE OF MASS CELL fC

$j f b=$ INDEX FOR BOTTOM FACE OF MASS CELL jC

$j c=j z+j \bmod +\operatorname{mcell}-2$

$j \mathrm{~cm}=j c-1$

$j c p=j c+1$

$j f t=j c-j \bmod$

$j f b=j f t+1$

$a l p c=a l p n(j c)$

rhoc $=$ rhon $(j c)$

c

c

CALCULATE VOLUME AVERAGE VELOCITY FOR MOMENTUM CELL

$u j z=u z(j z)$

uavg $=u f z * \operatorname{aratz}(j z)$

$\mathrm{c}$

C-..- FOR TOP FACE: CALCULATE AREA (ACZ), PHASIC AREA (ACz*alpk), -..-

C (ACz*alpk*rhok), MASS FLOWRATE (ACz*alpk*rhok"uk) \&

c MOMENTUM $(A C z * a l p k * r h o k * u k * u k)$

1f (jc .eq. 1) then

SINGLE (TOP Section) OR MULTIPLE (Bottom Section) INLET(S)

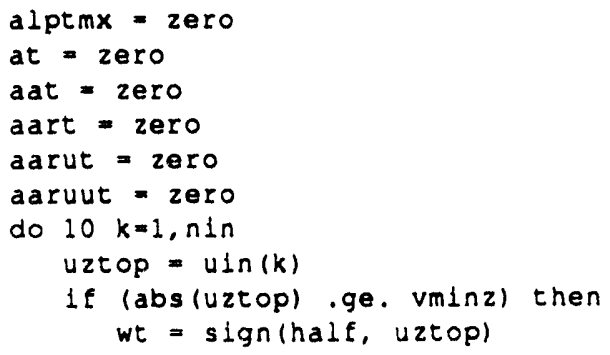




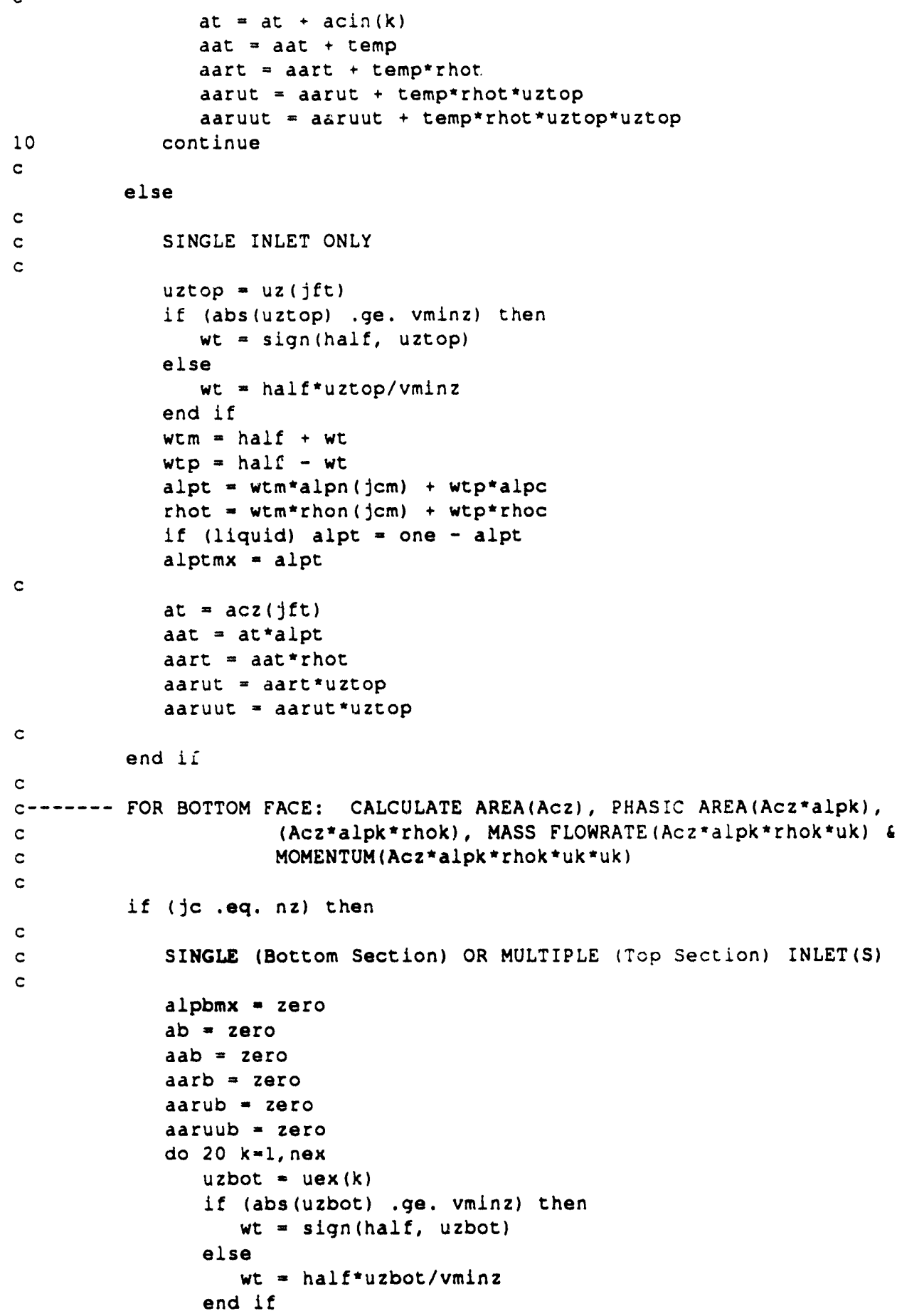


c

20

c

$c$

c

c

c

$c$

$c$

c

c

c

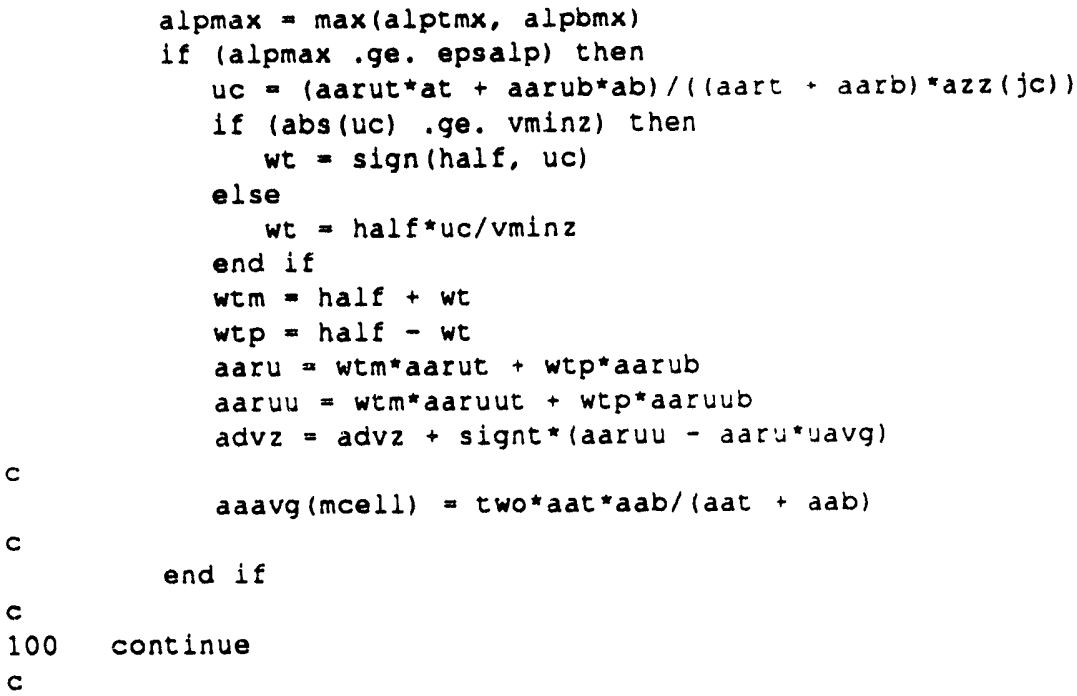

$w t m=h a l f+w t$ 


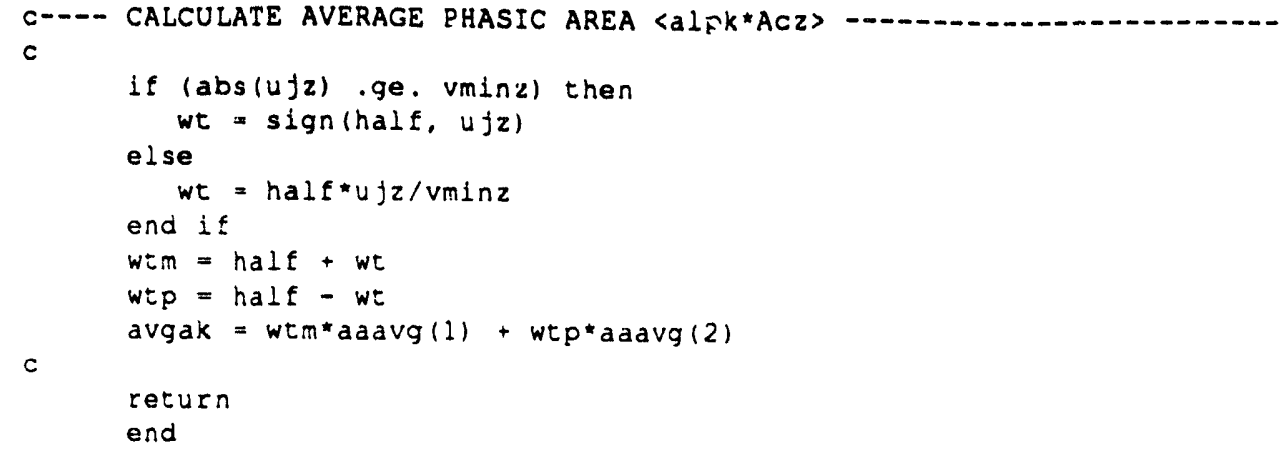




\begin{tabular}{|l|lll|l|}
\hline $2 / 15 / 93$ & WSRC-TR-93-086 Rev. 0 & FLOWTRAN-TF v1.2 Source Code & Pg. 19 of 354 \\
\hline
\end{tabular}

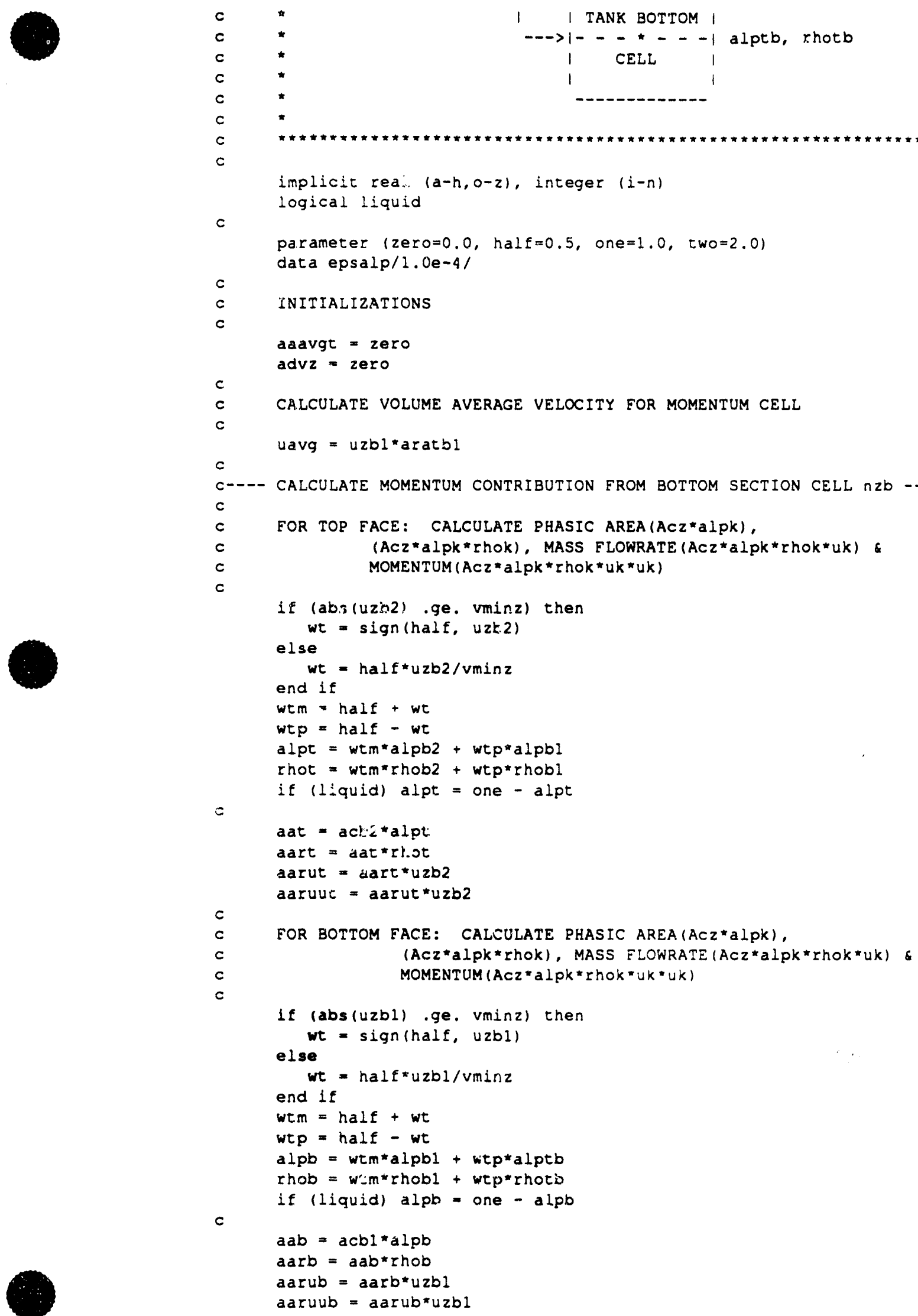

$\mathrm{c}$

if $(a b, s(u z \$ 2)$.ge. vminz) then

$w t=\operatorname{sign}($ half, uzk.2) 


\begin{tabular}{|l|ll|r|}
\hline Pg. 20 of 354 & WSRC-TR-93-086 Rev. 0 & FLOWTRAN-TF v1.2 Source Code & $2 / 15 / 93$ \\
\hline
\end{tabular}

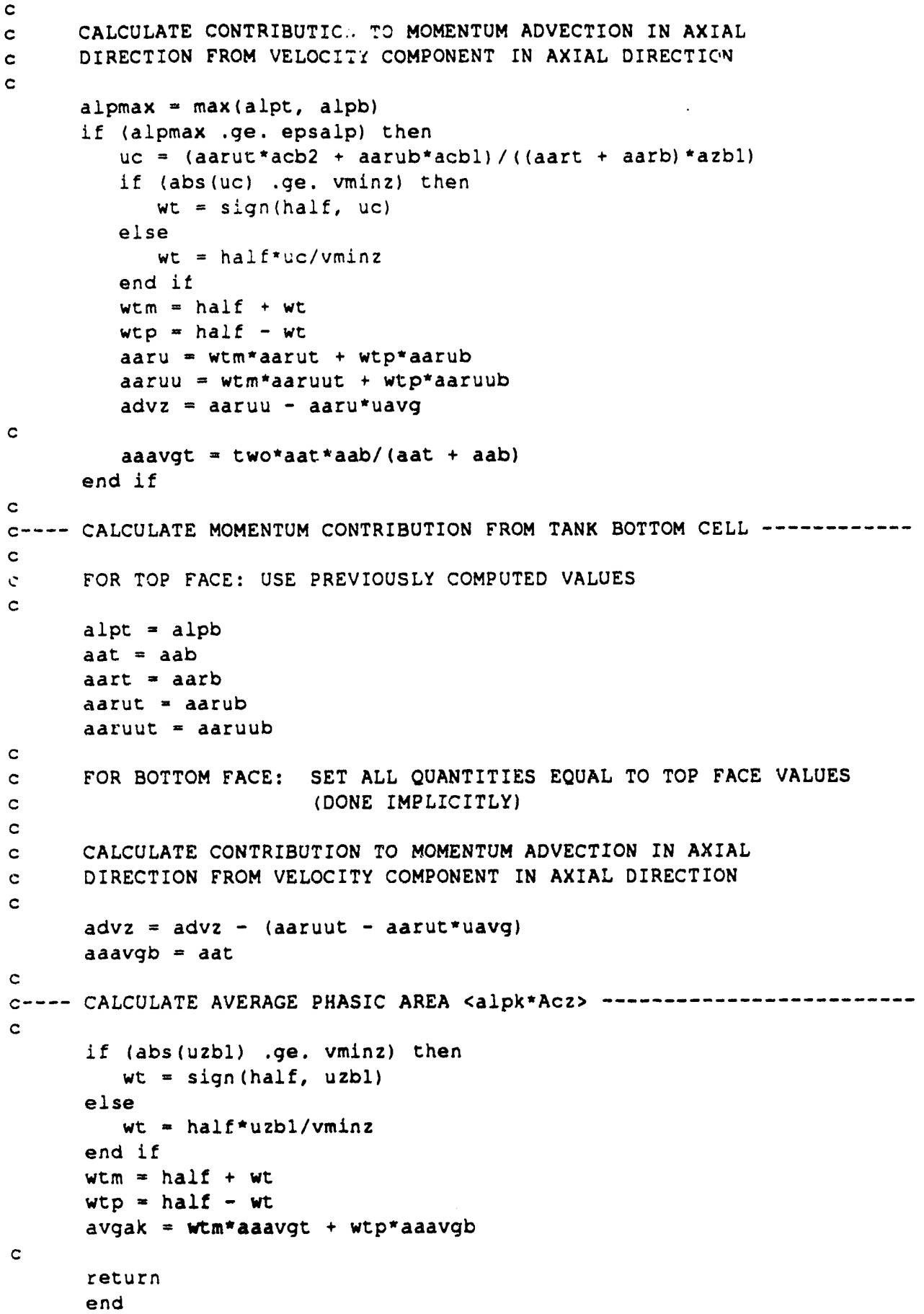

\section{AVECT}

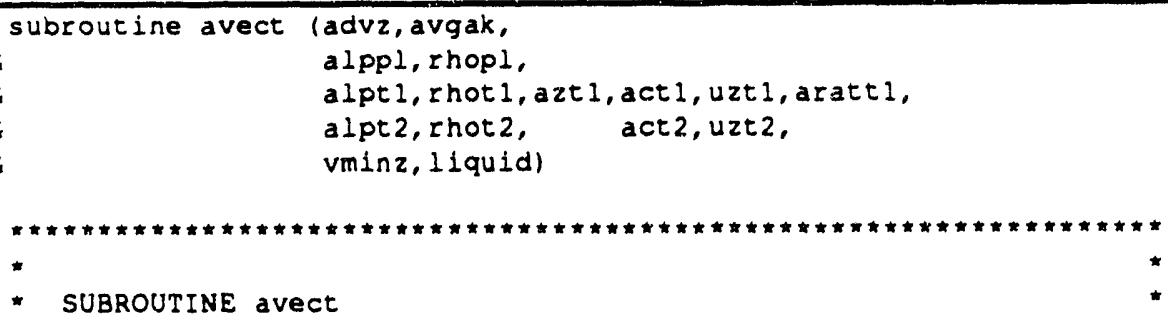




\begin{tabular}{|l|ll|l|}
\hline $2 / 15 / 93$ & WSRC-TR-93-086 Rev. 0 & FLOWTRAN-TF v1.2 Source Code & Pg. 21 of 354 \\
\hline
\end{tabular}

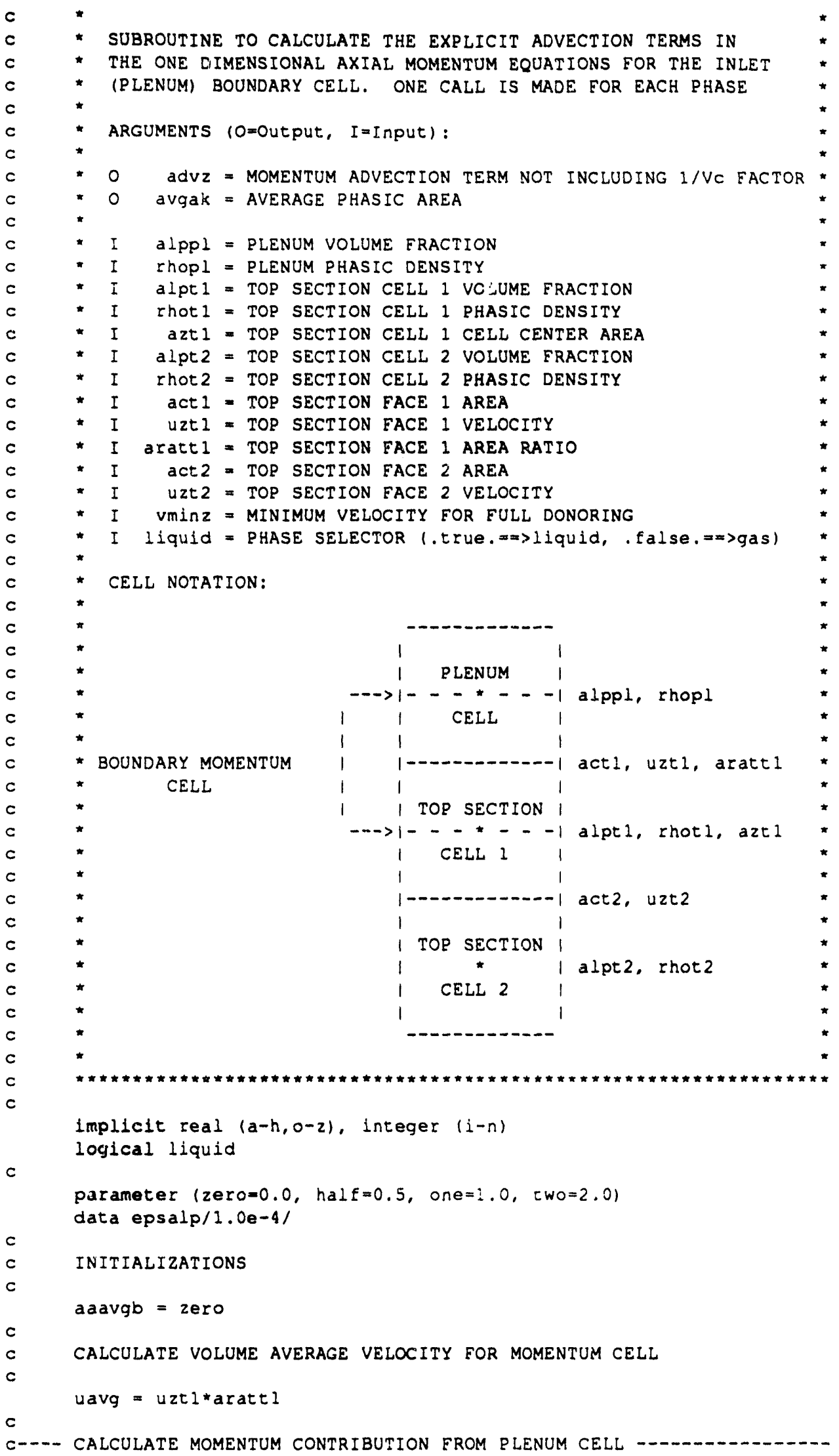




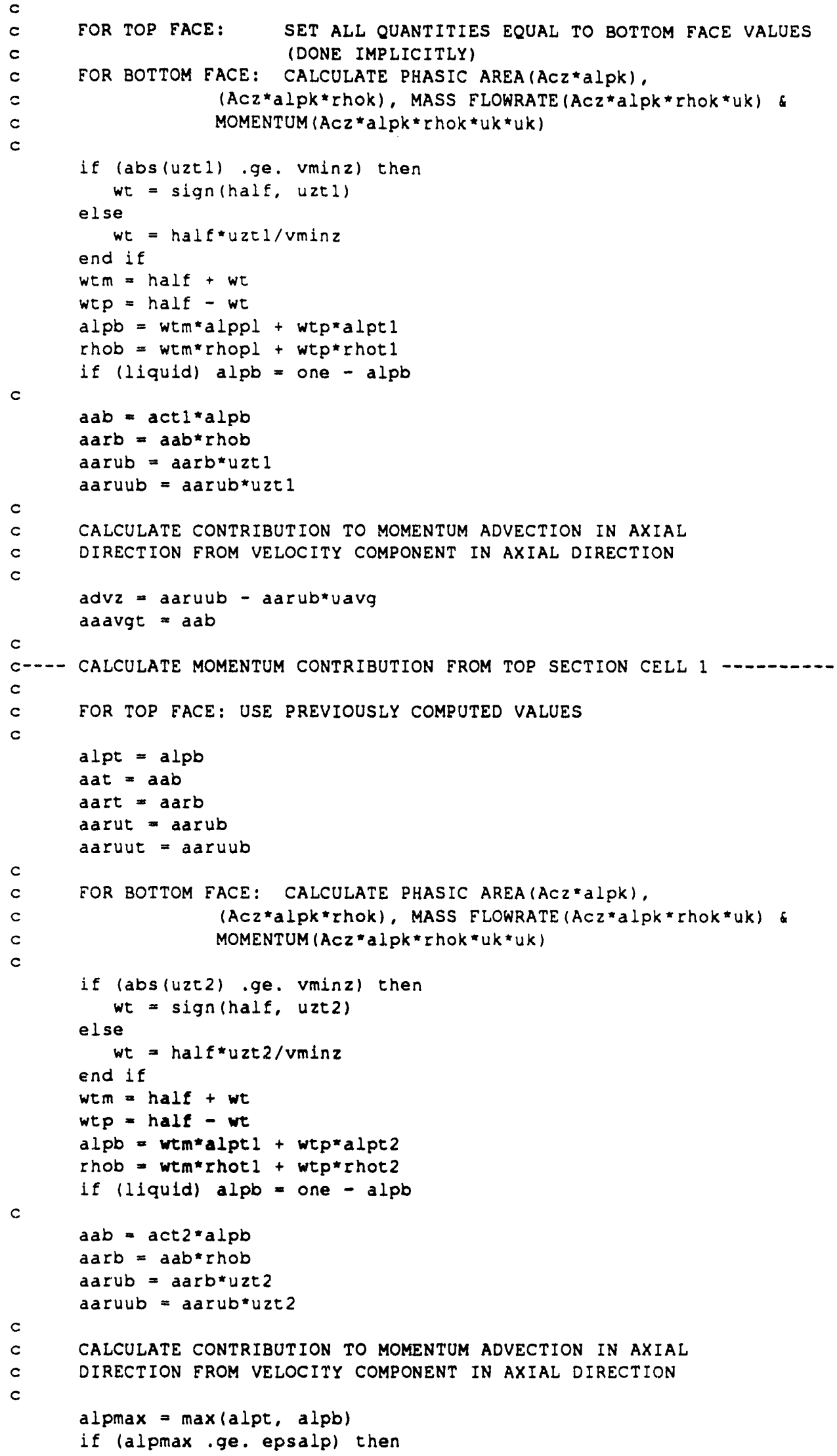




\begin{tabular}{|l|ll|l|}
\hline $2 / 15 / 93$ & WSRC-TR-93-086 Rev. 0 & FLOWTRAN-TF v1.2 Source Code & Pg. 23 of 354 \\
\hline
\end{tabular}

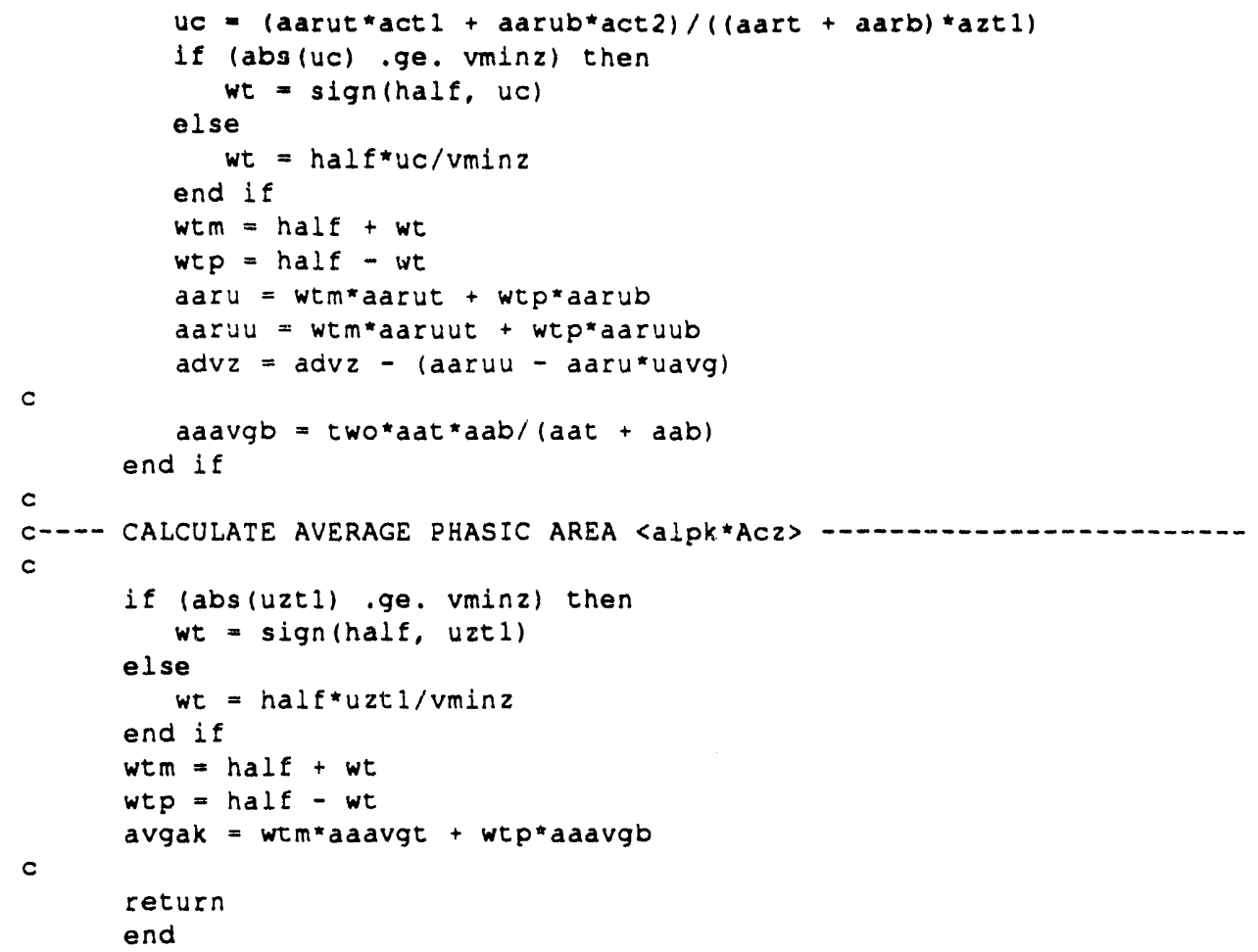

\section{AVECX}

subroutine avecx (advx, avgax, acx, acz,axx, aratx, wx, uz, alpn,

\& thon, vminz, vminx, ix, $k, i c c, n z p 1, n z p 2, n z p 3$, $n \times \max , n c h n, 1$ iquid) 


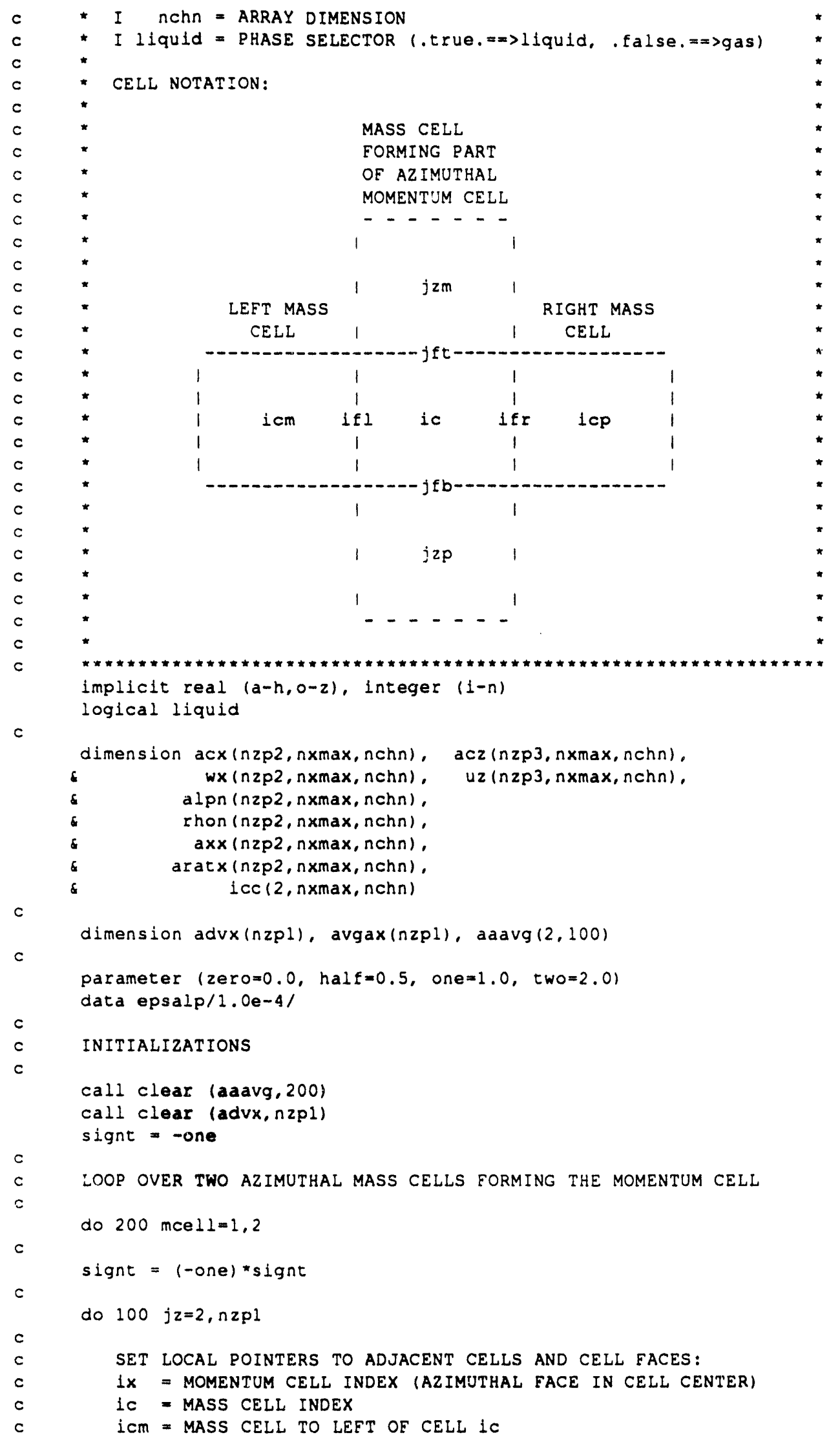



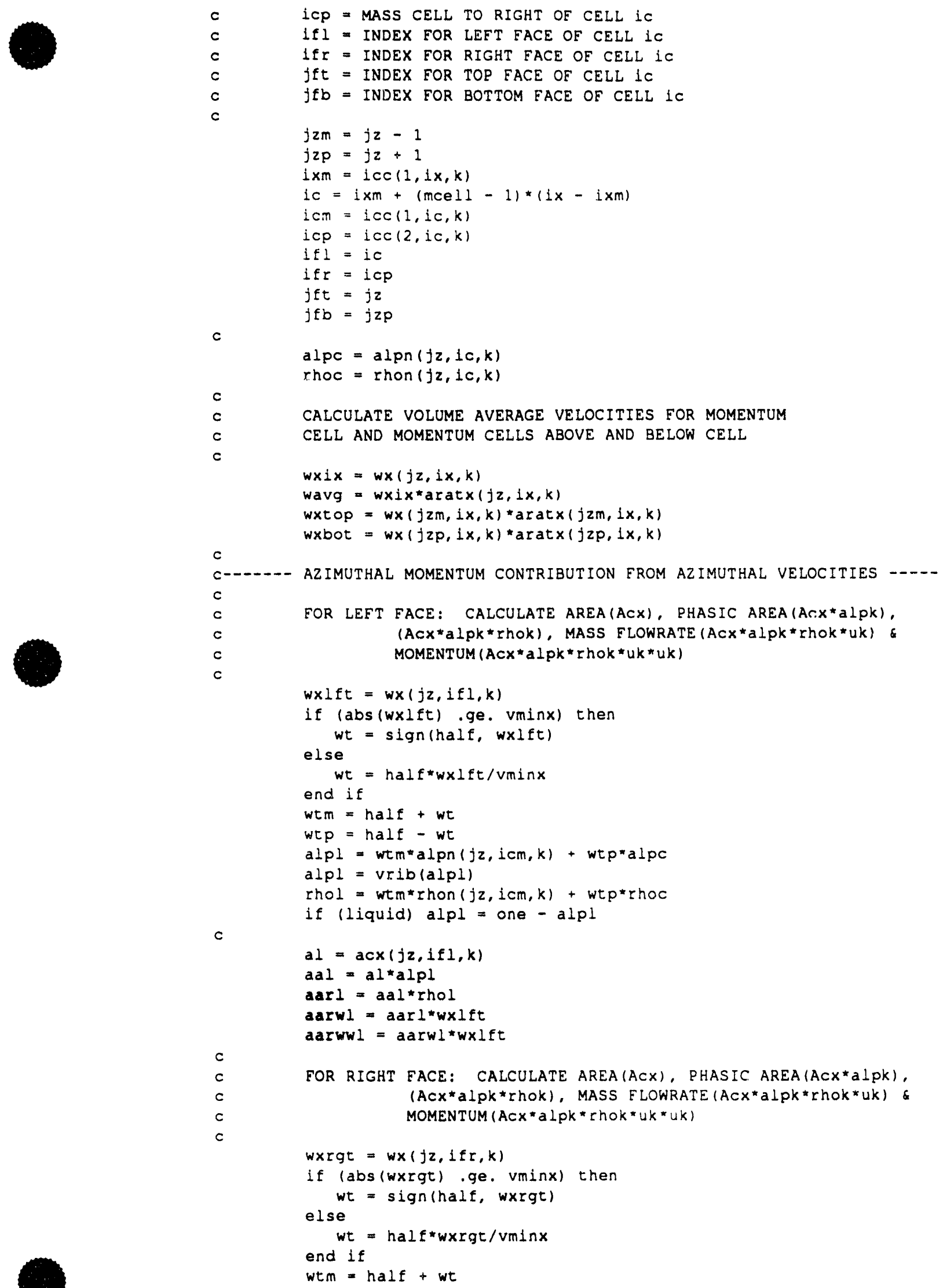


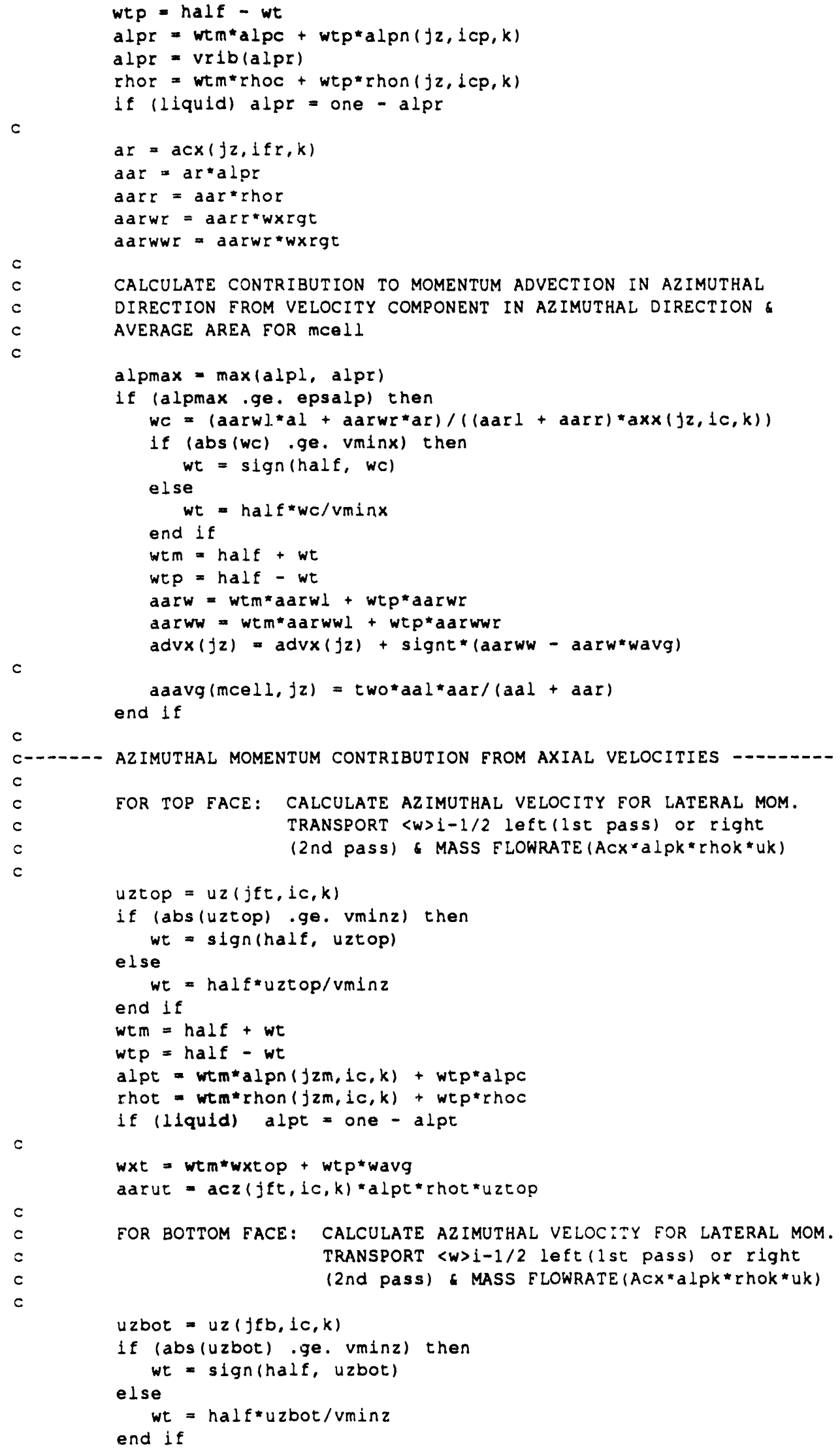




\begin{tabular}{|l|ll|l|}
\hline $2 / 15 / 93$ & WSRC-TR-93.086 Rev. 0 & FLOWTRAN-TF v1.2 Source Code & Pg. 27 of 354 \\
\hline
\end{tabular}

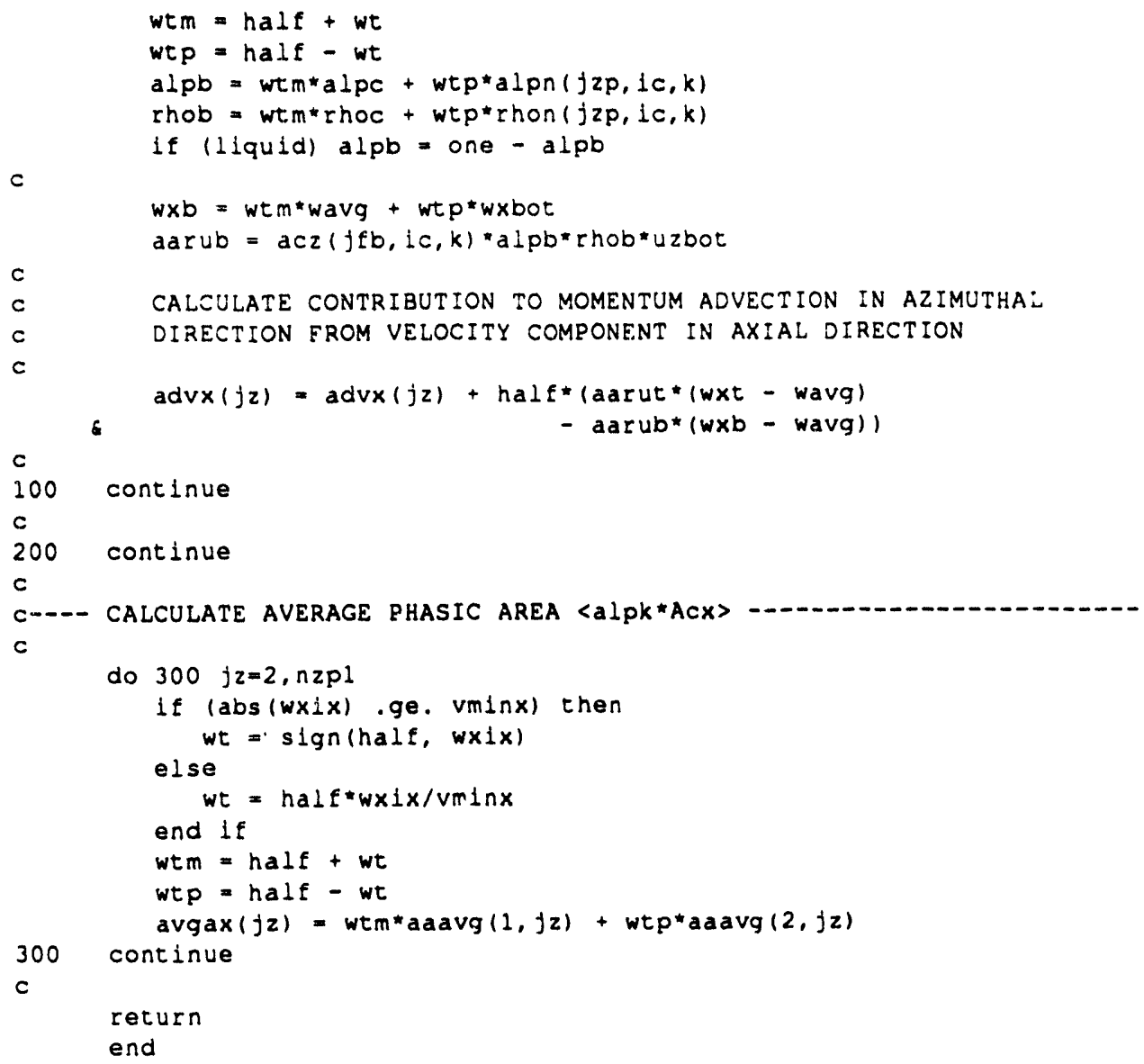

\section{AVECz}

subroutine avecz (advz, avgaz, acx, acz,azz, aratz, wx, uz, alpn,

- rhon, alpin, rhoin, aipex, rhoex, vminz, vminx, ix,

6 k, icc,nzp2,nzp3, nxmax, nchn, liquid)

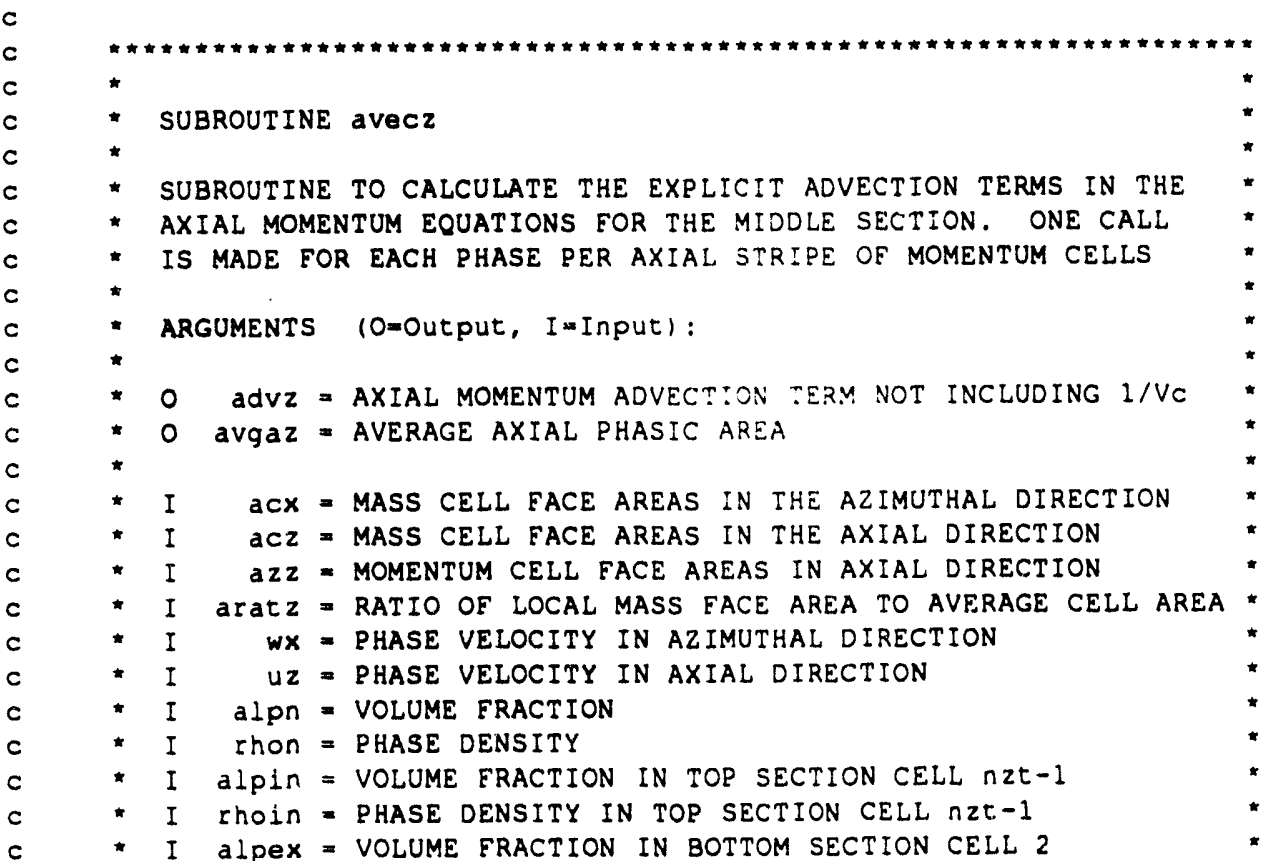




\begin{tabular}{|l|ll|r|}
\hline Pg. 28 of 354 & WSRC-TR-93-086 Rev. 0 & FLOWTRAN-TF V1.2 Source Code & $2 / 15 / 93$ \\
\hline
\end{tabular}

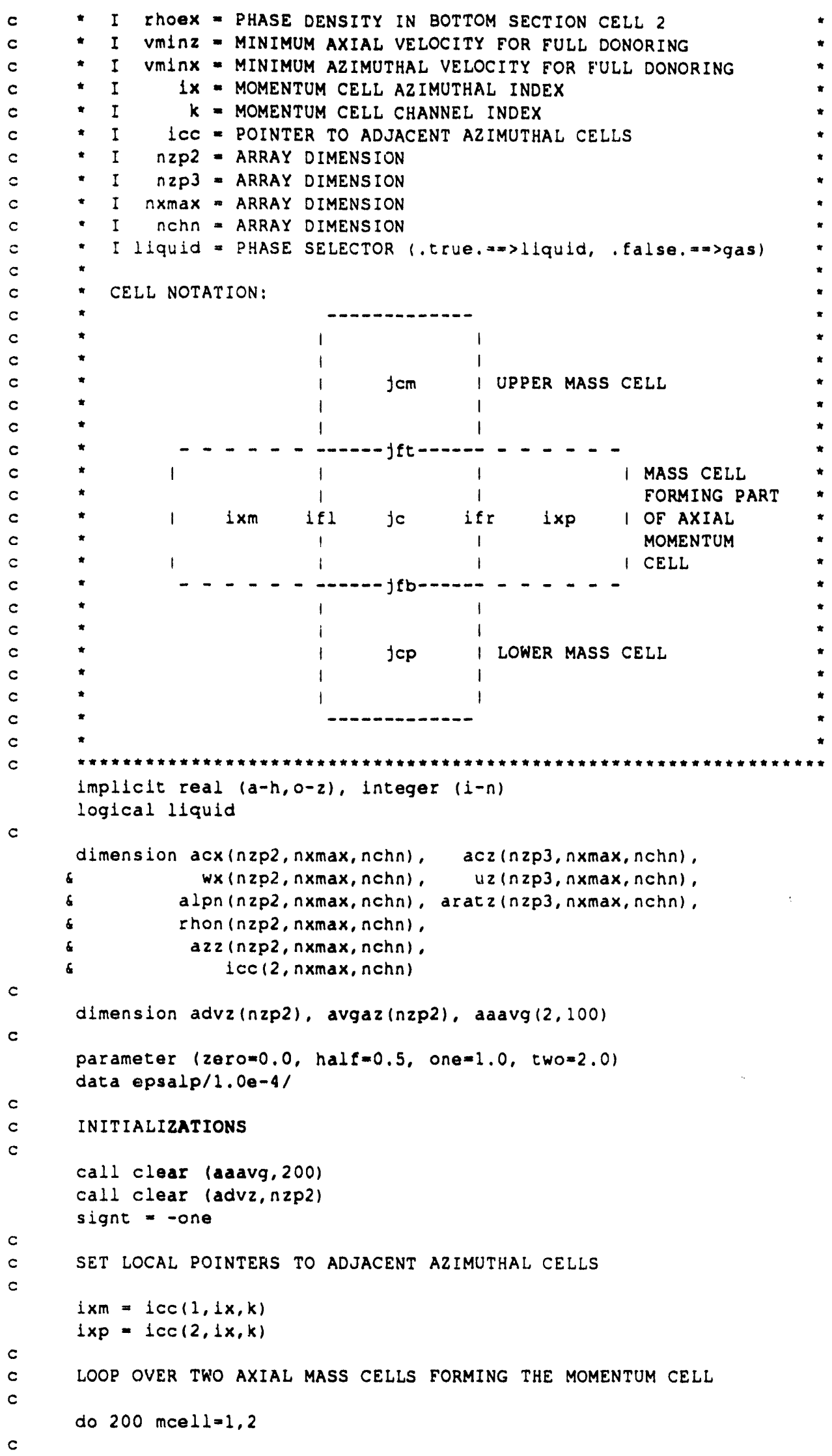




\begin{tabular}{|l|lll|l|}
\hline $2 / 15 / 93$ & WSRC.TR-93-086 Rev. 0 & FLOWTRAN-TF V1.2 Source Code & Pq. 29 of 354 \\
\hline
\end{tabular}

c

$c$

c

$c$

C

$c$

c

$c$

c

signt = (-one) $\operatorname{signt}$

do $100 j z=2, n z p 2$

SET LOCAL POINTERS TO ADJACENT CELLS AND CELL FACES:

$\mathrm{Jz}^{2}$ = MOMENTUM CELL INDEX (AXIAL FACE AT CELL CENTER)

JC = MASS CELL INDEX

JCM = MASS CELL ABOVE CELL JC

JCP = MASS CELL TO BELOW CELL JC

$j t t=$ INDEX FOR TOP FACE OF CELL jC

$j \in b=$ INDEX FOR BOTTOM FACE OF CELL jC

If 1 - INDEX FOR LEFT FACE OF CELL JC

If $\mathrm{Y}$ = INDEX FOR RIGHT FACE OF CELL JC

$j c=j z-2+\operatorname{mcel} 1$

$j \mathrm{~cm}=j \mathrm{c}-1$

$j c p=j c+1$

$j f t=j c$

$j f b=j c+1$

$1 f 1=1 x$

if $r=1 \times p$

$a l p c=a l p n(j c, 1 x, k)$

rhoc $=\operatorname{rhon}(j c, \mid x, k)$

CALCULATE VOLUME AVERAGE VELOCITIES FOR MOMENTUM CELL AND MOMENTUM CELLS TO LEFT AND RIGHT

$u z j z=u z(j z, 1 x, k)$

uavg =uzjz aratz $(j z, i x, k)$

$u z l f t=u z(j z, 1 \times m, k)$ aratz $(j z, 1 \times m, k)$

$u z$ rgt $=u z(j z, 1 \times p, k) \neq \operatorname{arat} z(j z, i x p, k)$

AXIAL MOMENTUM CONTRIBUTION FROM AXIAL VELOCITIES

FOR TOP FACE: CALCULATE AREA (ACZ), PHASIC AREA (ACz"alpk), (ACz"alpk*rhok), MASS FLOWRATE (ACz*alpk*rhok*uk) MOMENTUM (ACz*alpk*rhok*uk*uk)

uztop $=u z(j \in t, i x, k)$

if (abs (uztop) ,ge. vminz) then

else

$w t=\operatorname{slgn}$ (half, uztop)

$w t=h a l f * u z t o p / v m i n z$

end if

$w t m=$ half + wt

wtp = half - wt

if (jcm. It. 1) then

if (nchn ne. 3) then

alpm $=a \operatorname{lp} 1 \mathrm{n}$

else

alpm $=\operatorname{alpn}(j c, i x, k)$

end if

$a l p t=w t m * a l p m+w t p * a \perp p c$

else

rhot = wtm*rhoin + wtp*rhoc

$a \perp p t=w t m * a l p n(J c m, 1 x, k)+w t p * a \perp p c$

end if

rhot = wtm*rhon $(j \mathrm{~cm}, i x, k)+$ wtp*rhoc

if (liquid) alpt = one - alpt

$a t=\operatorname{acz}(j f t, 1 x, k)$

aat $=a t * a l p t$

aart $=$ aat*rhot 


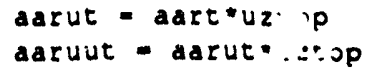

CALCULATE CONTRIBUTION TO MOMENTUM ADVECTION IN AXIAL DIRECTION FROM VELOCITY COMPONENT IN AXIAL DIRECTION 6 


\begin{tabular}{|l|ll|l|}
\hline $2 / 15 / 93$ & WSRC-TR-93-086 Rev. 0 & FLOWTRAN-TF v1.2 Source Code & Pg. 31 of 354 \\
\hline
\end{tabular}

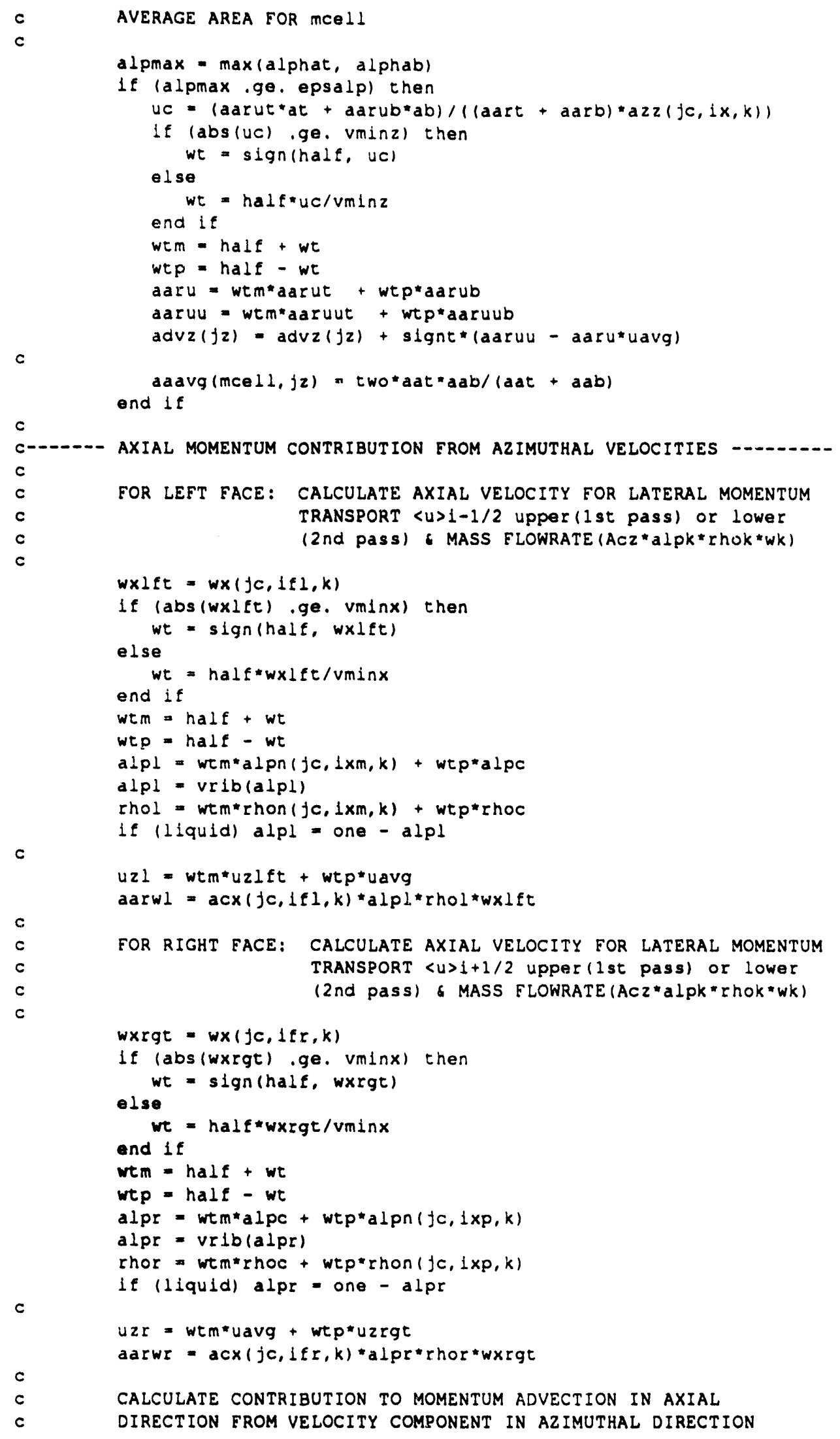




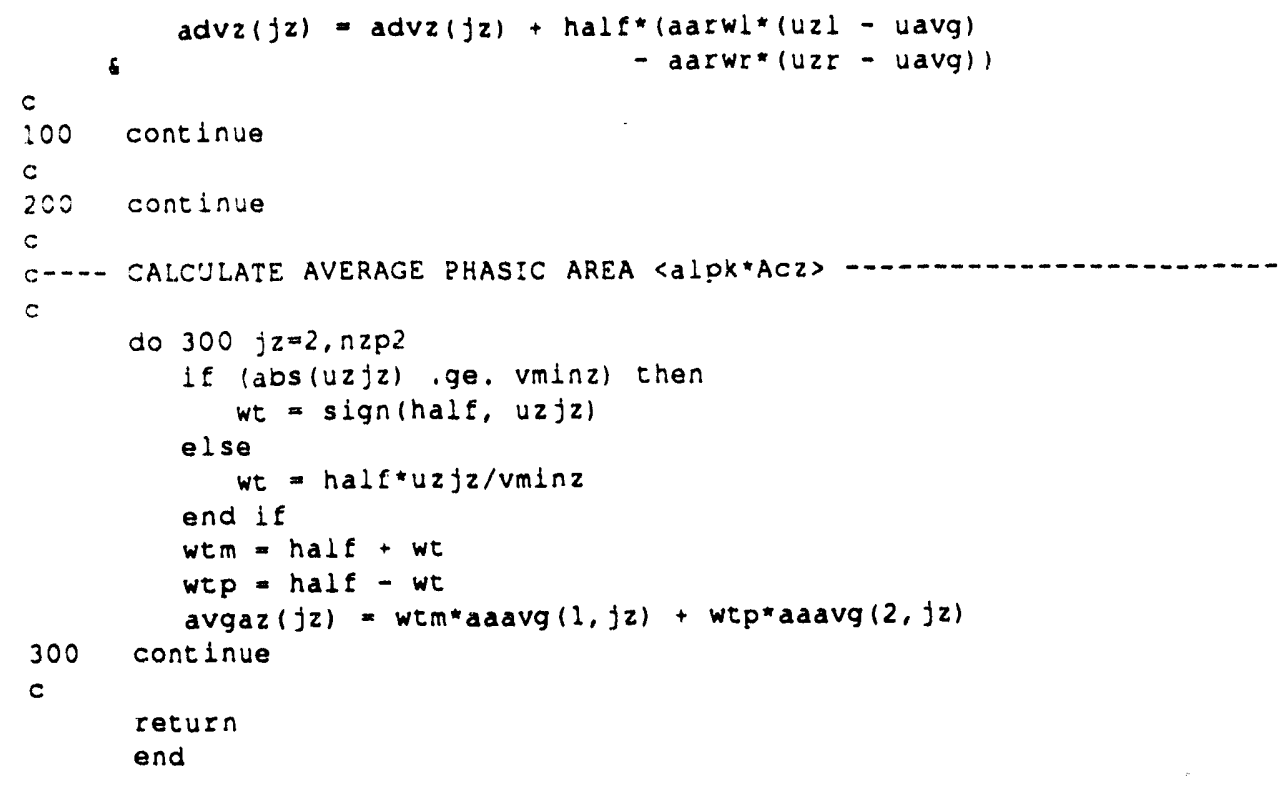

\section{AXIALP}

subroutine axialp laxlal, axalo, itimex, itime, timet,tsec,rdz,nz,

6 laxlp, ntp, intp, 1type, xtp, ytp, ztp, ntpmax, ntptot,

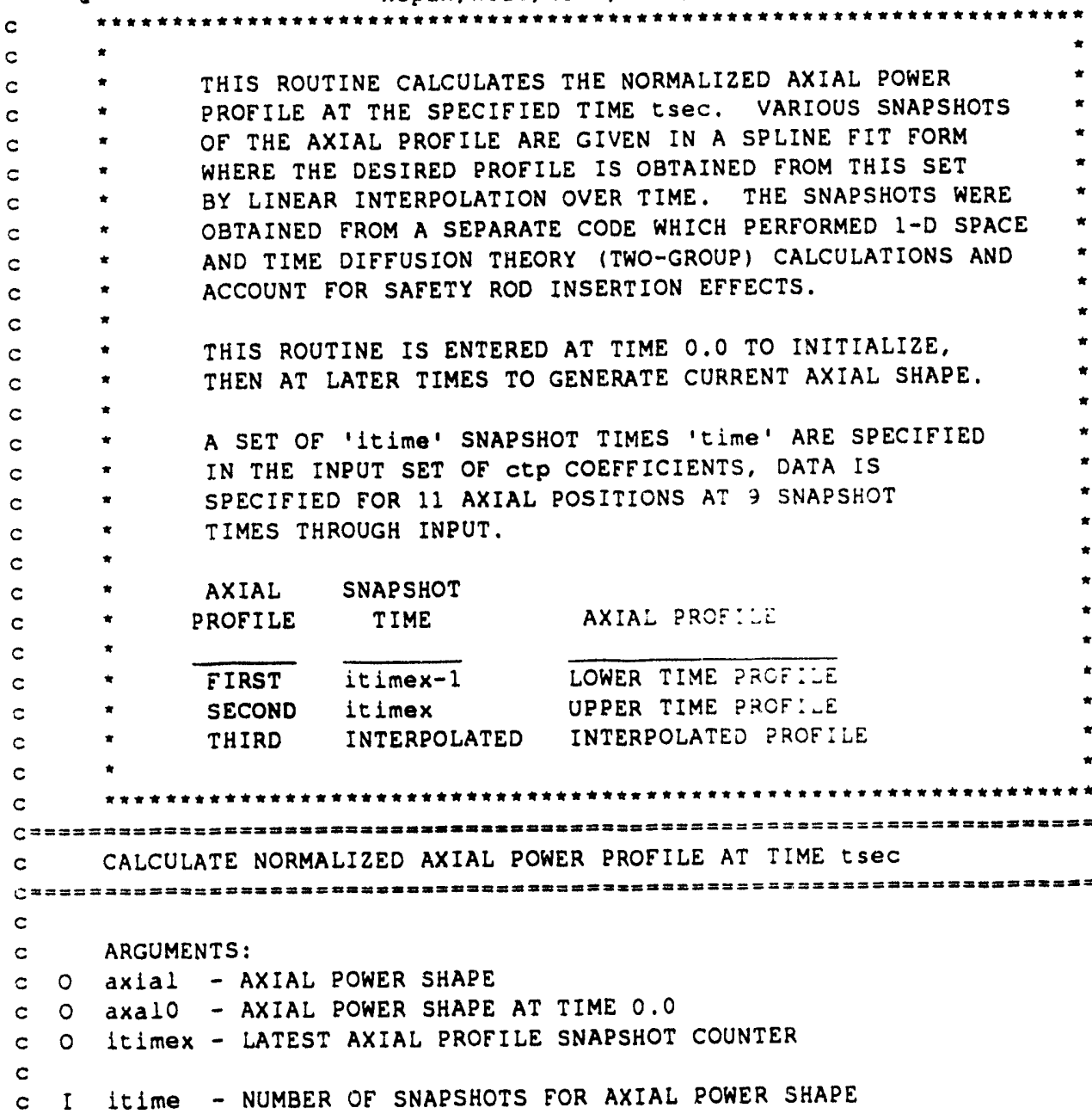




\begin{tabular}{|l|lll|l|}
\hline $2 / 15 / 93$ & WSRC-TR-93-086 Rev.0 & FLOWTRAN-TF v1.2 Source Code & Pg. 33 of 354 \\
\hline
\end{tabular}

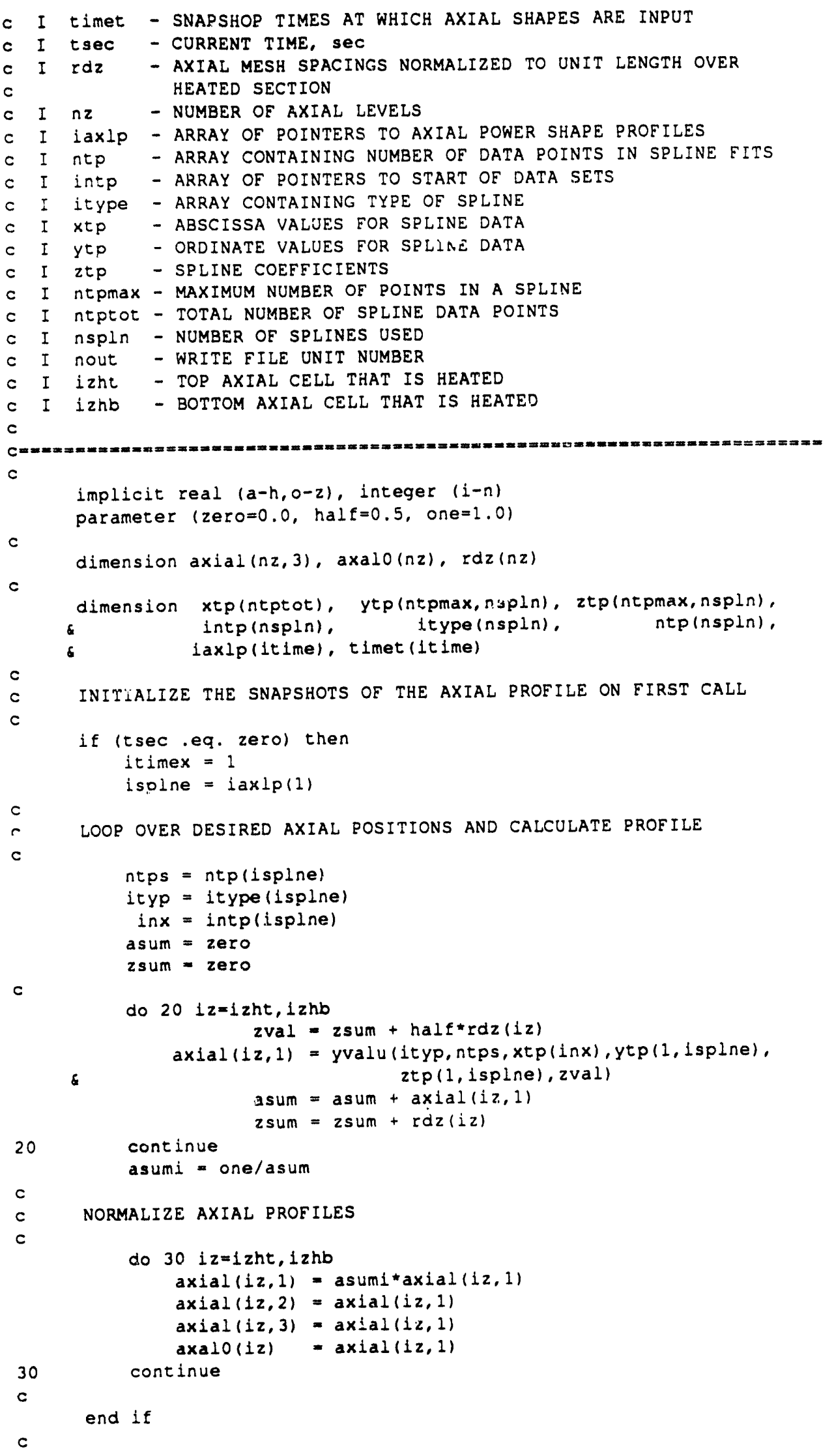




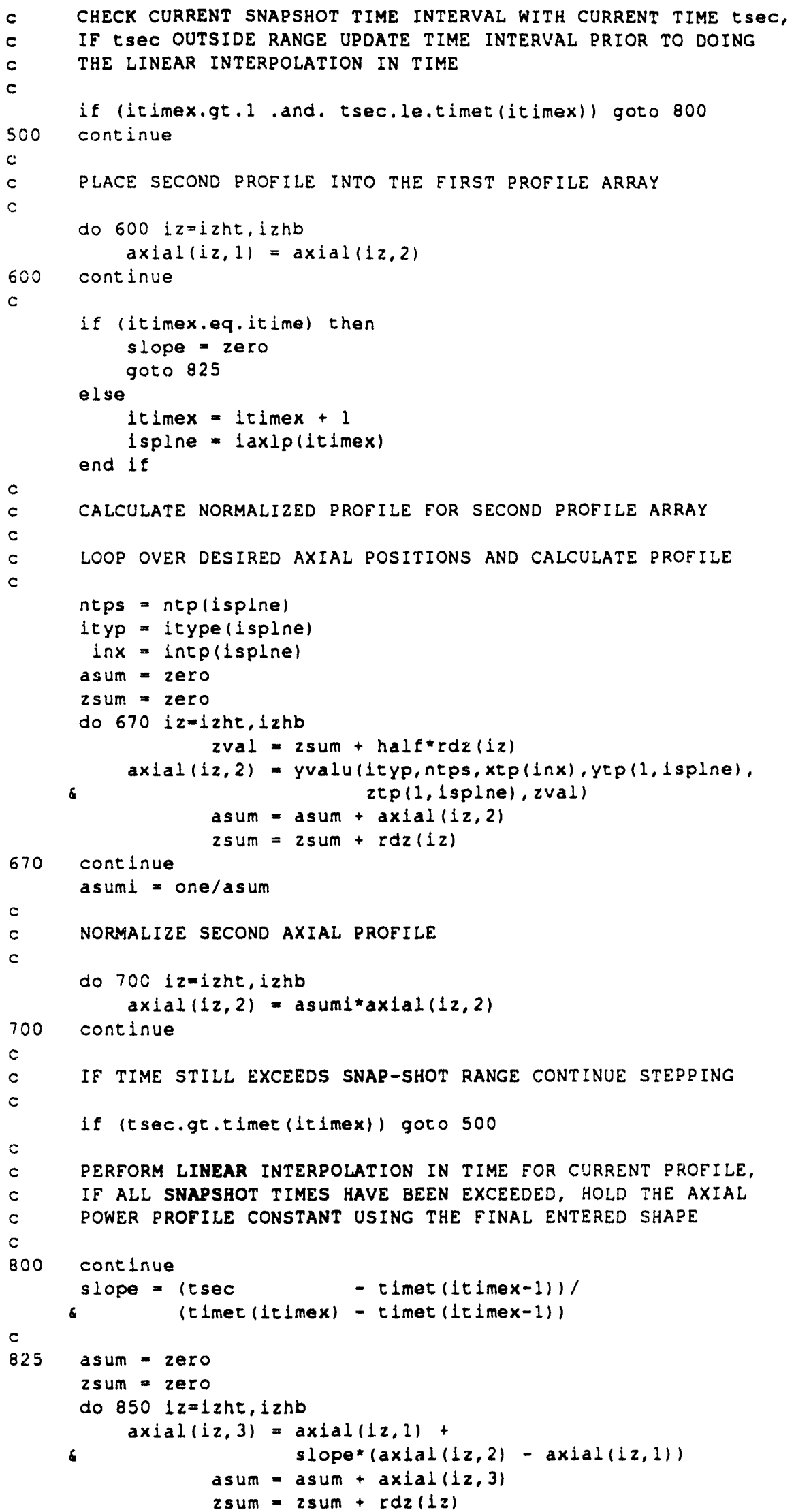




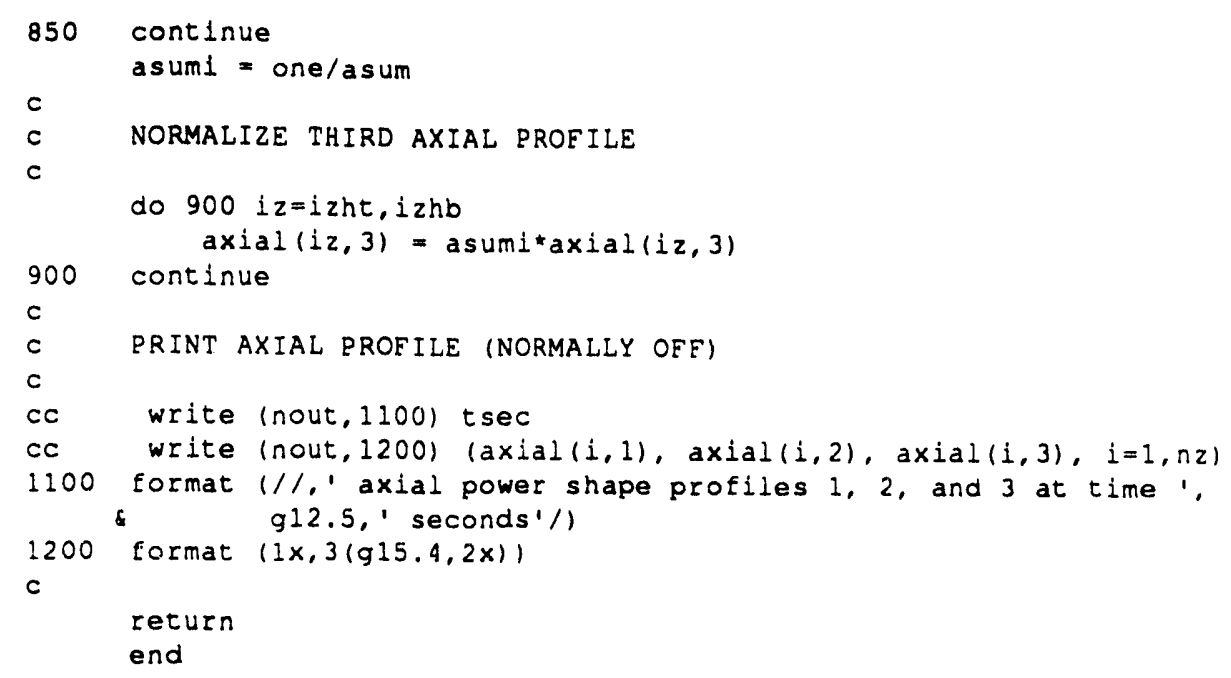

\section{BOUND}

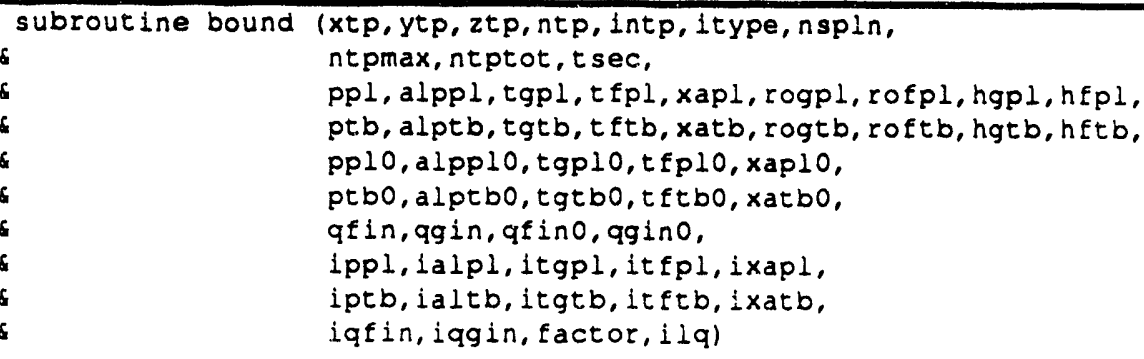




\begin{tabular}{|l|ll|r|}
\hline Pg. 36 of 354 & WSRC-TR-93-086 Rev. 0 & FLOWTRAN-TF v1.2 Source Code & $2 / 15 / 93$ \\
\hline
\end{tabular}

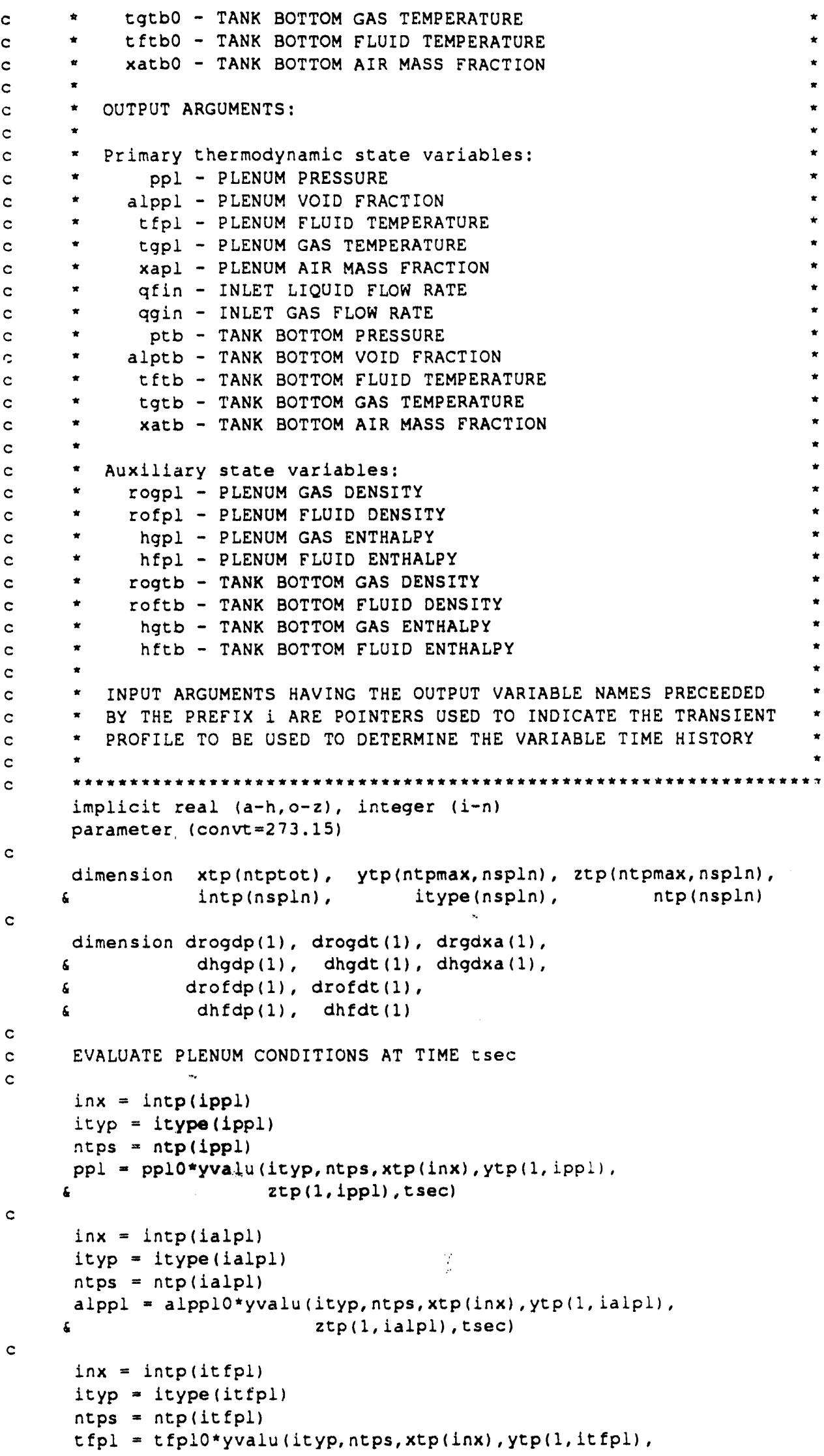


$\operatorname{ztp}(1$, itfpl), tsec) + convt

inx $=\operatorname{intp}(1 \mathrm{tgpl})$

ityp = itype (itgpl)

$n t p s=n t p(i t g p l)$

$t g p l=t g p l 0 * y v a l u(i t y p, n t p s, x t p(\ln x), y t p(1, i t g p l)$,

$z t p(1, i t g p 1), t \sec )+$ convt

$\operatorname{lnx}=\operatorname{intp}(i x a p l)$

ityp = itype (ixapl)

$n t p s=n t p(i x a p l)$

xapl $=$ xaplo*yvalu(ityp, ntps, $x t p(i n x), y t p(1, i x a p l)$,

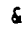

ztp $(1, i x a p l), t s e c)$

C

DETERMINE DENSITY AND ENTHALPY AT PLENUM

call state (ppl,tgpl,tfpl, xapl, factor, 1lq,

\& $1,1,1,1,1,1,1$

rogpl, drogdp, drogdt, drgdxa,

hgpl, dhgdp, dhgdt, dhgdxa,

rofpl, drofdp, drofdt,

hfpl, dhfdp, dhfdt)

EVALUATE LIQUID AND GAS INLET FLOWS:

(These values are always used for flow initialization purposes

regardless of B.C. optior. selected)

$\ln x=\operatorname{lntp}(i q f i n)$

ityp = itype (iqfin)

$n t p s=n t p(i q f i n)$

qfin $=$ qfino*yvalu(ityp, ntps, xtp(inx),ytp(1,iqfin), $z \in p(1$, iqfin), tsec $)$

$c$

inx $=$ intp(iqgin)

ityp = itype (iqgin)

ntps $=n t p(i q g i n)$

qgin = qgino*yvalu(ityp, ntps, xtp(inx),ytp(1,iqgin).

\&

$\operatorname{ztp}(1, i q g i n)$, tsec $)$

$c$

c

EVALUATE TANK BOTTOM CONDITIONS AT TIME tsec

$\operatorname{inx}=\operatorname{intp}(i p t b)$

ityp $=$ itype (iptb)

ntps $=n t p(i p t b)$

ptb $=p t b 0^{\star} y v a l u(1 t y p, n t p s, x t p(i n x), y t p(1, i p t b)$,

\& ztp $(1, i p t b), t \sec )$

$\ln x=\operatorname{intp}(1 a \operatorname{ltb})$

ityp = itype (ialtb)

ntps $=n t p(i a l t b)$

$a l p t b=a l p t b 0 * y v a d u(i t y p, n t p s, x t p(\vdots n x), y t p(1, i a \perp t b)$,

ztp (l, ialtb), tsec)

$c$

$\ln x=\operatorname{lntp}(i t f t b)$

ityp = itype (itftb)

ntps $=n t p(i t f t b)$

$t f t b=t f t b o$ yvalu (1typ, ntps, $x t p(i n x), y \in p(1, i t f t b)$,

\&

$2 \operatorname{te}(1, i t f(b), t \sec )+$ conve

c

$\operatorname{inx}=\operatorname{intp}(i t g t b)$

ityp $=$ itype (itgtb)

$n t p s=n t p(i t g t b)$

$t q t b=t g t b 0^{*} y v a l u(i t y p, n t p s, x t p(i n x), y t p(1, i t g t b)$,

5

$2 t p(1, i t g t b), t \sec )+$ convt 


\begin{tabular}{|l|ll|r|}
\hline Pg. 38 of 354 & WSRC-TR-93-086 Rev.0 & FLOWTRAN-TF v1.2 Source Code & $2 / 15 / 93$ \\
\hline
\end{tabular}

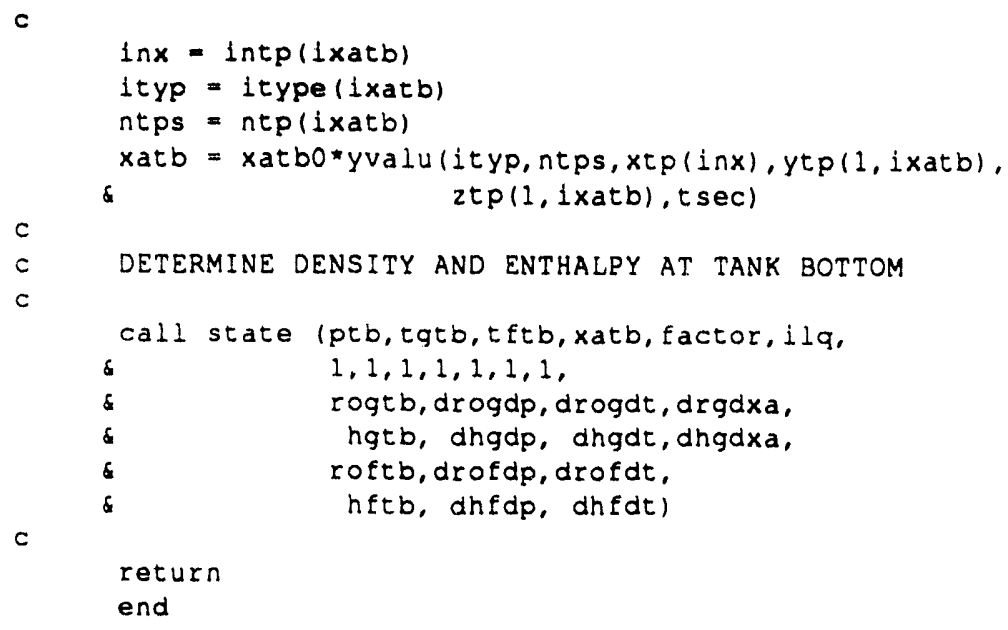

\section{COFCN}

subroutine cofen (alp,cob,c0s,co,cg,ann)

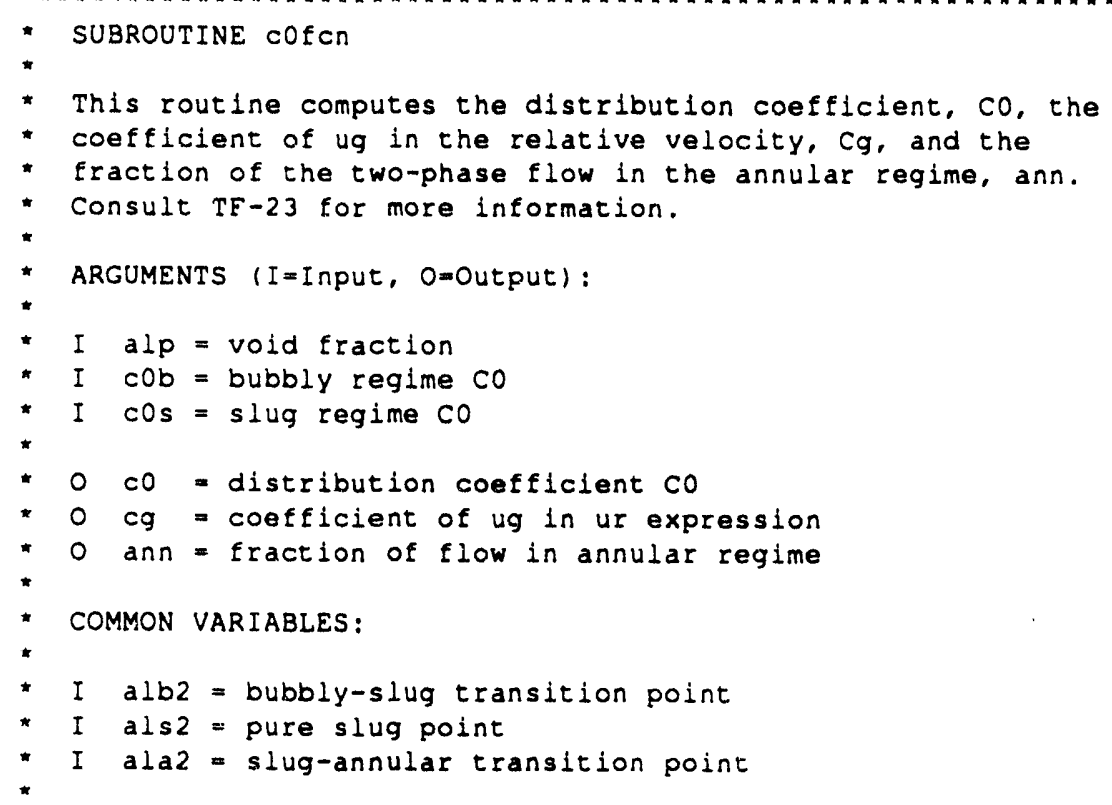




\begin{tabular}{|l|ll|l|}
\hline $2 / 15 / 93$ & WSRC-TR-93-086 Rev.0 & FLOWTRAN-TF v1.2 Source Code & Pg. 39 of 354 \\
\hline
\end{tabular}

$c$

$a l s=(a l p-a l b l) /(0 n e-a l b l)$

$a l b=a l p-a l s$

ann $=$ zero

$c 0=(a \perp b / a \perp p) * c O b+(o n e-a l b / a \perp p) * c 0 s$

c

$c g=(o n e-c o * a l p) /(o n e-a l p)$

$c$

else if (alp. le. ala2) then

$a_{i} a l=a l a 2 *((a l p-a l s 2) /(a l a 2-a l s 2)) * * \operatorname{expsa}$

$a l s=(a l p-a l a l) /(0 n e-a l a l)$

$a l a=a l p-a l s$

c

$a n n=a l a / a l p$

$c 0=($ one $-a n n) * c 0 s+a n n$

$c q=\left(o n e-c 0^{*} a l p\right) /($ one $-a l p)$

c

else

c

ann $=$ one

co = one

$\mathrm{cg}=$ one

c

end if

c

return

end

\section{CDENS}

subroutine cdens

(qg, dqgdt, vapor, $h f c, t w a 1 k, t s a t, u 11 q$, ugas rhol, rhog, condl, cpl, visl, visg, dhy, hfg,

$\delta$

tgas, alfa, docond)

SUBROUTINE cdens

subroutine to calculate condensation regime heat fluXes

OUTPUT ARGUMENTS:

qg - SURFACE HEAT FLUX TO GAS, $W / m * 2$

dqgdt - DERIVATIVE OF GAS HEAT FLUX WITH RESPECT TO WALL TEMPERATURE

vapor - VAPOR GENERATION RATE, $\mathrm{kg} / \mathrm{m} * 2-\mathrm{s}$

hCON - HEAT TRANSFER COEFFICIENT

INPUT ARGUMENTS:

tWalK - WALL TEMPERATURE, $K$

uliq - liquid phasic velocity, $\mathrm{m} / \mathrm{sec}$

ugas - gas phasic velocity, $\mathrm{m} / \mathrm{sec}$

alfa - void fraction

rhol - LIQUID DENSITY, $\mathrm{kg} / \mathrm{m} * 3$

rhog - GAS DENSITY, $\mathrm{kg} / \mathrm{m} * 3$

cONAl - LIQUID THERMAL CONDUCTIVITY, W/m-C

CPI - LIQUID HEAT CAPACITY, J/kg-C

cPg - GAS HEAT CAPACITY, J/kg-C

visI - LIQUID VISCOSITY, $\mathrm{kg} / \mathrm{m}-\mathrm{s}$

visg - GAS VISCOSITY, $\mathrm{kg} / \mathrm{m}-\mathrm{s}$

dhY - CELL HYDRAULIC DIAMETER, $m$

hfg - LATENT HEAT OF VAPORIZATION, J/kg

docond - LOGICAL FLAG TO INDICATE CALCULATION OF TERMS

IN CORRELATION THAT ARE CONSTANT FOR FLUID CELL 


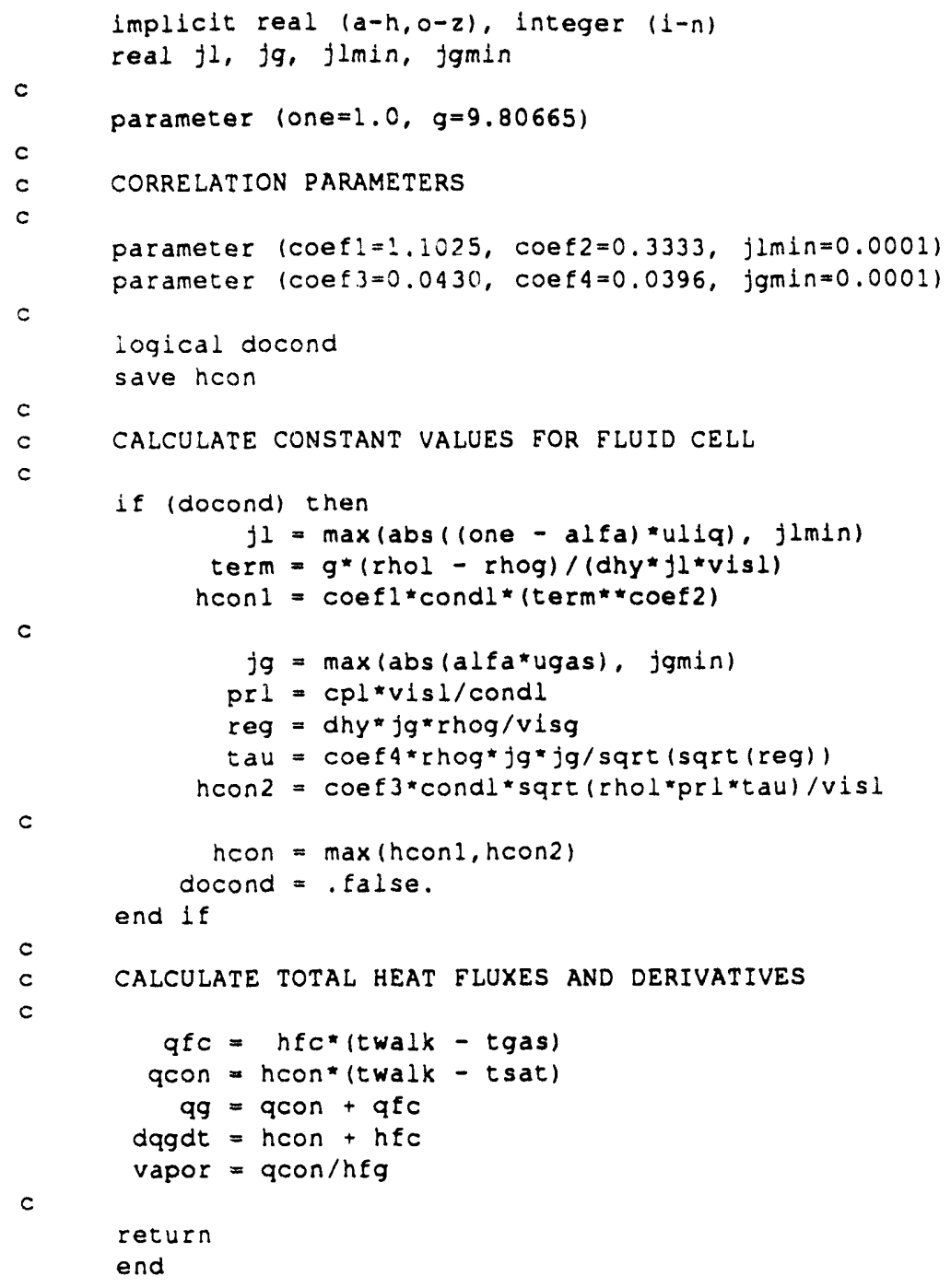

\section{CHEN}

subroutine chen (qflux, dqdtw, vapor, qchf, tchf, hfc, twall, tliq,
tgas, tsat, pres, alfa, beta, xalr, gliq, ggas, rhol,
rhog, visl, v1sg, condl, cpl, dhy, hfg, hisat, hgsat,
sigma, dochen, dochf, ichf, ilq)

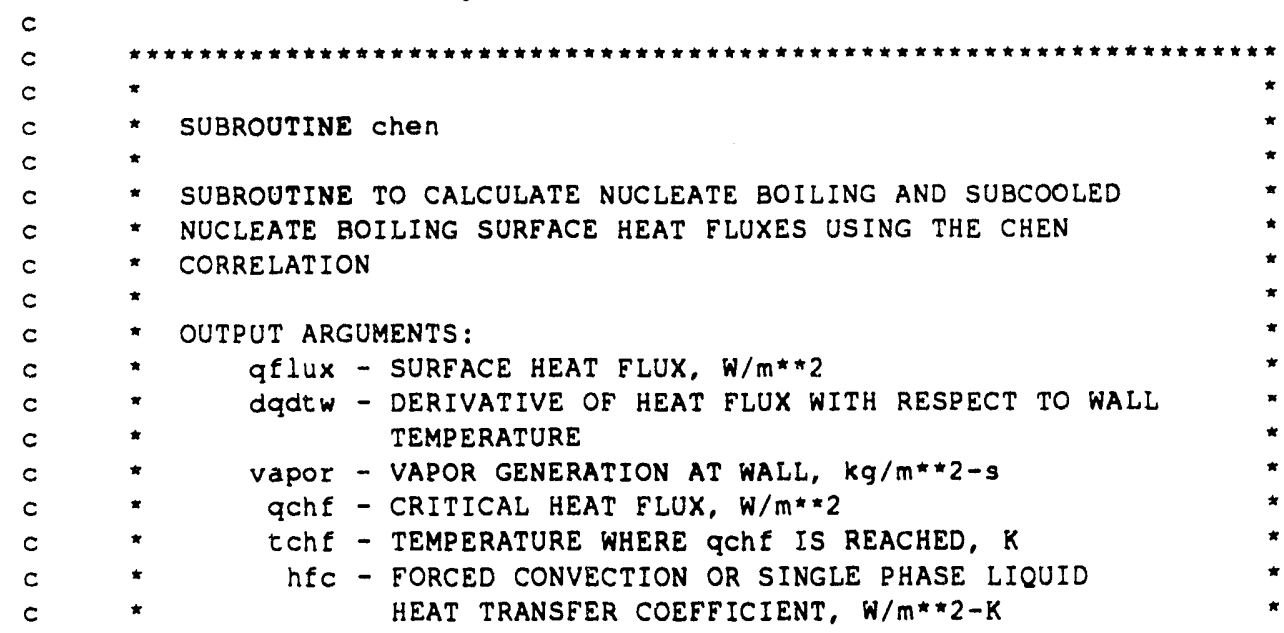




\begin{tabular}{|l|ll|l|}
\hline $2 / 15 / 93$ & WSRC-TR-93-086 Rev. 0 & FLOWTRAN-TF v1.2 Source Code & Pg. 41 of 354 \\
\hline
\end{tabular}

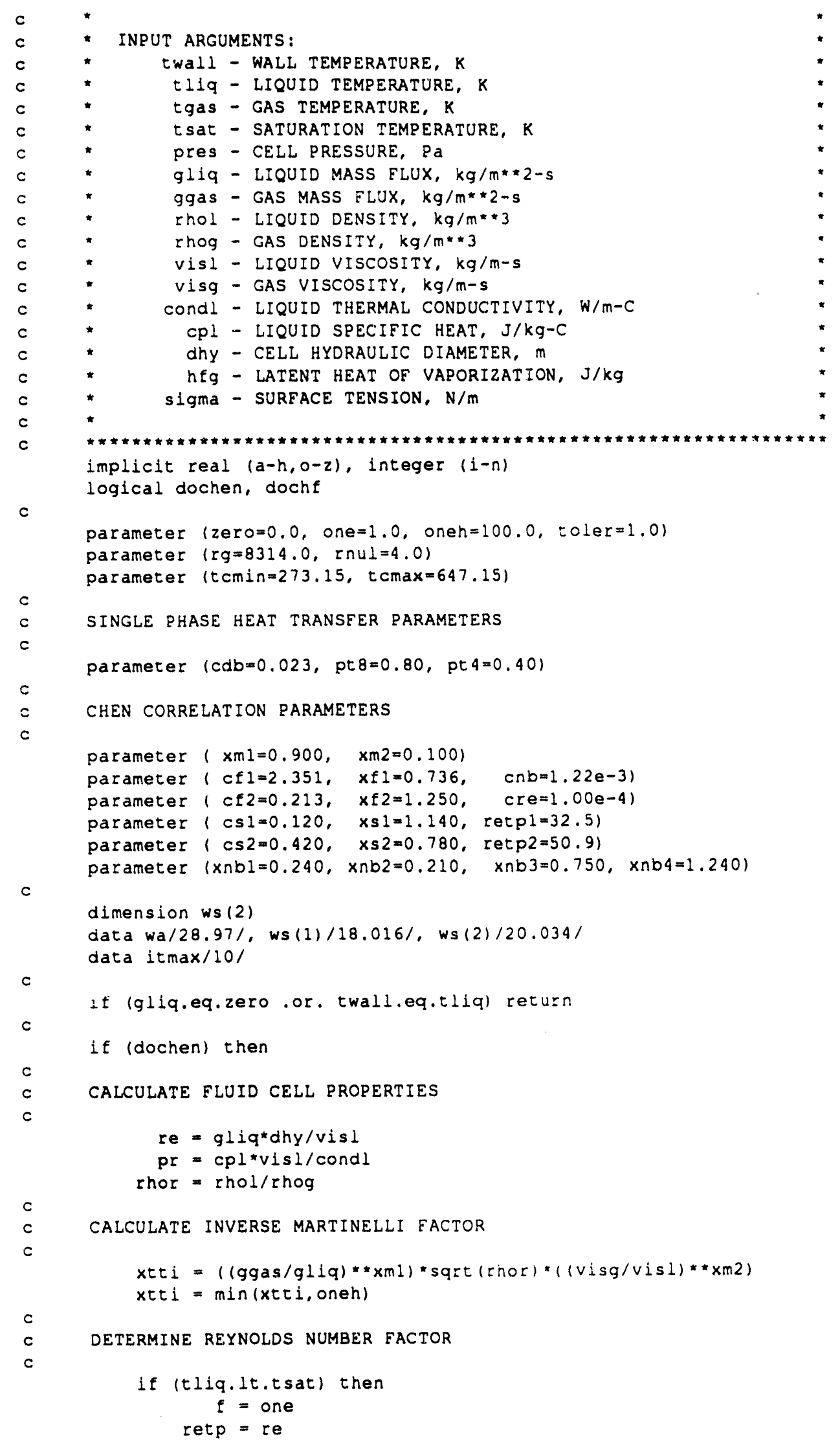




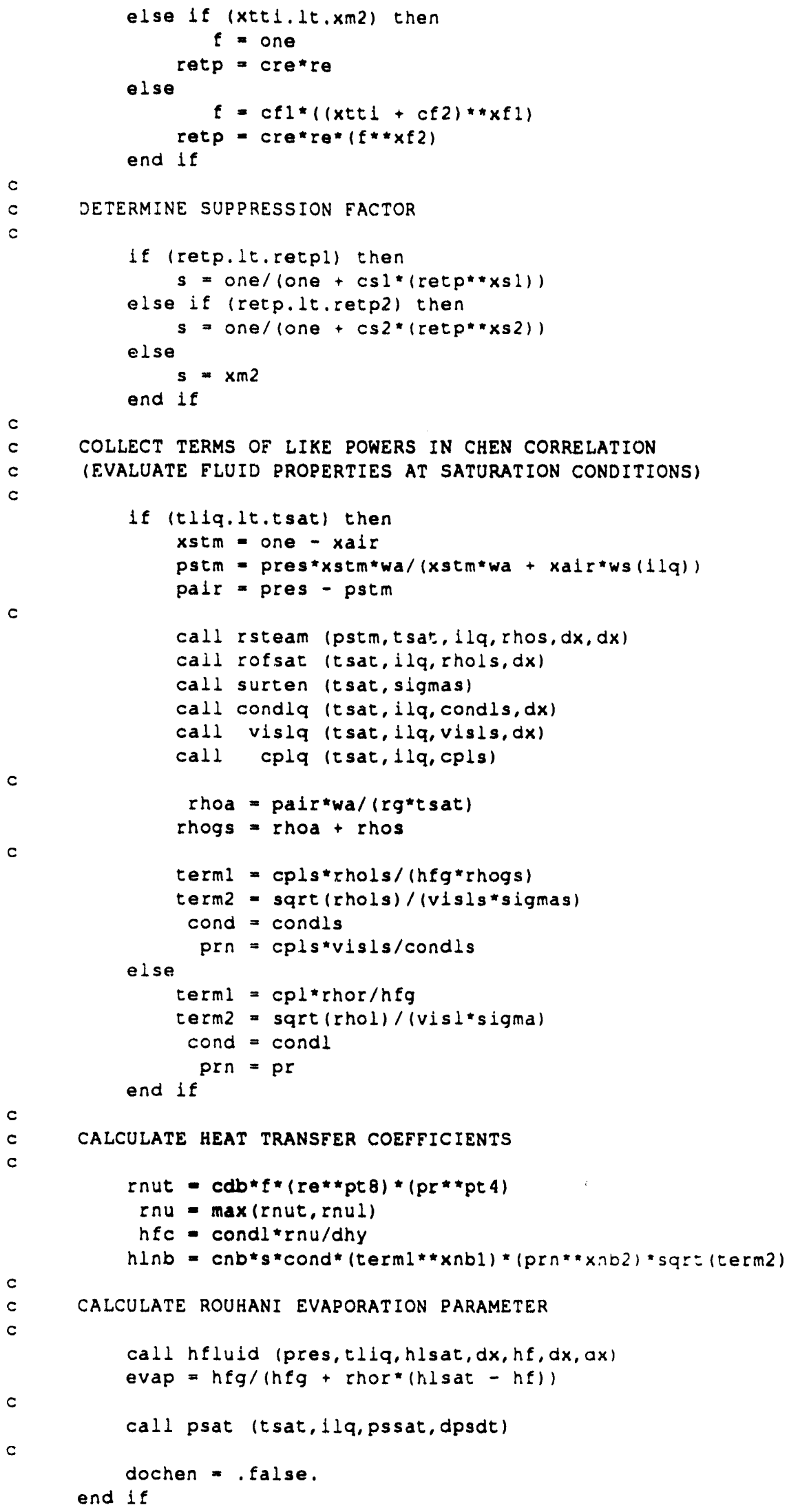




\begin{tabular}{|l|lll|l|}
\hline $2 / 15 / 93$ & WSRC-TR-93.086 Rev. 0 & FLOWTRAN-TF v1.2 Source Code & Pg. 43 of 354 \\
\hline
\end{tabular}

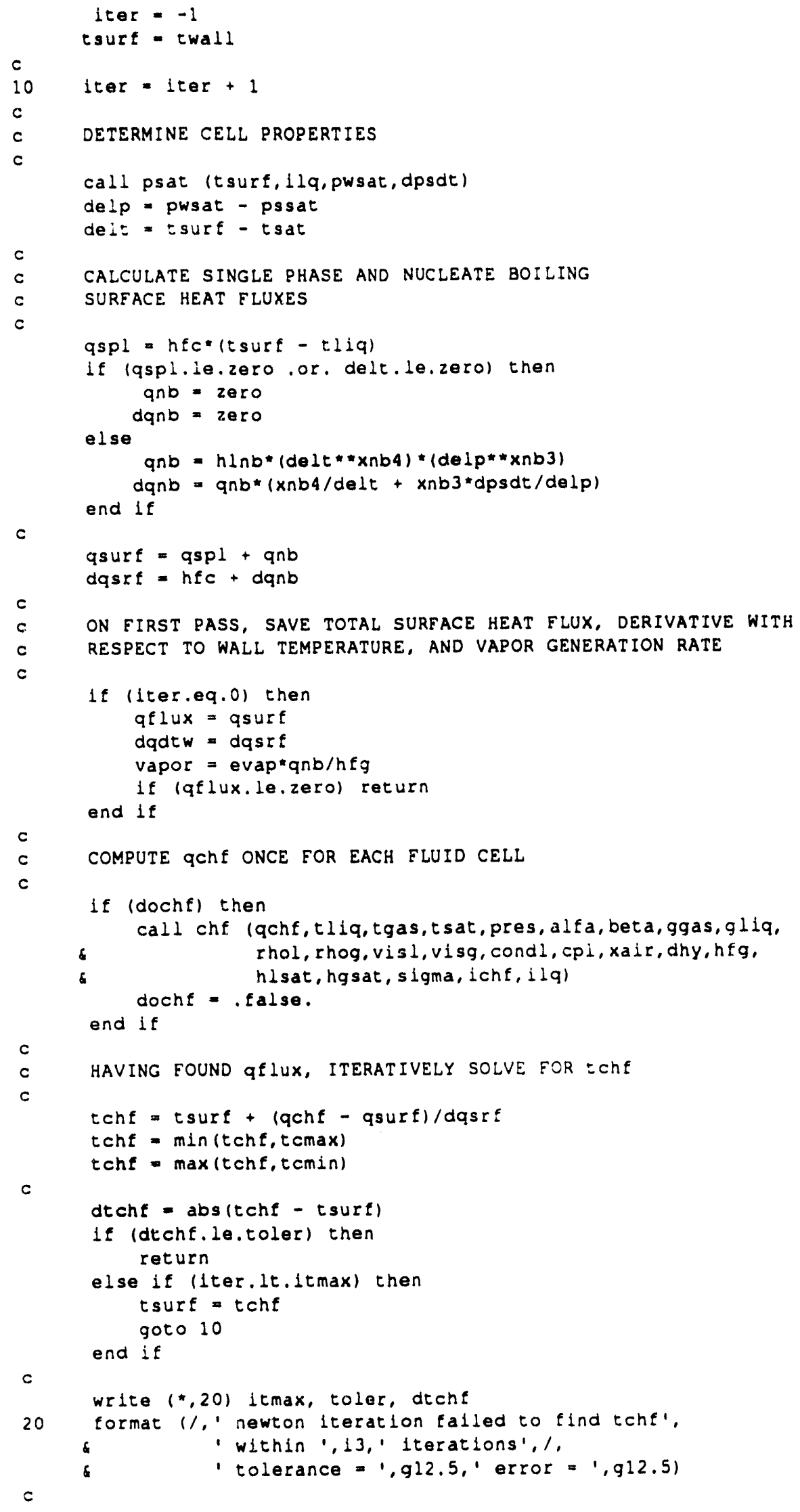




\begin{tabular}{|l|ll|r|}
\hline Pg. 44 of 354 & WSRC-TR-93.086 Rev. 0 & FLOWTRAN-TF v1.2 Source Code & $2 / 15 / 93$ \\
\hline
\end{tabular}

return

end

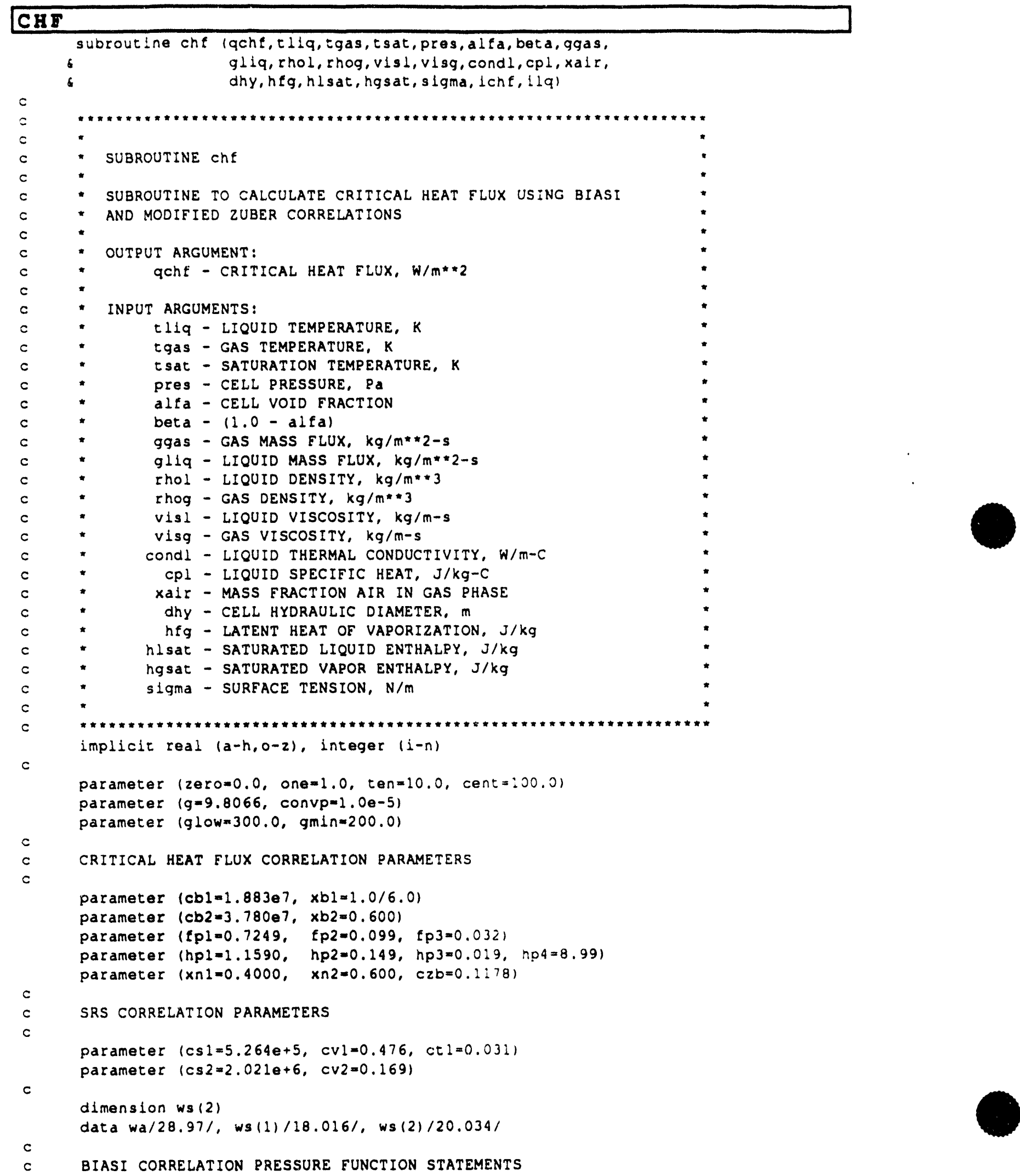




\begin{tabular}{|l|lll|l|}
\hline $2 / 15 / 93$ & WSRC-TR-93.086 Rev.0 & FLOWTRAN-TF v1.2 Source Code & Pg. 45 of 354 \\
\hline
\end{tabular}

$c$

$f p(p)=+f p\}+p *(\{p 2 * \exp (-\{p\} * p)\}$

$h p(p)=-h p 1+p *\left(h p 2 * \exp \left(-h p 3^{*} p\right)+h p 4 /(t e n+p * p)\right)$

If (1cht.eq.1) then

else

$g m i x=g l i q$

$g m 1 x=g 11 q+g g a s$

end $1 \mathrm{f}$

c

FOR LOW FLOWS, USE MODIFTED ZUBER POOL BOILING DNB CORREIATION

If (gmix.lt.glow) then

gchf = czb*beta*hfg*sqrt(rhog*sqrt (g*sigma*(rhol - rhog))

if (gmix.le.gmin) return

qzubr $=$ qch $f$

end 16

c

FORCED CONVECTION DNB CORRELATIONS

flow $=\max (g 11 q, g$ low)

if (ichf.eq.1) then

USE SRS CRITICAL HEAT FLUX CORRELATIONS

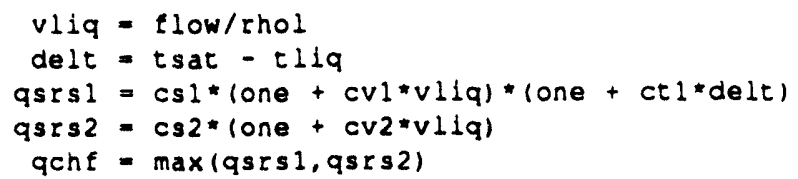




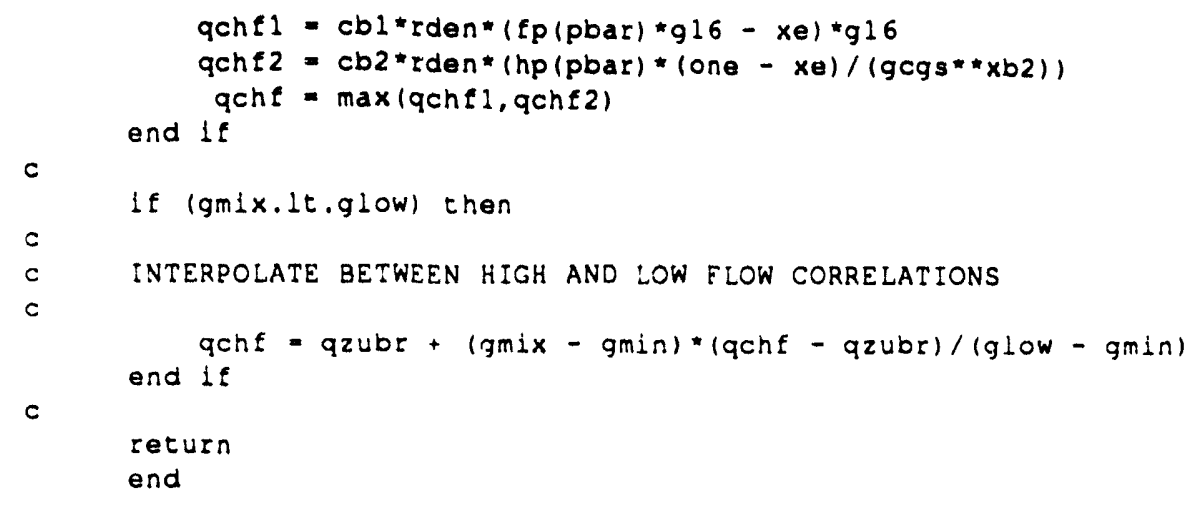

\section{CHISKI}

subroutine chlski (amat, $n r, n r l, b v c t$ )

$\star$

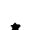

$\star$

$+$

$\star$

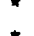

$+$

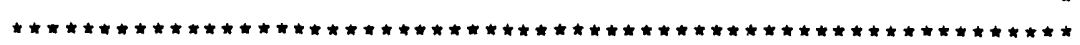

implicit real $(a-h, 0-z)$, integer $(1-n)$

parameter $(z e r o=0.0, \operatorname{maxp}=25, \operatorname{maxp} 1=\max p+1)$

dimension amat (maxp, maxpl), bvet (maxp)

$c$

CALCULATE EIRST ROW OF UPPER UNIT TRIANGULAR MATRIX.

3

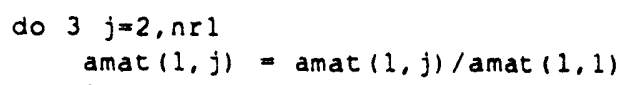



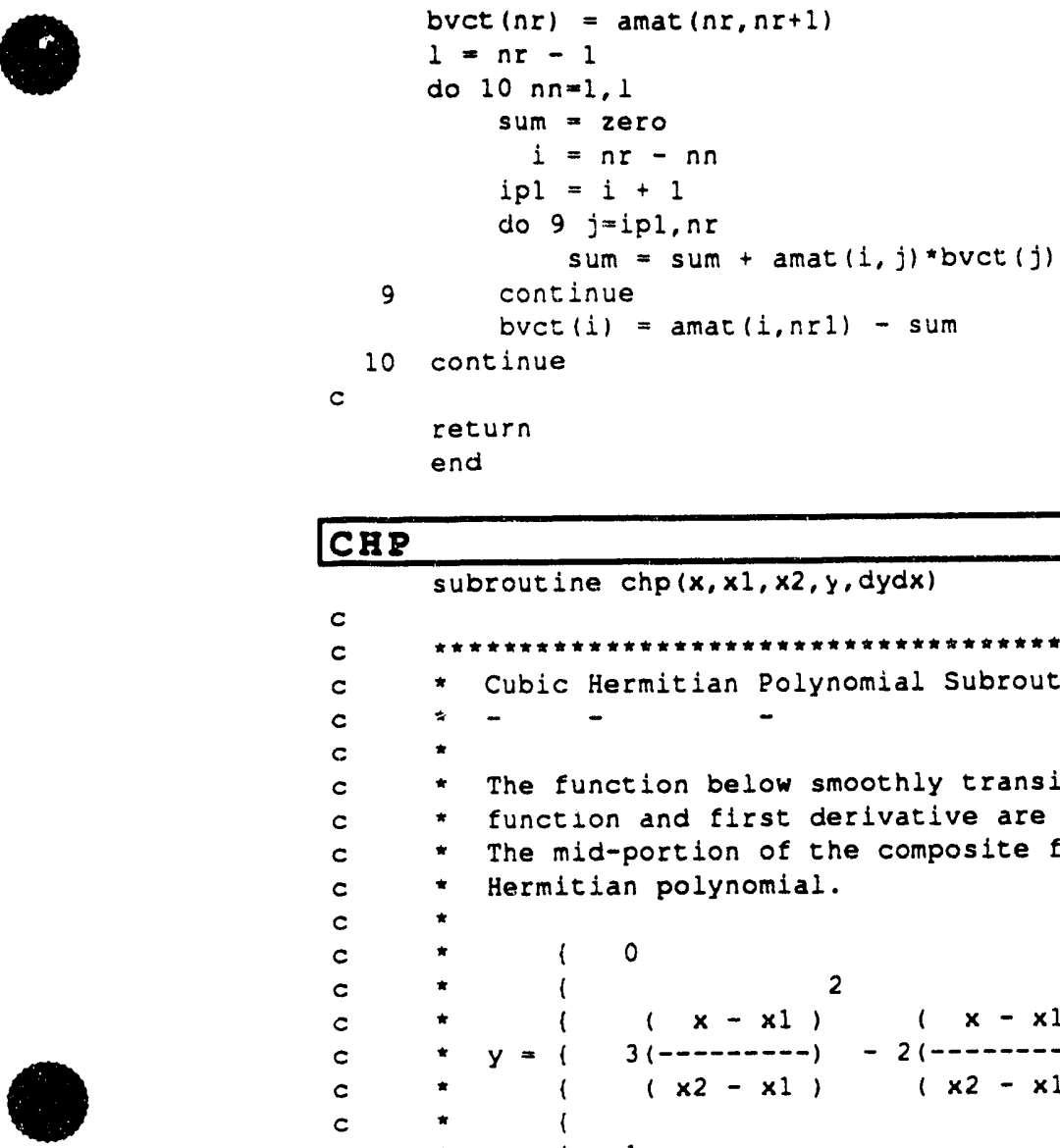

\section{CBP}

subroutine $\operatorname{chp}(x, x 1, x 2, y, d y d x)$

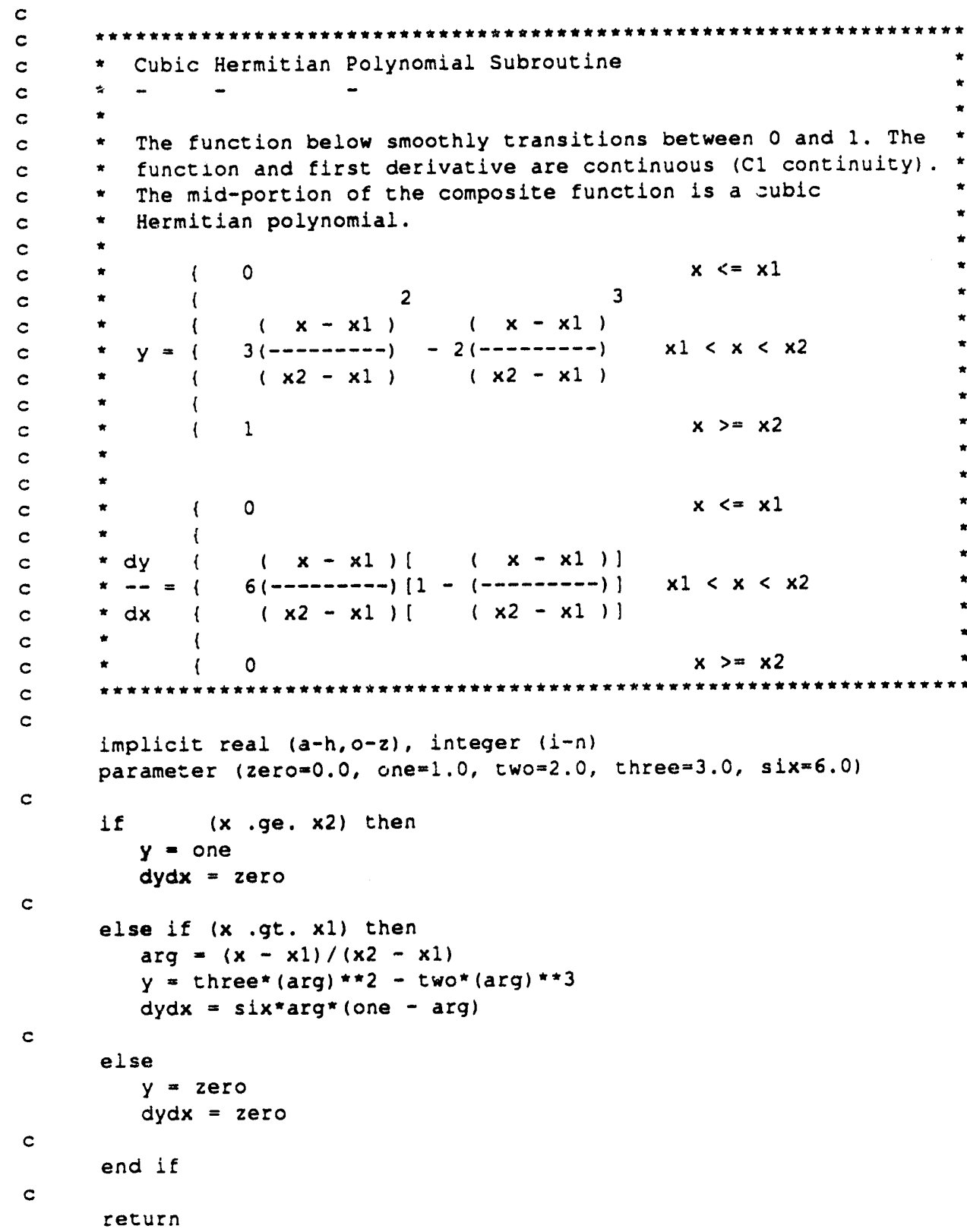


end

\section{CIA}

real function cia(aia, alpha, del, flowa, pia, rhog, urabs, visg)

$\star$

* FUnction cia

* this eunction computes the generalized interfacial

* FRICTION COEFFICIENT FOR the anNular flow REgime.

* arguments (O=output, I=Input):

- O cia - generalized interfacial friction coefficient,

* O cla

$+$

$\star$

* I aia - interfacial area per unit volume $\left(m^{\wedge}-1\right)$

* I alpha - void fraction

- I del - film thickness (m)

* I flowa - flow area $\left(m^{\wedge} 2\right)$

* I pia - interfacial perimeter (m)

- I rhog - gas density ( $\left.\mathrm{kg} / \mathrm{m}^{\wedge} 3\right)$

- I urabs - absolute relative velocity $(\mathrm{m} / \mathrm{s})$

* I visg - gas dynamic viscosity

$*$

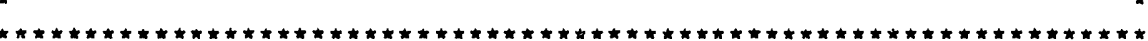

implicit real $(a-h, 0-z)$, integer $(1-n)$

real mgplus

parameter $($ zero $=0.0$, half $=0.5$, one $=1.0$, two $=2.0$, four $=4.0$ )

parameter $(c 1=0.046, c 2=-0.20, c 3=0.045, c 4=5.9$, regmin=0.01)

PRODUCT OF HALF THE GAS DENSITY AND INTERFACIAL AREA

factor $=$ half*rhog*aia

COMPUTE THE GAS PHASE FRICTION FACTOP FOR A SMOOTH PIPE

$r e g=f o u r$ *alpha flowa rhog $u r a b s / v i s g / p i a$

if (reg .ge. regmin) then

$f s=c 1 \star r e g * \star c 2$

else

cia $=$ factor* $(c 1 *$ regmin**c2)

return

end if

COMPUTE THE FILM REYNOLDS NUMBER

rem $=$ del*rhog*urabs/visg

SOLVE QUADRATIC EQUATION FOR SqIT(EI)

$b=-c 3 * \mathrm{rem} / \mathrm{sqrt}(\mathrm{two}) * \mathrm{fs}$

$c=-\left(\right.$ one $\left.-c 3^{\star} c 4\right) * f s$

sqrefr $=\operatorname{half} *\left(-b+\operatorname{sqrt}\left(b * \star 2-f\right.\right.$ our $\left.\left.{ }^{\star} c\right)\right)$

DETERMINE IF ET IS IN SMOOTH OR RIPPLE REGIME

mgplus $=\mathrm{rem} / \mathrm{sqrt}(\mathrm{two}) * \mathrm{sqrtfr}$

$f_{x}=f \mathrm{~s}$ "maxione, one + $\mathrm{c} 3$ =imgpius - c4)

$c i a=f a c t o r * f$ 


\begin{tabular}{|l|ll|l|}
\hline $2 / 15 / 93$ & WSRC-TR-93-086 Rev. 0 & FLOWTRAN-TF v1.2 Source Code & Pg. 49 of 354 \\
\hline
\end{tabular}

c

return

end

\section{CLEAR}

subroutine clear $(a x, n x)$

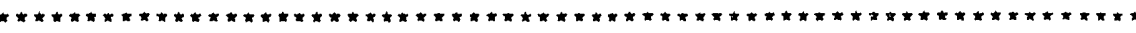

*

* suboutine clear

* routine to set all values in a real array to zero

$*$

- input arguments:

- ax = real array to be cleared

* $n x=$ DIMENSION OF $x$

$\star$

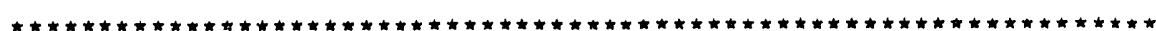

implicit real $(a-h, 0-z)$, integer $(1-n)$

parameter (zero $=0.0)$

dimension $\mathrm{ax}(\mathrm{n} \mathbf{x})$

$c$

do 10 i $x=1, n x$

$a x(i x)=$ zero

10 continue

c

return

end

\section{CLEARI}

subroutine clearl $(a x, n x)$

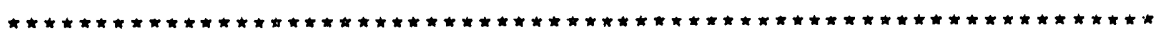

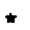

- suboutine clear1

$*$

* routine to set all values in a real array to one

* INPUT arguments:

- ax = REAL ARRAY TO BE CLEARED

* $n \mathrm{x}=$ DIMENSION OF $\mathrm{x}$

$\star$

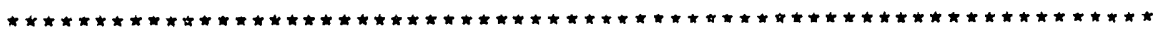

implicit real $(a-h, 0-z)$, integer $(i-n)$

parameter (one $=1.0)$

dimension $\mathrm{ax}(\mathrm{nx})$

c

do $10 i x=1, n x$

$a x(i x)=$ one

10 continue

c

return

end

\section{CONDIO}

subroutine condlg $(t, 1$, cond $l, d e d t)$

$c$
$c$
$c$
$c$
$c$
$c$
$c$

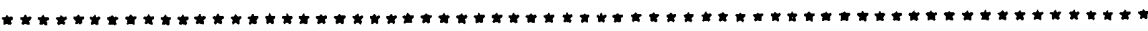

infut:

- $t=$ TEMPERATURE IN $k$

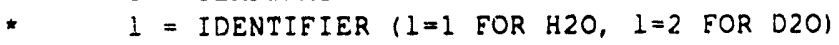




\begin{tabular}{|l|ll|r|}
\hline Pg. 50 of 354 & WSRC-TR-93-086 Rev. 0 & FLOWTRAN-TF v1.2 Source Code & $2 / 15 / 93$ \\
\hline
\end{tabular}

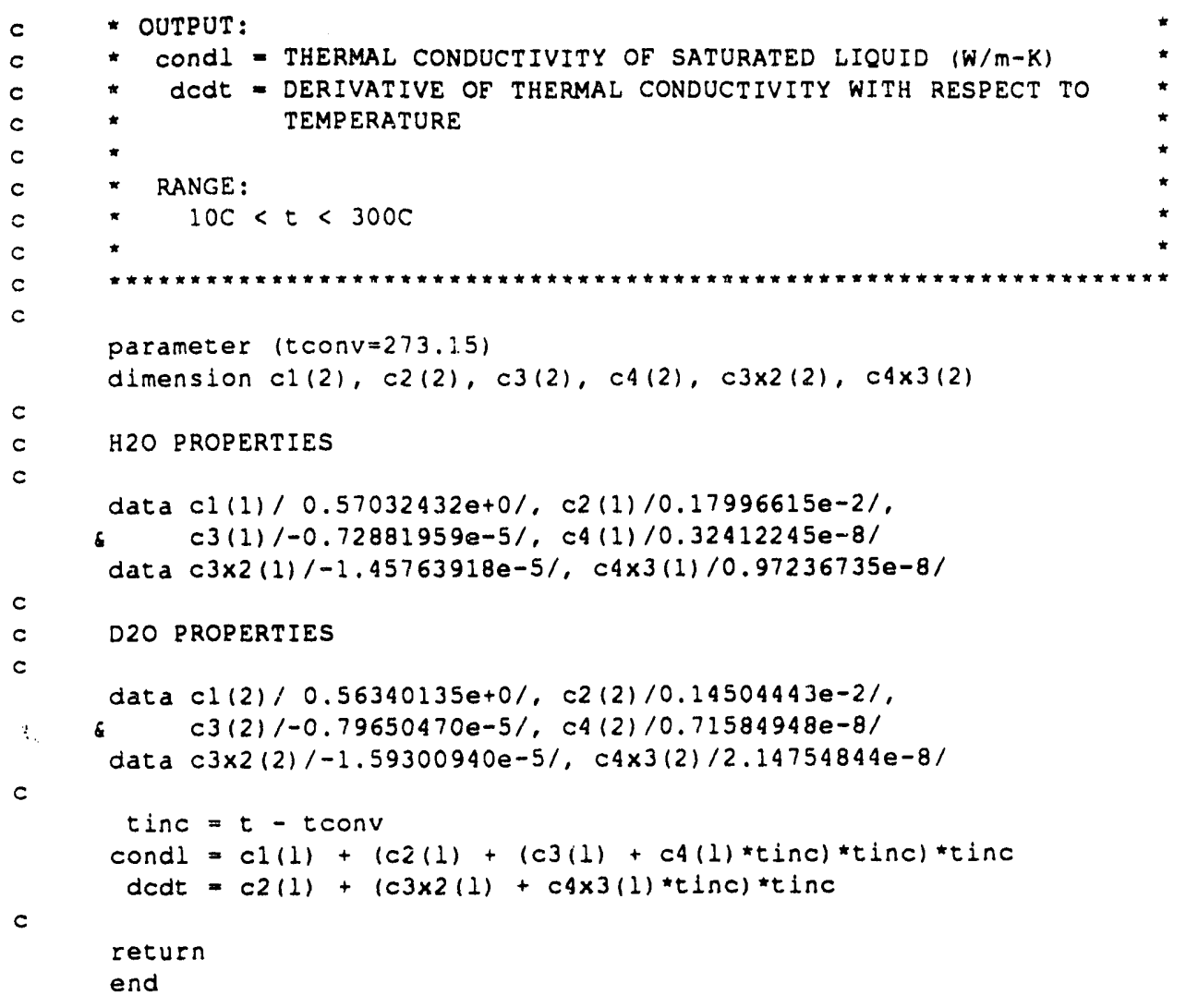

\section{CONDT}

function condt (mat, $t$, wtpc)

$$
\star
$$$$
\star
$$

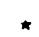$$
\star
$$$$
\star
$$

FUNCTION CONDT: THIS FUNCTION RETURNS THERMAL CONDUCTIVITY OF THE MATERIAL INDICATED BY mat AT THE TEMPERATURE $t$ IN UNITS OF $W / m-K$. FOR ALLOYS, WTPC GIVES EITHER THE WEIGHT PERCENT LITHIUM IN ALUMINUM OR THE WEIGHT PERCENT URANIUM IN ALUMINUM

THE FOLLOWING MATERIALS ARE AVAILABLE:

1 - URANIUM

2 - ALUMINUM

3 - 304L STAINLESS STEEL

4 - LITHIUM-ALUMINUM ALLOY

5 - URANIUM-ALUMINUM ALLOY

6 - ALUMINUM 6063

7 - MACOR

8 - INCONEL 600

9 - FLAME SPRAYED OXIDE

*

implicit real $(a-h, 0-z)$, integer $(1-n)$

parameter (one $=1.0$ )

dimension coef(36), clial(2), cual(3)

include 'knobs.ine' 


\section{CONLAW}

subroutine conlaw (p,tg,tf, xa, $1, n, n z, v i s g, c p g$, condg, diffm,

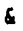$$
\text { visl, cpl, condl, sigma) }
$$

\section{ALLOY WEIGHT PERCENT COEFEICIENTS}

data clial/ 3.130461e-02, 1.281840e-00/

data cual/-5.69000e-04, 4.500000e-06, 1.90000e-07/

CONVERSION FACTOR FOR THERMAL CONDUCTIVITY TO SI UNITS

$\operatorname{data} \operatorname{csik} / 6.230645 e+03 /$

CALCULATE THERMAL CONDUCTIVITY IN ENGLISH UNITS

$\begin{aligned} m f & =4 * m a t-3 \\ c o n d t & =\operatorname{coef}(m f)+(\cos f(m f+1)+(\operatorname{coe} f(m f+2)+\operatorname{coe} f(m f+3) \star t) \star t) \star t\end{aligned}$

INCLUDE FUNCTIONS OF WEIGHT PERCENT ALUMINUM FOR ALLOYS

if (mat.eq.4) then

condt $=$ condt $+\operatorname{clial}(1) /($ one $+\operatorname{clial}(2) * w t p c)$

else if (mat.eq.5) then

condt $=$ condt $+($ cual $(1)+\operatorname{cual}(2) * w t p c+\operatorname{cual}(3) * t) * w t p c$

end if

CONVERT THERMAL CONDUCTIVITY TO SI UNITS

NOTE: xkmet IS A SENSITIVITY MULTIPLIER

condt $=x k m e t \star c s i k \star c o n d t$

return

end

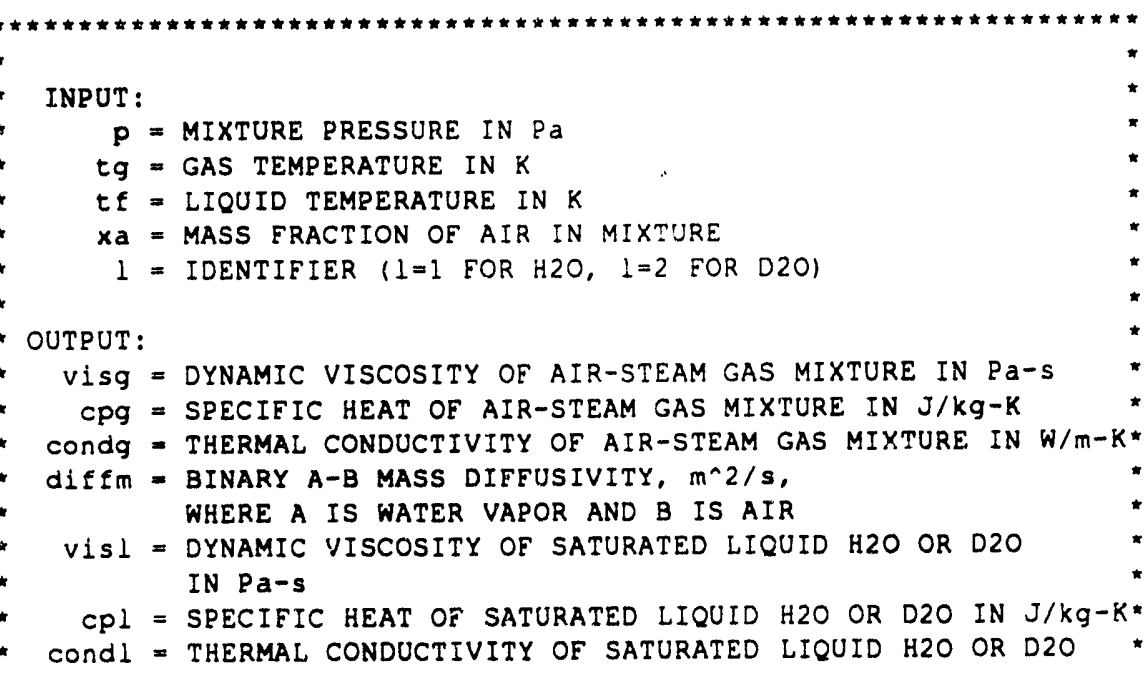




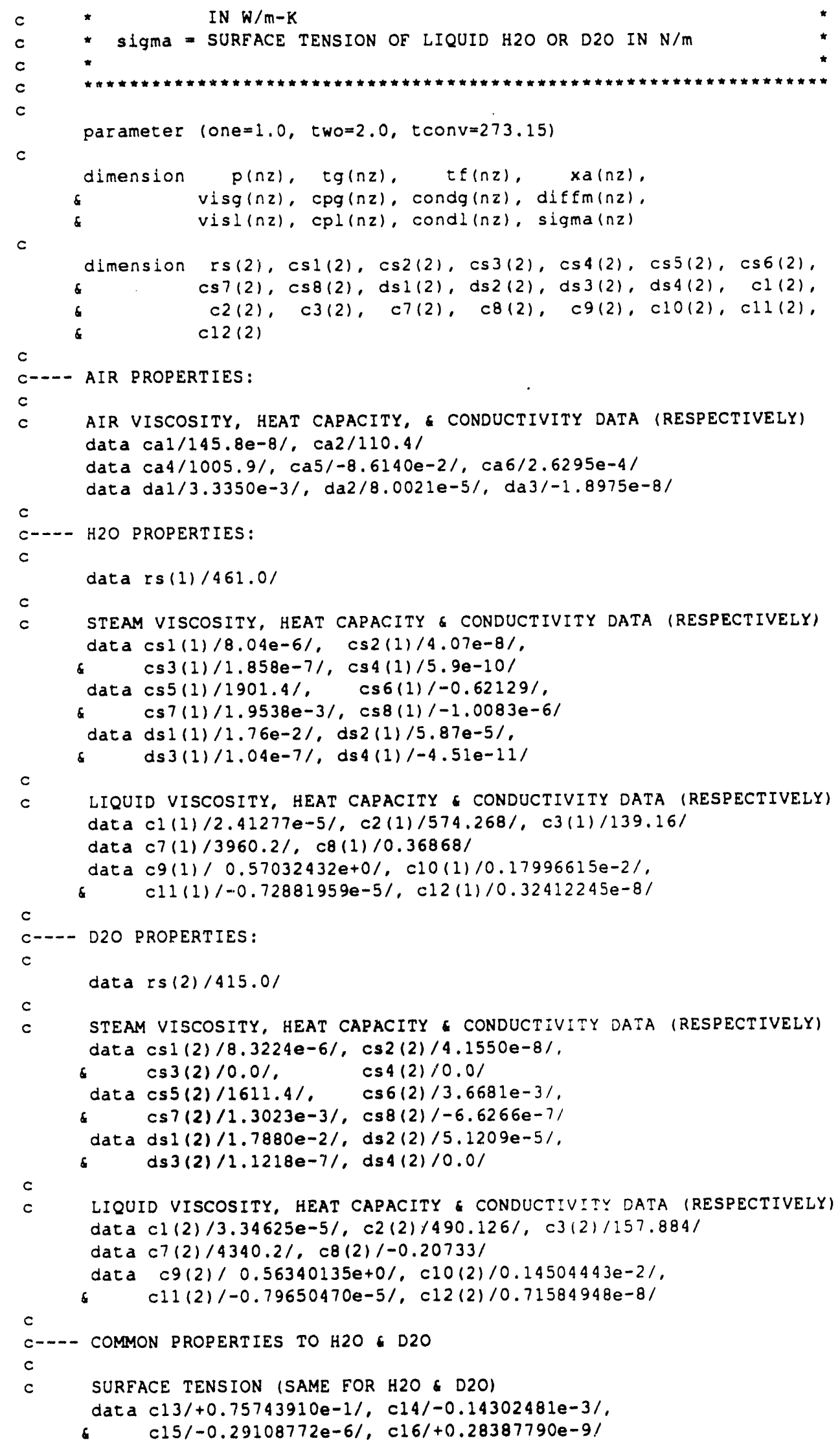




\begin{tabular}{|l|ll|l|}
\hline $2 / 15 / 93$ & WSRC-TR-93-086 Rev. 0 & FLOWTRAN-TF v1.2 Source Code & Pg. 53 of 354 \\
\hline
\end{tabular}

C

c

c

c

c

$c$

c

c

c

c

$c$

c

C

100 continue

c

DIFFUSIVITY FARAMETERS (ASSUMED SAME FOR H2O D2O)

data convet/1.01325e-4/, al/0.187/, sl/2.072/, ah/2.75/, sh/1.632/

do $100 j=n, n z$

AIR TRANSPORT PROPERTIES

visa $=\operatorname{cal} *(\operatorname{sqct}(\operatorname{tg}(j))) * 3 /(\operatorname{tg}(j)+\operatorname{ca} 2)$

conda $=d a 1+(d a 2+d a 3 * t g(j)) * t g(j)$

$c p a=c a 4+(c a s+c a 6 * t g(j)) * t g(j)$

STEAM TRANSPORT PROPERTIES

$\operatorname{ros}=p(j) /(\operatorname{rs}(1) * t g(j))$

tinc $=t g(j)-t \operatorname{conv}$

viss $=\operatorname{cs} 1(1)+\operatorname{cs} 2(1) \star \operatorname{tinc}-\cos *(\operatorname{cs} 3(1)-\operatorname{cs} 4(1) \star \operatorname{tin} c)$

conds $=(d s 1(1)+(d s 2(1)+(d s 3(1)+d s 4(1) * \operatorname{tinc}) * t i n c) * t i n c)$

$\operatorname{cps}=\operatorname{css}(1)+(\operatorname{cs} 6(1)+(\operatorname{cs} 7(1)+\operatorname{cs} 8(1) * \operatorname{tg}(j)) * \operatorname{tg}(j)) \star \operatorname{tg}(j)$

GAS TRANSPORT PROPERTIES - WEIGHT BY MASS FRACTION

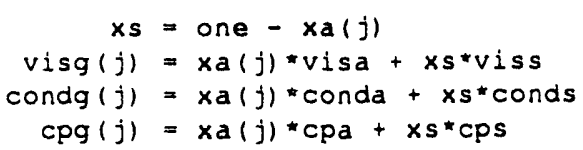

LIQUID TRANSPORT PROPERTIES

$t$ inc $=t E(j)-t \operatorname{conv}$

$\operatorname{visl}(j)=c l(l) \star \exp (c 2(1) /(t f(j)-c 3(1)))$

$c p l(j)=c 7(1)+t w o * c 8(1) * t f(j)$

condl $(j)=c 9(1)+(c 10(1)+(c 11(1)+c 12(1) * \operatorname{tinc}) * \operatorname{tinc}) * \operatorname{tinc}$

SURFACE TENSION

$\operatorname{sigma}(j)=c 13+(c 14+(c 15+c 16 * \operatorname{tinc}) * \operatorname{tinc}) * \operatorname{tinc}$

MASS DIFFUSIVITY

$\operatorname{diffm}(j)=\min (a l *(t g(j) * s l) * \operatorname{convrt} / p(j)$,

$a h *(t g(j) * \operatorname{sh}) * \operatorname{convrt} / p(j) 1$

return

end

\section{CONST}

subroutine const (ptn, alptn, tgtn, tftn, xatn, rogtn, roftn, volt,

pn, alpn, tgn, ten, xan, rogn, rofn, volc,

pbn, alpbn, tgbn, tfbn, xabn, rogbn, rofbn, volb,

ugt, uft, act, defft, dhe, cost, fldmt, fldct,

cobt, cost, ckbt, ckst,

ugz, ufz, acz, deffz, dhz, $\cos z, f \operatorname{dm} z, f l d c z$,

cObz, c0sz, ckbz, cksz,

$u g b, u f b, a c b$, deffb, $d h b, c o s b, f l d m b, f l d c b$,

$c O b b, c 0 s b, c k b b, c k s b$,

hgit, hfit, akgit, ysit, citn,

hgi, hfi, akgi, ysi, cizn,cixn,

hgib, hfib, akgib, ysib, cibn,

ppln, alppln, tgpln, tfpln, xapln,

rogpln, rofpln, volpl.

ptbn, alptbn, tgtbn, tftbn, xatbn, 


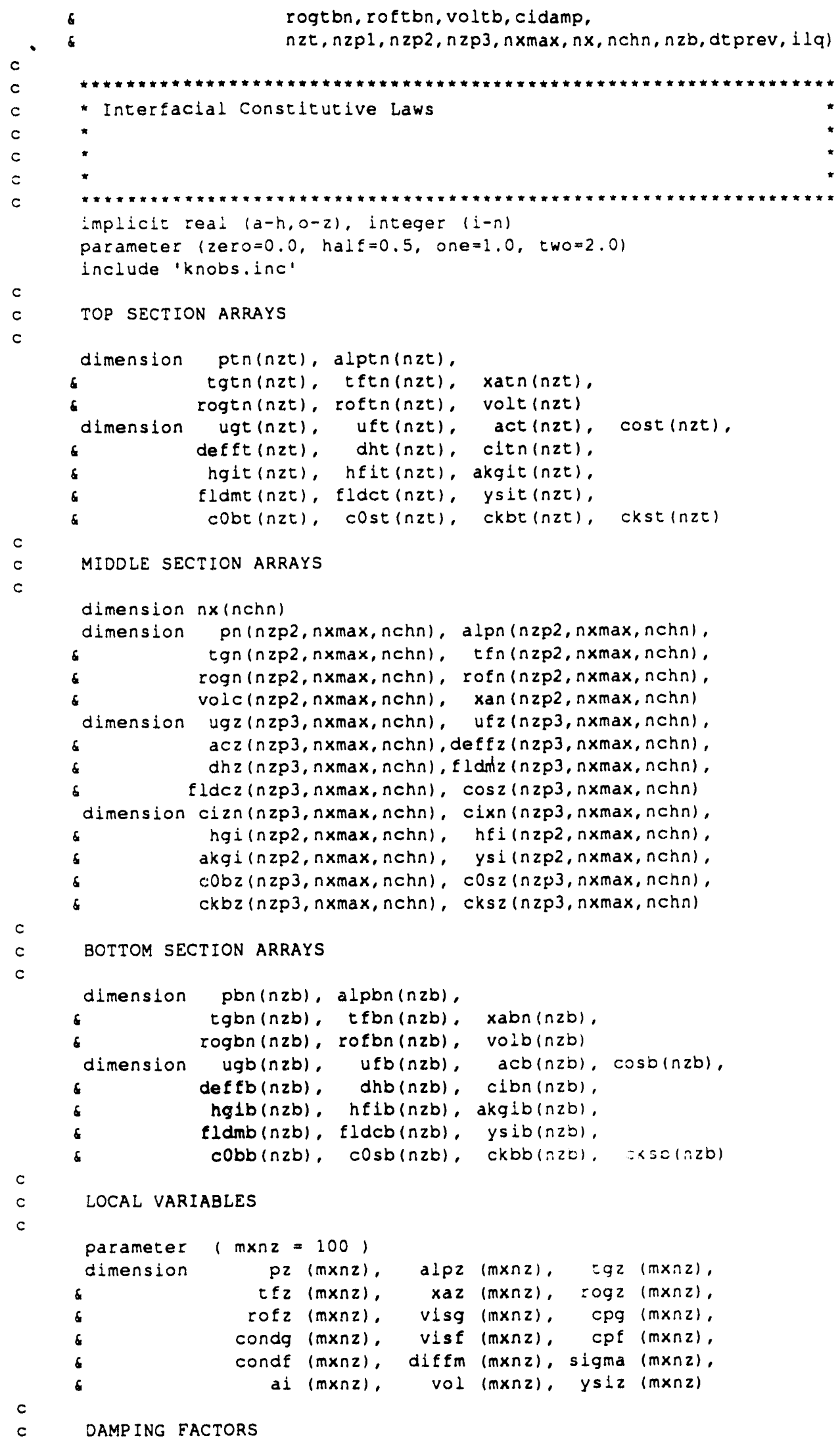


$c$ else 
200 cont inue

call conlaw $(p z, t g z, t f z, x a z, 11 q, 2, n z p 2, v 1 s q, c p q$, condg, diffm, visf, cpf, condf, slgma)

$c$

do $205 j z=2, n z p 2$

$j z m=j z-1$

cali intara $(a i(j z)$, citemp, akgin, hgin, hfin,

alpz $(j z)$, deffz(jz,ix, lc), fldmz $(j z, i x, i c)$,

$f i d c z(j z, i x, i c), \operatorname{cobz}(j z, i x, 1 c), \cos z(j z, i x, 1 c)$. $c k b z(j z, i x, i c), c k s z(j z, 1 x, 1 c), d h z(j z, 1 x, 1 c)$, $\operatorname{vol}(j z), \operatorname{acz}(j z, i x, i c), \cos z(j z, i x, 1 c), \operatorname{rof} z(j z)$, $\operatorname{rog} z(j z), \operatorname{sigma}(j z), u \in z(j z, i x, 1 c), u g z(j z, 1 x, i c)$.

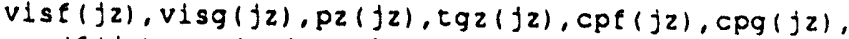

c condf $(f z)$, condg $(f z), d f f f(f z), y s i z(f z), x a z(j z))$

citemp $=-\min (c 1 z f a c, z e r o)+\max (c 1 z f a c, z e r o) * c i t e m p$

if (cldamp.ge, zero) then else

$\operatorname{cizn}(f z, 1 x, 1 c)=\operatorname{decay} 1 * \operatorname{clzn}(j z, 1 x, 1 c)+\operatorname{decay} 2 * c 1 t e m p$

$\operatorname{cizn}(j z, i x, i c)=\max (\min (c i t e m p, c i z n(j z, i x, i c) /$ decayl),

end if

clzn $(j z, i x, 1 c) \star$ decay 1$)$

4

$c i x n(j z, i x, i c)=\max (c i x f a c, z e r o)$

- min(cixfac, zero)*cizn(jz, ix, ic)

$h f i(j 2 m, i x, 1 c)=h f i(j 2 m, 1 x, 1 c)$ thalf*hfin

$h g i(j 2 m, 1 x, i c)=h g i(j 2 m, 1 x, 1 c)$ + half* hgin

akgi(jzm,ix, ic) =akgl(jzm,ix,ic) +half*akgin

$c$

if (cidamp, ge. zero) then

$h \in 1(j z m, 1 x, 1 c)=\operatorname{decay} 1 * h f m i d+\operatorname{decay} 2 * h f 1(j z m, 1 x, 1 c)$

$h g i(j z m, 1 x, i c)=$ decayl*hgmid + decay 2 *hgl(jzm, ix, ic)

else

akgi(jzm, ix,ic) = decayl*akgmid + decay $2 * a k g i(j z m, i x, i c)$

$\sqrt{2}$

$h f i(j z m, 1 x, 1 c)=\max (\min (h f i(j z m, 1 x, 1 c), h f m i d / d e c a y l)$, $h f m i d *$ decayl)

$h g i(j z m, i x, i c)=\max (m i n(\operatorname{hgi}(j z m, i x, i c)$, hgmid/decayl), hạid*decay 1 )

$\operatorname{akgi}(j z m, i x, i c)=\max (\min (\operatorname{akg} i(j z m, i x, i c), a k g m i d / d e c a y l)$,

end if c

$h f m i d=h f i(j z, i x, i c)$

hgmid $=h g 1(j z, 1 x, 1 c)$

$\operatorname{akgmid}=\operatorname{akgl}(j z, 1 x, 1 c)$

c

$h \in 1(j z, 1 x, 1 c)=h a l f$ h $h f 1 n$

$\operatorname{hg} 1(j z, 1 x, 1 c)=$ half* hgin

$\operatorname{akg} 1(j z, 1 x, 1 c)=\operatorname{half*akgin}$

$c$

it (jz .eq. 2) then

wht $=$ half*volc $(1, i x, i c) / \operatorname{volt}(n z t)$

hfit (nzt) = helt (nzt) + wght "hfin

hgit $(n z t)=h g i t(n z t)+$ wght* hgin

akgit $(n z t)=\operatorname{akgit}(n z t)+$ wght*akgin

c

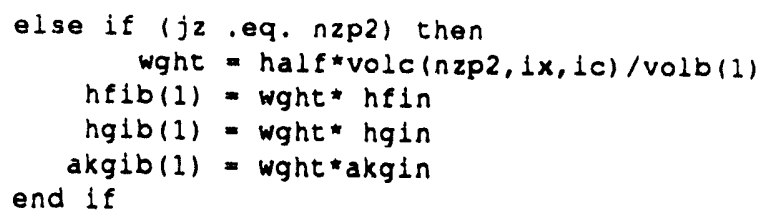




\begin{tabular}{|l|lll|l|}
\hline $2 / 15 / 93$ & WSRC.TR-93-086 Rev. 0 & FLOWTRAN-TF v1.2 Source Code & Pg. 59 of 354 \\
\hline
\end{tabular}

diffm, visf, cpf, condf, sigma)

$c$

do $305 f z=1, n z b$

$j z p=j z+1$

call intara (al $(j z)$, clcemp, akgin, hgin, hfin,

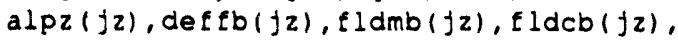
$\operatorname{cobb}(j z), c 0 s b(j z), c k b b(j z), c k s b(j z)$, $\operatorname{dhb}(j z), \operatorname{vol}(j z), a c b(j z), \operatorname{cosb}(j z), \operatorname{rof} z(j z)$. $\operatorname{rog} z(j z), s \operatorname{lgma}(j z), u f b(j z), u g b(j z), v i s f(j z)$, visg $(j z), p z(j z), \operatorname{tgz}(j z), \operatorname{cpt}(j z), \operatorname{cpg}(j z)$, condf $(j z), \operatorname{condg}(j z), \operatorname{diffm}(j z), y \operatorname{siz}(j z), x a z(j z))$

$c$

citemp $=-\min (c i z f a c, z e r o)+\max (c \downarrow z f a c, z e r o)$ "citemp

If (cldamp.ge. zero) then

clbn $(f z)=\operatorname{decay} 1 * \operatorname{cibn}(j z)+\operatorname{decay} 2 * c i t e m p$ else

$c \mid b n(j z)=\max (\operatorname{mln}(c i t e m p, c i b n(j z) / \operatorname{decay} 1), c i b n(j z)$ decay 1$)$

c end if

$h f i b(j z)=h \in i b(j z)+h a l f * h \in \ln$

$h g(b(f z)=h g i b(f z)+h a l f * h g i n$

$a k g i b(j z)=a k g i b(j z)+h a l f * a k g i n$

C

if (cldamp ge. zero) then

$h \in(b(j z)=$ decayl *hfbot + decay $2 * h f i b(j z)$

$n g i b(j z)=\operatorname{decay1}$ * ngbot + decayz*hgib(jz)

$a k g 1 b(j z)=\operatorname{decay} 1 * a k g b o t+\operatorname{decay} 2 * a k g i b(j z)$

else

$h f i b(j z)=\max (\min (h f i b(j z), h f b o t / \operatorname{decay} 1), h f b o t * \operatorname{dec} a y 1)$

$h g i b(j z)=\max (\min (\mathrm{hg} \mid \mathrm{b}(j z)$, hgbot/decayl), hgbot*decayl)

$a k g i b(j z)=\max (\min (a k g i b(j z), a k g b o t / d e c a y 1), a k g b o t *$ decay 1$)$

c end if

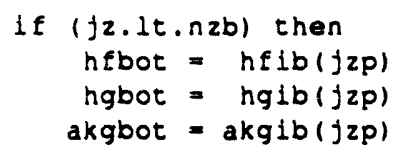

c

$h \in 1 b(j 2 p)=h a l f * h \in i n$

hgib(jzp) = half* hgin

end $1 f$

akgib $(j z p)=$ half*akgin

$c$

305 continue

c

return

end

\section{CPGS}

subroutine cpgs $(t, x a, l, c p g)$

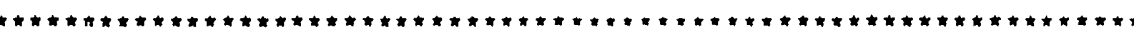

INPUT:

$t=$ TEMPERATURE IN $K$

Xa = MASS FRACTION OF AIR IN MIXTURE

$1=\operatorname{IDENTIFIER~}(1=1$ FOR H2O, $1=2$ FOR D2O

OUTPUT:

- $\quad$ p = SPECIFIC HEAT OF AIR-STEAM GAS MIXTURE IN J/kg-K

RANGE:

$275 K<t<700 K$ 


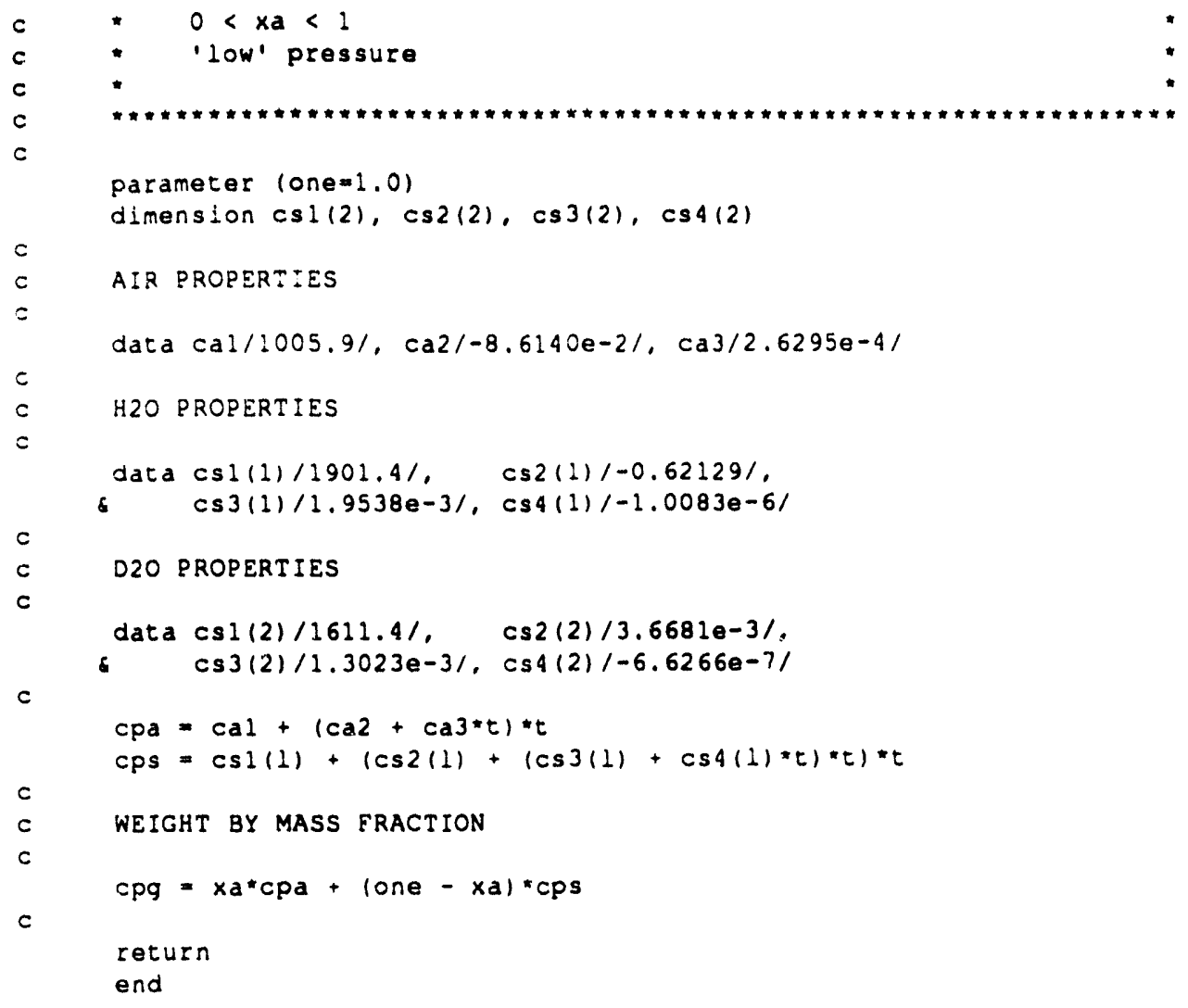

\section{CPIQ}

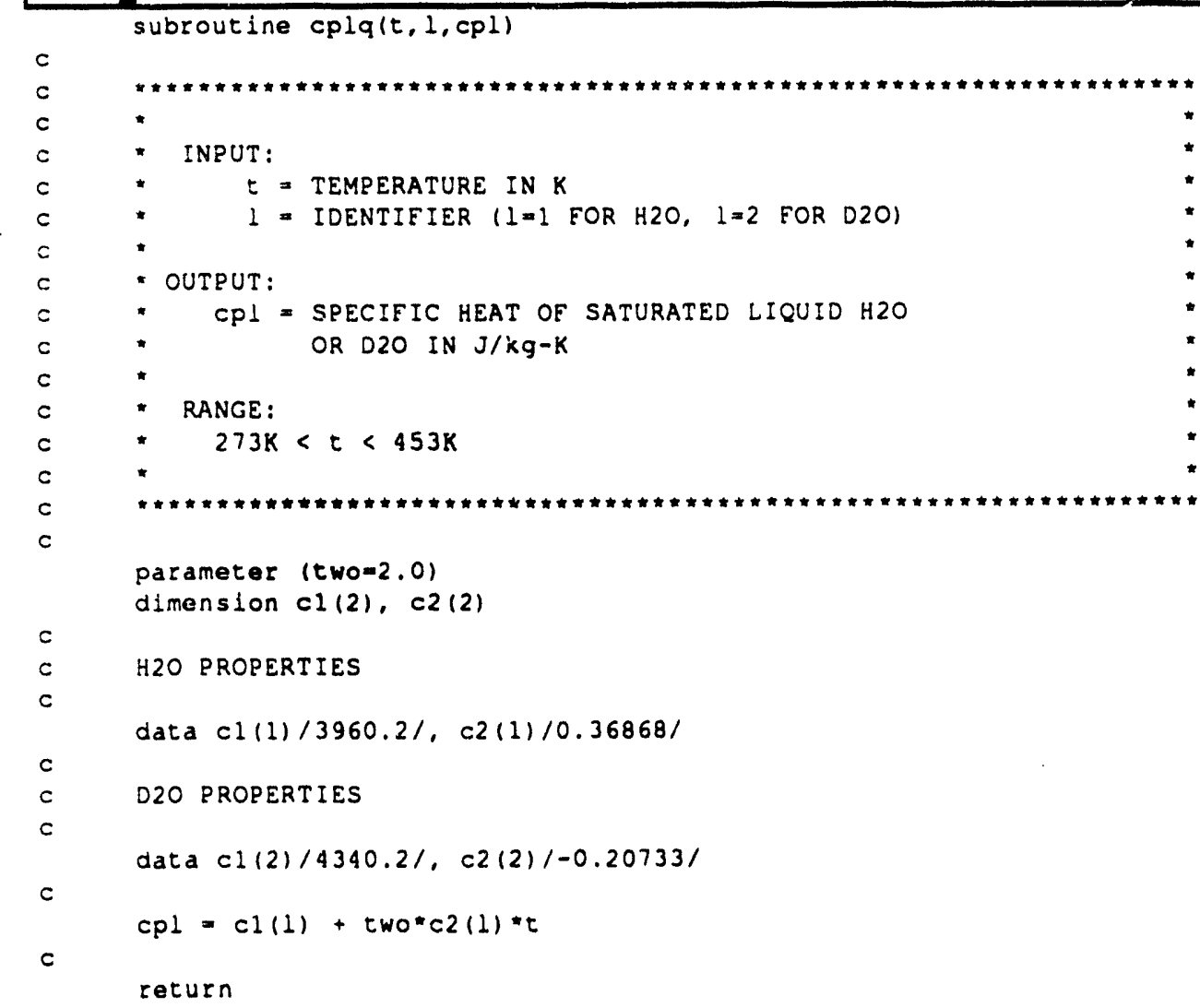

subroutine $\operatorname{cplq}(t, 1, \operatorname{cpl})$ 


\begin{tabular}{|l|lll|l|}
\hline $2 / 15 / 93$ & WSRC-TR-93-086 Rev. 0 & FLOWTRAN-TF v1.2 Source Code & Pg. 61 of 354 \\
\hline
\end{tabular}

end

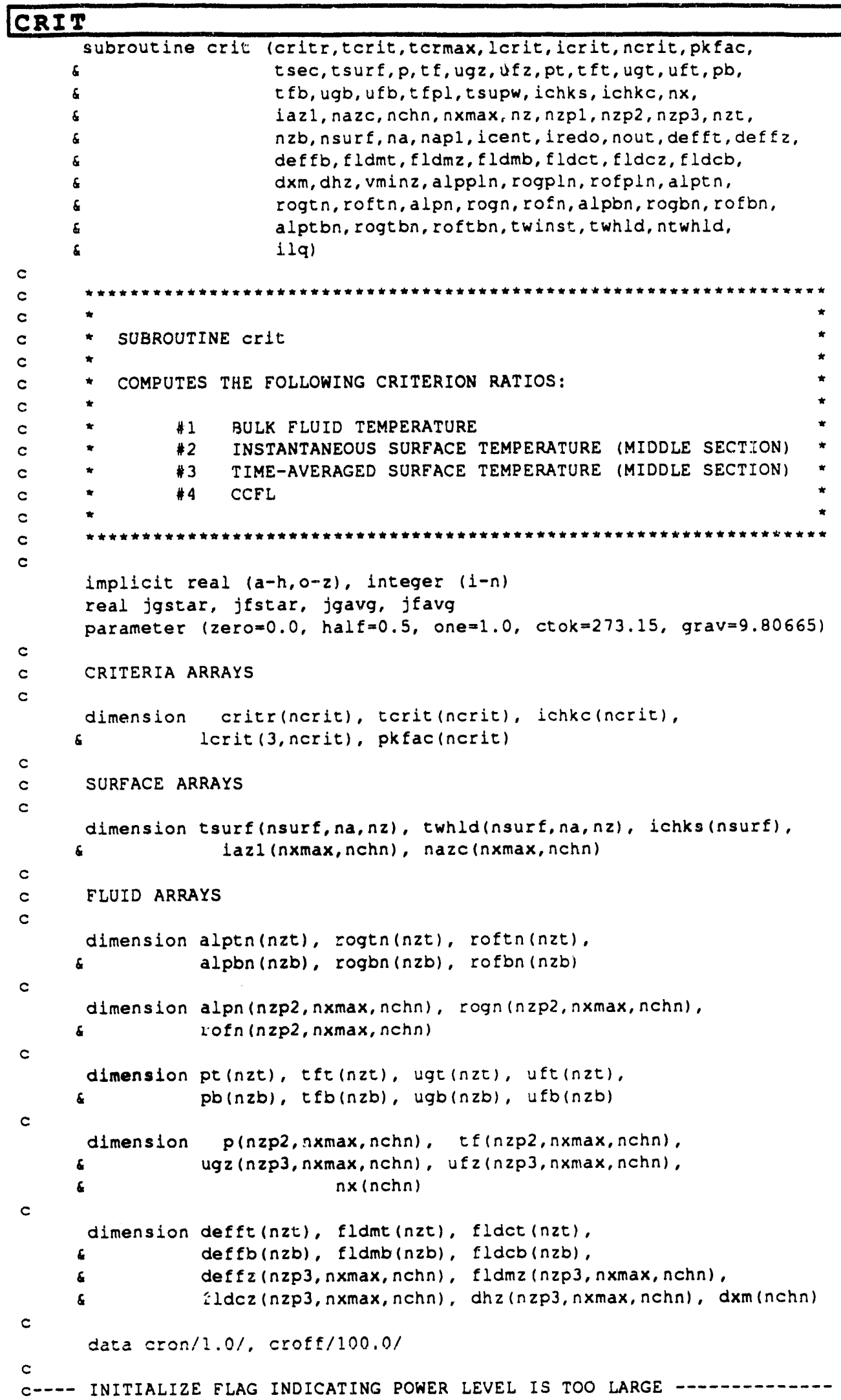


$c$

iredo $=0$

twinst $=$ zero

c

c

c

$c$

c

$c$

$c$

$c$

c

DO CRITERIA CHECKING IF CURRENT TIME GREATER THAN

CRITICAL SPECIFIED TIME; CONIINUE INITIALIZIN'

if $(t \sec .1 t$. tcrmax) return

critg $(3)=$ zero

ntwhld $=$ ntwhld +1

IF CRITERIA CHECKING FOR WALL SATURATION IS ON, LIMIT THE

FLUID SATURATION RATIO < 1 TO ACCELERATE ITERATIONS

if (ichkc(2) .eq. 1) then

crmax $=$ cron

else

$c r \max =\operatorname{croff}$

end if

$c$

$\mathrm{c}$

c

$c$

$c$

c

$c$ 
$c$

c

c

c

c-n-

c else
10 continue

$a l p m=\operatorname{alptn}(j z m)$

$\operatorname{rogm}=\operatorname{rogtn}(j \mathrm{zm})$

end if

$\operatorname{rofm}=\operatorname{roftn}(j 2 m)$

$f a c g=\operatorname{sign}($ half, $\operatorname{ugt}(j z)$ )

fact $=\operatorname{sign}($ half, uft $(j z))$

if (abs(ugt(jz)). It. vminz) facg = half*ugt (jz)/vminz

if $\left(\operatorname{abs}(u f t(j z))\right.$. Lt. vminz) facf $=\operatorname{nal} f^{*} u f t(j z) / \mathrm{vminz}$

wtgm $=$ half $+\mathrm{Eacg}$

wt $f m=$ half + fact

wtgp = one - wtgm

wtfp = one - wtfm

$w a l p g=w t g m * a l p m+w t g p \star a \perp p t n(j z)$

$w a l p f=w t f m * a l p m+w t f p * a l p t n(j z)$

wrog $=w t g m * \operatorname{rogm}+w t g p * \operatorname{rogtn}(j z)$

wrof $=w t f m * r o f m+w t f p * r o f t n(j z)$

drogd $=($ wrof - wrog $)$ grav*defft $(f z)$

jgstar $=\quad$ walpg *ugt $(j z) * s q r t(w r o g / d r o g d)$

ffstar $=($ one-walpf)*uft $(j z) * \operatorname{sqrt}($ wrof/drogd)

if (jfstar.gt. zero. and. jgstar. It. zero) then

$c n=\operatorname{sqrt}(a b s(j g s t a r))+f \operatorname{ldmt}(j z) * \operatorname{sqrt}(a b s(j f s t a r))$

$c_{n}=$ zero

end if

ratio $=p k f a c(4) * c n / f l d c t(j z)$

if (ratio.gt. critr(4)) then

tcrit $(4)=t \sec$

critr (4) = ratio

1 crit $(1,4)=0$

lcrit $(2,4)=1$

$\operatorname{lorit}(3,4)=j z$

end if

PRINT NONDIMENSIONAL SUPERFICIAL VELOCITIES

IF CRITERIA PRINT IS REQUESTED AND CRITERION EXCEEDED

if (icrit.eq.1 and. ratio.gt.one) then

write (nout, 25) tsec, jz, jgstar, jfstar

write $(*, 25)$ tsec, jz, jgstar, jfstar

end if

MIDDLE SECTION

do $601 \mathrm{c}=1$, nchn

Istet $=2$ *ic- $1+i \operatorname{cen} t$

isrfo = isrfi +1

$n s u b=n \times(i c)$

c

do $50 \quad i x=1$, nsub

iazf $=1 \mathrm{az} l(i x, i c)$

$i a z l=1 a z f+\operatorname{nazc}(1 x, i c)-1$

c

do $40 j z=2, n z p 1$

$j z 1=j z-1$

COMPUTE CRITERION RATIO \# 1 (BULK FLUID TEMPERATURE)

$t b u l k=t f(j z, i x, i c)$

cail tsat (p(jz,ix,ic), 1lq, tisat, xjunk) 


\begin{tabular}{|l|ll|r|}
\hline Pg. 64 of 354 & WSRC-TR-93-086 Rev.0 & FLOWTRAN-TF v1.2 Source Code & $2 / 15 / 93$ \\
\hline
\end{tabular}

c

c

$c$

c

c

c

c

c

c

c

c

1111

c

c

C

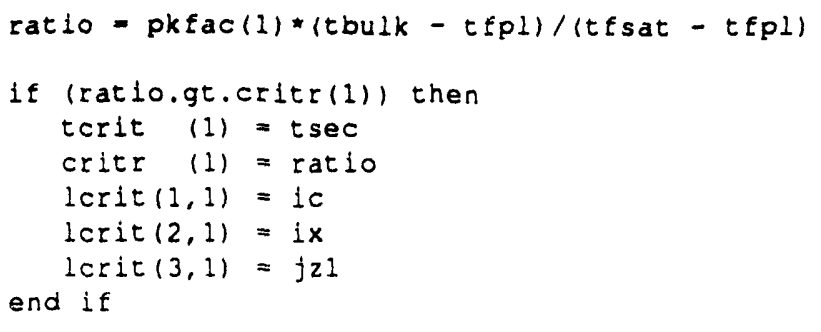

PRINT BULK FLUID TEMPERATURE AND SATURATION TEMPERATURE

IF CRITERIA PRINT IS REQUESTED AND CRITERION EXCEEDED

if (icrit.eq.1 and. ratio.gt.one) then

write (nout, 35) tsec, ic, ix, jzl, tfsat-ctok, tbulk-ctok

write $(*, 35)$ tsec, ic, ix, j2l, tfsat-ctok, tbulk-ctok

end if

IF CRITERION \#I EXCEEDED,

STOP TRANSIENT CALCULATIONS (IF CRITERION \#2 CHECKED)

if (ratio.ge. crmax) then

iredo $=1$

return

end if

COMPUTE CRITERION RATIO \#4 (CCEL)

if (ix.eq.1 and. jz.gt. 2$)$ then

jgavg $=$ zero

jfavg $=$ zero

do 1111 ixx=1, nsub

$j z m=j z-1$

facg $=\operatorname{sign}(h a 1 f, u g z(j z, i x x, 1 c))$

facf $=\operatorname{sign}($ half, $u f z(j z, 1 \times x, 1 c)$ )

if (abs(ugz(jz,ixx, ic)) . It . vminz)

$f a c g=\operatorname{half} u g z(j z, 1 x x, 1 c) / v m i n z$

if (abs (ufz(jz, ixx, 1c)) . It. vminz)

facf $=$ half*ufz(jz,1xx,1c)/vminz

wtgm $=$ half $+f a c g$

wt $f m=$ half + facf

wtgp = one - wtgm

wt $f p=$ one $-w t f m$

walpg =wtgmalpn $(j z m, 1 x x, 1 c)+w t g p * a \perp p n(j z, 1 x x, 1 c)$

walpf = wt $f m^{\star a} a p n(j z m, i x x, i c)+w t f p * a ! p n(j z, i x x, 1 c)$

wrog $=w t g m * r o g n(j 2 m, i x x, i c)+w t g p * \operatorname{rogn}(j z, i x x, i c)$

wrof = wt fmærofn(jzm, ixx,ic) + wtfp*rofn(jz,ixx,ic)

Jgavg = jgavg + walpg *ugz(jz,ixx,ic)

$j f a v g=j f a v g+($ one-walpf $) \neq u f z(j z, i x x, i c)$

continue

drogd $=(w r o f-w r o g) * g r a v \star d e f f z(j z, i x, i c)$

jgstar = jgavg/float (nsub) *sqrt (wrog/drogd)

jfstar = jfavg/float (nsub) *sqrt (wrof/drogd)

if (jfstar.gt. zero and. Jgstar. 1t. zerol then

$c_{n}=\operatorname{sqrt}(a b s(j g s t a r))+\operatorname{fldmz}(j z, i x, i c){ }^{*} \operatorname{sqrt}(a b s(j f s t a r))$

else

$c n=$ zero

end if

ratio $=p k f a c(4) * c n / f l d c z(j z, i x, i c)$ 


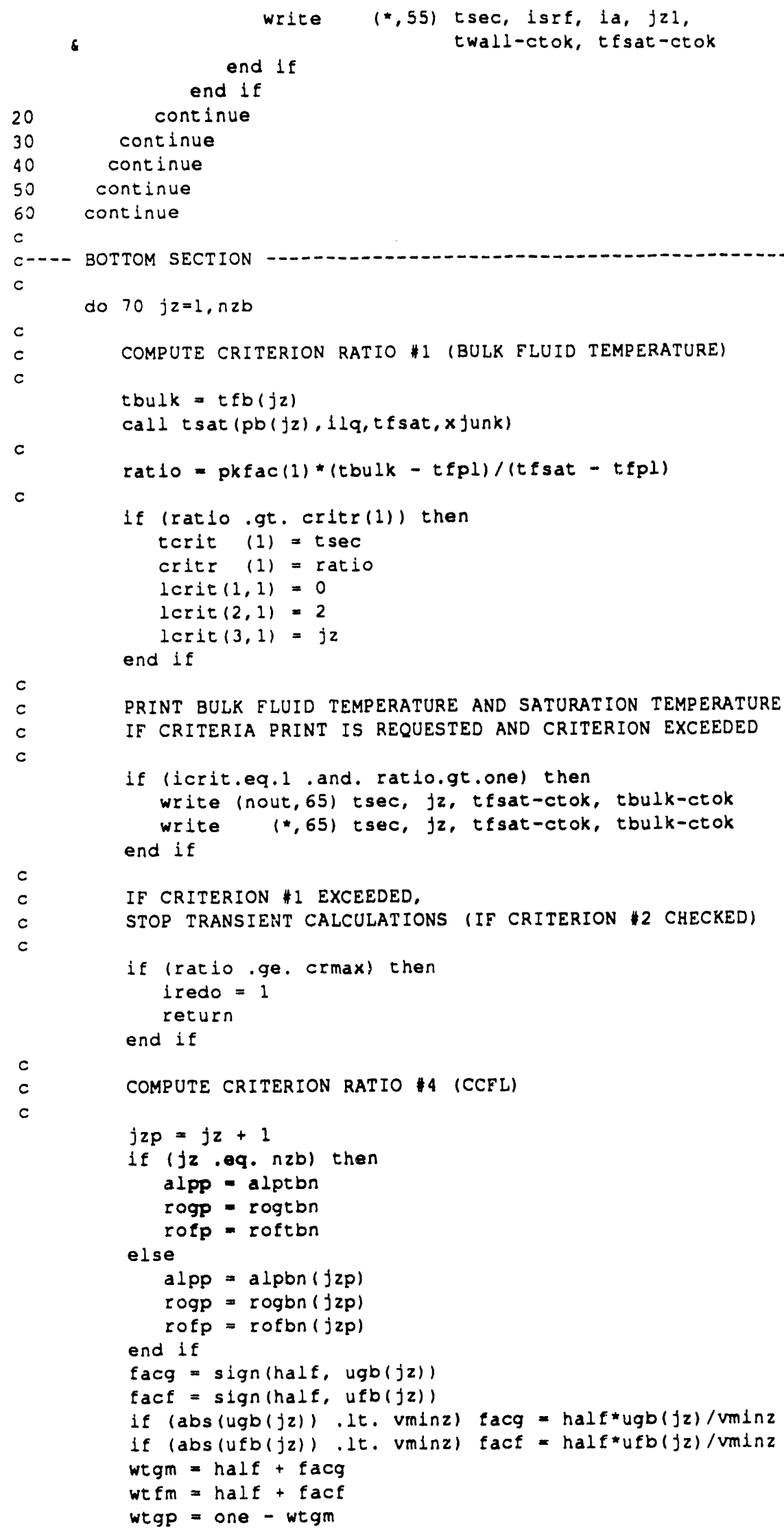


c

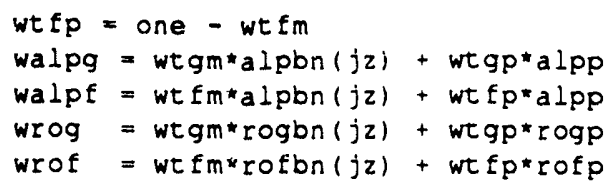




\begin{tabular}{|l|ll|r|}
\hline Pg. 68 of 354 & WSRC-TR-93-086 Rev. 0 & FLOWTRAN-TF v1.2 Source Code & $2 / 15 / 93$ \\
\hline
\end{tabular}

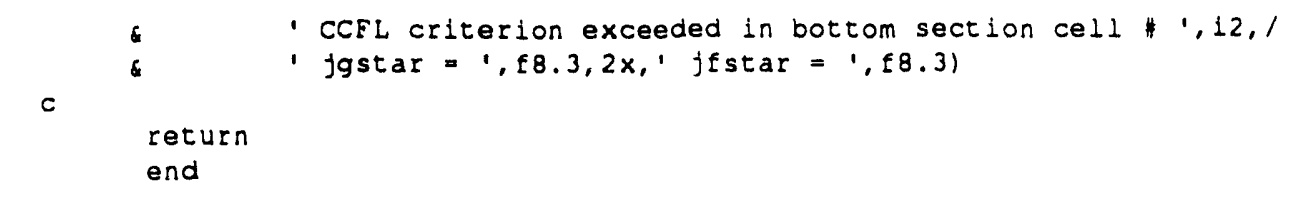

\section{CYINDR}

subroutine cylndr (ts, tsn, pows, tsurf,tsrfn, radc, ar, dr, zfacm,

vols, hcap, cond, chs, diag, cra,crc, ca, cb, trhs,

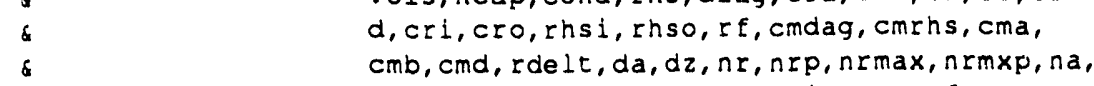

\& nsurf, nz, ncyln, icyln,wss, icent, qwf, qwg,

\& dqfdw, dqgdw, laxial, ncms)

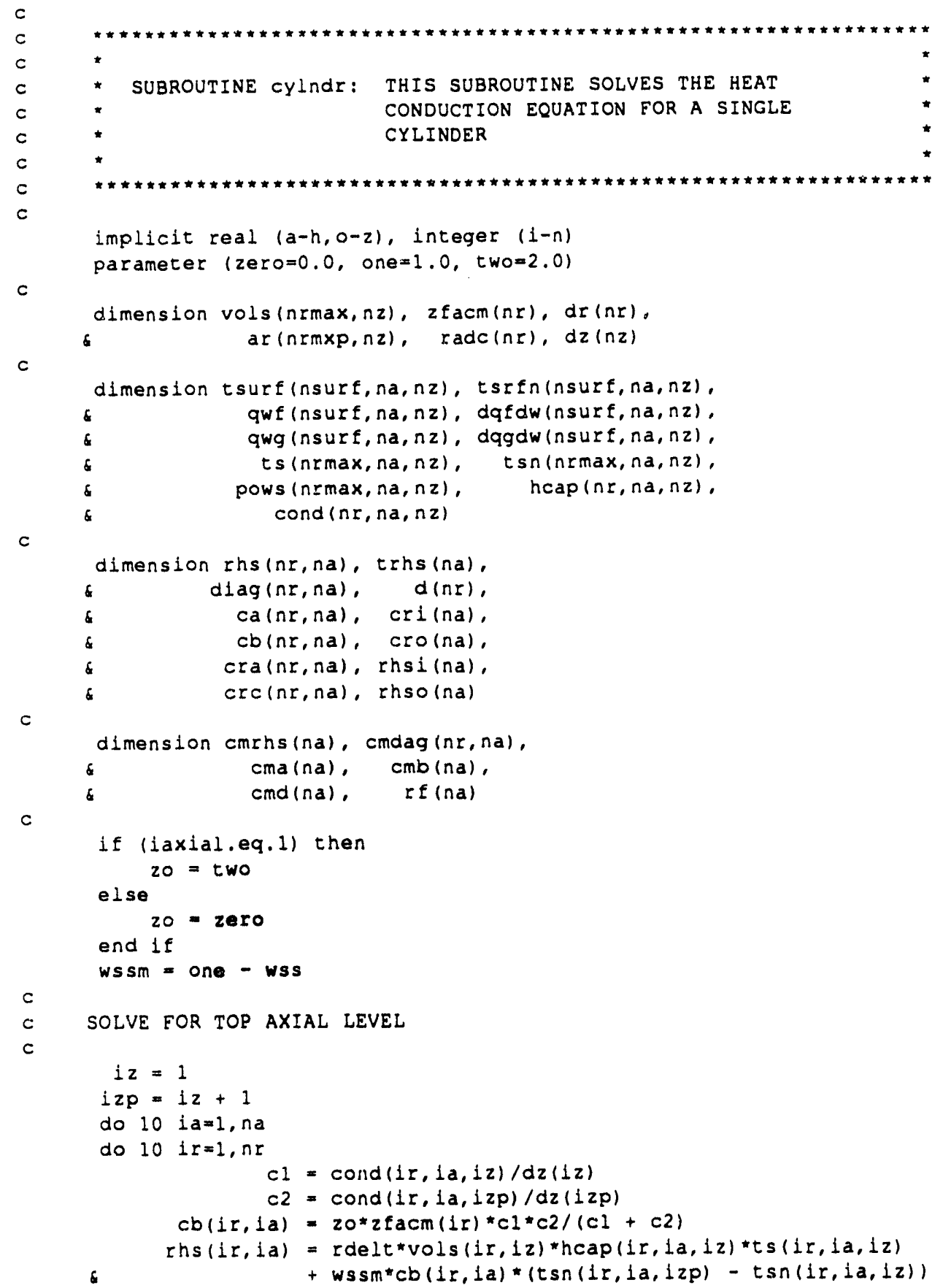




\begin{tabular}{|l|ll|l|}
\hline $2 / 15 / 93$ & WSRC-TR-93-086 Rev. 0 & FLOWTRAN-TF v1.2 Source Code & Pg. 69 of 354 \\
\hline
\end{tabular}

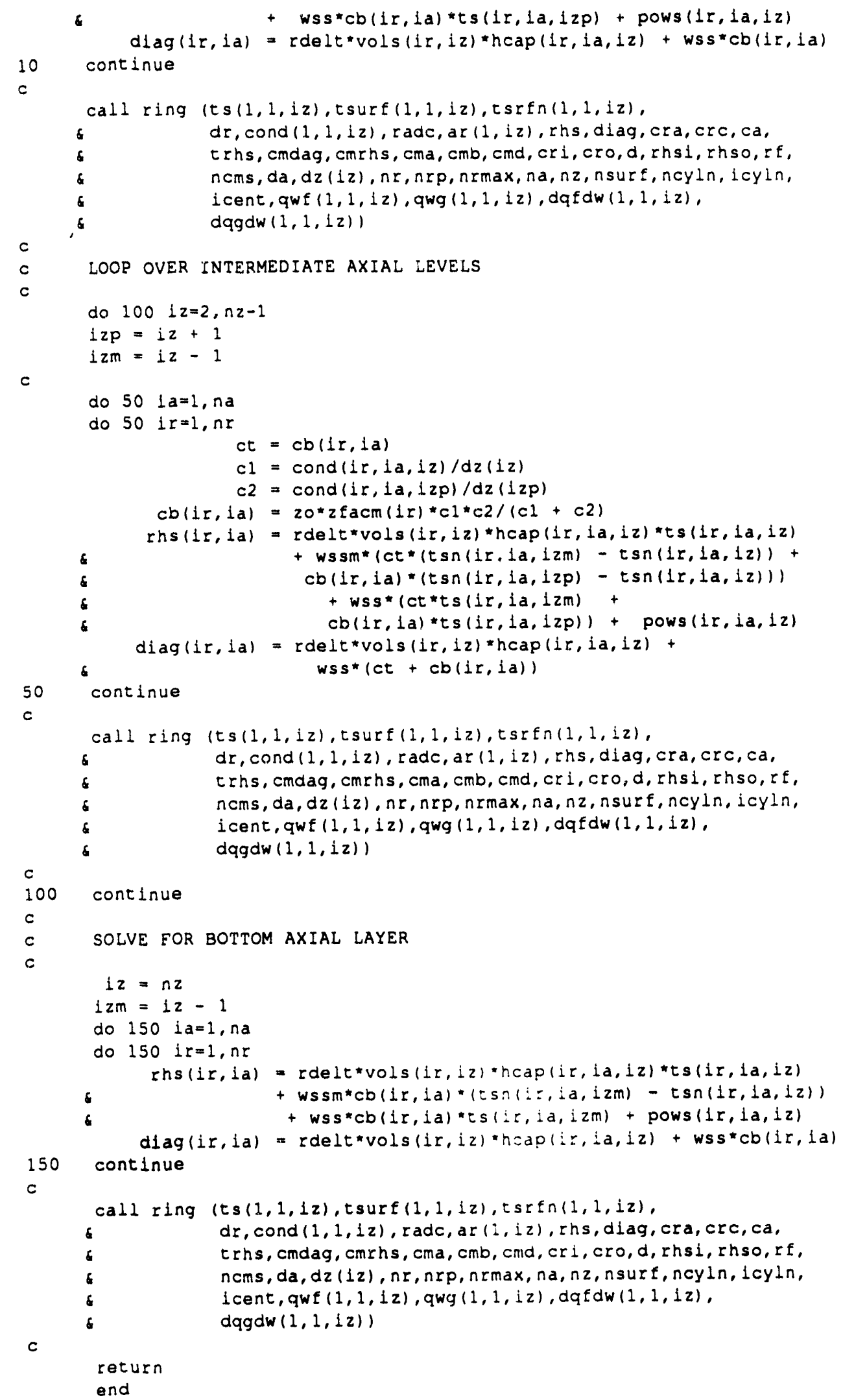




\section{DMPRIT}

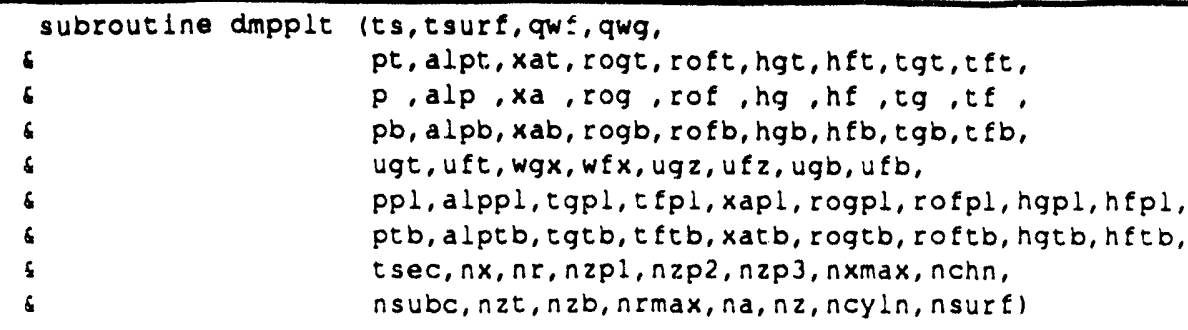




\begin{tabular}{|l|ll|l|}
\hline $2 / 15 / 93$ & WSRC-TR-93-086 Rev.0 & FLOWTRAN-TF v1.2 Source Code & Pg. 71 of 354 \\
\hline
\end{tabular}

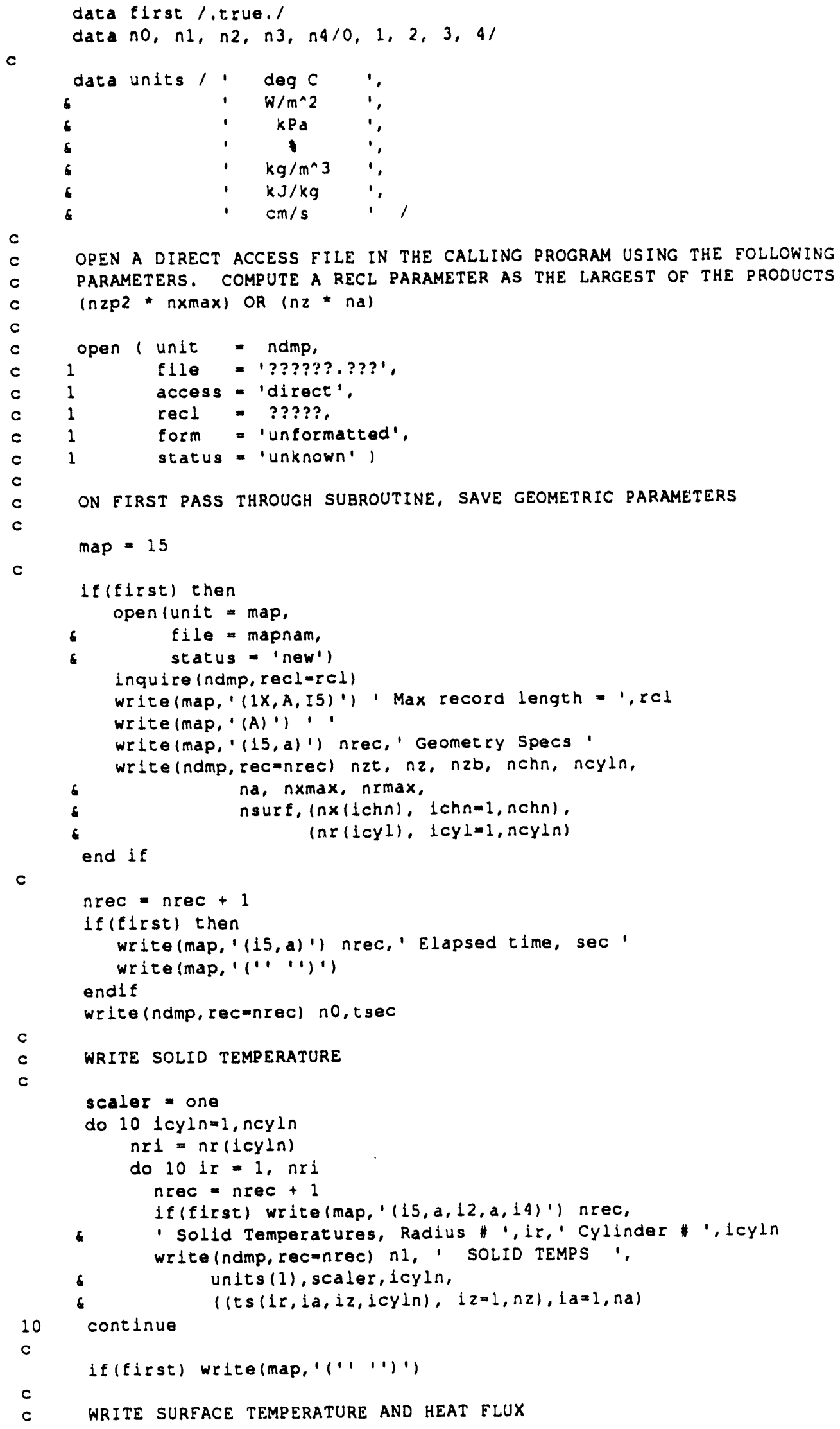


$c$

do 11 1surf $=2$, nsurf

nrec = nrec + 1

If(flrst) write(map, ' (i5, a, 12)') nrec,

- ' Temperatures, surface ' Isurf

write (ndmp, recanrec) n1, 'SURFACE TEMPS',

- units(1), scaler, isurf,

$\therefore$ continue (tsurf(1surf,1a,iz), $1 z=1, n z), 1 a=1$, na)

if(first) write(map, '(1' 1')

$c$

scaler $=$ one

do 12 lsurf $=2$, nsurf

nrec = nrec + 1

if(first) write(map, '(i5, a, i2)') nrec,

- 'Liq Heat Flx, Surface " isurf

write (ndmp, recenrec) nl, ' SURF HEAT FLUX',

- units(2), scaler, 1surf,

12

continue

( (qwf (1surf, 1a, iz), $i z=1, n z), 1 a=1, n a)$

if (first) write(map, ' $1, \cdots)$ ')

c

scaler = one

do 13 isurf $=2$, nsurf

nrec $=$ nrec +1

if (first) write(map, '(15,a, i2)') nrec

- 'Gas hear Flx, Surface ', isurf

write (ndmp, rec=nrec) nl, 'SURF HEAT FLUX',

f units (2), scaler, isurf,

\& $($ qwg (isurf,ia, iz), iz=1,nz), ia=1, na)

13

continue

if (first) write (map, '("' '1)')

WRITE LOCAL PRESSURE, VOID FRACTION, AIR MASS ERACTION,

GAS TEMPERATURE, FLUID TEMPERATURE, GAS DENSITY,

FLUID DENSITY, GAS ENTHALPY, FLUID ENTHALPY, AND

GAS AND FLUID VELOCITIES

TOP SECTION

$n$ rec $=n$ rec +1

if(first) write(map, '(15,a/,a)') nrec,

- 'pt, alpt, xat, tgt, $t f t$, rogt, roft, hgt, hft, ugt, uft,',

6 ' ppl,alppl, xapl,tgpl,tfpl, rogpl, rofpl, hqpl,,$f p l$ '

write (ndmp, rec=nrec) $n 2,(p t(j z), \operatorname{alpt}(; z), x a t(j z)$,

\&

1

$c$

if(first) write(map, '(1' '1)')

MIDDLE SECTION

scaler $=c k i l o$

do $20 \mathrm{ic}=1$, nchn

$n$ rec $=n$ rec +1

$n s u b=n \times(i c)$

If(flrst) write(map, ' (15,a,12)') nrec,

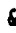

- Pressure, Mid Section, Channel \#, ic 


\begin{tabular}{|l|ll|l|}
\hline $2 / 15 / 93$ & WSRC-TR-93-086 Rev. 0 & FLOWTRAN-TF v1.2 Source Code & Pg. 73 of 354 \\
\hline
\end{tabular}

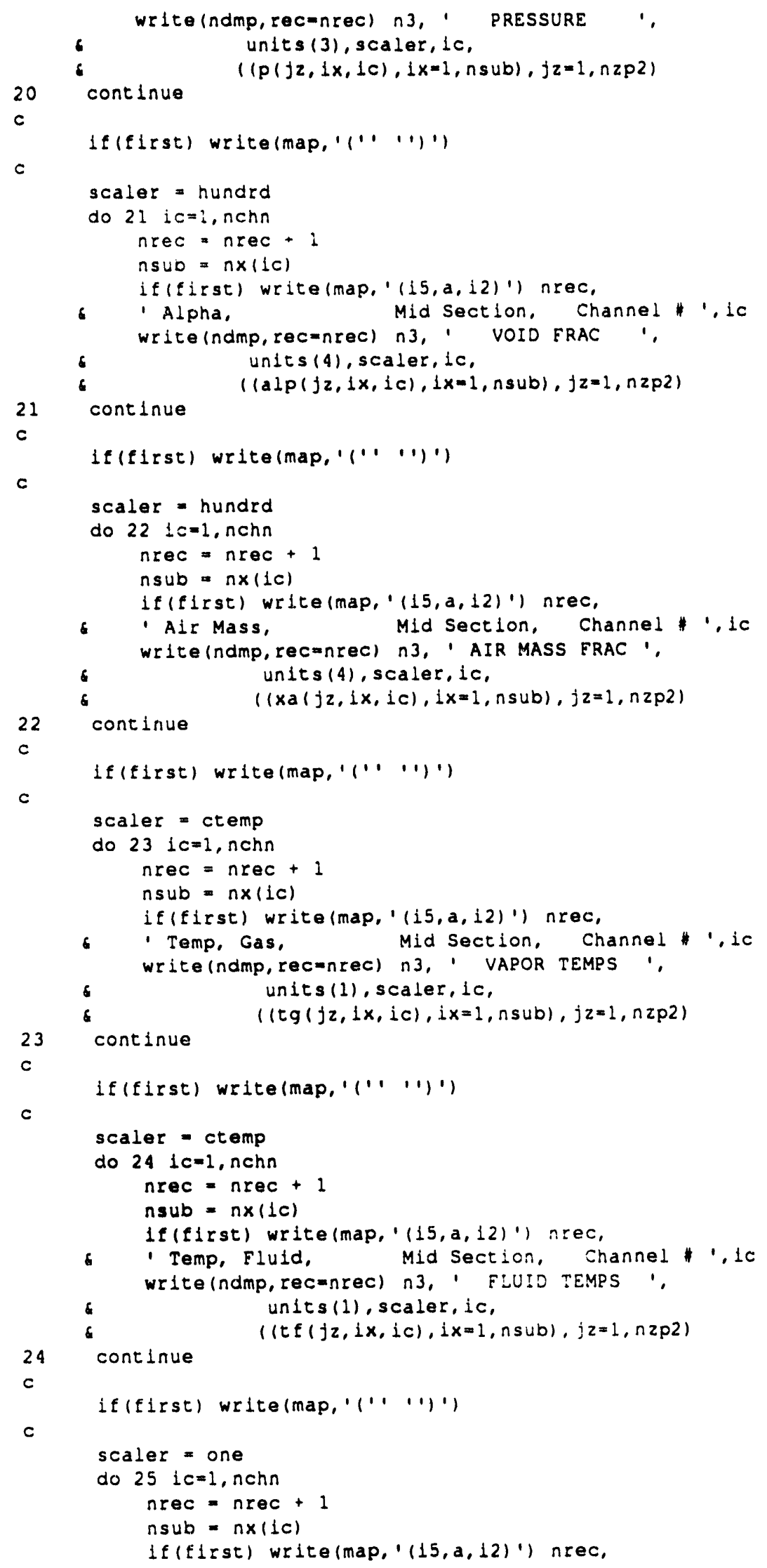




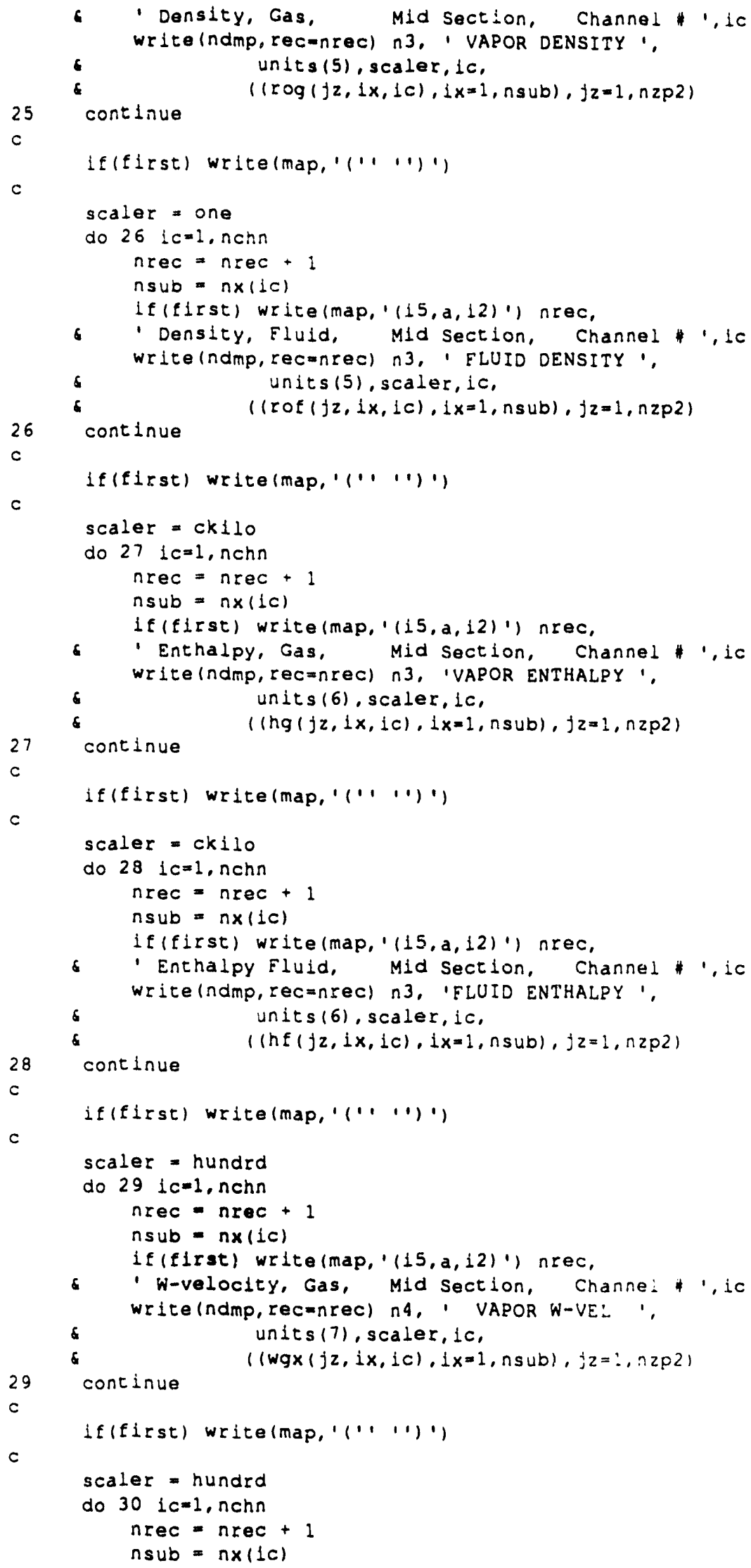



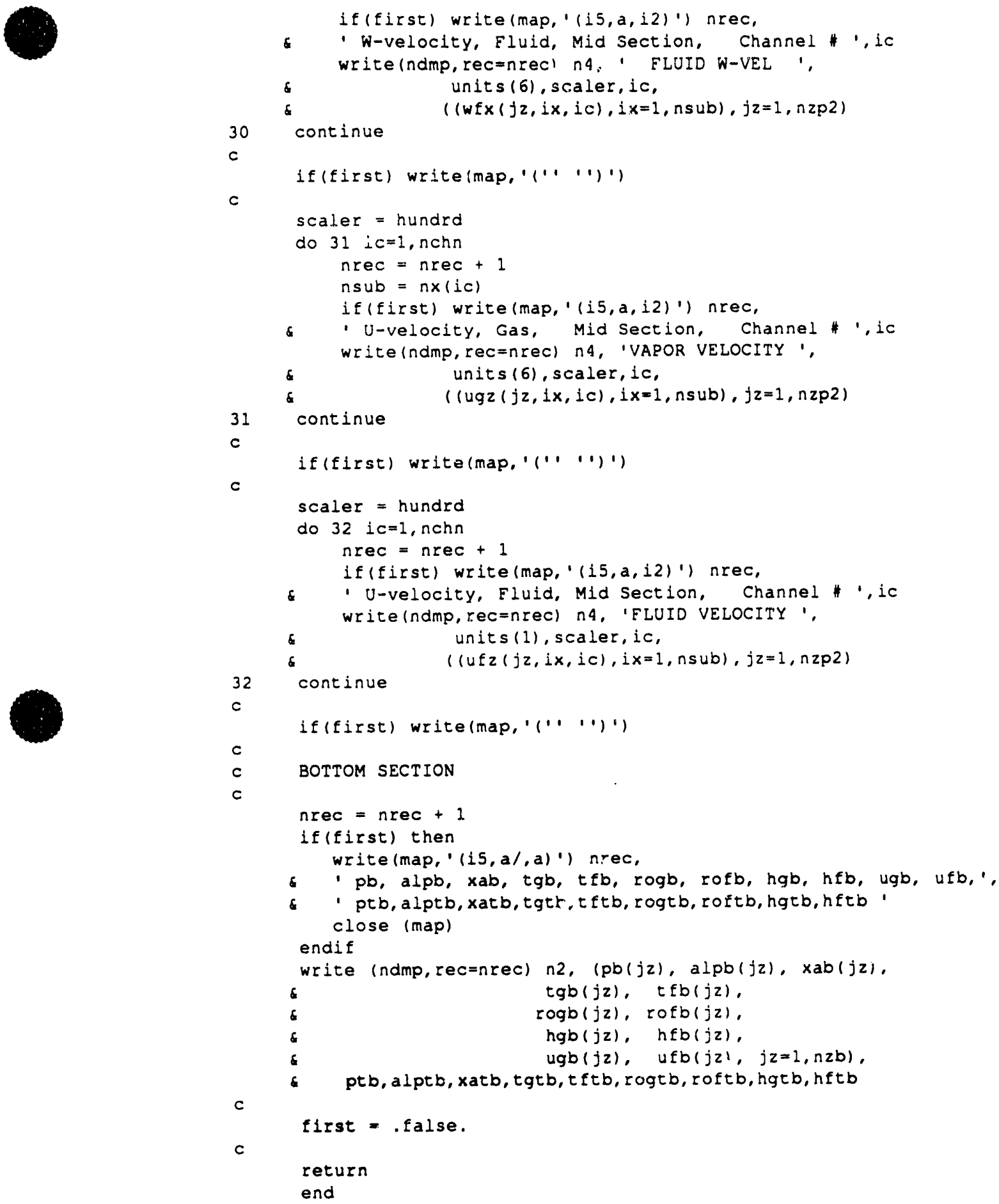

\section{DONORE}

subroutine donorblalpbn, xabn, rogbn, rofbn, hgbn, hebn,

* alptbn, xatbn, rogtbr, roftbn hgtbn, hftbn,

\& $u g b, u \in b, b g b, c g b, b f b, c \in b$,

\& $a c b, a d v b b, a d v c b, v m i n z$.

nzb) 


\begin{tabular}{|l|ll|r|}
\hline Pg. 76 of 354 & WSRC-TR-93.086 Rev.0 & FLOWTRAN-TF v1.2 Source Code & 2/15/93 \\
\hline
\end{tabular}

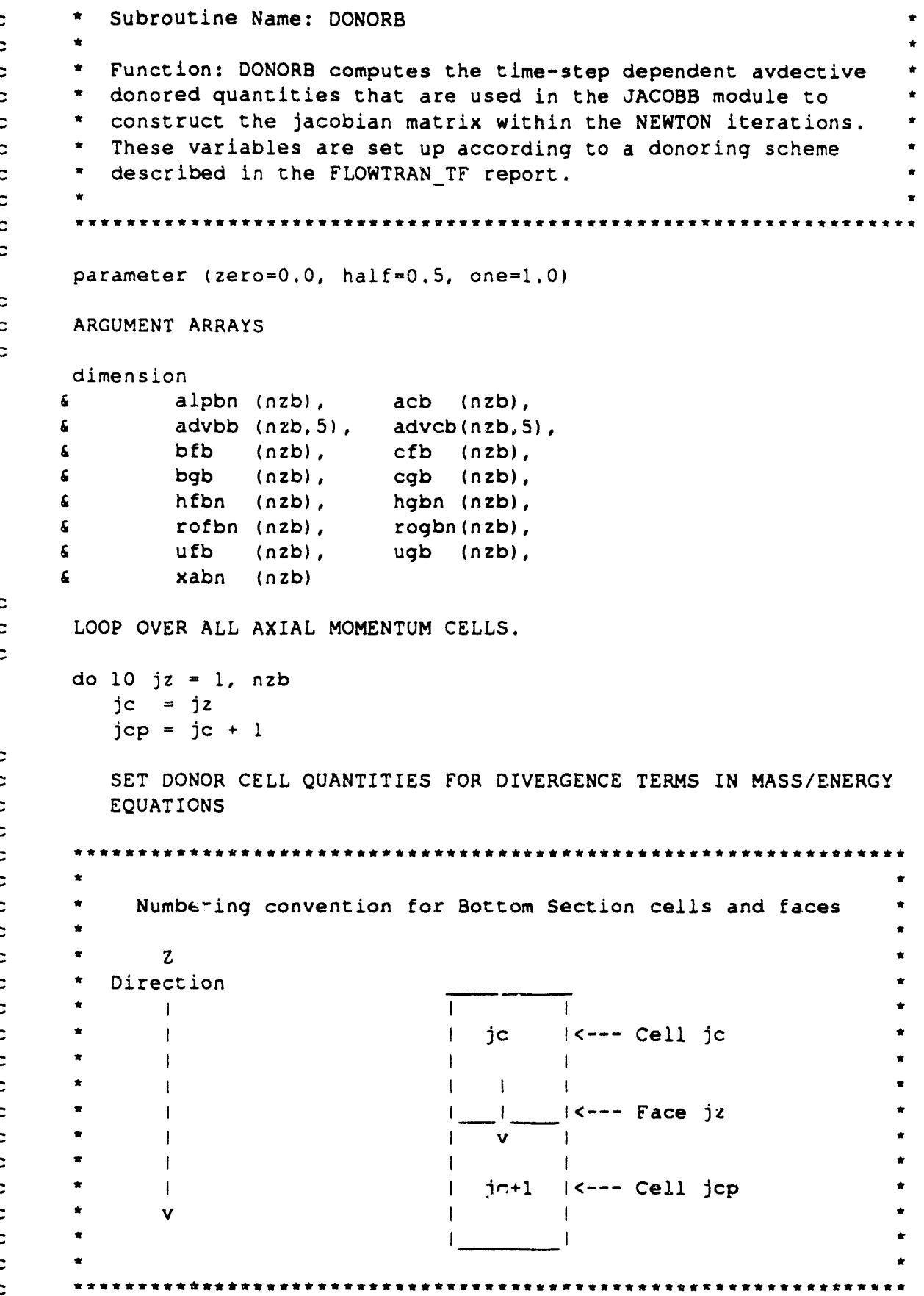

COMPUTE DONORING WEIGHTS FOR MASS/ENERGY CELLS ABOVE AND BELOW GIVEN HOHENTUM ERCE (I.E. JC ANO jCP)

NOTE THAT: FOR VELOCITIES OF MAGITUDE LESS THAN VminZ LINEAR 
INTERPOLATION IS EMPLOYED. FOR VELOCITIES GREATER THAN Vminz IN MAGITUDE FULL DONORING IS EMPLOYED 


Pg. 78 of 354
CWSRC-TR-93-086 Rev.0 FLOWTRAN-TF v1.2

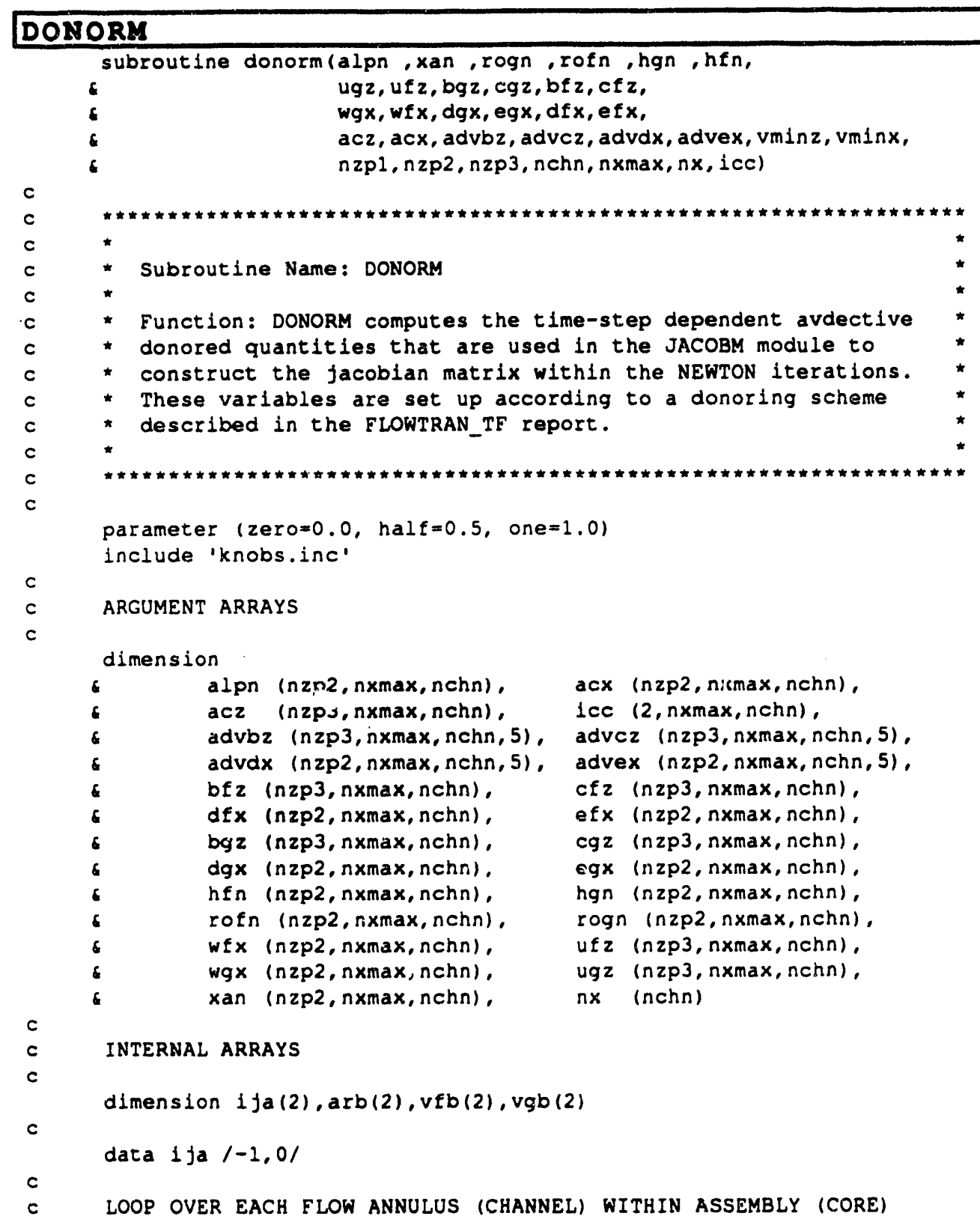


c c c c c
c
c

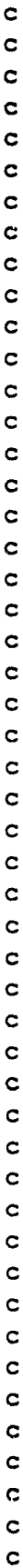

do $40 k=1$, nchn LOOP OVER ALL AZIMUTHAL MASS/ENERGY SECTORS WITHIN 2D CHANNEL

$n s u b=n x(k)$

do $30 i=1$, nsub

LOOP OVER ALL AXIAL MASS/ENERGY CELLS WITHIN AZ IMUTHAL SECTOR

do 20 jc $=2, \mathrm{nzp} 2$

$j x=j c$

SET DONOR CELL QUANTITIES FOR DIVERGENCE TERMS IN MASS/ENERGY EQUATIONS

Convention for positive velocities (incoming and outgoing from a specific mass/energy cell c)
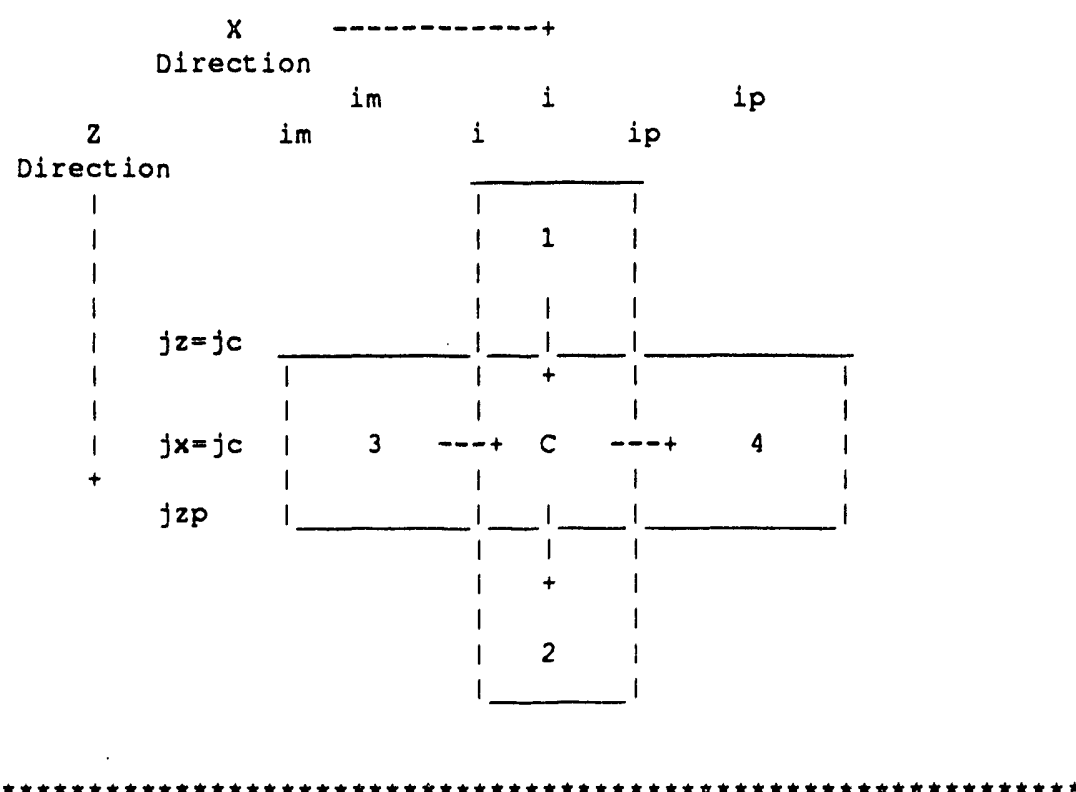

$m=1$, Compute donoring at horizontal faces

$m=2$, Compute donoring at vertical faces

MIDDLE SECTION - channel $k$

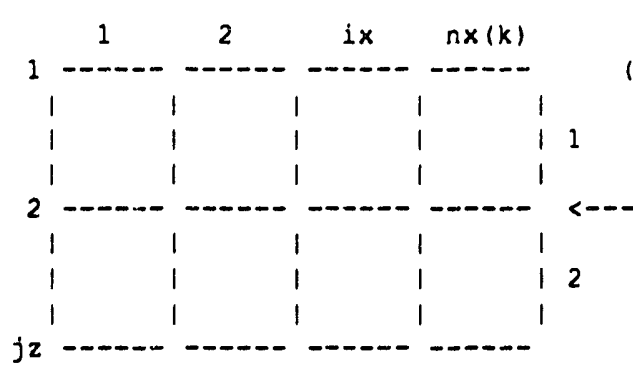

(Fictitious cells overlapping bottom cell. of top section)

Connects to top section 
$m=2$

SET la AND Ja INDEXES ACCORDING TO WHICH FACE BEING PROCESSED

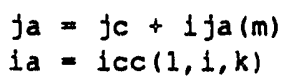

COMPUTE DONORING WEIGHTS FOR MASS/FNERGY CELLS ABOVE AND BELOW GIVEN MOMENTUM FACE. A POSI'IIVE VELOCITY IMPLIES INCOMING FLOW INTO GIVEN MASS/ENERGY CELL $(j c, i, k)$

NOTE THAT: FOR VELOCITIES OF MAGITUDE LESS THAN VMIN LINEAR INTERPOLATION IS EMPLOYED. FOR VELOCITIES GREATER THAN VMIN IN MAGNITUDE FULL DONORING IS EMPLOYED

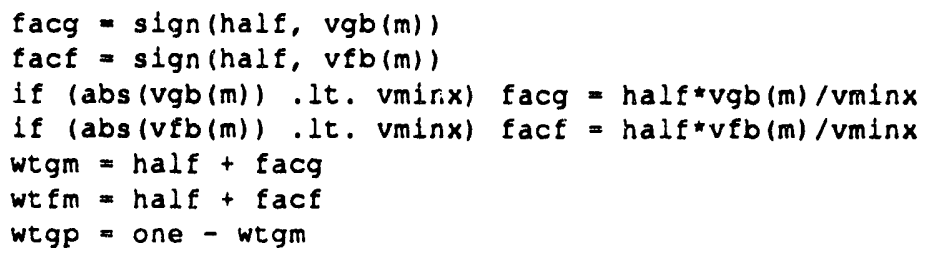




\begin{tabular}{|l|lll|l|}
\hline $2 / 15 / 93$ & WSRC-TR-93-086 Rev. 0 & FLOWTRAN-TF v1.2 Source Code & Pg. 83 of 354 \\
\hline
\end{tabular}

nzt)

* Subroutine Name: DONORT

* Function: DONORT computes the time-step dependent avdective

* donored quantities that are used in the JACOBT module to

* construct the jacobian matrix within the NEWTON iterations.

* These varlables are set up according to a donoring scheme

* described in the ELOWTRAN TE report.

$\star$

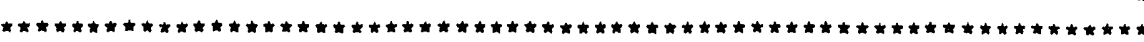

parameter $(z e r o=0.0$, half $=0.5$, one=1.0)

ARGUMENT ARRAYS

dimension

- alptn (nzt), act (nzt),

( advbt $(n z t, 5), \quad \operatorname{advct}(n z t, 5)$,

c bft (nzt), cft (nzt),

\& bgt (nzt). cgt (nzt),

\& hetn (nzt), hgtn (nzt),

- roftn (nzt), rogtn(nzt),

c uft (nzt), ugt (nzt),

c

c

c

c

C

c

C

c

c

c

c

c

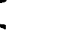

c

c

c

c

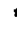

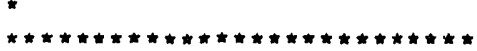

COMPUTE DONOR CELL QUANTITIES

SET FACE AREA, LIQUID AND MIXTURE GAS VELOCITIES

$\operatorname{art}=\operatorname{act}(j z)$

$v f t=u f t(j z)$

$v g t=\operatorname{ugt}(j z)$

LOOP OVER ALL AXIAL MOMENTUM CELLS

do $10 \mathrm{jz}=1$, nzt

$j c=j z$

$\mathrm{fcm}=\mathrm{jc}-1$

SET DONOR CELL QUANTITIES FOR DIVERGENCE TERMS IN MASS/ENERGY EQUATIONS

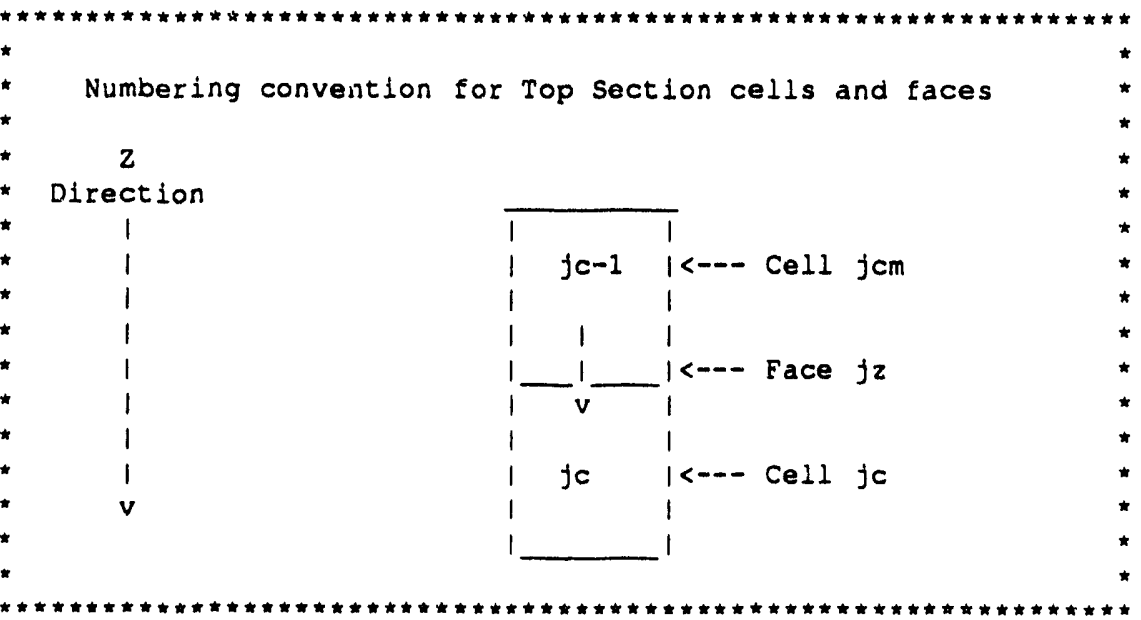




\begin{tabular}{|l|ll|r|}
\hline Pg. 84 of 354 & WSRC-TR-93-086 Rev. 0 & FLOWTRAN-TF v1.2 Source Code & $2 / 15 / 93$ \\
\hline
\end{tabular}

COMPUTE DONORING WEIGHTS FOR MASS/ENERGY CELLS ABOVE AND BELOW GIVEN MOMENTUM FACE (I.E. jC AND jcm)

NOTE THAT: FOR VELOCITIES OF MAGITUDE LESS THAN VIIIZ LINEAR INTERPOLATION IS EMPLOYED. FOR VELOCITIES GREATER THAN VMInZ IN MAGITUDE FULL DONORING IS EMPLOYED.

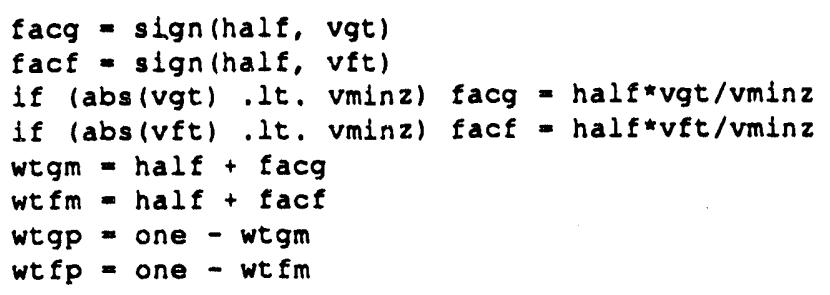

SET CELL PROPERTIES FOR JCM MASS CELL

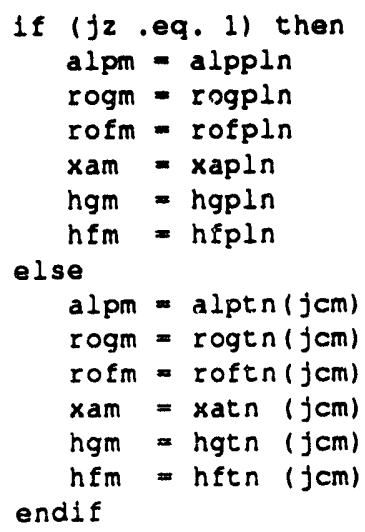




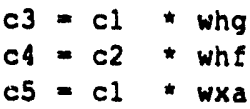

c

c

c

10

c

return

end

\section{EOSSID}

COMPUTE DONORED ADVECTIVE QUANTITIES FOR CELL FACE fz

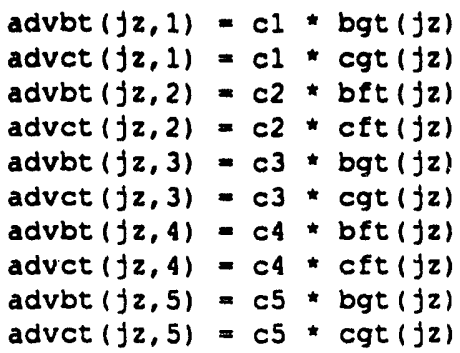

subroutine eossld (hcap, cond, mat, ts, wtpc, nr, na, nz, nrmax) 
c

c

c

c

c

c

c

c

c

c

c

c

c

c

c

$c$

c

parameter loneh=0.01, one $=1.01$

Include 'knobs.inc'

dimension hcap $(n r, n a, n z)$, ts(nrmax, na,nz),

6 cond (nr, na, nz), mat (nrmax, nz)

LOCAL COEFFICIENT ARRAYS

dimension coefl(18), $\operatorname{coef} 2(9), \cos 3(27)$

DENSITY ALLOY WEIGHT PERCENT COEFFICIENTS

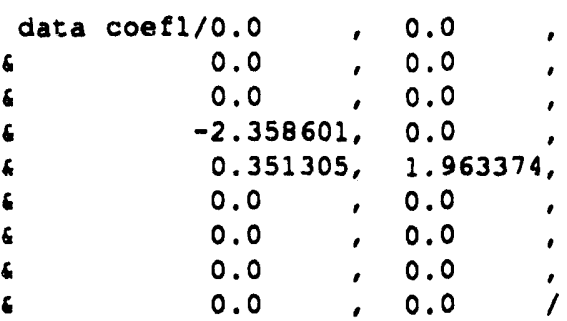

DENSITY TEMPERATURE COEFFICIENTS (LBM/FT*\#3 AT $20 \mathrm{C}$ )

data coef2/1186.7700,

168.8400 ,

500.4400 ,

168.6200 ,

171.6500 ,

168.0

157.0

525.0

$254.0 \%$

HEAT CAPACITY TEMPERATURE COEFEICIENTS (BTU/LBM-F)

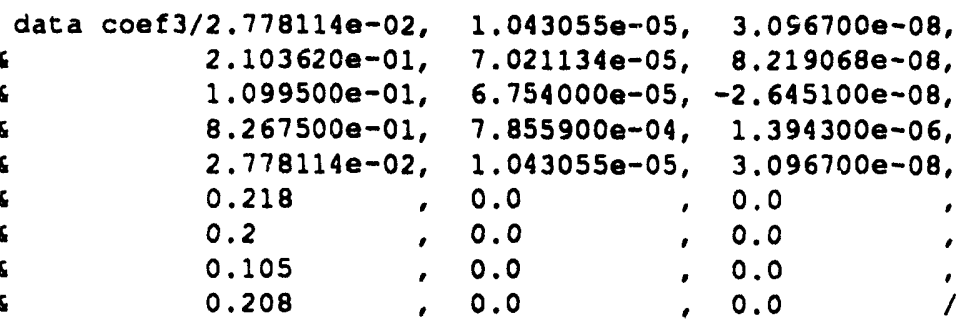

CONVERSION FACTORS FOR THERMAL CONDUCTIVITY, DENSITY, AND HEAT CAPACITY FROM ENGLISH (SRS) UNITS TO SI UNITS

data csik/6.230645e+03/, csid/16.01846/, csic/4.18400e+03/

do 100 i $a=1, n a$

do $1001 r=1, n r$

do $100 \quad 12=1, n 2$

$t s i d=t s(i, r, 1 a, 1 z)$

$n s i d=\operatorname{mat}(1 r, 1 z)$

rhoo $=\operatorname{coef} 2$ (nsld)

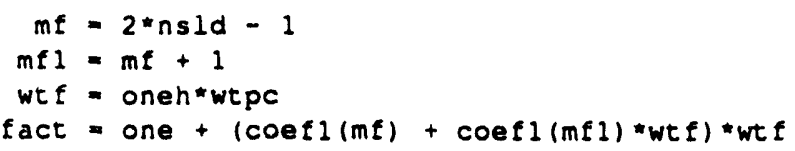


c

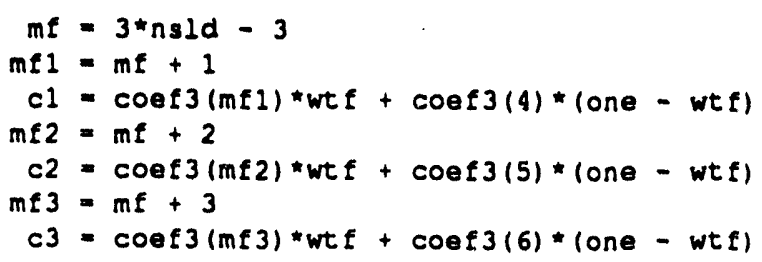

c

c

c

c

c

c

c

c

100 continue

c

CALCULATE DENSITY AND HEAT CAPACITY AND CONVERT TO SI UNITS

rho = csid*fact*rhoo

$c v=\operatorname{csic} *(c 1+(c 2+c 3 * t s 1 d) * t s i d)$

$\operatorname{hcap}(1 r, 1 a, 1 z)=x c v m e t * r h o * c v$

CALCULATE THERMAL CONDUCTIVITY IN SI UNITS

$\operatorname{cond}(1 r, 1 a, 1 z)=\operatorname{condt}(n s l d, t s l d, w t p c)$

return

end

\section{EXPL1D}

subrout ine explld lbgz,bfz,cgz, cfz,

acz, arat $z, d h z, \cos z, d z m, u g z, u f z$,

absrz, fmltz, formz, frez,cizn, c0bz, c0sz,

$a z z, v o l c, p n, a l p n, t g n, t f n, x a n$, rogn, rof $n$, acin, ugin, ufin, alpin, rogin, rofin, acex, ugex, ufex, alpex, rogex, rofex, rdelt, vminz,

$n z, n i n$, nex, jstrt, jend, Jmod, ierr, msgld, ilq)

SUBROUTINE explld

THIS ROUTINE COMPUTES THE NEW TIME STEP FLUID VELOCITIES

AT EACH CELL FACE AS A LINEAR FUNCTION OF THE NEW TIME

STEP PRESSURE DIFFERENCE ACROSS THE CELL FACE FOR THE ONE

DIMENSIONAL TOP AND BOTTOM SECTIONS OF THE ASSEMBLY. THE

COEFFICIENTS FORMING THESE LINEAR EXPRESSIONS ARE FUNCTIONS

OF OLD TIME STEP QUANTITIES; THEREFORE, THIS CALCULATION

- is only done once at the beginNing of each time step.

- THE EXPRESSIONS HAVE THE FOLLOWING FORM:

FOR GAS PHASE:

$$
u_{g, j+1 / 2}^{n+1}=B^{n} g, j+1 / 2+c^{n} g_{j} j+1 / 2 *\left(P^{n+1}-P^{n+1}{ }_{j+1}\right)
$$

FOR LIQUID PHASE:
$n+1$
$u$
f. $j+1 / 2$

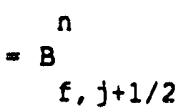
$+c^{n}, j+1 / 2$
$=\left(p^{n+1}-p^{n+1}\right)$

WHERE: $\quad B=$ bgz(Z-DIFECTION)

9

$B=b E 2(2-D I R E C T I O N)$

f

$C=\operatorname{cgz}(Z-D I R E C T I O N)$

g 


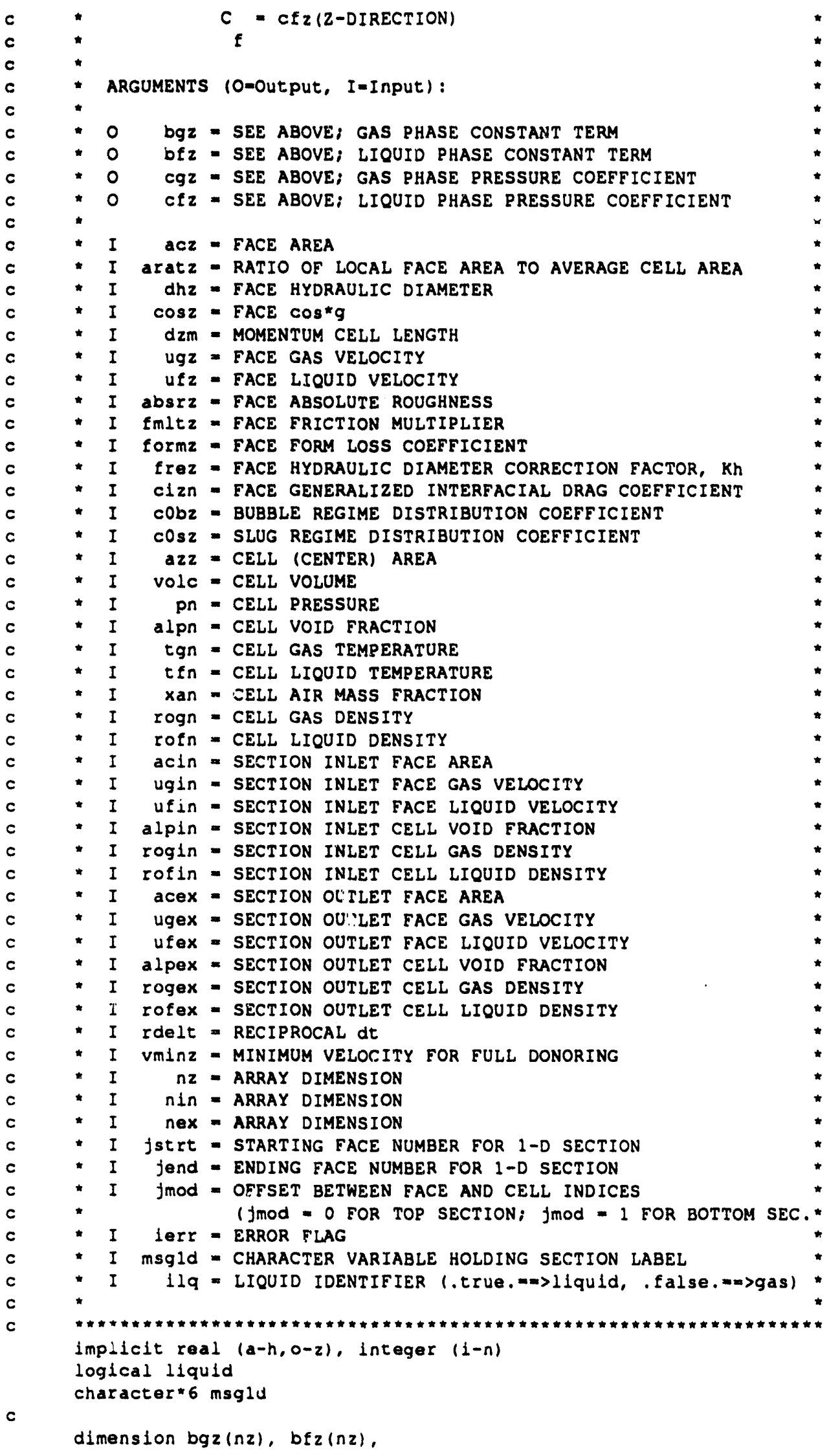


$\operatorname{cg} z(n z), \operatorname{cfz}(n z)$

dimension acz(nz), aratz(nz), dhz(nz), cosz(nz), $d z m(n z)$, - ugz(nz), ufz(nz), absrz(nz), fmltz(nz), formz(nz),

c

- $\quad \operatorname{frez}(n z), \operatorname{cizn}(n z), \operatorname{cobz}(n z), \cos z(n z)$

dimension azz(nz), vole(nz), pn(nz), alpn(nz), tgn(nz),

c

$\operatorname{tfn}(n z), \quad \operatorname{xan}(n z), \quad \operatorname{rog} n(n z), \operatorname{rof} n(n z)$

dimension

$\operatorname{acin}(n i n), \operatorname{acex}(n e x)$, ugin(nin), ugex(nex), $u f \ln (n \mid n)$, ufex(nex), alpin(nin), alpex(nex), rogin(nin), rogex(nex), rofin(nin), rofex(nex)

c

c

c

c

c

c$$
\text { c }
$$$$
\text { c }
$$$$
\text { c }
$$

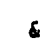$$
\text { c }
$$$$
\text { c }
$$

parameter $(z e r o=0.0$, half $=0.5$, one $=1.0$, two $=2.0$, three $=3.0)$

data epsv/0.001/, eps1/0.001/, eps2/0.01/, epsdet/1.0e-20/

LOOP OVER AXIAL CELLS IN TOP OR BOTTOM SECTION

do $100 \mathrm{jz}=j$ strt, jend

SET POINTERS TO MASS CELLS FORMING TOR (jzt)

AND BOTTOM (jzb) OF MOMENTUM CELL;

jmod $=0$ FOR TOP SECTION

jmod $=1$ FOR BOTTOM SECTION

$j z b=j z+j \bmod$

$j z t=j z b-1$

$u g=u g z(j z)$

$u f=u f z(j z)$

COMPUTE VOLUME FRACTIONS FOR TOP AND BOTTOM CELLS

rvolz $=t$ wol $(\operatorname{volc}(j z t)+\operatorname{volc}(j z b))$

$f t=\operatorname{volc}(j z t) /(\operatorname{volc}(j z t)+\operatorname{volc}(j z b))$

$f b=$ one - ft

CALCULATE VOLUME AVERAGED PROPERTIES

$p c=f t * p n(j z t)+f b * p n(j z b)$

$\operatorname{tgc}=f t \star \operatorname{tgn}(j z t)+f b * \operatorname{tgn}(j z b)$

$t f c=f t * t f n(j z t)+f b * t f n(j z b)$

$x a c=f t * x a n(j z t)+f b * x a n(j z b)$

$a l p g=f t * a l p n(j z t)+f b * a l p n(j z b)$

$r h o g=f t * r o g n(j z t)+f b * \operatorname{rogn}(j z b)$

$a \operatorname{cog}=f t * a \operatorname{lpn}(j z t) * \operatorname{rog} n(j z t)+f b^{\star} a \operatorname{lpn}(j z b) * \operatorname{rog} n(j z b)$

alpf = one - alpg

rhof $=f t * \operatorname{rofn}(j z t)+f b * \operatorname{rofn}(j z b)$

$\operatorname{arof}=f t *($ one $-\operatorname{alpn}(j z t)) * \operatorname{rofn}(j z t)+$

$f b *($ one $-a \operatorname{lpn}(j z b)) * \operatorname{rofn}(j z b)$

CALCULATE ADVECTION TERMS

liquid $=$. false.

call avecl ladvg, avgag, acz, azz, aratz, ugz, alpn, rogn, acin, ugin, alpin, rogin, acex, ugex, alpex, rogex, vminz, jz, jmod, nz, nin, nex, liquid) 


\begin{tabular}{|l|l|l|l|}
\hline Pg. 90 of 354 & WSRC-TR-93-086 Rev. 0 & FLOWTRAN-TF v1.2 Source Code & 2/15/93 \\
\hline
\end{tabular}

c

c

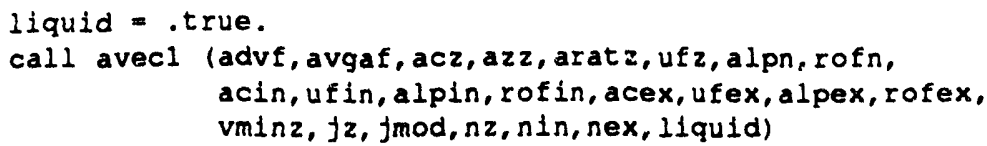

EVALUATE ADVECTION TERM CUT-OFF FUNCTIONS

phig $=\min (o n e, \max (z e r o,(a l p g$-eps1)/(epe2-eps1))) phif $=\min (o n e, \max (z e r o,(\operatorname{alpf-eps} 1) /($ eps2-eps 1$)))$

COMPUTE $\mathrm{CO}, \mathrm{Cg}$ \& ANNULAR FRACTION ann

call cofen (alpg, cobz $(j z), \operatorname{cosz}(j z), c 0, c g, a n n)$

COMPUTE GAS AND LIQUID WALL DRAG AND DERIVATIVES

theta $=$ phif

alpgdz = avgag*rvolz

alpfdz = avgaf*rvolz

call fwallz (fwg, fwf, dfgdug, dffduf, dhz $(j z)$, thet $a, f m l t z(j z)$, formz $(j z), \operatorname{absrz}(j z)$, frez(jz), ug, uE, alpgdz, alpfdz, $p c, t g c, t f c, x a c, r h o g$, rhof, ilq)

ADD FALLING FILM CORRECTION TERM TO LIQUID WALL DRAG IF LIQUID FLOW IS DOWNWARD (NOT USED CURRENTLY!)

$\cos g=\cos z(j z) * \max (z e r o, \operatorname{sign}($ one, $\cos z(j z) \star u f))$

fwf = fwf + theta*ann*alpf*(rhof-rhog)*cosg

*(one - two*alpf/(three*frez(fz)))

COMPUTE INTERFACIAL DRAG AND DERIVATIVES

urel $=c g \star u g-c 0 \star u f$

fidz $=\operatorname{cizn}(j z) *(a b s(u r e l)+$ epsv)*urel

dfddug $=\operatorname{cizn}(j z) \operatorname{cg}^{\star}$ (two*abs (urel) + epsv)

dfdduf $=-\operatorname{cizn}(j z) * c 0 *($ two*abs (urel) + epsv)

alpmin $=\min (a l p n(j z t), \operatorname{alpn}(j z b))$

phiw = min(one, $\max (z e r o,(a)$ pmin-eps1)/(eps2-eps1)))

coef $=($ one - ann $) \star a l p g * p h i w$

$f i w z=c o e f *(f w g+f w f)$

$d f w d u g=$ coef $* d f g d u g$

dfwduf $=$ coef ${ }^{*} d f d u f$

$f i=f i d z+f i w z$

dfidug = dfddug $+d f w d u g$

dfiduf = dfdduf + dfwduf

CALCULATE 2 X2 SYSTEM GAS MOMENTUM EQUATION COEFFICIENTS

$d t z=$ rdelt*aratz $(j z)$

fiterm $=f i-d f 1$ dug*ug - dfiduf*uf

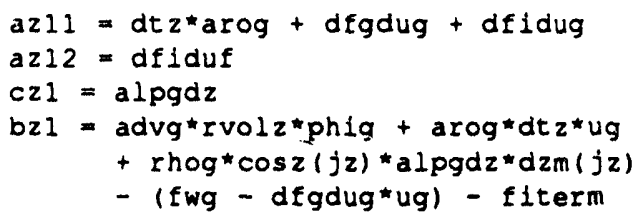

CALCULATE $2 \times 2$ SYSTEM LIQUID MOMENTUM EQUATION COEFFICIENTS 


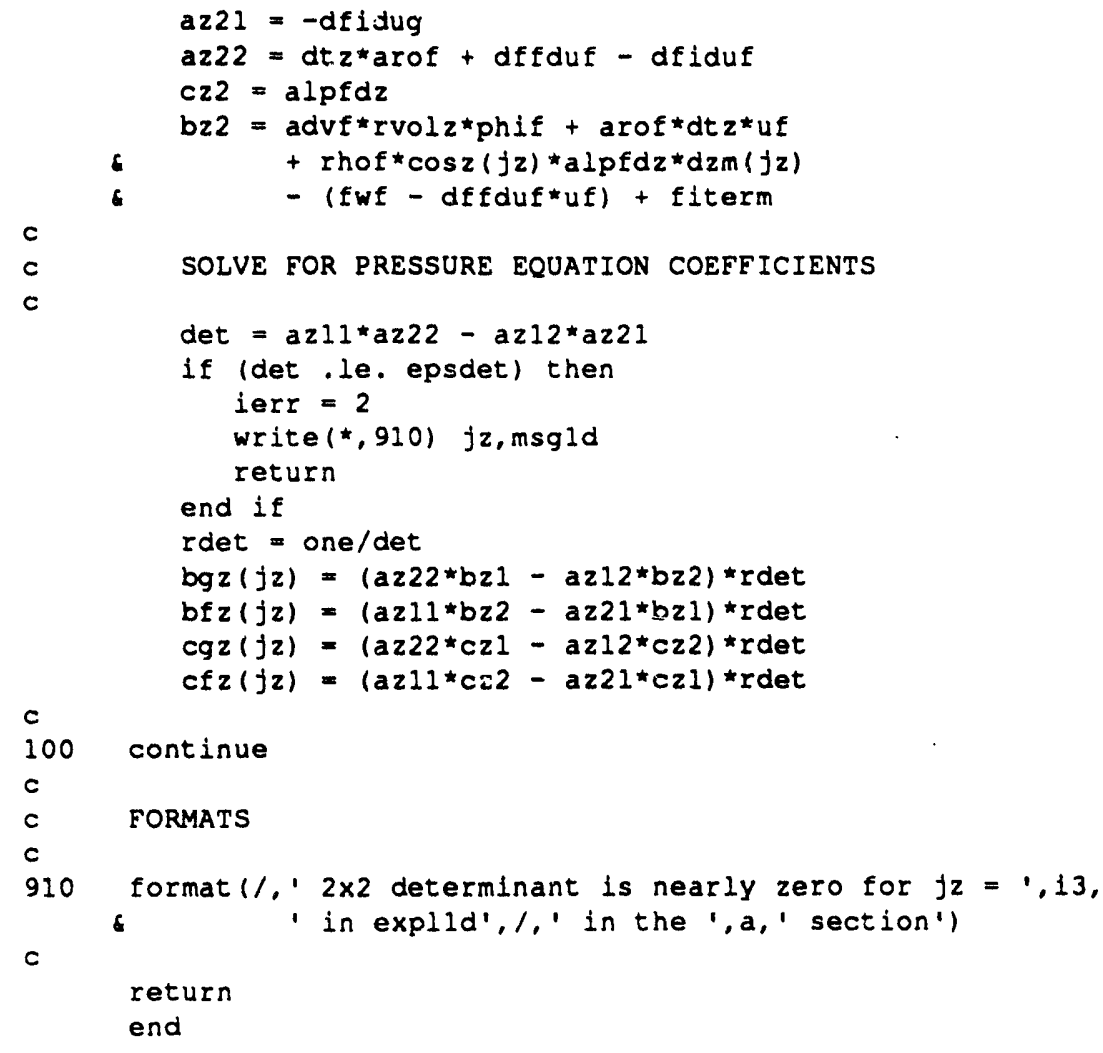

\section{EXP L2D}

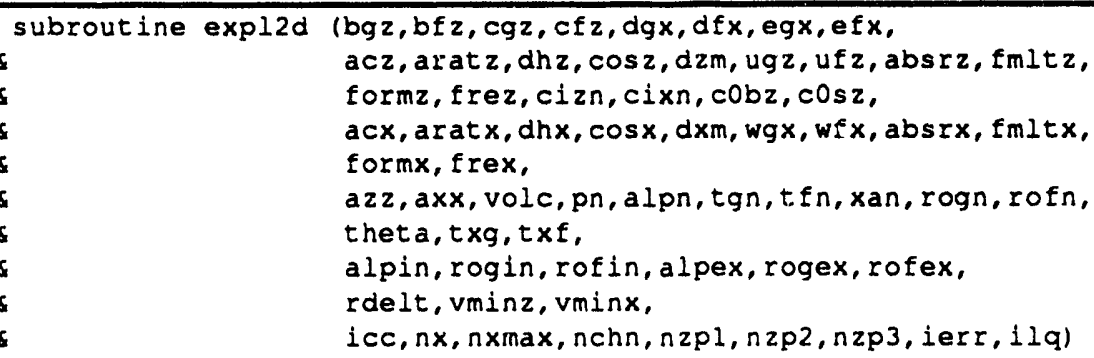


FOR LIQUID PHASE:

$$
\begin{aligned}
& u_{f, j+1 / 2}^{n+1}=B_{f, j+1 / 2}^{n}+c_{f, j+1 / 2}^{n} *\left(P^{n+1}-P_{j+1}^{n+1}\right) \\
& \left.w_{f, i+1 / 2}^{n+1}=D_{f, i+1 / 2}^{n}+E_{f, i+1 / 2}^{n}{ }^{n} P^{n+1}-P_{1+1}^{n+1}\right)
\end{aligned}
$$

WHERE :

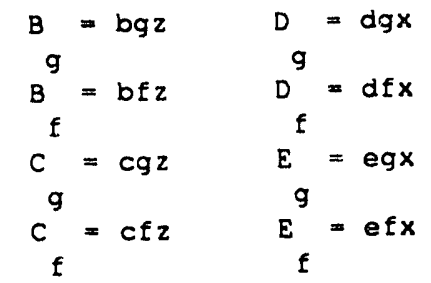

ARGUMENTS (O-Output, I=Input):

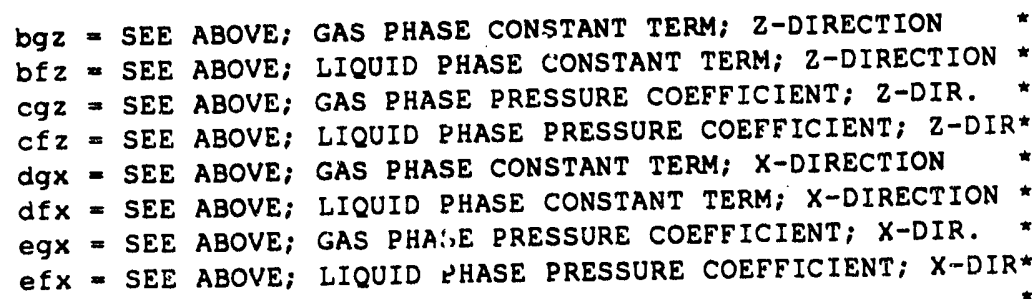




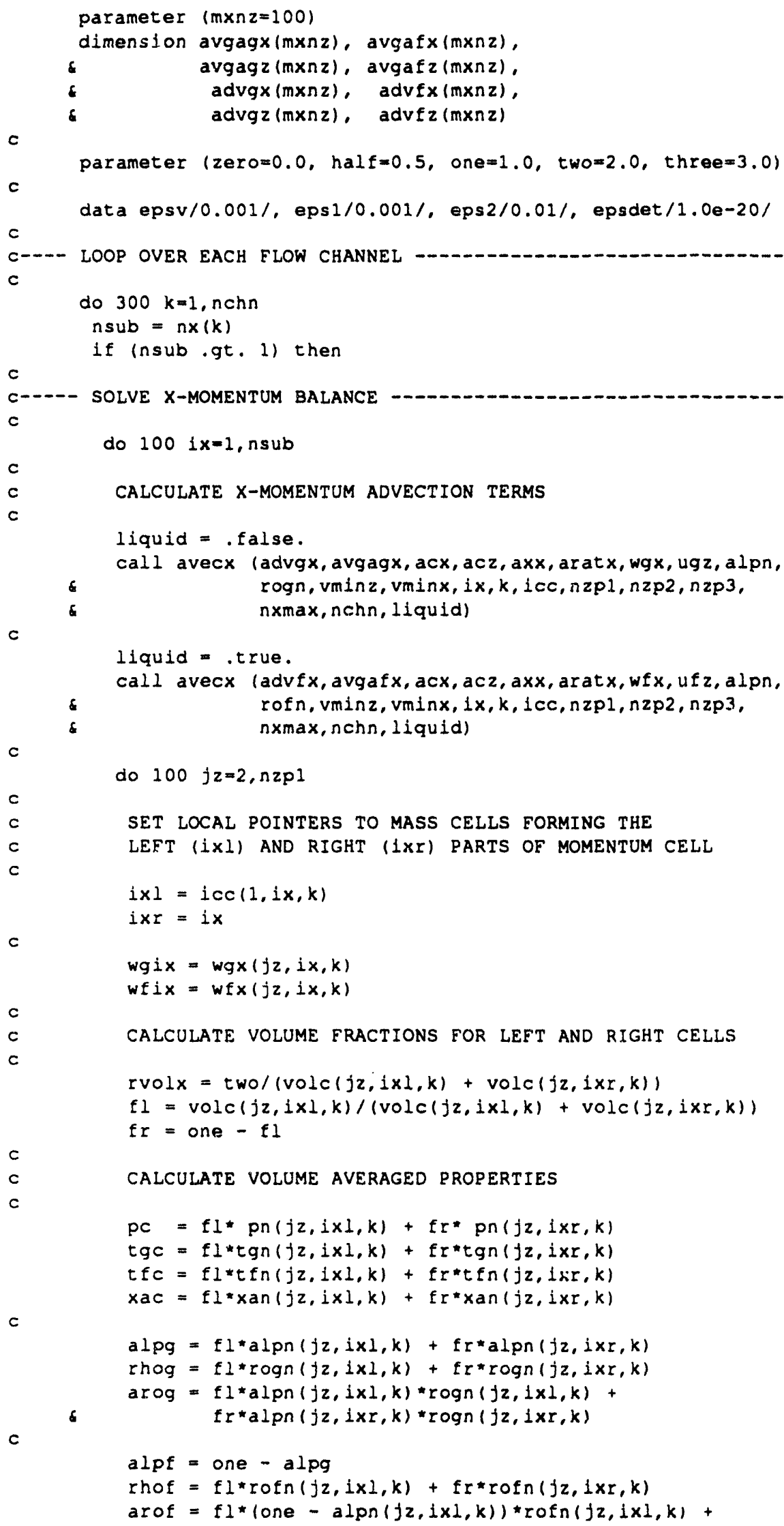


c

c

c

$c$

c

$c$

c

c

$f r *$ (one $-\operatorname{alpn}(j z, i x r, 1)) \star \operatorname{rofn}(j z, i x r, k)$

thet $a x=f l^{\star t h e t} a(j z, i x l, k)+f r * t h e t a(j z, i x r, k)$

EVALUATE ADVECTION TERM CUT-OFF FUNCTIONS

phig $=\min ($ one, $\max (2 \operatorname{ero},($ alpg-eps1)/(eps2-eps1)))

phif $=\min (o n e, \max (z \operatorname{ero},(\operatorname{alpf-eps1)/(eps2-eps1))}$

COMPUTE GAS AND LIQUID WALL DRAG AND DERIVATIVES

alpgdx $=\operatorname{avgagx}(j z) * r v o l x$

alpfdx = avgafx(jz)*rvolx

call fwallx (fwg, fwf, dfgdwg, dffdwf,

dhx $(j z, 1 x, k)$, thetax, fnitx $(j z, 1 x, k)$, formx $(j z, i x, k)$, absrx $(j z, i x, k), f \operatorname{rex}(j z, i x, k), w g \perp x, w f \perp x$, $a l p g d x, a l p f d x, p c, t g c, t f c, x a c$, rhog, rhof, $11 q)$

COMPUTE INTERFACIAL DRAG AND DERIVATIVES

$\operatorname{cixl}=\operatorname{half}{ }(\operatorname{cixn}(j z, i x l, k)+\operatorname{cixn}(j z+1, i x l, k))$

$\operatorname{cixr}=\operatorname{half} *(c i x n(j z, i \times r, k)+\operatorname{cixn}(j z+1, i \times r, k))$

clxavg $=f l{ }^{\star} c i x l+f{ }^{*} \operatorname{cixr}$

wrel $=w g i x-w f i x$

$f i=$ cixavg*(abs (wrel) + epsv)*wrel

$d f l d w g=c^{*} x_{a v g}(t w o * a b s(w r e l)+e p s v)$

$d f i d w f=-d f i d w g$

CALCULATE $2 \times 2$ SYSTEM GAS MOMENTUM EQUATION COEFFICIENTS

$d t x=r d e l t \star a r a t x(j z, i x, k)$

fiterm $=f i-d f i d w g * w g i x-d f i d w f * w f i x$

$a \times 11=d t x^{\star} a r o g+d f g d w g+d f i d w g$

$a \times 12=d f i d w f$

ex1 = alpgdx

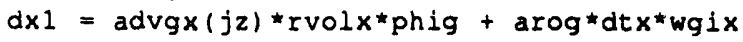

$+r h \circ{ }^{\star} \cos x(j z, i x, k){ }^{\star} a l p g d x x^{*} d x m(k)$

- (fwg - dfgdwg*wgix) - fiterm

CALCULATE $2 \times 2$ SYSTEM LIQUID MOMENTUM EQUATION COEFFICIENTS

$a \times 21=-d f i d w g$

$a \times 22$ = $d t \times$ *arof + dffdwf - dfidwf

ex2 $=$ alpfdx

$d x 2=\operatorname{advfx}(j z) *$ rvolx*phif + arof*dtx*wfix

$+\operatorname{rhof} * \cos x(j z, i x, k) * a \operatorname{lpfdx} * d x m(k)$

- (fwf - dffdwf*wfix) + fiterm

SOLVE FOR PRESSURE EQUATION COEFFICIENTS

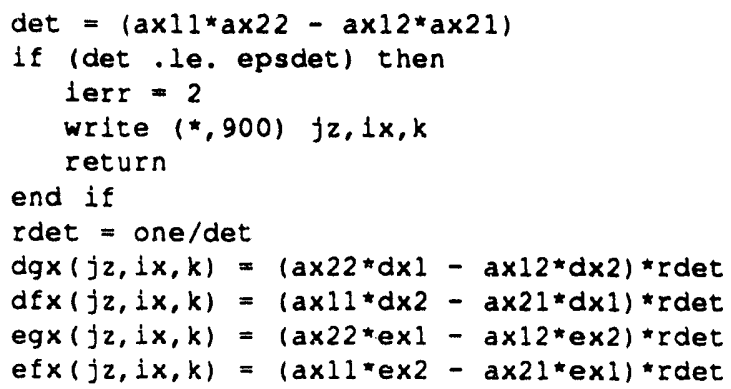




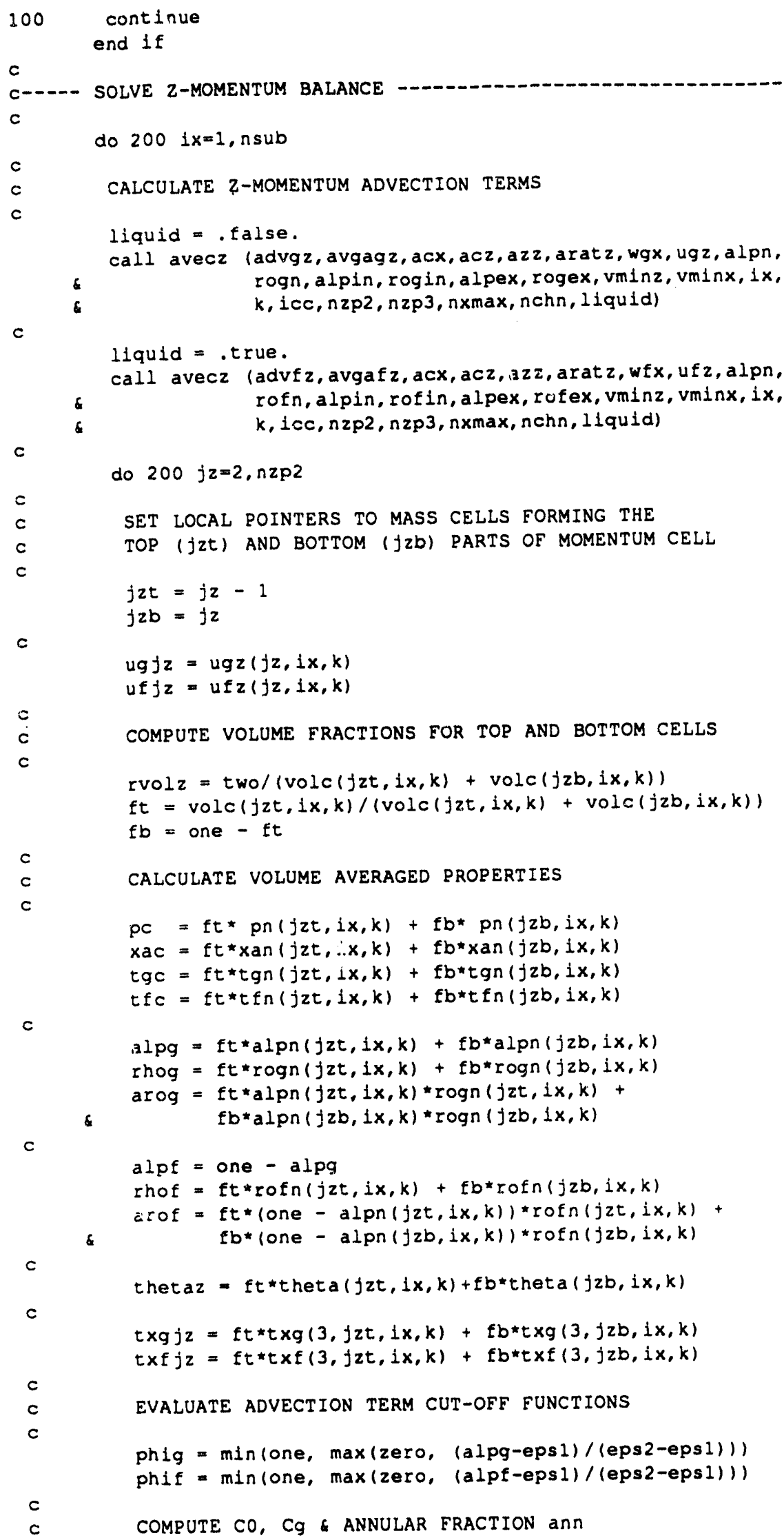




\begin{tabular}{|l|ll|l|}
\hline $2 / 15 / 93$ & WSRC-TR-93-086 Rev. 0 & FLOWTRAN-TF v1.2 Source Code & Pg. 97 of 354 \\
\hline
\end{tabular}

c

c

c

c

c

c

c

c

$c$

$c$

$c$

$\operatorname{call} \operatorname{cofcn}(a \operatorname{lpg}, \operatorname{cobz}(j z, i x, k), \cos z(j z, i x, k), c 0, c g, a n n)$

COMPUTE GAS AND LIQUID PHASE WALL DRAG AND DERIVATIVES

alpgdz $=\operatorname{avgagz}(f z) \star r v o l z$

alpfdz $=\operatorname{avgafz}(j z) * r v o l z$

call fwallz (fwg, fwf, dfgdug, dffduf,

dhz $(j z, i x, k)$, thetaz, fmltz $(j z, i x, k)$, formz $(j z, i x, k)$, absrz $(j z, i x, k), f r e z(j z, i x, k), u g j z$, uf $j z$, alpgdz, alpfdz, pc, tgc, tfc, xac, rhog, rhof, ilq)

ADD FALLING FILM CORRECTION TERM TO LIQUID WALL DRAG IF LIQUID FLOW IS DOWNWARD (NOT USED CURRENTLY!)

$\operatorname{cosg}=\operatorname{cosz}(j z, i x, k){ }^{*} \max (z e r o, \operatorname{sign}(o n e, \operatorname{cosz}(j z, i x, k) \star u f j z))$

$f w f=f w f+t h e t a z * a n n \star a l p f \star(r h o f-r h o g) \star c o s g$

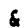

*(one - two*alpf/(three*frez $(j z, i x, k)))$

COMPUTE INTEREACIAL DRAG AND DERIVATIVES

urel $=c g \star u g j z-c 0 * u f j z$

$f i d z=\operatorname{cizn}(j z, j x, k) *(a b s(u r e l)+e p s v) * u r e l$

dfddug $=\operatorname{cizn}(j z, i x, k) \star c g *(t w o * a b s(u r e l)+e p s v)$

dfdduf $=-\operatorname{cizn}(j z, i x, k) \star c 0 \star(t w o * a b s($ urel) + epsv)

alpmin $=\min (a l p n(j z t, i x, k), \operatorname{alpn}(j z b, i x, k))$

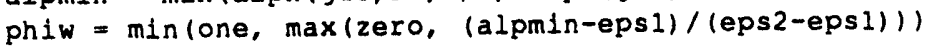

coef $=$ (one - ann)*alpg*phiw

$f \pm w z=\operatorname{coe} f *(f w g+f w f)$

dfwdug $=$ coef ${ }^{\star} d f g d u g$

dfwduf $=$ coef $\star d f f d u f$

$f i=f i d z+f i w z$

dfidug = dfdaug + dfwdug

dfiduf $=$ dfdduf + dfwduf

CALCULATE $2 \times 2$ SYSTEM GAS MOMENTUM EQUATION COEFFICIENTS

$d t z=r d e l t * a r a t z(j z, i x, k)$

fiterm $=f i-d f i d u g \star u g j z-d f i d u f \star u f j z$

$\operatorname{az} 11=d t z^{*}$ arog $+d f g d u g+d f i d u g$

az12 = dfiduf

$c z l=a l p g d z$

$b z l=\operatorname{advgz}(j z) * r v o l z \star p h i g+\operatorname{arog}{ }^{*} d t z z^{\star} u j z$

\& $+\{h \circ g * \cos z(j z, i x, k) \star a l p g d z \star d z m(j z)$

c - (fwg - dfgdug*ugjz) - fiterm - txgjz

CALCULATE $2 \times 2$ SYSTEM LIQUID MOMENTUM EQUATION COEFFICIENTS

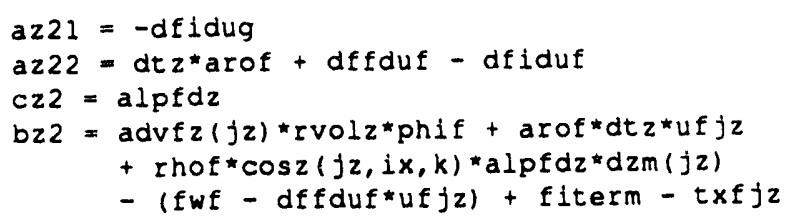

SOLVE FOR PRESSURE EQUATION COEFFICIENTS

det $=(a z 11 * a z 22-a z 12 * a z 21)$

if (det.le. epsdet) then

iert $=2$ 


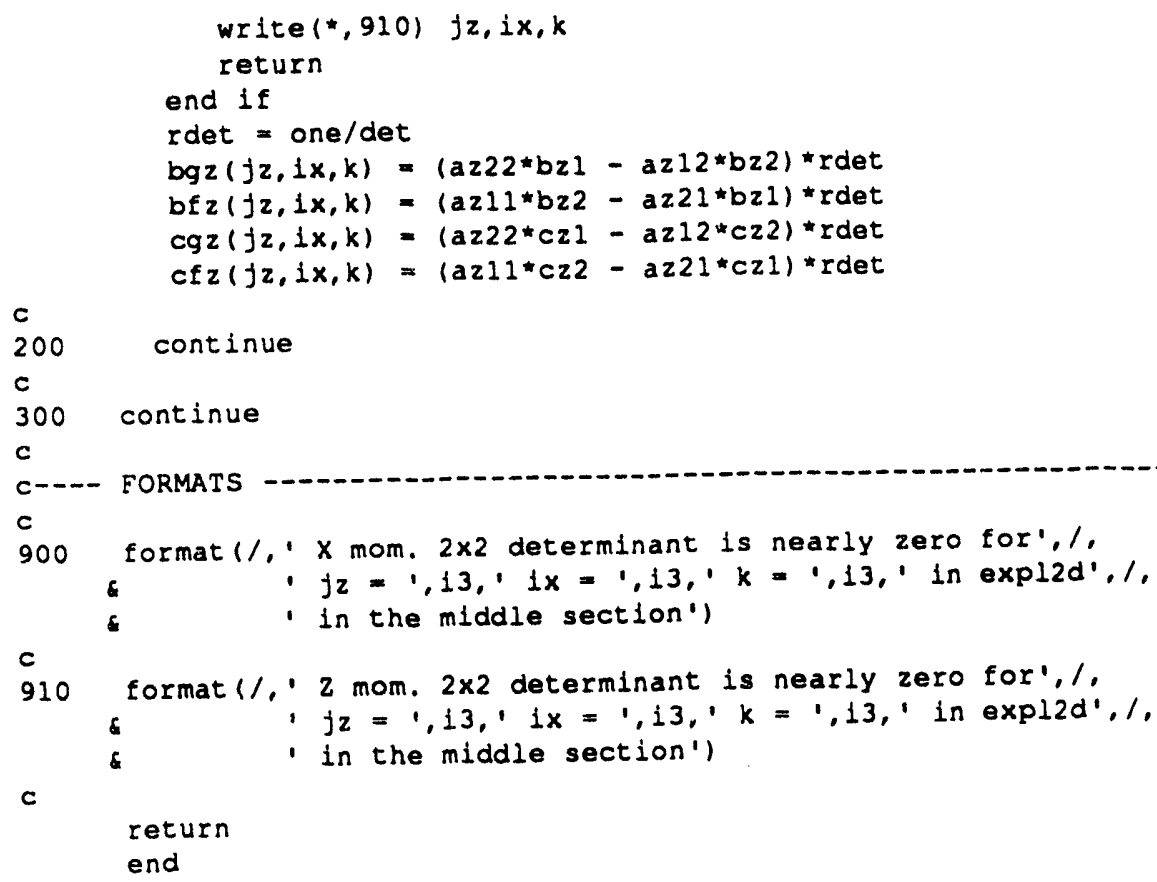

\section{EXPICT}

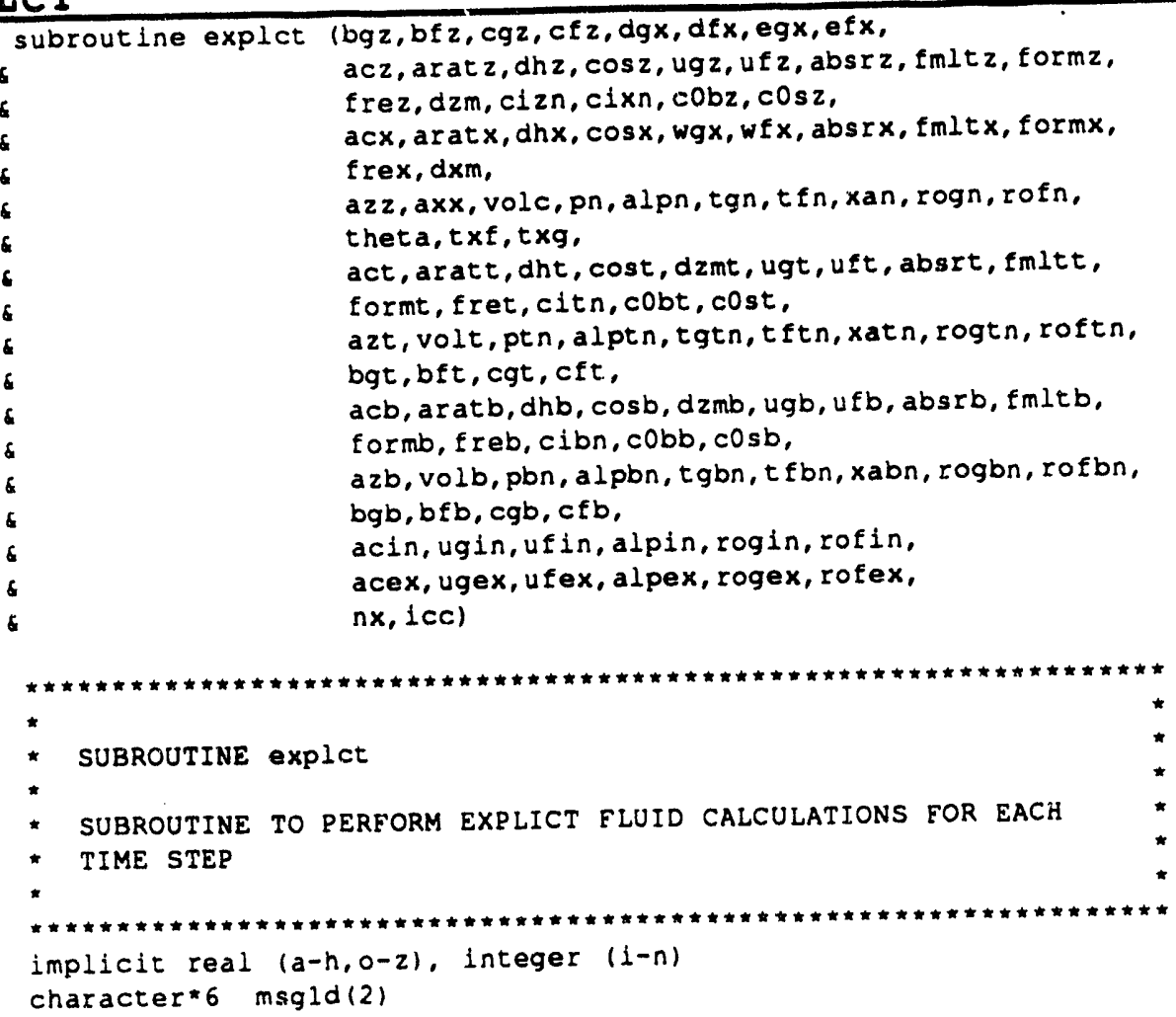

* subroutine to perform explict fluid calculations for each * 
c

dimension azz(nzp2, nxmax, nchn). 6

6

6

4

6

6

c

dimension $\operatorname{bgz}(n z p 3, n \times m a x, n c h n)$. 6 bfz (nzp3, nxmax, nchn). - $\operatorname{cgz}(n z p 3, n \times \max , n c h n)$. \& cfz(nzp3,nxmax, nchn), theta (nzp2, nxmax, nchn),

\begin{abstract}
ufz (nzp3, nxmax, nchn). absrz (nxp3, nxmax, nchn), fmltz (nzp3, nxmax, nchn), formz (nzp), nxmax, nchn). frez (nzp3, nxmax, nchn), $\operatorname{dzm}(n z p 3)$.

c12n (nzp3, nxmax, nchn). cixn (nzp3, nxmax, nchn). cObz (nzp3, nxmax, nchn), $\operatorname{cosz}(n z p 3, n \times \max , n c h n)$
\end{abstract}

vole (nzp2, nxmax, nchn) , alpn (nzp2, nxmax, nchn), tfn (nzp2, nxmax, nchn), rogn (nzp2, nxmax, nchn),

txg (4, nzp2, nxmax, nchn), $1 \operatorname{cc}(2, n \times \max , n \operatorname{ch} n)$, wfx (nzp2, nxmax, nchn). absrx (nzp2, nxmax, nchn). fmltx (nzp2, nxmax, nchn), formx (nzp2, nxmax, nchn), frex (nzp2, nxmax, nchn). dxm (nchn).

$\operatorname{axx}(n z p 2, n \times m a x, n c h n)$, pn (nzp2, nxmax, nchn), $\operatorname{tgn}(n z p 2, n \times \max , n c h n)$, xan (nzp2, nxmax, nchn), rofn (nzp2, nxmax, nchn),

$t x f(4, n z p 2, n x \max , n c h n)$. $n \times(n c h n)$

dgx (nzp2, nxmax, nchn), dfx (nzp2, nxmax, nchn), egx (nzp2, nxmax, nchn), efx (nzp2, nxmax, nchn)
TOP SECTION ARRAYS

dimension act (nzt), aratt (nzt), \& dzmt (nzt), ugt (nzt), c fmltt (nzt), formt (nzt),

6

c

dimension azt(nzt), volt (nzt).

$\&$

6

c

dimension bgt(nzt).

bft (nzt),

\section{BOTTOM SECTION ARRAYS}

c

dimension

$a c b(n z b)$,

c.

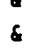

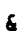

c

dimension fmltb(nzb), formb (nzb), $\mathrm{cObb}(n z b)$. $\operatorname{cosb}(n z b)$

$a z b(n z b), \quad \operatorname{volb}(n z b)$,

dht (nzt), cost (nzt), uft (nzt), absrt (nzt), fret (nzt), citn(nzt).

⿷

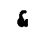
$\operatorname{tgbn}(n z b), t f b n(n z b)$.

ptn (nzt), alptn (nzt), $\operatorname{xatn}(n z t), \operatorname{rogtn}(n z t)$.

dimension $b g b(n z b), \quad b f b(n z b), \quad c g b(n z b), \quad c f b(n z b)$

ARRAYS TO HOLD INLET AND OUTLET PARAMETERS

dimension acin(nsubc), acex(nsubc),

\& ugin (nsubc), ugex(nsubc).

\& ufin (nsubc). ufex(nsubc),

- alpin (nsubc), alpex(nsubc),

- rogin (nsubc), rogex(nsubc).

- rofin (nsubc), rofex(nsubc)

c

include 'params.inc'

c

parameter $($ zero $=0.0$, half $=0.5$, one=1.0)

data msgld(1)/'top $1 /$. 


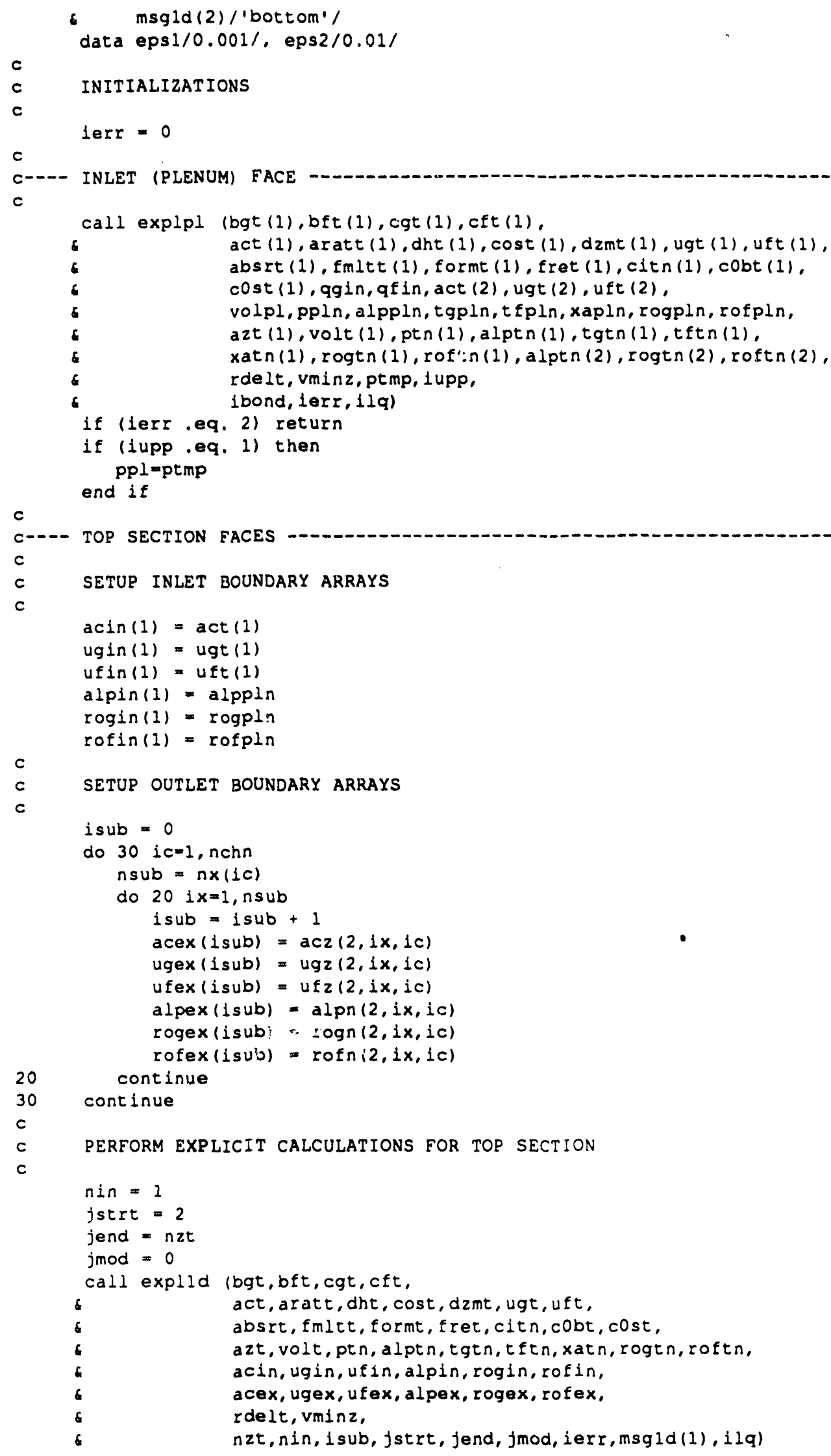


if (ierr .eq. 2) return

c

c

c

DEFINE WETTED WALL PARAMETER FOR FICTICIOUS CELLS

alpft $=$ one - alptn(nzt)

alpfb = one - alpbn(1)

phift $=\min (o n e, \max (z e r o,(a) p f t$-eps1)/(eps2-eps1)))

phifb $=$ tiin (one, $\max ($ zero, (alpfb-eps1)/(eps2-eps1)))

c

do $351 \mathrm{c}=1$, nchn

do $351 x=1, n x(1 c)$

thetal $1, i x, 1 c)=$ phift

35

thet $a(n z p 2, i x, 1 c)=$ phifb

c DEFINE WETTED WALL PARAMETER FOR MIDDLE SECTION CELL

C IF NO SOLID CALCIJLATIONS ARE BEING PERFORMED

c

if (isolid eq. 0 ) then

do 36 ic=1, nchn

do $36 i x=1, n x(i c)$

do $36 j z=2, n z p 1$ alpf = one - alpn $(j z, i x, i c)$ theta $(j z, i x, i c)=\min (o n e, \max (z e r o$.

a

continue

end if

(alpf-eps1) /(eps2-eps1)))

c

SETUP BOUNDARY PARAMETERS

if (nzt .eq. 1) then

alpi $=$ alppln

$\operatorname{rog} 1=$ rogpln

rof $1=$ rofpln

else

alpi $=\operatorname{alptn}(n z t-1)$

$\operatorname{rog} i=\operatorname{rogtn}(n z t-1)$

$\operatorname{rof} 1=\operatorname{roftn}(n z t-1)$

end if

c

if (nzb .eq. 1) then

alpx $=$ alptbn

rog $x=$ rogtbn

rofx $=$ roftbn

else

alpx $=$ alpbn (2)

$\operatorname{rog} x=\operatorname{rog} b n(2)$

$\operatorname{rof} x=\operatorname{rofbn}(2)$

end 14

c

PERFORM EXPLICIT CALCULATIONS FOR MIDDLE SECTION

$c$

call expl2d (bgz,bfz, cgz, cf $z, d g x, d f x, e g x, e f x$,

6

acz, aratz, dhz, cosz, dzm, ugz, uf $z$, absrz, fmltz, formz, frez, cizn, cixn, cobz, cosz,

acx, arat $x$, dhx, cos $x, d x m, w g x, w f x$, absrx, fmltx,

formx, frex,

$a z z, a x x$, volc, pn, alpn, tgn, tf $n, x a n, r o g n$, rof $n$,

theta, txg, txf,

alpi, rog1, rof 1, alpx, $\operatorname{rog} x, \operatorname{rof} x$,

rdelt, vminz, vminx,

icc, nx, nxmax, nchn, nzpl, nzp2, nzp3, ierr, ilq) 
1f (1ers.eq. 2) return

C

C-- BOTTOM SECTION FACES -

SETUP INLET BOUNDARY ARRAYS

isub $=0$

do 50 ic $=1$, nchn

$n s u b=n \times(i c)$

do 40 ix $=1$, nsub

isub $=$ isub +1

$\operatorname{acin}(1 s u b)=\operatorname{acz}(n z p 2,1 x, i c)$

$u g i n(i s u b)=u g z(n z p 2, i x, i c)$

$u f \ln (i s u b)=u f z(n z p 2, i x, i c)$

alpin (1sub) =alpn (nzpl, ix, 1c)

$\operatorname{rogin}(1 s u b)=\operatorname{rogn}(n z p l, i x, i c)$

40

$\operatorname{rofin}(1 \operatorname{sub})=\operatorname{rofn}(n z p l, 1 x, 1 c)$

continue

50 continue

c

c

c

SETUP OUTLET BOUNDARY ARRAYS

$\operatorname{acex}(1)=a c b(n 2 b)$
$u g e x(1)=u g b(n 2 b)$
$u f e x(1)=u f b(n z b)$
alpex $(1)=a$ lptbn
rogex $(1)=$ rogtbn
rofex $(1)=$ roftbn

c PERFORM EXPLICIT CALCULATIONS FOR BOTTOM SECTION

$\dot{c}$

nex $=1$

jstrt $=1$

jend $=n z b-1$

jmod $=1$

call explld (bgb, bfb, cgb, cfb,

6

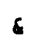

$a c b, a$ r $a t b, d h b, c o s b, d z m b, u g b, u f b$, absrb, fmltb, formb, freb, cibn, cObb, cosb,

$a z b, v o l b, p b n, a l p b n, t g b n, t f b n, x a b n$, rogbn, rofbn,

acin, ugin, ufin, alpin, rogin, rofin,

acex, ugex, ufex, alpex, rogex, rofex,

rdelt, vminz,

nzb, isub, nex, jstrt, fend, jmod, ierr, msgld (2), ilq)

if (ierr.eq. 2) return

C

C---- OUTLET (TANK BOTTOM) FACE

c

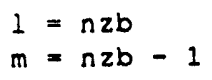

c

return

end 


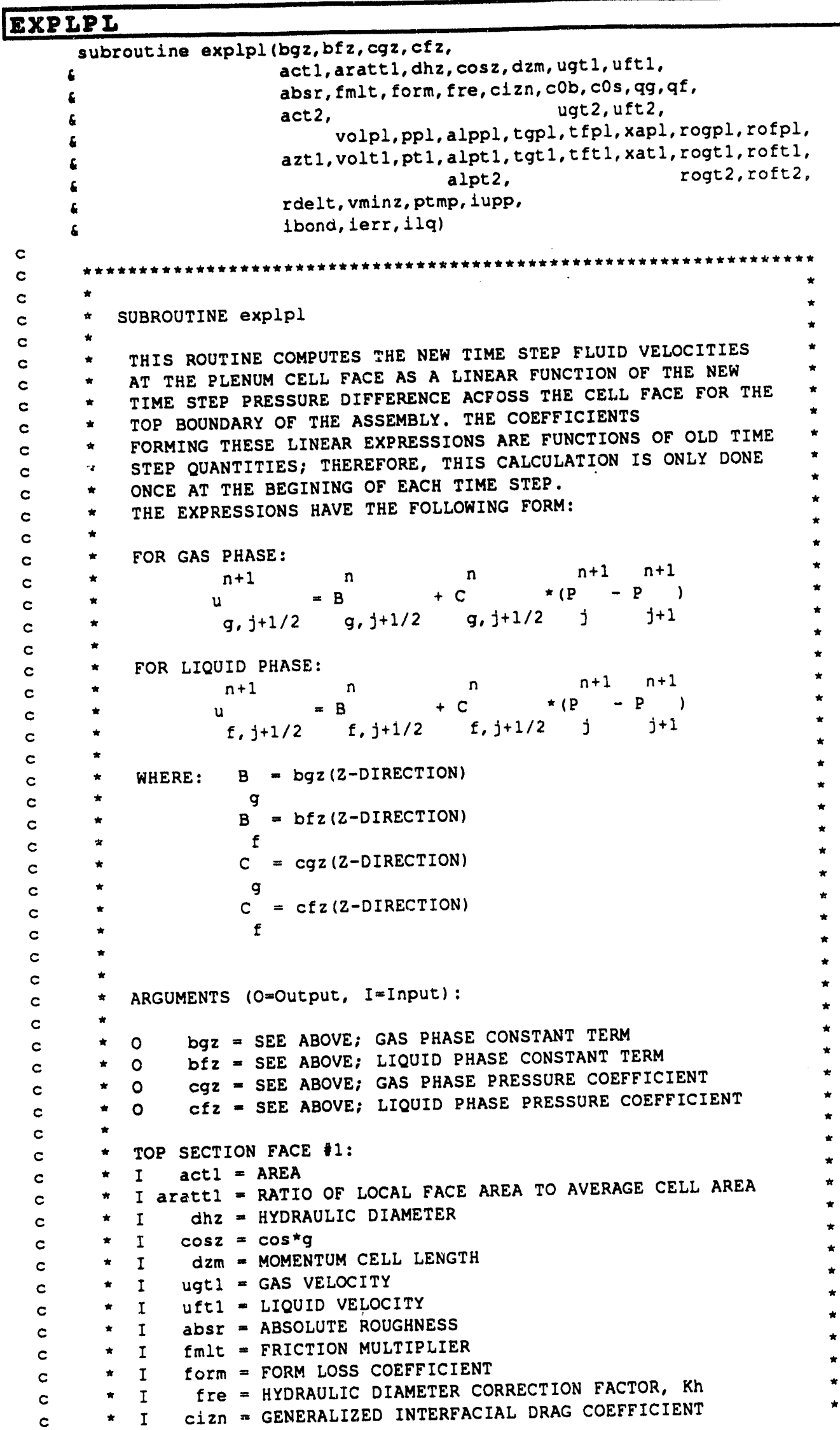




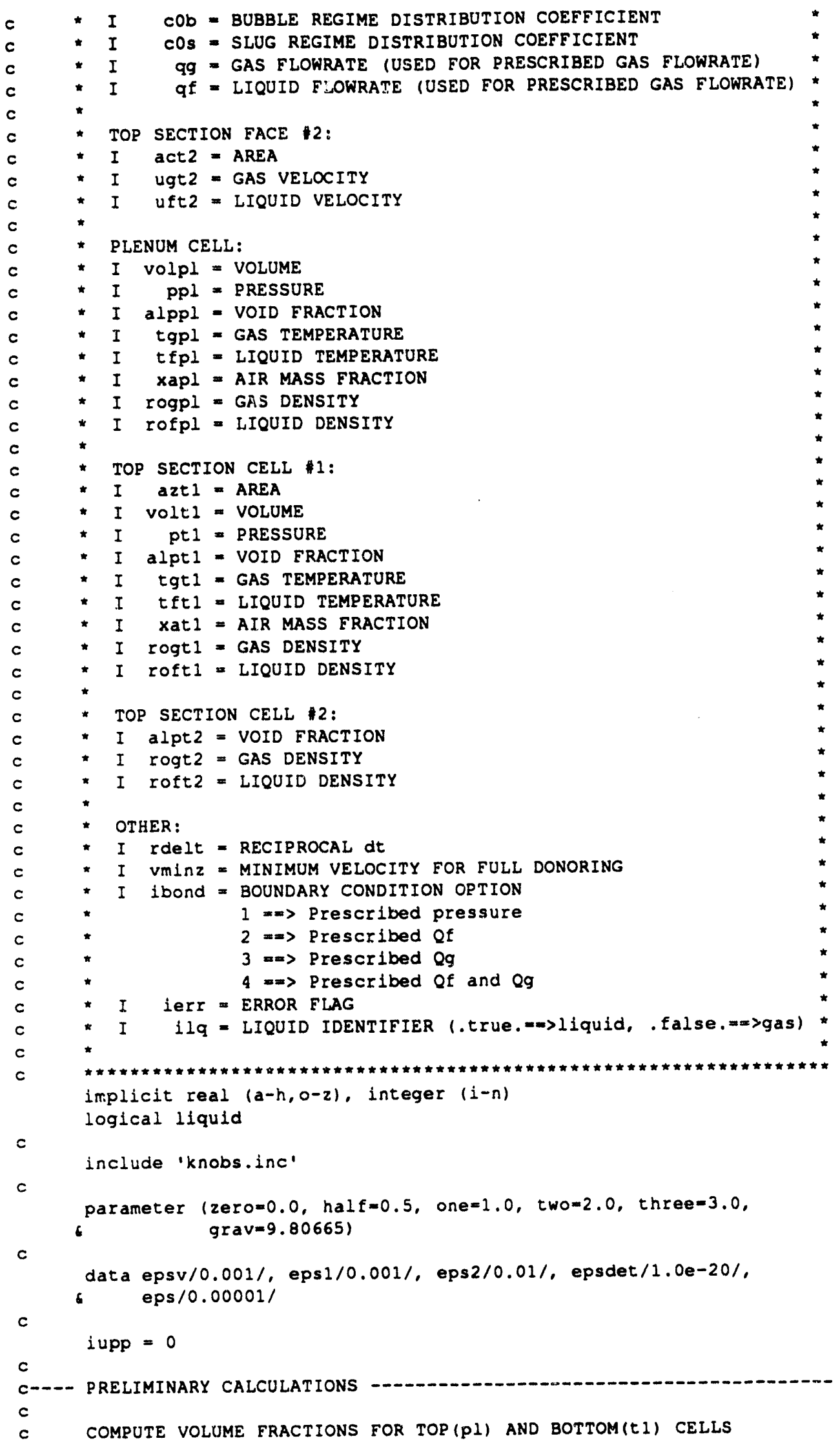


$r$ rolz $=t w o /($ volpl + volt 1$)$

$f t=v o l p l /(v o l p l+v o l t 1)$

$\mathrm{fb}=$ one $-\mathrm{ft}$

c

c

c

CALCULATE VOLUME AVERAGED PROPERTIES

$p c=f t * p p l+f b * p t l$

$t g c=f t * t g p l+f b * t g t l$

$t f c=f t * t f p l+f b * t f t l$

$x a c=f t * x a p l+f b * x a t 1$

$a l p g=t t^{\star a} a p p 1+f b * a l p t 1$

rhog $=f t \star r o g p l+f b$ rogt $1^{\prime}$

$\operatorname{arog}=f t * a l p p l * r o g p l+f b * a l p t 1 * r o g t 1$

alpf $=$ one - alpg

rhof $=f t$ rofpl $+f b^{\star}$ roft 1

$a r o f=f t *(o n e-a l p p l) * r o f p l+f b *(o n e-a l p t 1) *$ roft 1

$c$

c

$c$

CALCULATE ADVECTION TERMS

liquid $=$. false.

call avect ladvg, avgag,

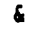

$\varepsilon$

6

c

liquid $=$.true.

call avect (advf, avgaf,

c.

6

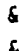

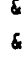

C

$c$

$c$

c

c

c

c

c

alpp], rogp1,

alpt 1, rogt 1, azt 1, act 1, ugt 1 , aratt 1 ,

alpt2, rogt2, act 2 , ugt 2 ,

vminz, liquid)

EVALUATE ADVECTION TERM CUT-OFF FUNCTIONS

phig $=\min (o n e, \max (z e r o,($ alpg-eps1)/(eps2-eps1)))

phif $=\min (o n e, \max (z e r o,($ alpf-epsi) $/($ eps 2 -eps 1$)))$

SET INTERFACIAL DRAG PARAMETERS AND FORM LOSS COEFFICIENT

if (cosz.eq.zero .and. plnht.ne.zero

call cofen (one, cob,cos, co,cg, ann)

$c 1=\operatorname{cipln}$

formpl $=$ formhs

else

call cofcn (alpg, cob, cos, co,cg, ann)

ci $=$ cizn

formpl $=$ form

end if

COMPUTE GAS AND LIQUID WALL DRAG AND DERIVATIVES

theta $=$ phif

alpgdz = avgag*rvolz

alpfdz = avgaf*rvolz

call fwallz (fwg, fwf, dfgdug, dffduf,

dhz, theta, fmlt, formpl, absr, fre, ugt 1 , uft 1 ,

alpgdz, alpfdz, pc, tgc, tfc, xac, rhog, rhof, ilq)

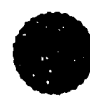

c 


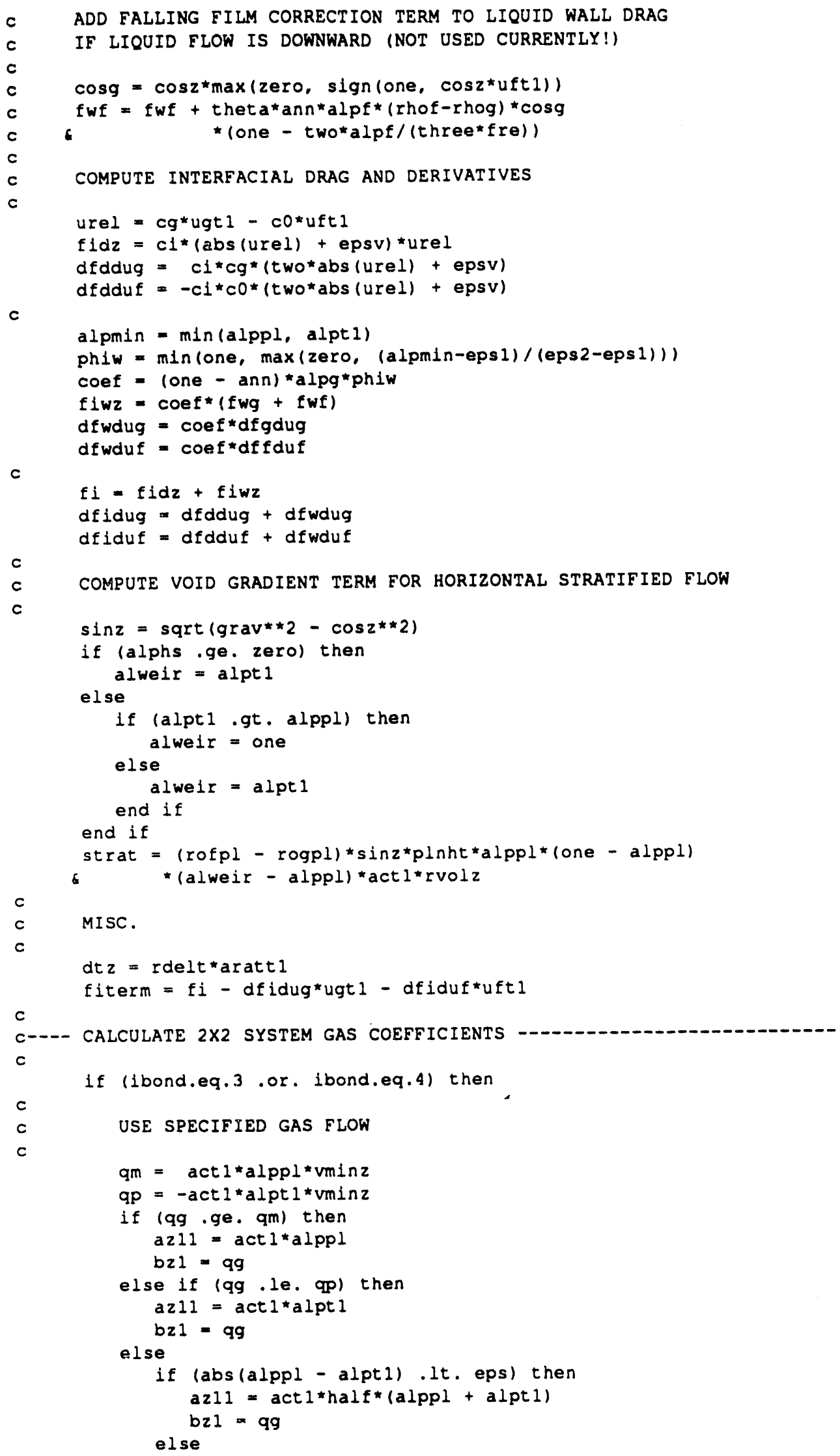



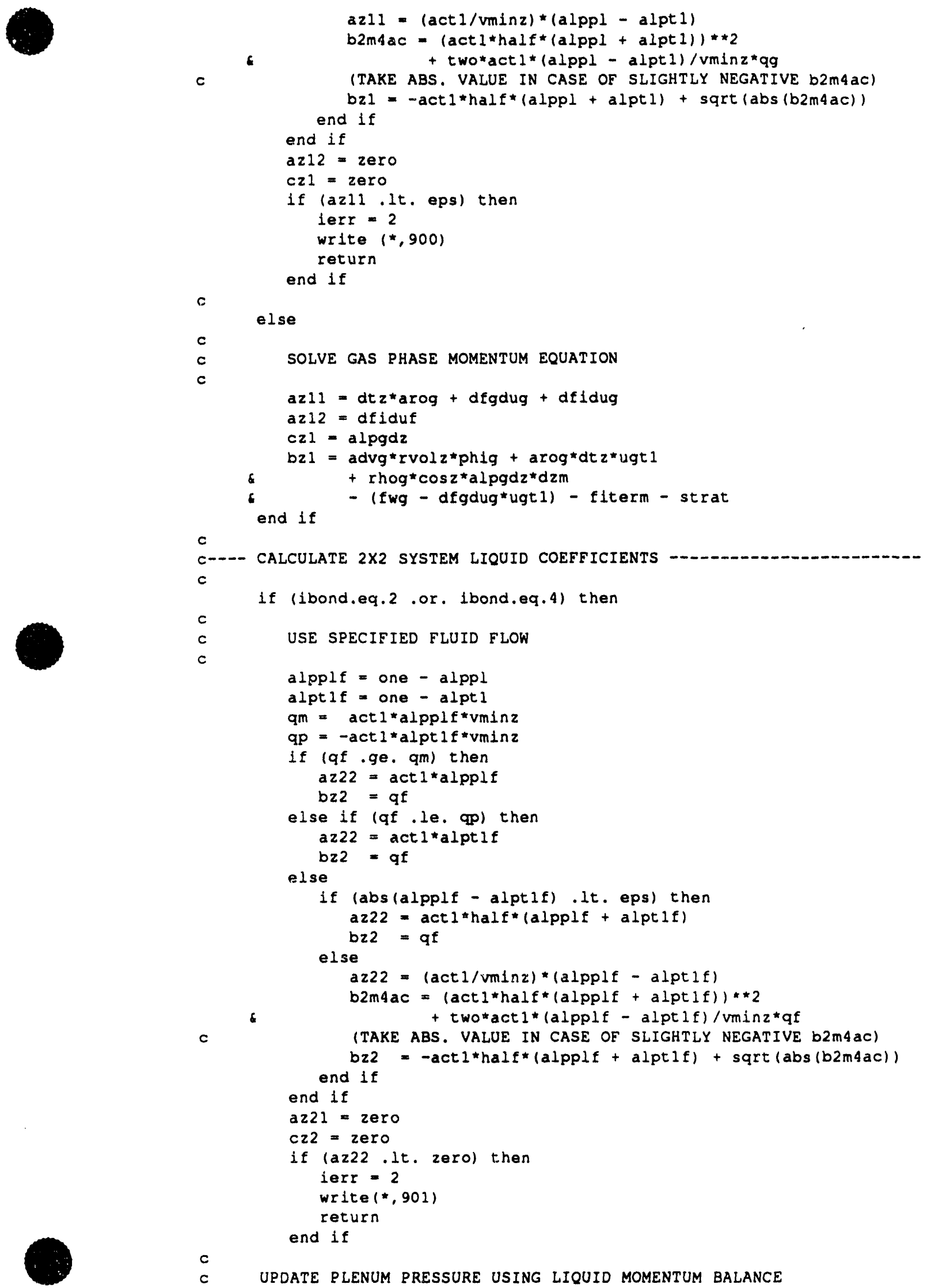
c

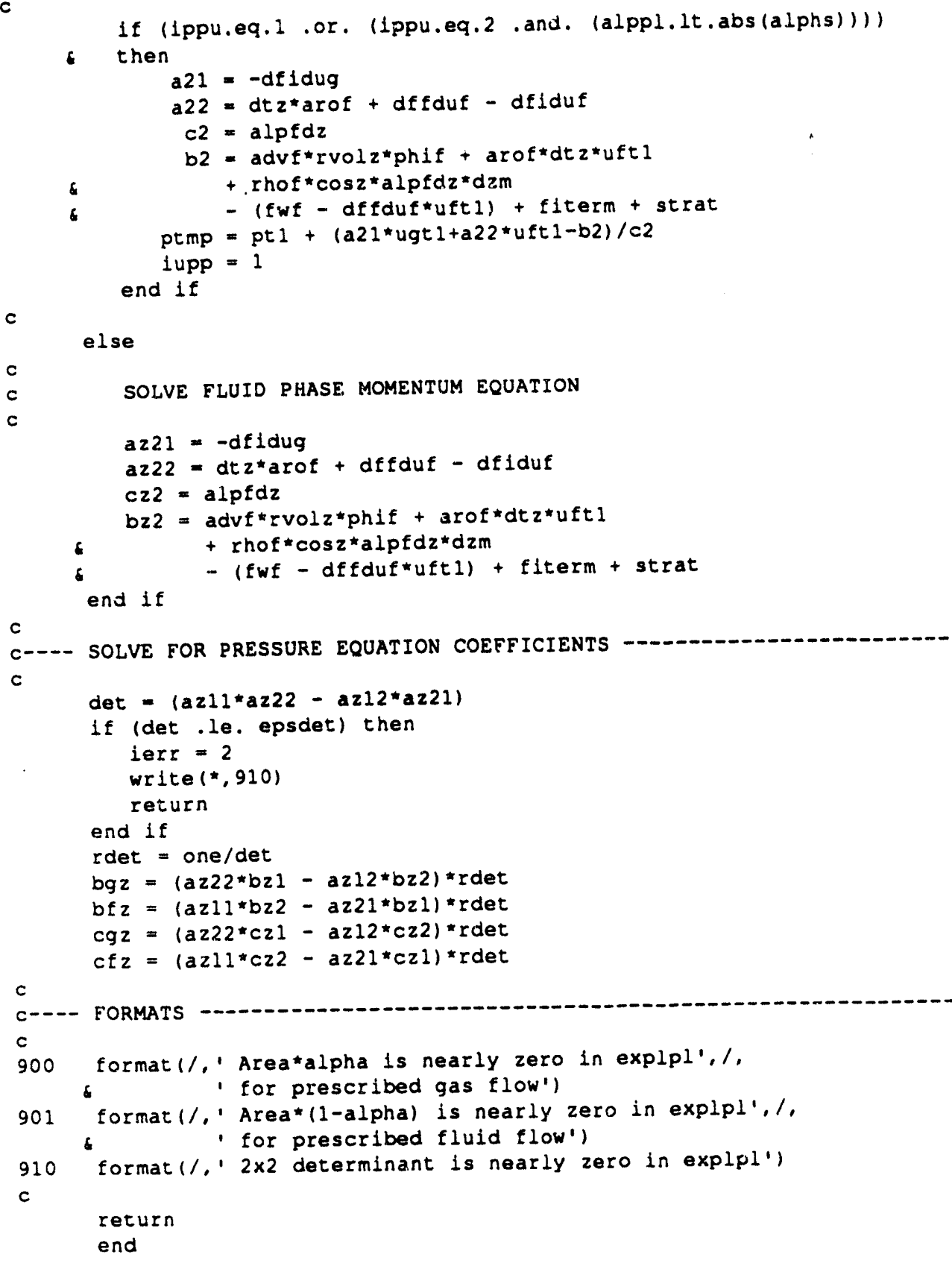

\section{EXPITB}

subroutine expltb(bgz,bfz,cgz,cfz $a c b l, a r a t b l, d h z, \cos z, d z m, u g b l, u f b l$, absr, fmlt, form, fre, c12n, cob, c0s. acb2, ugb2, ufb2, voltb, ptb, alptb, tgtb, tftb, xatb, rogtb, roftb, $a z b 1, v o l b 1, p b 1, a l p b 1, t g b 1, t f b 1, x a b 1$, rogbl, rofb1, alpb2, rogb2, rofb2,

rdelt, vminz, iexr, 11q)

$c$
$c$
$c$
$c$

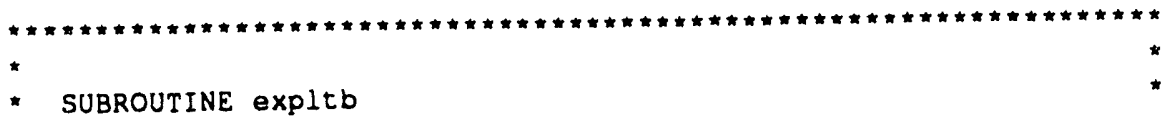




\begin{tabular}{|l|ll|l|l|}
\hline $2 / 15 / 93$ & WSRC-TR-93-086 Rev. 0 & FLOWTRAN-TF v1.2 Source Code & Pg. 109 of 354 \\
\hline
\end{tabular}

THIS ROU'LINE COMPUTES THE NEW TIME STEP FLUID VELOCITIES at the tank bottom cell face as a linear function of the new * TIME STEP PRESSURE DIFFERENCE ACROSS THE CELL FACE FOR THE BOTTOM BOUNDARY OF THE ASSEMBLY. TAE COEFFICIENTS FORMING THESE LINEAR EXPRESSIONS IRE FUNCTIONS OF OLD TIME STEP QUANTITIES; THEREFORE, THIS CALCULATION IS ONLY DONE ONCE AT THE BEGINING OF EACH TIME STEP.

THE EXPRESSIONS HAVE THE FOLLOWING FURM:

FOR GAS PHASE:

$u_{g, j+1 / 2}^{n+1}=B_{g, j+1 / 2}^{n}+c_{g, j+1 / 2}^{n} *\left(p^{n+1}-p^{n+1}{ }_{j+1}\right)$

FOR LIQUID PHASE:

$$
u_{t, j+1 / 2}^{n+1}=B_{f, j+1 / 2}^{n}+c_{f, j+1 / 2}^{n}{ }^{n}\left(^{n+1}-P^{n+1}{ }_{j+1}\right)
$$

WHERE: $\quad B=b g z(2-D I R E C T I O N)$

$$
\begin{aligned}
& { }_{B}^{g}=b f z(Z-D I R E C T I O N) \\
& f \\
& C=\operatorname{cg} Z \text { (Z-DIRECTION) } \\
& \text { g } \\
& C_{f}=\operatorname{cfz}(Z \text {-DIRECTION) }
\end{aligned}
$$

ARGUMENTS (0=Output, I=Input):

O bgz = SEE ABOVE; GAS PHASE CONSTANT TERM

O bf $z$ SEE ABOVE; LIQUID PHASE CONSTANT TERM

O $\quad$ CgZ = SEE ABOVE; GAS PHASE PRESSURE COEFFICIENT

- 0 ef $Z$ = SEE ABOVE; LIQUID PHASE PRESSURE COEFFICIENT

- bottom section face "nzb (TERMEd 'bl' in thIs subroutine):

$$
\text { I } a c b 1=A R E A
$$

- I aratbl = RATIO Of local face AREa to AVERAGe CELl aREa

- I dhz = HYDRAULIC DIAMETER

- I $\cos z=\cos { }^{*} g$

* I $\quad \mathrm{dzm}=$ MOMENTUM CELl LENGTH

* I Ugbl = GAS VELOCITY

- I U U

* I abSr $=$ ABSOLUTE ROUGHNESS

- I fmIt = FRICTION MULTIPLIER

- I fOIm = FORM LOSS COEFFICIENT

- I fIe * HYDRAULIC DIAMETER CORRECTION FACTOR, Kh

* I cIzn = gENERALIZED INTERFACIAL DRAG COEFFICIENT

- I $\quad C O b=$ BUBBLE REGIME DISTRIBUTION COEFFICIENT

- I cOs = SLUG REGIME DISTRIBUTION COEFFICIENT

* Bottom section face "nzb-1 (TERmed 'b2' in this subroutine):

- I acb2 = AREA

* I ugb2 a GAS VELOCITY

* I ufb2 = LIQUID VELOCITY

- tank bottom cell:

- I voltb = VOLUME

- I ptb = PRESSURE

- I alptb = VOID FRACTION

- I tgtb = GAS TEMPERATURE

- I $t f t b=$ LIQUID TEMPERATURE 


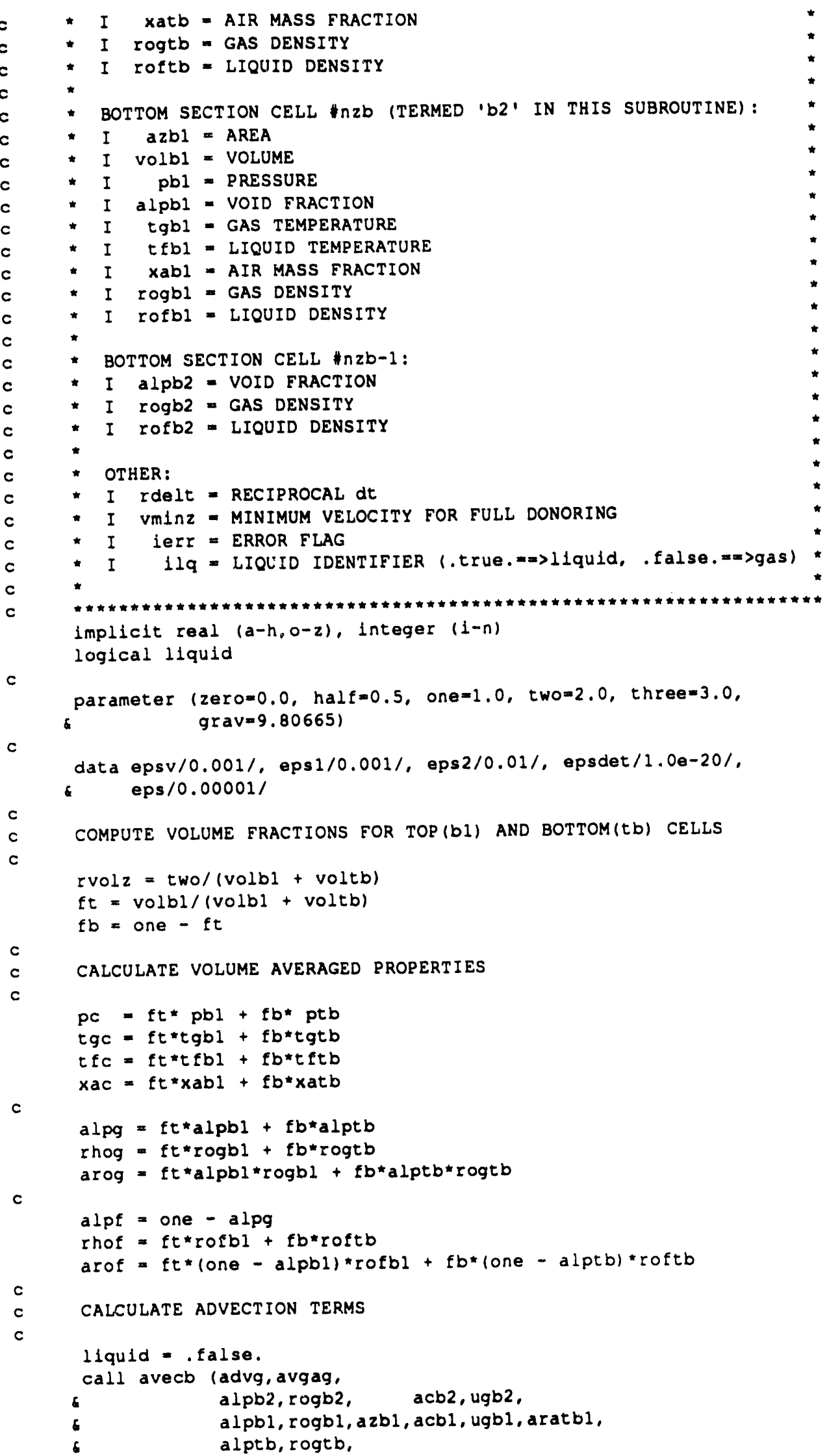


$\operatorname{vminz}, 11 q u 1 d)$

c

liquid $=$.true.

call avecb ladvf, avgaf,

- alpb2, rofb2, acb2,ufb2,

\& alpbl, rofbl, azbl, acbl, ufbl, aratbl,

s alptb, roftb.

- vminz, liquid)

EVALUATE ADVECTION TERM CUT-OFF FUNCTIONS

phlg $=\min (o n e, \max (z e r o,(a) p g-e p s 1) /($ eps2-eps1)))

phif $=\min (o n e, \max (z e r o,($ a.pf-eps1)/(eps2-eps1)))

COMPUTE co, cg, ANNULAR FRACTION ann

call $\operatorname{cofcn}(a 1 p g, c 0 b, c 0 s, c 0, c g, a n n)$

COMPUTE GAS AND LIQUID WALL DRAG AND DERIVATIVES

theta $=$ phif

alpgdz = avgag*rvolz

alpfdz = avgaf*rvolz

$c$

call fwallz (fwg, fwf, dfgdug, dffduf,

- ahz, theta, fmlt, form, absr, fre, ugbl, ufbl,

6 alpgdz,alpfdz, pc,tgc,tfc, xac, rhog, rhof, 1lq)

ADD FALLING FILM CORRECTION TERM TO LIQUID WALL DRAG

IF LIQUID FLOW IS DOWNWARD (NOT USED CURRENTLY!)

$\operatorname{cosg}=\cos z * \max (z e r o, \operatorname{sign}(o n e, \cos z * u f b 1))$

$f w f=f w f+$ theta*ann*alpf* (rhof-rhog)*cosg

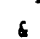

* (one - two*alpf/(three*fre))

COMPUTE INTERFACIAL ORAG AND DERIVATIVES

urel $=c g^{\star u g b l}-c 0 * u f b 1$

$f i d z=c i z n *(a b s$ (urel) + epsv)*urel

dfddug $=c 12 n * c g *($ two*abs (ure $l)+$ epsv)

dfdduf $=-c i z n * c 0 *$ (two*abs (urel) + epsv)

c

alpmin $\min (a l p b l$, alptb)

phiw = min(one, $\max (z e r o,($ alpmin-eps1)/(eps2-eps1))

coef $=($ one - ann $) * a l p g * p h i w$

$f i w z=\operatorname{coe} f *(f w g+f w f)$

$d f w d u g=c o e f * d f g d u g$

dfwduf = coef $d f f d u f$

C

$f i=f i d z+f i w z$

dfidug = dfddug + dfwdug

$d f$ t.tuf $=$ dfdduf + dfwduf

c

CALCULATE $2 \times 2$ SYSTEM GAS MOMENTUM COEFFICIENTS

$d t z=r d e l t \star a r a t b l$

fiterm = fi - dfidug*ugbl - dfiduf*ufbl

c

$a z 11=d t z$ arog + dfgdug + dfidug

az12 = dfiduf

$c z 1=a l p g d z$

$b z l=a d v g^{*} r v o l z^{*} p h i g+\operatorname{arog}{ }^{*} d t z^{*} u g b l$

$6 \quad+$ rhog*cos $z^{*} a l p g d z * d z m$

- - (fwg - dfgdug*ugbl) - flterm 
c

c

$c$

c

900 format (1, ' Area*alpha is nearly zero in expltb', /,

6 ' for prescribed gas flow')

901 format (1,' Area*(1-alpha) is nearly zero in expltb', /,

- 'for prescribed fluid flow')

910 format (/,' 2 2 2 determinant is nearly zero in expltb')

c

return

end

\section{FIIMB}

$$
\begin{aligned}
& \text { subroutine flimb (qf, dqfdt, qg, dqgdt, vapor, wet, qchf, tchf, twalk, } \\
& \text { tliq, tgas, tsat, pres, alfa, beta, xa, uliq, ugas, } \\
& \text { rhol, rhog, condg, cpg, visg, dhy, hfg, hlsat, hgsat, } \\
& \text { sigma, dofilm, 1lq) }
\end{aligned}
$$

$*$

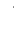$$
\text { * GORSLUND-ROHSENOW DROP IMPINGEMENT CORRELATION FOR LIQUID, }
$$$$
\text { * and dougall-Rohsenow correlation for gas }
$$ 


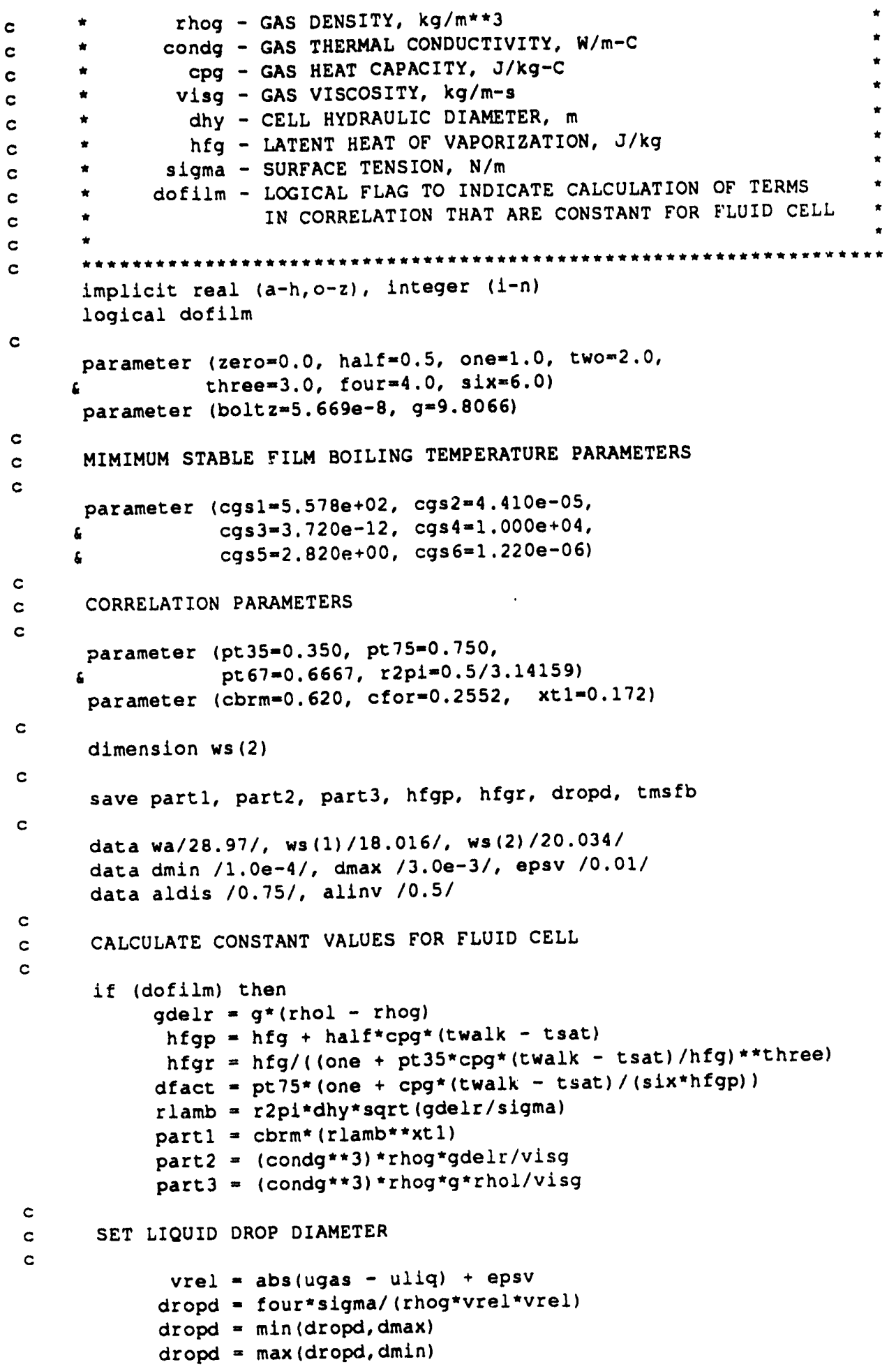

DETERMINE MINIMUM STABLE FILM BOILING TEMPERATURE, tmS fb USING GROENEVELD-STEWART CORRELATION

$$
\begin{aligned}
& \text { xstm }=\text { one }-x a \\
& \text { palr }=\text { pres*xa/(xa+xstm*wa/ws(11q)) }
\end{aligned}
$$

EVALUATE AIR, STEAM, AND LIQUID ENTHALPIES (hair, hstm, hfld) 
c visg, cundg, cpg, visg, zero, 11

c

c

c

c

C

c:

c

c

c

c

c

$$
\text { end if }
$$

FILM BOILING REGIME: USE FILM HEAT FLUX RESULTS

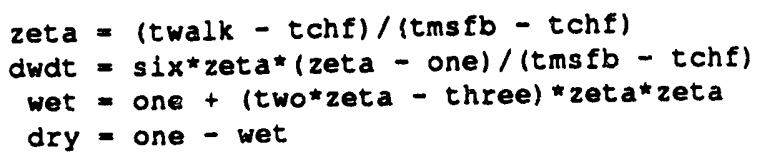

\section{EWALLX}

subrautine fwalix(fwg, Ewf, dfgdwg, dffdwf,

6 dh, theta, fmlt, kmlt, absr, fre, wg, wf,

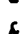

alpgdx, alpfdx, $p, t g, t f, x a$, rhog, rhof, ilq)$$
\approx
$$

c

c

c

c

c

c

$c$

c

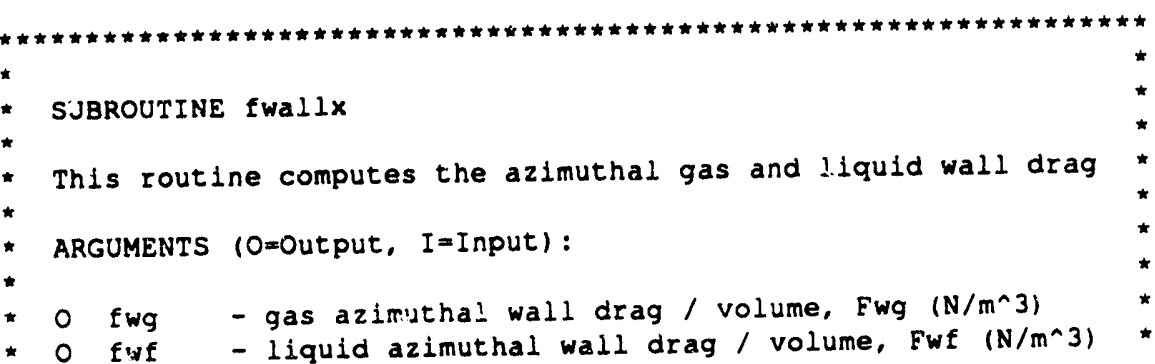




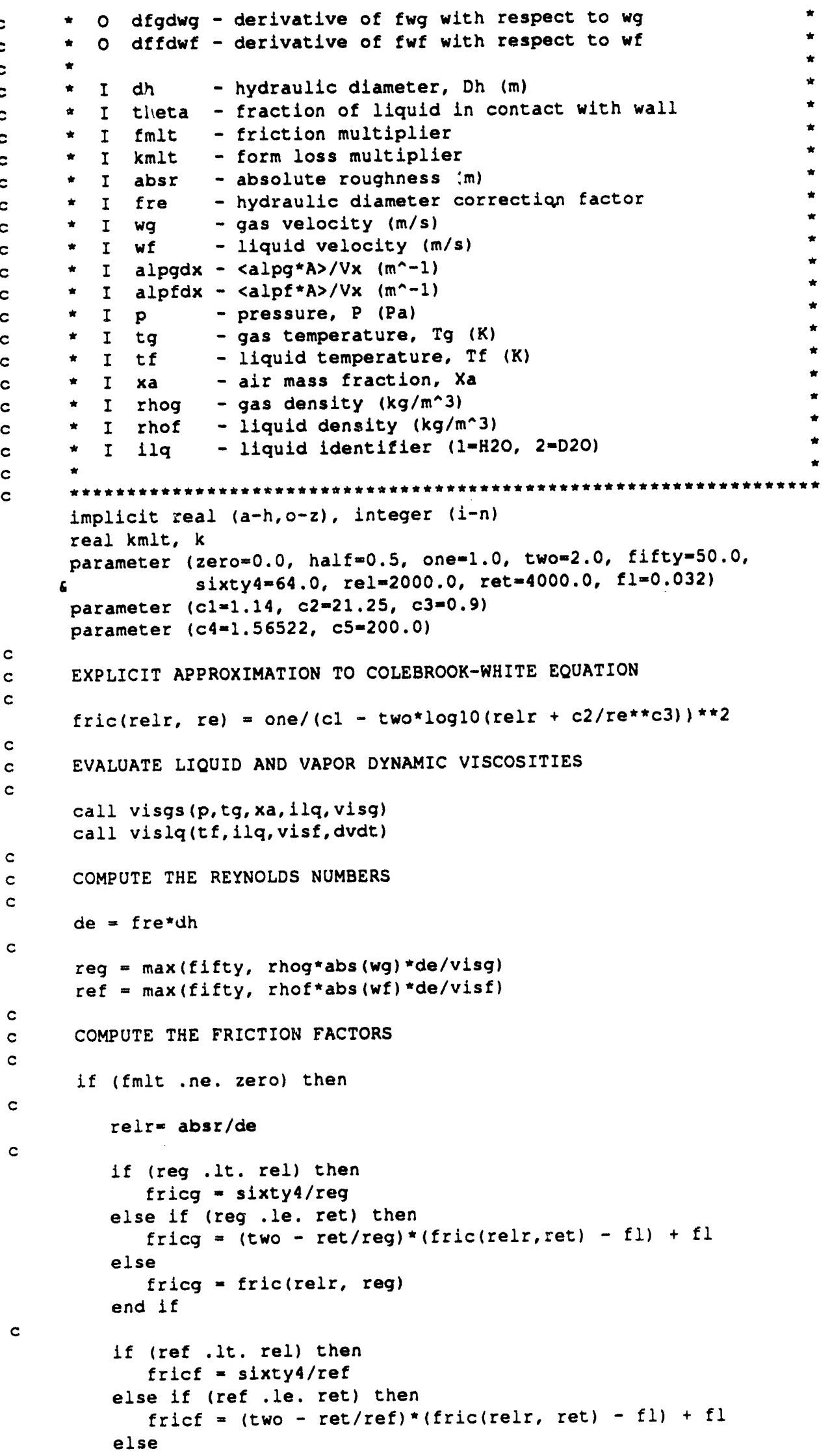




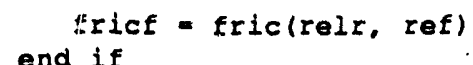

c

c

c

C

c

end if

COMPUTE NOMINAL FORM LOSS COEFEICIENT

$k=c 4+c 5 /$ ref

COMPUTE WALL FRICTION \& DERIVATIVE FOR EACH PHASE

dfgdwg $=($ (one-theta)*fmlt*fricg/dh + kmlt*alpgdx*k)*rhog*abs (wg)

dffdwf $=(\quad$ theta *fmlt*fricf/dh+kmit*alpfdx*k)*rhof*abs (wf)

$f w g=d f g d w g * h a l f \star w g$

fwf $=$ dffdwf*half*wf

return

end

\section{FWALLZ}

subroutine fwallz(fwg, fwf, dfgdug, dffduf,

dh, theta, fmlt, form, absr, fre, ug, uf,

8

alpgdz, alpfdz,p,tg,tf,xa, rhog, thof,ilq)

SUBROUTINE Ewallz

This routine computes the axial gas and liquid wall drag

EXCLUDING the laminar falling film correction component of

the liquid wall frictional wall drag. Consult TF-28 for more *

information.

ARgUMENTS (O=Output, I=Input):

- fwg - gas axial wall drag per unit volume, Fwg $\left(\mathrm{N} / \mathrm{m}^{\wedge} 3\right)$

- Ewe - liquid axial wall drag per unit vol., Fwf (N/m^3)

- dfgdug - derivative of fwg with respect to ug

- dffduf - derivative of fwf with respect to uf

I dh - hydraulic diameter, Dh (m)

* I theta - fraction of liquid in contact with wall

* I fmlt - friction multiplier

* I form - form loss coefficient

* I absr - absolute roughness (m)

* I fre - hydraulic diameter correction factor, $\mathrm{Kh}$

- I ug - gas velocity $(\mathrm{m} / \mathrm{s})$

- I uf - liquid velocity $(\mathrm{m} / \mathrm{s})$

*I alpgdz - <alpg*A>/Vz $\left(m^{n}-1\right)$

- I alpfdz - <alpf*Az/Vz $\left(m^{\wedge}-1\right)$

* I $P$ - pressure, $P$ (Pa)

* I tg - gas temperature, Tg (K)

- I tf - liquid temperature, Tf (K)

* I xa - air mass fraction, $x a$

- I rhog - gas density $\left(\mathrm{kg} / \mathrm{m}^{\wedge} 3\right)$

- I rhof - liquid density $\left(\mathrm{kg} / \mathrm{m}^{\wedge} 3\right)$

I $11 q$ - liquid identifier $(1=\mathrm{H} 20,2=\mathrm{D} 20)$

COMMON VARIABLES

I xeric - multiplier to friction factors for sensitivity studies 
c

c

c

c

c

c

c

c

c

c

$c$

$c$

$c$

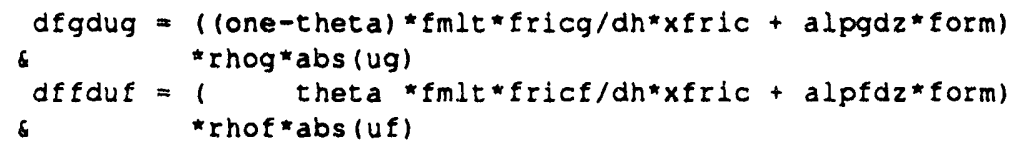

$f w g=d f g d u g * h a l f * u g$

fwf $=$ dffduf half $u f$

return

end

Implicit real $(a-h, 0-z)$, integer $(1-n)$

parameter (zerom 0.0 , half $=0.5$, one $=1.0$, two $=2.0$, fift $y=50.0$,

parameter $(c)=1.14, c 2=21.25, c 3=0.9)$

Include 'knobs.inc'

EXPIICIT APPROXIMATION TO COLEBROOK-WHITE EQUATION

fric(relr, re) $=$ one $/(c)-t w o * \log 10($ relr $+c 2 /$ re**c3) $) * 22$

If (fmlt ne. zero) then

EVALUATE LIQUID AND VAPOR DYNAMIC VISCOSITIES

call visgs (p,tg, xa, 1lq,visg)

call vislq(tf, 1lq, visf, dvdt)

O.C. JONES LAMINAR FLOW FRICTION CORRECTION

de $=f r e^{\star} d h$

$r e l r=a b s r / d e$

COMPUTE THE REYNOLDS NUMBERS

reg $=\max (f i f t y$, rhog*abs (ug)*de/visg)

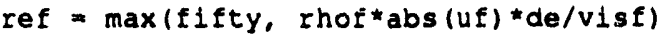

COMPUTE THE FRICTION FACTORS

if (reg . lt. rel) then

fricg = sixty4/reg

else if (reg.le. ret) then else

fricg $=($ two $-r e t / r e g) *(f r i c(r e l r, r e t)-f l)+f 1$

frlcg $=$ frlc(relr, reg)

end if

if (ref..1t. rel) then

fricf = sixty $4 /$ ref

else if (ref.le. ret) then

else

fricf $=$ fric (relr, ref)

end if

end if

COMPUTE WALL FRICTION \& DERIVATIVE FOR EACH PHASE

(DOES NOT INCLUDE LAMINAR FALLING FILM CORRECTION)$$
\text { end }
$$ 


\section{GAJOR5}

subroutine gajor5 (ncol,ca)

$\star$

* Performs gauss-jordan inversion of a $5 \times 5[\mathrm{~cm}]$ matrix to

* eliminate alp, tg, tf, and $x a$ for a pressure only problem.

$\star$

* The following is a limited gauss-jordan technique for a

* 5x5 matrix with an arbitrary number of pressure coupling

* coefficients.

*

$\star$

* Ta a a al c $\mid$ b. . . .

* 1

la a a a al c|

I 1 l 1 auss-

la a a a al c l b... jordan

l 1 l 1 l operated

la a a a a

to

$\overline{11} 000001$ e 1 d. . .

10200001 e 1 d...

10010001 e 1 a...

$\begin{array}{llllllll}0 & 0 & 0 & 1 & 0 & 0 & \text { e } & \text { d d . . . I }\end{array}$

la a a a al c l b...

$\begin{array}{lllllllll}1 & 0 & 0 & 1 & 1\end{array}$

1000011 e 1 d...1

1

final result (after gauss-fordan inversion) is of the form:

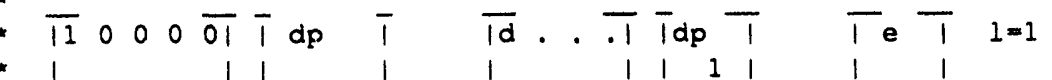

1

11

$\begin{array}{lllllll}10 & 1 & 0 & 0 & 01 & 1 & \text { dalp }\end{array}$

1

$\begin{array}{llllllll}10 & 0 & 1 & 0 & 01 & 1 & \mathrm{dtg}\end{array}$

1

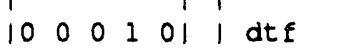

1

$\begin{array}{llllllll}1 & 0 & 0 & 0 & 1 & 1 & 1 & d x a\end{array}$

1d. .11 .1

$-11$

1

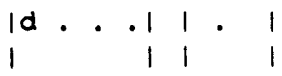

ld. .11 .1

ld. . . I lap I

$1_{-} \quad-11_{-} n_{-}$

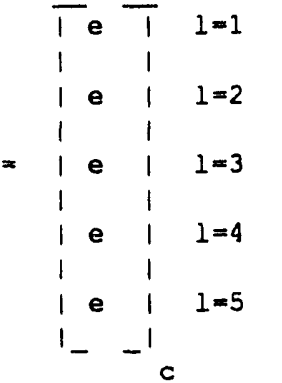

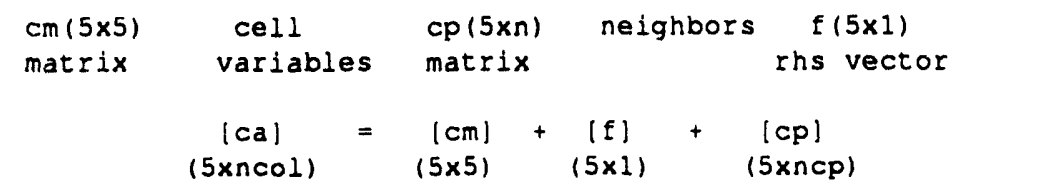

Note that: the elements contained within the upper tri-angular portion of [cm] are not operated upon. Only those element members employed in later computations (1.e. [cp] and (f]) are altered.

implicit double precision $(a-h, 0-z)$, integer $(i-n)$

parameter (one $=1.0)$

dimension ca $(5, \mathrm{ncol})$

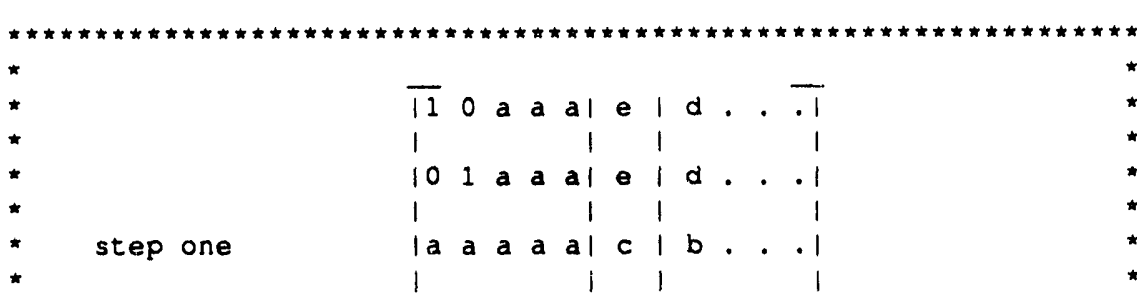


$r d=o n e /(c a(1,1) * c a(2,2)-c a(1,2) * c a(2,1))$

do $10 \mathrm{k}=3, \mathrm{ncol}$

temp $=c a(2,2) * c a(1, k)-c a(1,2) * c a(2, k)$

$c a(2, k)=r^{*}(c a(1,1) * c a(2, k)-c a(2,1) * c a(1, k))$

$c a(1, k)=r d * t e m p$

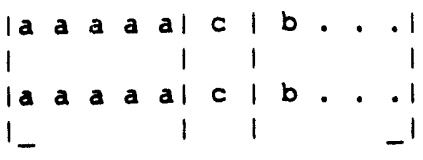

\section{"}




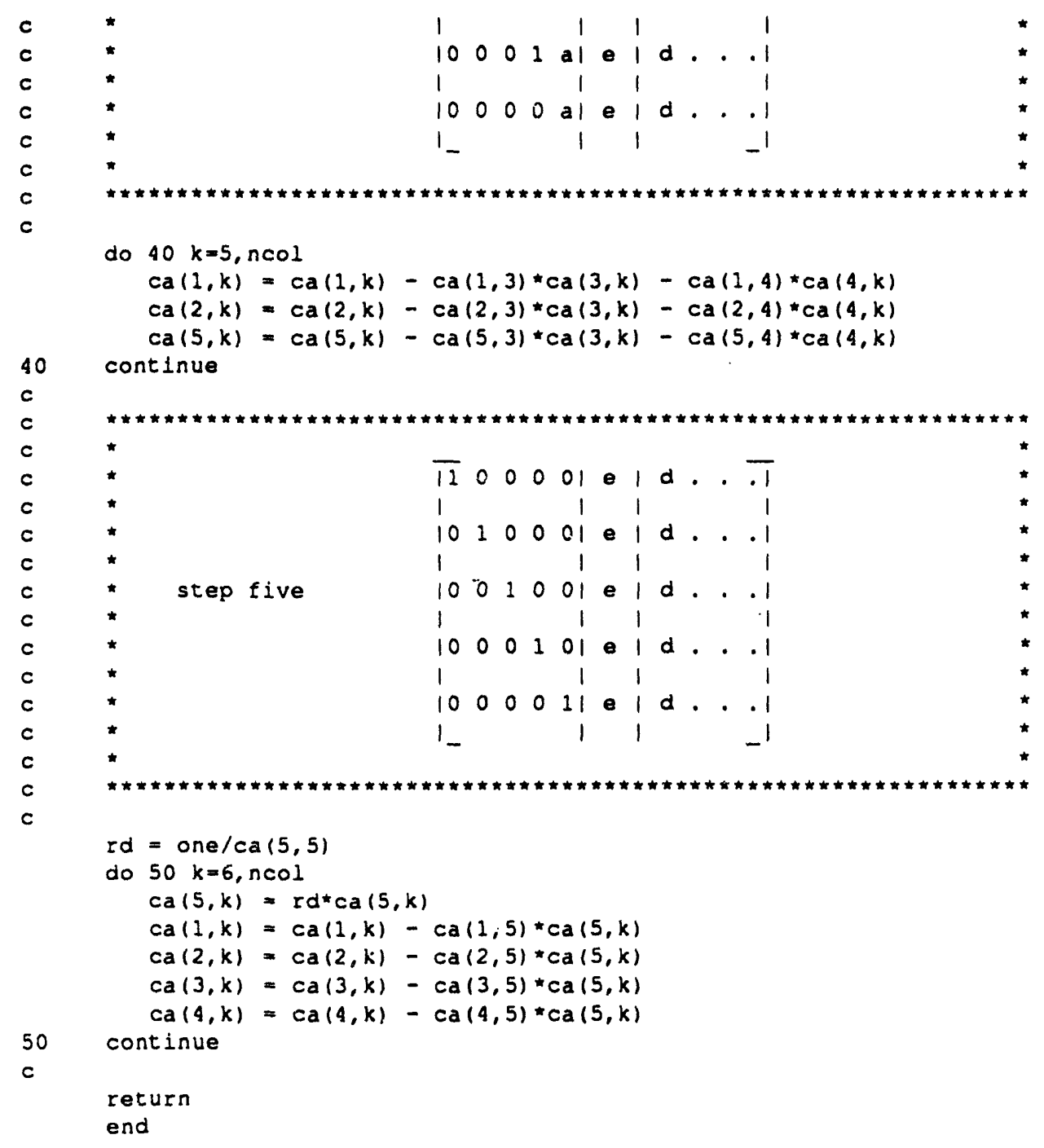

\section{GAMMAA}

subroutine gammaa(p,alpha, tg, xa, xao,
ga, dgada, dgadxa,
ea, deadp, deada, deadtg, deadxa)

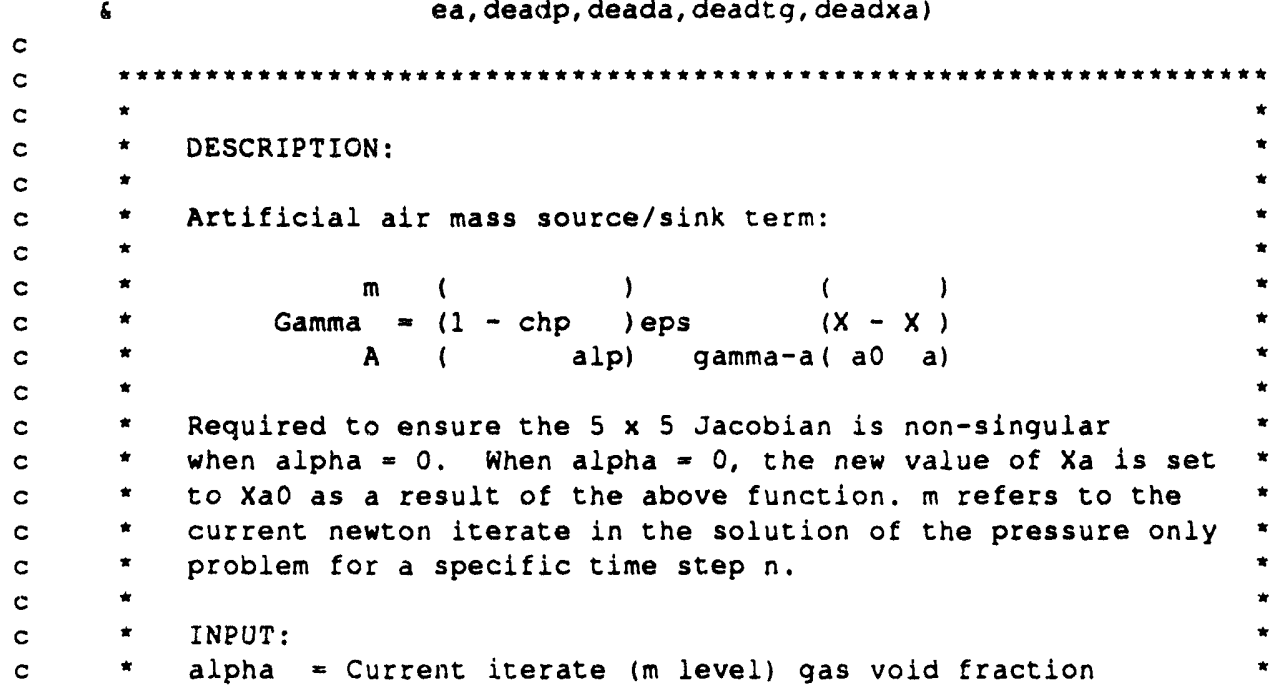




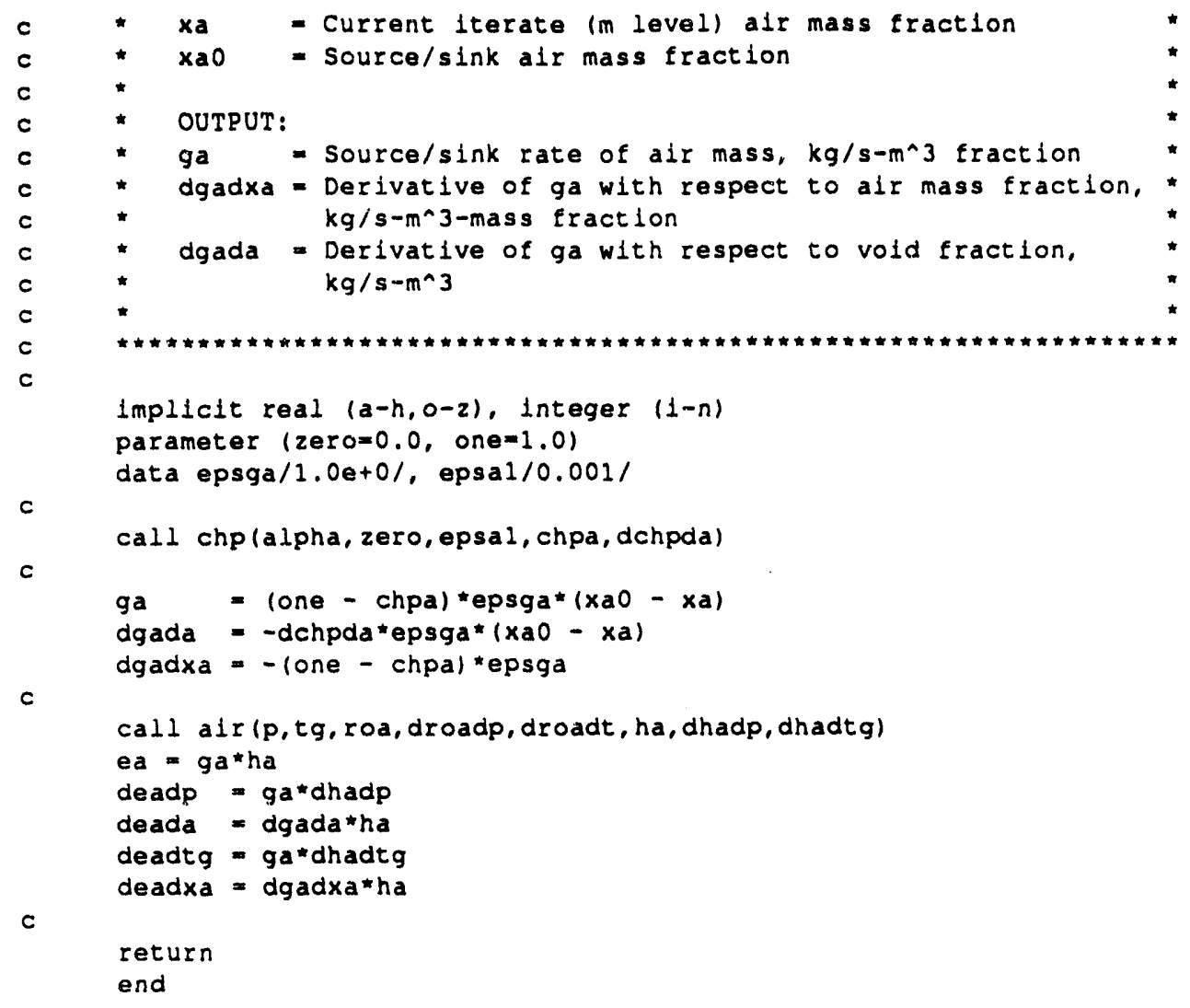

\section{GAMMAI}

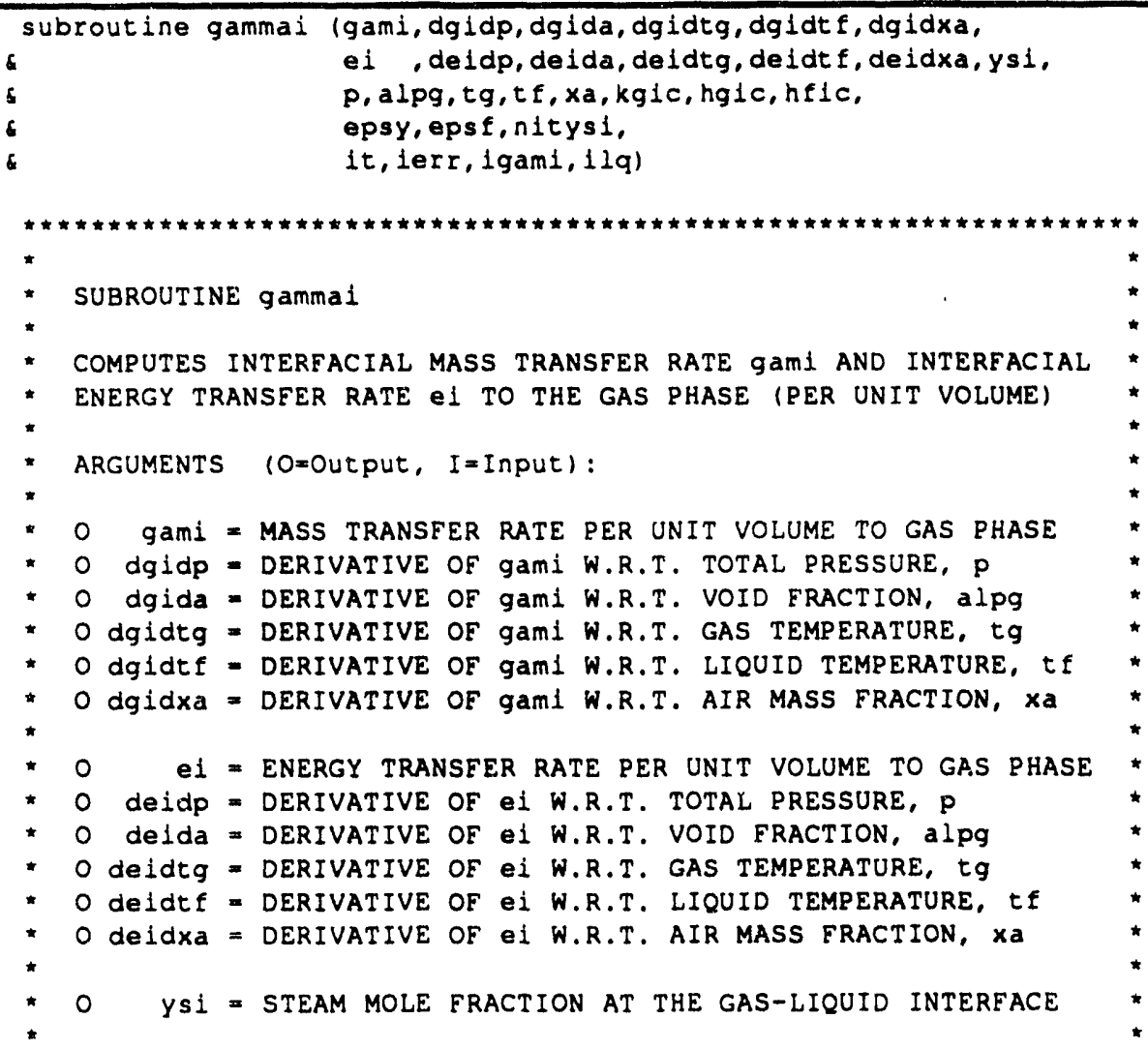




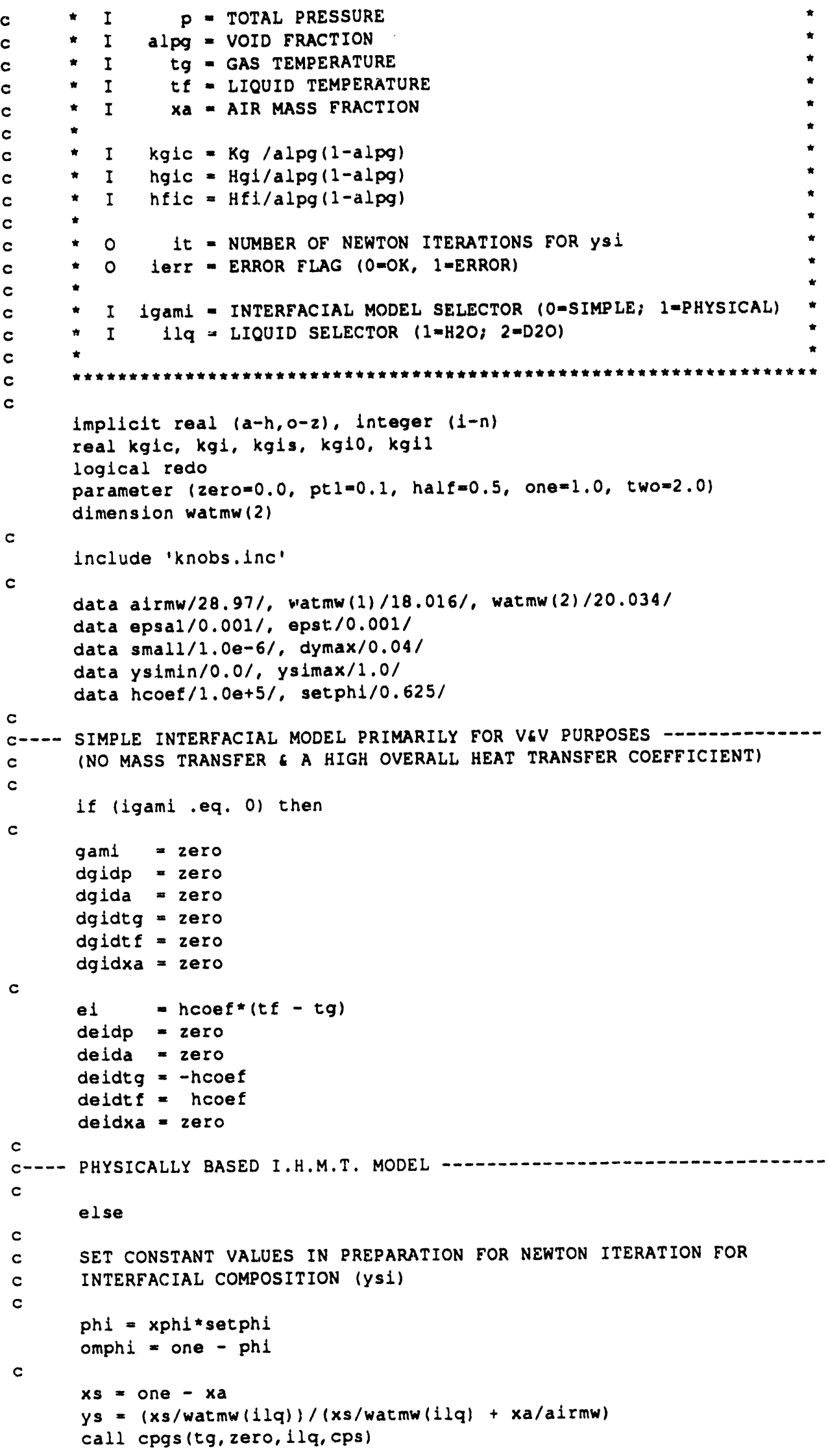


c

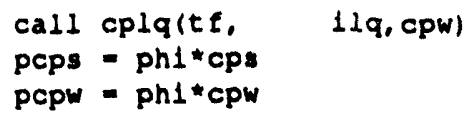


c

c

c

$c$

c

c

30

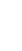

6

$$
\text { dhfdt } 1=+ \text { dptedt (one - phlag) (phlaf*hfis - hf10) }
$$

rdnom = one/(hs1 - hwl + pcpw"deltf - pcps"deltg)

gami =-rdnom* (hgi*deltg + hfl*deltf)

fg $=h g 1+$ angdt 1 tdeltg - gaml"pcps

ff $=h f 1+\operatorname{dhfdt} 1$ deltf + gaml*pcpw

dgldt $1=-\operatorname{rdnom} *(g a m 1 *(d h s i d t-d h w 1 d t)+f g+t f)$

$$
\begin{aligned}
& \text { fy = one - ysi + phi*delys } \\
& \text { fysl = gaml*fy - kg1*delys }
\end{aligned}
$$

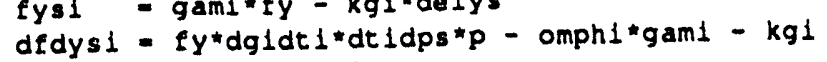

$$
\begin{aligned}
& \text { dysi = fysi/dfdysi }
\end{aligned}
$$

tol $=\max ($ epsy*ysi, small)

If (abs (dys1).1t.tol and. abs (fysi). It.epsf) then

BAIL OUT W/O UPDATE SO THAT ABOVE QUANTITIES ARE

BAIL OUT W/O UPDATE SO THAT ABO REDUNDANT CODING BELOW)

$$
\text { go to } 100
$$

ADJUST INITIAL GUESS AND TRY AGAIN IF YSI IS AT AN EXTREME VALUE, IF YSI IS AT AN INTERMEDIATE VALUE AND THE ITERATION FAILED TO CONVERGE, TRY AN AVERAGE OF THE LAST TWO VALUES AS AN INITIAL GUESS IN CASE A LIMIT CYCLE HAS BEEN REACHED

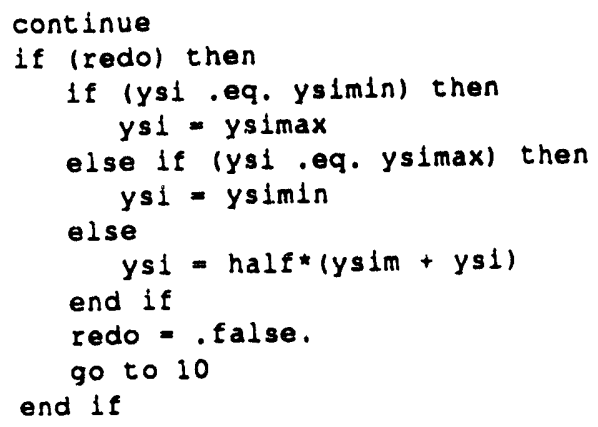




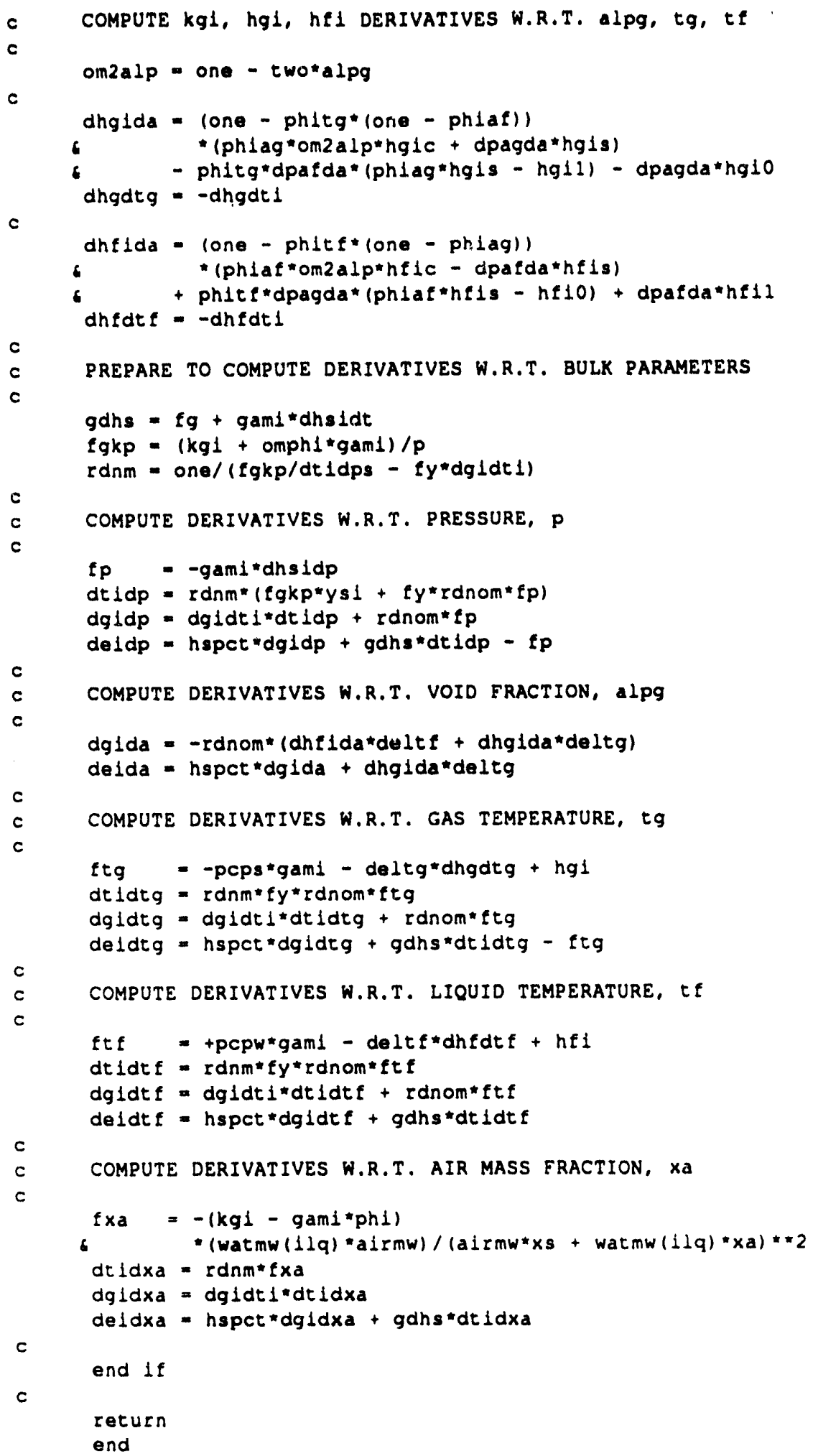

\section{GEOELD}

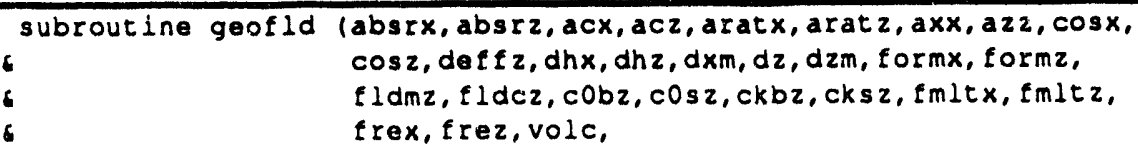


absrt, act, aratt, azt, cost, defft, dht, dzt, dzmt, formt, fldmt, fldct, cObt, cOst, ckbt, ckst, fmltt, eret, volt, $a b s r b, a c b, a r a t b, a z b, c o s b$, deffb, dhb, dzb, dzmb, formb, fldmb, fldcb, cObb, c0sb, ckbb, cksb, fmltb. freb, volb, acpl, volpl, actb, voltb, 1az1, 1 cc, na, nazc, nx, nchn, nxmax, nz, nzp1, nzp2, nzp3, nzt, nzb, bot $1 n$, subln, topln, 1units, 1stop)

C
$c$
$c$
$c$
$c$
$c$
$c$
$c$
$c$
$c$
$c$

\begin{tabular}{|c|c|c|}
\hline dimension & $\begin{array}{r}\text { absrt }(n z t), \\
\operatorname{act}(n z t),\end{array}$ & $\begin{array}{r}a b s r b(n z b), \\
a c b(n z b),\end{array}$ \\
\hline c & $\operatorname{aratt}(n z t)$, & $\operatorname{aratb}(n z b)$, \\
\hline c & $\operatorname{azt}(n z t)$ & $a z b(n 2 b)$ \\
\hline$\leftarrow$ & $\cos t(n z t)$. & $\cos b(n z b)$. \\
\hline 6 & defft $(n z t)$, & deffb $(n z b)$. \\
\hline 6 & $\operatorname{dht}(n z t)$ & $d h b(n z b)$ \\
\hline 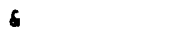 & $d z t(n z t)$, & $d z b(n z b)$, \\
\hline 6 & $d z m t(n z t)$ & $d z m b(n z b)$, \\
\hline 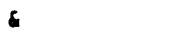 & fldmt $(n z t)$, & $f 1 \mathrm{dmb}(n z b)$ \\
\hline$c$ & fldct $(n z t)$ & $f l d c b(n z b)$, \\
\hline 6 & cobt $(n z t)$ & $c O b b(n z b)$, \\
\hline c & $\operatorname{cost}(n z t)$, & $\operatorname{cosb}(n z b)$, \\
\hline 6 & ckbt $(n 2 t)$. & $c k b b(n 2 b)$, \\
\hline 6 & ckst $(n 2 t)$. & $\operatorname{cksb}(n z b)$, \\
\hline$c$ & fmltt (nzt) & fmltb $(n z b)$, \\
\hline 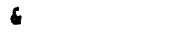 & $f \operatorname{ret}(n z t)$ & $\operatorname{reb}(n z b)$ \\
\hline
\end{tabular}

$\operatorname{absrx}(n z p 2, n \times \max , n c h n)$. $\operatorname{acx}(n z p 2, n \times m a x, n c h n)$, aratx (nzp2, nxmax, nchn). $\operatorname{axx}(n z p 2, n \times m a x, n c h n)$. $\cos x(n z p 2, n x \max , n c h n)$, $\operatorname{volc}(n z p 2, n x \max , n c h n)$, dhx (nzp2, nxmax, nchn), formx (nzp2, nxmax, nchn). fldcz (nzp3, nxmax, nchn). $\operatorname{cosz}$ (nzp3, nxmax, nchn). cksz (nzp3, nxmax, nchn), fmltx (nzp2, nxmax, nchn). frex (nzp2, nxmax, nchn), $d z(n z p 2), d x m(n c h n)$ 


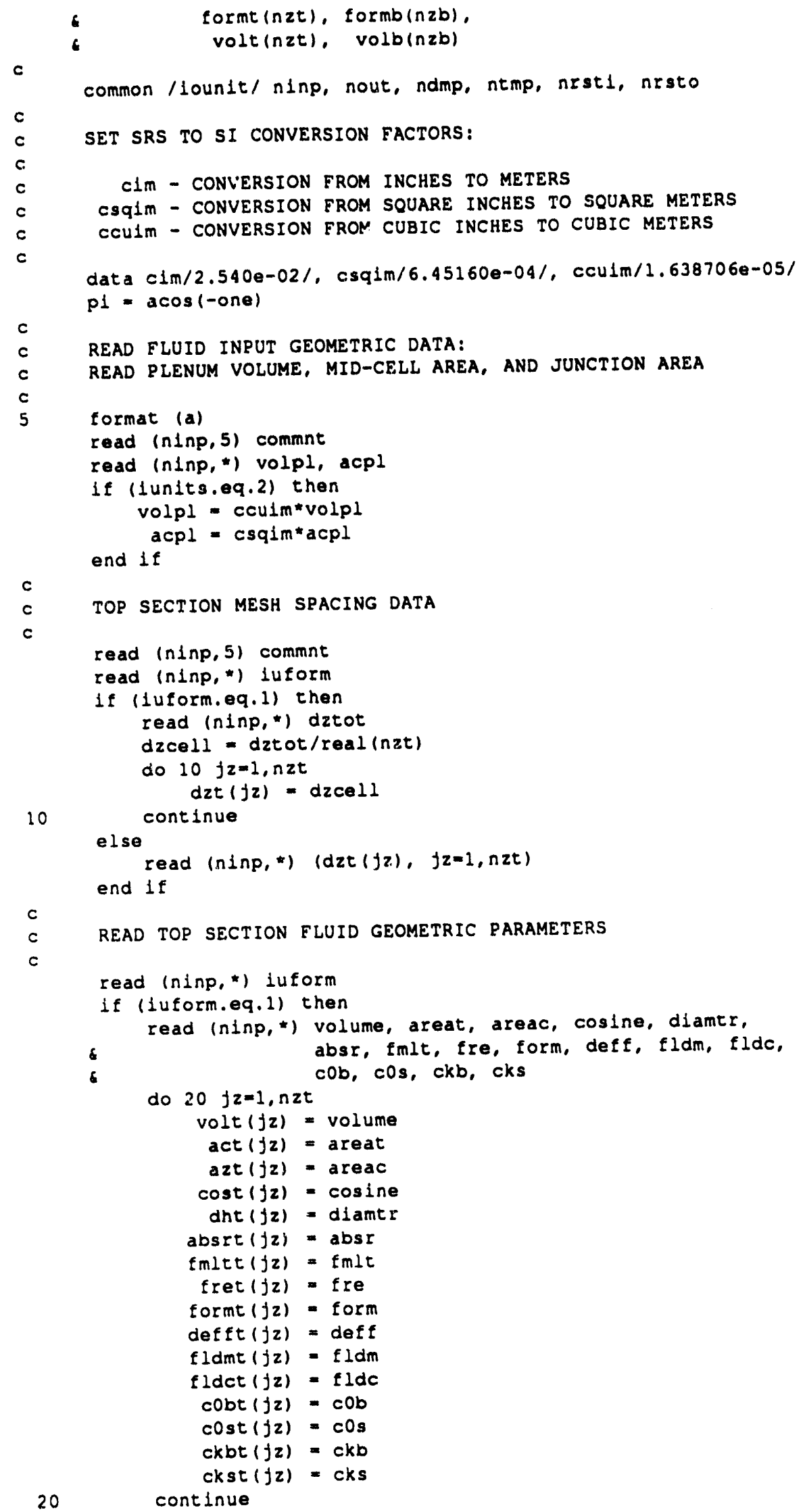




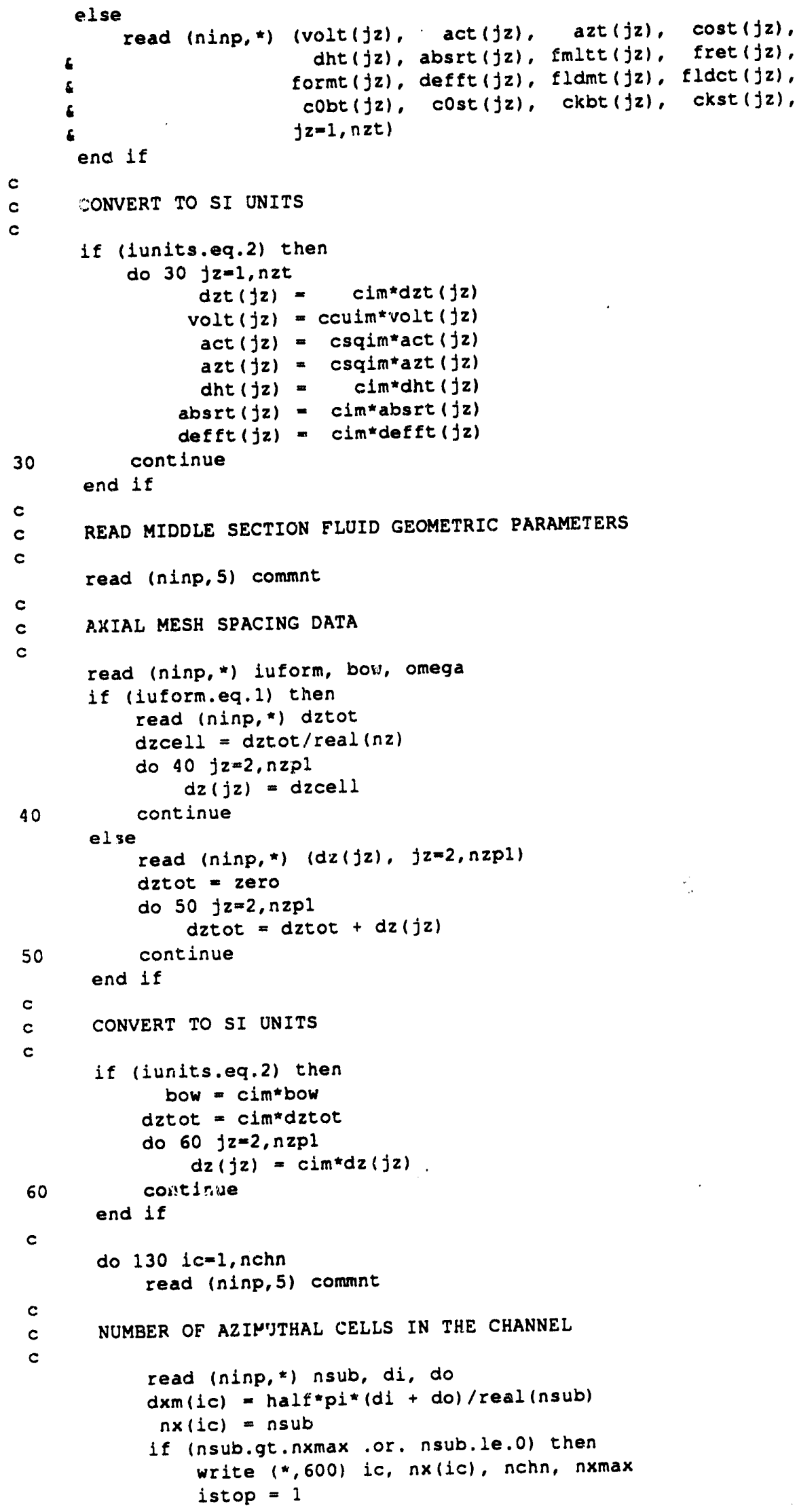


and if

write (nout, 500) nsub, ic

c

c

c

c

6

AZIMUTHAL GEOMETRY DATA:

(TEMPORARILY HOLD RIB GAP WIDTH IN ACX AREA ARRAY)

read (ninp, 5) commnt

read (ninp,*) iuform

if (iuform.eq.1) then

read (ninp,*) gapl, gap2, cosine, diamtr,

do 80 ix $=1$, nsub absr, fmit, fre, form

do $70 \quad j z=2, n z p 1$

$\operatorname{acx}(j z, i x, i c)=$ gapl

$\operatorname{axx}(j z, i x, i c)=$ gap2

$\cos x(j z, i x, i c)=\operatorname{cosine}$

$\operatorname{dhx}(j z, i x, i c)=d i a m t r$

$\operatorname{absrx}(j z, i x, i c)=a b s r$

$f m I t x(j z, i x, i c)=f m l t$

frex $(j z, 1 x, 1 c)=f r e$

continue

formx $(j z, i x, i c)=$ form

continue

else if (iuform.eq.2) then

read (ninp, $(\operatorname{acx}(2, i x, i c), \operatorname{axx}(2, i x, i c)$. $\cos x(2,1 x, 1 c), \quad d h x(2,1 x, 1 c)$, $\operatorname{abs} \mathrm{Ix}(2,1 x, 1 c), \operatorname{fm} 1 t x(2,1 x, 1 c)$, frex $(2, i x, i c)$, formx $(2, i x, i c)$.

do $85 i x=1, n s u b$ $i x=1, n s u b)$

do $75 j z=3, n z p 1$

$\operatorname{acx}(j z, i x, i c)=\operatorname{acx}(2, i x, 1 c)$

$\operatorname{axx}(j z, 1 x, i c)=\operatorname{axx}(2,1 x, 1 c)$

$\cos x(j z, i x, i c)=\cos x(2, i x, i c)$

$\operatorname{dhx}(j z, i x, i c)=d h x(2, i x, i c)$

$\operatorname{abs} x x(j z, 1 x, i c)=\operatorname{absrx}(2,1 x, 1 c)$

$f m l t x(j z, i x, i c)=f \operatorname{lt} x(2, i x, i c)$

$f \operatorname{rex}(j z, i x, i c)=\operatorname{frex}(2, i x, i c)$

formx $(j z, i x, i c)=f \operatorname{orm} x(2, i x, i c)$ continue

else

continue

read (ninp, *) ( $\operatorname{acx}(j z, i x, i c), \quad \operatorname{axx}(j z, i x, 1 c)$, $\cos x(j z, i x, i c), \quad d h x(j z, i x, i c)$, absrx $(j z, i x, i c), \operatorname{fmltx}(j z, i x, i c)$, frex $(j z, i x, i c)$, formx $(j z, i x, i c)$,

end if

$i x=1, n s u b), j z=2, n z p \mid$ )

AXIAL GEOMETRY DATA

read (ninp, 5) commnt

read (ninp,") luform

if (iuform.eq.1) then

read (ninp, ") areaf, areac, cosine, diamtr, absr, fmlt, fre, form, deft,

do $100 \quad 1 x=1$, nsub fldm, fldc, cOb, cos, ckb, cks

do $90 \quad j z=2, n z p 2$

$\operatorname{acz}(j z, i x, i c)=$ areaf

$\operatorname{azz}(j z, i x, i c)=$ areac

$\cos z(j z, i x, i c)=\operatorname{cosine}$

$\operatorname{dhz}(j z, i x, i c)=$ diamt $r$

$\operatorname{absrz}(j z, i x, i c)=\operatorname{absr}$ 
90

100

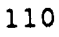

120

6

130

c

c

c

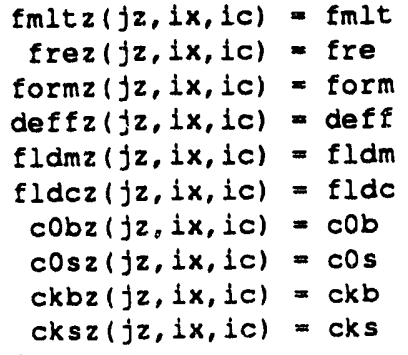

CONVERT TO SI UNITS

if (iunits.eg.2) then

do 160 ic $=1$, nchn

$d x m(i c)=c^{\star} i m^{\star} d x m(i c)$

nsub $=n \times(1 c)$

do 150 ix $=1$, nsub

do $140 \quad j z=2, n z p l$

$\operatorname{acx}(j z, i x, i c)=c i m * a c x(j z, i x, i c)$

$\operatorname{acz}(j z, 1 x, i c)=c s q i m \star a c z(j z, i x, 1 c)$

$\operatorname{axx}(j z, i x, i c)=c i m \star a x x(j z, i x, i c)$

$\operatorname{azz}(j z, i x, i c)=c s q i m \star a z z(j z, i x, i c)$ 


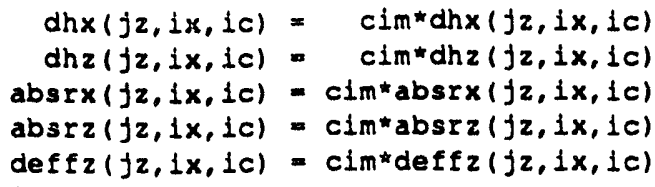


ADJUST AXIAL DIRECTIONAL COSINES FOR BOWING

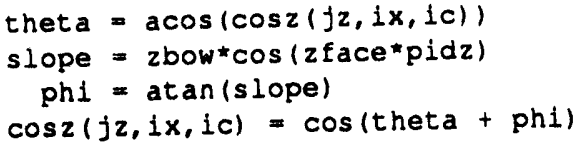


c

c

$c$

c

c

c

c

CALCULATE MOMENTUM CELL AXIAL LENGTHS IN TOP SECTION AND CONVERT DIRECTION COSINES TO GRAVITY VECTORS

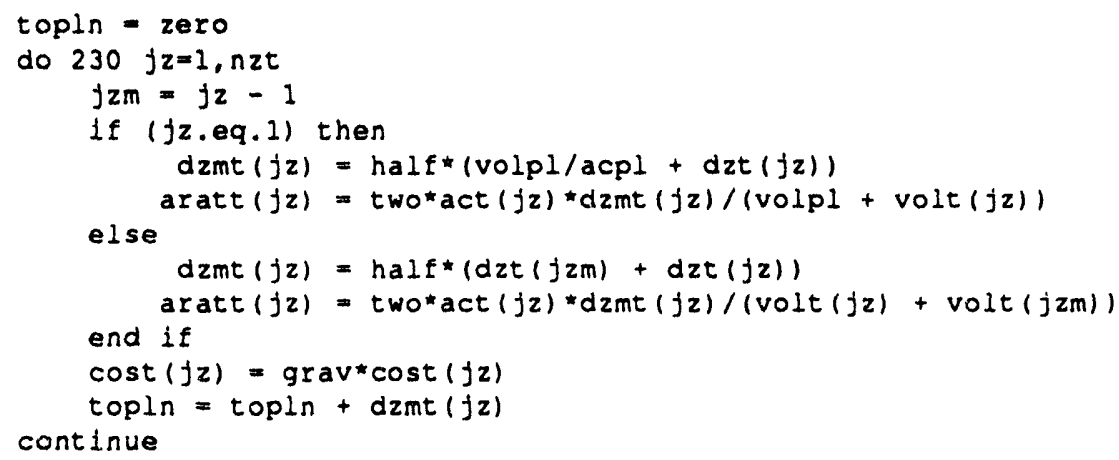

SET UP AZIMUTHAL INTERCONNECTION ARRAYS

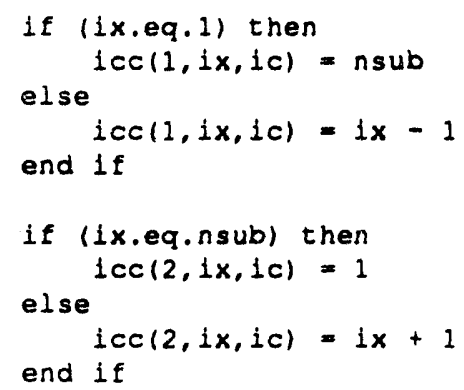

SET UP AZIMUTHAL CELL PARAMETERS FOR SUBCHANNEL 
c

$1 \mathrm{faz}=1+n \operatorname{set} *(1 \mathrm{x}-1)$

if $(\bmod (1 c, 2)$.eq. 0 and. nsub.gt.1 and. nchn.gt.1) then ifaz $=1 \mathrm{faz}-\mathrm{na} / 8$

do $250 \quad j z=2, n z p 1$

$$
j z p=j z+1
$$
$\operatorname{volc}(f z, i x, i c)=\operatorname{half}(\operatorname{acz}(j z, 1 x, 1 c)+$

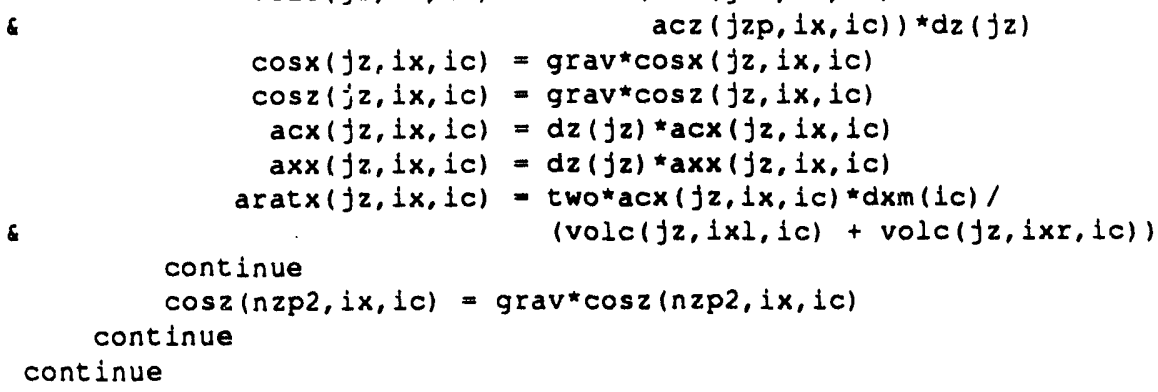

4

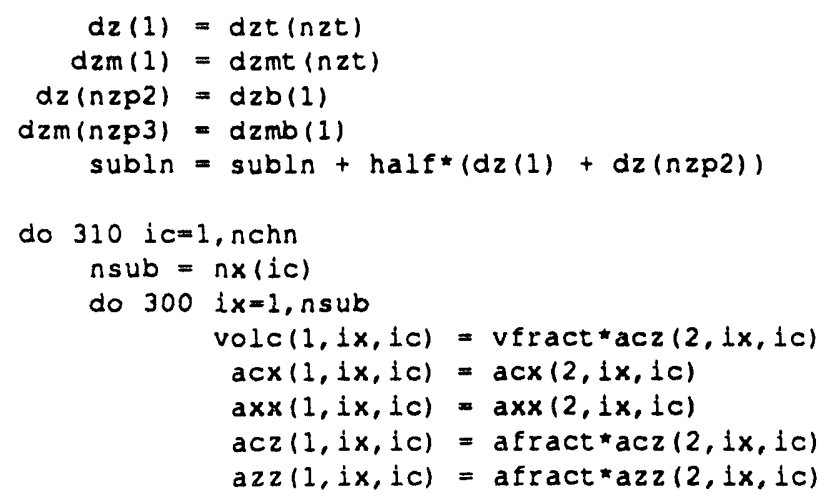


c

c

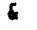
continue continue

300

310

c

c c 500 c

c $c$

c $\cos 2(1,1 x, 1 c)=\cos t(n z t)$

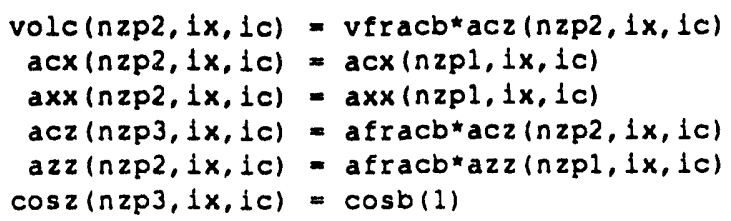

\section{GEOSLD}

subroutine geosid (diam, wt, mat, rlbl, ribt, iorib, loxid, nr, ichks, c ncyln, nsurf, nrmax, nrmxp, nz, iunits, istop)

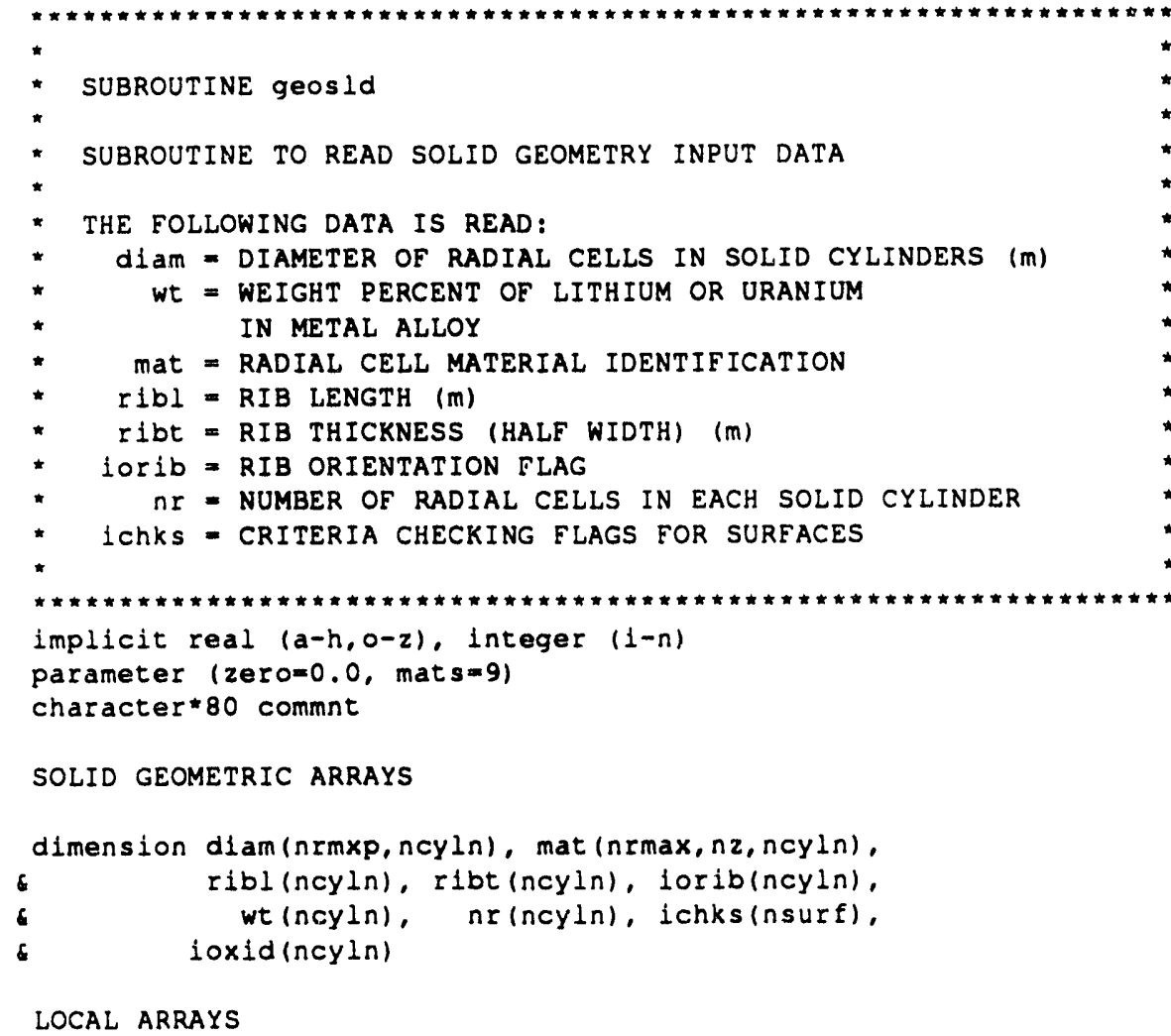




\begin{tabular}{|l|lll|l|}
\hline $2 / 15 / 93$ & WSRC-TR-93-086 Rev. 0 & FLOWTRAN-TF v1.2 Source Code & Pg. 137 of 354 \\
\hline
\end{tabular}

c

c

c

c

c

c

c

c

c

c

C

c

C

c

30

40

c

50

c

dimension wtmin(2), wtmax (2)

common /lountt/ ninp, nout, ndmp, ntmp, nrst1, nrsto

data wtmin $10.0,0.0 /$, wtmax $/ 10.0,30.5 /$

SET SRS TO SI CONVERSION FACTOR:

cIm - CONVERSION FROM INCHES TO METERS

data cim/2.540e-02/

READ IN CYLINDER RADIAL NODALZATION:

nsect - NUMBER OF RADIAL SECTORS IN THE CYLINDER

ncell - NUMBER OF RADIAL CELLS WITHIN THE SECTOR

do 50 icyln=1, ncyln

read $(n i n p, 5)$ commnt

read (ninp, *) dinner, nsect

$n I(\operatorname{Icy} \ln )=0$

LOOP OVER RADIAL SECTORS AND CALCULATE CELL DIAMETERS

$1 d=1$

$\operatorname{diam}(1 d, 1 c y l n)=\operatorname{dinner}$

do 20 isect $=1$, nsect

read ( $n$ inp, *) douter, ncell

$n r(1 c y l n)=n r(1 c y l n)+n c e l l$

dcell = (douter - dinner)/ncell

do $101 \operatorname{ce} 11=1$, nce 11

$1 d=1 d+1$

dlam $(1 d, 1$ cyln) = dinner + lcell*dcell

continue

dinner = douter

continue

READ IN RIB GEOMETRY AND ALLOY WEIGHT PERCENT:

$n$ sect - NUMBER OF AXIAL SECTORS IN THE CYLINDER

read (ninp, 5) commnt

read $(n i n p, *)$ iorib(icyln), ribl(icyln), ribt (icyln)

read (ninp, 5) commnt

read (ninp, *) ioxid(1cyln), wt (icyln), nsect

LOOP OVER AXIAL SECTORS AND READ MATERIAL IDENTIFICATIONS

$i z 1=1$

$n r \dot{1}=\operatorname{nr}(1 \mathrm{cy} \perp \mathrm{n})$

do 40 isect $=1$, nsect

read (ninp, *) iz2, (mat (ir,izl,icyln), ir $=1, n r i)$

If $(122 . g t .121)$ then

$121 p=121+1$

do $301 z=1 z 1 p, i z 2$

do 30 ir $=1$, n $r 1$

$\operatorname{mat}(1 \mathrm{r}, 1 z, 1$ cyln $)=\operatorname{mat}(1 \mathrm{r}, 1 z 1,1$ cyln)

end if

$i z 1=122+1$

continue

continue

READ IN CRITERIA CHECKING FLAG FOR EACH SOLID SURFACE 
$c$

CHECK THAT WEIGHT PERCENTS FOR ALLOYS ARE WITHIN ALLOWED BOUNDS, CHECK THAT MATERIAL IDENTIFICATION NUMBERS ARE ALL > 0 , AND WRITE MATERIAL IDENTIFICATION NUMBERS TO OUTPUT FILE

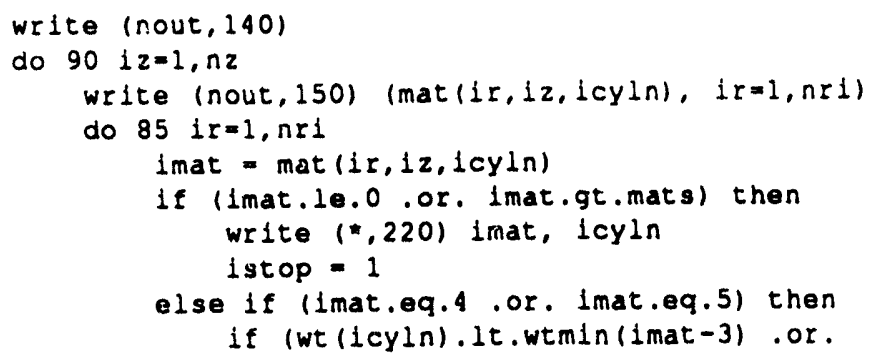




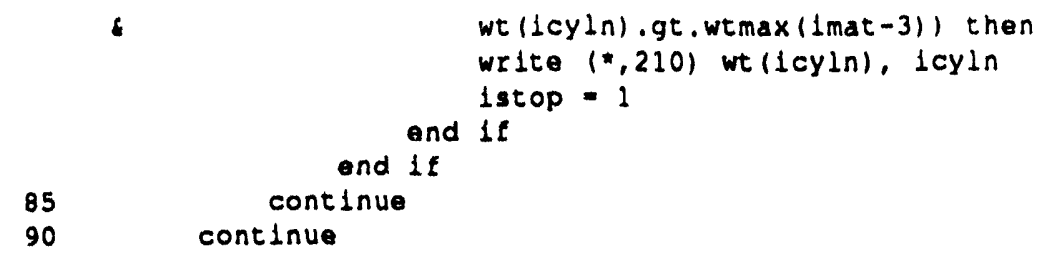

- wt $(1 c y l n)$.gt.wtmax $(1 \operatorname{mat}-3))$ then

FOR REACTOR ASSEMBLIES, CHECK THAT UNIFORM MATERIAL IS INDICATED

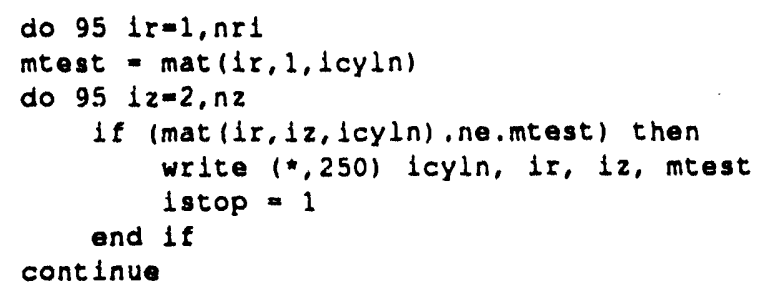

\section{HCONV}

subrout ine hconv (qflux, dqdtw, hfc, twall, tfld, gfld, dhy, visf,

6 


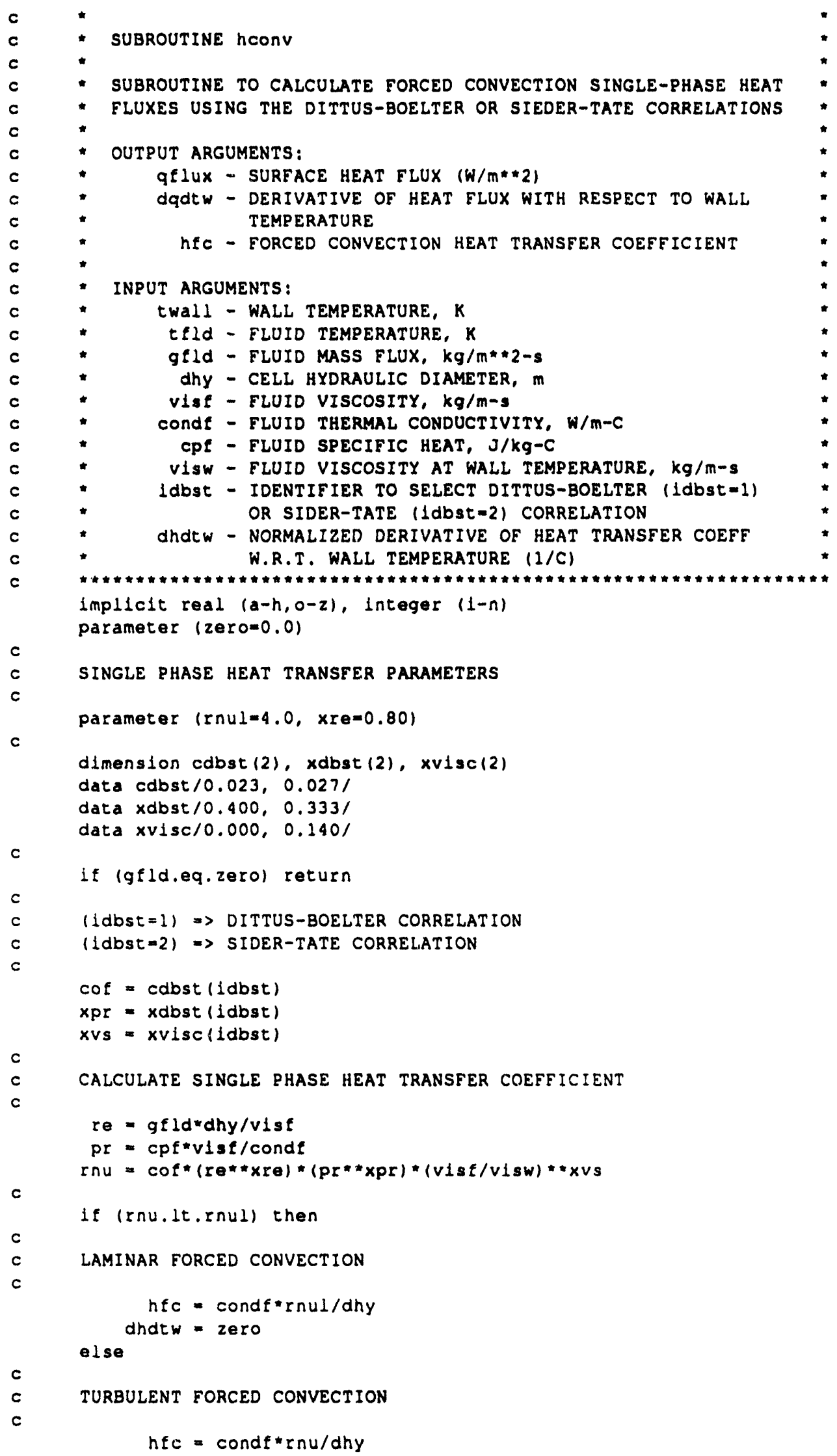


end if

dhdtw = -xvs"dvdtw

c
c

CALCULATE HEAT FLUX AND DERIVATIVE

delt = twall - tfld

qflux = hfc"delt

$d q d t w=h f c+q f l u x * d h d t w$

$c$

return

end

\section{BFTA}

subroutine hfla (uf, rhof, thermf, viscf, cpf, delta, hf, length)

THIS SUBROUTINE COMPUTES THE HEAT AND MASS TRANSFER

COEFFICIENTS ON THE LIO SIDE FOR ANNULAR OR RIB FLOW

INPUT: rhof - liquid density, $\mathrm{kg} / \mathrm{m}^{\wedge} 3$

uf - liquid velocity, $\mathrm{m} / \mathrm{s}$

thermf- liquid thermal conductivity, $w / m-K$

viscf - liquid viscosity, $\mathrm{kg} / \mathrm{m}-\mathrm{s}$

cpf - liquid heat capacity, J/kg-K

delta - liquid film thickness, $m$

length- length of annular $f 11 \mathrm{~m}, \mathrm{~m}$

(note: this parameter is used only if the film Reynolds number is laminar; therefore it is reasonable to assume it equal to the overall channel length)

OUTPUT: he - heat transfer coef., $w / m^{\wedge} 2-K$

INTERNAL:

re - film Reynolds number based on liquid properties

pr - liquid Prandt 1 number

pe - Peclet number for heat transfer

BASIS IS:

$N U=((3 /(2 * P I)) *(D E L / L) * P E) * .5 \quad$ FOR RE $<1200$

SEE TREYBAL, R.E., MASS TRANSFER OPERATIONS, 3RD.ED.,

PP. 52-54, 675 .

$N U=1.76 E-5 *(R E * 1.506) *(P R * 0.5) \quad F O R 1300<R E<8300 *$

$N U=N U(R E<1200)+0.01 *(N U(R E>1300)-N U(R E<1200)) *$ *

$$
(R E-1200)
$$

LINEAR INTERPOLATION FOR $1200<$ RE $<1300 *$

therm = thermal conductivity, rhof = liq.density, viscf=1iq.visc*

$c p f=1 i q$. heat capacity, uf =1iq.velocity, $u g=$ gas velocity

diffe thermal diffusivity, pe= peclet no., anu= nusselt no. $h=$ heat transfer coefficient

real length

parameter (four $=4.0, \mathrm{pl}=3.14159$ )

parameter (cnst $=3.0 /(2.0 * \mathrm{p} 1))$

parameter (fract $=0.01$ ) 
c

data $a 2 / 1.76 e-5 /, a 3 / 1.506 /, 501 \mathrm{am} / 1200.0 /$, retur/1300/, eps/0.1/

re - four* rhof "abs (uf) *delta/visct

pr $=$ cpt*viscf/thermf

If (re.1t.relam) then

pe = re"pr

anu = sqrt (cnst"pe*delta/length)

hf = sqrt (cnst "four"rho:"max (abs (uf), eps) "pr

6 ( (visct*length)) *thermf

else if (re.gt.retur) then

anu $=a 2 *\left(r e^{* * a}\right) *$ sqrt $(p r)$

ht = anu*thermf/delta

else

pe $=r e^{*} p r$

anul = sqrt (enst"pe*delta/length)

anuh $=a 2 *(r e * * a) * \operatorname{sqrt}(p r)$

anu $=$ anul + fract*(anuh - anul)*(re - relam)

end $1 \mathrm{t}$

he = anuthermf/delta

return

end

\section{AEIB}

subroutine hfib (uf, ug, rhof, thermf, cpf, dia, hf, visf, visg)

SUBROUTINE hEIb:

THIS SUBROUTINE COMPUTES THE HEAT TRANSFER

COEFFICIENT ON THE LIQUID SIDE FOR BUBBLES

INPUT :

OUTPUT:

INPUT: rhof - liquid density, $\mathrm{kg} / \mathrm{m}^{\wedge} 3$

uf - liquid velocity, $\mathrm{m} / \mathrm{s}$

ug - mixture gis velocity, $\mathrm{m} / \mathrm{s}$

thermf - thermal conductivity, $W / m-K$

dia - average bubble diameter, $m$

cpf - liquid heat capacity, J/kg-K

visf - dynamic viscosity of Llquid, Pa-s

visg - dynamic viscosity of gas, Pa-s

NOTE: THE ABOVE ARE MIXTURE GAS PROPERTIES TO BE EVALUATED AT BULK GAS TEMPERATURE AND PRESSURE.

OUTPUT: he - heat transfer coef., $W / m^{\wedge} 2-K$

INTERNAL:

difft- 11quid thermal diffusivity, $m^{\wedge} 2 / s$

watmw- molecular welght of light water, $\mathrm{kg} / \mathrm{kgmole}$

Le - Lewis Number, Sc/Pr

HEAT TRANS. COEFF. LOGIC:

FOR RE < 1, BASIS IS:

$N U=2+0.5 * P E+0.25 *(2 * V I S F+3 * V I S G) /(3 * V I S F+$ $3 * V I S G) * P E * 2 * L N(P E)$ 


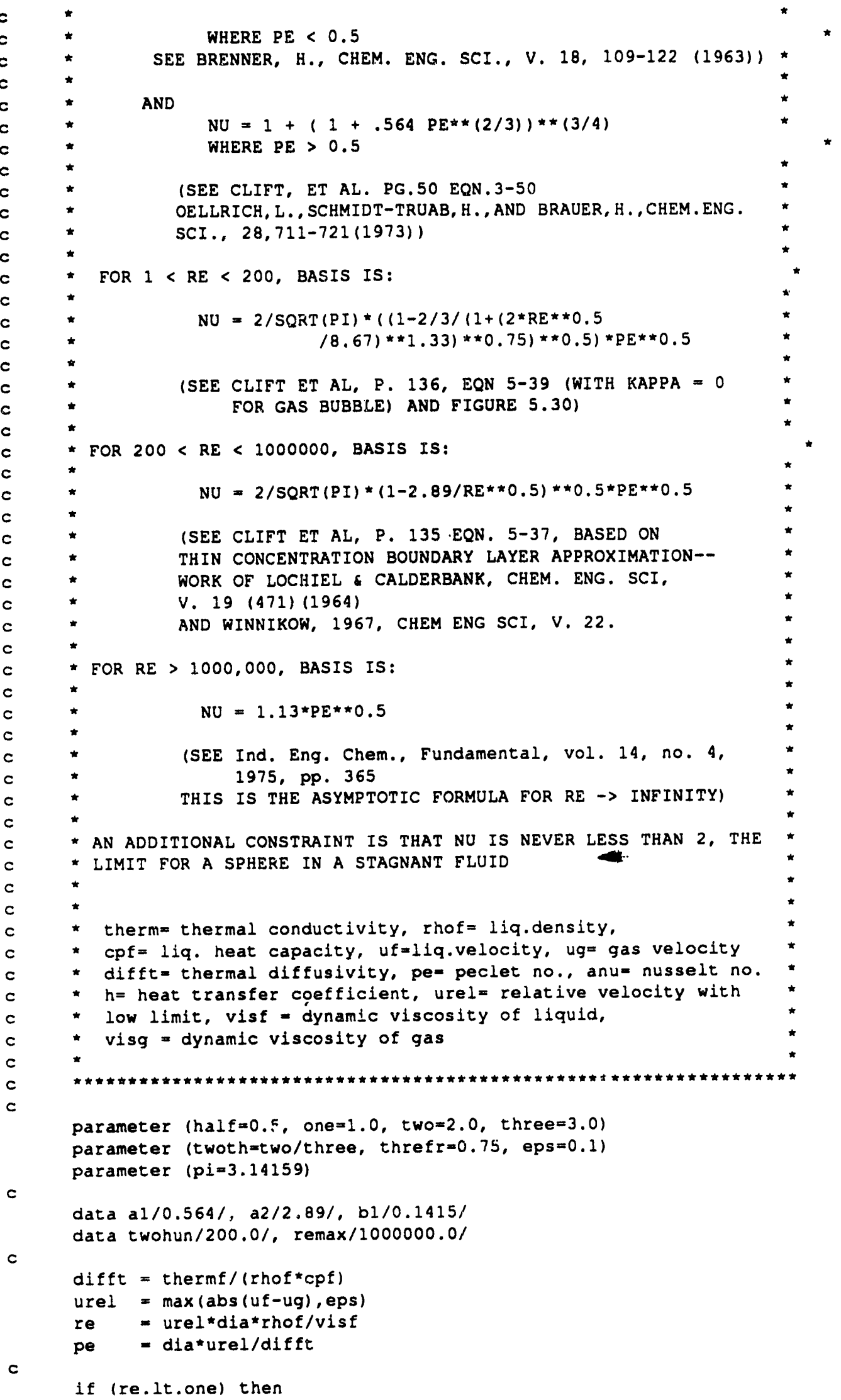




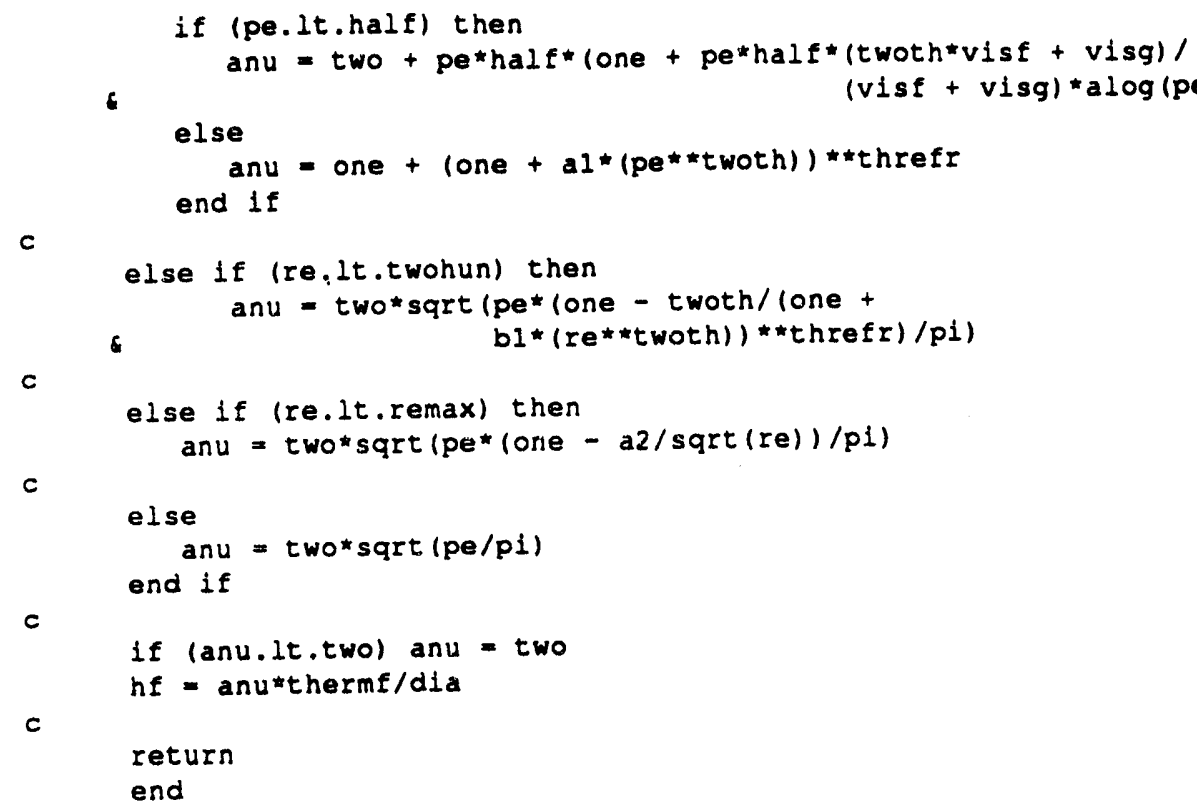

\section{BEIS}

subroutine hfis (viscf, rhof, thermf, cpf, dhydrl, hf)

SUBROUTINE hEIS:

THIS SUBROUTINE COMPUTES THE HEAT TRANSFER COEFFICIENT ON THE LIOUID SIDE FOR TAYLOR BUBBLES

INPUT: viscf - liquid viscosity, $\mathrm{kg} / \mathrm{m}-\mathrm{s}$

rhof - liquid dinsity, $\mathrm{kg} / \mathrm{m}^{\wedge} 3$

thermf - thermal conductivity, $w / m-K$

cpf - liquid heat capacity, J/kg-K

dhydrl - hydraulic diameter, m

NOTE: THE ABOVE ARE MIXTURE GAS PROPERTIES TO BE EVALUATED AT BULK GAS TEMPERATURE AND PRESSURE.

OUTPUT: hf - heat transfer coef., $w / m^{\wedge} 2-K$

heat trans. COEFE, logic

$\mathrm{NU}=\mathrm{CS}^{\star D S} \mathrm{DS}(\mathrm{G} / \mathrm{LS}) \star \star 0.25 / \mathrm{DIFFT} * 0.5$

BASIS IS

VAN heUVen, J. W., AND BEek, W. J., 1963, CHEM. ENG. SCI., V. 18 (377) WHERE IT IS ASSUMED THAT DS/LS > 1 , SO CS $=1$ AND LS IS SET EQUAL TO 4 CM--SEE TF DOCUMENTATION FOR JUSTIFICATION OF THIS VALUE

anu = nusselt number, hf = heat transfer coefficient

parameter $(g=9.81)$ 


\begin{tabular}{|l|ll|l|}
\hline $2 / 15 / 93$ & WSRC-IR-93-086 Rev. 0 & FLOWTRAN-TF v1.2 Source Code & Pg. 145 of 354 \\
\hline
\end{tabular}

data a1/0.88/, a2/4.50/

$c$

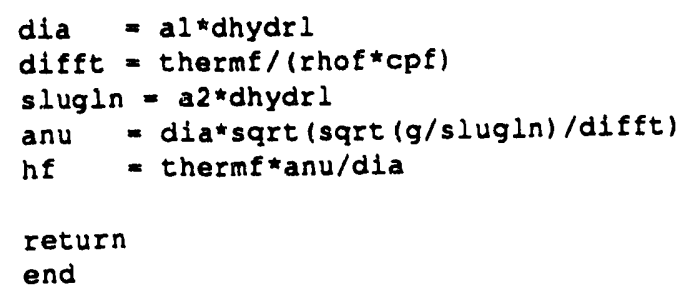

\section{BEIUID}

subroutine hfluid(p,t,hfs, dhfsdt, hf, dhfdp, dhfdt)

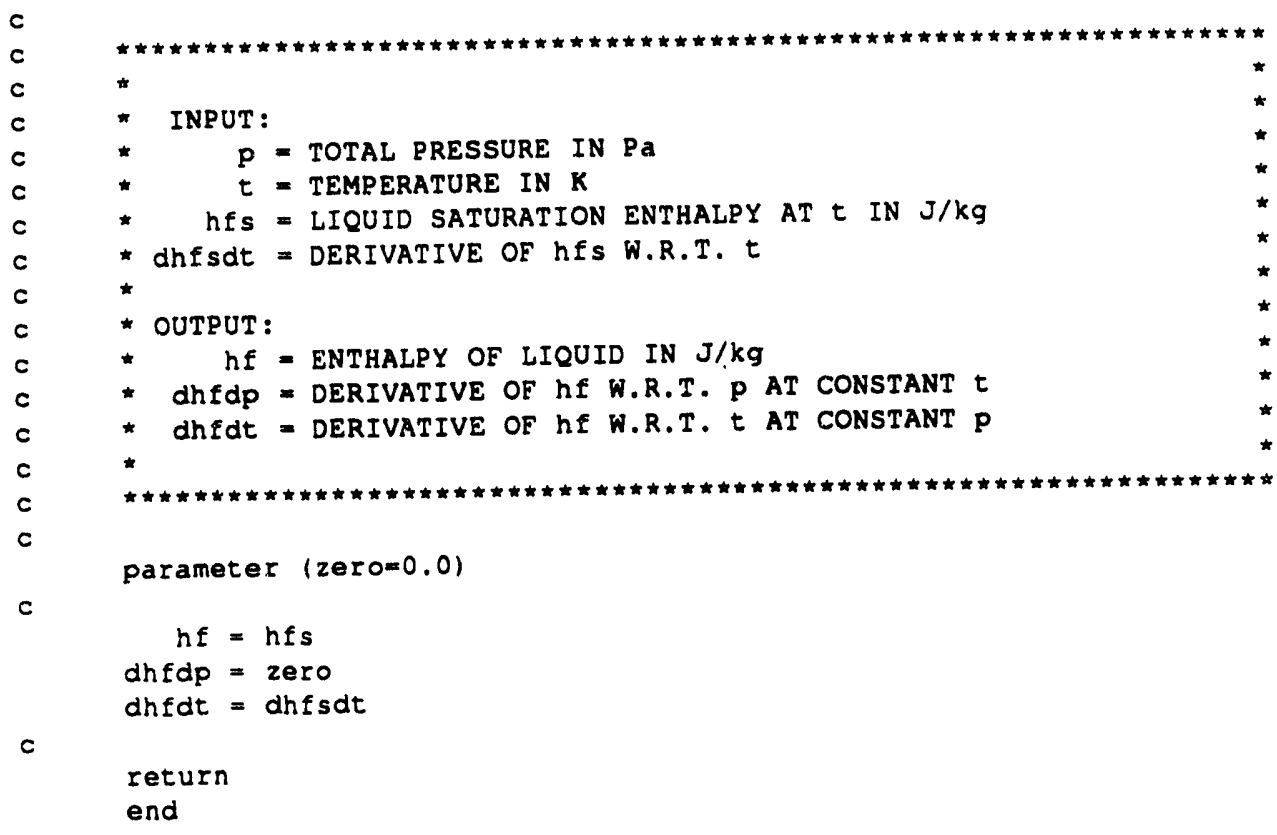

\section{HESAT}

subroutine hfsat $(t, 1, h f s$, dhfsdt $)$

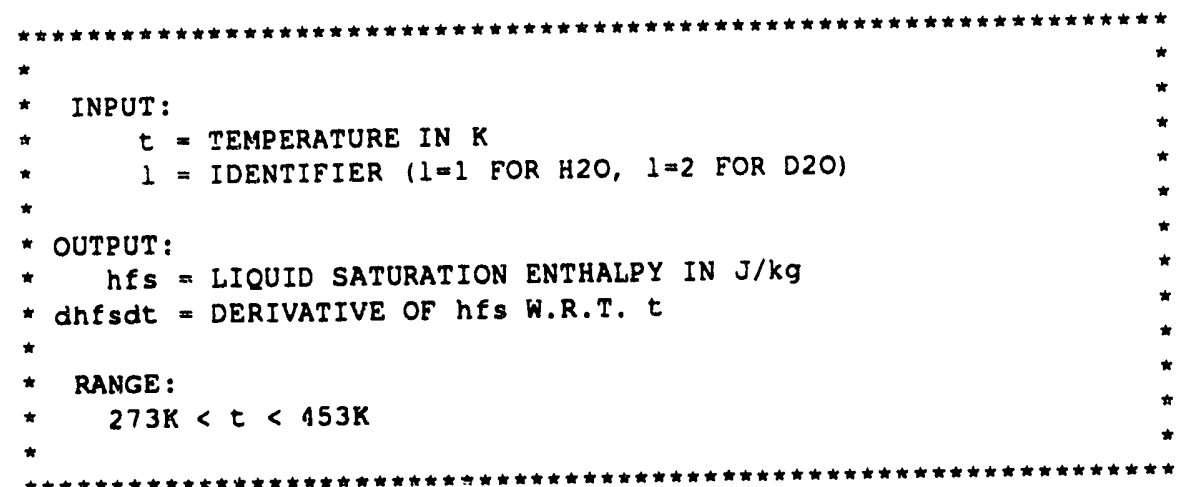


c

c

c

D20 PROPERTIES

$\operatorname{data} c 1(2) /-1.1856 e+6 /, c 2(2) / 4340.2 /, c 3(2) /-0.20733 /$

$h \in s=c l(1)+(c 2(1)+c 3(1) \star t) \star t$

dhfsdt $=c 2(1)+t_{\text {wo*c }}(1) * t$

return

end

\section{HGIA}

subroutine hgia ( $p, t g, u g$, hhog,viscg,ysi,ysb, thermg,cpg,

\&

diffm, dhydrl, hg, akg, deltal

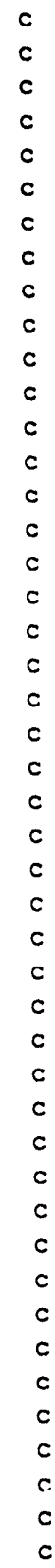

THIS SUBROUTINE COMPUTES THE HEAT AND MASS TRANSFER

COEFEICIENTS ON THE GAS SIDE FOR RIB FLOW

INPUT: rhog - mixture gas density, $\mathrm{kg} / \mathrm{m}^{\wedge} 3$

p - pressure, Pa

tg - bulk gas temperature, $K$

ug - gas velocity, $\mathrm{m} / \mathrm{s}$

viscg - mixture gas viscosity, $\mathrm{kg} / \mathrm{m}-\mathrm{s}$

dhydrl- hydraulic diameter, $m$

thermg- thermal conductivity, w/m-K

diffm - mixture gas mass diffusivity, $m^{\wedge} 2 / s$

cpg - mixture gas heat capacity, J/kg-K

uf - liquid velocity, $\mathrm{m} / \mathrm{s}$

NOTE: THE ABOVE ARE MIXTURE GAS PROPERTIES TO BE EVALUATED AT BULK GAS TEMPERATURE AND PRESSURE.

OUTPUT: hg - heat transfer coef., $w / m^{\wedge} 2-K$

akg - mass transfer coef.., (kgmole gas $\mathrm{mix}$ ) $-\mathrm{m} / \mathrm{s}$

therm = thermal conductivity, rhof = liq.density,

$c p f=1 i q$. heat capacity, uf=liq.velocity, ug= gas velocity

diff = thermal diffusivity, pex peclet no., anu= nusselt no.

$h=$ heat transfer coefficient

COEFFicient LOGIC:

$S H=0.023 * R E * 0.83 * S C * 0.44 / Y 1 m$

gilliland ANd SHERwood, IND. ENG. CHEM., V. 26, 1934

* AS QUOTED IN SHERWOOD, PIGFORD, WILKE, MASS TRANSFER, 1975

* $P$. NOTE RE is based on Gas Velocity RELATIVE to Wall, NOT*

* To liouid Velocity, and that the RANGe is Dictated by the FILM *

* REYNOLDS NUMBER, = 4*MASS FLOW/UNIT WIDTH/VISC

* y im is the log mean mole fraction of the non-diffusing

* PHASE, based on interface and bulk partial pRessures

* the chilton-colburn analogy is USEd to apply the relations to

* heat transfer (SH*ylm is equivalent to nU)

*

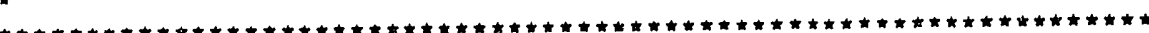

parameter (half $=0.5$, one $=1.0$, third $=1.0 / 3.0$,

$c$ pt $8=0.8$, rcon $=8314.4$ )

data a1/0.023/,a2/0.83/, a3/0.44/

data epsya/1.0e-4/, remin/2000.0/ 
$\operatorname{cof}=a 1 *(r e * \star a 2)$

$\operatorname{sh}=\operatorname{cof} * s c * a 3 / y l m$

anu $=\operatorname{cof} \star p r \star \star a 3$

c

hg = anu*thermg/dhydrl

$a k g=s h \star d i f f m / d h y d r l$

$a k g=a k g{ }^{\star c} c$

return

end

\section{BGIB}

subroutine hgib (dia, rhog,p,tg, thermg, diffm, cpg, hg, akg)

SUBROUTINE hgib:

THIS SUBROUTINE COMPUTES THE HEAT AND MASS TRANSFER COEFFICIENTS ON THE GAS SIDE FOR BUBBLES.

INPUT: dia - bubble diameter, $m$

rhog - mixture gas density, $\mathrm{kg} / \mathrm{m}^{\wedge} 3$

$p$ - fluid cell pressure, $\mathrm{Pa}$

tg - bulk mixture gas temperature, $K$

thermg- thermal conductivity, $W / m-K$

diffm - mixture gas mass diffusivity, $m^{\wedge} 2 / s$

$\mathrm{cpg}$ - mixture gas heat capacity, $\mathrm{J} / \mathrm{kg}-\mathrm{K}$

Note: The above are mixture gas properties to be evaluated at bulk gas temperature and pressure.

OUTPUT: hg - heat transfer coef.., w/m^2-K

akg - mass transfer coef., (kgmole of gas phase) m/s

INTERNAL:

rcon - ideal gas constant, $8314.4 \mathrm{~Pa}-\mathrm{m}^{\wedge} 3 / \mathrm{K}-\mathrm{kgmole}$

difft-mixture gas thermal diffusivity, $m^{\wedge} 2 / s$

watmw- molecular weight of light water, $\mathrm{kg} / \mathrm{kgmole}$

Le - Lewis Number, $\mathrm{Sc} / \mathrm{Pr}$

c - molar concentration of gas mixture based on

ideal gas law, $\mathrm{p} /\left(\mathrm{rcon} \mathrm{tg}^{\mathrm{g}}\right), \mathrm{kgmole} / \mathrm{m}^{\wedge} 3$

HEAT TRANSFER COEFFICIENT LOGIC

BASIS: THAT BUBbLE SIDE hEAT AND MASS TRANSFER IS CONSIDERED * 

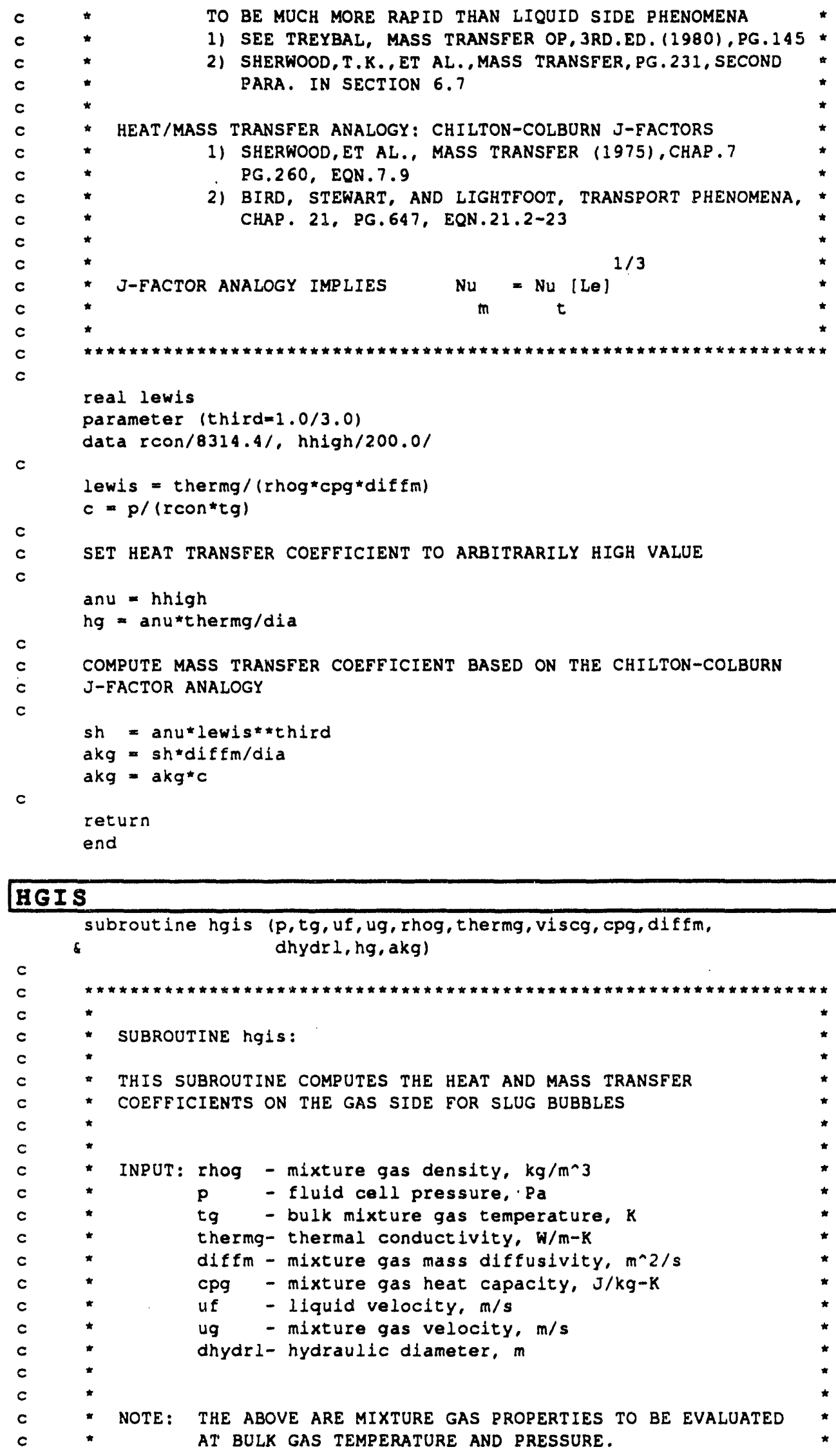


\begin{tabular}{|l|ll|l|}
\hline $2 / 15 / 93$ & WSRC-TR-93-086 Rev.0 & FLOWTRAN-TF v1.2 Source Code & Pg. 149 of 354 \\
\hline
\end{tabular}

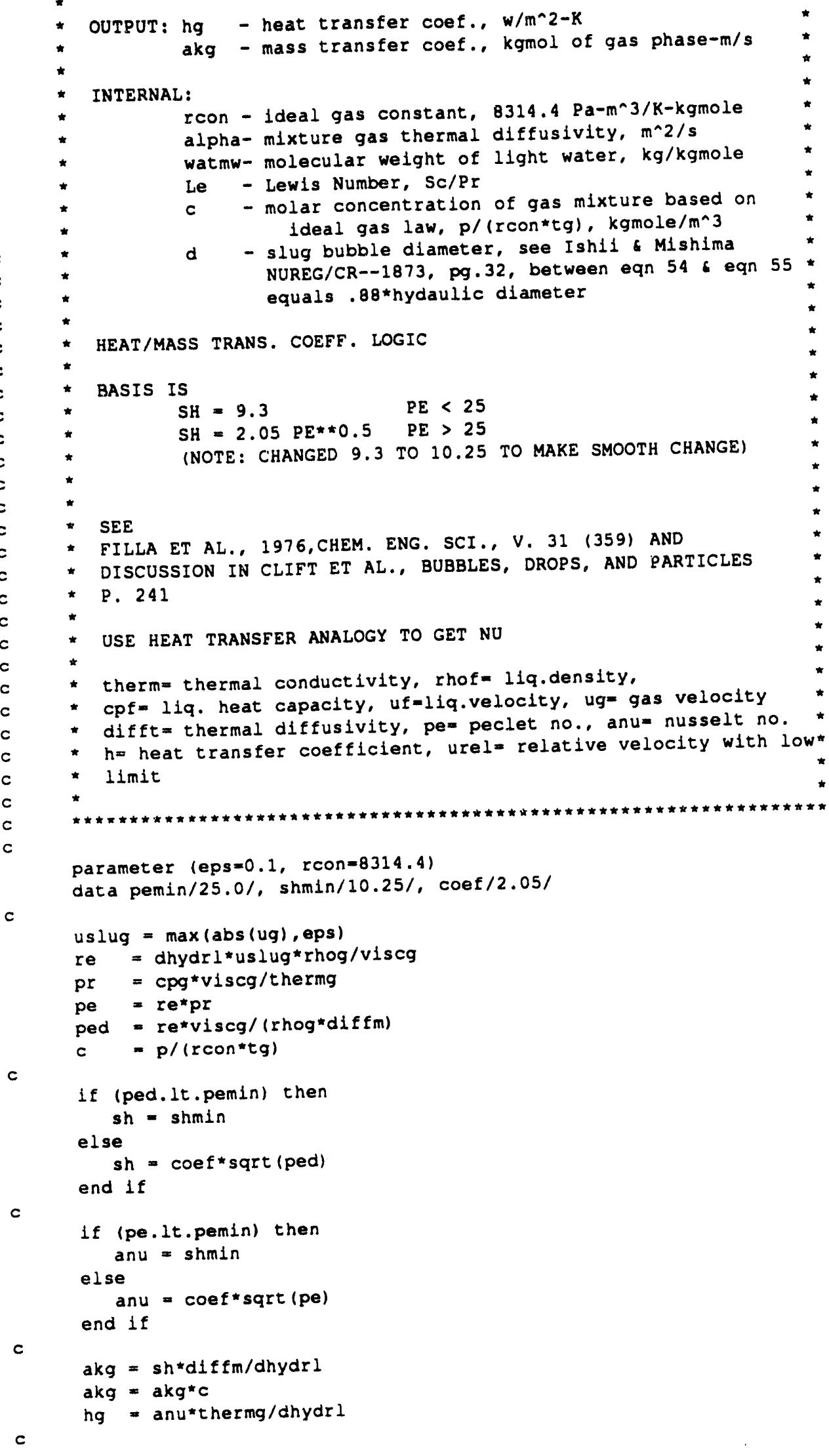


return

end

\section{BSSAT}

subrout ine hssat (p, 1, hss, dhssdp)

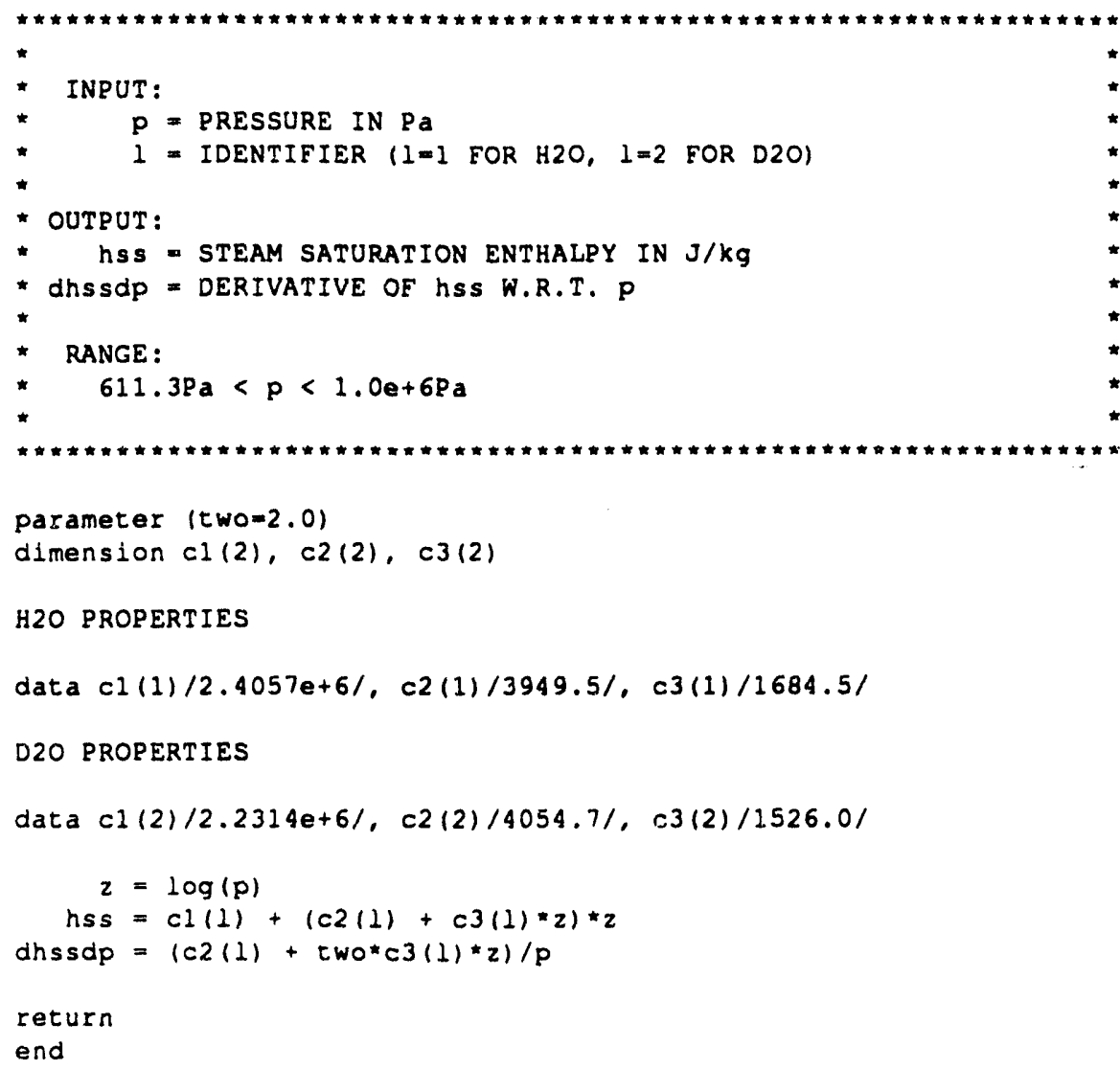

\section{ASTEAM}

subroutine hsteam(t,ts, dtsdp, hss, dhssdp, 1, hs, dhsdp, dhsdt)

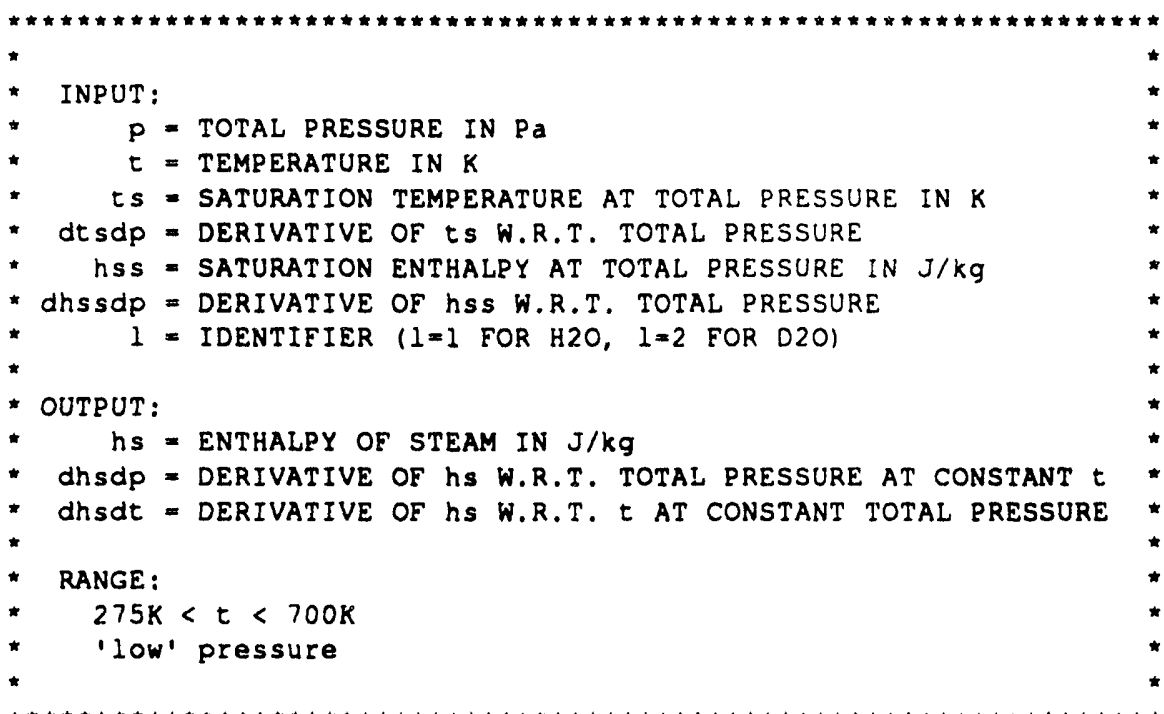




\begin{tabular}{|l|ll|l|l|}
\hline $2 / 15 / 93$ & WSRC-TR-93-086 Rev. 0 & FLOWTRAN-TF v1.2 Source Code & Pg. 151 of 354 \\
\hline
\end{tabular}

$c$

parameter $(t w o=2.0$, three $=3.0$, four $=4.0)$

dimension $c 1(2), c 2(2), c 3(2), c 4(2)$

c

c

H2O PROPERTIES

data c1(1)/1901.4/, c2 (1)/-0.62129/,

c c3(1)/1.9538e-3/, c4 (1)/-1.0083e-6/

c

c

D2O PROPERTIES

data c1 (2)/1611.4/, c2(2)/3.6681e-3/,

c

c $\quad c 3(2) / 1.3023 e-3 /$, c4 $(2) /-6.6266 e-7 /$

$h s=h s s+(c 1(1)+((c 2(1) / t w o)+($ (c3 $(1) /$ three $)$

c $\quad+(c 4(1) /$ four $) \star t) \star t) \star t) \star t$

- $\quad-(c 1(1)+((c 2(1) /$ two $)+($ (c) $(1) /$ three $)$

( $+(c 4(1) /$ four $) \star(s) \star t s) \star(t s) \star t s$

dhsdp $=$ dhssdp

6 $\quad-(c 1(1)+(c 2(1)+(c 3(1)+c 4(1) * t s) * t s) * t s) * d t s d p$

dhsdt $=c 1(1)+(c 2(1)+(c 3(1)+c 4(1) * t) * t) * t$

c

return

end

\section{INBOND}

subrout ine inbond (ppl0, alpplo, tgplo,tfplo, xaplo

- ptbo,alptbo, tgtbo,tftbo, xatbo,

- qfino, qgino, fsplt, tnklvo,

ippl, ialpl, itgpl, it fpl, ixapl,

iptb, ialtb, itgtb, itf tb, ixatb,

iqfin, iqgin, itnklv, tcrmax, pkfac,

ichkc, nchn, nsubc, nspln, ncrit, ibond,

iunits, istop)

c

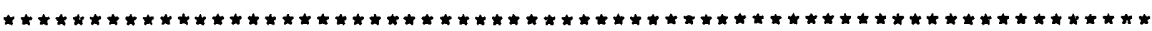

$\star$

* subroutine inbond

SUBROUTINE TO PROCESS THERMAL-HYDRAULIC BOUNDARY CONDITIONS INPUT BOUNDARY CONDITIONS FOR FLUID-PHASE CALCULATIONS

istop - 0, OK; >0, TERMINATE PROBLEM AFTER READING INPUT

SPLINE COEFFICIENT ARGUMENTS:

PP1O - PLENUM PRESSURE

alPP10 - PLENUM VOID FRACTION

TEPIO - PLENUM FLUID TEMPERATURE

tgP10 - PLENUM GAS TEMPERATURE

XaP1O - PLENUM AIR MASS FRACTION

PEDO - TANK BOTTOM PRESSURE

a LPEDO - TANK BOTTOM VOID FRACTION

tftbO - TANK BOTTOM FLUID TEMPERATURE

tgtbO - TANK BOTTOM GAS TEMPERATURE

xatbO - TANK BOTTOM AIR MASS FRACTION

gfinO - INLET LIQUID FLOW RATE

qgInO - INLET GAS FLOW RATE

tnklvo - INITIAL TANK LEVEL

POINTERS TO TRANSIENT PROFILES:

IPPL - PLENUM PRESSURE

IAIPL - PLENUM VOID FRACTION 


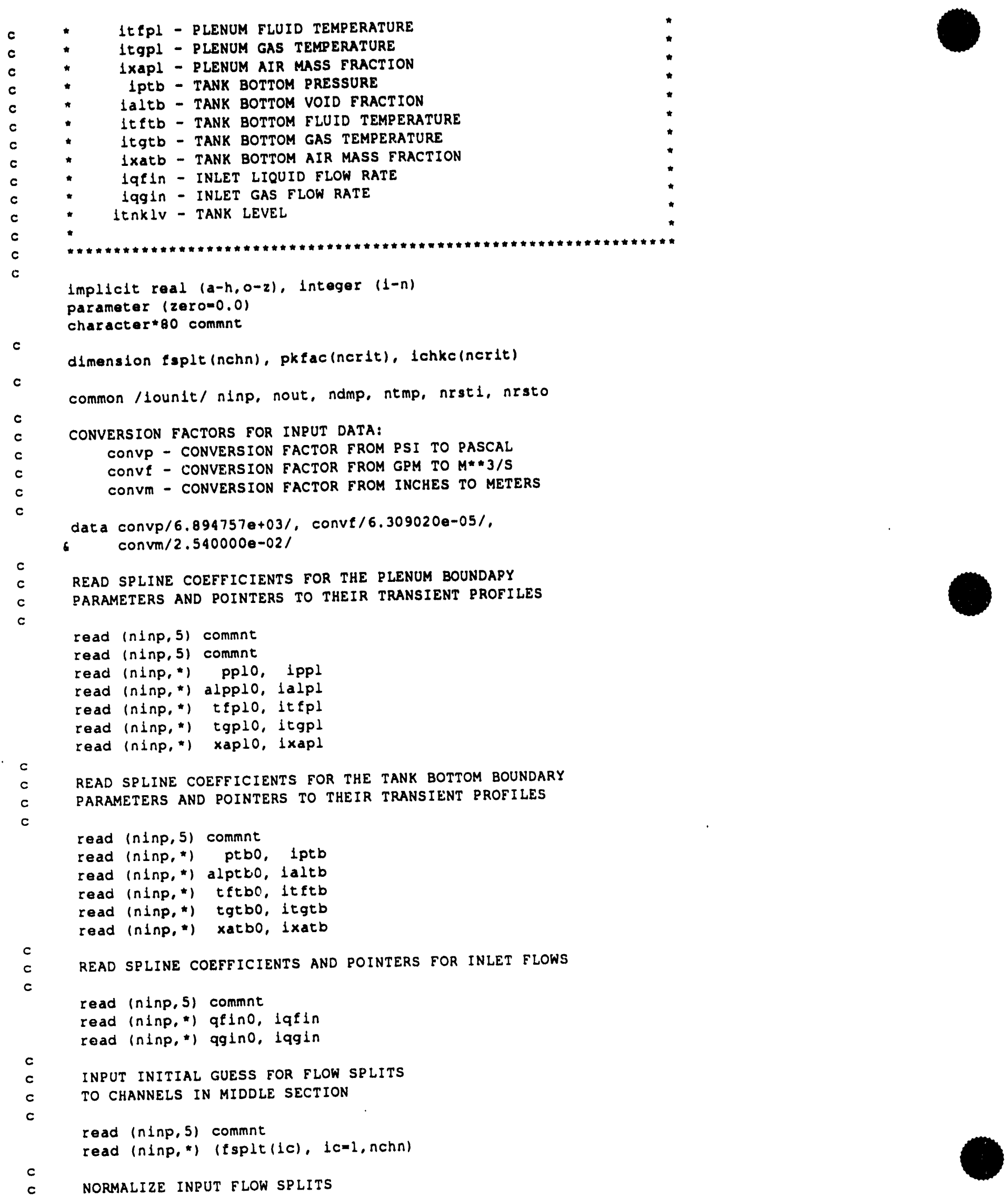


c

total $=2 e r 0$

do $101 \mathrm{c}=1$, nchn

total = total + tsplt (ic)

10

cont Inue

c

do $201 \mathrm{c}=1$, nchn

fsplt $(1 c)=f \operatorname{splt}(1 c) /$ total

20

continue

READ COEFFICIENT AND POINTER TO TANK LEVEL TRANSIENT PROFILE

read (ninp, 5) commnt

read (nlnp,*) tnklvo, lenklv

READ IN CRITERIA CHECKING FLAGS AND PEAKING FACTORS

read $(n \operatorname{lnp}, 5)$ commnt

read (nInp, *) (1chkc(1c), 1c=1,ncr 1t)

read (nInp, *) (pkfac(1c), 1c=1,ncrit)

wr1te (nout, 120) (1c, lehke(1c), pkfac(1c), lc=l,ncrit)

c

READ IN TIME AT WHICH CRITERIA CHECKING BEGINS.

$$
\begin{array}{lll}
\text { read }(n i n p, 5) & \text { commnt } \\
\text { read (ninp, }) & \text { tcrmax } \\
\text { write (nout, 130) } & \text { tcrmax }
\end{array}
$$

c

c

CONVERT SRS UNITS TO SI FOR CALCULATIONS

1f (1units.eq.2) then pp10 = convp*ppl0 ptbo = convp*ptbo

qfino = convf*qfino

qgino = convt*qgino

end if

tnklvo = convm"tnklvo

$c$

c

c

CHECK THAT THE TRANSIENT POINTERS ARE CORRECT

If (ippl.le.0.or. lppl.gt.nspln) then

write (nout, 110) ippl, nspln

istop $=1$

end if

c

if (lalpl.le.0 .or, lalpl.gt.nspln) then write (nout, 110) lalpl, nspln

istop $=1$

end $1 f$

C

If (Itfpl.1e.0 .or. Itfpl.gt.nspln) then write (nout, 110) 1tfpl, nspln istop $=1$

end if

c

$1 f$ (1tgpl.1e.0 .or. 1tgpl.gt.nspln) then write (nout, 110) 1tgpl, nspln 1stop $=1$

end if

If (Ixapl.1e.0 .or. ixapl.gt.nspln) then

write (nout, 110) ixapl, nspln

istop $=1$

end if 
c

$1 f$ (1ptb.1e.0 .or. 1ptb.gt.nspln) then wrlte (nout,110) 1ptb, nspln

end if 1stop $=1$

c

If (1altb.le.0 .or. laltb.gt.nspln) then write (nout, 110) laltb, nspln 1stop $=1$

c

end if

$1 f$ (1tftb.1e.0 .or. Itftb.gt.nspln) then write (nout, 110) ltttb, nspin 1stop $=1$

and $1 \mathrm{~s}$

$c$

1f (1tgtb.10.0.or. 1tgtb.gt.nspln) then write (nout, 110) itgtb, nspln Istop $=1$

end if

$c$

If (1xatb.1e.0 .or. 1xatb.gt.nspln) then write (nout, 110 ) 1xatb, ispln 1stop $=1$

c

end if

If (1tnklv.le.0 .or. itnklv.gt.nspln) then write (nout, 110) ltnklv, nspln 1stop $=1$

c

and if

if (libond.eq.2 .or. Ibond.eq.4) and.

- (iqfin.le.0.or. iqfin.gt.nspln)) then write (nout, 110) latin, nspin 1stop $=1$

else if ((1bond.eq.3 .or. Ibond.eq.4) .and.

- (lqgin.le.0 .or. lqgin.gt.nspin)) then write (nout, 110) lggin, nspln istop $=1$

end if$$
\text { c }
$$

$$
\begin{aligned}
& \text { c } \\
& \text { c } \\
& 5
\end{aligned}
$$$$
\text { format (a) }
$$$$
110 \text { format } l^{\prime} \text { 《 warning } \gg \text { Inbond encountered a transient array', }
$$$$
6 \text { "pointer of }, 13,1, \text { ' pointer values must be }>0 \text { '. }
$$$$
6 \text { and }<1,13,11
$$

c

\section{INDPWR}
subroutine indpwr (ninp, nout, ncyln, mnrp, nrp, nrmax, nr, na,
6 perct, rphi, nregt, nfret, nlast, nrgtm,
6 expose, iwtdry, istopl

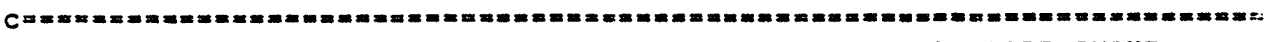

c PROCESS WET OR MODERATED TANK DECAY POWER RADIAL SHAPE INPUT 


\begin{tabular}{|l|ll|l|}
\hline $2 / 15 / 93$ & WSRC-TR-93.086 Rev.0 & FLOWTRAN-TF v1.2 Source Code & Pg. 155 of 354 \\
\hline
\end{tabular}

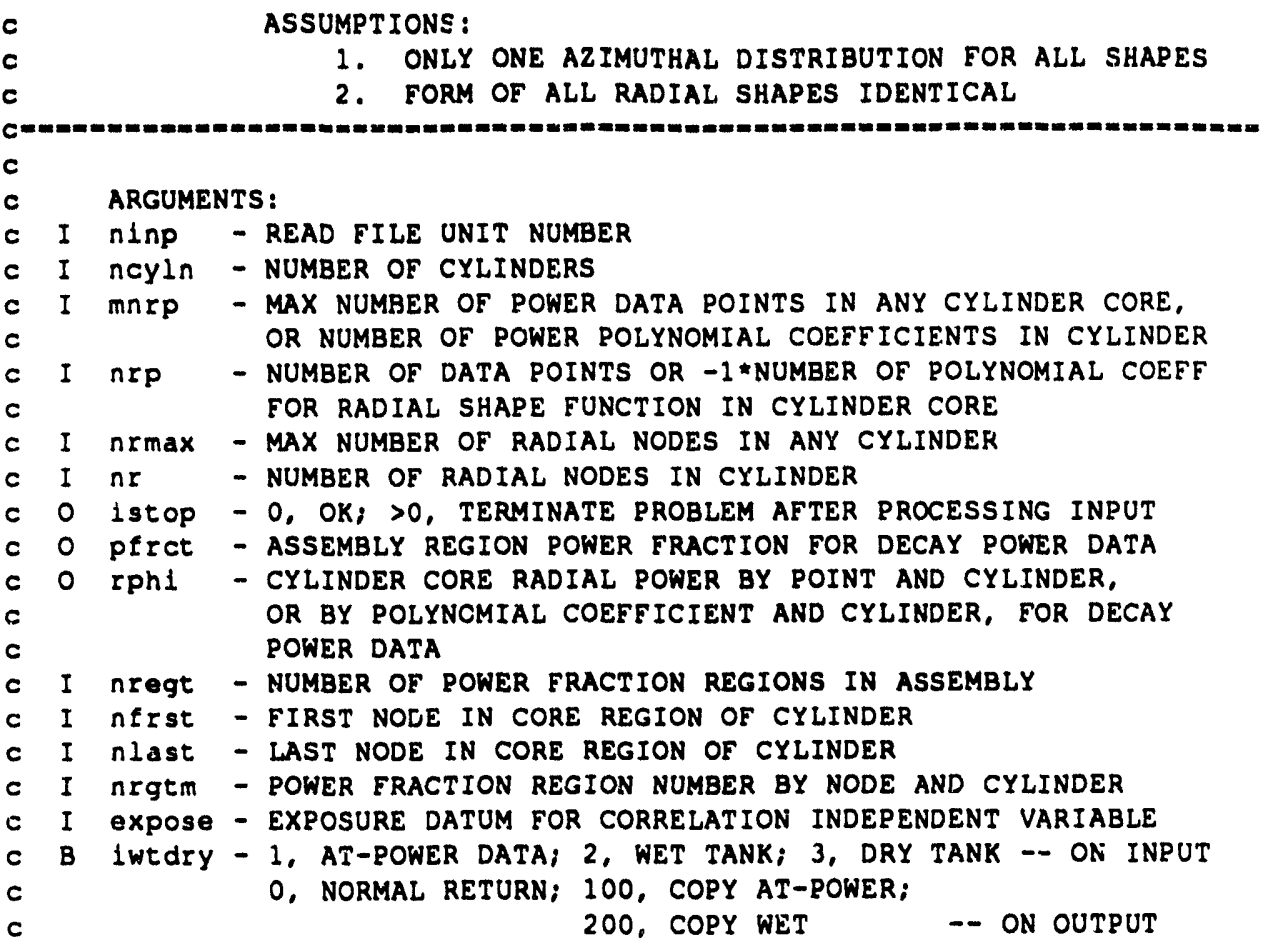

NOTE THAT rPh1 AND pEract ARE BEING USED AS DUMMY ARRAYS HERE. THEIR "CORRECT" IDENTITIES ARE CONTROLLED BY THE CALL FROM INPOWR.

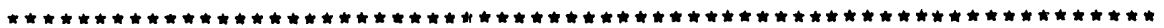

implicit real $(a-h, 0-z)$, integer $(1-n)$

parameter (zerom0.0, one=1.0. pfbig=1.0e+10,

$t m i n=0.9999, t \max =1.0001)$

dimension pfrct (30), rphi(mnrp, ncyln), nrgtm(nrmax, ncyln)

dimension $n \in(n c y l n)$, nfrst (ncyln),

character*80 commnt $n x p(n c y l n)$, nlast (ncyln)

LOCAL ARRAYS

PfCOIE - COEFFICIENTS FOR POWER FRACTION POLYNOMIAL IN EXPOSURE FOR CORE REGION IN CYLINDER

PEIIINT - COEFFICIENTS FOR POWER FRACTION POLYNOMIAL IN EXPOSURE FOR INNER CLAD REGION IN CYLINDER

PFOUTI - COEFFICIENTS FOR POWER FRACTION POLYNOMIAL IN EXPOSURE FOR OUTER CLAD REGION IN CYLINDER

nCYl - TABLE OF CYLINDER NUMBERS BY CYLINDER SET

cOeff - COEFFICIENTS FOR RADIAL POWER POLYNOMIAL IN EXPOSURE BY POINT AND CYLINDER SET

LOCAL ARRAYS

dimension pfcore $(5,20)$, pfinnr $(5,20)$, pfoutr $(5,20)$,

\& ncyl (5), coeff $(20,20,5)$

character*3 threex, blank, leditl(30), ledit $2(30), \operatorname{ledit} 3(30)$

character*14 wetnes (3)

character*11 region (3)

$c$

data threex/'xxx/, blank/ '

data wetnes/. at-power ' 'wet tank decay', 'dry tank decay'/ 
data region/" core ' 'Inner clad', 'outer clad'/

c

c

c

c

c

c

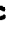

C

c

c

c

c

c

c

c

C

read (ninp, *) commnt

if (1wtdry.ne.1) then

READ SKIP CONTROL

ISKlP = 0 , JUST USE AT-POWER SHAPE DATA

= 1, READ IN AND PROCESS WET/DRY TANK DECAY DATA

- 2, USE WET TANK SHAPE FOR DRY TANK

read (ninp, *) 1skip

write (nout, 994) wetnes (iwtdry), 1skip

If (1skip.ne.0 and. 1sk1p.ne.1 and. 1sk1p.ne.2) then

write (nolit, 87) 1skip

istop $=1$

goto 940

end if

ISKIP POINTS TO AT-POWER SHAPE FOR WET/DRY DECAY POWER

If (iskip.eq.0) goto 940

ISKIP POINTS TO WET DECAY SHAPE FOR DRY DECAY POWER

end if

if (iwtdry.eq.3 and. 1skip.eq.2) goto 950

PROCESS THE DEFINED INPUT

read (ninp, *) ncylnp, ncoeff

if (ncylnp.ne.ncyln) then

write (nout, 912 ) ncylnp, ncyin

istop $=1$

goto 35

end $1 f$

if (ncoeff.gt.20) then

write (nout, 921) ncoeff

istop $=1$

goto 35

end $1 f$

c

do 1 lcyln=1, ncyln

read (ninp,") (pfcore(1cyln, ncf), pfinnr(icyln, ncf),

6

pfoutr (1cyln, ncf), ncf =1, ncoef $f$ )

1

c

write (nout, 998) wetnes(iwtdry), ncylnp, ncoeff

do 996 icyln=1, ncyln

write (nout, 997) (icyln, ncf, pfcore(1cyln,ncf),

6

?

continue

pelinnr (1cyln, ncf). pfoutr (1cyln, ncf), nct $=1$, ncoerf $)$

MAKE REGION ASSIGNMENTS 
8

c

$c$

$c$

c

c

\&

c

$c$
$c$
$c$
$c$
$c$
$c$

10

$c$

$c$

c

12

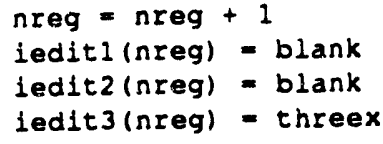

CONSTANT TERM

pfrct (nreg) = pfoutr(1cyln, 1)

if (expose.gt.zero) then

DO REST OF EXPOSURE POLYNOMIAL

do 10 ncf $=2$, ncoeff

fact $=$ expose* exct -1$)^{*}$

pfrct (nreg) $=\operatorname{pfrct}(n r e g)+\operatorname{pfoutr}(10 y l n$, ncf) *fact

continue

end if

CHECK NODAL ASSIGNMENT

if (nrl.gt.0 and. nl.1t.nri) then

$n l p=n l+1$

do 12 ir $=n l p, n r 1$

if (1wtdry.eq.1) then nrgtm (ir, icyln) = nreg

else if (nrgtm(ir, icyln). ne.nreg) then

write (nout, 908) ir, icyln, region(3).

istop $=1$

end if

continue

end if

c

22

c

continue

if (iwtdry.eq.1) then

nregt $=$ nreg

else if (nreg.ne.nregt) then

write (nout, 911 ) nreg, nregt

istop $=1$

end $1 f$

C

$c$

c

c

c

c

c

$c$

CHECK IF ANY POWER FRACTIONS ARE IN 1.0-X FORM.

FLAG IS PFRCT>E+10. ASSUMES ONLY ONE OTHER NONZERO

PERCT WILL BE PRESENT, AND SO SETS VALUE TO 1.0 MINUS IT.

LOOP OVER ALL REGIONS LOOKING FOR VERY LARGE PFRCT

do $230 \mathrm{nrg}=1$, nreg

FOUND YERY LARGE pfrCt 
c

c

C

c

c

c

c

225

230

235

c

c

c

25

c

c

$c$

c

$c$

$c$

$c$

c28

c29

c

$c$

c

32

34

$c$

c

35

$c$

if (pfrct(nrg). It.pfbig) goto 230

LOOP OVER ALL OTHER REGIONS LOOKING FOR NONZERO PFRCT

do 225 ireg=1, nreg

if (1reg.eq.nrg) goto 225

if (pfrct(ireg).eq.zero) goto 225

FOUND NONZERO PFRCT, SET VERY LARGE PFRCT TO

1. 0 MINUS IT, AND EXIT

pfrct (nrg) = one - pfrct (ireg) goto 235

continue

continue

continue

EDIT OUT RESULTING POWER FRACTIONS

write (nout, 23) wetnes (iwtdry), expose

do $25 \mathrm{nrg}=1, \mathrm{nreg}$

write (nout, 24) nrg, perct (nrg), leditl(nrg),

$c$

continue

WRITE OUT NODAL POWER FRACTION ASSIGNMENTS (NORMALLY OFF)

write (nout, 26) wetnes (iwtdry)

do 29 icyln=1, ncyln

$n r i=n r(i c y l n)$

do 28 ir $=1$, n $r i$

write (nout, 27) icyln, ir, nrgtm(ir,icyln)

continue

continue

CHECK THAT PFRCT SUM TO 1.0, NORMALIZE IF NOT

ptot $=$ zero

do $321=1$, nreg

ptot $=$ ptot + pfrct $(i)$

continue

if (ptot.lt.tmin .or. ptot.gt.tmax) then

ptot = one/ptot

write (nout, 33) ptot

do $34 i=1,18$

perct (i) $=$ ptot*pfrct (1)

end if cont inue

READ RADIAL SHAPE DATA, GENERATE IPhI ARRAY

read (ninp, *) commnt

read (ninp,*) nset, nrpa, ncoeff

if (nrpa.le.0) then

nrps $=1$

nrpm $=$ ncoeff

ntest $=-$ ncoeff

write (nout, 41) wetnes (iwtdry), nset, ncoeff

else

nrps $=n$ rpa

nrpm = nrpa

ntest $=\mathrm{nrpa}$ 
end $1 f$

write (nout, 40) wetnes(iwtdry), nset, nrpa, ncoeff

read (ninp, *) (ncyl(1), $i=1, n c y)$

read (ninp, *) (( (coeff(nc,np,ns), nc=1, ncoeff), $n p=1, n$ rps), $n s=1, n s e t$ )

c

write (nout, 42) (1, ncyl (1), $1=1$, ncyln)

write (nout, 44) (( $n s, n p, n c, \operatorname{coeff}(n c, n p, n s), n c=1, n c o e f f)$,

a $n p=1, n$ rps), $n s=1, n s e t$ )

c

c

c

ERROR CHECKING

if (nset.gt.5) then

write (nout, 913) nset

istop $=1$

goto 85

end if

$c$

if (nrps.gt.20) then

write (nout, 914) nrps

1stop $=1$

goto 85

end if

if (nrpa.gt.0 and. nrps.gt.mnrp) then

write (nout, 712) nrps, mnrp

istop $=1$

goto 85

end if

if (nrpa.le.0 and. ncoeff.gt.mnrp) then

write (nout, 712) ncoeff, mnrp

istop $=1$

goto 85

end if

c

if (ncoeff.gt.20) then

write (nout, 915) ncoeff

istop $=1$

goto 85

end if

LOOP OVER CYLINDERS AND SET UP RADIAL DATA SETS

do 70 icyln=1, ncy $1 n$

ERROR CHECK

if (iwtdry.eq.1) then

$n \operatorname{rp}(1 c y l n)=$ ntest

else

$n$ rp2 $=\operatorname{abs}(n \operatorname{rp}(i c y l n))$

If (nrpm.ne.nrp2) then

write (nout, 916) wetnes(1wtdry), nrpm, nrp2

istop $=1$

goto 85

end if

end if

c

iset $=n c y l(i c y l n)$

LOOP OVER COEFEICIENTS IN CYLINDER CORE, LOAD CONSTANTS 
45

c

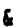

50

60

$c$

70

c

c

85

C

160

170

c

c

c

c

$c$

940

$c$

95

iwtdry $=200$

return

c

c

c

23

WRITE FORMATS

format 1 'O', a,' power fraction assignments in assembly:',

6 ' exposure = $, 912.5,1.1$-reg- -power',

( fract----core--- - in clad- -out clad- ')

c

24 format (' $, 13,4 x, g 12.5,3(6 x, a, 3 x))$

c

26

c

27

if (nrpa.le.0) then

do $45 n c=1$, ncoef $f$

$\operatorname{rph} 1(n c, 1$ cyln $)=\operatorname{coeff}(n c, 1$, iset $)$

else

do $60 \mathrm{np}=1$, nrps

$\operatorname{rphi}(n p, i c y l n)=\operatorname{coeff}(1, n p, 1$ set $)$

if (expose.eq.zero) goto 60

DO REST OF EXPOSURE POLYNOMIAL

do $50 n c=2$, ncoeff

fact $=$ expose**(nc-1)

$\operatorname{rphi}(n p, i c y l n)=\operatorname{rphi}(n p, 1 c y \mid n)+$

continue

coeff (nc, np, iset $) * f$ act

continue

end if

continue

EDIT OUT POWER DATA

write (nout, 130) wetnes(iwtdry), expose

write (nout, 150)

do 170 icyln $=1$, ncyln

do 160 np=1, nrpm

write (nout, 155) icyln, np, rphi(np,icyln)

continue

continue

RETURN HAVING PROCESSED RADIAL DECAY SHAPE DATA

iwtdry $=0$

return

RETURN TO LOAD AT-POWER SHAPE INTO DECAY SHAPE

continue

$y=100$

return

format ('0', a,' power fraction region assignments by node', 1 , - $\quad$-cyln-- -radial node-- --pf region-- ')

format (' $, 2 x, 13,11 x, 13,15 x, 13)$ 


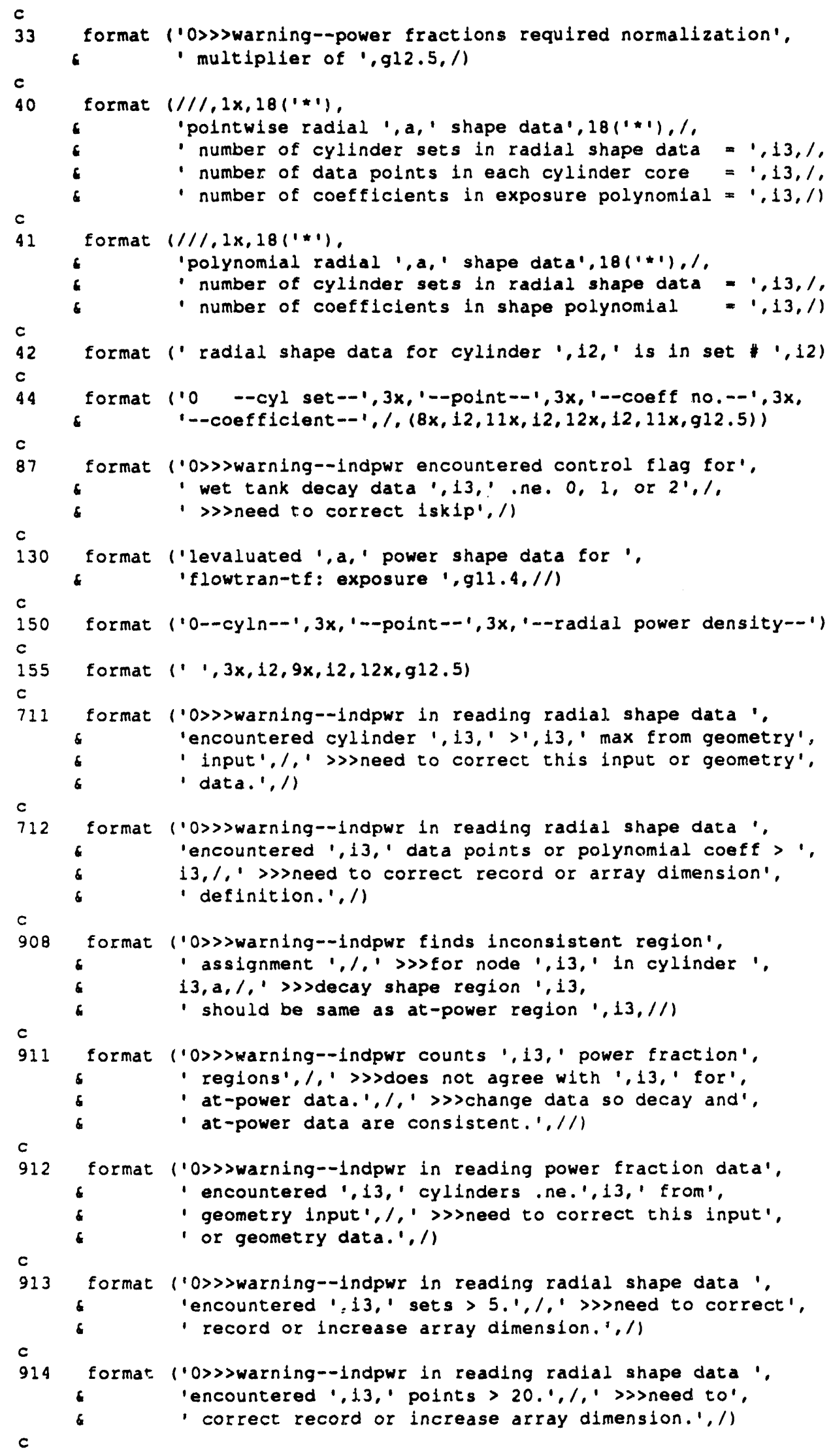



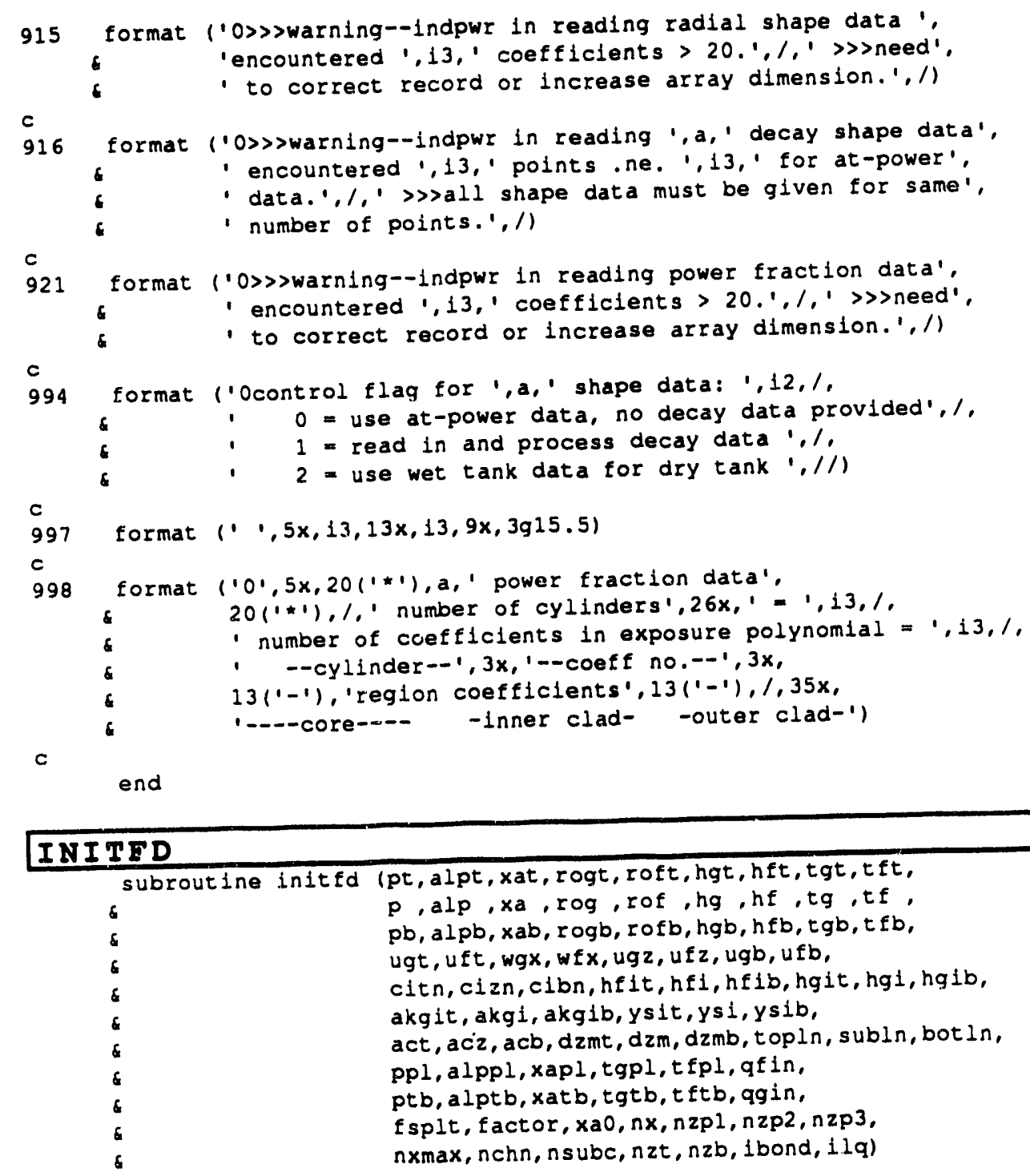

$c$

$c$

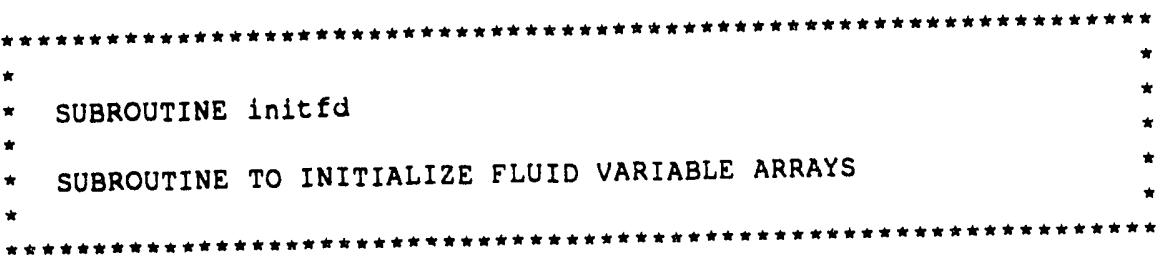

implicit real $(a-h, 0-z)$, integer $(1-n)$

parameter (zero $=0.0$, one $=1.0$, hundrd $=100.0$ )

MIDDLE SECTION PHYSICAL PARAMETERS

\begin{tabular}{|c|c|c|}
\hline ime & n) & 031 \\
\hline 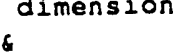 & $\begin{array}{l}p(n z p 2, n x \max , n c n n), \\
\operatorname{alp}(n z p 2, n x \max , n c h n),\end{array}$ & xa (nzp2, nxmax, nchn). \\
\hline 6 & $\operatorname{rog}(n z p 2, n \times \max , n c h n)$. & $\operatorname{rof}(n z p 2, n x \max , n c h n)$, \\
\hline f & $h g(n z p 2, n x \max , n c h n)$. & $h f(n z p 2, n \times \max , n \in h n)$, \\
\hline & $\operatorname{tg}(n z p 2, n x \max , n \operatorname{chn})$ & $t f(n z p 2, n x \max , n c h n)$, \\
\hline & ugz $\operatorname{lnzp} 3, n \times \max , n c h$ & ufz(nzp), nxmax, nchn), \\
\hline & wgx (nzp2, nxmax, nchn), & wfx (nzp2, nxmax, nchn), \\
\hline & $\operatorname{acz}(n z p 3, n \times m a x, n c h n)$, & $y \operatorname{si}(n z p 2, n x \max , n c h n)$, \\
\hline & $2 p 3, n x \max , n(h n)$, & $n \in 1$ ( $n 2 p 2, n \times \max , n$ \\
\hline & & $n x \max , n$ \\
\hline
\end{tabular}




\begin{tabular}{|l|ll|r|}
\hline Pg. 164 of 354 & WSRC-TR-93-086 Rev. 0 & FLOWTRAN-TF v1.2 Source Code & $2 / 15 / 93$ \\
\hline
\end{tabular}

c

c

c

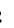

parameter $(\operatorname{mxnz}=100)$

dimension drogdp (mxnz), drogdt (mxnz), drgdxa (mxnz),

6

c

6
TOP SECTION PHYSICAL PARAMETERS

dimension pt(nzt), dzmt(nzt), alpt (nzt), $\quad$ at (nzt), roge (nzt), roft (nzt), hgt (nzt), $n f t(n z t)$, tgt (nzt). $t \in t(n z t)$, ugt (nzt), uft (nzt), act (nzt), ysit (nzt). citn(nzt), $n f i t(n z t)$, hglt (nzt), akg1t (nzt)

dimension watmw(2)

data airmw/28.97/, watmw(1)/18.016/, watmw(2)/20.034/

data epsal/0.001/

$r t m w=o n e-w a t m w(i l q) / a 1 r m w$

c

BEGIN FLUID INITIALIZATION:

CALCULATE PROPERTY DIFFERENTIALS ACROSS ASSEMBLY

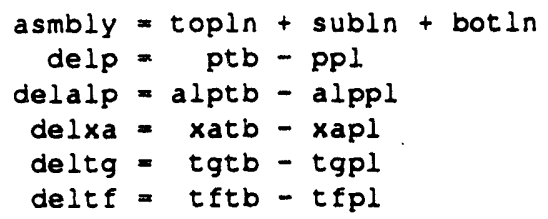

INTERPOLATE BEWTEEN PLENUM AND TANK BOTTOM CONDITIONS TO SET LOCAL PRESSURE, GAS TEMPERATURE, FLUID TEMPERATURE, VOID FRACTION, AND AIR MASS FRACTIONS

TOP SECTION

fract $=$ zero

do $10 j z=1, n z t$

fract $=$ fract + dzmt $(f z) / a s m b l y$

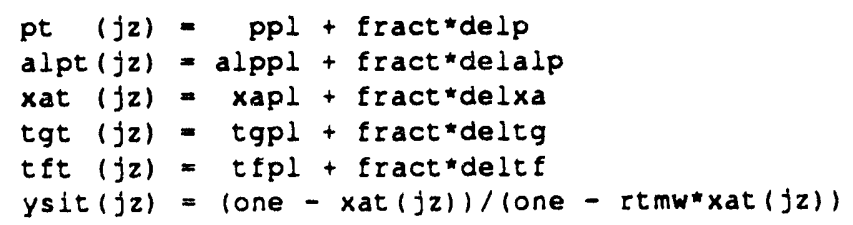


c

c

10

c

$c$

c

c

c

20

30

40

c

alpf a one - alpt (jz)

if (alpt (fz). It. epsal) then

$w t=\operatorname{alpt}(f z) /$ epsal

$x a t(j z)=($ one $-w t) * x a 0+w t * x a t(f z)$

$y s i t(j z)=($ one $-x a t(j z)) /($ one $-\operatorname{rtmw} * x a t(j z))$

psi $=y \operatorname{sit}(j z) * p t(f z)$

call tsat (psi, $11 q, t 1, d t 1 d p s)$

$\operatorname{tgt}(j z)=($ one $-w t) * t i+w t \star \operatorname{tg} t(j z)$

end if

if (alpf..1t. epsal) then

$w t=a l p f / e p s a l$

psi $=y s i t(f z) * p t(f z)$

call tsat (ps1,11q, t1, dt 1dps)

$t f t(j z)=($ one $-w t) * t 1+w t * t f t(j z)$

end $1 f$

hfit $(j z)=$ hundrd

hg1t $(j z)=$ hundrd

akgit $(j z)=$ hundrd

continue

MIDDLE SECTION

fract $=$ topln/asmbly

do $40 \quad j z=2, n z p 1$

fract $=$ fract $+d z m(j z) / a s m b l y$

do 30 lc $=1$, nchn

nsub $=n \times(1 c)$

do $201 x=1$, nsub

$p(j z, i x, i c)=p p l+f r a c t * d e l p$

$\operatorname{alp}(j z, i x, i c)=a l p p l+f r a c t * d e l a l p$

$x a(j z, 1 x, 1 c)=x a p l+f r a c t \star d e l x a$

tg $(j z, i x, 1 c)=t g p l+f r a c t * d e l t g$

$t f(j z, 1 x, 1 c)=t f p l+f r a c t \star d e l t f$

$y s 1(j z, 1 x, 1 c)=($ one $-x a(j z, 1 x, 1 c)) /($ one $-\operatorname{rtmw} x a(j z, 1 x, 1 c))$

alpf $=$ one $-\operatorname{alp}(j z, i x, i c)$

if (alp(jz,ix,ic) . It. epsal) then

$w t=\operatorname{alp}(j z, i x, i c) / e p s a 1$

$x a(j z, i x, i c)=($ one $-w t) * x a 0+w t * x a(j z, i x, 1 c)$

$y s i(j z, i x, 1 c)=(o n e-x a(j z, 1 x, i c)) /$

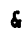

(one - $\operatorname{rtmw*xa}(j z, 1 x, i c))$

psi $=y s i(j z, 1 x, 1 c) * p(j z, 1 x, 1 c)$

call tsat (psi,1lq, t1, dt 1dps)

$t g(j z, i x, i c)=($ one $-w t) \star t 1+w t \star t g(j z, 1 x, 1 c)$

end if

if (alpf..1t. epsal) then

$w t=$ alpE/epsal

ps1 $=y s 1(j z, 1 x, 1 c) * p(j z, 1 x, 1 c)$

call tsat (ps $1,11 q, t 1, d t 1 d p s)$

$t f(j z, i x, 1 c)=($ one $-w t) \star t i+w t * t f(j z, 1 x, i c)$

end if

hfi $(j z, i x, i c)=$ hundrd

hgi $(j z, i x, i c)=$ hundrd

$\operatorname{akg} 1(j z, 1 x, i c)=$ hundrd

continue

continue

continue

BOTTOM SECTION 
c

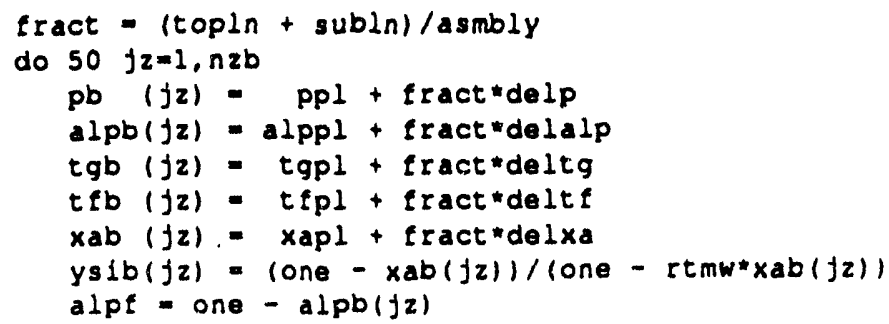

MIDDLE SECTION:

do $90 \mathrm{ic}=1$, nchn

nsub $=n \times(1 c)$

do $80 \quad 1 x=1$, nsub

call state (p,tg,tf, xa, factor, ilq,

2, nzpl, nzp2, nxmax, nchn, ix, ic, rog, drogdp, drogdt, drgdxa,

hg, dhgdp, dhgdt, dhgdxa, $r \circ f, d r o f d p, d r o f d t$,

continue

$h f$. dhfdp, dhfdt)

continue

BOTTOM SECTION :

call state (pb,tqb,ttb, xab, factor, 1lq, $1, n z b, n z b, 1,1,1,1$, rogb, drogdp, drogdt, drgdxa, 


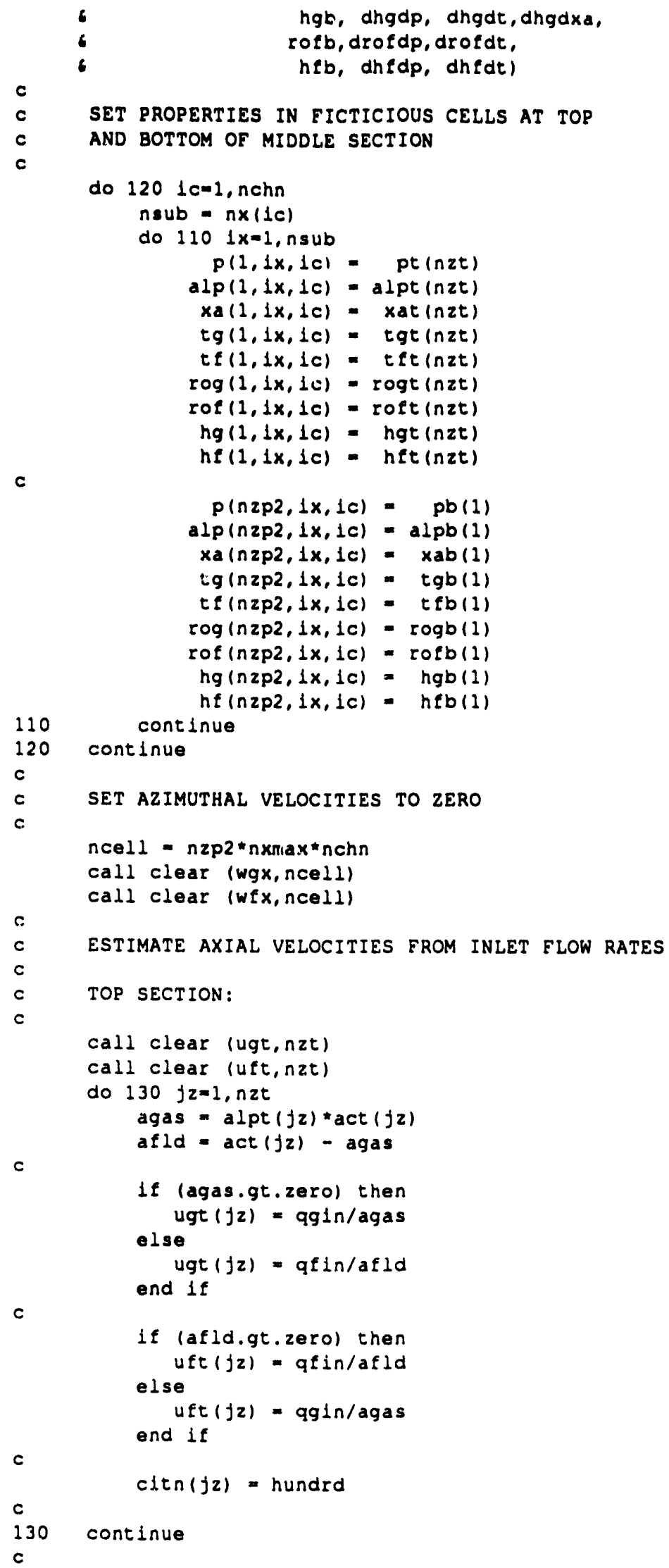




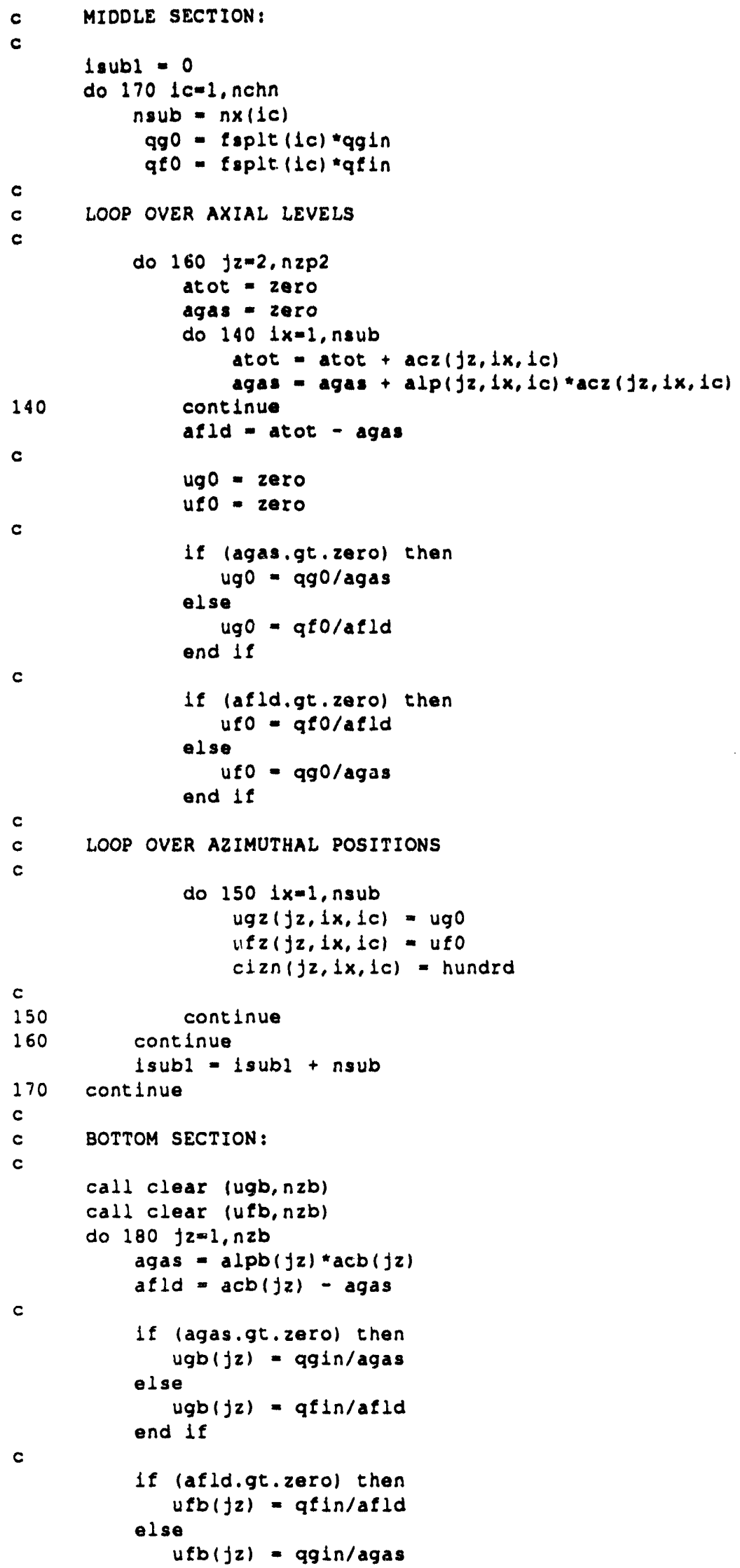




\begin{tabular}{|l|lll|l|}
\hline $2 / 15 / 93$ & WSRC-TR-93-086 Rev. 0 & FLOWTRAN-TF v1.2 Source Code & Pg. 169 of 354 \\
\hline
\end{tabular}

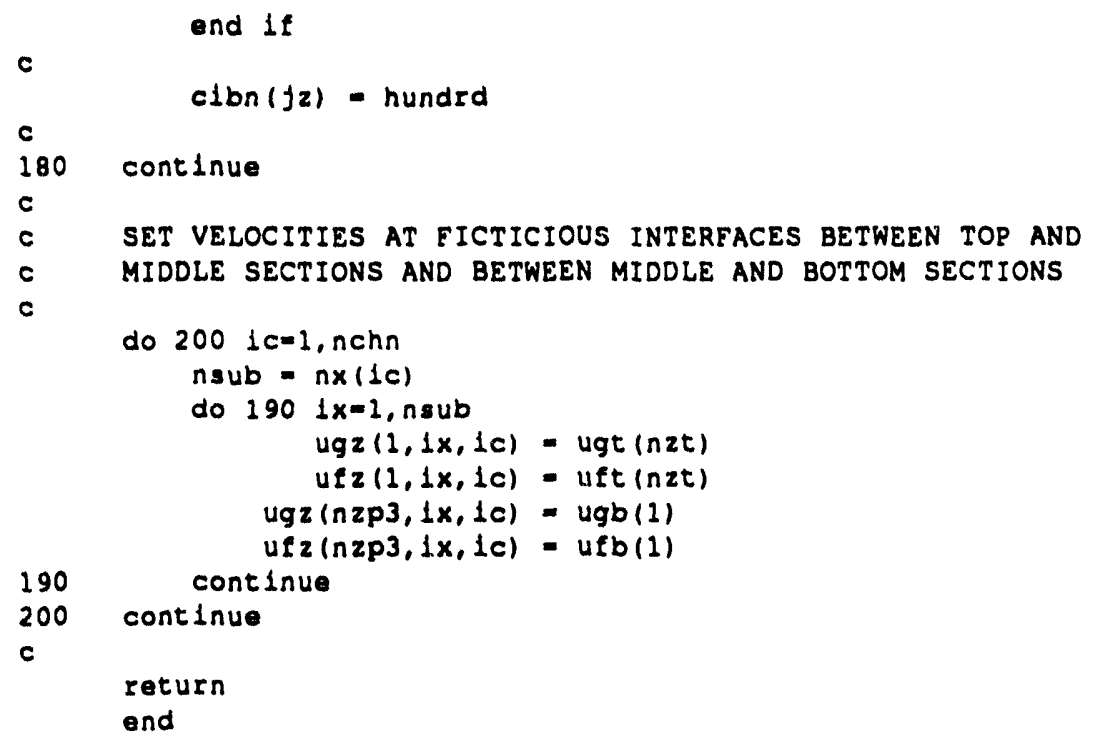

\section{INITI}

subroutine $\ln 1 t 1$

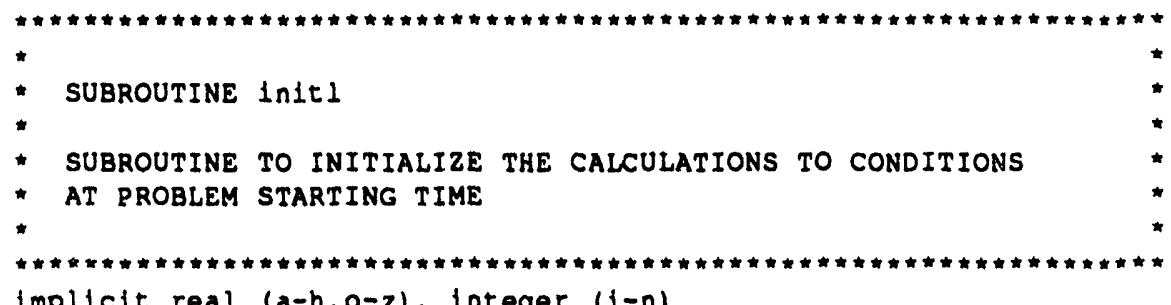

implicit real $(a-h, 0-z)$, Integer $(1-n)$

parameter (zero=0.0, half $=0.5$, fifty=50.0)

common /iounlt/ ninp, nout, ndmp, ntmp, nrsti, nrsto 


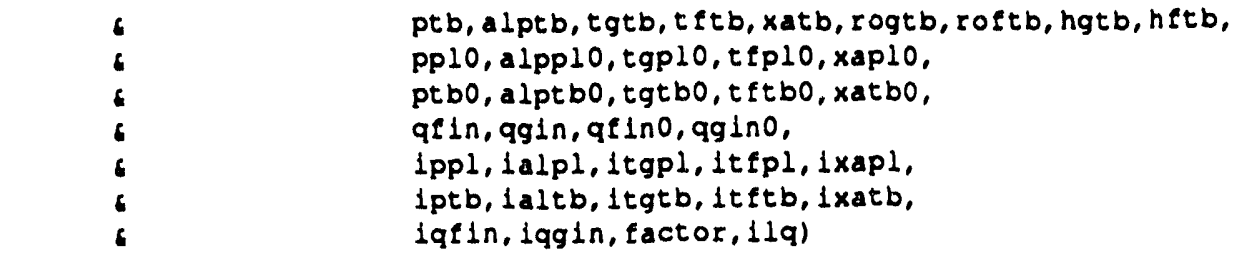

c

SETUP FLUID VARIABLES FROM INPUT FILE INITIAL CONDITIONS

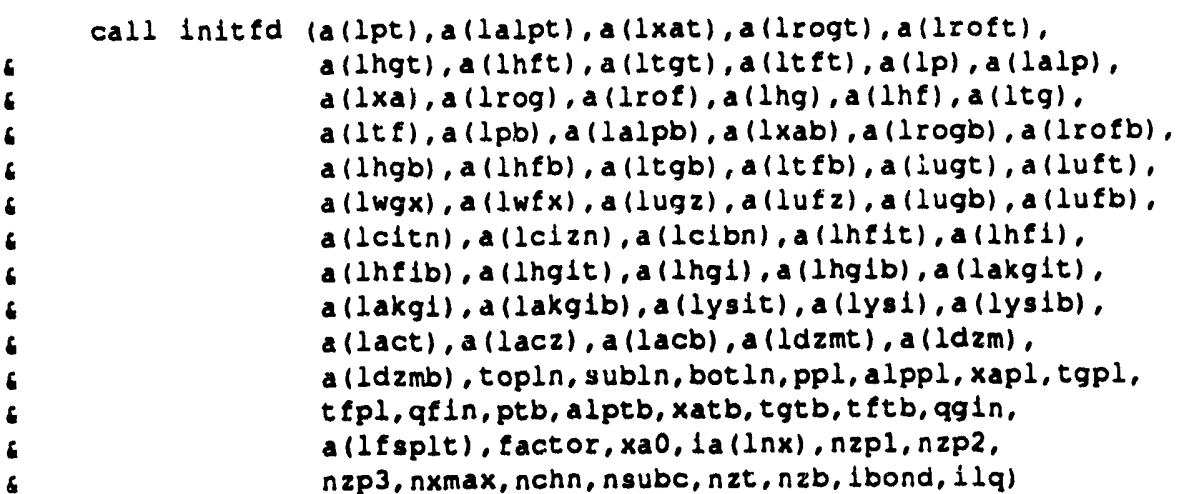

SET OLD TIME (n) VALUES OF VARIABLES FOR FIRST TIME STEP

call reset (a(1ts),a(1tsurf),a(1tsn),a(1tsrfn), $a(1 p), a(1 a l p), a(1 \times a), a(1 r \circ g), a(1 r \circ f), a(1 h g)$, $a(1 h f), a(1 t g), a(1 t f), a(1 p n), a(1 a \mid p n), a(1 \times a n)$, $a(\operatorname{lrogn}), a(1 \operatorname{rof} n), a(1 h g n), a(1 h f n), a(1 \operatorname{tgn}), a(1 t(n)$. $a(1 p t), a(1 a l p t), a(1 \times a t), a(1 r o g t), a(1 r o f t), a(1 h g t)$. $a(1 h f t), a(1 t g t), a(1 t f t), a(1 p t n), a(1 a l p t n)$, $a(1 \times a t n), a(1$ rogt $n), a(1$ roftn), a (1hgtn), a (1hftn), $a(1 t g t n), a(1 t f t n), a(1 p b), a(i a \perp p b), a(1 \times a b)$, $a(1 r \circ g b), a(1 r o f b), a(1 h g b), a(1 h f b), a(1 t g b)$. $a(1 t f b), a(1 p b n), a(1 a \mid p b n), a(1 \times a b n), a(1 r \circ g b n)$, $a$ (1rofbn), a (1hgbn), a (1hfbn), a (1tgbn), a (1tfbn), ppl .xapl, rogpl , rofpl, hgpl, hfpl, tgpl, tfpl . ppln, xapln, rogpln, rofpln, hgpln, hfpln, tgpln, tfpln, ptb , xatb , rogtb , roftb, hgtb , hftb, tgtb , tftb , ptbn, xatbn, rogtbn, roftbn, hgtbn, hetbn, tgtbn, tftbn, alppl, alppln, alptb, alptbn, nzp2, nxmax, nchn, nzt, $n z b, n$ max, na, nz, ncyln, nsurf)

else

SETUP FLUID AND SOLID VARIABLES FROM DATA IN RESTART FILE

call restrt (a(1ts), a(1tsurf), a(1tsn), a(1tsrtn), $a(l q w f), a(l q w g), a(1 d q f d w), a(1 d q g d w), a(1 p t), a(1 a l p t)$, $a(1 \times a t), a(1 r \circ g t), a(1 r \circ f), a(1 h g t), a(1 h f t), a(1 t g t)$. $a(1 t f t), a(1 p t n), a(1 a$ lpt $n), a(1 \times a t n), a(1$ rogtn), $a(1$ roftn), a (1hgtn), a(lhftn), a(1tgtn), a (1tftn), a(lp), $a(1 a l p), a(1 \times a), a(1 r o g), a(1 r o f), a(1 h g), a(1 h f), a(1 t g)$, $a(1 t f), a(1 p n), a(1 a \mid p n), a(1 \times a n), a(\operatorname{lr} \circ g n), a(1<\circ f n)$. $a(1 h g n), a(1 h f n), a(1 \in g n), a(1 t f n), a(1 p b), a(1 a l p b)$, $a(1 \times a b), a(1<\circ g b), a(1<\circ b b), a(1 h g b), a(1 h f b), a(1<g b)$, $a(1 t f b), a(1 p b n), a(1 a \mid p b n), a(1 \times a b n), a(1 r \circ g b n)$, $a(1$ rofbn), a (1hgbn), a (1hfbn), a (1tgbn), a (1tfbn). $a(1 \cup g t), a(1 \cup f t), a(1 w g x), a(l w f x), a(1 \cup g z), a(l \cup(z)$, $a(1 u g b), a(1 u f b), a(1 c i t n), a(1 c i z n), a(1 c i \times n), a(1 c 1 b n)$, $a(\operatorname{lnf}(t), a(\operatorname{lnf} 1), a(\ln f \mid b), a(\operatorname{lng} i t), a(\operatorname{lng} 1), a(\operatorname{lng} 1 b)$, $a(\operatorname{lakg} 1 t), a(\operatorname{lakg} 1), a(\operatorname{lakg} \mid b), a(\lg i t), a(\lg 1), a(\lg i b)$, 


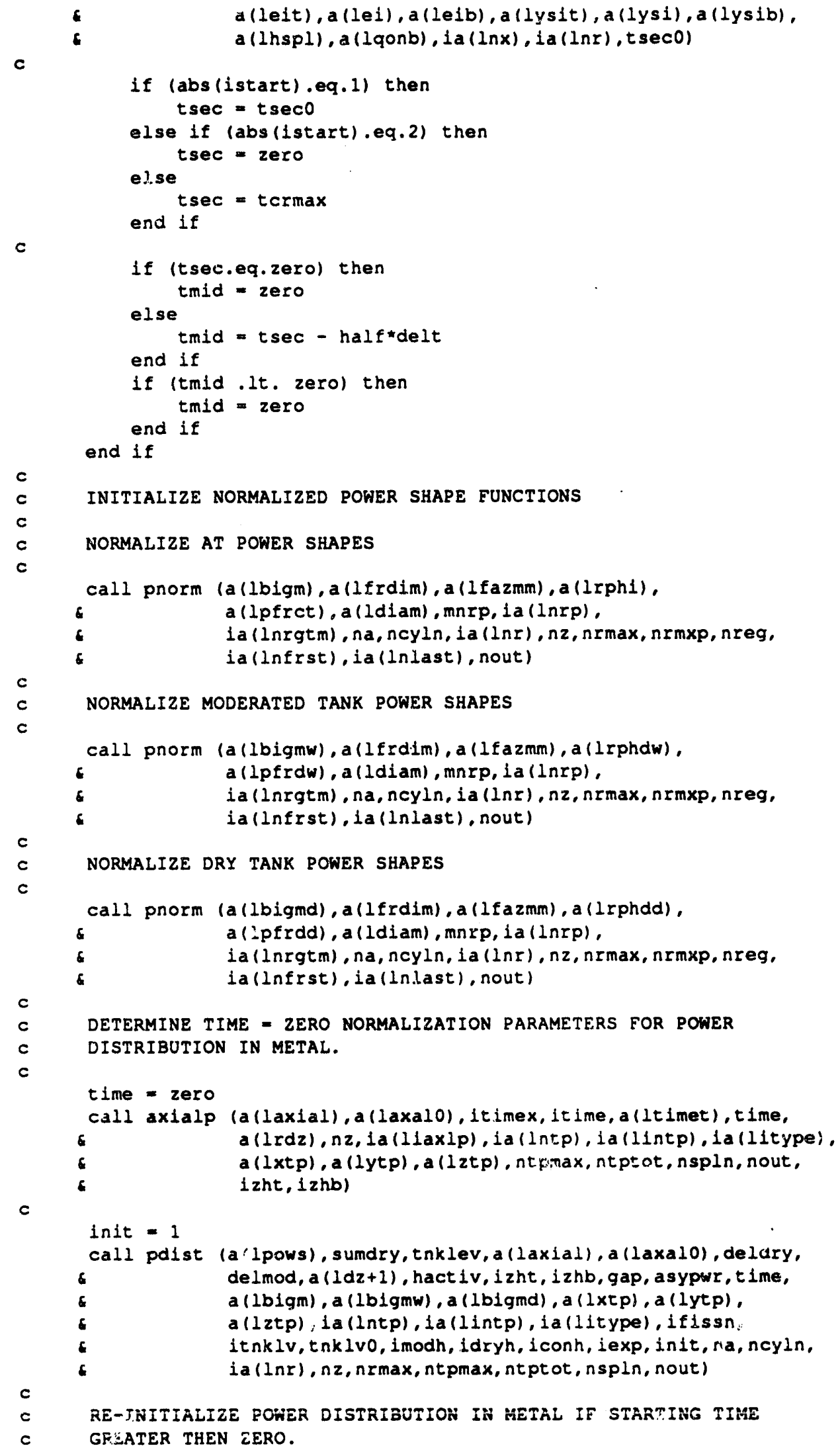

INITIALIZE NORMALIZED POWER SHAPE FUNCTIONS

NORMALIZE AT POWER SHAPES

call pnorm (a(1bigm), a (lfrdim), a (lfazmm),a (lrph1), 


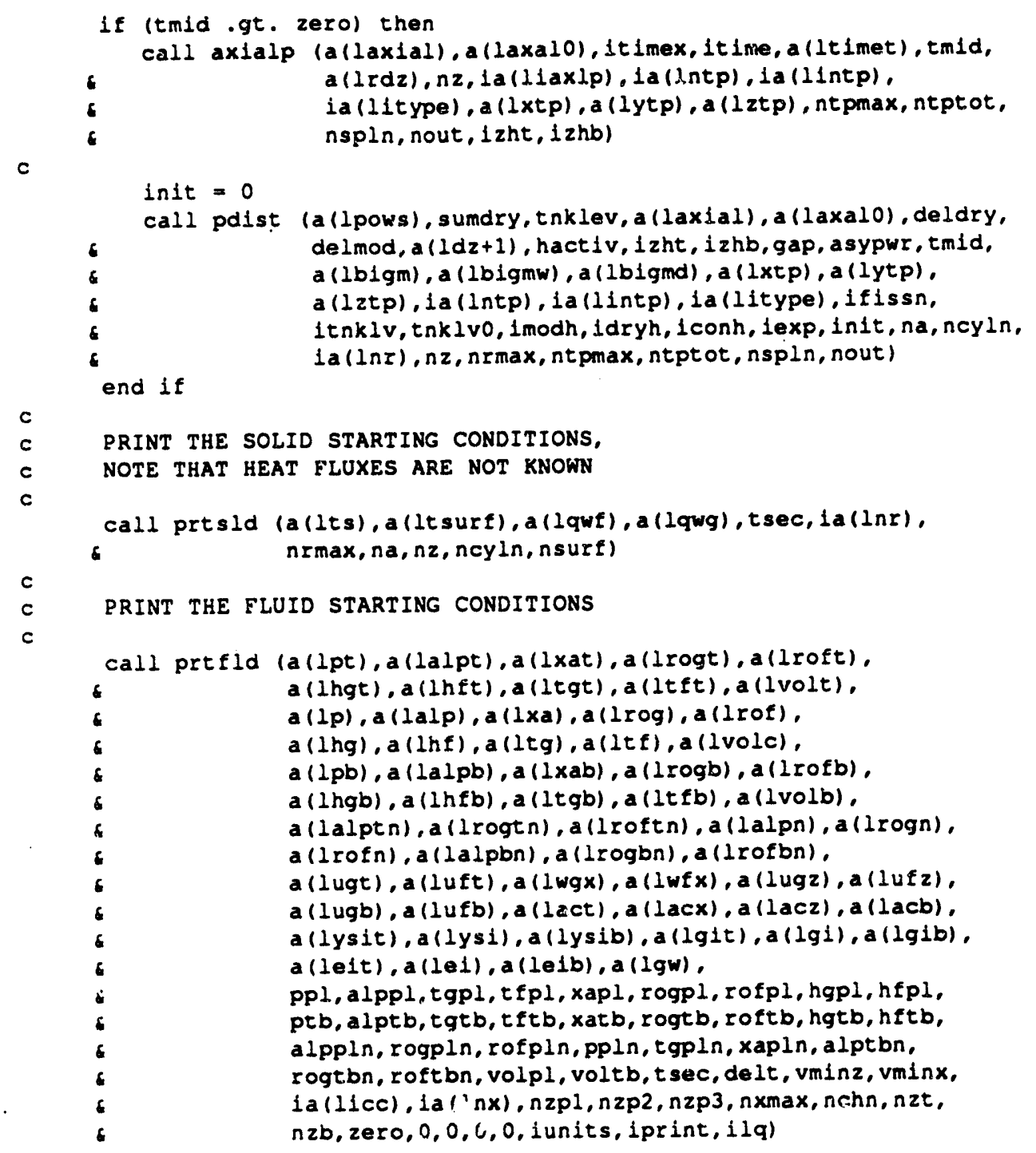

PRINT THE POWER DISTRIBUTION STARTING CONDITIONS

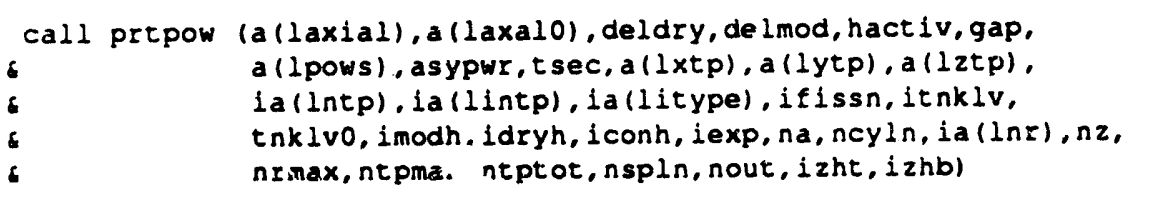

IF A DATA DUMP FOR PLOTTING WILL BE CREATED, ALSO INCLUDE THE STARTING DATA IN THE PLOT DUMP FILE

if (dtpdmp.1t.runsec) then

call dmpplt (a(lts), a (ltsurf), a (lqwf), a (lqwg), a(lpt), $a(1 \mathrm{a}$ pt), a (lxat), a(lrogt), a (lroft), a (1hgt), $a(1 h f t), a(1 t g t), a(1 t f t), a(1 p), a(l a l p), a(1 \times a)$, $a(1\ulcorner\circ g), a(1 r \cap f), a(1 h g), a(\ln f), a(1 t g), a(1 t f)$, $a(1 p b), a(1 a l p b), a(1 \times a b), a(1 r \circ g b), a(1 r \circ f b)$, $a(1 h g b), a(1 h f b), a(1 t g b), a(1 t f b), a(1 u g t), a(1 u f t)$. $a($ ( wgx), $a((w f x), a(1 \cup g z), a(1 \cup f z), a(l u g b), a(1 u f b)$, ppl, alppl, lgpl, tfpl, xapl, rogpl, rofpl, hgpl, hfpl, ptb, alptb, tgtb, $t f t b, x a t b$, rogtb, roftb, hgtb, hftb, tsec, ia $(\ln x)$, ia $(\ln r), n z p 1, n z p 2, n z p 3, n x \max , n c h n$, 


\begin{tabular}{|l|lll|}
\hline $2 / 15 / 93$ & WSRC-TR-93-086 Rev. 0 & FLOWTRAN-TF v1.2 Source Code & Pg. 173 of 354 \\
\hline
\end{tabular}

end $1 f$ nsubc, nzt, nzb, nrmax, na, nz, ncy $n$, nsurf)
return
end

\section{INITSD}

subroutine initsd (ts,tsurf, qwf, qug, dqfdw, dqgdw, hspl, qonb,

6

tsolld, tsrfac, nr, mat, wt, nsurf, nrmax, na

$n z, n c y \mid n)$

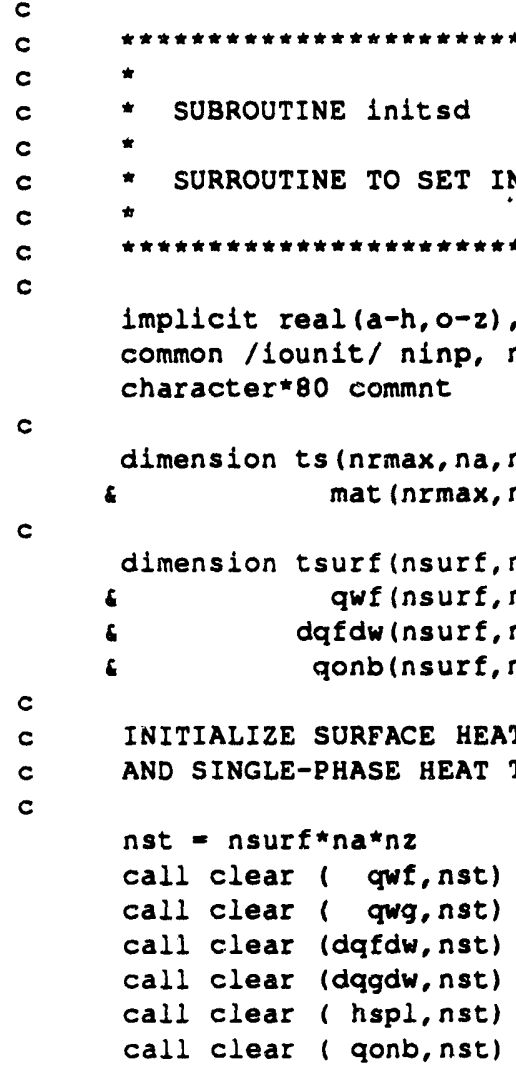

$c$

c

c

10

20

c

c

c

30

c

INITIALIZE SOLID NODE TEMPERATURES

do 20 icyln=1, ncyln

$n r i=n r(1 c y l n)$

do 10 ia=1, na

do $10 \quad 1 r=1, n r i$

do $10 \quad \mathrm{z}=1, \mathrm{nz}$

continue

ts $(1 r, i a, i z, i c y l n)=t s o l i d$

continue

INITIALIZE SOLID SURFACE TEMPERATURES

do 30 ia=1, na

do 30 isurf $=1$, nsurf

do $30 \quad i z=1, n z$

tsurf(izurf,ia,iz) = tsrfac

continue

return

end 
INWER

$$
\begin{aligned}
& \text { subroutine inner (dp, axm1, axm2, azml, azm2, rhs, sz, sx, dpt, atm1, rhst, } \\
& \text { st, dpb, abm1, rhsb, sb, } t, d p m, \text { icc, cmesh, nx, nxmax, } \\
& \text { nzm, nzpl, nzp2, nzt, nzb, nchn, nlarg, epsin, errin, } \\
& \text { pref, initmx, initct, ischem, irebal, ncmr, lerr) }
\end{aligned}
$$

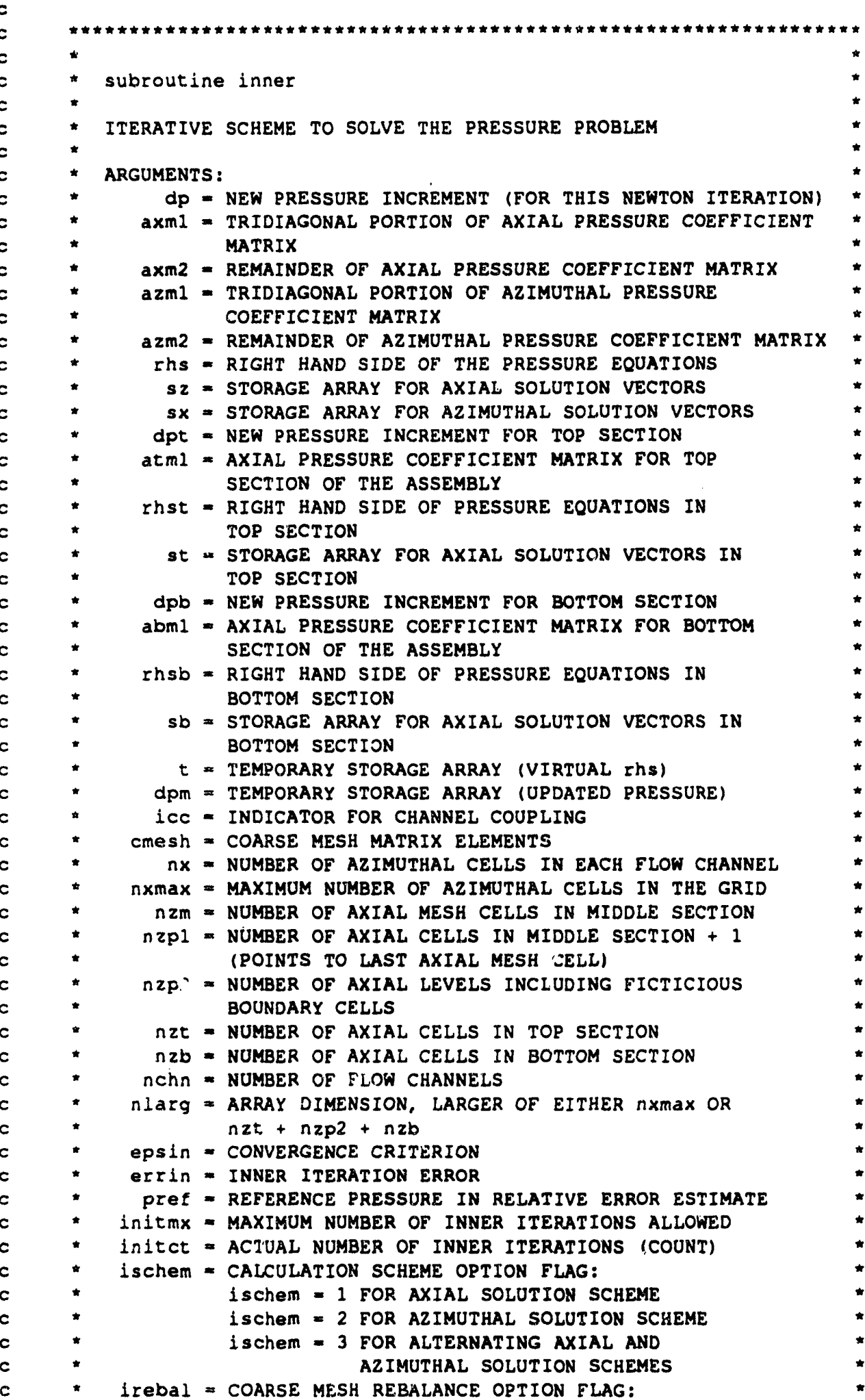




\begin{tabular}{|l|ll|l|}
\hline $2 / 15 / 93$ & WSRC-TR-93-086 Rev.0 & FLOWTRAN-TF v1.2 Source Code & Pg. 175 of 354 \\
\hline
\end{tabular}

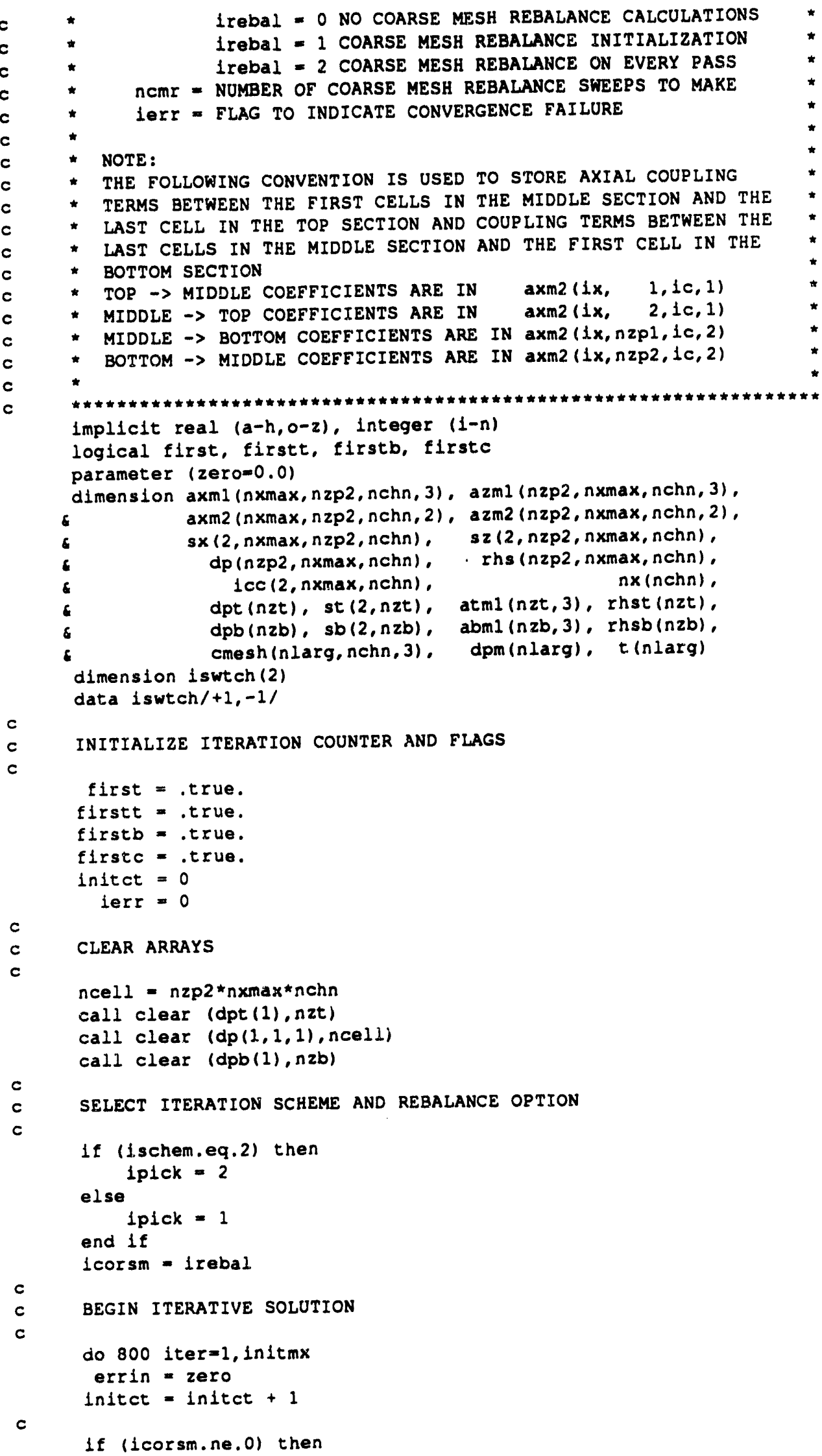


$c$

c

c

c

$c$

c

c

c

110
$\mathrm{c}$
$\mathrm{c}$
$\mathrm{c}$

PERFORM COARSE MESH CALCULATIONS TO OBTAIN AN

INITIAL ESTIMATE FOR THE PRESSURE SOLUTION

do 270 icmr=1, ncmr

LOOP OVER ALL FLOW CHANNELS

do $2601 c=1$, nchn

$n s u b=n \times(1 c)$

if (ipick.eq.1) then

SET UP AXIAL REBALANCE ARRAYS AND PERFORM

COARSE MESH CALCULATION

if (firstc) then

ON FIRST PASS, SET UP INVARIENT ELEMENTS OF COARSE MESH ARRAYS: INSERT TOP SECTION COEFFICIENTS INTO THE COARSE MESH ARRAY

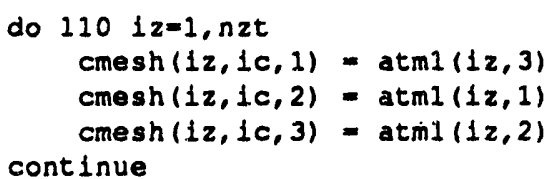

INSERT MIDDLE SECTION COEFEICIENTS INTO THE COARSE MESH ARRAY

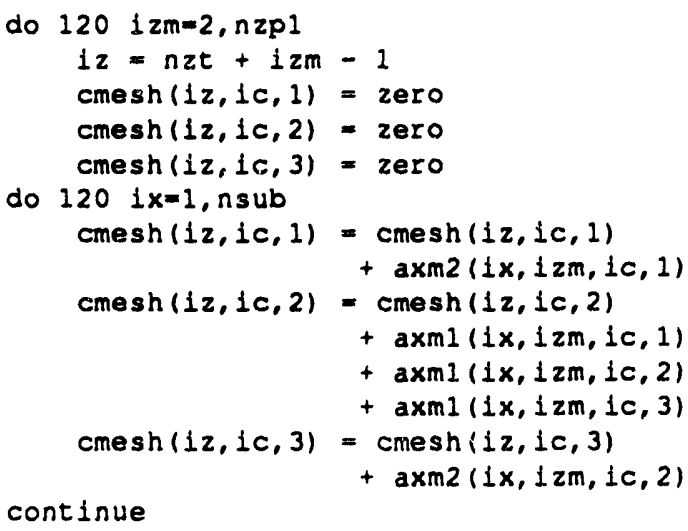

INSERT BOTTOM SECTION COEFFICIENTS INTO THE COARSE MESH ARRAY

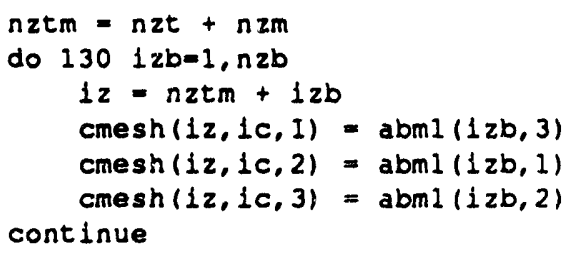

INCLUDE TOP TO MIDDLE AND BOTTOM TO MIDDLE COUPLING TERMS

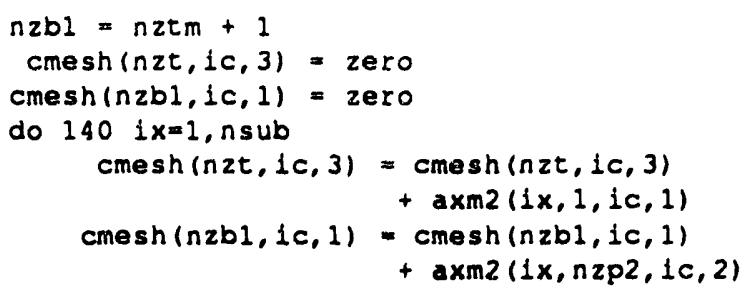




\begin{tabular}{|l|ll|l|}
\hline $2 / 15 / 93$ & WSRC-TR-93-086 Rev. 0 & FLOWTRAN-TF v1.2 Source Code & Pg. 177 of 354 \\
\hline
\end{tabular}

140
$c$
$c$
$c$

150
$c$
$c$
$c$

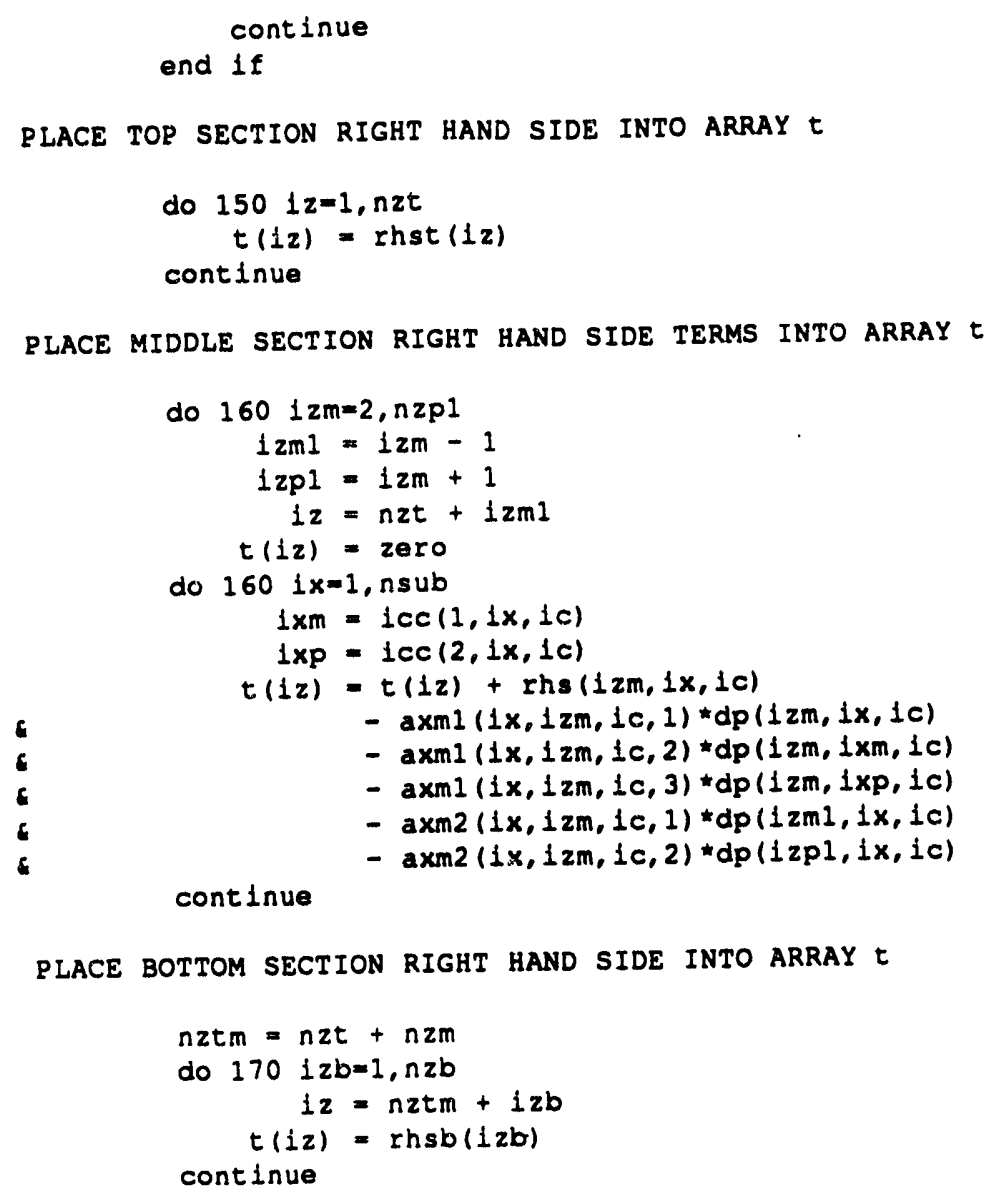


c

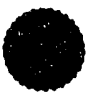

6

call tridag (firstc, l, nsub, nsub, $\operatorname{cmesh}(1,1 \mathrm{l}, 1$ ), cmesh $(1,1 c, 2)$, cmesh $(1,1 c, 3)$,

$t, d p m, s z(1,1,1,1 c))$

c

ADJUST PRESSURE SOLUTION BY REBALANCE FACTORS

c

250

260

270

$c$

c

c

c

310

c

c

c

c

c 


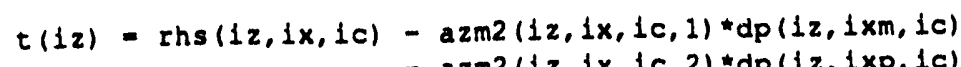

INCLUDE AXIAL COUPLING TERMS LINKING THE FIRST CELL IN THE MIDDLE SECTION TO THE LAST CELL IN THE TOP SECTION AND THE LAST CELL IN THE MIDDLE SECTION TO THE FIRST CELL IN THE BOTTOM SECTION

$$
\begin{aligned}
t(2) & =t(2)-\operatorname{axm2}(1 x, 2,1 c, 1) \star d p t(n z t) \\
t(n z p 1) & =t(n z p 1)-\operatorname{axm2}(1 x, n z p 1,1 c, 2) \star d p b(1)
\end{aligned}
$$

SOLVE TRIDAIGONAL AXIAL EQUATIONS FOR NEW PRESSURE INCREMENTS

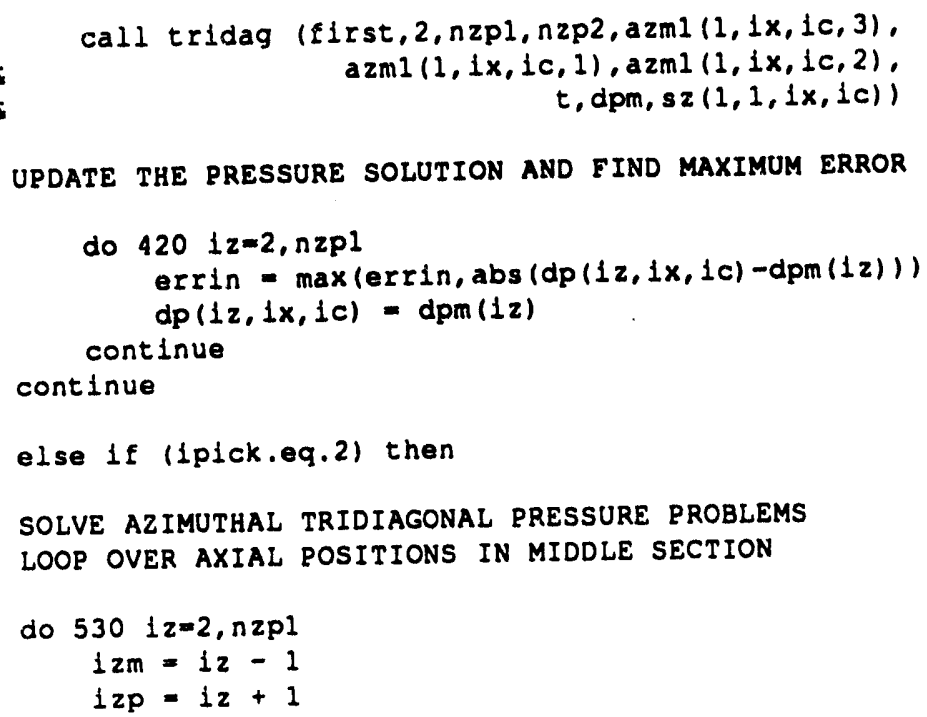




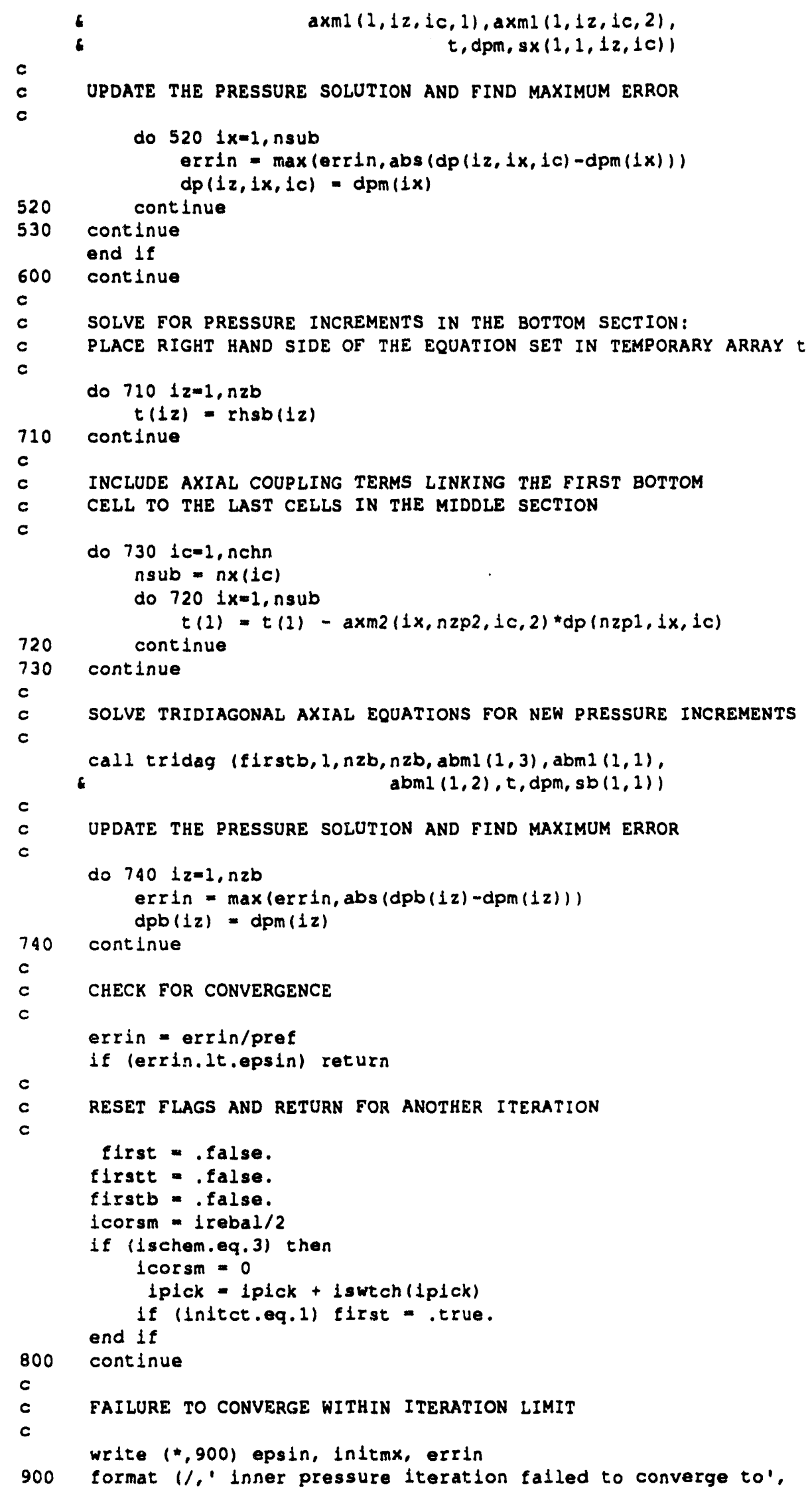




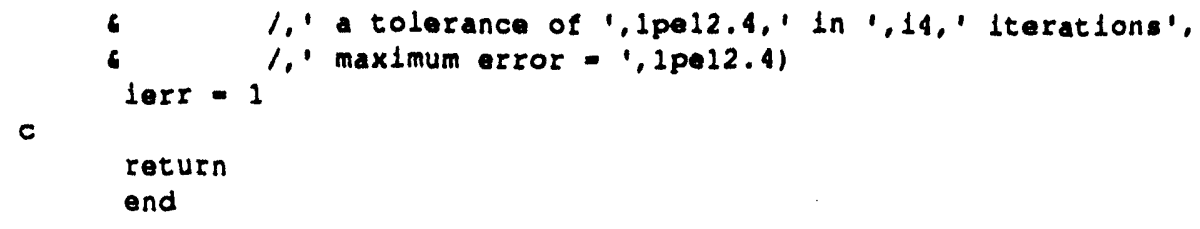

\section{INOPTS}

subrout Ine inopts (1cmax, Ipmax, npdat, nset)$$
\text { c }
$$

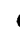$$
\text { c }
$$$$
\text { c }
$$$$
\text { c }
$$$$
\begin{aligned}
& c \\
& c \\
& c
\end{aligned}
$$$$
\text { c }
$$$$
\text { c }
$$$$
\text { c }
$$$$
\text { c }
$$$$
\text { c }
$$$$
\text { c }
$$$$
\text { c }
$$$$
\text { c }
$$$$
\text { c }
$$$$
\text { Iinplicit real }(a-h, 0-z) \text {, Integer }(i-n)
$$ 
1ppu

READ IN REFERENCE PRESSURE, COMPRESSIBILITY FACTOR, MINIMUM VELOCITY FOR FULL DONORING, ACCURACY CHECKING TOLERANCE, AND OTHER MISCELLANEOUS MODEL PARAMETERS, FOR SRS INPUT UNITS, CONVERT PRESSURE TO SI UNITS

read (ninp, 10) commnt

read (ninp, *) 119, pref, factor, uminz, vminx, tol, tolss, nstdy, 6 tsupw, htdamp, cldamp, xdelt, xturb, xao

READ IN BOILING CURVE AND INTERFACIAL WALL AND BULK OPTIONS

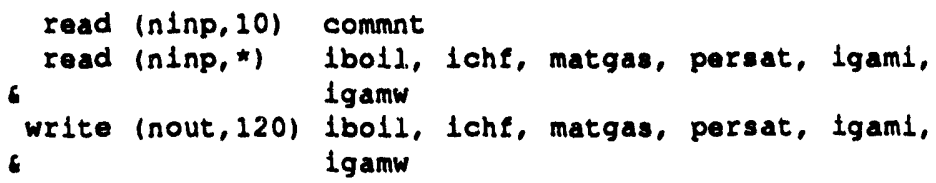


c

c c

c

c

c

c

c 
c

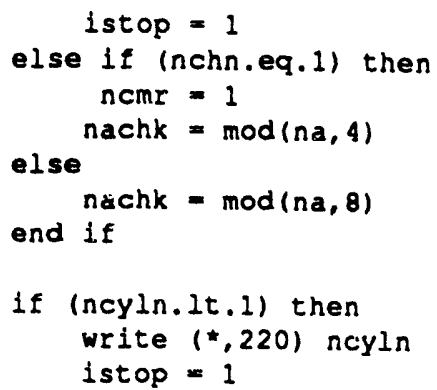

$\begin{array}{ll}\text { format } & 1 \\ 6 & \text { : * } \\ 6 & 1 \\ 6 & 1\end{array}$

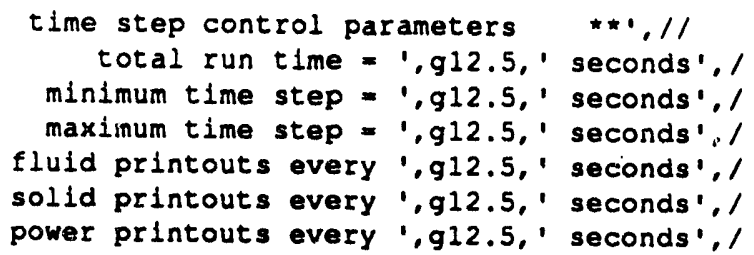




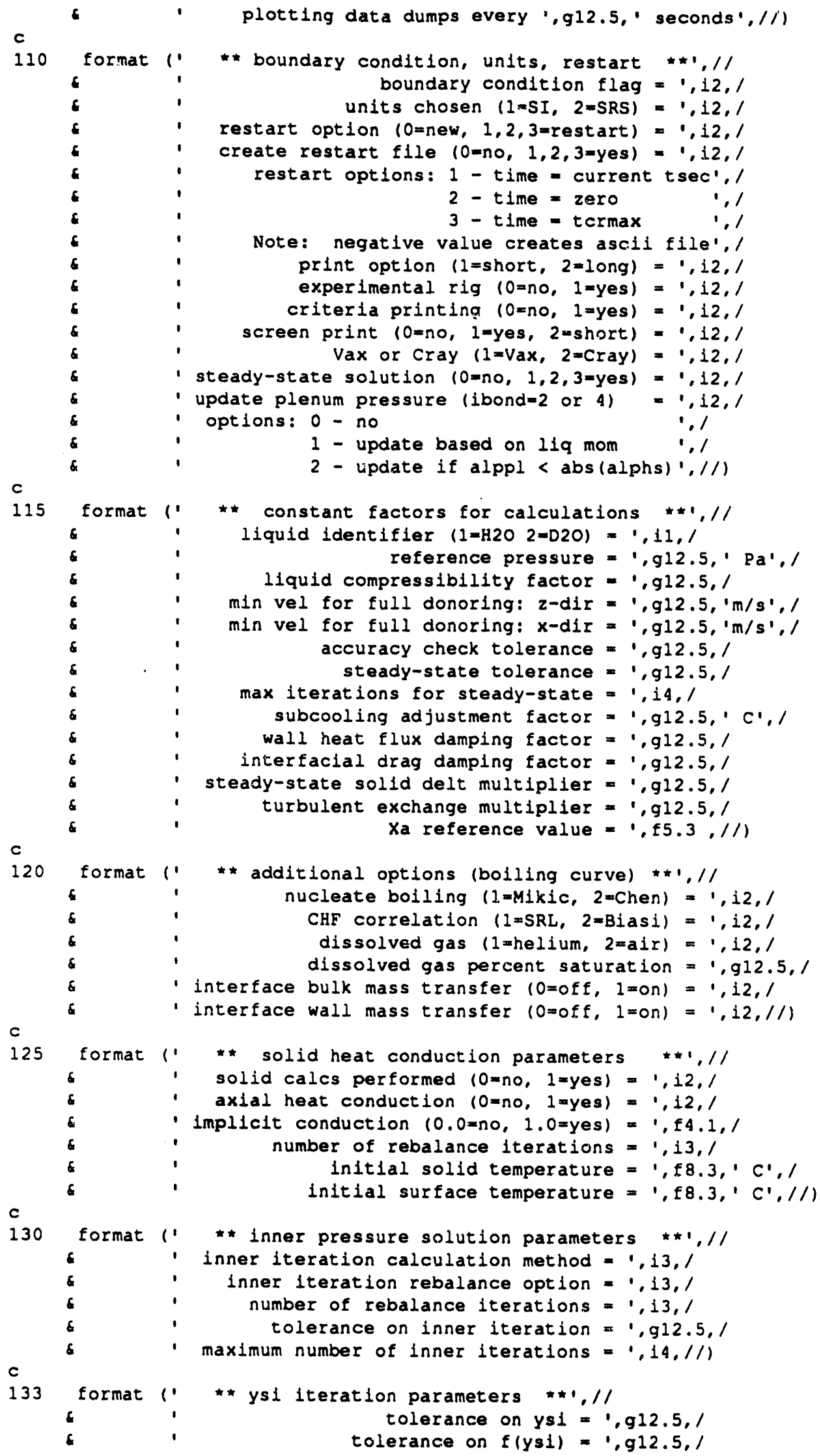


c

spline profile specifications $\quad \star \star 1, / /$ number of spline data sets specified $=1,14,1$ number of spline profiles used $=1,14,1$ total number of points in all profiles $=1,14,1$ number of points in radial power shapes $=1,14,1$ number of times for axial power shapes $=1, i 4, / / 1$

$c$

145 format (' * geometric input data for problem ** .,/ number of flow channels $=1,13,1$ maximum number of fluid azimuthal cells $=1,13,1$ number of metal cylinders $=1,13,1$ fixed number of solid azimuthal cells $=1,13,1$ maximum number of radial solid cells $=1,13 . /$ number of axial cells in top section $=1,13,1$ number of axial cells in middle section $=1,13,1$

- number of axial cells in bottom section $=1,13, / / 1$

$$
\text { c }
$$

* active core input data for problem **',//

first heated cell in active core $=1,13,1$

last heated cell in active core $=1,13,1$

gap from active core to tank bottom $=1,912.5, \mathrm{~m}^{\prime} \mathrm{m}^{\prime}$,

inttial moderator temperature $=1,912.5, \mathrm{C}^{\prime} \mathrm{C}, 1$

moderator heat transfer coefficient $=1,912.5$,

radiation b. c. flag $(0=0 f f, 1=0,=1,11,1$ $\omega / m * 2-c^{\prime}, /$

diameter of radiating surface $=1, \mathrm{~g} 12.5, \mathrm{~m}^{\prime}, /$

enissivity of inner surface $=1.912 .5,1$

emissivity of outer surface $=1, g 12.5, / / 1$

c

155 format $($ '

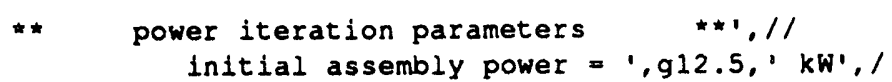




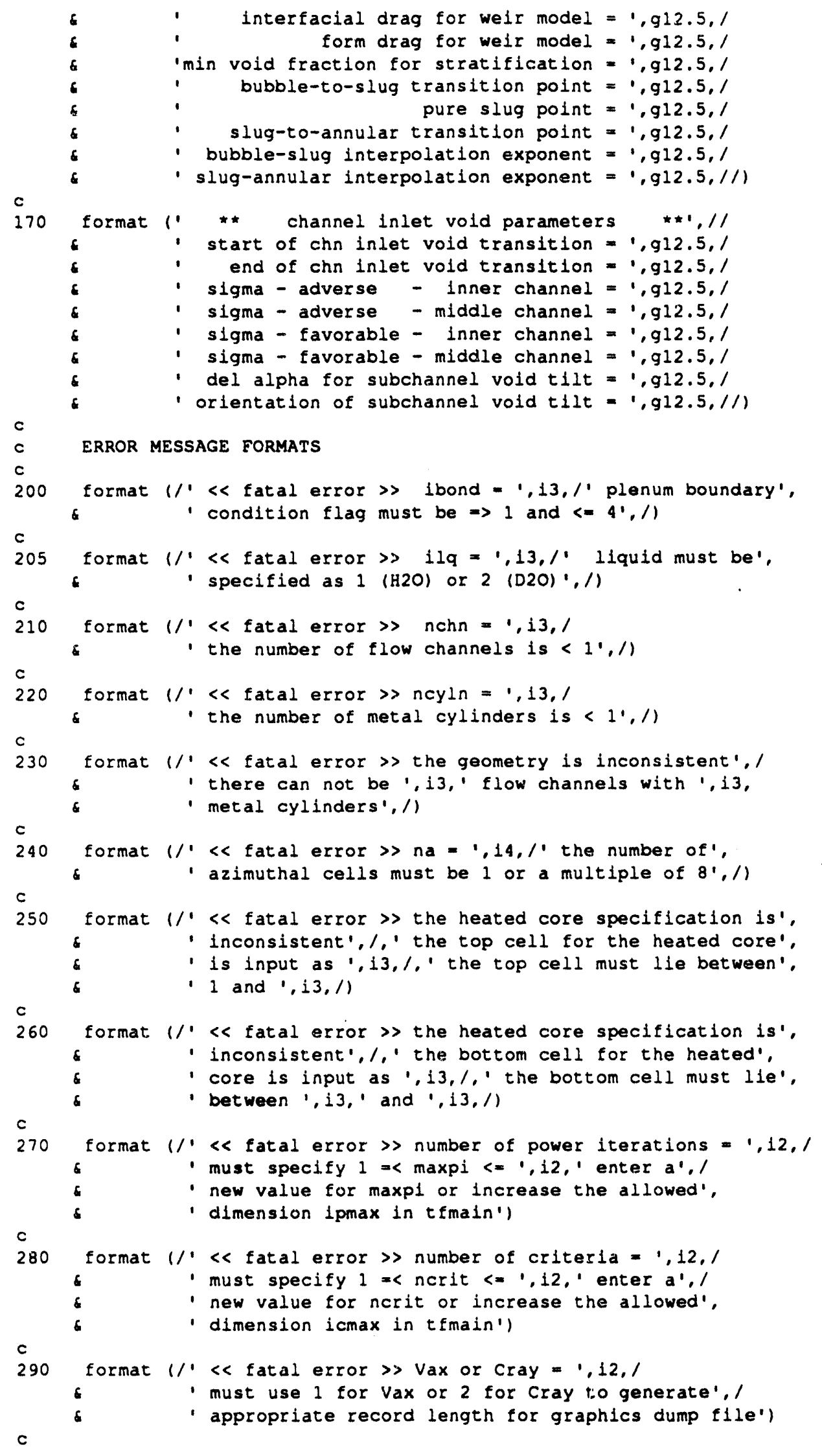




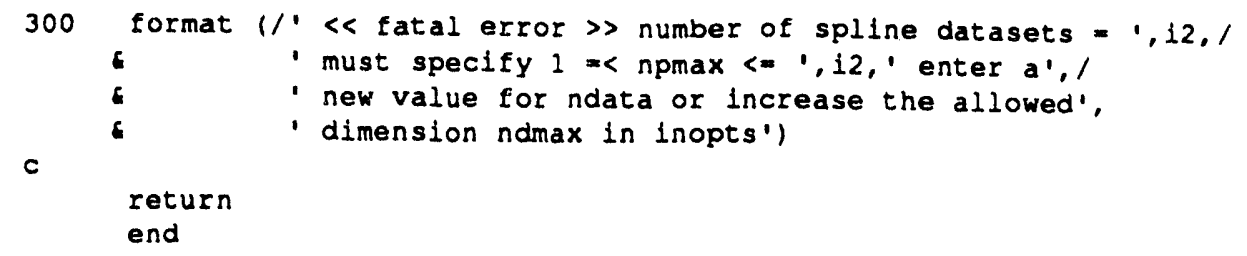

\section{INPOWR}

subroutine inpowr (ninp, nout, na, ncyln, mnrp, nrp, nrmax, nr,
nz, itime, istop, iexp, fazmm, timet, nreg,
nfrst, nlast, nrgtm, deldry, delmod,
ifissn, iaxlp, imodh, idryh, iconh,
pfrct, pfrdd, pfrdw, rphi, rphdd, rphdw)

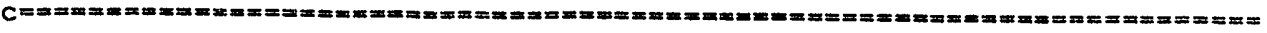

c PROCESS POWER INPUT REQUIRED FOR ONE FLOWTRAN CALCULATION

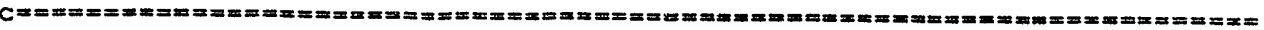

$c$

ARGUMENTS:

I nINP - READ FILE UNIT NUMBER

I nout - WRITE FILE UNIT NUMBER

I na - NUMBER OF AZIMUTHS

I nCYIn - NUMBER OF CYLINDERS

I mIIP - MAX NUMBER OF PONER DATA POINTS IN ANY CYLINDER CORE,

OR NUMBER OF POWER POLYNOMIAL COEFFICIENTS IN CYLINDER

O nIP - NUMBER OF DATA POINTS OR - 1 *NUMBER OF POLYNOMIAL COEFF FOR RADIAL SHAPE FUNCTION IN CYLINDER CORE

I nImaX - MAX NUMBER OF RADIAL NODES IN ANY CYLINDER

I $n I$ - NUMBER OF RADIAL NODES IN CYLINDER

I itime - NO. OF SNAPSHOTS, AXIAL POWER SHAPE

0 istop - 0, OK; >0, TERMINATE PROBLEM AFTER PROCESSING INPUT

O pfrCt - ASSEMBLY REGION POWER FRACTION FOR AT-POWER DATA

O IPhI - CYLINDER CORE RADIAL POWER BY POINT AND CYLINDER, OR BY POLYNOMIAL COEFFICIENT AND CYLINDER, FOR AT-POWER DATA

- fazmm - AZIMUTHAL METAL POWER BY AZIMUTH AND CYLINDER

0 timet - SNAPSHOT TIMES FOR AXIAL POWER DATA

O nreg - NUMBER OF POWER FRACTION REGIONS IN ASSEMBLY

I nfISt - FIRST NODE IN CORE REGION OF CYLINDER

I nlast - LAST NODE IN CORE REGION OF CYLINDER

O nrgtm - POWER FRACTION REGION NUMBER BY NODE AND CYLINDER

O deldrY - DRY TANK FRACTION OF POWER DUE TO DECAY HEAT

O delmod - MODERATED TANK FRACTION OF POWER DUE TO DECAY HEAT

I LeXP - MODE FLAG (0=REACTOR LIMIT, I=EXPERIMENTAL MOCKUP)

O pfrdd - ASSEMBLY REGION DRY DECAY POWER FRACTION

O pfrdw - ASSEMBLY REGION WET DECAY POWER FRACTION

O rphdd - CYLINDER CORE DRY DECAY RADIAL POWER SHAPE

O rphdW - CYLINDER CORE WET DECAY RADIAL POWER SHAPE

Implicit real $(a-h, 0-z)$, integer $(1-n)$

parameter (zero=0.0, one $=1.0$, $\operatorname{maxset}=40$ )

character 80 commnt

C

dimension pfrct (30), rphl(mnrp,ncyln).

\& perdd (30), rphdd (mnrp, ncyln).

c

pfrdw (30), rphdw (mnrp, ncyln)

dimension timet(1time), 1axlp (itime),

- nfrst (ncyln), nlast (ncyln). $\operatorname{nr}(\operatorname{ncyl} n), \quad n \operatorname{rp}($ ncyln)

dimension nrgtm(nrmax, ncyln), fazmm(na, ncy $\ln$ ) 


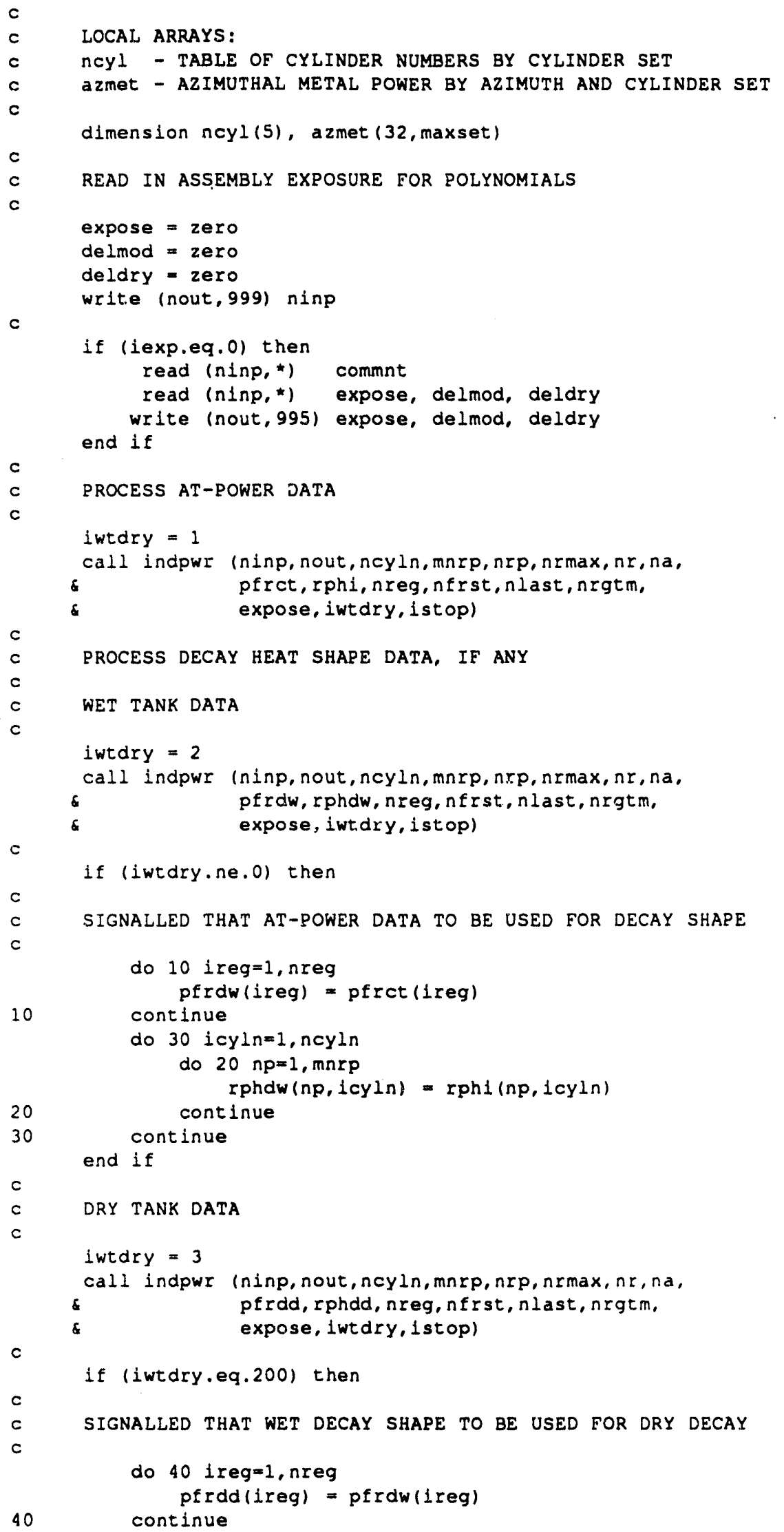


50

60

c

c

$c$

70

$c$

80

90

c

$c$

c

$c$

c

100

1.10

c

c

c

c

$c$ 
do 130 icy $l n=1$, ncyln

iset $=n \operatorname{cyl}(i c y l n)$

$c$

$c$

120

130

c

140

150

160

c

$c$

$c$

C

c

c

c

810

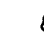

$c$

812 format (" stape data for cylinder ',12,' is in set " 12)

c

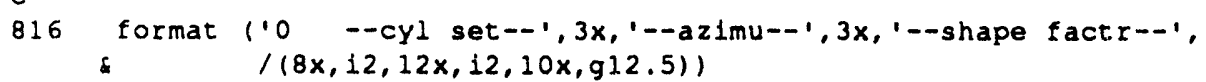




\begin{tabular}{|l|ll|l|}
\hline $2 / 15 / 93$ & WSRC-TR-93-086 Rev. 0 & FLOWTRAN-TF v1.2 Source Code & Pg. 193 of 354 \\
\hline
\end{tabular}

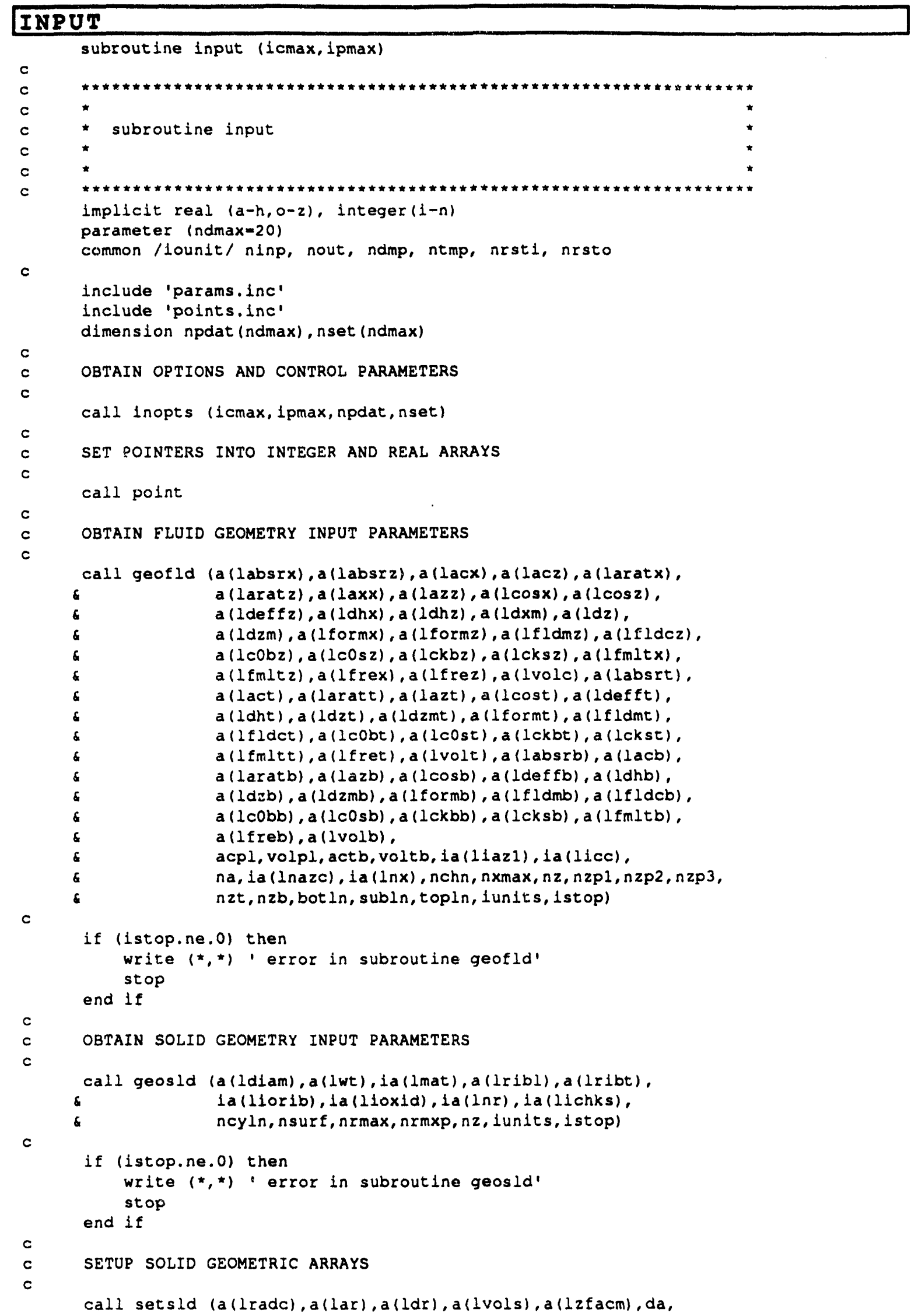




$$
\begin{aligned}
& \text { c a (ldiam), } a(1 d z+1), a(1 \mathrm{rdz}) \text {, hactiv, } \\
& \text { la (lmat), 1a (110x1d), 1a (lnfrst), 1a (1nlast), } \\
& \text { la (lnr), nz, na, nrmax, n rmxp, ncy ln, 1zht, 1 zhb, } \\
& \text { icent, Iradbc, douts, emis1, emiso, crad1) }
\end{aligned}
$$

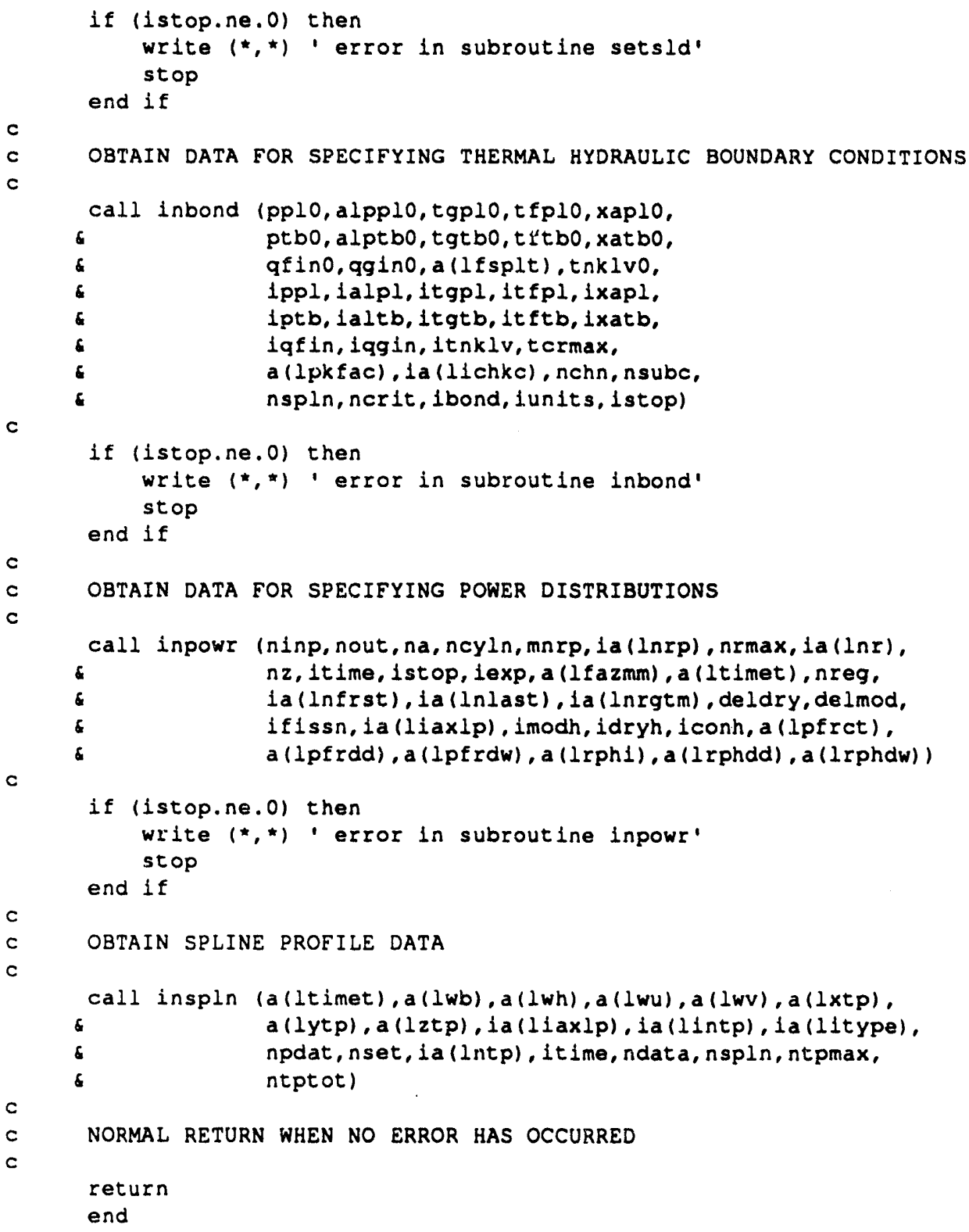

\section{INSPLN}

subroutine inspln (timet, wb, wh, wu, wv, xtp,ytp, ztp, iaxlp, intp,

- itype, npdat, nset, ntp, itime, ndata, nspln, ntpmax. \& ntptot)

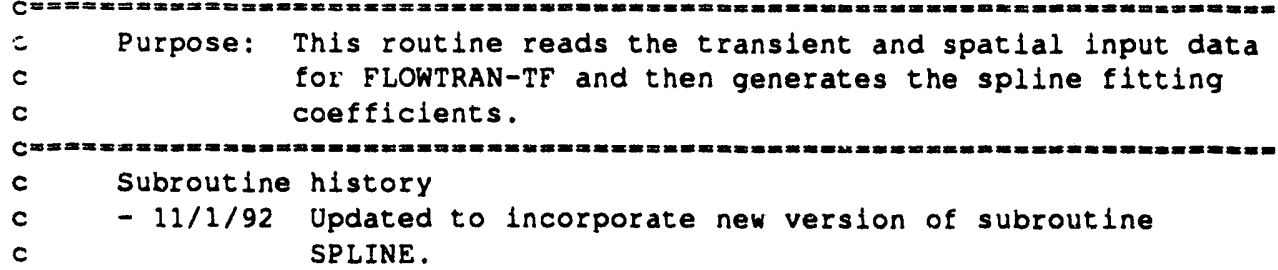




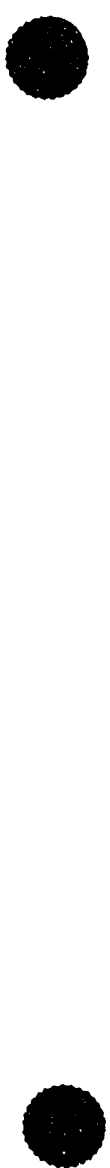

Arguments

\begin{tabular}{|c|c|c|c|c|}
\hline NAME & $I / 0$ & TYPE & DIM & DESCRIPTION \\
\hline$--\infty-\infty-\infty$ & $-\infty-$ & --- & ---- & 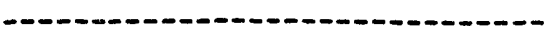 \\
\hline timet & $\mathbf{I}$ & $R \star 4$ & it ime & $\begin{array}{l}\text { Snapshot times of axial power } \\
\text { shapes }\end{array}$ \\
\hline wh, wb, wu, wv & I & $R \star 4$ & ntpmax & Intermediate working arrays \\
\hline laxlp & I & $I * 4$ & it ime & Polnters to axial power shapes \\
\hline 1type & I & $I \star 4$ & nspln & $\begin{array}{l}\text { Identifier of type of spline } \\
=0 \text { (Natural cubic spline) } \\
=1 \text { (First-degree spline) }\end{array}$ \\
\hline npdat & I & $I \star 4$ & ndata & Number of points per dataset \\
\hline nset & I & $I \star 4$ & ndata & Number of splines per dataset \\
\hline 1.time & I & $I * 4$ & & $\begin{array}{l}\text { Number of snapshots for axial } \\
\text { power shape }\end{array}$ \\
\hline ndata & I & $I * 4$ & & Number of spline datasets \\
\hline nspln & I & $I \star 4$ & & Number of splines \\
\hline ntpmax & I & $I \star 4$ & & $\begin{array}{l}\text { Maximum number of points per } \\
\text { spline }\end{array}$ \\
\hline ntptot & I & $I \star 4$ & & Total number of data points \\
\hline$x \in p$ & 0 & $R * 4$ & ntptot & Abscissa values or knots \\
\hline$y \in p$ & 0 & $R \star 4$ & $\begin{array}{l}\text { (ntpmax, } \\
\text { nspln) }\end{array}$ & Ordinate values \\
\hline$z t p$ & 0 & $R \star 4$ & $\begin{array}{l}\text { (ntpmax, } \\
\text { nspln) }\end{array}$ & $\begin{array}{l}\text { Slopes of first-degree splines } \\
\text { or second derivatives of } \\
\text { natural cubic splines at the } \\
\text { knots }\end{array}$ \\
\hline intp & 0 & $I \star 4$ & nspln & $\begin{array}{l}\text { Pointers to start of spline } \\
\text { data }\end{array}$ \\
\hline ntp & 0 & $I * 4$ & nspln & Number of points per spline \\
\hline
\end{tabular}

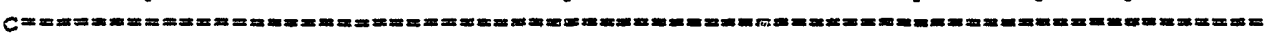

implicit real $(a-h, 0-z)$, integer $(i-n)$

character $\star 80$ commnt, label

character ${ }^{*}$ xvalu

common /iounit/ ninp, nout, ndmp, ntmp, nrsti, nrsto

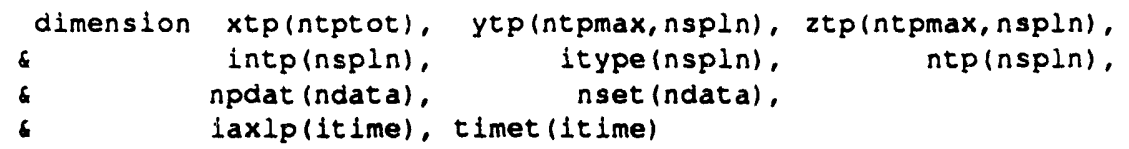




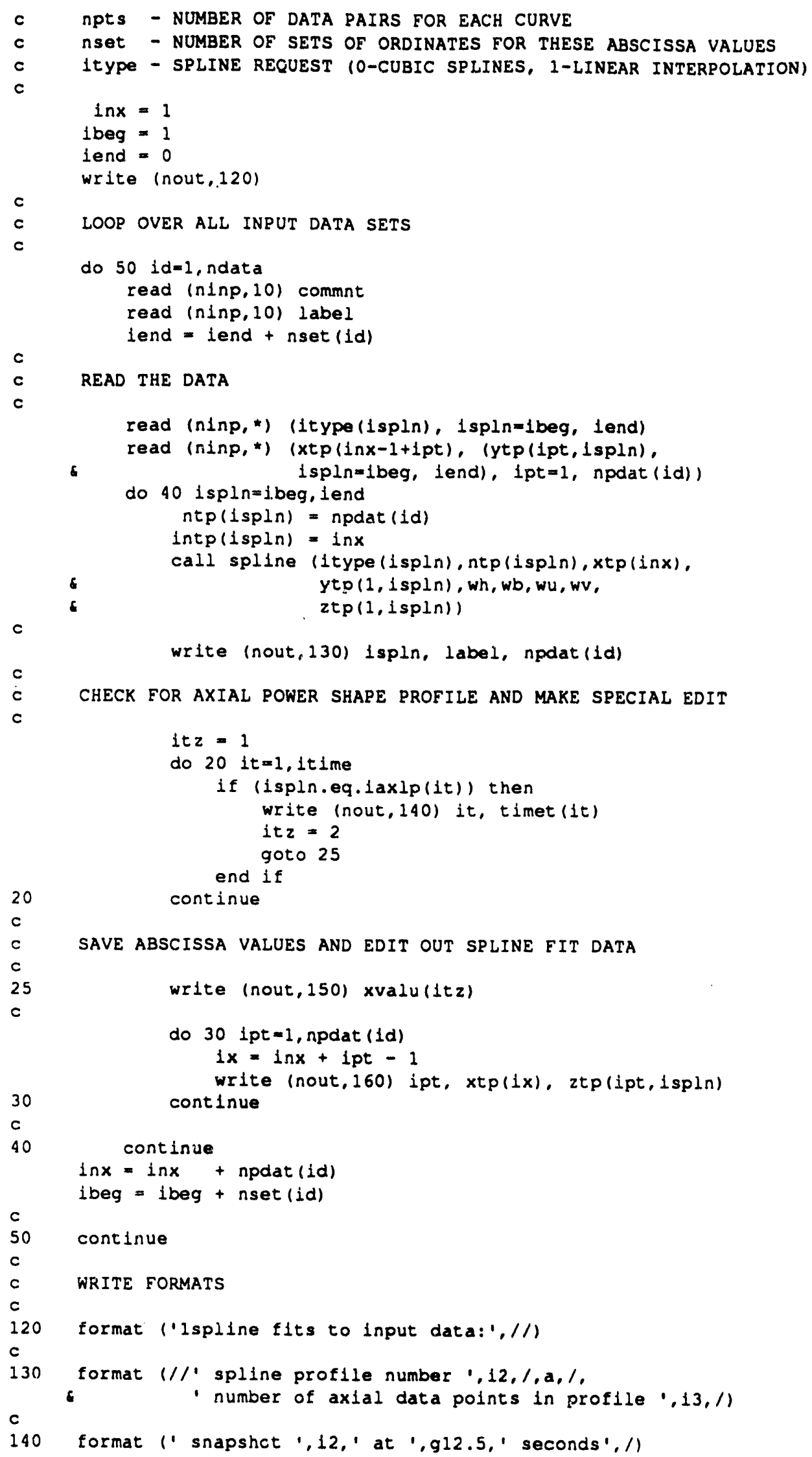




\begin{tabular}{|l|ll|l|}
\hline $2 / 15 / 93$ & WSRC-TR-93-086 Rev. 0 & FLOWTRAN-TF v1.2 Source Code & Pg. 197 of 354 \\
\hline
\end{tabular}

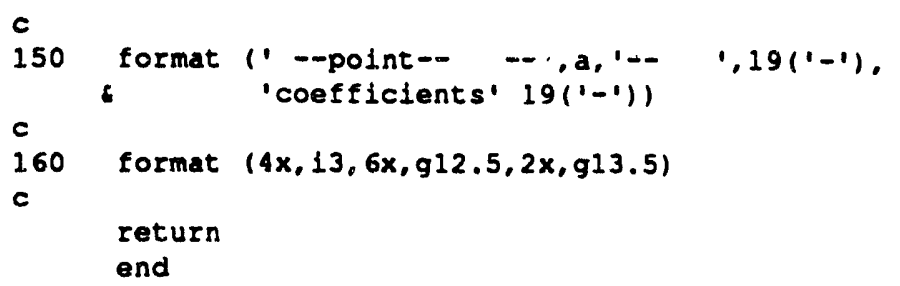

\section{INTARA}

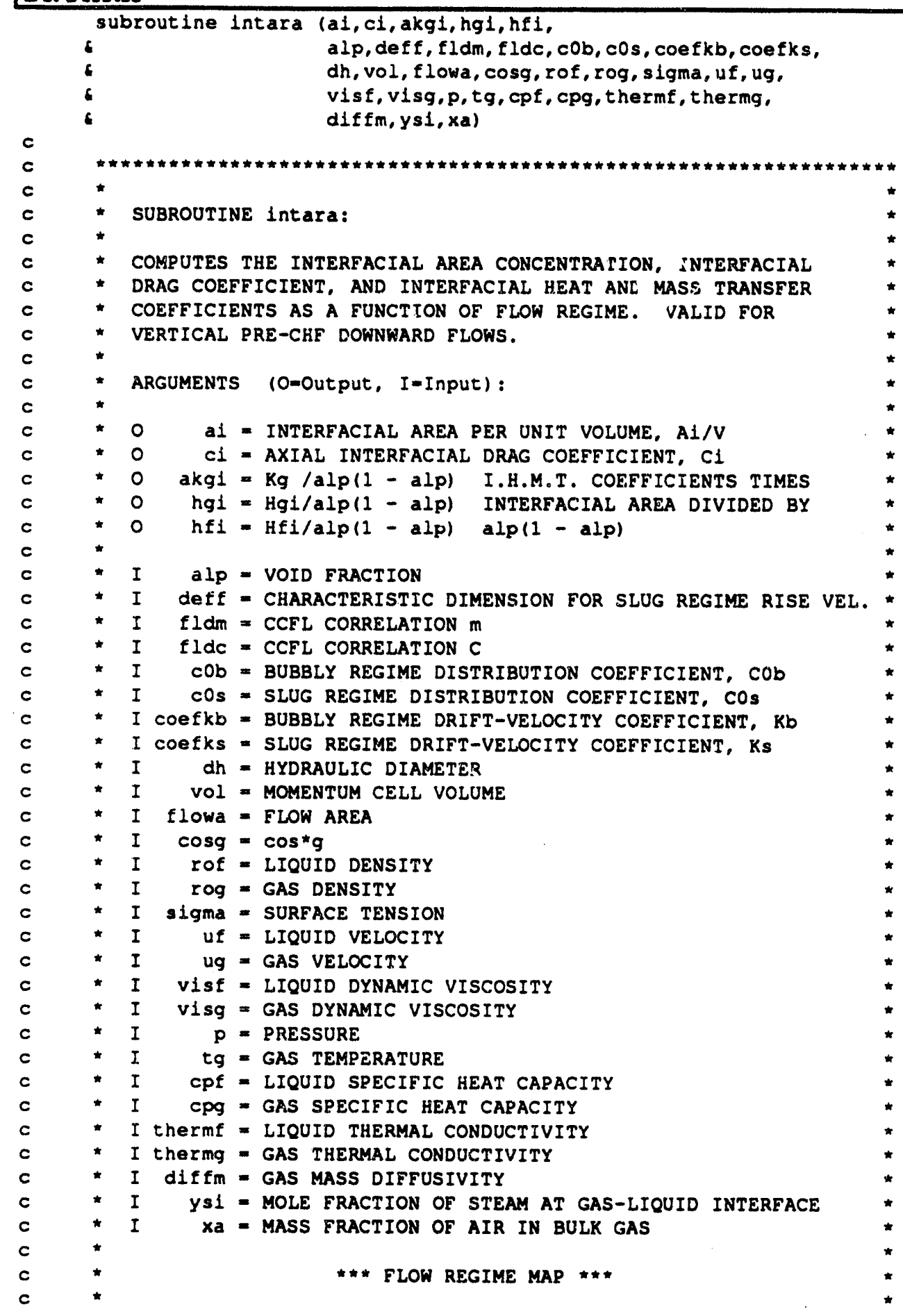




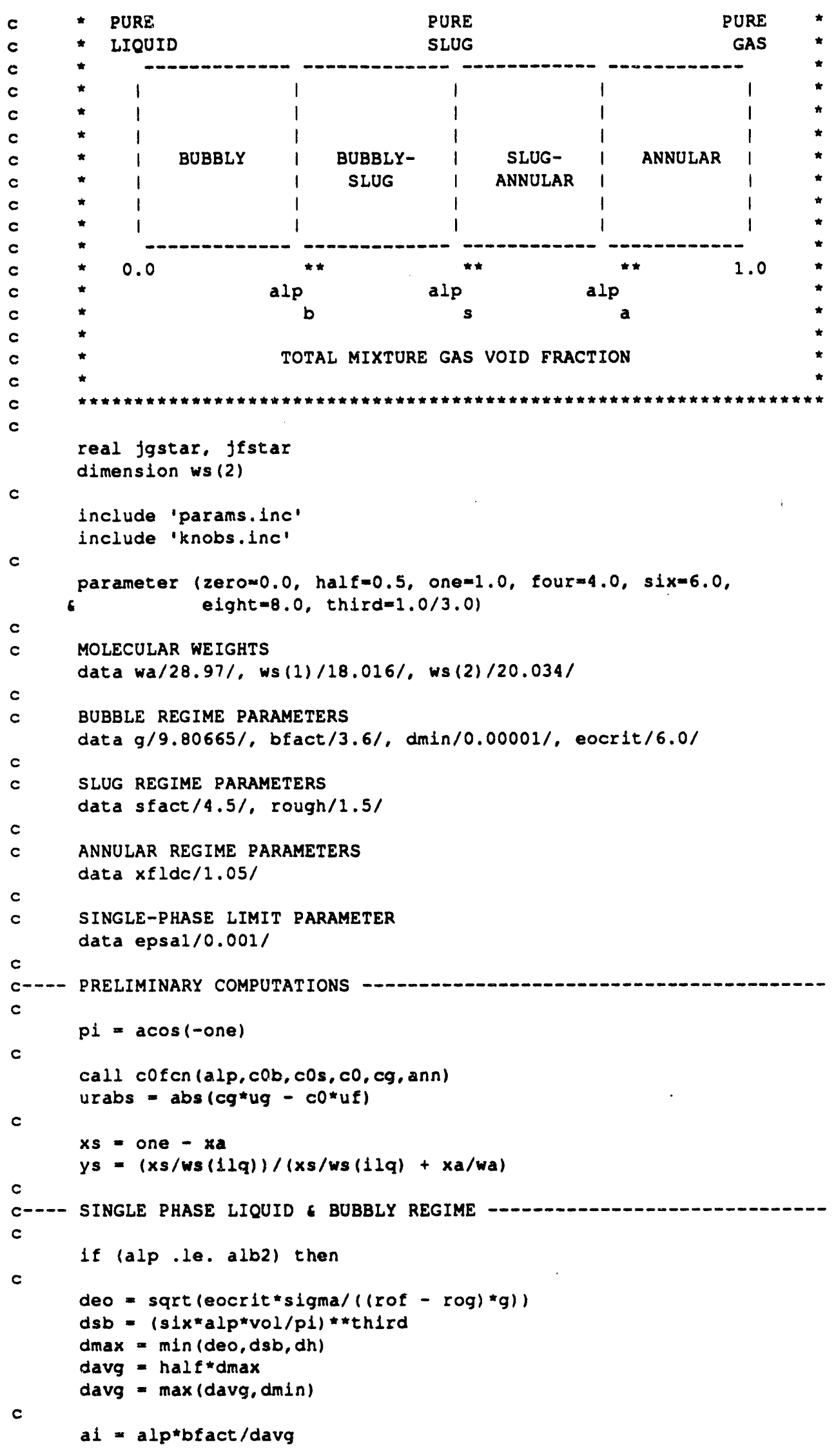


$c$

$f r b=(b f a c t / d a v g) /(o n e-a l p)$

$c 1=\max (e p s a l, a l p) \star(o n e-a l p) \star * 3 *$ rof $/$ coef $k b * * 2$

c

* *sqrt ((rof - rog)*g/sigma)

call hfib (uf, ug, rof, thermf, cpf, davg, hfb, visf, visg)

$h f 1=f r b * h f b$

$c$

call hgib (davg, rcg,p,tg, thermg, diffm, cpg, hgb, akgb)

hgl $=$ frb*hgb

$a k g i=f r b \star a k g b$

c

C--- BUBBLY \& SLUG REGIME -

c

c

else if (alp. le. als2) then

deo $=\operatorname{sqrt}($ eocrit*sigma/((rof - rog $) * g))$

$d s b=\left(s i x{ }^{*} a l p * v o l / p i\right) *$ third

$d \max =\min (d e o, d s b, d h)$

davg = half*dmax

davg $=\max ($ davg, $\operatorname{dm} i n)$

c

$a 1 b 1=a 1 b 2 *((a) s 2-a l p) /(a 1 s 2-a l b 2)) * \star$ expbs

als $=(a l p-a l b l) /($ one $-a l b)$

c

$a l b=a l p-a l s$

$a i b=a l b * b f a c t / d a v g$

als = als*sfact*rough/dh

$a i=a i b+a i s$

$f r b=a l b /(a l p *(o n e-a l p))$

$f r s=a i s /(a l p *(o n e-a l p))$

c

$c i=a l b *($ one $-a l p) * \star 3 * \operatorname{rof} / \operatorname{coef} k b * * 2 * s q r t((r \circ f-\operatorname{rog}) * g / s i g m a)$

c

\& $+a l s *(o n e-a l p) * \star 3 * \operatorname{rof} /(\operatorname{coefks} * \star 2 * \operatorname{def} f)$

call hfib (uf,ug, rof, thermf, cpf, davg, hfb, visf,visg)

call hfis (visf, rof, thermf, cpf, dh, hfs)

$c$

$h f 1=f r b \star h f b+f r s{ }^{*} f s$

call hgib (davg, rog, p, tg, thermg, diffm, cpg, hgb, akgb)

call hgis ( $p, t g, u f, u g$, rog, thermg, visg, cpg, diffm, dh, hgs, akgs)

$h g i=f r b h^{\star} g b+f r s^{\star} h g s$

$a k g i=f r b b^{\star} a k g b+f r s^{\star} a k g s$

$c$

C---- SLUG \& ANNULAR REGIME

c

$c$

else if (alp.le. alaz) then

ala1 $=$ alaz*((alp - als2) $/($ alaz - als2 $))^{* * e x p s a}$

als = (alp - alal)/(one - alal)

c

ala = alp - als

ais $=$ als*sfact*rough/dh

pw = four flowa/dh

del $=($ one $-a l a) * f l o w a /$ pw

pia = pw - eight*del

ala $=a l a * p l a /(f l o w a * a l p)$

frs $=a i s /(a l p *(o n e-a l p))$

$f r a=a i a /(a l p *(o n e-a l p))$

$a i=a i s+a i a$

c

$c 1=a l s *$ ione - alp)**3*rof/(coefks**2*deff)

c

- + cla(aia,alp,del, flowa, pla, rog, urabs, visg) 


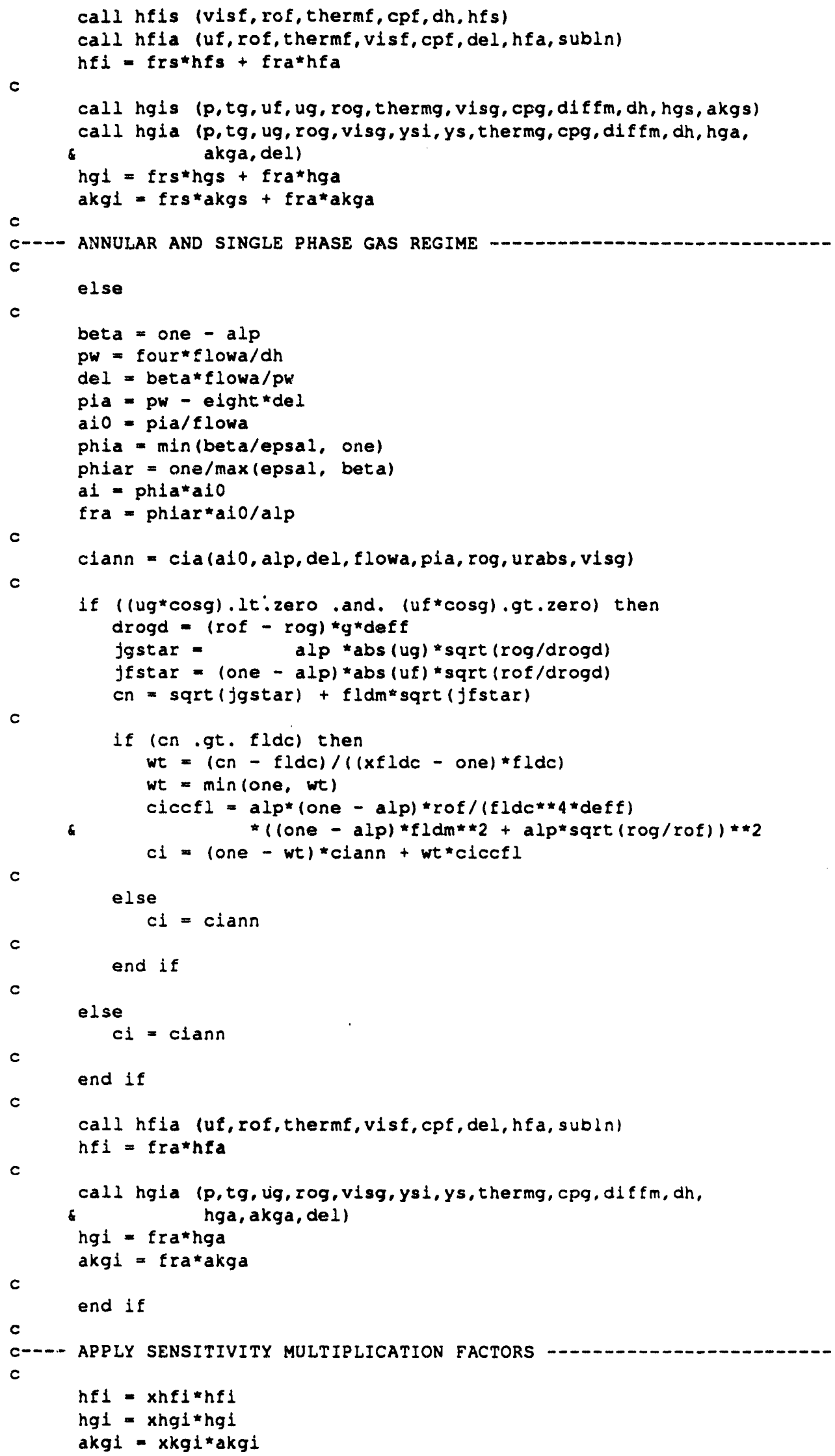


c

return

end

\section{JACOBB}

subrout ine jacobb(pbn, alpbn, tgbn, tfbn, xabn, rogbn, rofbn, hgbn, hfbn,

6

c

c.

$c$

$c$

$c$

$c$

$c$

c

c

c

$c$

$c$

c

$c$

$c$

c

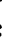

cvax

$c$

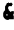

4

6

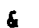

cpt f

cpxa

cpab

cptgb

cpt fb

cpxab

$c$

dimension

- gib

- heib

akgib

hgb

heb

hgbn

$h \in b n$

$n \times$

$\mathrm{pb}$

pbn

$p$

rhsb

dvbz

vc2 $\mathrm{pb}, \mathrm{alpb}, \mathrm{tgb}, \mathrm{tb}, x a b$, rogb, rofb, hgb, hfb ,

gib,eib, hfib, hgib, akgib, ysib.

ptb, p, rdelt, factor, abm1, rhsb, azml.

cpab, cptgb, cptfb, cpxab, fab, ftgb, ftfb, fxab,

cpa , cptg, cptf , cpxa .

volb, advbb, advcb, advbz, advcz,

xa0, epsy, epsf, nitysi.

nzb, nzp2, nzp3, ncĭn, nxmax, nx, Igami, ierr, 1lq)

SUBROUTINE JACOBB

is routine computes the jacobian matrix at the current

rate $m$ for the bottom section of a three-section reactor

The mathematical and coupling considerations

LOWTRAN_TF manual and the FORTRAN of JACOBM should be

This subroutine is compatible only with the FLOWTRAN_TF

rogram and the tank bottom section of a three-section

actor-assembly simulation. (nzp3, nxmax, nchn, 5), (4, nzp2, nxmax, nchn), (4, nzp2, nxmax, nchn), (4, nzp2, nxmax, nchn), (4, nzp2, nxmax, nchn). $(2, n z b)$ 。

$(2, n z b)$.

$(2, n 2 b)$,

$(2, n z b)$,

$\begin{array}{ll}\text { abml } & (n z b, 3), \\ \text { azml } & (n z p 2, n x m a x, n c h n, 3), \\ \text { alpb } & (n z b), \\ \text { alpbn } & (n z b), \\ \text { advbb } & (n z b, 5), \\ \text { advcb } & (n z b, 5), \\ \text { fab } & (n z b), \\ \text { ftgb } & (n z b), \\ \text { ft } f b & (n z b), \\ \text { fxab } & (n z b) \\ & \\ \text { eib } & (n z b), \\ \text { hgib } & (n z b), \\ \text { ysib } & (n z b), \\ \text { rofb } & (n z b), \\ \text { rogbn } & (n z b), \\ \text { rofbn } & (n z b), \\ \text { tfbn } & (n z b), \\ \text { tgbn } & (n z b), \\ \text { tfb } & (n z b), \\ \text { tgb } & (n z b), \\ \text { xab } & (n z b), \\ \text { xabn } & (n z b), \\ & \end{array}$


- rogb (nzb), volb (nzb)

c

c

c

10 continue

Update densities and enthalples as well as thelr derivatives.

call state ( $p b, t g b, t f b, x a b$, factor, $11 q$,

$\begin{array}{ll}\text { \& } & \text { rogb, drgdp, drgdtg, drgdxa, hgb, dhgdp, dhgdtg, dhgdxa, } \\ \text { rofb, drfdp, drfdtf, hfb, dhfdp, dhfdtf) }\end{array}$

c

c

Loop over all axial mass/energy cells within bottom section.

do 80 ic $=1, \mathrm{nzb}$

$\mathrm{jcm}=\mathrm{Jc}-1$

$j c p=j c+1$

$j z=j c$

$j z m=j c m$

Set the number of columns in the [ca] and [cp] matrices.

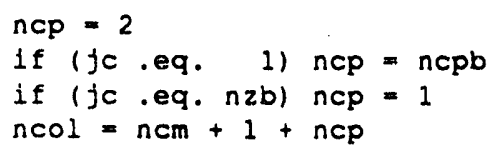


c
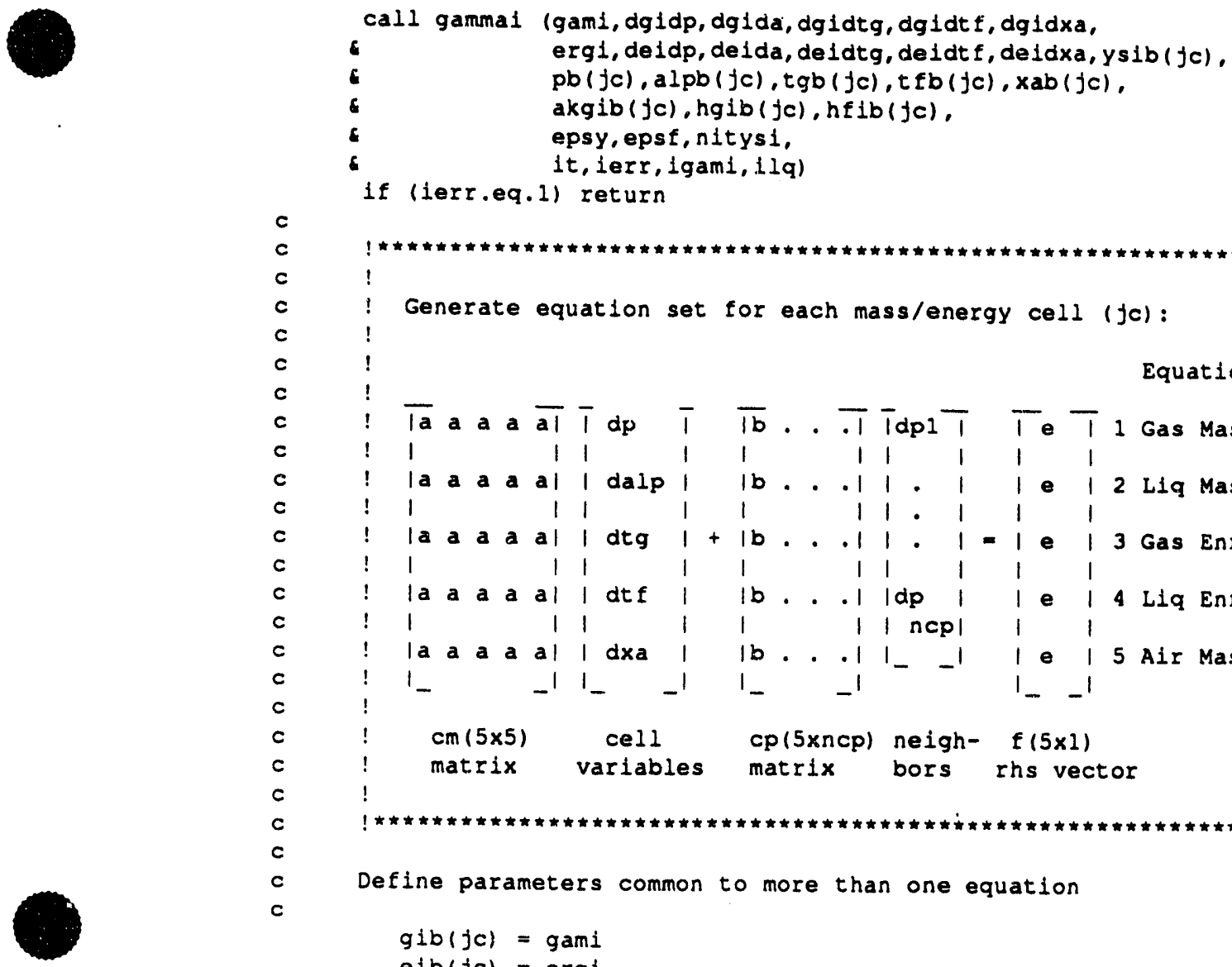

$c$

c

!

Generate equation set for each mass/energy cell (fc): Equation 1 la a a a al | dalp 1 1

c

$r f n=\operatorname{rofbn}(j c)$

$r f m=r o f b(j c)$

$x n=x a b n(f c)$

$x m=x a b(j c)$

c

$d p j p=p r m-p t b$

if $(j c . l t . n z b) d p j p=p r m-p b(j c p)$

if (jc.ne. 1) $d p j m=p b(j c m)-p r m$ 


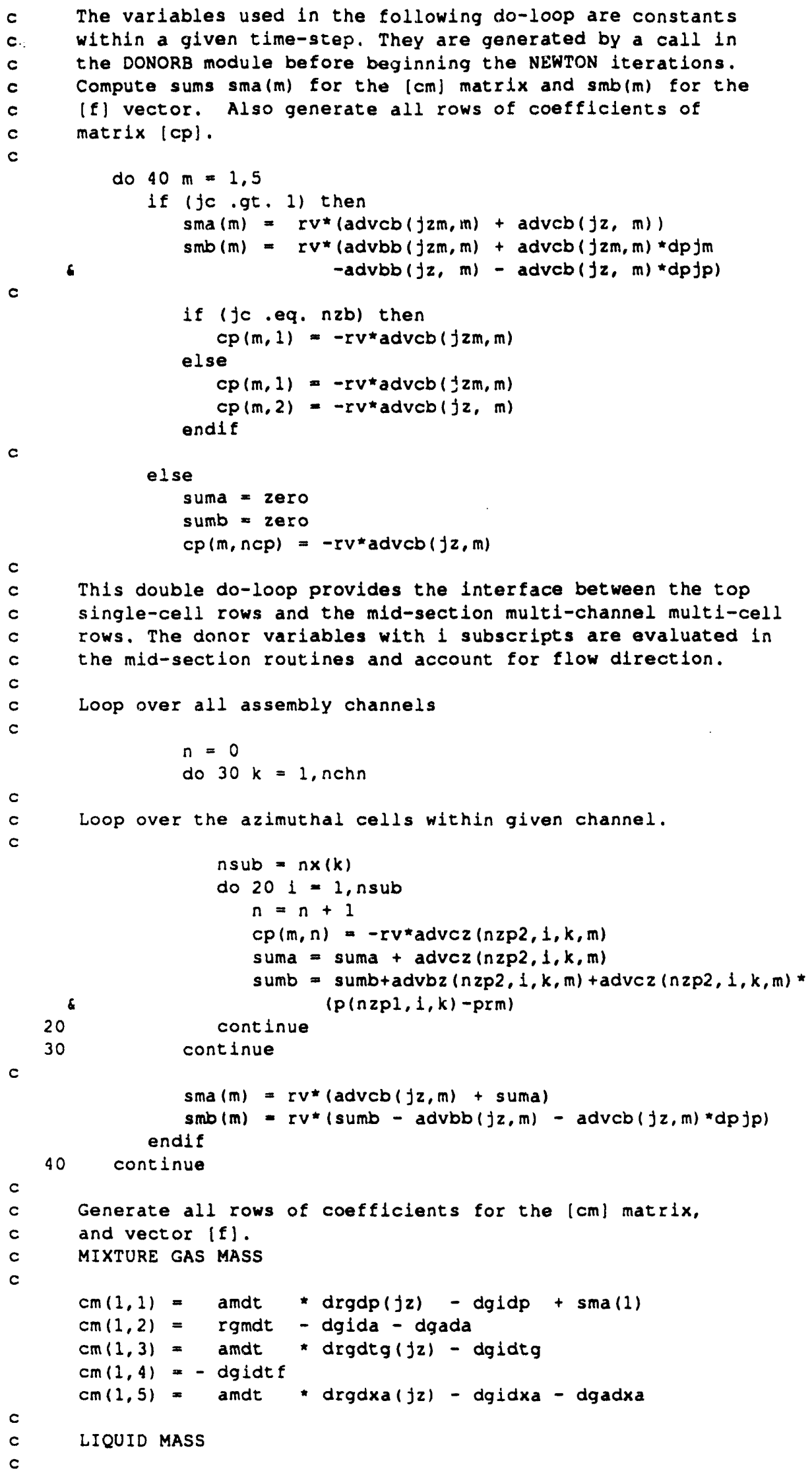


c

$\mathbf{c}$

c

c

c

c

C

c

c

c

c

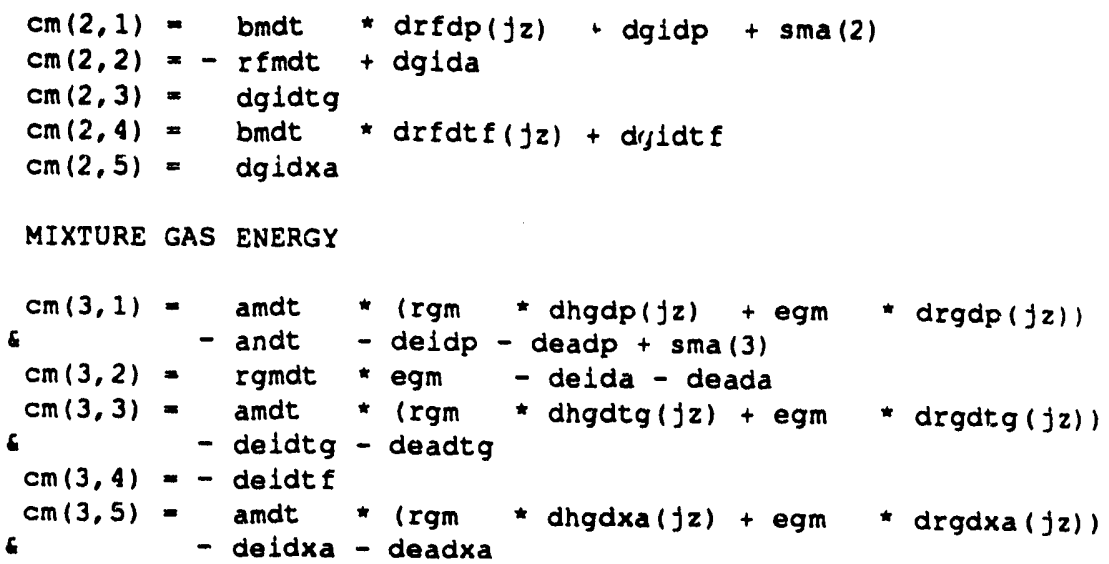


c

c

c

c

c

c

c

c

$c$

c

c

$c$

c

c

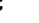

ddom $=\operatorname{abs}(c p(1,1))$

if (ddom .ge. one) go to 90

$\operatorname{abml}(J c, 1)=$ one

$\operatorname{abml}(j c, 2)=$ zero

$\operatorname{abml}(J c, 3)=c p(1,1)$

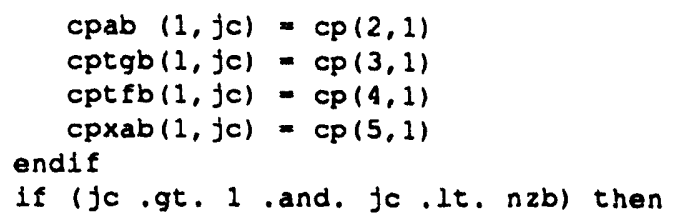




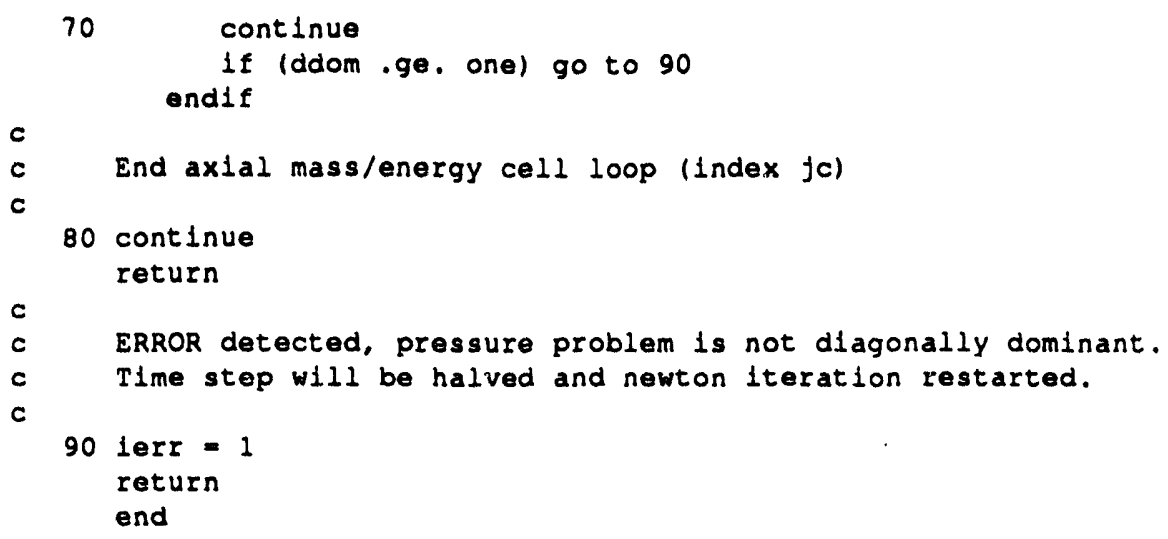

\section{JACOBX}

subrout ine facobm(pn, alpn, tgn, tf $n, x a n$, rogn, rof $n$, hgn, hf $n$,

c $\quad p$,alp, tg,tf ,xa , rog, rof , hg , hf ,

a gi,ei, hfl,hgi akgi,ysi,gw, hspl,

rdelt, factor,

azm1, azm2, rhs, qwg, qwf, tsurf, ar,

cpa, cptg, cptf, cpxa, fa, ftg, ftf, fxa,

volc, advbz, advcz, advdx, advex, txg, txf,

xao, epsy, epsf, nitysi,

iazl, nazc, na, napl, nr, nrmxp, nz, nsurf, icent, $n z p 1, n z p 2, n z p 3, n c h n, n x \max , n x, n c y l n, 1 c c, 1 g a m 1$. igamw, ierr,11q)

This routine computes the facobian matrix at the current iterate $m$. It is obtained by linearizing the densities and internal energies vs temperatures, pressure, and air mass fraction in the mixture gas, liquid, and air mass equations, and mixture gas and liquid energy equations. It is assumed that phase velocities are known linear functions of their appropriate pressure driving forces. Upon substitutions to eliminate phase velocities, a pressure only poisson equation is obtained.

This subroutine is compatible only with the FLOWTRAN TF program and the mid_section (core) of a three-section reactor-assembly modelel.

$!$

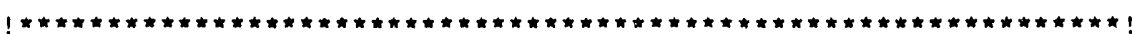

cvax double prectsion $f, c a, c m, c p$

dimension $f(5), c a(5,26), \mathrm{cm}(5,5), \mathrm{cp}(5,20)$

parameter $(z e r o=0.0$, one $=1.0)$

ARGUMENT ARRAYS

dimension

\begin{tabular}{|c|c|c|c|c|}
\hline \\
\hline c & a $2 \mathrm{ml}$ & $(n z p 2, n x \max , n c h n, 3)$, & hgn & $(n z p 2, n x \max , n c h n)$, \\
\hline \$ & azm2 & $(n z p 2, n x \max , n c h n, 2)$, & $n \in n$ & $(n z p 2, n x \max , n c h n)$, \\
\hline - & alp & (nzp2,nxmax, nchn), & $i c c$ & $(2, n x \max , n c h n)$ \\
\hline \& & alpn & $(n z p 2, n \times \max , n c h n)$, & $\mathbf{p}$ & $(n z p 2, n \times \max , n c h n)$. \\
\hline$\leftarrow$ & $a d v b z$ & $(n z p 3, n x \max , n c h n, 5)$, & pn & $(n z p 2, n x \max , n c h n)$, \\
\hline 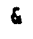 & advcz & (nzp3, nxmax, nchn, 5), & rhs & $(n z p 2, n x \max , n c h n)$, \\
\hline & $a d v d x$ & $(n \geq p 2, n \times \max , n c h n, 5)$, & rog & $(n z p 2, n \times \max , n c h n)$, \\
\hline
\end{tabular}




$\begin{array}{ll}\text { advex } \\ \text { cpa } \\ \text { cptg } \\ \text { cptf } \\ \text { cpxa } \\ \text { f } & \text { ftg } \\ \text { f } & \text { ftf } \\ & \text { fxa } \\ & \text { hg }\end{array}$

\section{dimension}

- 91

c hfi

a akg1

- gw

- hspl

- qwg

- lazl

c nr$$
\text { an }
$$

dimension $\operatorname{txg}(4, n z p 2, n x \max , n c h n), \operatorname{txf}(4, n z p 2, n \times m a x, n c h n)$

c

c

c

INTERNAL ARRAYS

dimension sma (5), smb(5)

parameter ( $\mathrm{mxnz}=100$ )

dimension drgdp (mxnz), drgdtg(mxnz), drgdxa(mxnz), 6

6

6 dhgdp (mxnz), dhgdtg(mxnz), dhgdxa(mxnz). $d r f d p(m \times n z)$, drfdtf $(m \times n z)$, dhfdp (mxnz), dhfdt $f(m \times n z)$

c

$$
\text { \& }
$$

equivalence

(nzp2, nxmax, nchn) , (nzp2, nxmax, nchn). (nzo2, nxmax, nchn). (nsurf, na, nz). (nsurf, na, nz), (nxmax, nchn).

rof rogn rofn

$t \in n$

$\operatorname{tgn}$

$t f$

tg

volc

xa

xan

$\mathrm{nx}$

(nzp2, nxmax, nchn), (nzp2, nxmax, nchn), (nzp2, nxmax, nchn) . (nzp2, nxmax, nchn). (nzp2, nxmax, nchn). (nzp2, nxmax, nchn). (nzp2, nxmax, nchn). (nzp2, nxmax, nchn). (nzp2, nxmax, nchn), (nzp2, nxmax, nchn) . (nchn)

e1 (nzp2, nxmax, nchn). hg1 (nzp2, nxmax, nchn). ysi (nzp2, nxmax, nchn). ar (nrmxp,nz, ncyln). tsurf (nsurf, na,nz), qwe (nsurf,na,nz). nazc (nxmax, nchn). c

c

c

c

c

c

c

$\operatorname{data} g a m w / 0.0 /$, ergw/0.0/

SET FIXED NUMBER OF COLUMNS IN [Ca], [cm], AND [CP] MATRICES

$$
\begin{aligned}
& \text { ncm }=5 \\
& \text { ncp }=4 \\
& \text { ncol }=n c m+1+n c p
\end{aligned}
$$

LOOP OVER EACH FLOW ANNULUS (CHANNEL) WITHIN ASSEMBLY (CORE)

do $60 \mathrm{k}=1, \mathrm{nchn}$

isrfi=2*k-1+1cent

isto = Iseft +1

icyli = Iste $1-k$

1 icylo = isto $-k$

LOOP OVER ALL AZIMUTHAL MASS/ENERGY SECTORS WITHIN 2D CHANNEL

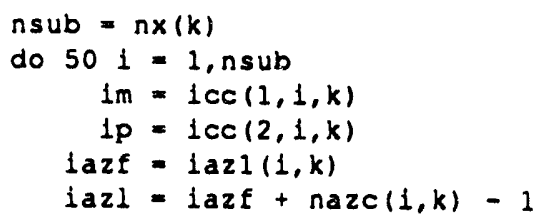

UPDATE DENSITIES AND ENTHALPIES AS WELL AS THEIR DERIVATIVES 


\begin{tabular}{|l|lll|l|}
\hline $2 / 15 / 93$ & WSRC-TR-93-086 Rev.0 & FLOWTRAN-TF v1.2 Source Code & Pg. 209 of 354 \\
\hline
\end{tabular}

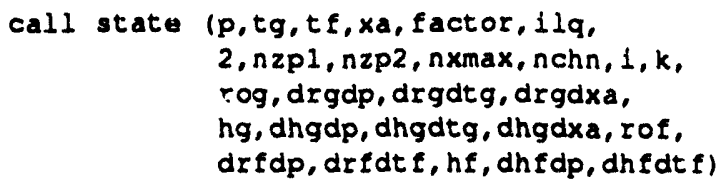

c

c

c

c

c

c

c

C

$c$

c

SET INTEREACIAL MASS SOURCE/SINK TERMS AT THE WALL

If (lgamw eq. 1) then

gamw $=g w(j c, 1, k) \pitchfork v v$

if (gamw.gt.zero) then

call hssat ( $(j c, 1, k), 11 q$, hvap, dum)

ergw = gamw*hvap

else if (gamw.It.zero) then

call hfsat (tf (jc, $1, k), 1 l q, h l i q, d u m$ )

else

ergw $=g a m w \star h l 1 q$

ergw $=$ zero

end $1 f$

end if

c

COMPUTE HEAT TRANSFER FROM INNER AND OUTER WALLS TO LIQUID AND GAS 
c

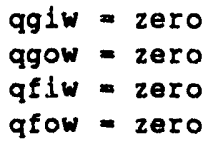

c

10

c

c

c

c

$c$

c

c

c

c

c

c

c

$c$

c

c

c

c

c

c

C

c

c

$c$

c

c

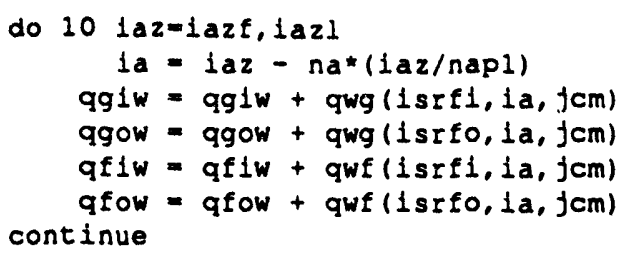
continue

DEFINE PARAMETERS COMMON TO MORE THAN ONE EQUATION

$g i(j c, 1, k)=\operatorname{gam} 1$

ei $(j c, i, k)=\operatorname{erg} 1$

andt $=\operatorname{alpn}(j c, i, k) * \operatorname{rdelt}$

andt $=\operatorname{alp}(j c, 1, k) *$ rdelt

bndt $=($ one $-\operatorname{alpn}(j c, i, k)) *$ rde $l t$

bmdt $=$ (one - alp $(j c, i, k)) *$ rdelt

$$
\begin{array}{ll}
\text { ggn } & =h g n(j c, i, k) \\
\text { egm } & =h g(j c, i, k)
\end{array}
$$$$
\text { efn } \quad=h f n(j c, 1, k)
$$$$
\text { efm }=h f(j c, 1, k)
$$

$\operatorname{gtxf}=\operatorname{txf}(1, j c, 1, k)$

$g t \times g=t \times g(1, j c, 1, k)$

$\operatorname{prn} \quad=\operatorname{pn}(j c, 1, k)$

prm $=p(j c, i, k)$

$q t \times f=t \times f(2, j c, i, k)$

qtxg $=\operatorname{txg}(2, j c, i, k)$

$\operatorname{rgn} \quad=\operatorname{rogn}(j c, 1, k)$ 


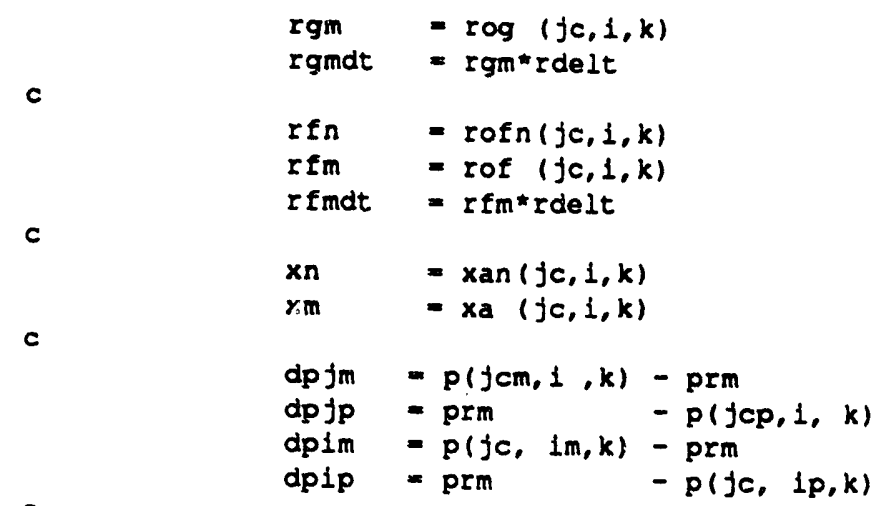

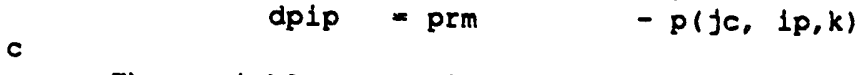

c The variables used in the following do-loop are constants c within a given time-step. They are generated by a call to c the DONORM module before beginning the NEWTON 1terations. c Compute sums sma(m) for the [cm] matrix and $s m b(\mathrm{~m})$ for the c [f] vector. Also generate all rows of coefficients of c maLrix [CP]. c do $20 \mathrm{~m}=1,5$

5

$c$

6

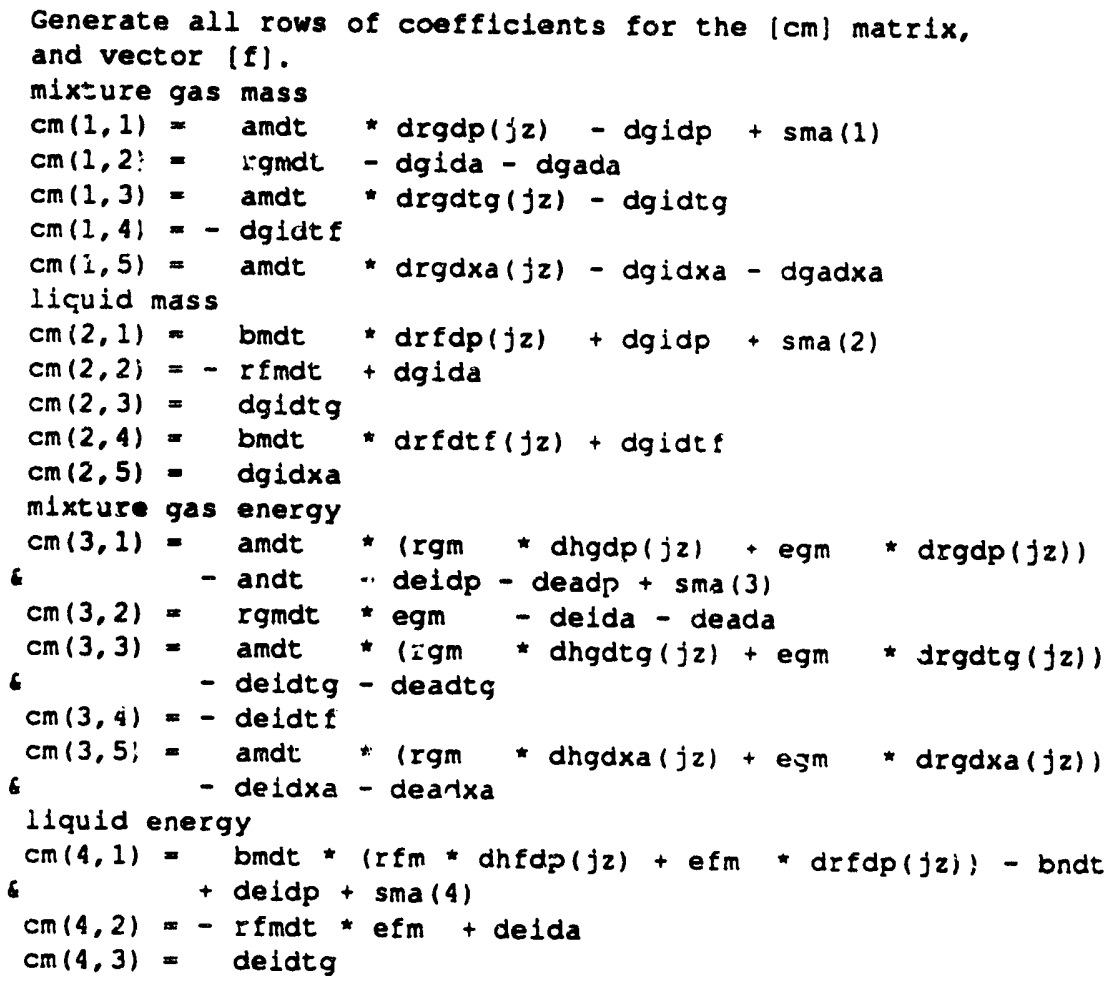




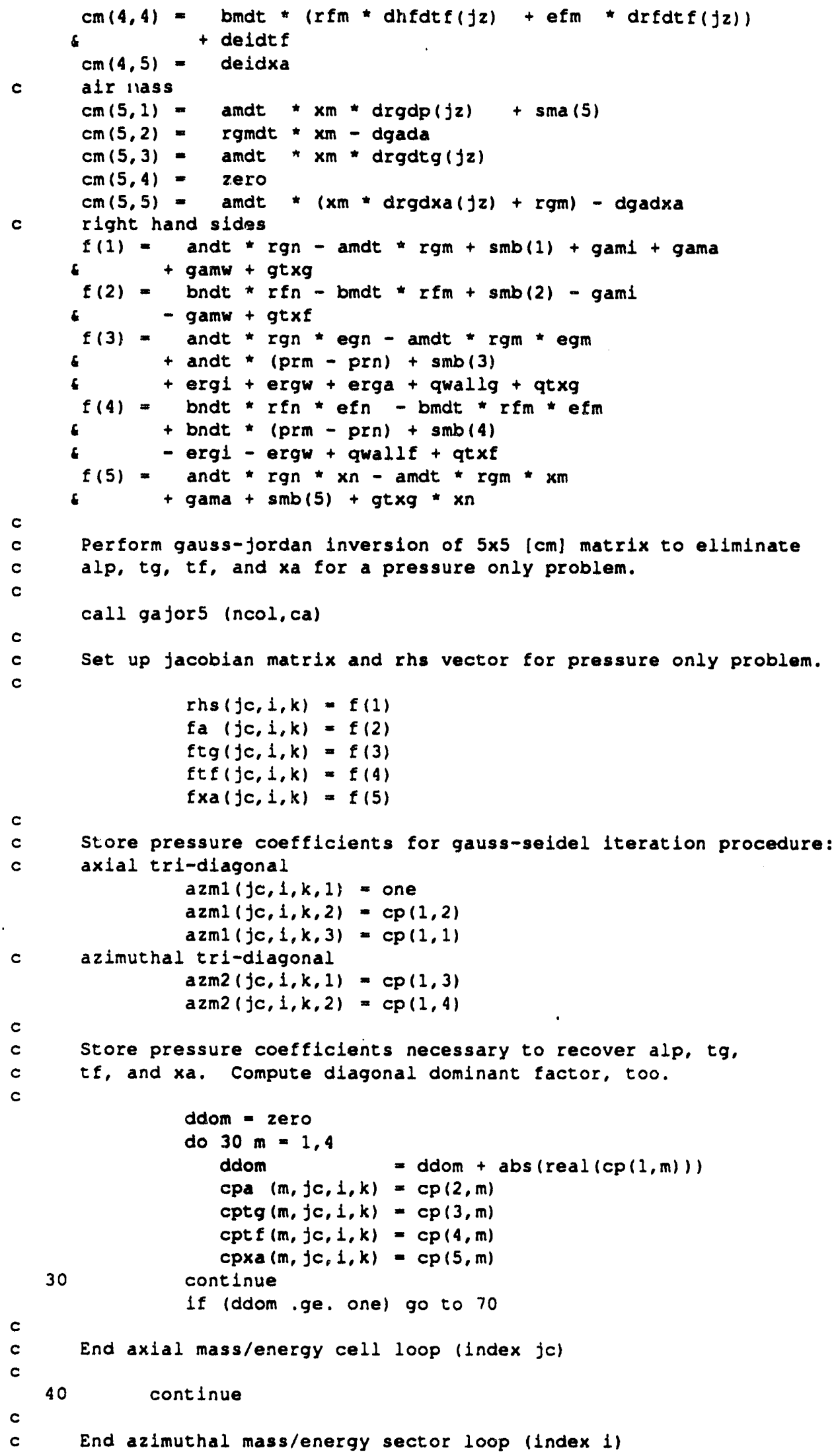




\begin{tabular}{|l|ll|l|}
\hline $2 / 15 / 93$ & WSRC-TR-93-086 Rev. 0 & FLOWTRAN-TF v1.2 Source Code & Pg. 213 of 354 \\
\hline
\end{tabular}

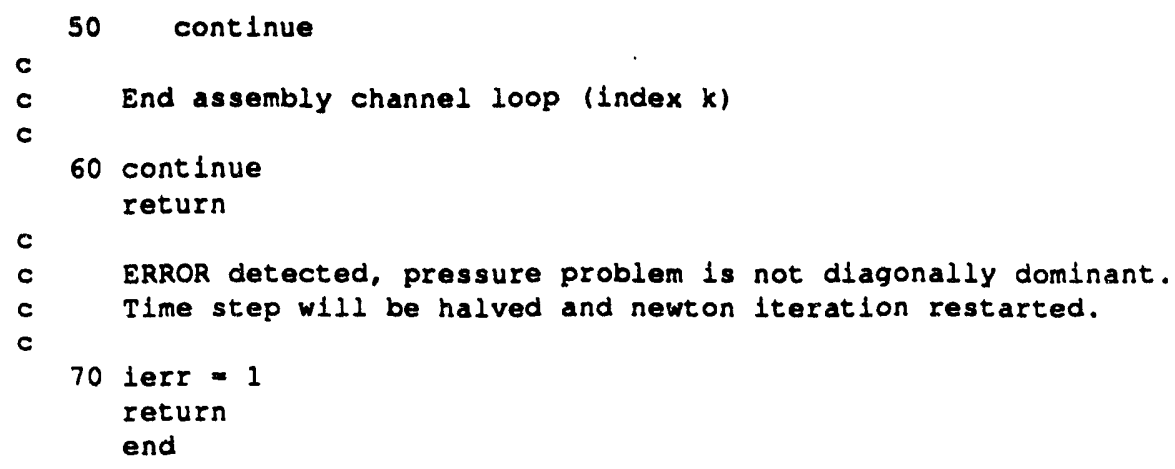

\section{JACOBT}

subrout ine jacobt (ptn, alpt $n, t g t n, t$ ft $n$, xat $n$, rogt $n$, roft $n$, hgt $n$, hft $n$,

6 pt, alpt, tgt, tft , xat , rogt, roft , hgt , hft ,

- git,eit, inflt, hglt, akgit,ysit.

c ppl,p, rdelt, factor, atm1, rhst, azm1,

c spat, cptgt, cptft, cpxat, fat, ftgt, ftft, fxat,

- cpa , cptg , cptf , cpxa.

- volt, advbt, advct, advbz, advcz,

\& xaO,epsy,epsf,nitysi,

c $n z t, n z p 2, n z p 3, n c h n, n x \max , n x, 1 \operatorname{gam} 1, i e r r, i l q)$

c

c

c

c

c

This routine computes the jacobian matrix at the current l.terate $m$ for the top section of a three-section reactor assembly. The mathematical and coupling considerations are the same as for the mid-section JACOBM. The FLOWTRAN TF manual and the FORTRAN of JACOBM should be examined for more solution detail.

This subroutine is compatible only with the FLOWTRAN TF program and the top_section (plenuin) of a three-section reactor-assembly simulation. ! (nzt, 3),

(nzp2, nxmax, nchn, 3)

(nzt),

(nzt)

(nzt, 5),

(nzt, 5),

(nzt).

(nzt).

(nzt),

(nzt)

eit (nzt).

holt (nzt). 
c$$
\text { c }
$$

c 
Call routines to define parameters needed in the equation set.

Set air mass source/sink terms.

call gammaa (pt (jc), alpt (jc), tgt ( fc), xat (jc), xao, 6 gama, dgada, dgadxa,

6 erga, deadp, deada, deadtg, deadxa)

Set interfacial heat and mass source/sink terms within the bulk.

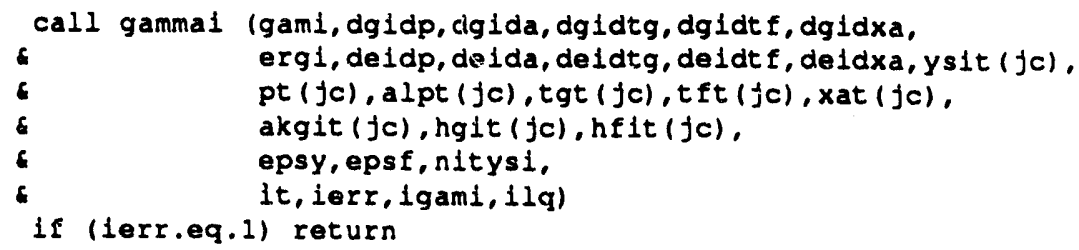




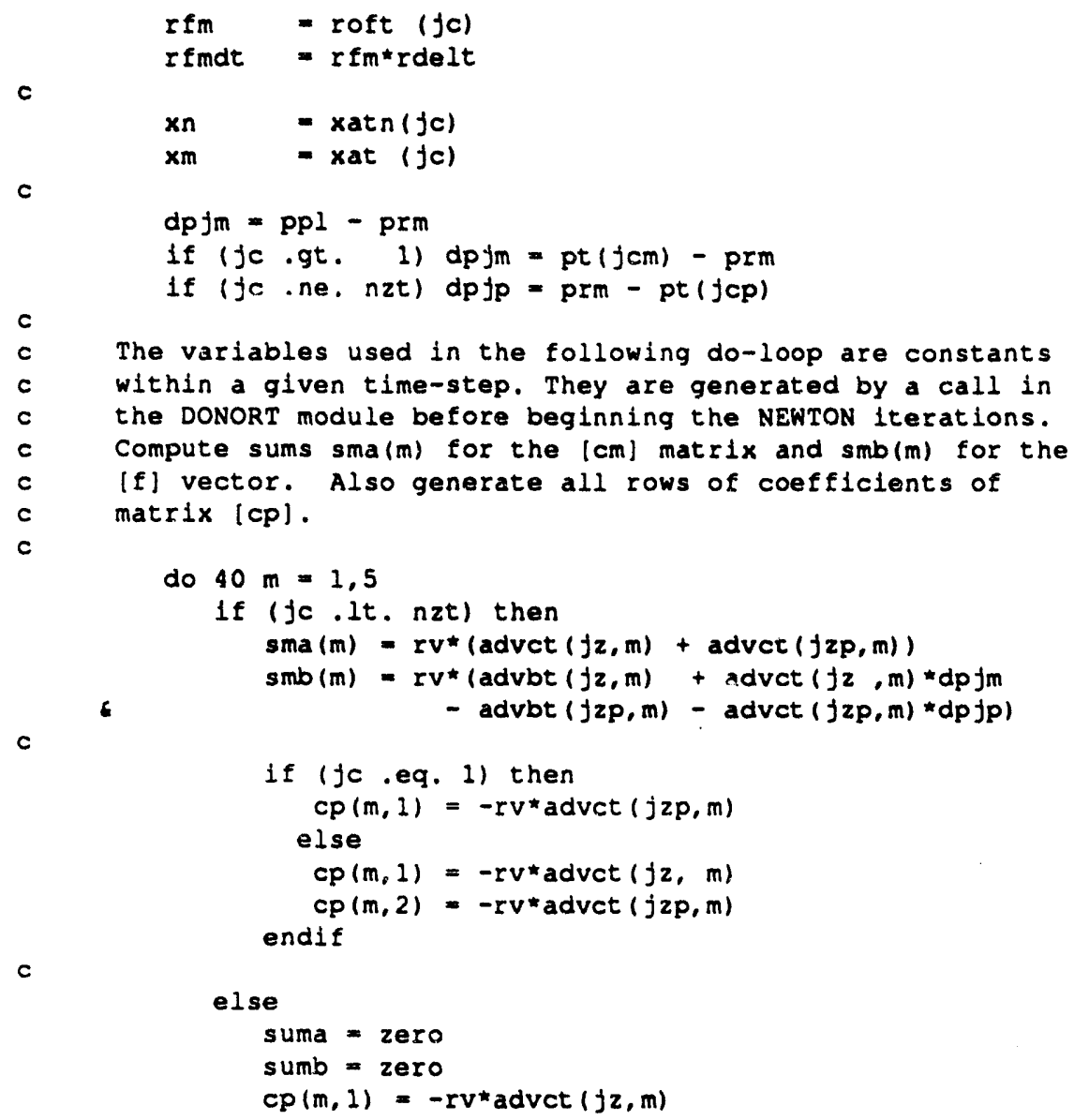




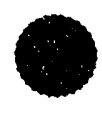

$c$
$c$
$c$
$c$
$c$

MIXTURE GAS MASS

$\mathrm{cm}(1,1)=$ amdt $* \operatorname{drgdp}(j z)-\operatorname{dgidp}+\operatorname{sma}(1)$

$\mathrm{cm}(1,2)=$ rgmdt - dgida - dgada

$\mathrm{cm}(1,3)=$ amdt $* \operatorname{drgdtg}(j z)-\operatorname{dg} 1 \mathrm{dtg}$

$\mathrm{cm}(1,4)=$ - dgidt $f$

$c m(1,5)=a$ andt * drgdxa(fz) - dgldxa - dgadxa

LIQUID MASS

$\mathrm{cm}(2,1)=$ bmdt * $d r f d p(f z)+d g 1 d p+s m a(2)$

$\mathrm{cm}(2,2)=-r f m d t+d g i d a$

$\mathrm{cm}(2,3)=d g i d t g$

$c m(2,4)=b m d t * \operatorname{drfdtf}(f z)+\operatorname{dg} i d t f$

$c m(2,5)=d g i d x a$

$c$

MIXTURE GAS ENERGY

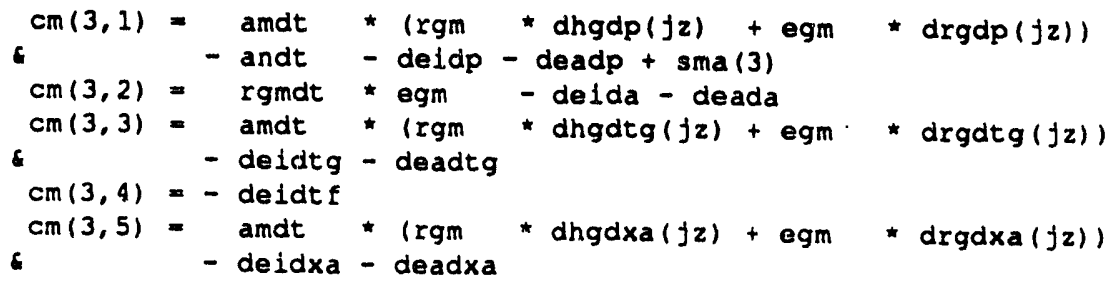


$f t g t(f c)=f(3)$

$f t f t(f c)=f(4)$

$f x a t(f c)=f(5)$

if (jc .eq. 1) then

Store pressure coefficients for gauss-seldel iteration procedure: axlal tri-diagonal only store pressure coefficlents necessary to recover alp, tg, $t f$, and $x a$ for top cell in the top section. Compute diagonal dominant factor, too.

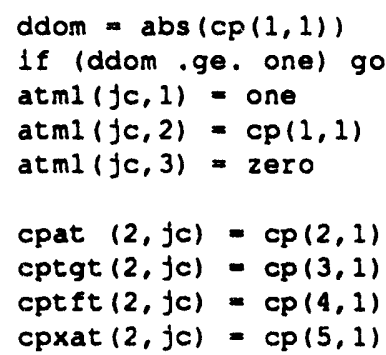




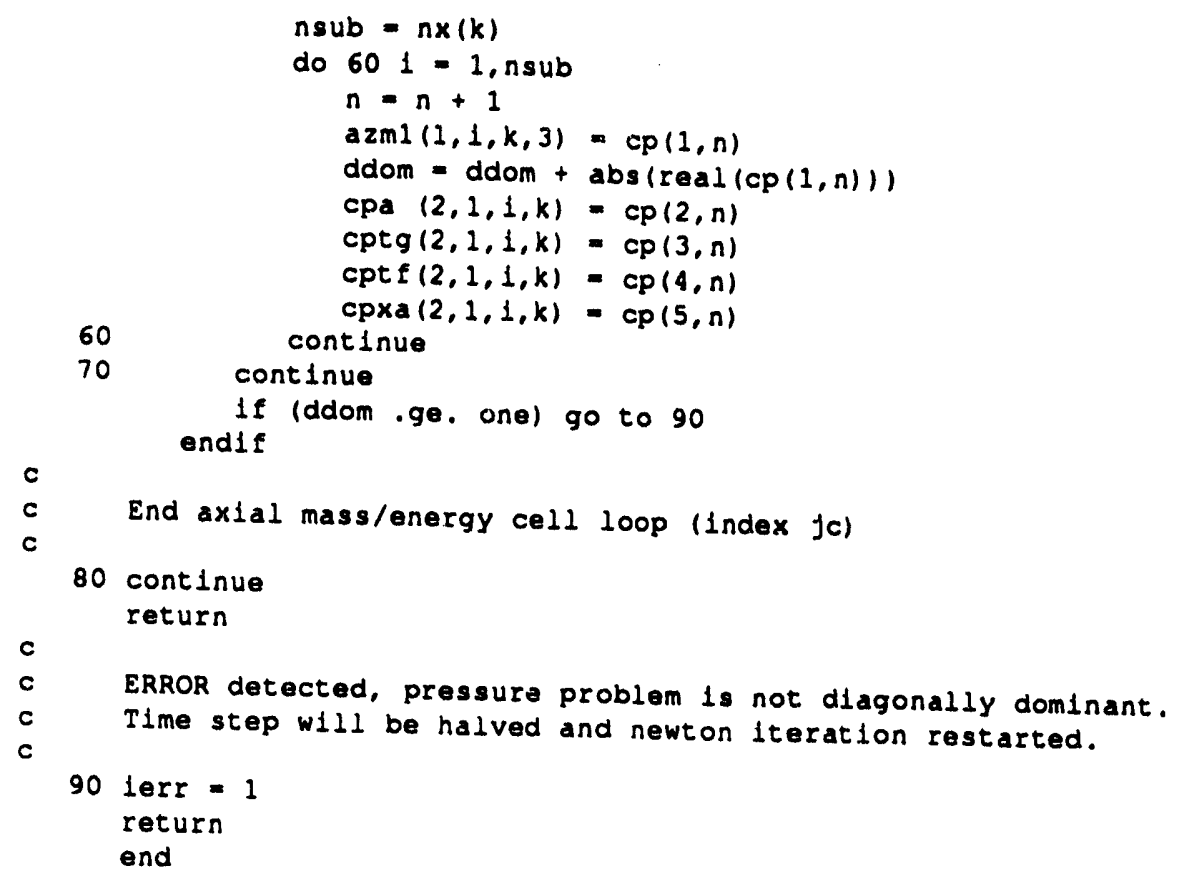

MIKIC

subroutine mikic lqflux, dqdtw, vapor, qchf, qonb, hfe, tchf, twall, tliq, tgas, tsat, pres, alfa, beta, xalr, gliq, ggas, rhol, rhog, visl, visg, condl, cpl, dhy, arib, leff, ribln, ribtk, mwall, hfg, hlsat, hgsat, slgma, matgas, persat, domiki, dochf, ichf, 1lq)

\section{SUBROUTINE mikiC}

THIS ROUTINE COMPUTES SURFACE HEAT FLUX AND THE DERIVATIVE WITH RESPECT TO WALL TEMPERATURE FOR LAMINAR AND TURBULENT SINGLE-PHASE FORCED CONVECTION WITH SUBCOOLED NUCLEATE BOILING (MIKIC AND ROHSENOW).

OUTPUT ARGUMENTS:

qfluX - SURFACE HEAT FLUX, $\omega / m * * 2$

dqdTW - DERIVATIVE OF HEAT FLUX WITH RESPECT TO WALL TEMPERATURE

vaPOT - VAPOR GENERATION AT WALL, $\mathrm{kg} / \mathrm{m} * 2-\mathrm{s}$

qChE - CRITICAL HEAT FLUX, W/ $m * 2$

qONb - HEAT FLUX AT ONSET OF NUCLEATE BOILING, $W / m * \star 2$

hfC - FORCED CONVECTION OR SINGLE PHASE LIOUID HEAT TRANSFER COEFFICIENT, $W / m * * 2-K$

tchf - TEMPERATURE WHERE qChf IS REACHED, $K$

INPUT ARGUMENTS:

tWall - WALL TEMPERATURE, $K$

tIIq - LIQUID TEMPERATURE, $K$

tgas - GAS TEMPERATURE, $K$

tsat - SATURATION TEMPERATURE, $K$

pres - CELL PRESSURE, Pa

alfa - GAS BULK VOLUME ERACTION

beta - LIQUID BULK VOLUME FRACTION

gliq - LIQUID MASS FLUX, $\mathrm{kg} / \mathrm{m} * 2-\mathrm{s}$

ggas - GAS MASS FLUX, $\mathrm{kg} / \mathrm{m} * 22-\mathrm{s}$

rhol - LIQUID DENSITY, $\mathrm{kg} / \mathrm{m} * 3$ 


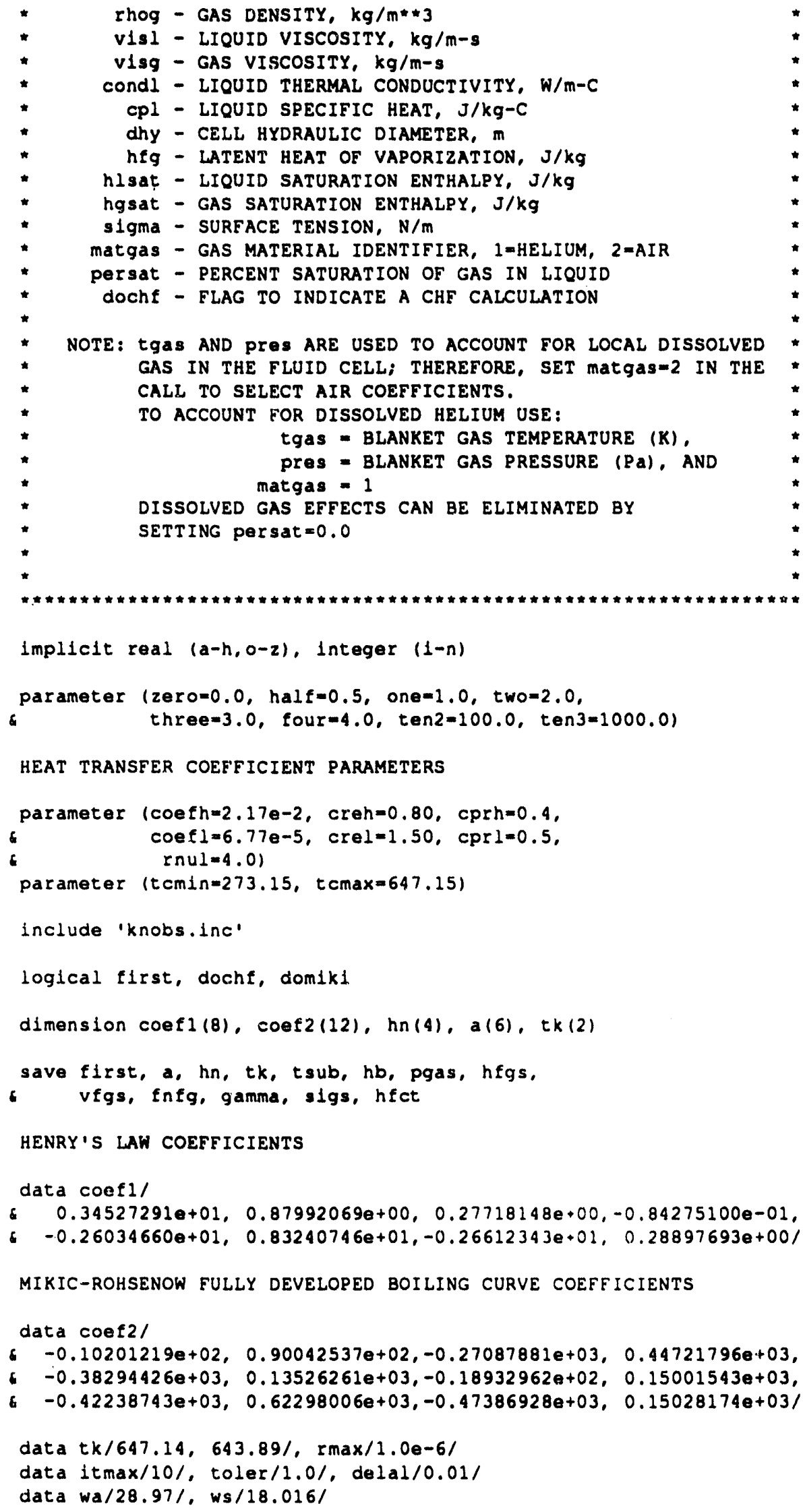


EVALUATE STEAM PHYSICAL PROPERTIES AT SATURATION

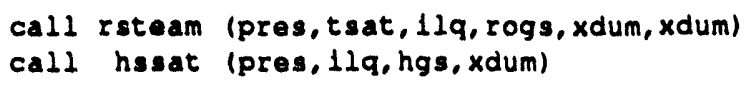

EVALUATE LIQUID PHYSICAL PROPERTIES AT SATURATION

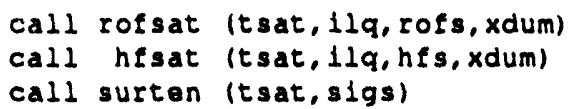

COMPUTE GAMMA TERM IN THE ONB CORRELATION

USING FLUID PROPERTIES AT SATURATION

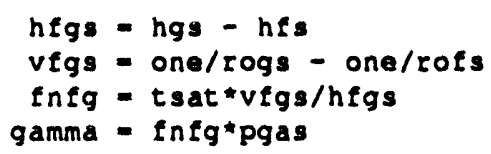

COMPUTE THE LIQUID SUPERFICIAL REYNOLDS NUMBER AND BULK FLUID PRANDTL NUMBER

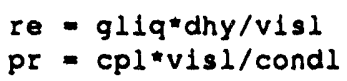

COMPUTE THE NUSSELT NUMBER FOR TURBULENT SINGLE PHASE FORCED CONVECTION BASED ON SRL DERIVED CORRELATION FOR TWO-PHASE FLOW IN ANNULAR GEOMETRIES

CHURN-TURBULENT CORRELATION

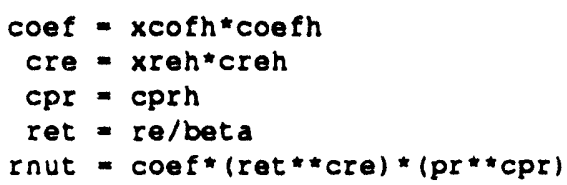

ANNULAR CORRELATION

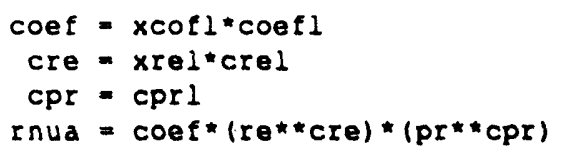

COMBINE CHURN-TURBULENT AND ANNULAR CORRELATIONS

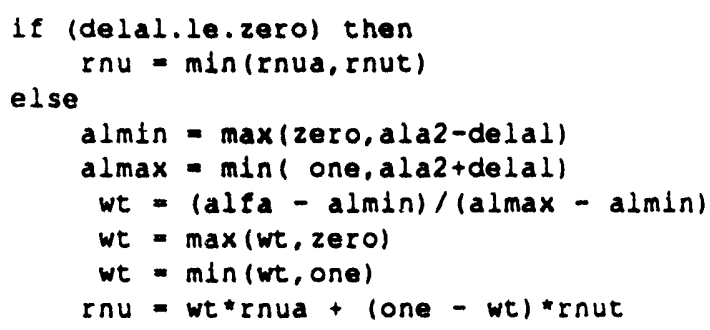




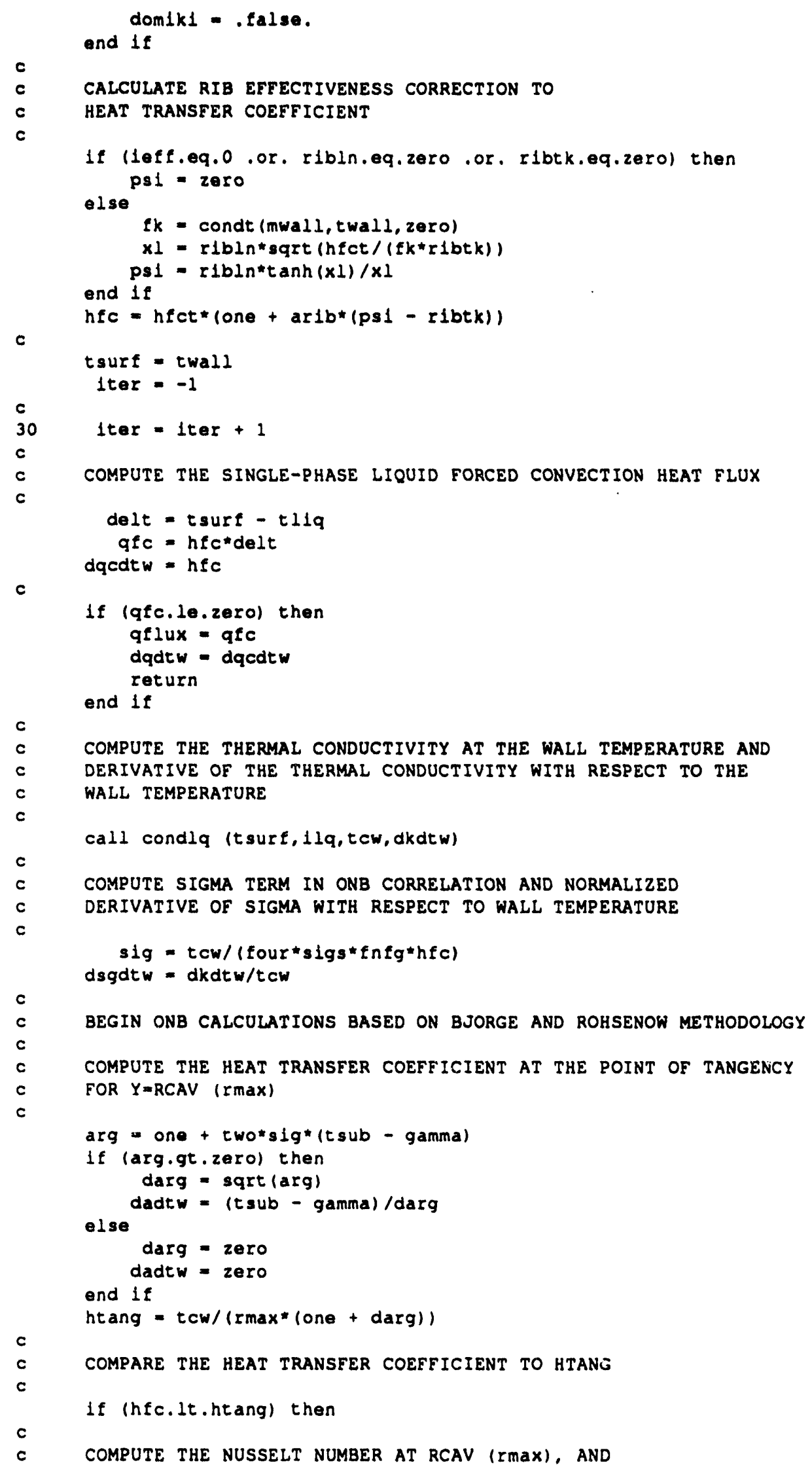




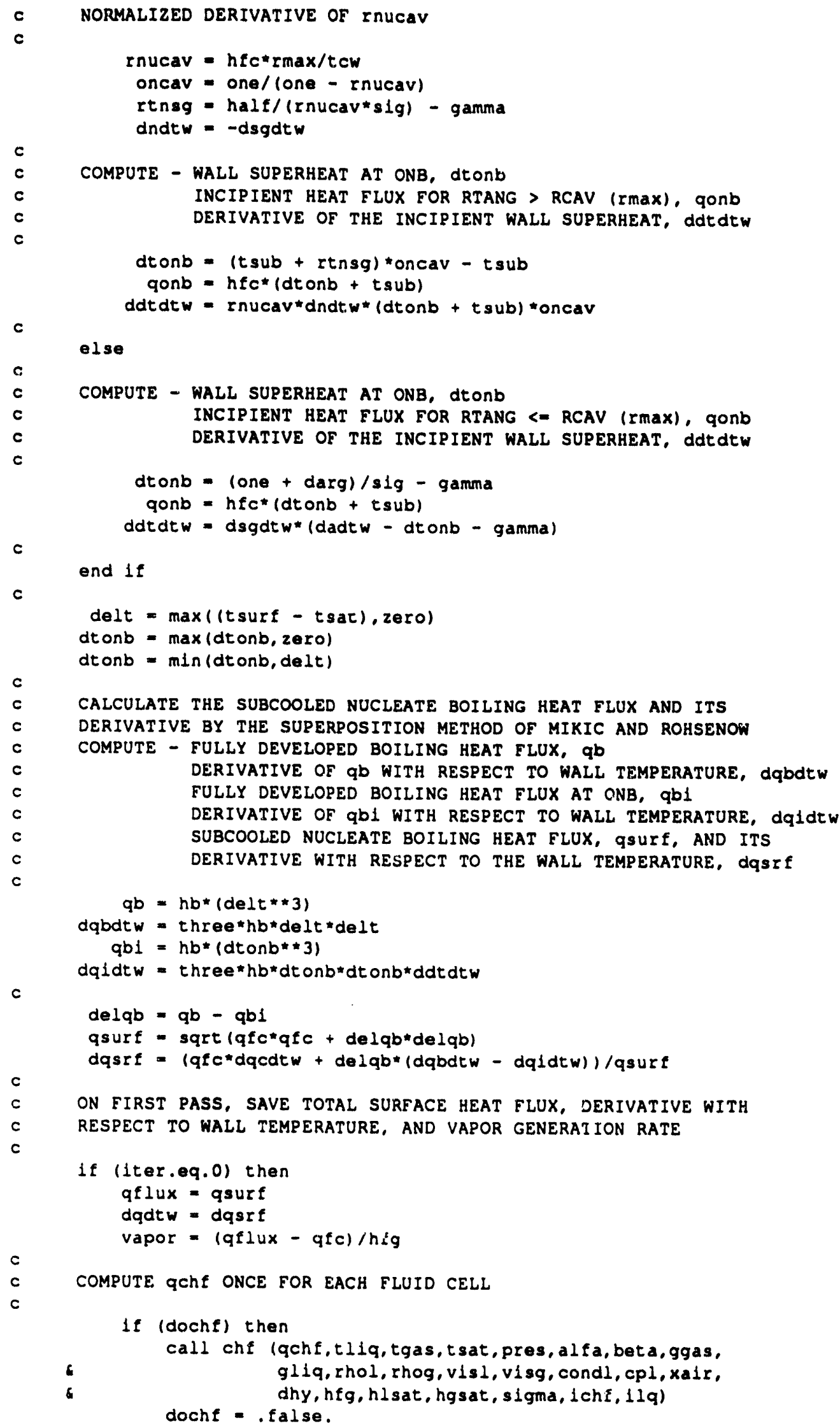

c

c

c

c

$c$

c

c

c 


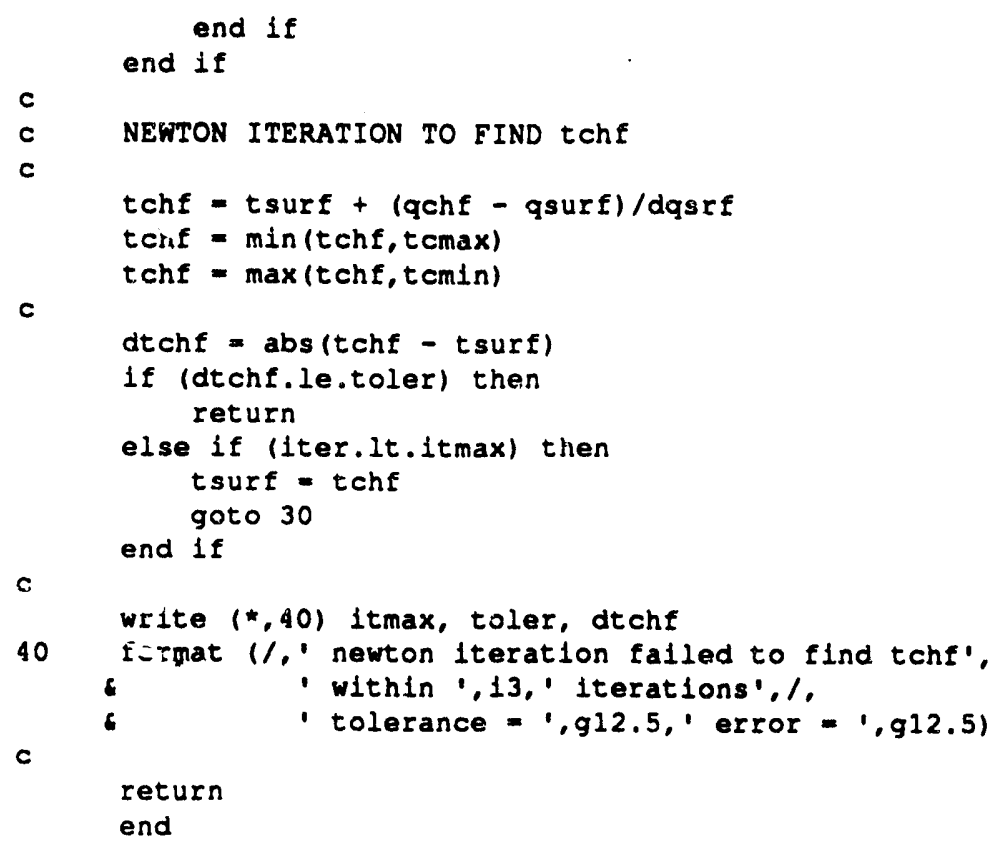

\section{MOVE}

subroutine move $(a, b, n)$

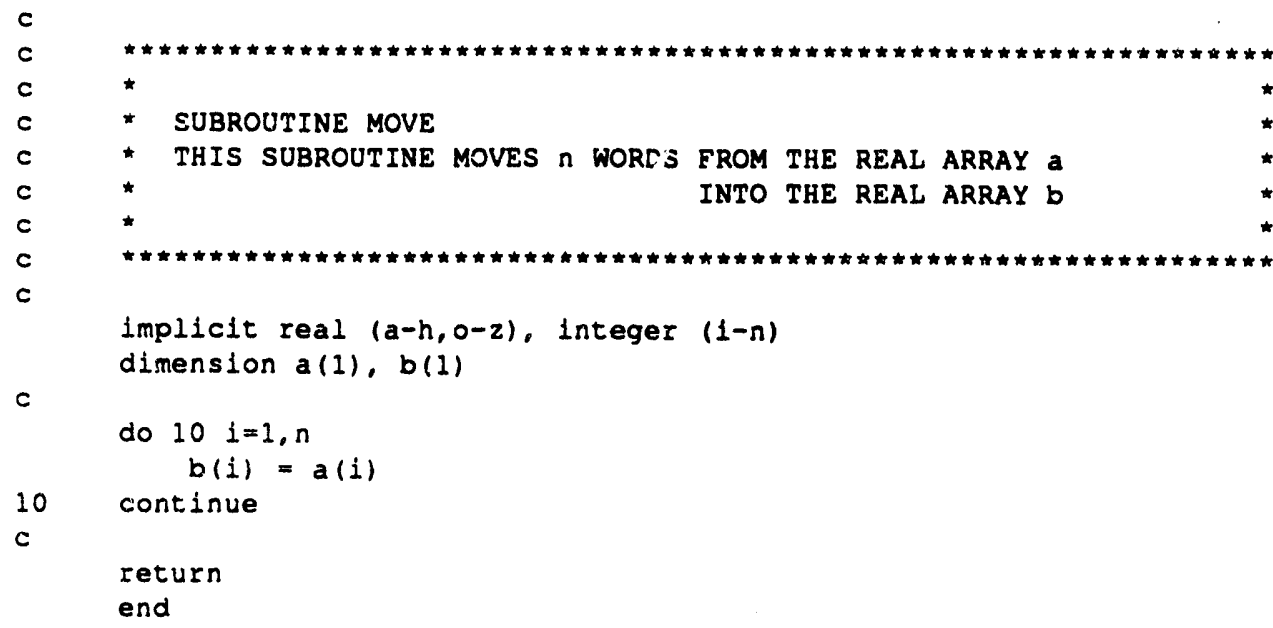

\section{NEWTON}

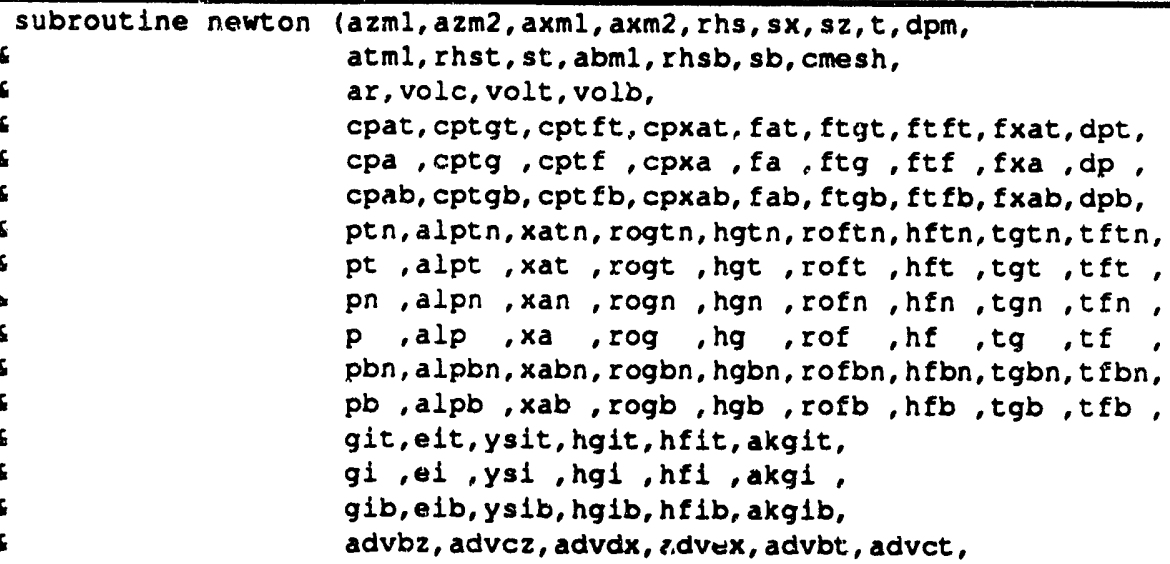




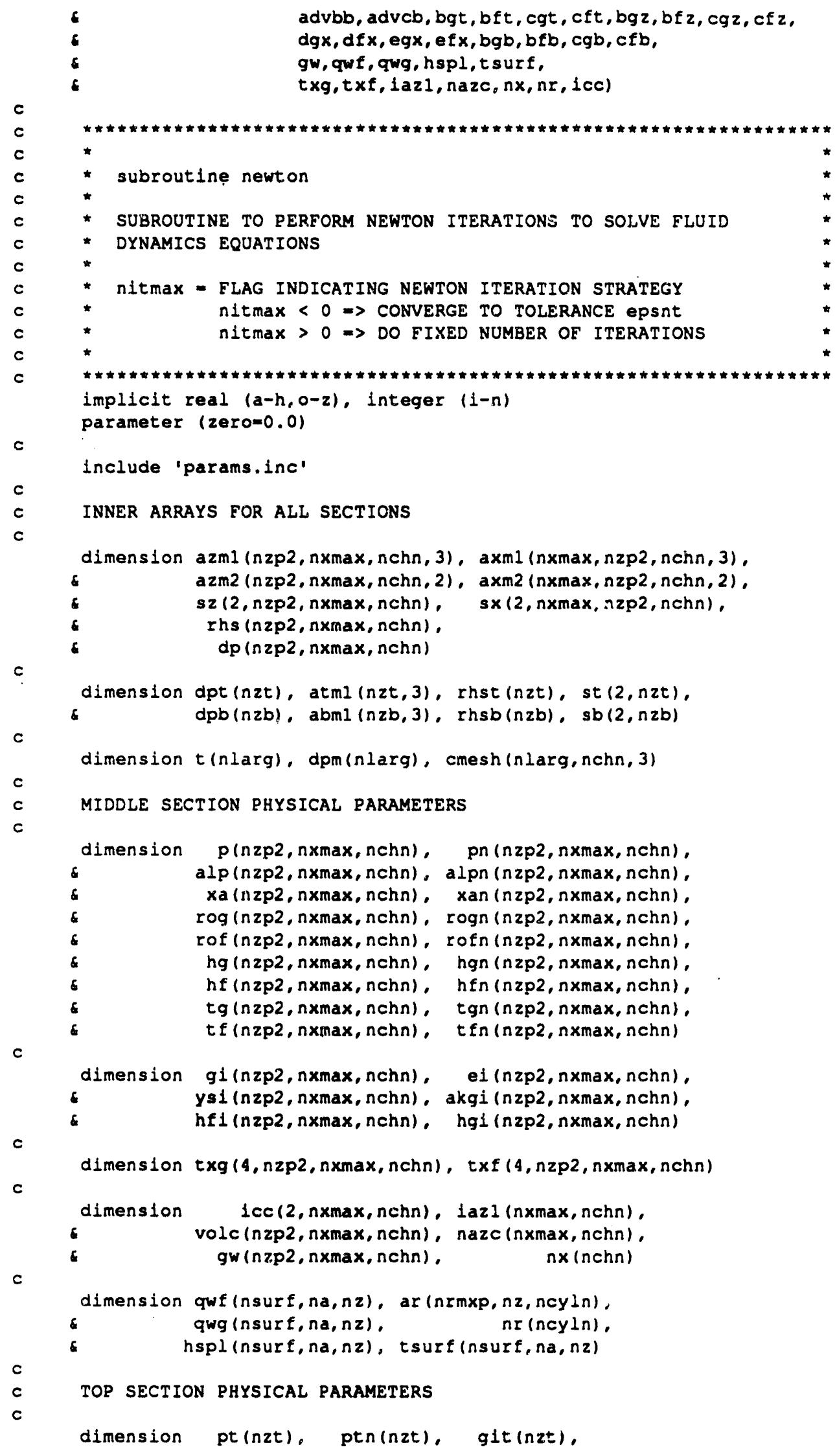




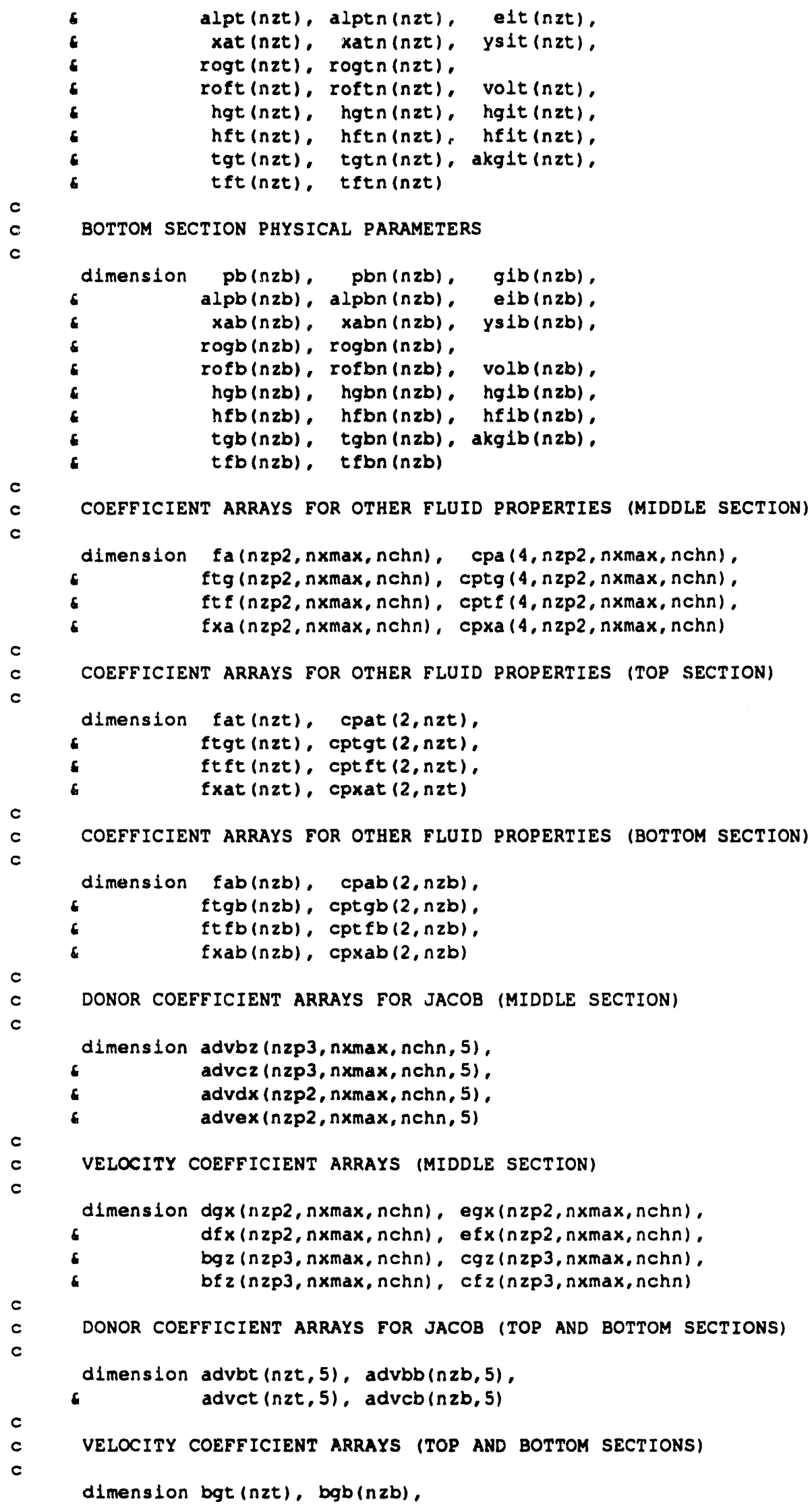




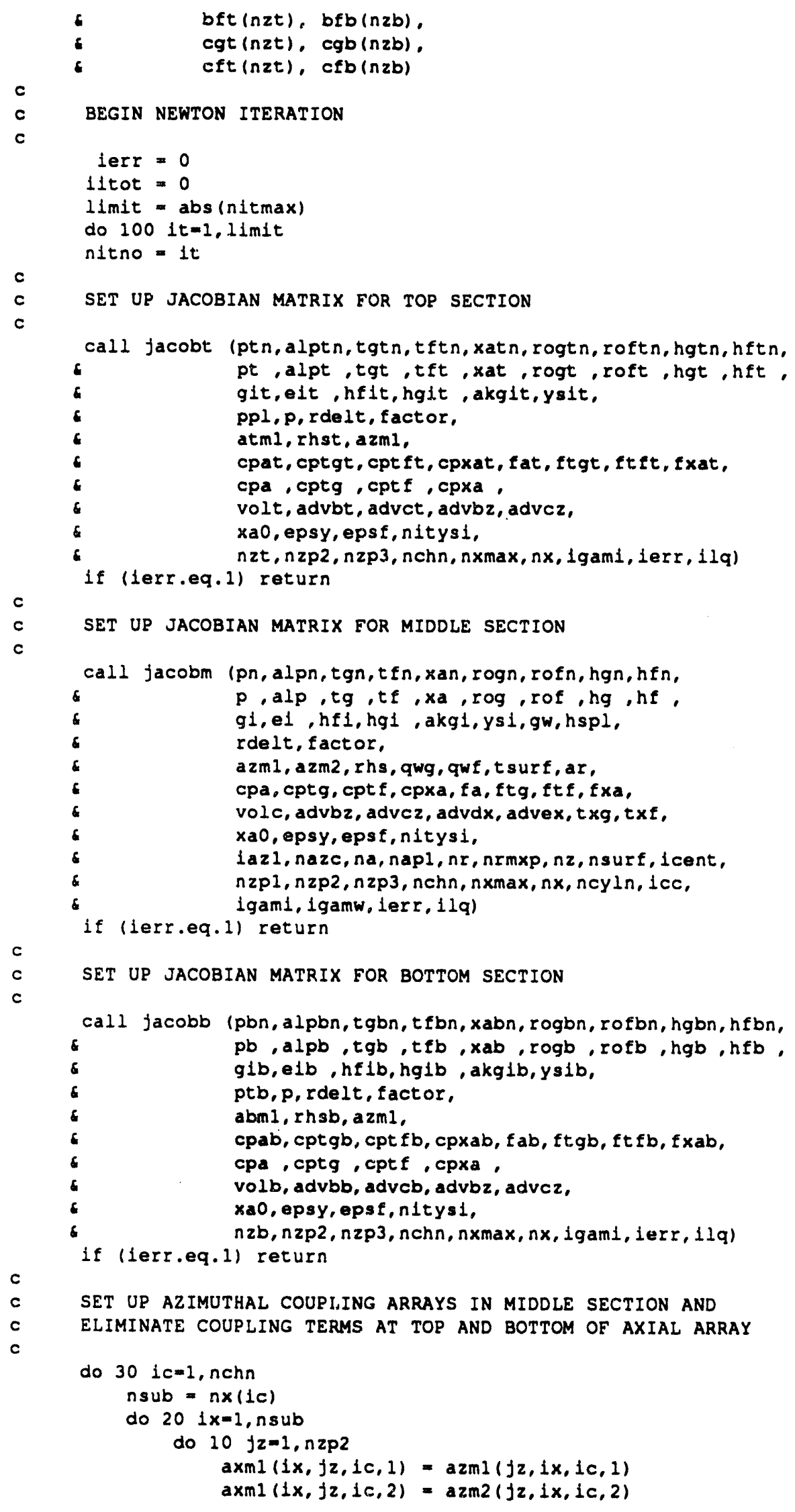




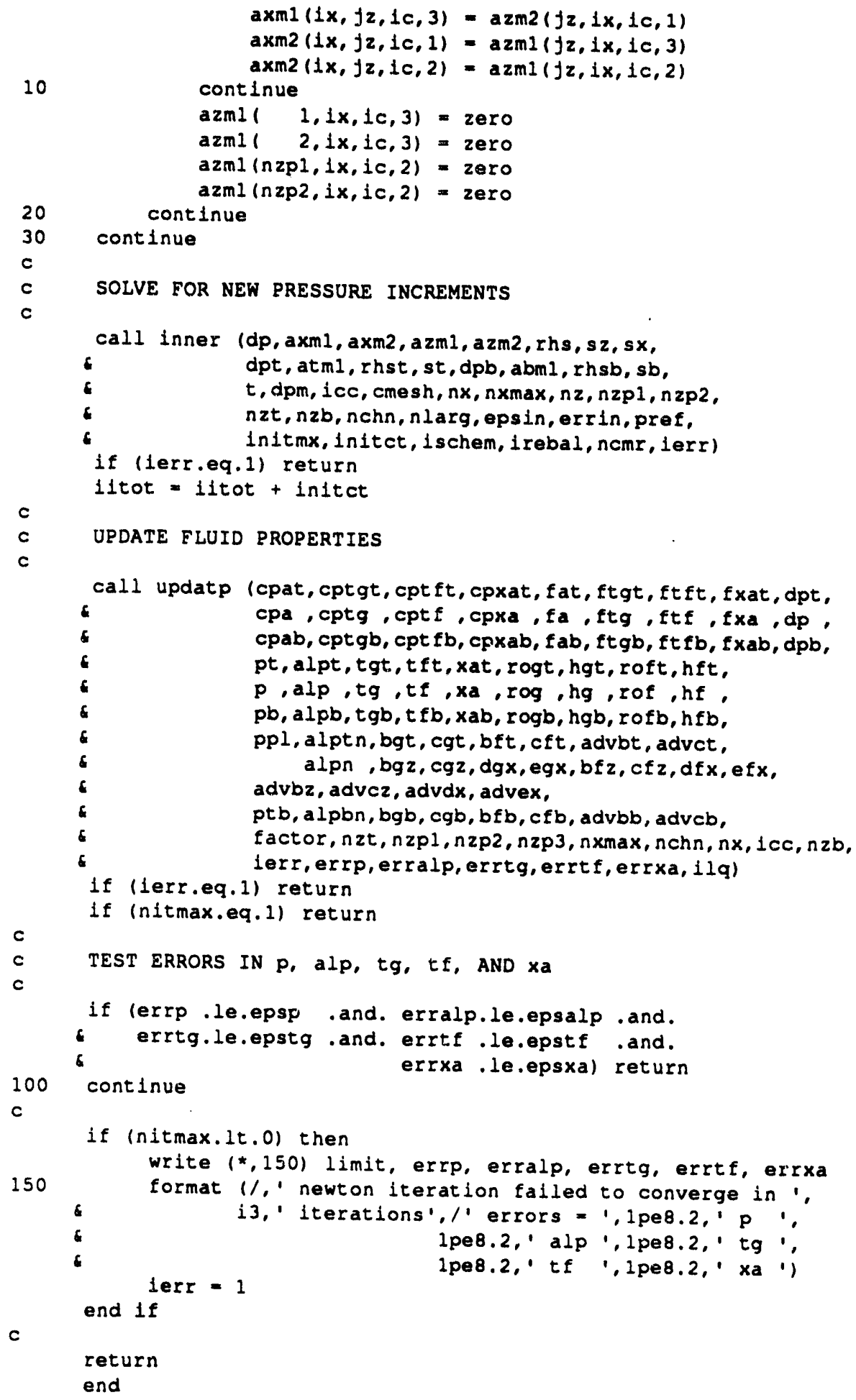




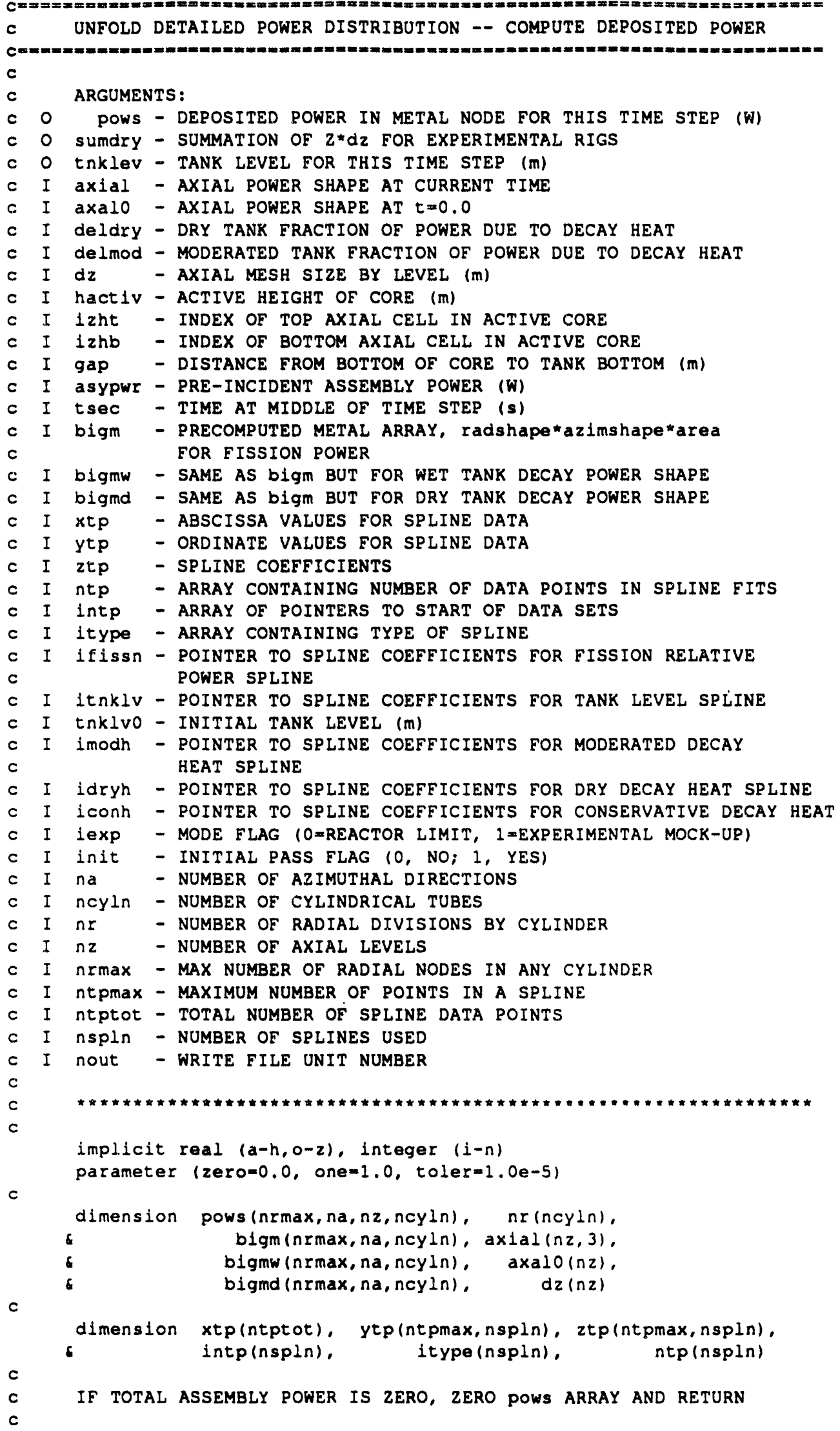


if (asypwr.eq.zero) then

call clear (pows, nrmax na*nz*ncyln)

end if return

$c$
$c$
$c$
$c$
$c$
$c$
$c$
$c$
$c$
$c$
$c$

EVALUATE TIME-DEPENDENT AMPLITUDE FACTORS:

fdkdYY, DRY TANK DECAY HEAT. NOTE--FOR THE EXPERIMENTAL MOCK-UP MODE, THIS PROVIDES NORMALIZED POWER VS TIME

fdkmod, MODERATED TANK DECAY HEAT

fdkCON, CONSERVATIVE DELTA-MULTIPLIER

ffissn, FISSION HEAT

tnklev, TANK LEVEL

hrat , NORMALIZED TANK LEVEL RATIO: (tnklev - gap)/hactiv

if (iexp.eq.1) then

deldry $=$ one

delmod $=$ zero

fdkmod $=$ zero

$f d k c o n=$ one

ffissn $=$ zero

tnklev $=$ zero

hrat $=$ zero

else

inx $=\operatorname{intp}(i \bmod h)$

ityp $=1$ type $(1 \bmod h)$

ntps $=n t p(1 \operatorname{modh})$

fdkmod $=$ yvalu $(1 t y p, n t p s, x t p(\operatorname{lnx}), y t p(1, i \operatorname{modh})$,

c

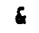
ztp (1, 1modh), tsec)

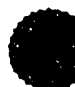

$$
\begin{aligned}
\operatorname{inx} & =\operatorname{intp}(i \operatorname{conh}) \\
\operatorname{ityp} & =i \operatorname{type}(i c o n h)
\end{aligned}
$$

$n t p s=n t p(i c o n h)$

fdkcon = yvalu(1typ, ntps, xtp(inx),ytp(l,iconh), $\operatorname{ttp}(1, i$ conh $), t \sec )$

$$
\begin{aligned}
\text { inx } & =i n t p(i f i s s n) \\
\text { ityp } & =i t y p e(i f i s s n) \\
n t p s & =n t p(1 f i s s) \\
\text { ffissn } & =\text { yvalu(ityp,ntps, xtp(inx),ytp(1,ifissn), }
\end{aligned}
$$

c $\operatorname{ztp}(1$, ifissn), tsec)

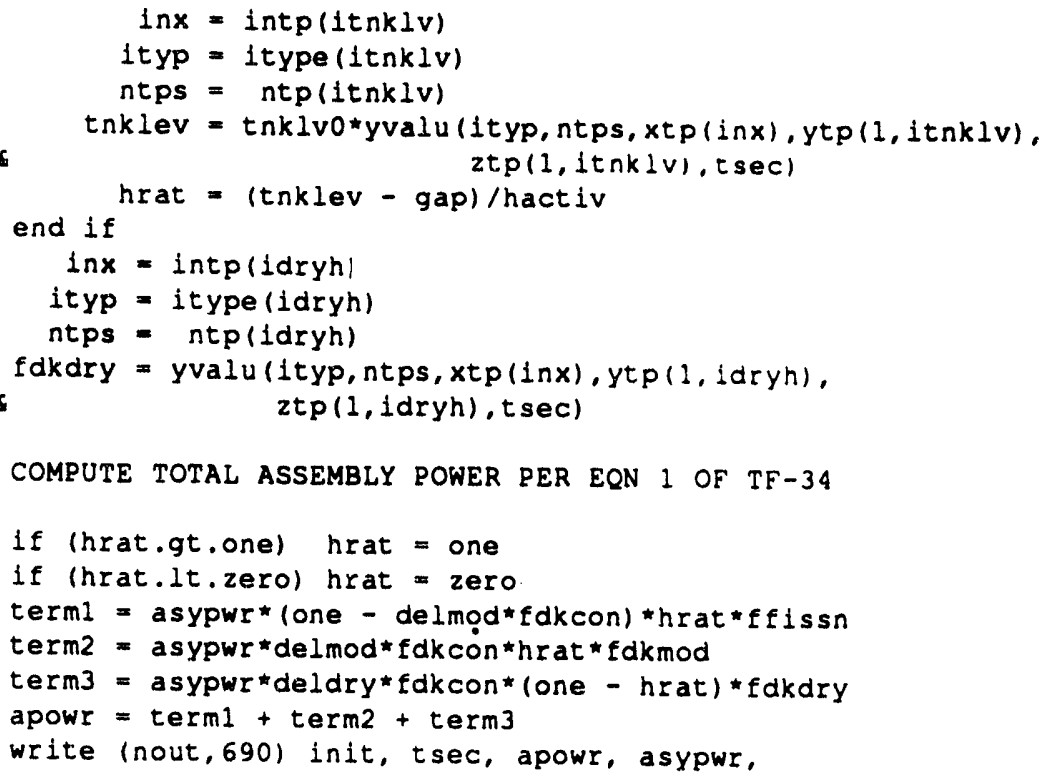




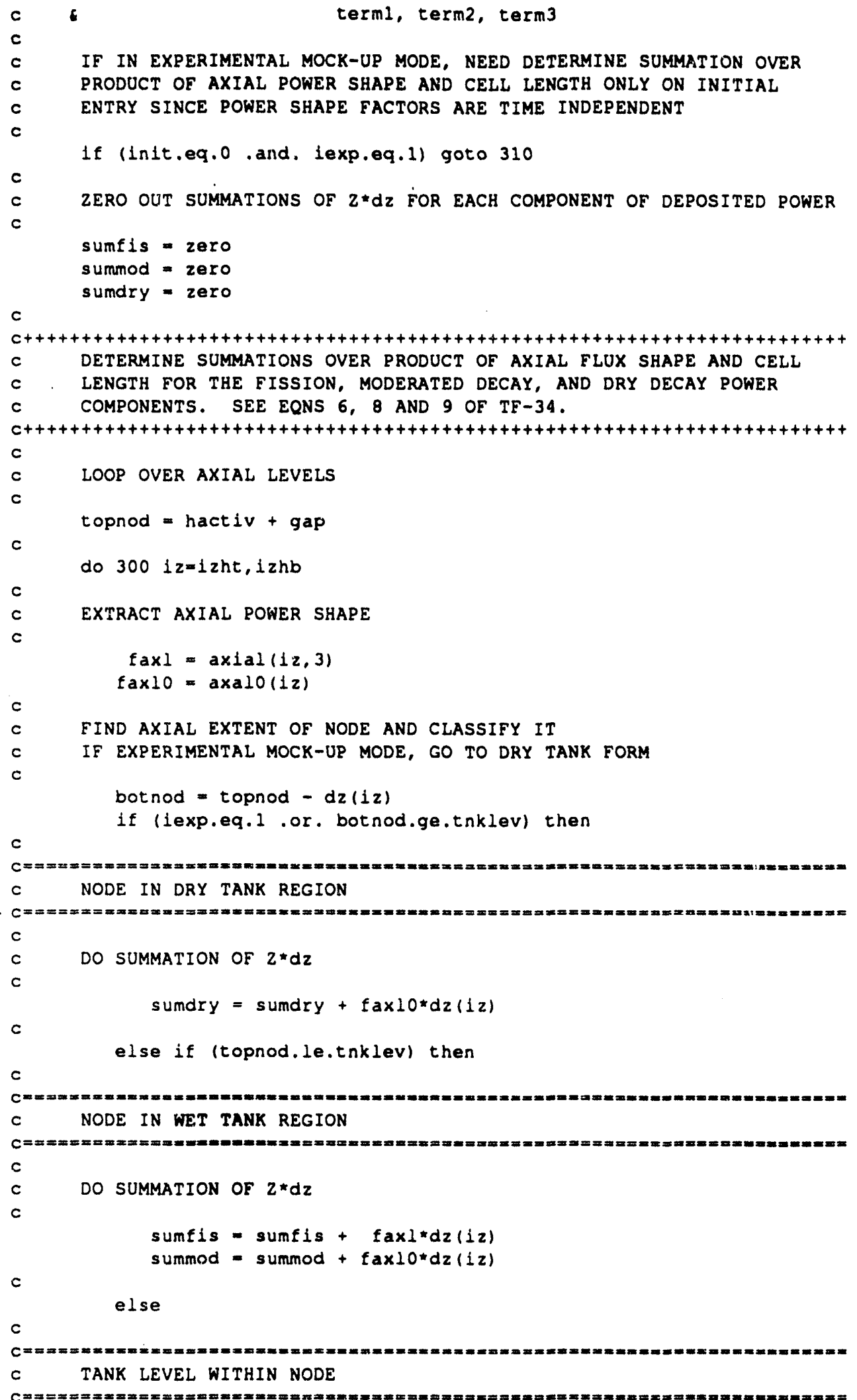


dzwet = tnklev - botnod

c dzdry = topnod - tnklev

c

DO SUMmation of $z$ *dz

sumfis $=$ sumfis $+f a x 1^{*} d z w e t$

summod $=$ summod $+f a \times 10 * d z$ wet

sumdry = sumdry + faxlowdzdry

c

end $1 f$

topnod - botnod

300 continue

c

310 continue

$\mathrm{c}$

$\mathrm{Ct+t+t+t+t+t+t+t+t+t+t+t+t+t+t+t+t+t+t+t+t+t+t+t+t+t+t+t+t+t+t+t+t+t+t+t+}$

C COMPUTE THE NORMALIZED POWER DISTRIBUTION, CONVERT TO POWER

C DEPOSITED IN NODE (w)

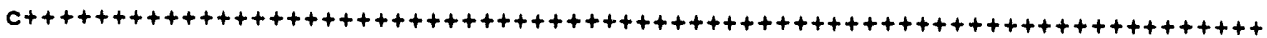

c

c LOOP OVER AXIAL LEVELS

c

$c$

topnod $=$ hact $1 v+$ gap

do 500 iz=izht, izhb

EXTRACT AXIAL POWER SHAPE

$c$

$\operatorname{tax} 1=\operatorname{axtal}(12,3)$

$f a \times 10=\operatorname{axalO}(1 z)$

FIND AXIAL EXTENT OF NODE AND CLASSIFY IT,

IF EXPERIMENTAL MOCK-UP MODE, USE DRY TANK FORM

botnod = topnod $-d z(1 z)$

if (lexp.eq.1 .or. botnod.ge.tnklev) then

$c$

$c=$

$c$

$c=$

$c$

NODE IN DRY TANK REGION

comb $=$ zero

combw $=$ zero

combd $=$ term 3

c

else if (topnod.le.tnklev) then

c

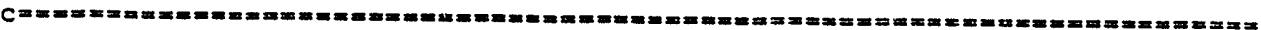

c NODE IN WET TANK REGION

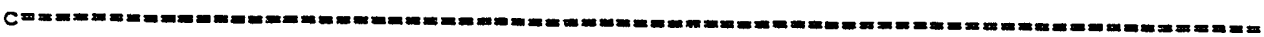

c

comb $=\operatorname{term} 1^{\star} f a x l^{\star} d z(1 z) /$ sumf is

combw $=\operatorname{term} 2 \star f a x 10 \star d z(1 z) /$ summod

combd $=$ zero

c

else

C

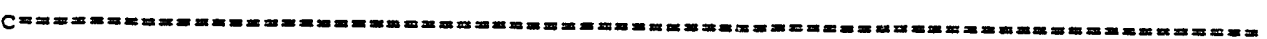

c TANK LEVEL LIES WITHIN NODE

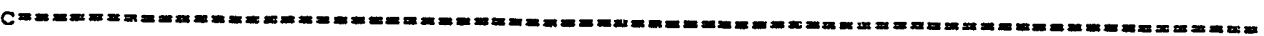

c

$\begin{aligned} \text { comb } & =\operatorname{term} 1 \star f a x 1^{\star} \mathrm{dzwet} / \text { sumf } 1 \mathrm{~s} \\ \text { combw } & =\operatorname{term} 2^{\star} \mathrm{fax} 10^{\star} \mathrm{dzwet} / \text { summod } \\ \text { combd } & =\operatorname{term} 3^{\star} \mathrm{fax} 10^{\star} \mathrm{dzdry} / \text { sumdry }\end{aligned}$ 
end $1 f$

c

c

c

c

c

c

c

c

c

c

c

420

430

500

c

c

c

c

c

c

c

c

c

c

c

c

c

$c$
$c$
7
780

(

COMPUTE NODAL POWER DEPOSITION

LOOP OVER CYLINDERS

do 430 lcy $\ln =1$, ncyln

$n r 1=n r(1 c y l n)$

LOOP OVER AZIMUTHAL DIVISIONS

do $420 \quad 1 a=1$, na

LOOP OVER RADIAL DIVISIONS

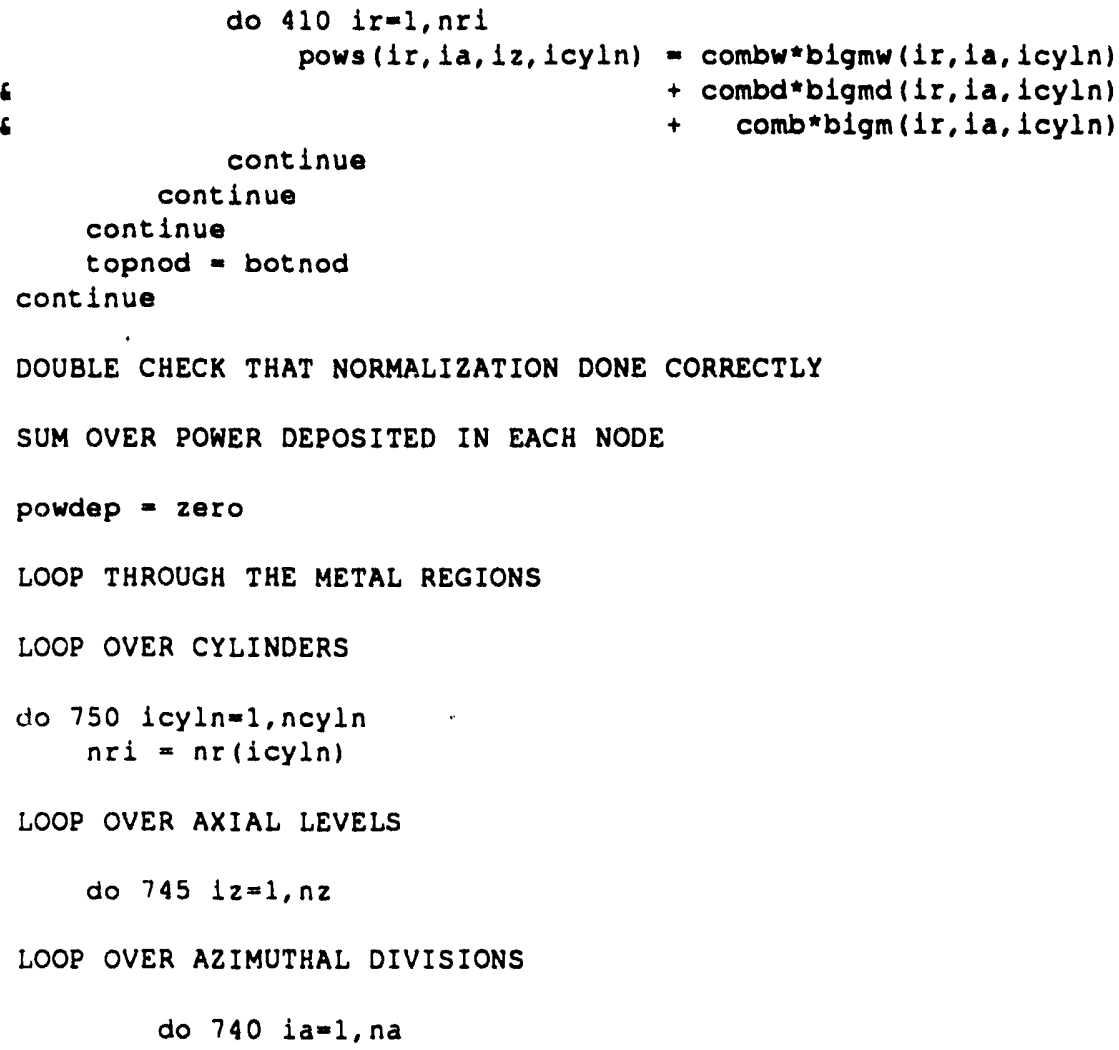




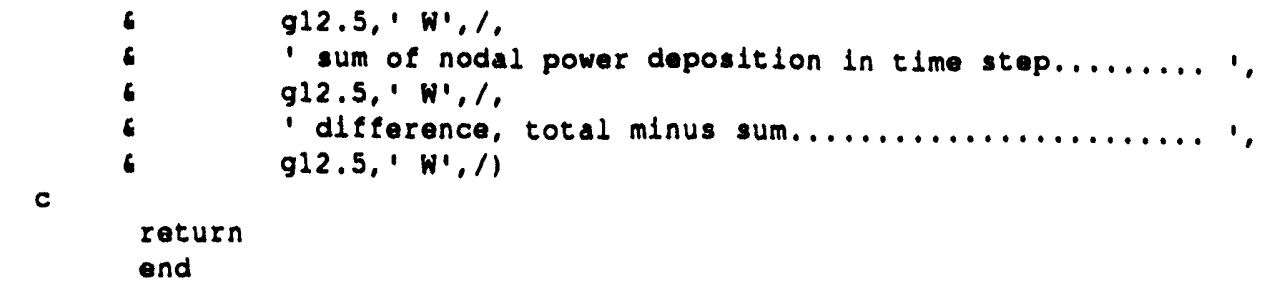

\section{PNORM}

subroutine pnorm lblgm, frdlm, fazmm, rphl, pfrct,dlam, mnrp, nrp,

- nrgtm, na, ncyln, nr, nz, nrmax, nrmxp, nreg, nfrst,

- nlast, nout)

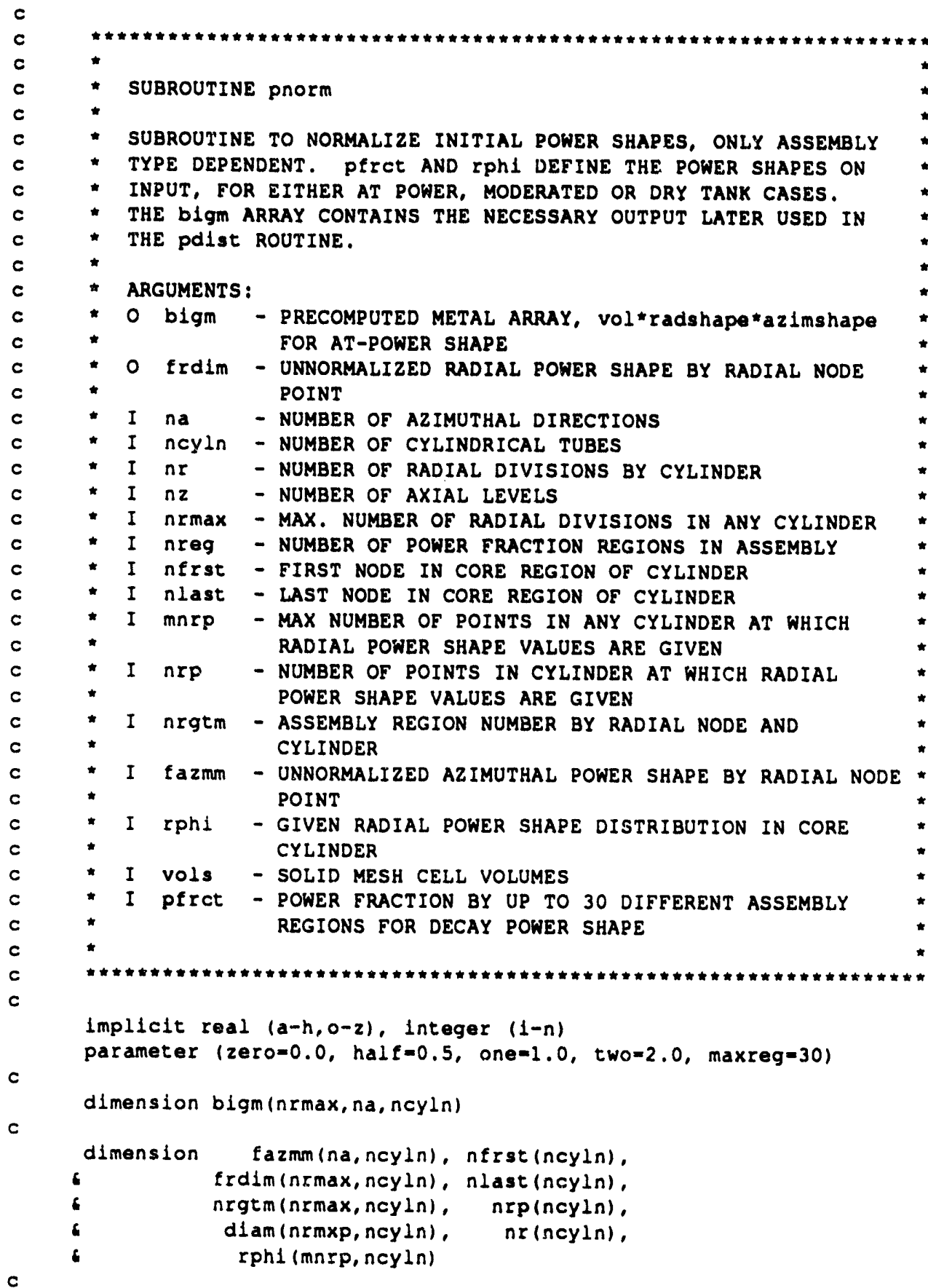


c

c

c

c

c

c

c

$c$

c

10

20

c

$c$

c

c

c

$c$

c

$c$

$c$

c

c

$c$

30

40

50

c

c

LOCAL ARRAYS

dimension preg (maxreg), pfrct (maxreg)

p1 - acos(-one)

$d a=t w o * p 1 /$ float (na)

CHECK FIXED ARRAY DIMENSION

If (nreg.gt.maxreg) then

wrlte (nout, 910) nreg stop

end if

CREATE NON-NORMALIZED NOCAL RADIAL POWER SHAPES BY INTERPOLATING IN POLYNOMIAL CREATED FROM GIVEN POWER SHAPE DATA

FIRST INITIALIZE RADIAL SHAPE FUNCTIONS

do 20 1cyln=1, ncyln

$n \times 1=n r(i c y l n)$

do $101 \mathrm{r}=1, n \mathrm{n} 1$

frdim $(15,1$ cyln $)=$ one

continue

continue

COMPUTE DETAILED NODAL SHAPES IN CORE REGION OF CYLINDERS

call radshp (frdim, rphl, diam, ncyln, nrmax, nrmxp, 6 nfrst, nlast, mnrp, nrp, nout)

CREATE THE PRODUCT AZIMUTHAL RADIAL SHAPE FUNCTION USED

IN PDIST (NEED BE DONE ONLY ONCE, TIME-INDEPENDENT)

FIRST, NORMALIZE POWER SHAPES TO PRESERVE POWER FRACTIONS

ZERO OUT REGION POWERS

call clear (preg, nreg)

LOOP OVER ALL METAL NODES

do 50 1cyln=1, ncyln

$n r i=n r(1 c y l n)$

if (nri.eq.0) goto 50

do $401 r=1, n r 1$

$n r g=n \operatorname{rgtm}(i r, i c y l n)$

frad $=\operatorname{trd1m}(1 r, 1 c y \mid n)$

rinner = half diam (1r,leyln)

router = half*diam $(1 r+1,1 c y \mid n)$

area $=$ da*half*(router $* * 2$ - rinner**2)

do 30 la=l, na

$\operatorname{blgm}(1 r, 1 a, 1$ cyln) = $\operatorname{rad} * \operatorname{tazmm}(1 a, 1 c y l n) * a r e a$

preg(nrg) $=\operatorname{preg}(n r g)+b 1 g m(1 r, 1 a, 1 c y l n)$

continue

continue

cont inue

COMPUTE NORMALIZATION FACTORS BY REGION

do 60 nrg=1, nreg

if (preg(nrg).eq.zero) then

if (pfrct(nrg).ne.zero) write(nout, 210) nrg, pfrct(nrg) 


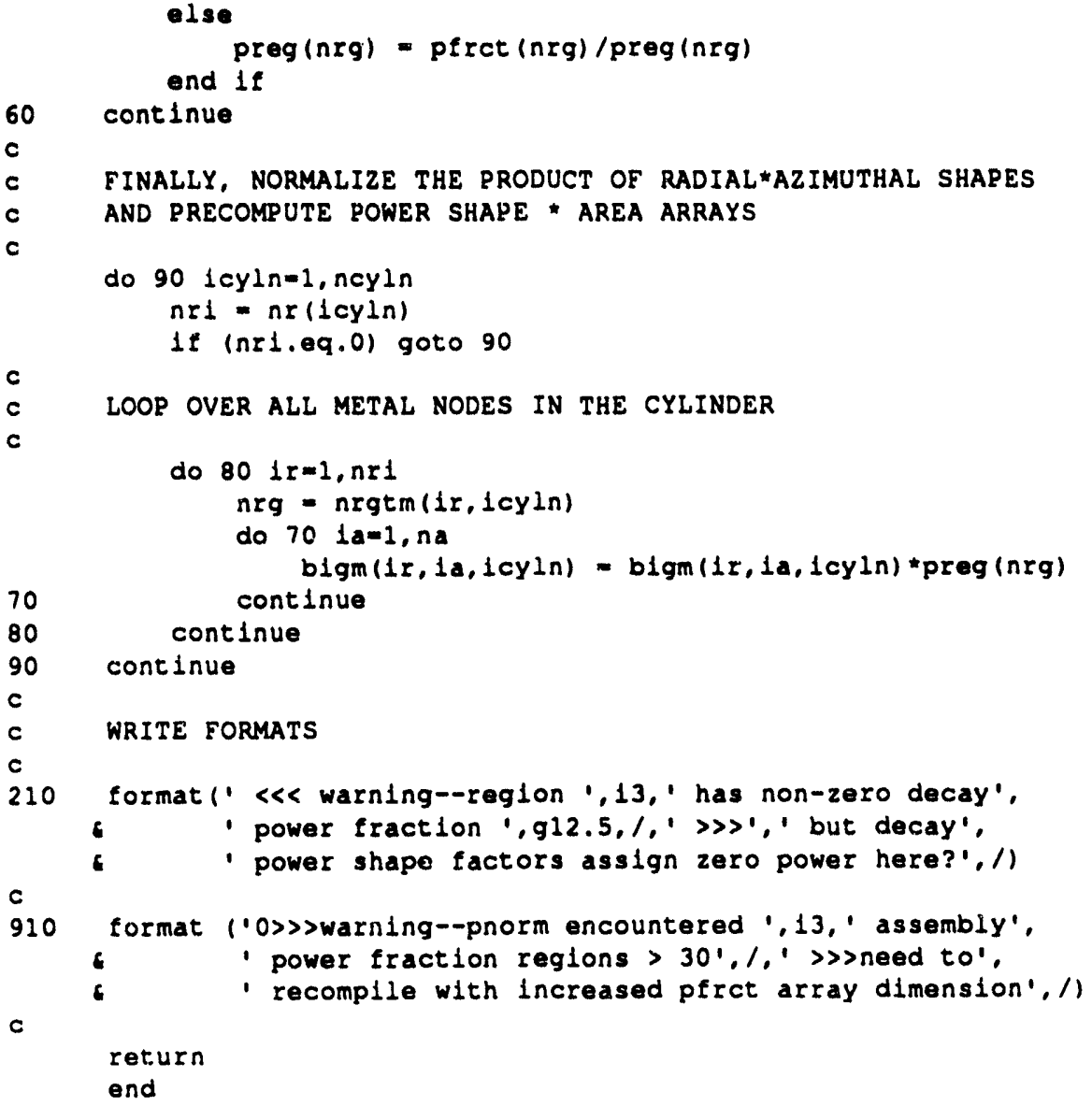

\section{POINT}

subroutine point 
c

Imat $=\operatorname{lnazc}+$ nsubc

$1 n t p=1$ mat $+n r \max { }^{*} n z z^{*} c y l n$

lintp = lntp + nspln

11type = 11ntp + nspln

11 axlp $=11$ type + nspln

c

lnrgtm = 11axlp + 1time

c

l1chks = lnrgtm + nrmax*ncyln

lichke - lichks + nsurf

lenia - lichkc + ncrlt

if (lenia.gt.maxia) then

write $(*, 100)$ lenia, maxia

100

format $1 /$ " fatal error $\gg$ integer arrays exceed storage',

- allocation $/ 1$ length of 1 a () $=1,16$,

- ' maximum $1 a(1=1,16 / 1$

else

c

CLEAR La ARRAY

do $101=1$, lenta

$1 a(1)=0$

10

continue

end $1 f$

SET POINTERS FOR REAL ARRAYS

ncell $=n 2 p 2 * n \times \max * n c h n$

$n f a c e=n z p 3^{*} n x \max * n c h n$

c

$n$ larg $=\max (n \times \max , n z t+n z p 2+n z b)$

$n r a z c=n r m a x{ }^{*} n a^{*} n z z^{*} n c y l n$

$n r a c=n r \max { }^{*} n{ }^{*} n c y l n$

$n r z c=n r \max * n z * n c y l n$

nac = na*ncyln

$n r c=n r \max { }^{*} n c y l n$

nsaz $=$ nsurf*na*nz

$n m n c=m n r p^{*} n c y l n$

$c$

$1 p k f a c=1$

INNER PRESSURE SOLUTION ARRAY POINTERS (MIDDLE SECTION)

$c$

6

C

c 
c

$c$

c

c

C

c

c

c

INNER RRESSURE SOLUTION ARRAY POINTERS (BOTTOM SECTION)

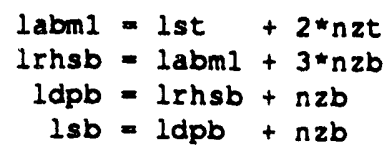

OLD TIME STEP VALUES

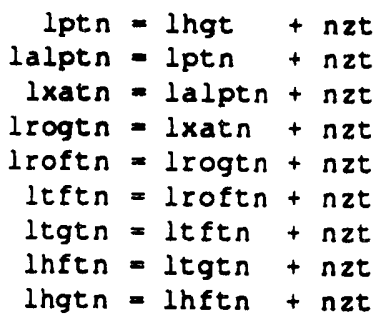


c

c

c

c

c

c

c

c

c

C

c

c

$1 \mathrm{bgb}=l \mathrm{cft}+\mathrm{nzt}$

$1 b f b=l b g b+n z b$

$1 c g b=1 b f b+n z b$

$1 c f b=1 c g b+n z b$

c

c

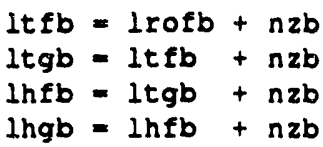


c

c

c

c

c

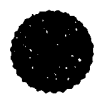

c

ladvet $=$ ladvbt $+5 * n z t$

$1 \mathrm{advbb}=1 \mathrm{advct}+5 * n z t$

$1 a d v c b=1 a d v b b+5 * n z b$

FLUID GEOMETRIC ARRAY POINTERS (MIDDLE SECTION)

$1 \cos x=1 \mathrm{advcb}+5 * n 2 b$

$1 \cos 2=1 \cos x+n \operatorname{cel} 1$

1 acx $=1 \cos 2+n f a c e$

lacz $=$ lacx + ncell

laratx = lacz + nface

laratz = larat $x+$ ncell

laxx = laratz + nface

$\operatorname{laz} 2=\operatorname{laxx}+$ ncell

ldeffz $=$ lazz + ncell

$1 d h x=1 d e f f z+n f a c e$

$1 \mathrm{dhz}=1 \mathrm{dhx}+$ ncell

labsrx $=1$ dhz + nface

labsrz = labsrx + ncell

$\operatorname{lfldmz}=1 \mathrm{absrz}+n f a c e$

$1 f l d c z=1 f l d m z+n f a c e$

$1 \mathrm{cObz}=1 \mathrm{fldcz}+\mathrm{nface}$

$1 \cos z=1 \mathrm{cobz}+$ nface

$1 c k b z=1 c 0 s z+n f a c e$

1 cksz $=1 \mathrm{ckbz}+\mathrm{nface}$

$1 \mathrm{fmltx}=1 \mathrm{cksz}+\mathrm{nface}$

lfmltz $=1 \mathrm{fmltx}+$ ncell 


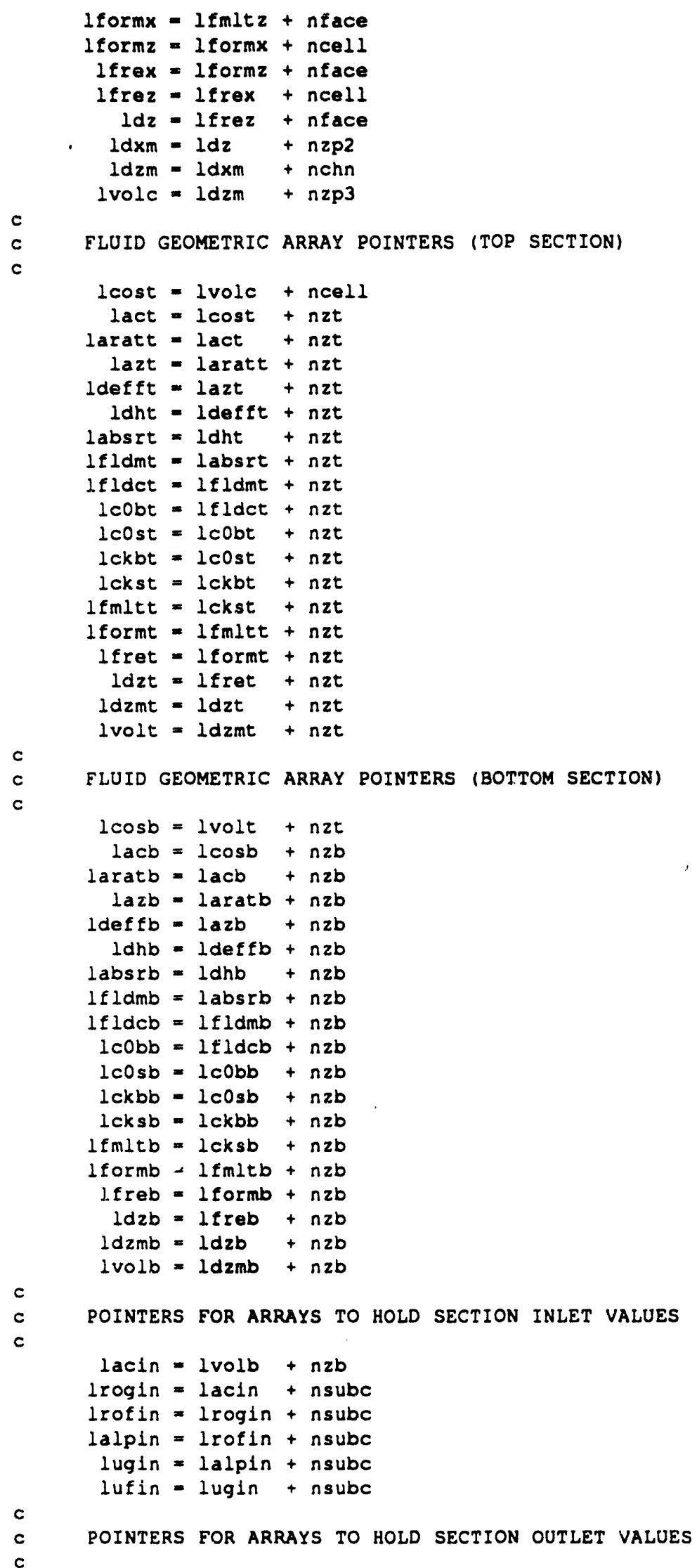




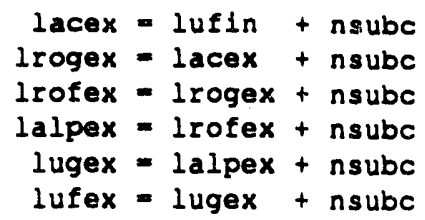

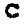

c

c

c

c

c

c

C

c

c

POINTERS TO INTERFACIAL DRAG COEFFICIENT ARRAYS

lcitn = lufex + nsubc

lcixn $=1 c 1 t n+n z t$

$1 c 1 z n=1 c 1 \times n+n f a c e$

$l c i b n=1 c i z n+n f a c e$

POINTERS TO TURBULENT EXCHANGE ARRAYS

$1 t \times g=1 c i b n+n z b$

$1 t \times f=1 t \times g+4 \pi_{n c e l l}$

POINTERS TO INTERFACIAL HEAT AND MASS TRANSFER ARRAYS (MIDDLE SECTION)

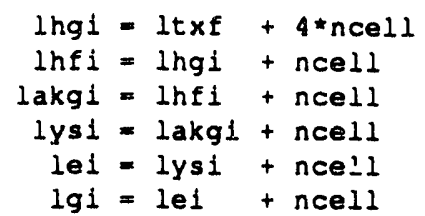




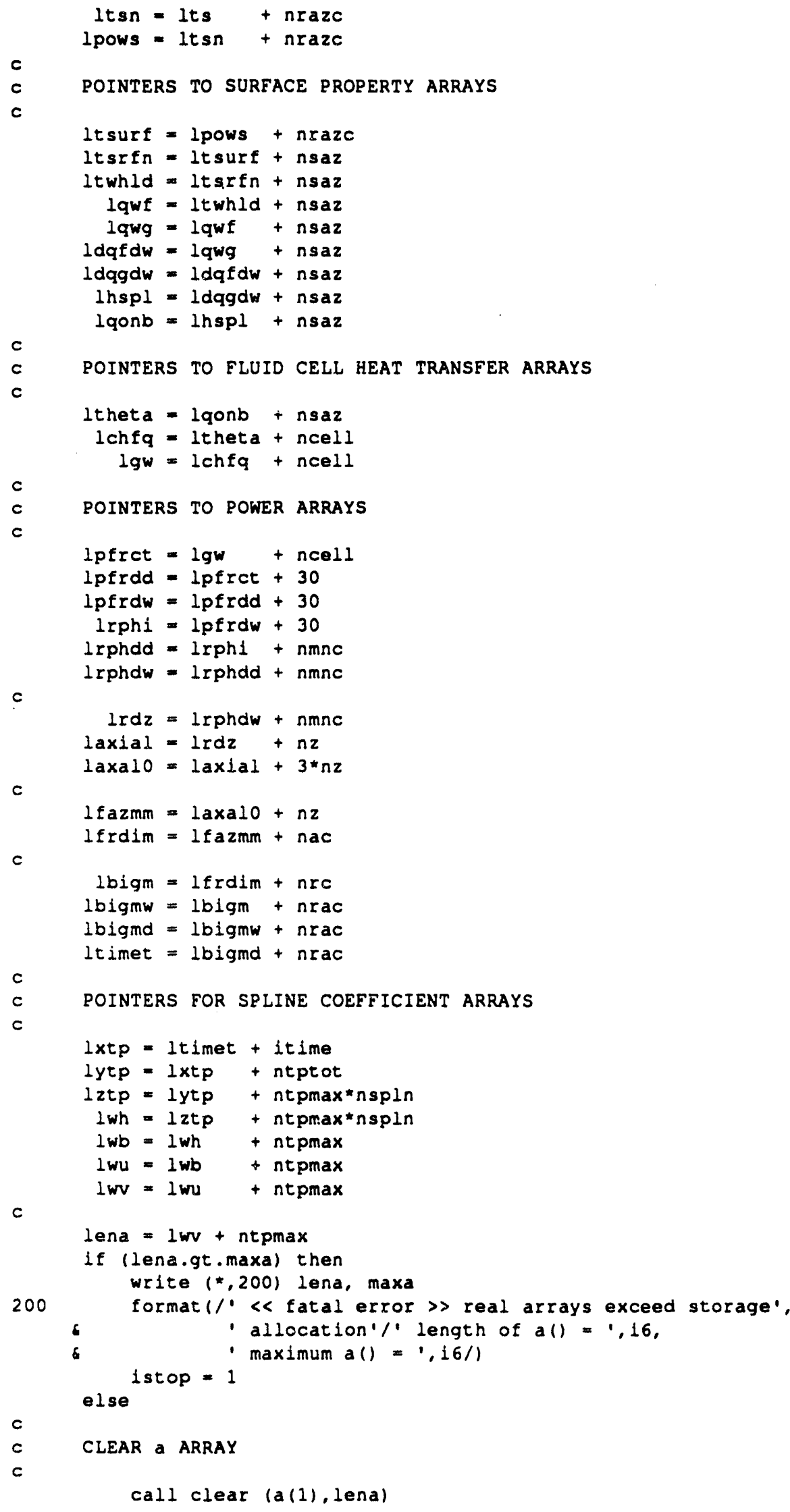

c

c

c

c

c

200

Iwv + ntpmax

If (lena.gt.maxa) then write $(*, 200)$ lena, maxa

lxtp = Itimet + itime

lztp $=1 y t p+n t p \max { }^{*} n s p l n$

Iwh $=12 t p+n t p \max n s p l n$

$1 w b=1 w h+n t p m a x$

lwu $=1 w b+n t p \max$

$I w v=l w u+n t p \max$

lpfrct $=1 \mathrm{gw}+\mathrm{ncel} 1$

lpfrdd $=1$ lpfrct +30

lpfrdw $=1$ pfrdd +30

lrphdd = Irphi + nmnc

laxial $=1 \mathrm{rdz}+n z$

1 fazmm $=1$ axalo $+n z$

lfrdim = lfazmm + nac

$1 \mathrm{bigmw}=1 \mathrm{blgm}+\mathrm{nrac}$

lbigmd = lbigmw + nrac

ltimet = lbigmd + nrac

format $(/$ < fatal error $\gg$ real arrays exceed storage'.

- 'allocation'/ length of a $(1=1,16$,

1 stop $=1$

maximum $a()=1, i 6 /)$

else

CLEAR a ARRAY

call clear (a(1), lena) 


\begin{tabular}{|l|ll|l|}
\hline $2 / 15 / 93$ & WSRC-TR-93-086 Rev.0 & FLOWTRAN-TF v1.2 Source Code & Pg. 245 of 354 \\
\hline
\end{tabular}

end $1 f$

return

end

\section{RONER}

subroutine power (asypwr, tolpow, critr, tcrit, lcrit, lchkc, iredo,

6

ncrit, maxpi, icmax, ipmax, ip, iquit, nout)$$
\text { c }
$$

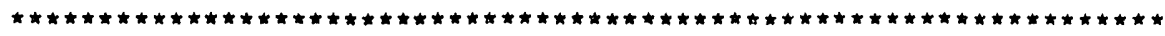

$\star$

* SUBRoUtine power

* subroutine to perform interpolarion to obtain a new guess

* FOR THE PRE-INCIDENT ASSEMBLy POWER AND TO CHECK FOR

* CONVERGENCE OF THE POWER ITERATION SCHEME

implicit real $(a-h, 0-2)$, integer $(1-n)$

cvax double precision $a a, b b, c c, c l, c 2, c 3, c k w, d i s c, d 1, d 2, f 1, f 2, f 3$,

cvax

cvax

p, p1,p2,p3, powest, root 1, root 2, temp,

zerc, half, one, four

parameter $($ zero $=0.0$, hal $f=0.5$, one $=1.0$, four $=4.0)$

parameter (zeros $=0.0$, half $s=0.5$ )

parameter $(\mathrm{pbig}=1.0 \mathrm{e} 12$, I $\mathrm{pwr}=20, c k w=0.001)$

logical iquit

dimension critr(1cmax, ipmax), lcrit (3, 1cmax, ipmax),

c

tcrit (1cmax, ipmax), ichkc(ncrit)

LOCAL ARRAY TO HOLD POWER ITERATION VALUES

dimension p(ipwr)

DO NOT PERFORM POWER ITERATIONS FOR ZERO INITIAL POWER

if (asypwr.eq.zeros) then

write (nout, 250)

iquit = .true.

return

end if

$c$

IF PREVIOUS POWER LEVEL RESULTED IN TSAT OR FLOODING

CRITERIA BEING EXCEEDED THEN REDO THE TRANSIENT

if (iredo.eq.1) then

if (1p.eq.1) then

else

asypwr = halfs*asypwr

end if

asypwr $=$ halfs*(asypwr $+p(i p-1))$

do 5 ic $=1$, nerit

$\operatorname{crttr}(1 c, 1 p)=$ zero

5

continue

return

end if

BEGIN CALCULATION OF MAXIMUM PREINCIDENT POWER:

FIRST UPDATE SOLUTION AND PRINT LAST RESULT

$p(1 p)=$ asypwr

6

write $(*, 200)$ ip-1, $p(1 p) * c k w,(\operatorname{critr}(1 c, 1 p), i c=1, n c r i t)$

(tcrit (ic,ip), ic $=1$, ncrit) 
10

c

$c$

c

20

c

c

c

$c$

c

$c$

c critm $=$ zeros

do 10 lc=l, ncrit

critm $=\max (c r i t m, 1 c h k c(i c) * \operatorname{critr}(1 c, i p))$

continue

CHECK FOR CONVERGENCE OR FAILURE OF ITERATION SEQUENCE

if (maxpi.eq.1) then

write (nout,*) ' no power search requested'

goto 110

else if (abs (critm-one). le.tolpow) then

write (nout, *) ' power search converged'

write $(*, *)$ ' power search converged'

goto 110

else if (ip.gt.maxpi) then

write (nout,*) ' power search falled'

goto 110

else if (1redo.eq. -1 ) then

write (nout, ") 'power search ending' goto 110

end if

powest $=$ pbig

if (ip.eq.1) then

OBTAIN SECOND ESTIMATE OF POWER ASSUMING:

A LINEAR DEPENDENCE FOR TSAT AS RATIO=C*POWER

OR A QUADRATIC DEPENDENCE FOR ONB AS RATIO=C*POWER *2

do 20 ic=1, nerit

if (lchkc(ic).eq.1) then

temp $=p(1) / c r i t r(1 c, 1)$

powest $=\min$ (powest, temp)

end if

continue

else

BEGIN QUADRATIC ITERATIONS FOR POWER

$1 C=1-\rightarrow$ TSAT POWER

$i c=2 \rightarrow$ ONB POWER

do 30 ic $=1$, ncrit

if (ichkc(ic).eq.1) then

p1 = one

$p 2=p(i p-1) / p(1 p)$

$c 1=\operatorname{critr}(i c, i p)$

$c 2=\operatorname{critr}(1 c, i p-1)$

if (ip.eq.2) then

p3 $=$ zero

$c 3=$ zero

else

$p 3=p(1 p-2) / p(1 p)$

end if

$c 3=\operatorname{critg}(1 c, 1 p-2)$

$f 1=c 1 /((p 1-p 2) *(p 1-p 3))$

$f 2=c 2 /((p 2-p 1) *(p 2-p 3))$

$f 3=c 3 /((p 3-p 1) *(p 3-p 2))$

$a a=f 1+f 2+f 3$

$b b=-f 1 *(p 2+p 3)-f 2 *(p 1+p 3)-f 3 *(p 1+p 2)$ 


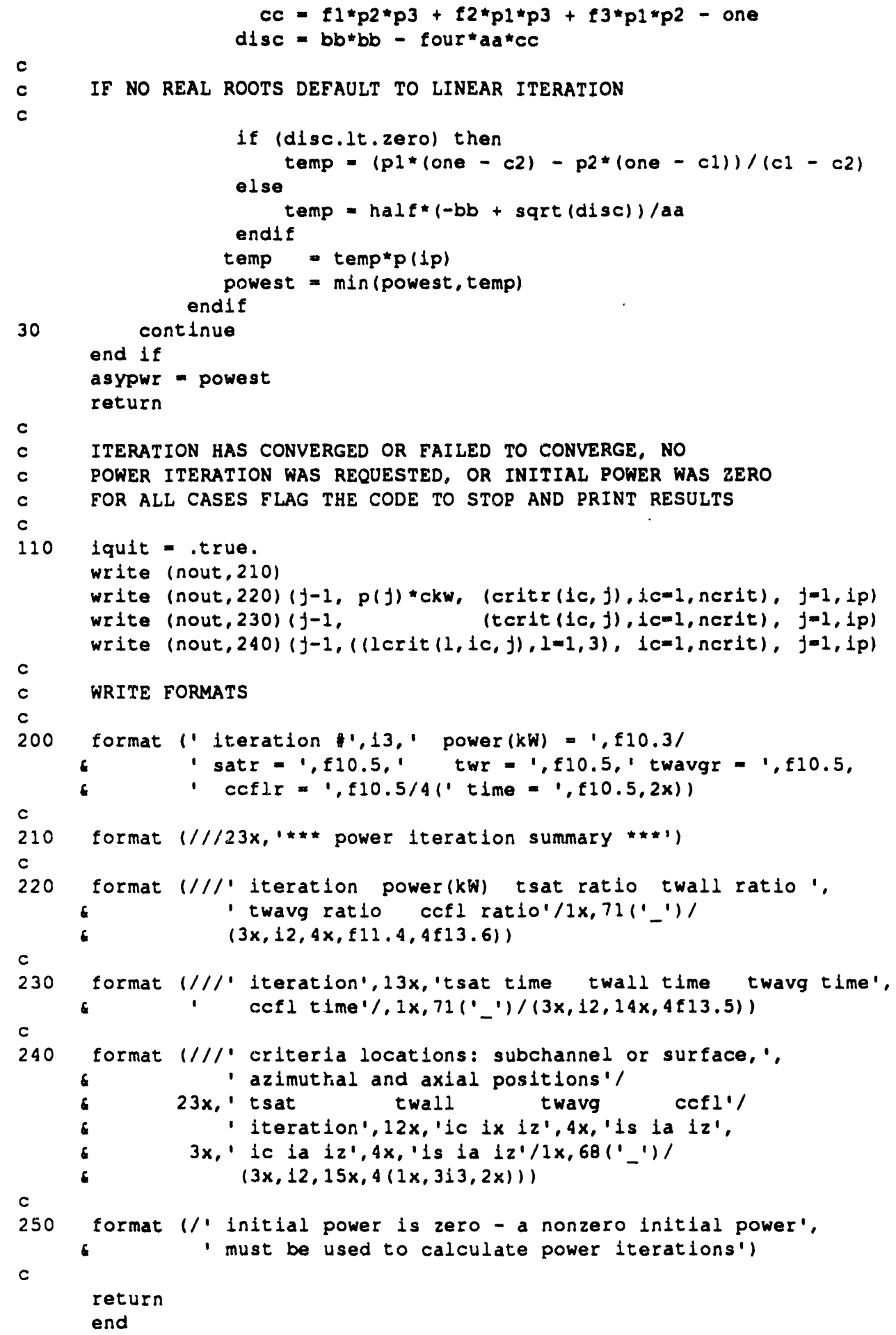

\section{PROPCK}

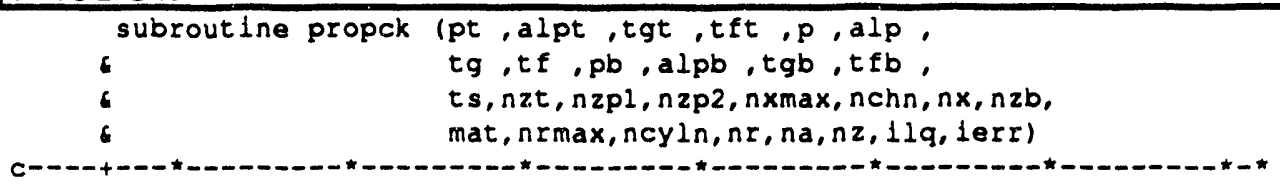




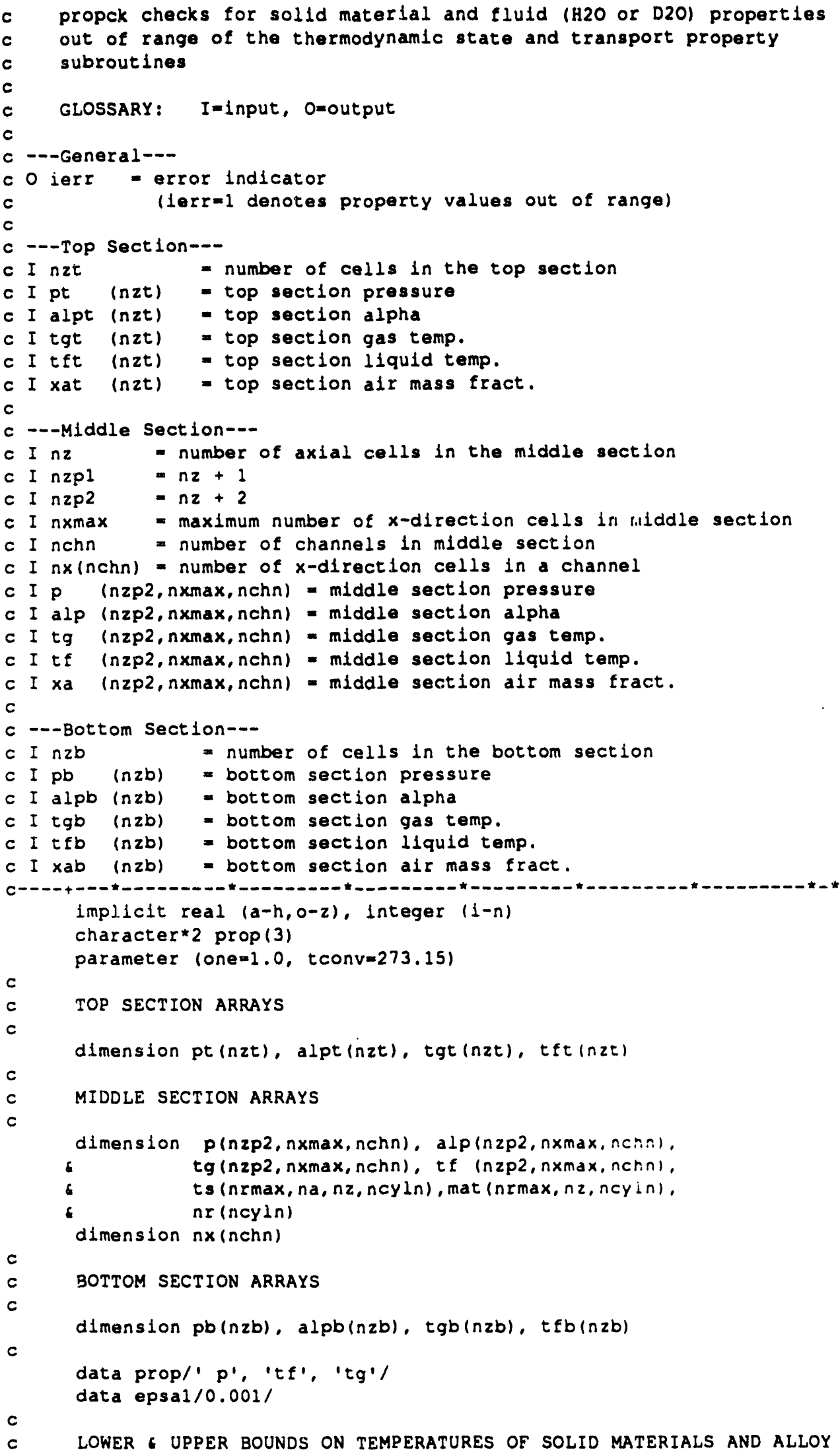




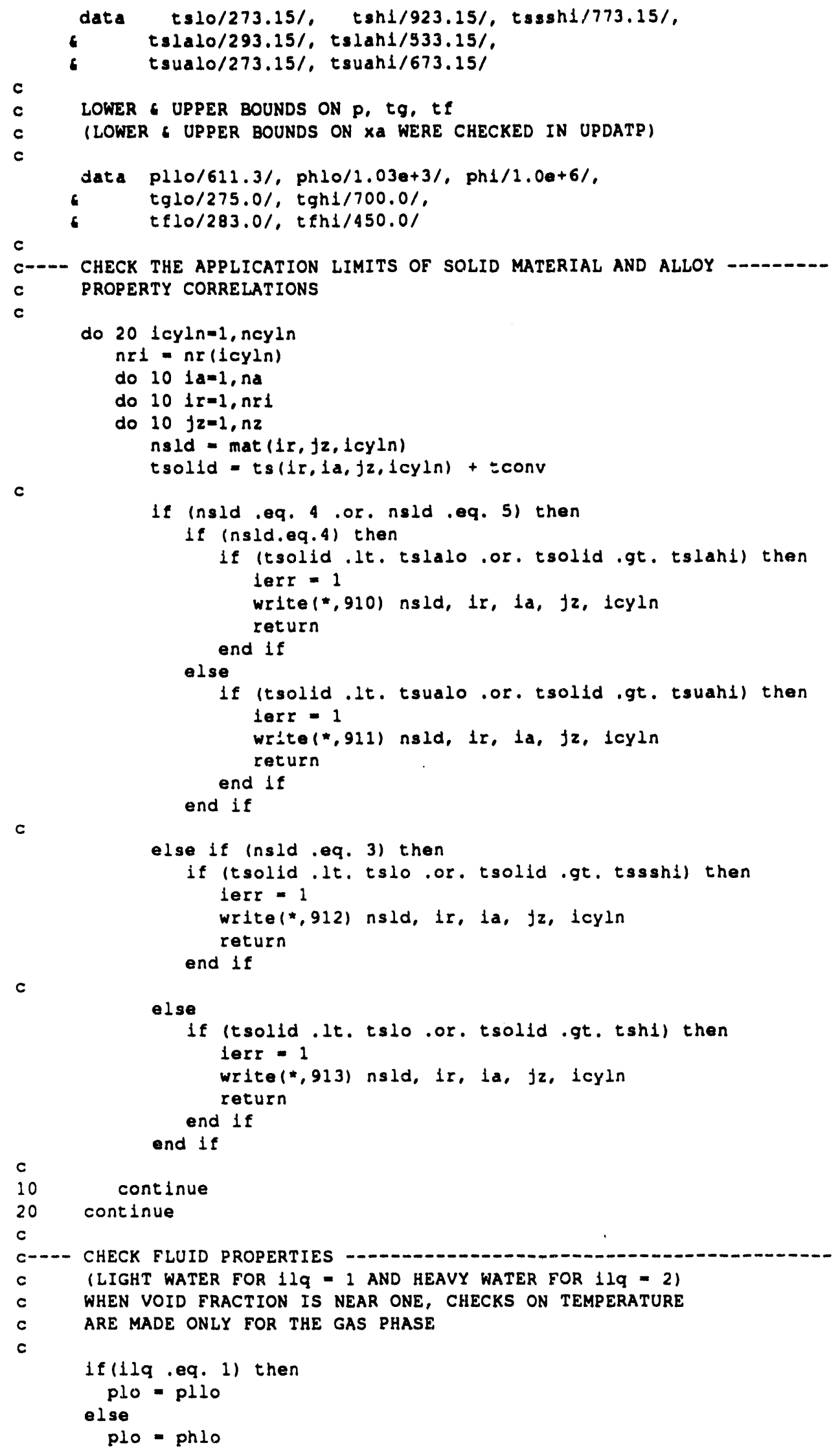




\section{\begin{tabular}{|l|ll|r|}
\hline Pg. 250 of 354 & WSRC-TR-93-086 Rev.0 & FLOWTRAN-TF v1.2 Source Code & 2/15/93 \\
\hline
\end{tabular}}

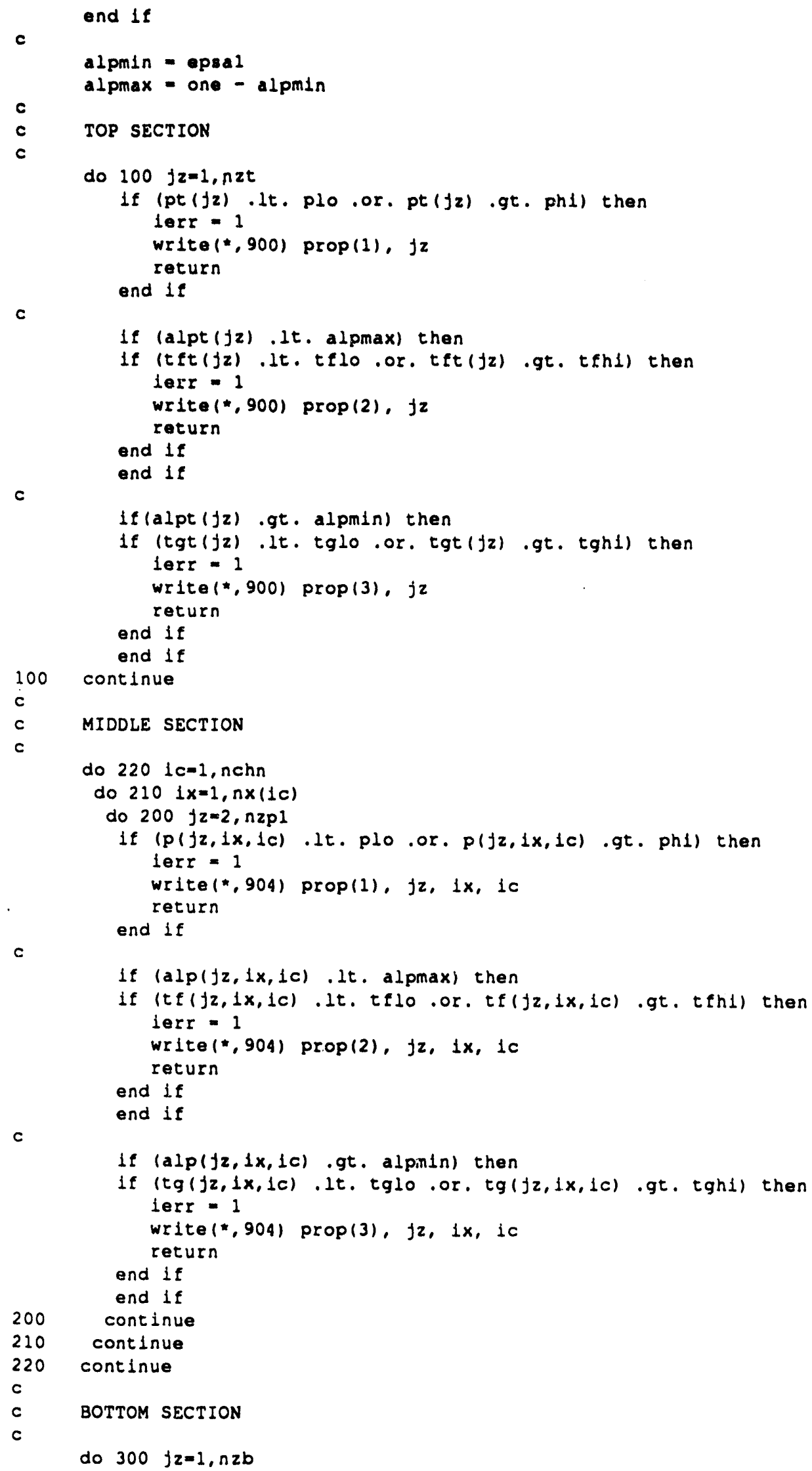




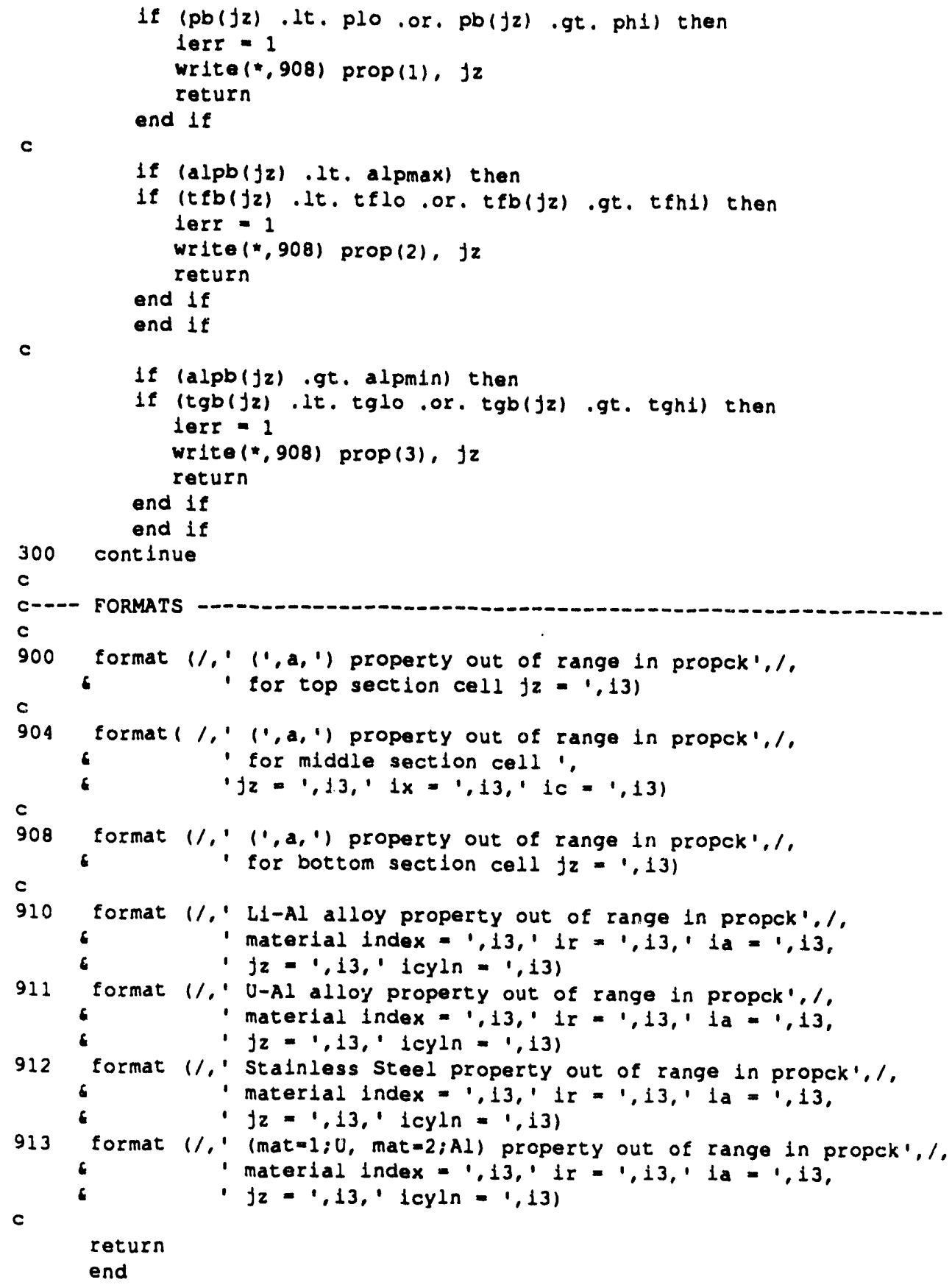

\section{PRTFID}

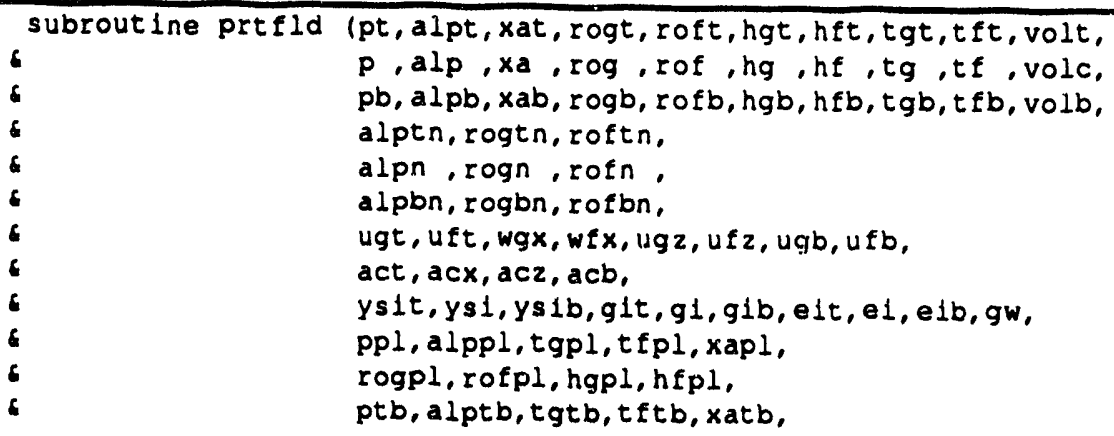




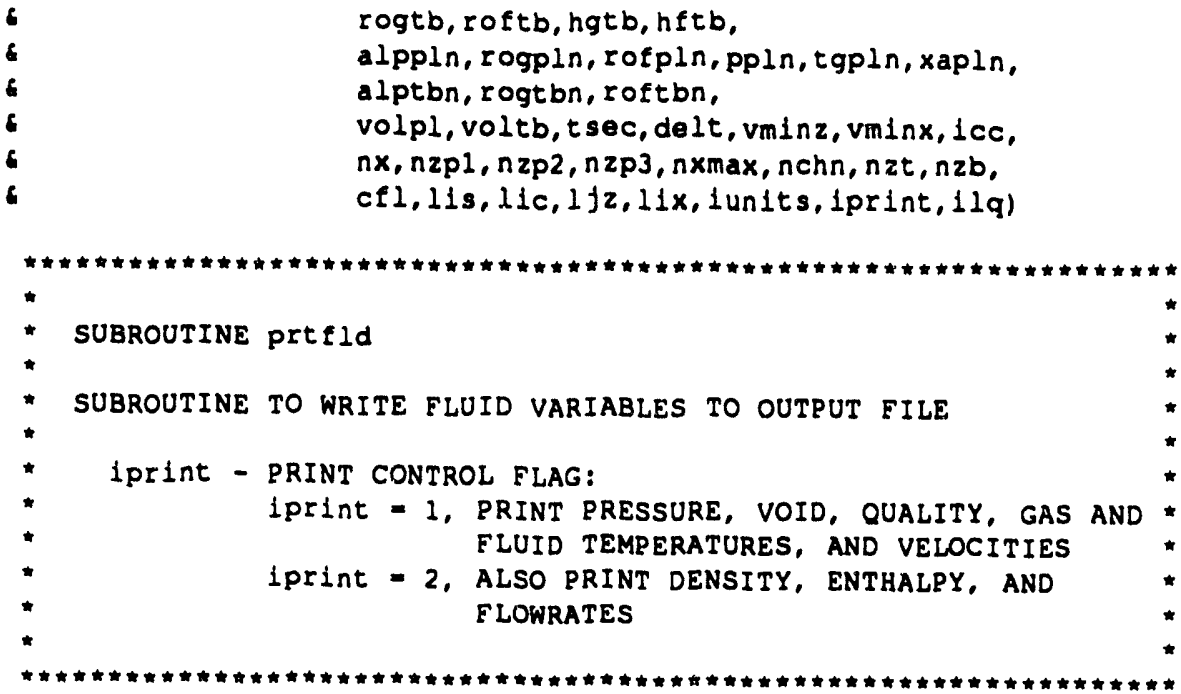

implicit real $(a-h, 0-2)$, integer $(1-n)$

real Jg, ff

logical first

parameter $(c k i 10=0.001$, thousa=1000.0, ccel=273.15)

parameter (zero=0.0, half $=0.5$, one $=1.0)$

parameter (cps $1=6894.757$, cft $=0.3048, c g p m=15850.32)$

parameter $(\operatorname{ccf} m=2.118880 e+03$, pst $p=101325.0$, tst $p=298.15)$

character* 3 top

character* 6 bottom

character 11 plenum, tank

character 6 upres, kpa,psia

character*5 uvelo, mps, fps

character*5 uflow, lps, gpm

c

c

MIDDLE SECTION PHYSICAL PARAMETERS

\begin{tabular}{|c|c|c|}
\hline \multirow{2}{*}{ dimension } & $n \times(n c h n)$, & $p(n z p 2, n \times m a x, n c h n)$, \\
\hline & $\operatorname{alp}(n z p 2, n \times \max , n c h n)$, & $x a(n z p 2, n x \max , n c h n)$, \\
\hline 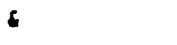 & $\operatorname{rog}(n z p 2, n x \max , n c h n)$, & rof $(n z p 2, n x \max , n c h n)$, \\
\hline 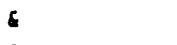 & $h g(n z p 2, n x \max , n c h n)$. & $h f(n z p 2, n x \max , n c h n)$, \\
\hline 6 & tg (nzp2, nxmax, nchn). & tf (nzp2, nxmax, nchn) \\
\hline \& & ug $2(n z p 3, n x \max , n c h n)$, & ufz $(n z p), n x \max , n c h n)$ \\
\hline & wgx (nzp2, nxmax, nchn), & wfx (nzp2, nxmax, nchn), \\
\hline 6 & ei (nzp2, nxmax, nchn), & $\begin{array}{l}g 1(n z p 2, n \times \max , n c h n) \\
g w(n z p 2, n \times \max , n c h n)\end{array}$ \\
\hline 6 & $\operatorname{acz}(n z p 3, n x \max , n c h n)$ & $\operatorname{acx}(n z p 2, n x \max , n c h$ \\
\hline 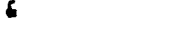 & volc(nzp2, nxmax, nch & $1 c c(2, n x \max , n c h n)$ \\
\hline
\end{tabular}

c

dimension alpn (nzp2, nxmax, nchn), rogn (nzp2, nxmax, nchn), 6 rofn (nxp2, nxmax, nchn)

dimension qmasfi(6)

TOP SECTION PHYSICAL PARAMETERS

\begin{tabular}{|c|c|c|c|}
\hline dimension & pt $(n z t)$, & alpt $(n z t)$, & $x a t(n z t)$ \\
\hline$\&$ & $\operatorname{rogt}(n z t)$ & roft $(n z t)$, & $y \operatorname{sit}(n z t)$ \\
\hline & hgt (nzt) & $h f t(n z t)$, & git $(n z t)$ \\
\hline & $\operatorname{tgt}(n z t)$, & $t \in t(n z t)$, & eit $\ln z t$ \\
\hline 6 & ugt (nzt), & uft (nzt), & \\
\hline
\end{tabular}




\begin{tabular}{|l|l|l|l|}
\hline $2 / 15 / 93$ & WSRC-TR-93-086 Rev.0 & FLOWTRAN-TF v1.2 Source Code & Pg. 253 of 354 \\
\hline
\end{tabular}

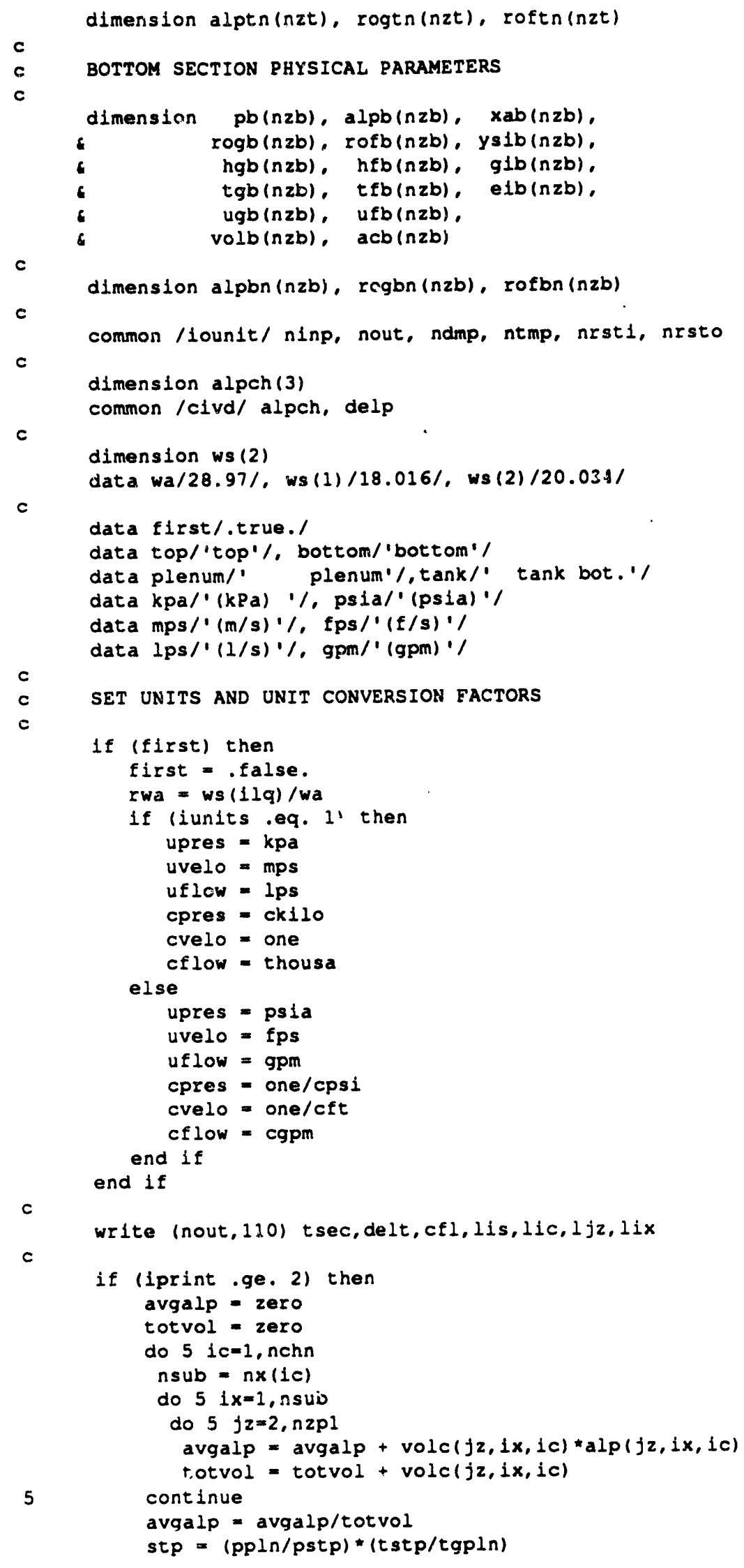


end if

write $(20,370)$ tsec, delt, ppln, tgpln, xapln, avgalp, stp

c

c

c

c

c

$c$

c
WRITE - PRESSURE, VOID FRACTION, QUALITY, GAS TEMPERATURE, FLUID TEMPERATURE, AND ALL GAS AND FLUID VELOCITIES

TOP SECTION

write (nout, 120) top, upres, uvelo, uvelo 


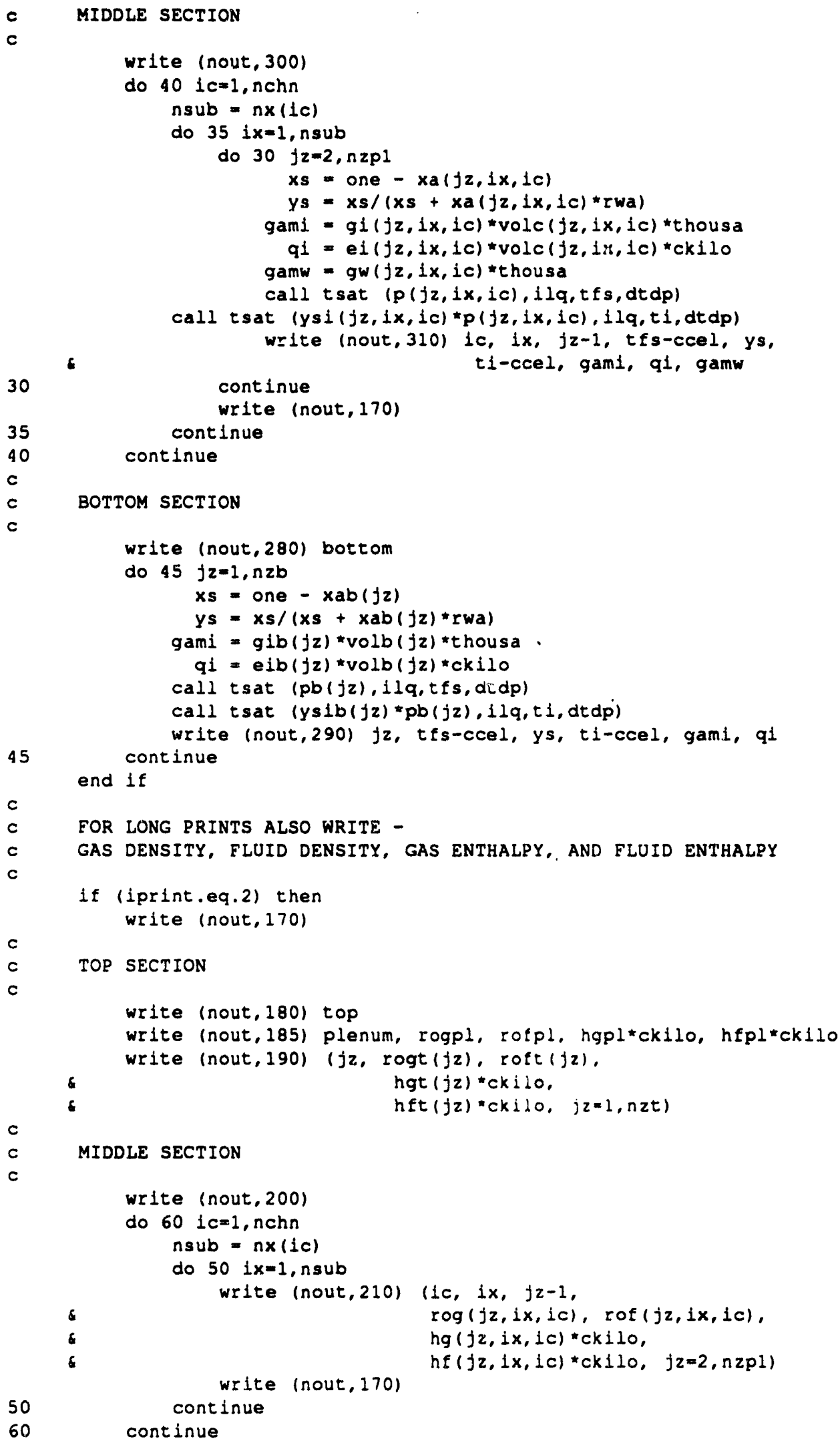


c

c

do $80 \quad j z=2, n z p 2$

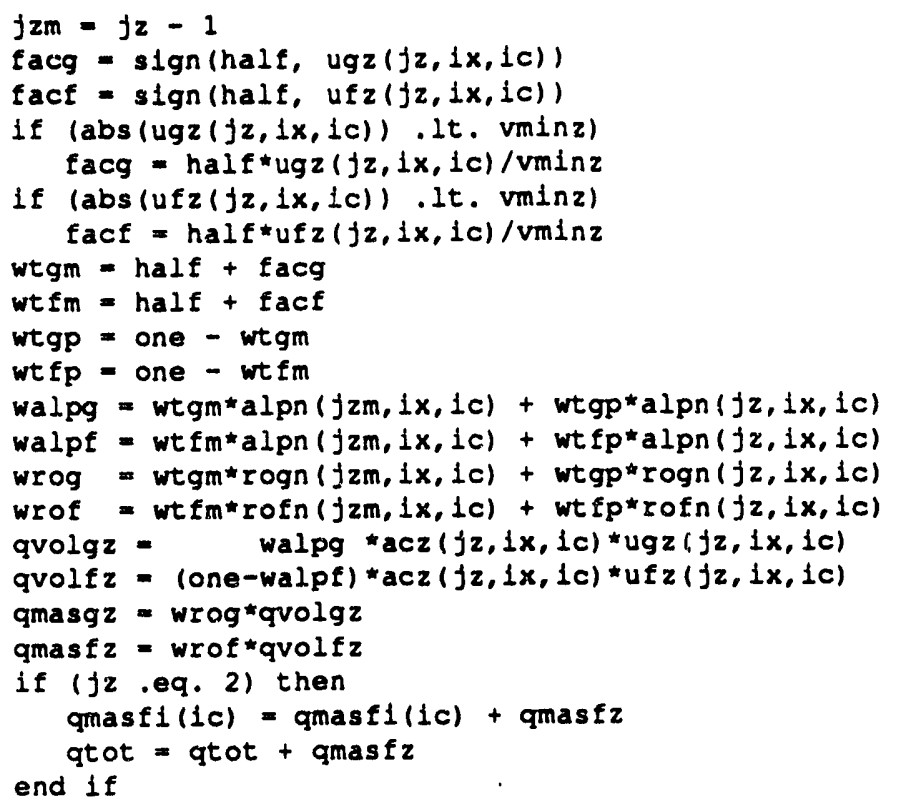
qvolgz $z^{\star c g p m}, q v o l f z$ * cgpm qvolgz*cgpm, quolf $z$ *cgpm

end if 


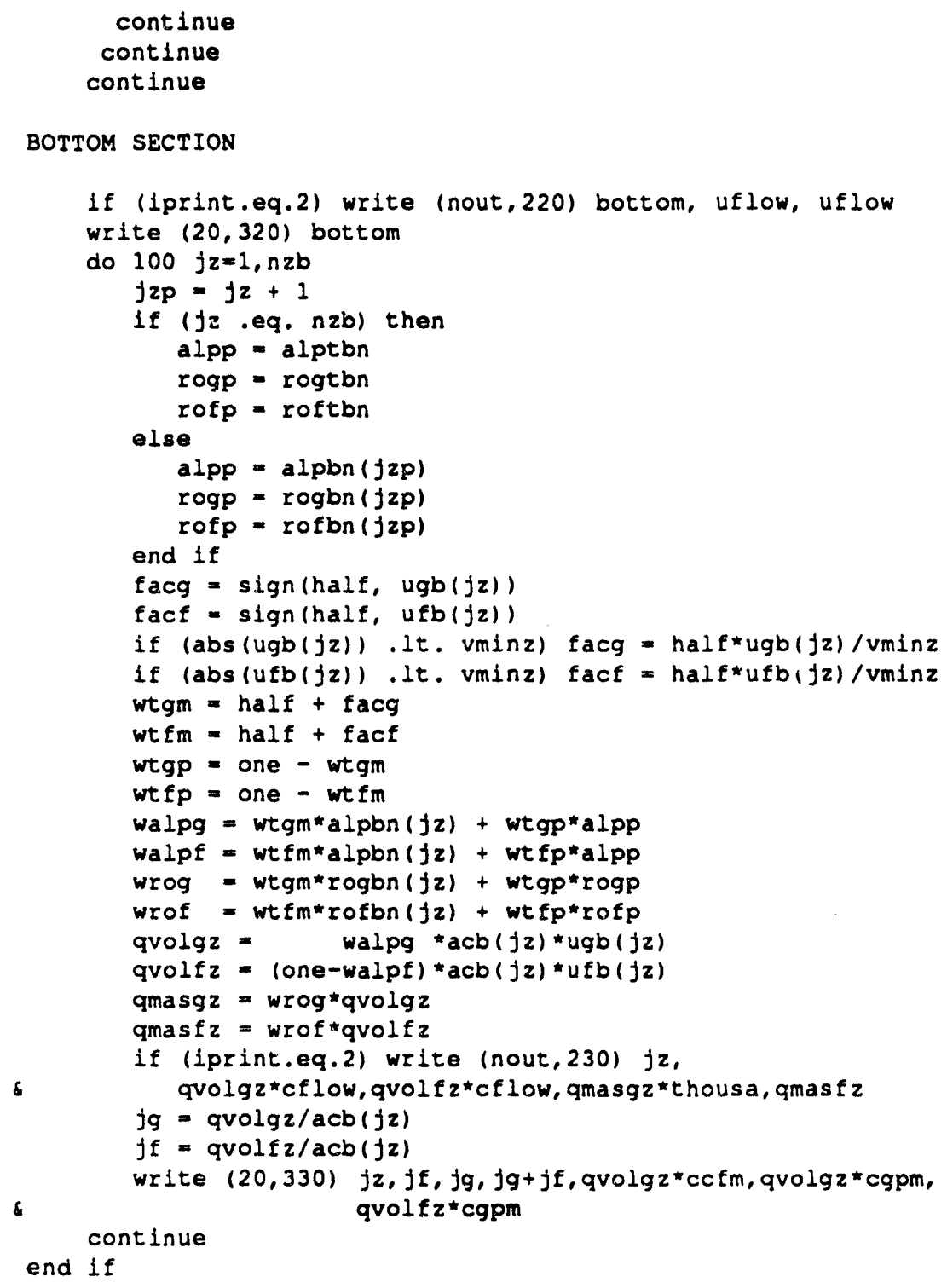



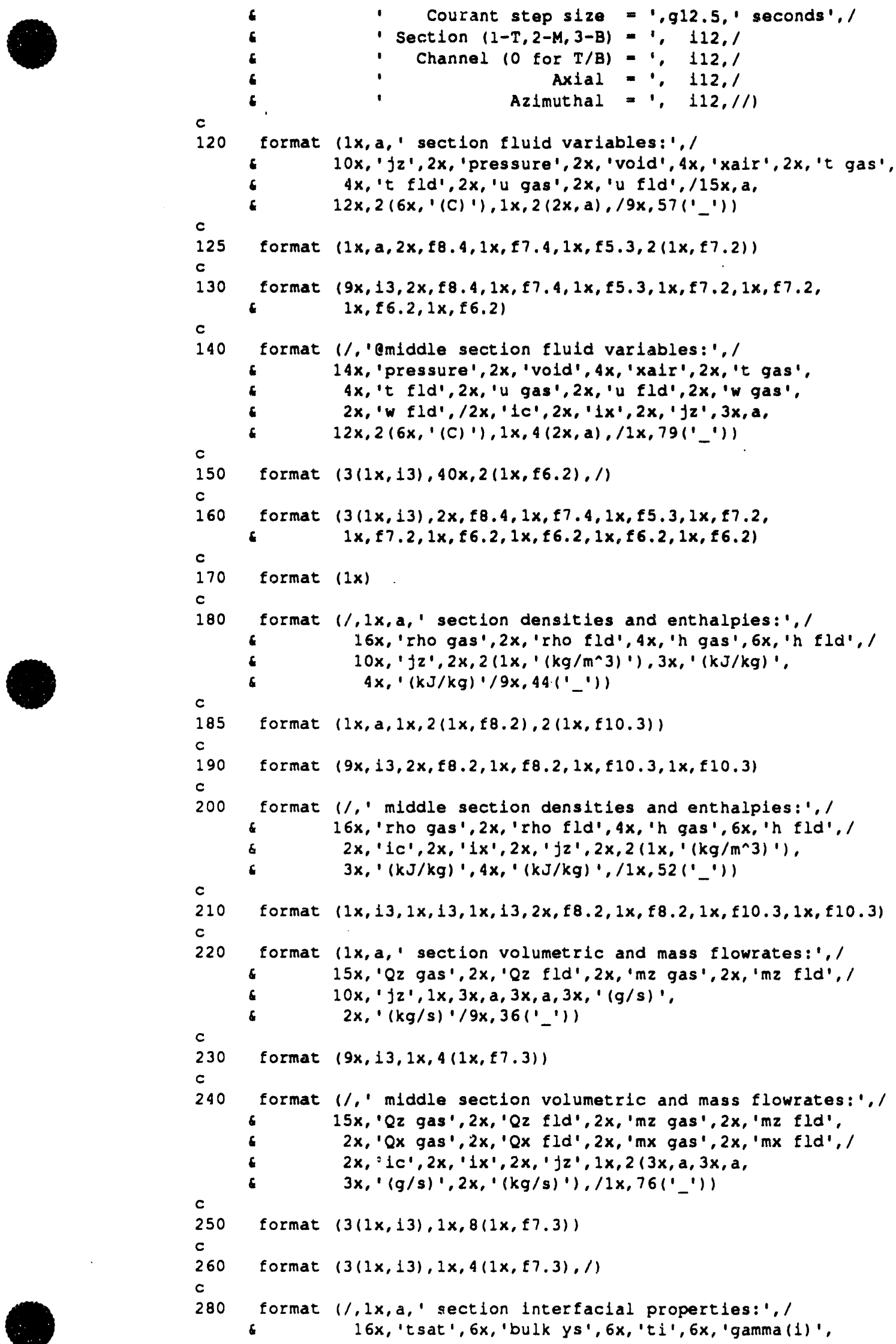


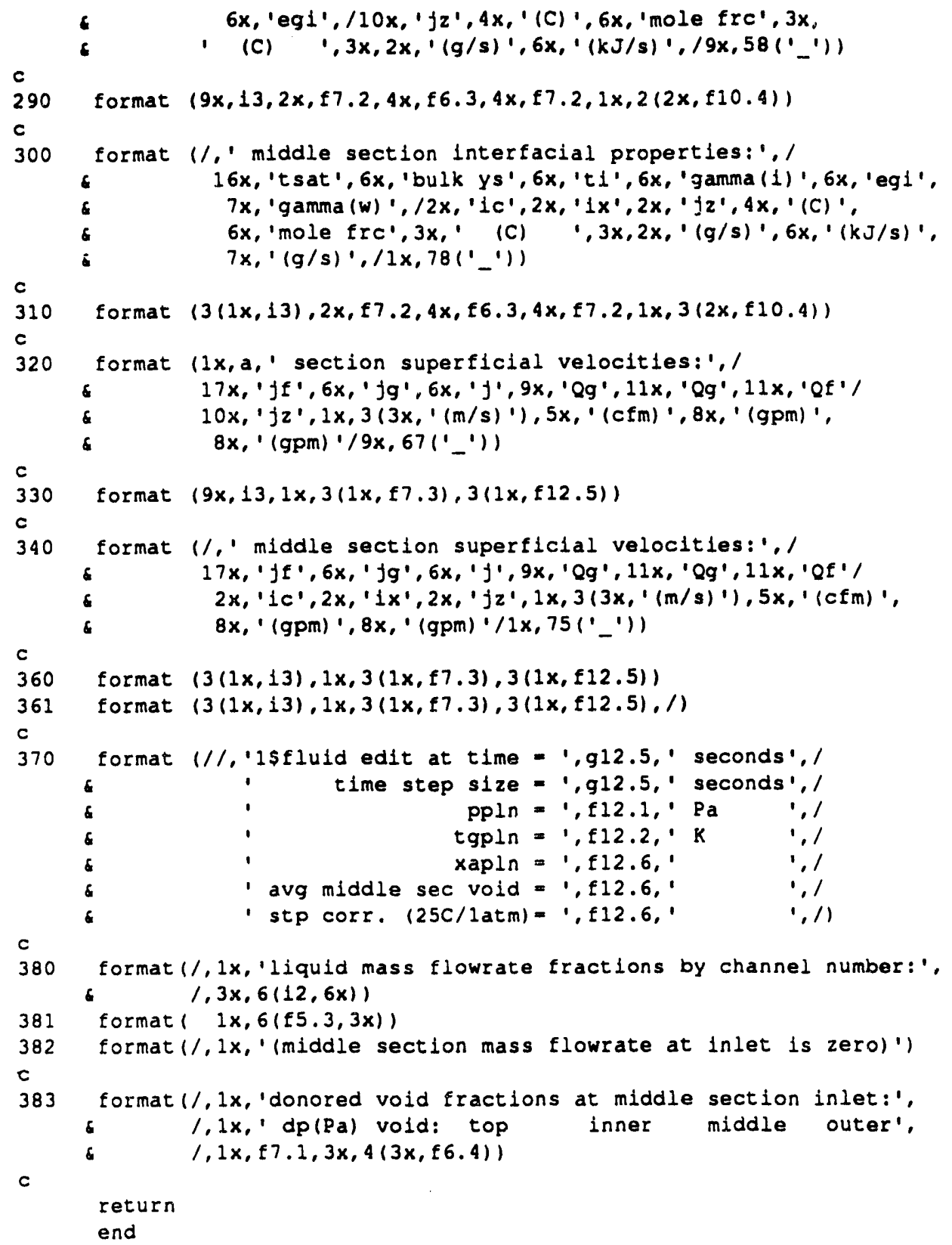




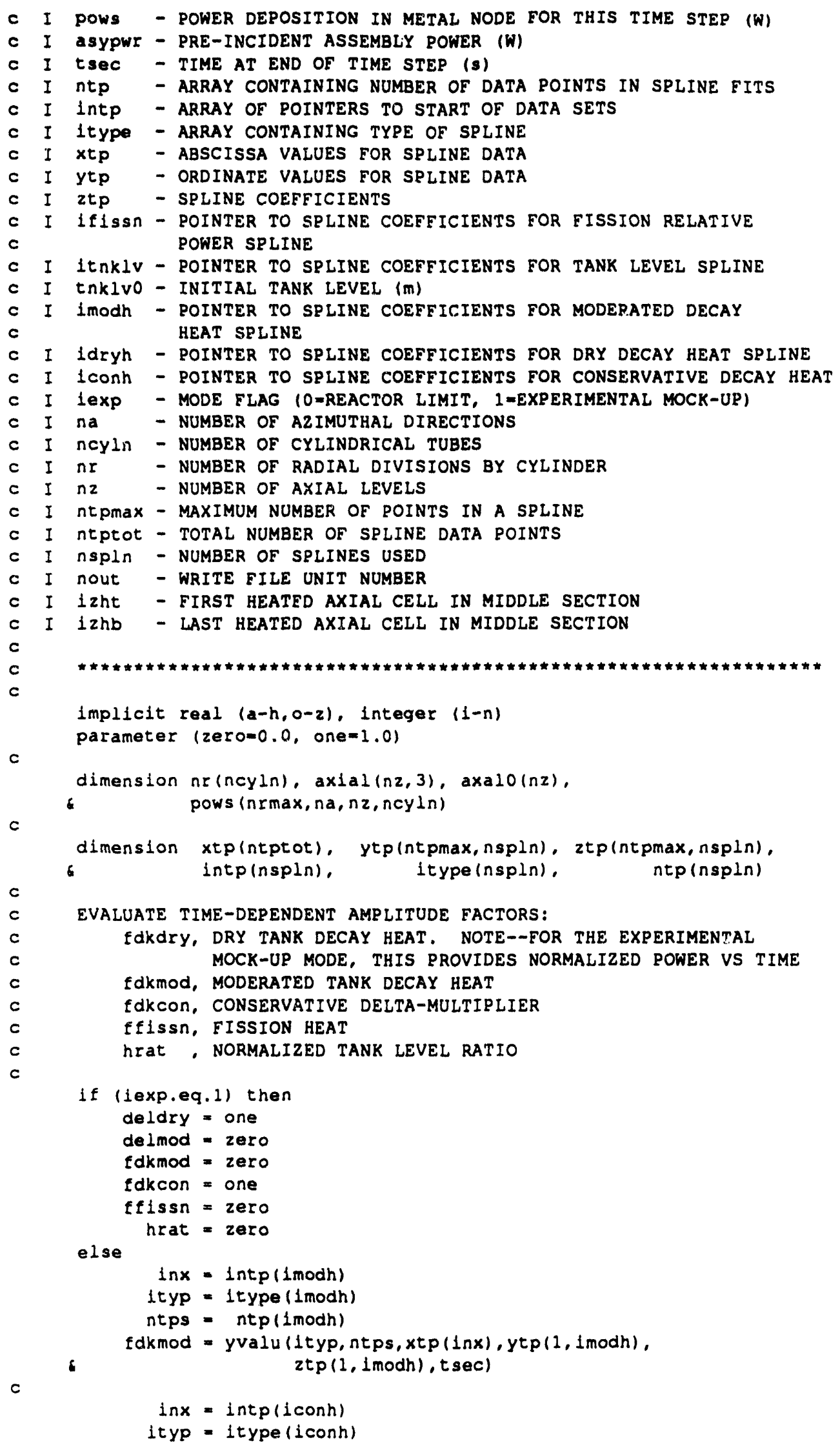




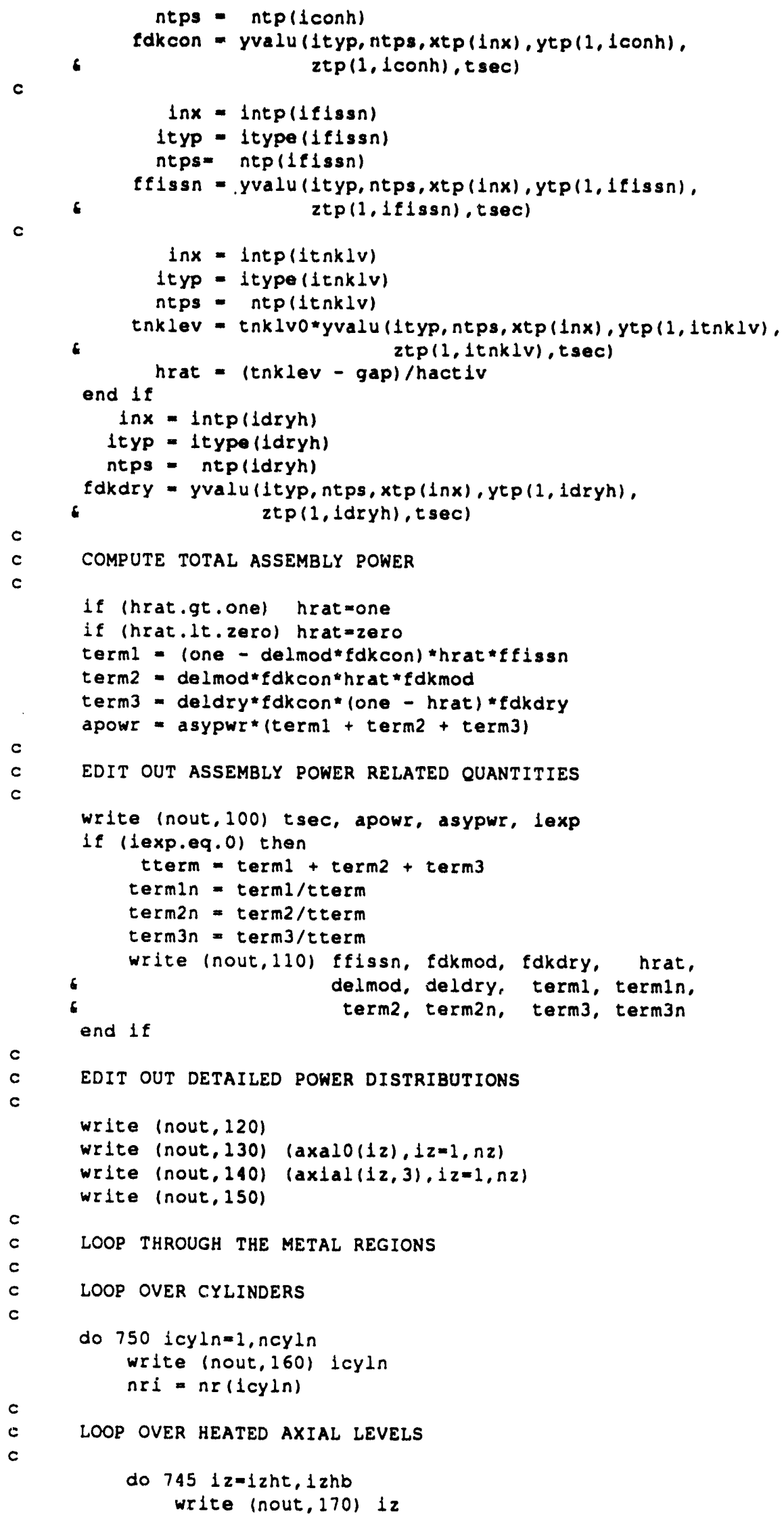


write (nout, 180)

c

c

c

c

740

745

750

c

c

c

100

c

LOOP OVER AZIMUTHAL DIVISIONS

do $740 \quad 1 a=1$, na

LOOP OVER RADIAL DIVISIONS

continue continue

cont inve

WRITE FORMATS

format ('1',20x,' power edit at time $1,912.5, \sec ^{\prime}, / / 1$,

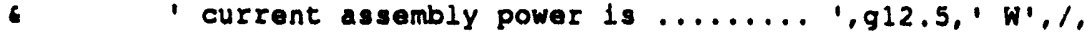

- pre-1ncldent assembly power was ... ', g12.5, W',

- 'mode flag ',12,' (0-reactor limit, 1mexpt mock-up)',/)

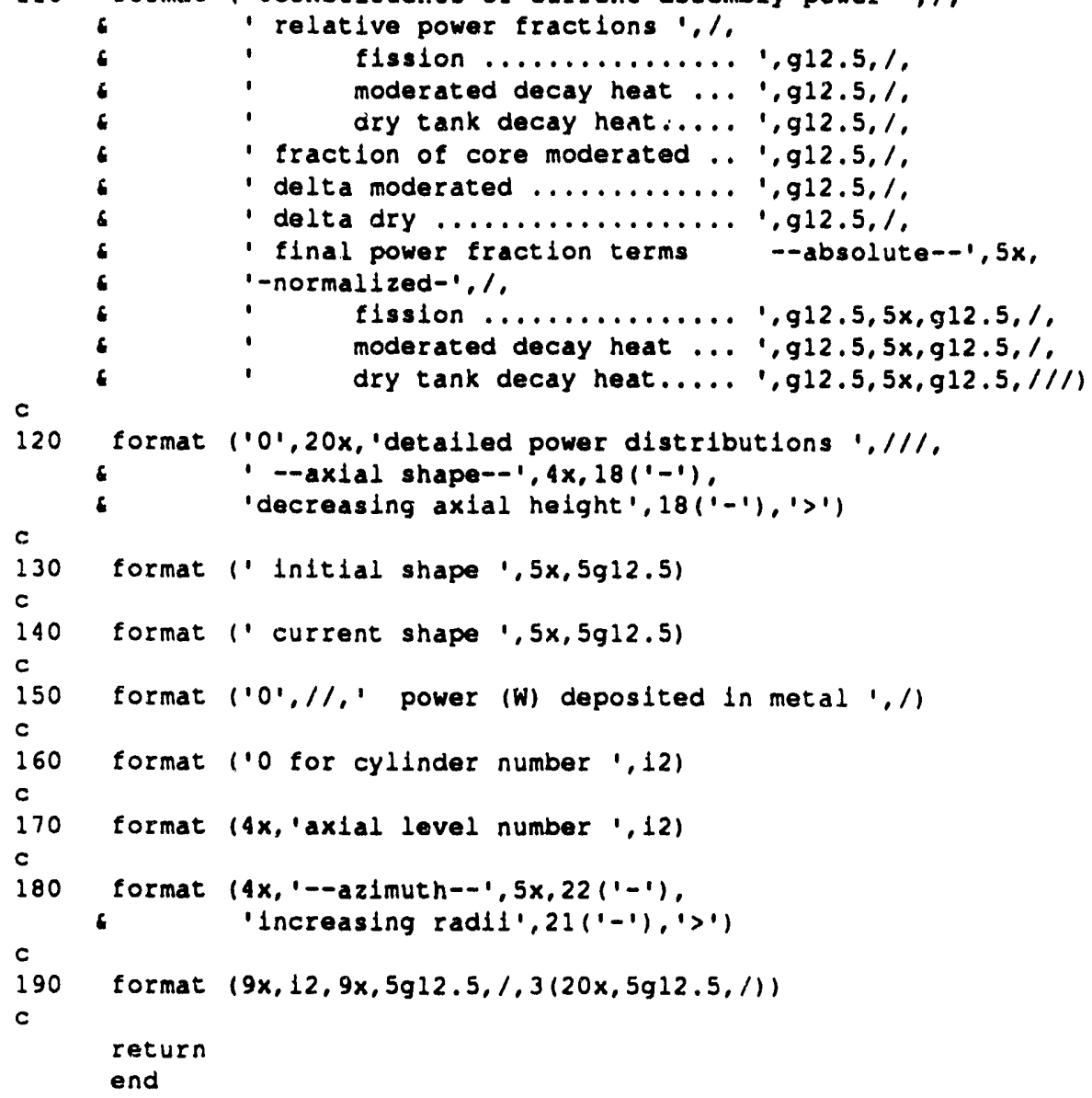

\section{PRT SID}

subroutine prtsid (ts, tsurf, qwf, qwg, tsec, nr, nrmax, na, nz, 1 ncy $\ln$, nsurf) 


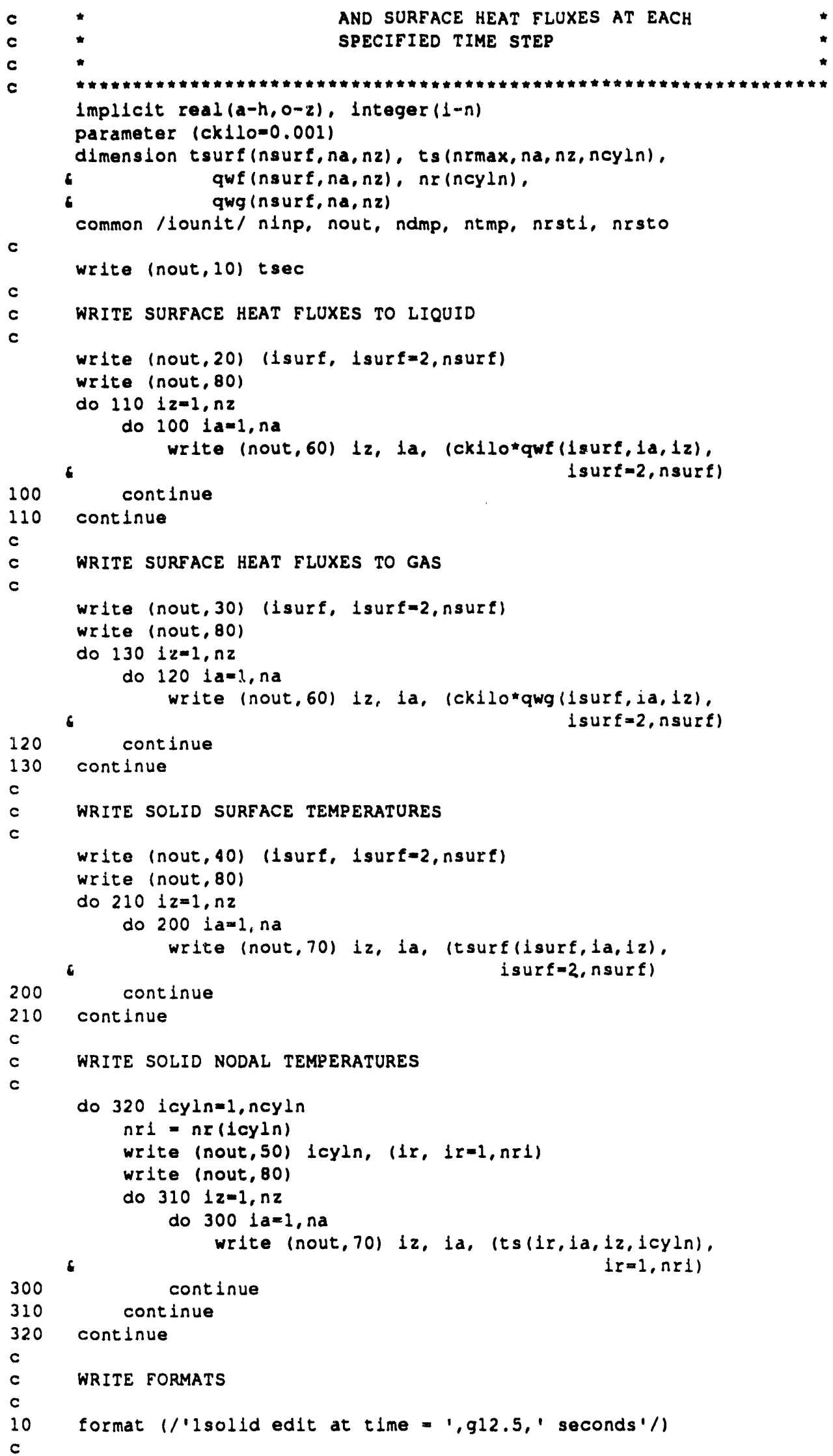




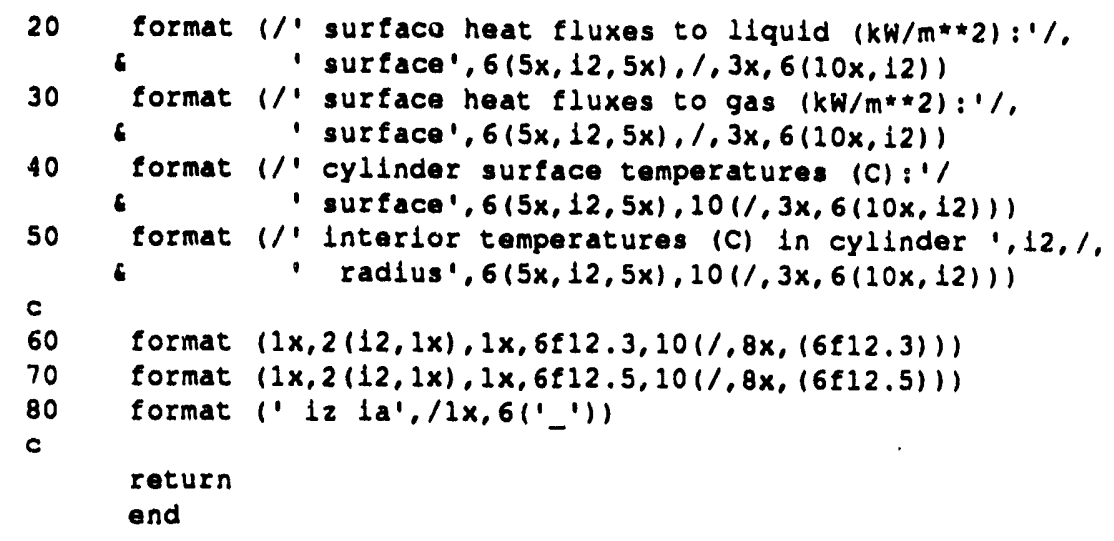

\section{RRTSRN}

subroutine prtsinfpt, alpt, tgt, $t$ ft, xat, rogt, roft, hgt, het,

c p,alp, tg, tf, xa, rog, rof , hg, hf.

6 pb, alpb,tgb,teb, xab, rogb, rofb, hgb, hfb,

- ppl,alppl,tgpl, tfpl, xapl, rogpl, rofpl, hgpl, hfpl, ptb, alptb, tgtb, tftb, xatb, rogtb, roftb, hgtb, hftb, ugt, uft, ugz, ufz, wgx, wf $x, u g b, u f b$,

nzt, nzpl, nzp2, nzp3, nxmax, nchn, nx, nzb, tsec, delt, dtmin, dtmax, nitno, 11tot, dif, difp, difalp, diftg, diftf, difxa, difts, delhm, ysit,ysi,ysib,g1t,g1,gib,

c critr, ncrit, iscrn, twinst, cfl, vminz, act l)

C

parameter $($ zero=0.0, half $=0.5$, one=1.0)

logical first

character 1 blank

character esc

c

dimension critr(ncrit)

TOP SECTION ARRAYS

dimension pt (nzt), rogt(nzt),

a alpt (nzt), hgt (nzt),

c tgt (nzt), roft (nzt).

1 $t \in t$ (nzt), het (nzt),

\& xat (nzt), ysit (nzt), git (nzt)

dimension ugt (nzt), uft (nzt)

c

MIDDLE SECTION ARRAYS

dimension $n \times(n c h n)$

dimension $p$ (nzp2, nxmax, nchn), rog(nzp2, nxmax,nchn),

c

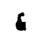

6

$c$

6 6

c

c

c alp (nzp2, nxmax, nchn), hg (nzp2, nxmax,nchn). tg (nzp2, nxmax, nchn), $\operatorname{rof}(n z p 2, n x \max , n c h n)$. tf (nzp2, nxmax, nchn), he (nzp2, nxmax, nchn), xa (nzp2, nxmax, nchn), ys1 (nzp2, nxmax, nchn), g1 (nzp2, nxmax, nchn)

dimension ugz(nzp3, nxmax, nchn), ufz(nzp), nxmax, nchn), wgx (nzp2, nxmax, nchn), wfx(nzp2, nxmax, nchn)

BOTTOM SECTION ARRAYS

dimension $\mathrm{pb}(n z b), \operatorname{rogb}(n z b)$,

- $\quad a l p b(n z b)$, hgb (nzb).

- $\quad t g b(n z b), \operatorname{rofb}(n z b)$.

- $t f b(n z b), h \in b(n z b)$. 
c

6 $\quad x a b(n z b), y s i b(n z b), g i b(n z b)$

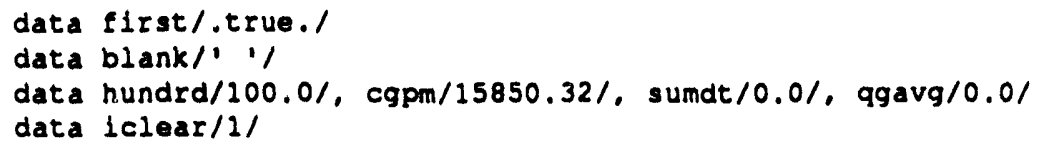




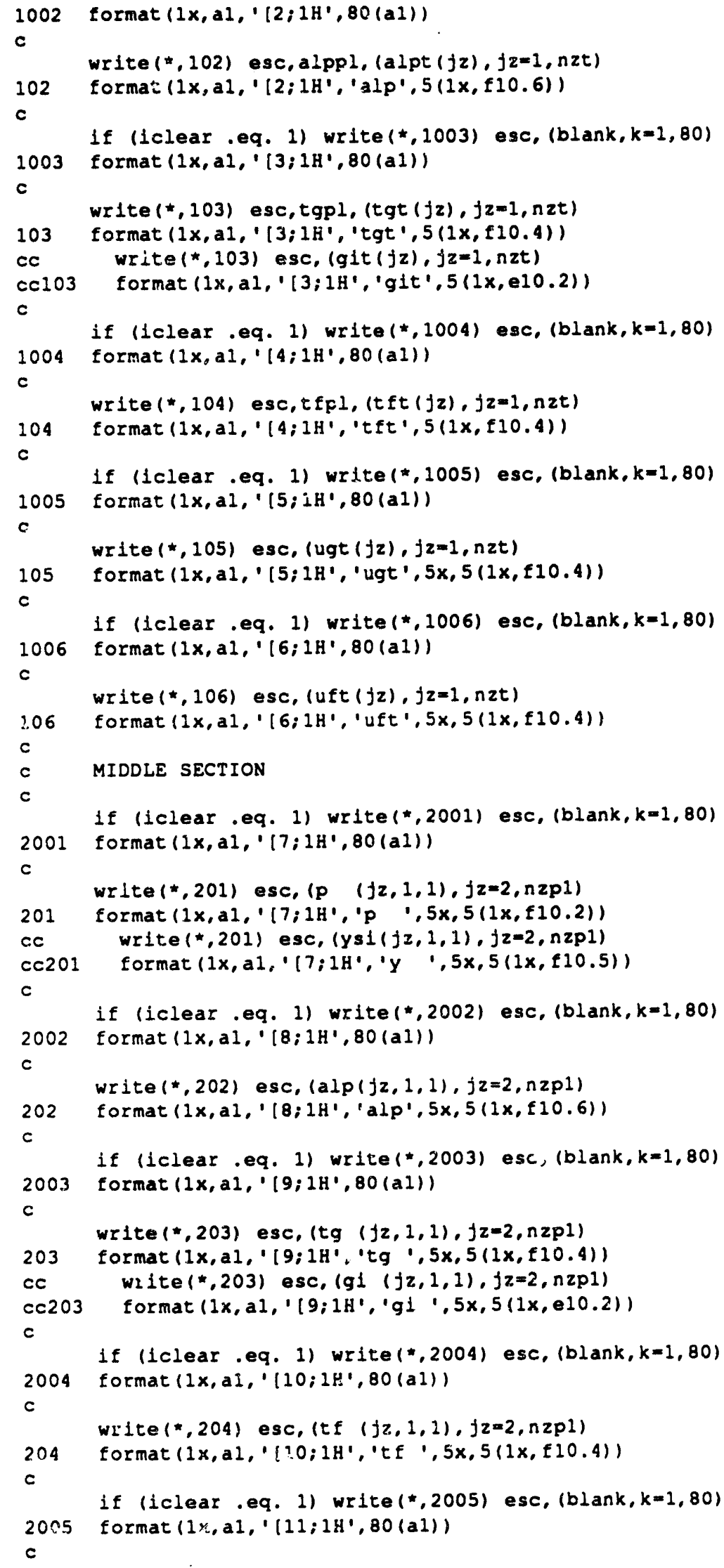




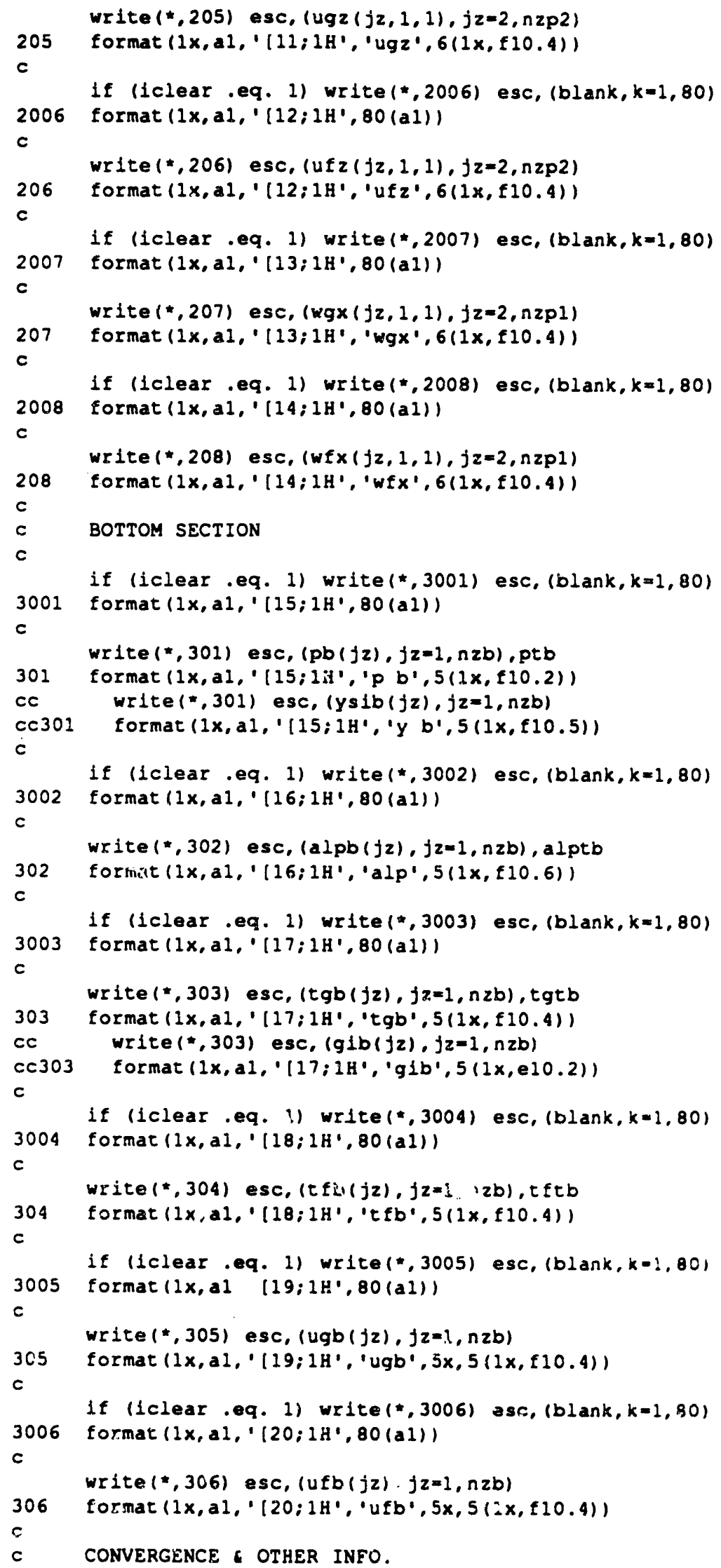




\begin{tabular}{|l|lll|l|}
\hline $2 / 15 / 93$ & WSRC-TR-93-086 Rev. 0 & FLOWTRAN-TF v1.2 Source Code & Pg. 269 of 354 \\
\hline
\end{tabular}

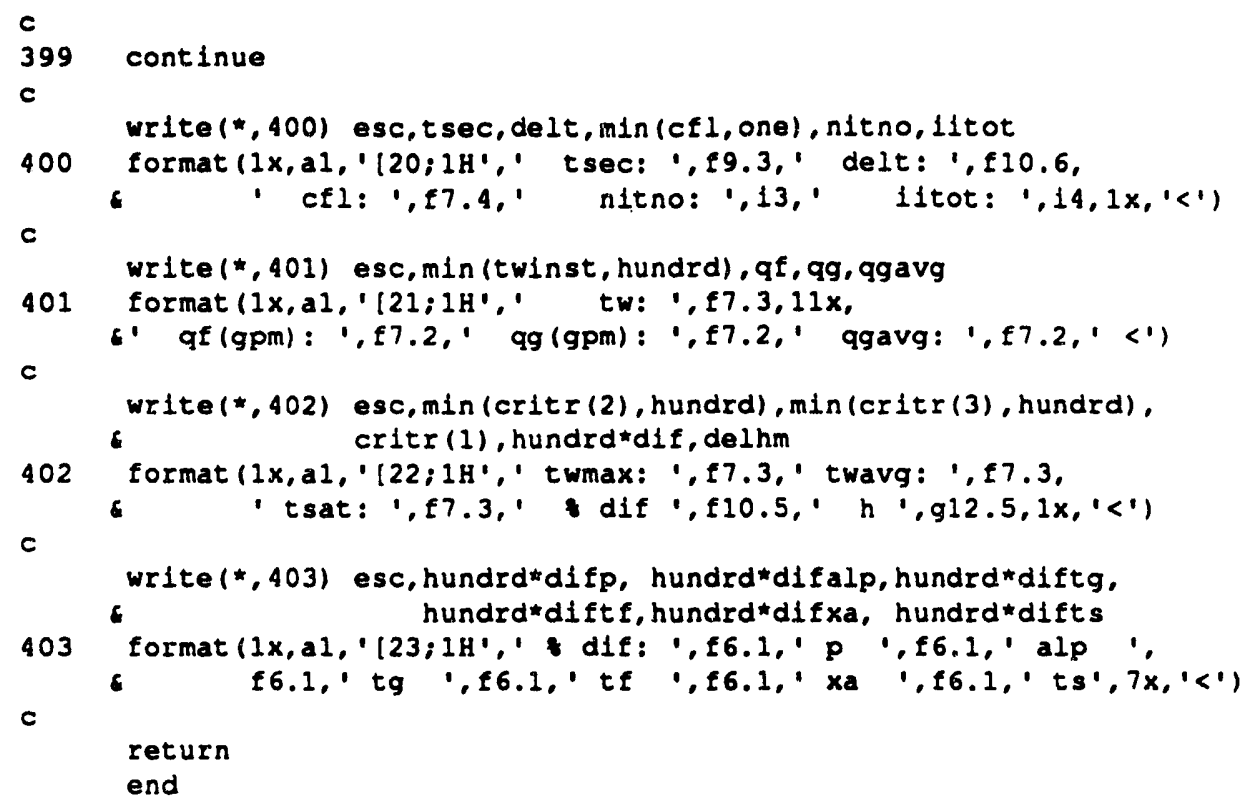

\section{PSAT}

subroutine psat $(t, 1, p s, d p s d t)$

c $\quad$ c

c $\quad$ INPUT:

* $t$ = MIXTURE Gas or LIQUid TEMPERATURE, $K$

* 1 = IDENTIFIER $(1=1$ EOR H2O, $1=2$ FOR D2O)

output:

PS = SATURATION PRESSURE OF H2O OR D2O, Pa

dpsdt = DERIVATIVE OF ps W.R.T. $t$

RANGE:

$273 \mathrm{~K}<t<453 \mathrm{~K}$

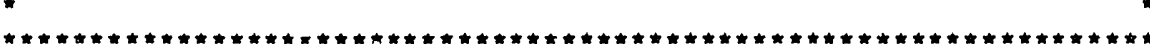

parameter $(t w o=2.0$, three $=3.0)$

dimension $c 1(2), c 2(2), c 3(2), c 4(2)$

H2O PROPERTIES

data cl (1)/-38.874/。 c2 (1)/0.29129/.

6 c3(1) $/-5.7014 \mathrm{e}-4 /$, c4 (1) $/ 4.0606 \mathrm{e}-7 /$

D2O PROPERTIES

data cl (2)/-39.686/, c2 (2)/0.29393/,

6 c3(2)/-5.7036e-4/, c4(2)/4.0230e-7/

$c$

ps $=\exp (c 1(1)+(c 2(1)+(c 3(1)+c 4(1) * t) * t) * t)$

dpsdt $=p s *\left(c 2(1)+\left(t w o * c 3(1)+\left(t h r e{ }^{*} c 4(1)\right) * t\right) * t\right)$

c

return

end

OHALI

subroutine qwall (qwf, qwg, dqfdw, dqgdw, theta, gw, hspl, qonb, chfq, 


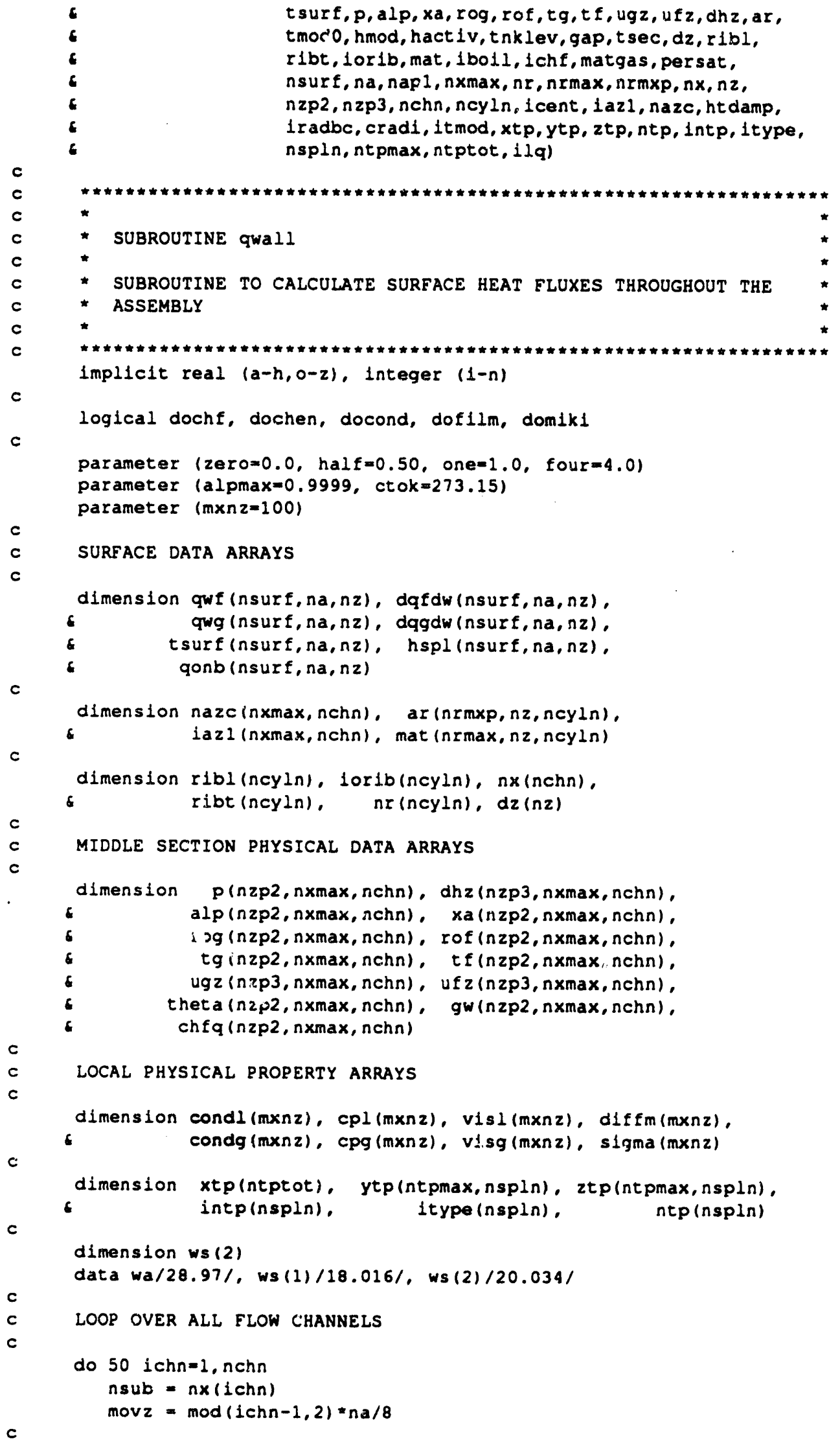




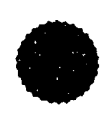

C FIND INNER AND OUTER SURFACE NUMBERS AND CORRESPONDING CYLINDERS,
C BYPASS CALCULATIONS OVER FICTITIOUS SURFACE NUMBR

c

1srfi $=2 * 1 \operatorname{chn}-1+1$ cent

isrfo = isrfi +1

if (isrfi.eq.1) isrfi=isrfo

1 cyl1 = 1srfi - 1chn

icylo = isrfo - ichn

$n r i=n r(i c y l 1)$

$n r p=n r 1+1$

DETERMINE IF RIBS ARE PRESENT IN THE FLOW CHANNEL AND SET THE RIB HALF THICKNESS ( $\mathrm{l}$ ibtk) AND LENGTH ( $\mathrm{I}$ ibln) AND THE EFFECTIVENESS CALCULATION FLAGS FOR INNER AND OUTER SURFACES

if (lorib(1cylo).eq. -1) then

ribln = ribl(icylo)

ribtk = ribt (1Cylo)

leffl $=0$

leffo $=1$

else if (lorib(lcyli).eq.t .and. icyli.ne.icylo) then

ribln = ribl(1cyli)

ribtk $=$ ribt (1cyli)

ieffi $=1$

Leffo $=0$

else

ribln $=$ zero

ribtk $=$ zero

leffl $=0$

leffo $=0$

end if

LOOP OVER ALL AZIMCTHAL SUBCHANNEL POSITIONS

do 40 ix=1, nsub lazf $=1$ azl $(1 x, 1 \mathrm{chn})$

$1 a z l=1 a z f+\operatorname{nazc}(i x, 1 c h n)-1$

CALCULATE PHYSICAL PROPERTIES IN AXIAL FLUID CELLS

call conlaw $(p(1, i x, i c h n), t g(1, i x, 1 c h n), t f(1, i x, i c h n)$ xa (1,ix, ichn), 1lq, 2, nzp2, visg, cpg, condg, diffm.

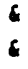
visl, cpl, condl, slgma)

LOOP OVER ALL AXIAL LEVELS

do 30 iz $=1, \mathrm{nz}$

$12 p 1=12+1$

$12 p 2=1 z+2$

dhyc $=\operatorname{half} *(d h z(1 z p 1,1 x, 1 c h n)+\operatorname{dhz}(1 z p 2, i x, 1 c h n))$

DETERMINE FLUID PROPERTIES WITHIN CELL

pres $=p(1 z p l, 1 x, i c h n)$

alfa $=\operatorname{alp}(12 p 1, i x, i c h n)$

$x a 1 r=x a(1 z p l, 1 x, 1 c h n)$

beta = one - alfa

xstm = one - xalr

pstm $=$ pres*xstm/(xstm +xa1r*ws(1lq)/wa)

call tsat (pres, 1lq, tfsat, xdum)

call tsat (pstm,1lq, tssat, xdum) 


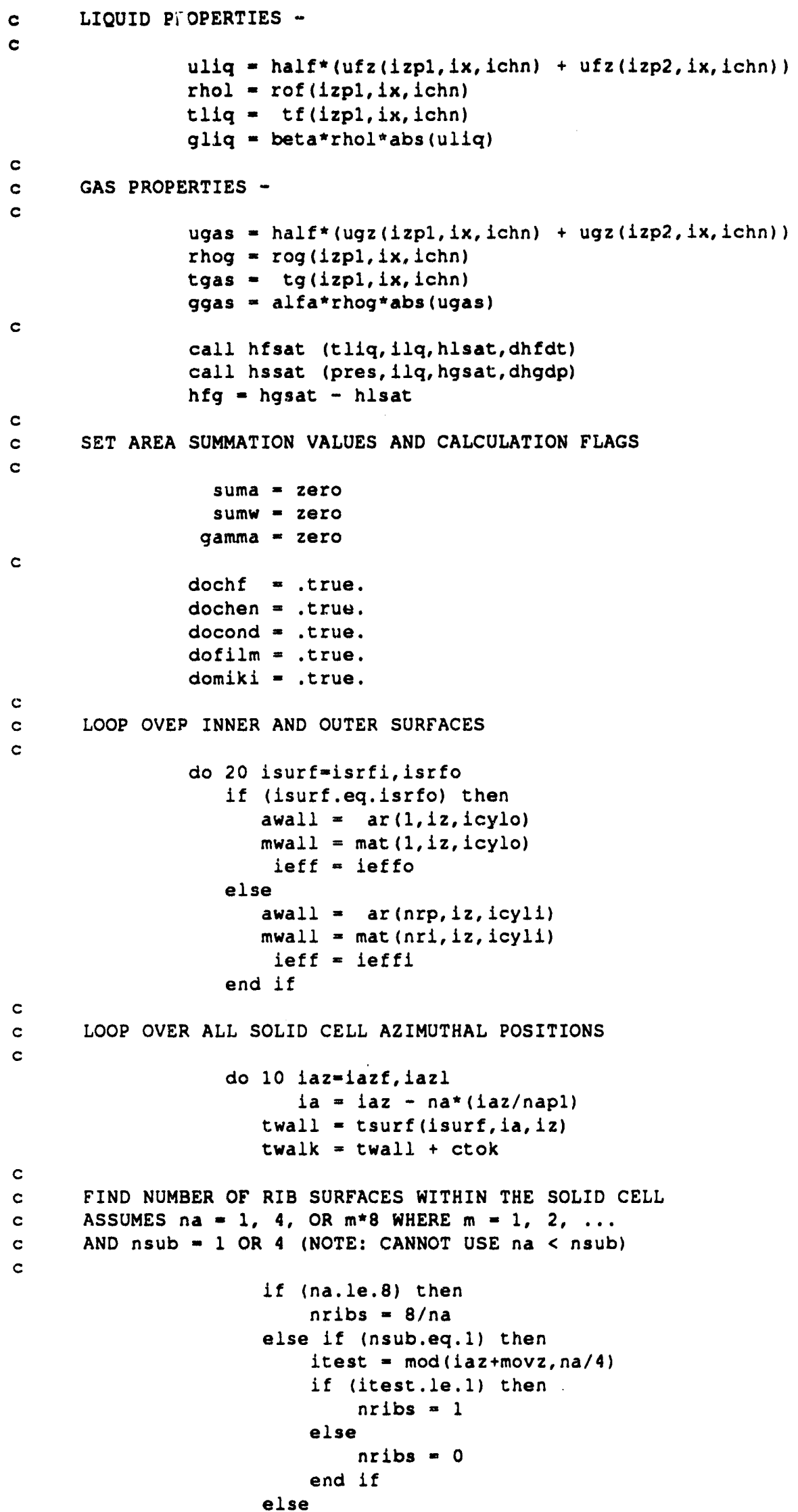




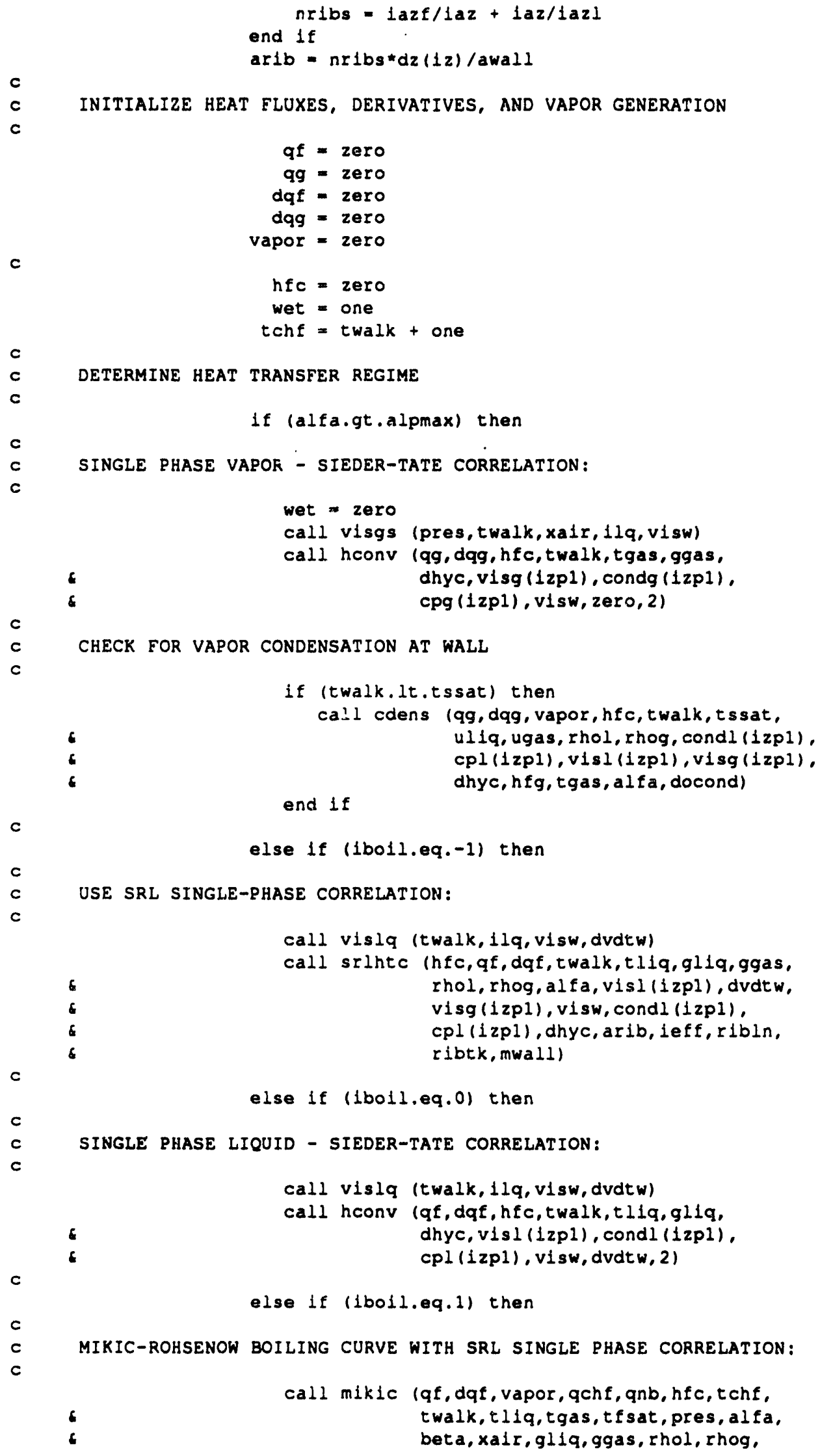


visl (1zpl), visg (1zpl), condl (izpl), cpl (1zp1), dhyc, arib, leff, ribln, ribtk, mwall, hfg, hlsat, hgsat, sigma (1zp1), matgas, persat, dom1k1, doch $f, 1 \operatorname{ch} f, 11 q)$

c

c

c

$c$

c

:

6

6

6

6

๘

6

end if

else

NUCLEATE BOILING - CHEN CORRELATION:

call chen (qf, dqf, vapor, qchf, tchf, hfc, twalk, tl1q, tgas, tfsat, pres, alfa, beta, xalr,gliq, ggas, rhol, rhog, visl(1zp1), visg(izpl), condl (1zpl), $\operatorname{cpi}(1 \mathrm{zp} 1)$, dhyc, hfg, hlsat, hgsat, slgma (12pl). dochen, docht, 1chf, 1lq)

if (twalk.gt.tchf) then

FILM OR TRANSITION BOILING -

BROMLEY-POMERANZ CORRELATION FOR LIQUID AND DOUGALL-ROHSENOW CORRELATION FOR GAS:

call filmb lqf, dqf, qg, dqg, vapor, wet, qchf, tchf, twalk, tilq, tgas, tfsat, pres, alfa, beta, xair, uliq, ugas, rhol. rhog, condg (izpl), cpg (1zpl), visg (izpl), dhyc, hfg, hlsat, hgsat,

end if slgma (izpl), dofilm, ilq)

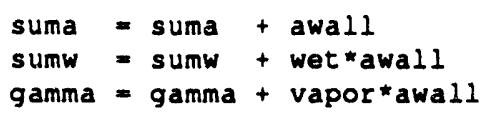

SET UP WEIGHTED HEAT FLUX AND DERIVATIVE ARRAYS AND STORE SINGLE PHASE HEAT TRANSFER COEFFICIENTS

wgtm = one - htdamp

qwf (1surf, $1 a, 1 z)=$ htdamp*qf +

wgtm*qwe (1surf, 1a, iz)

qwg (isurf, ia, 1z)

= htdamp*qg +

wgtm* qwg (isurf, $1 a, i z)$

dqfdw (isurf, ja, iz) = htdamp*dqf +

wgtm dqfiw (1surf, 1a, 1z)

dggdw (1surf, $1 a, 1 z)=$ htdamp*dqg +

wgtm*dqgdw (1surf, ia, 1z)

$h \operatorname{spl}(1$ surf, $1 \mathrm{a}, 1 \mathrm{z})=$ htdamp*hfc+

wgtm*hspl (1surf, ia, iz)

qonb(1surf, $1 a, 1 z)=$ htdamp qnb +

wgtm*qonb (isurf, $1 \dot{a}, i z)$

continue

continue

CALCULATE FRACTIONAL WETTED SURFACE AREA IN FLUID CELL, ALSO STORE WALL VAPOR GENERATION AND CHF TEMPERATURE

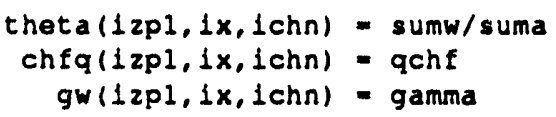




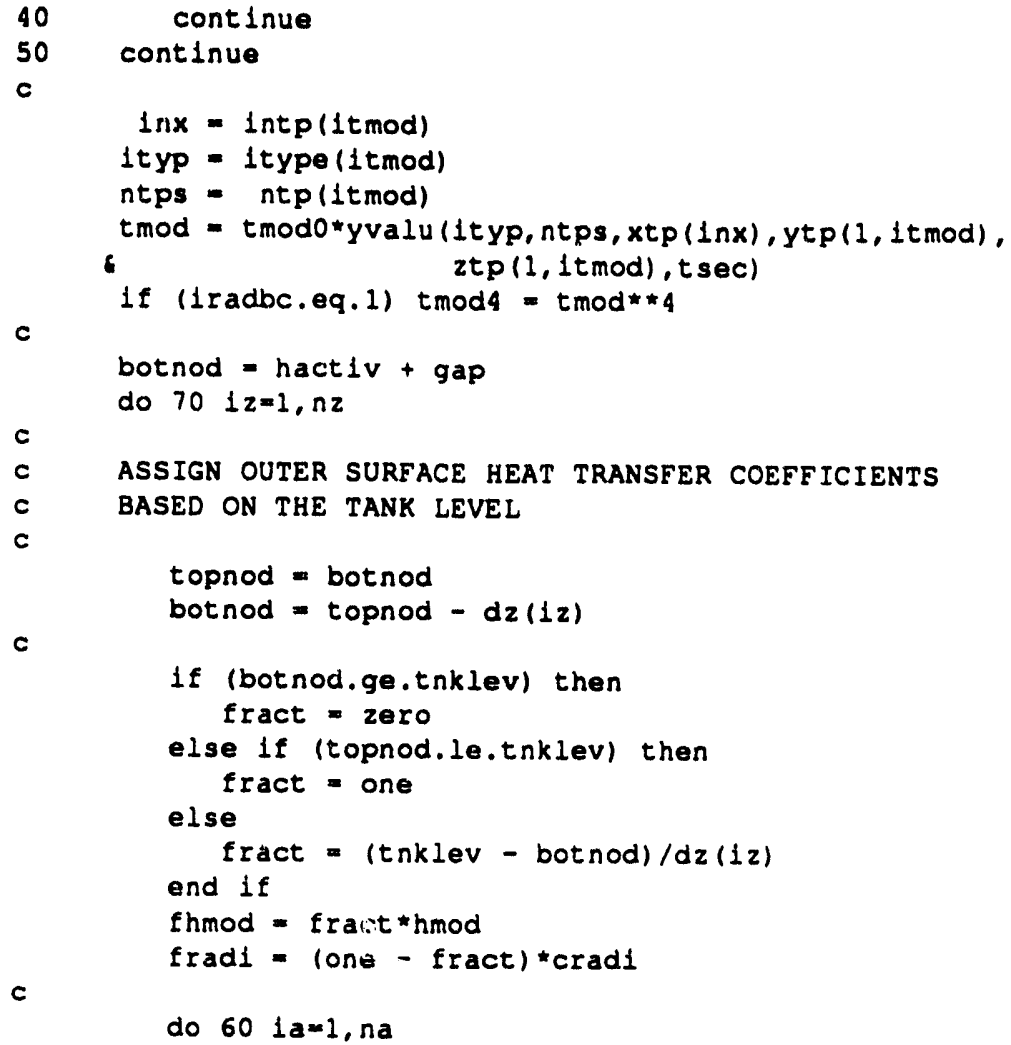




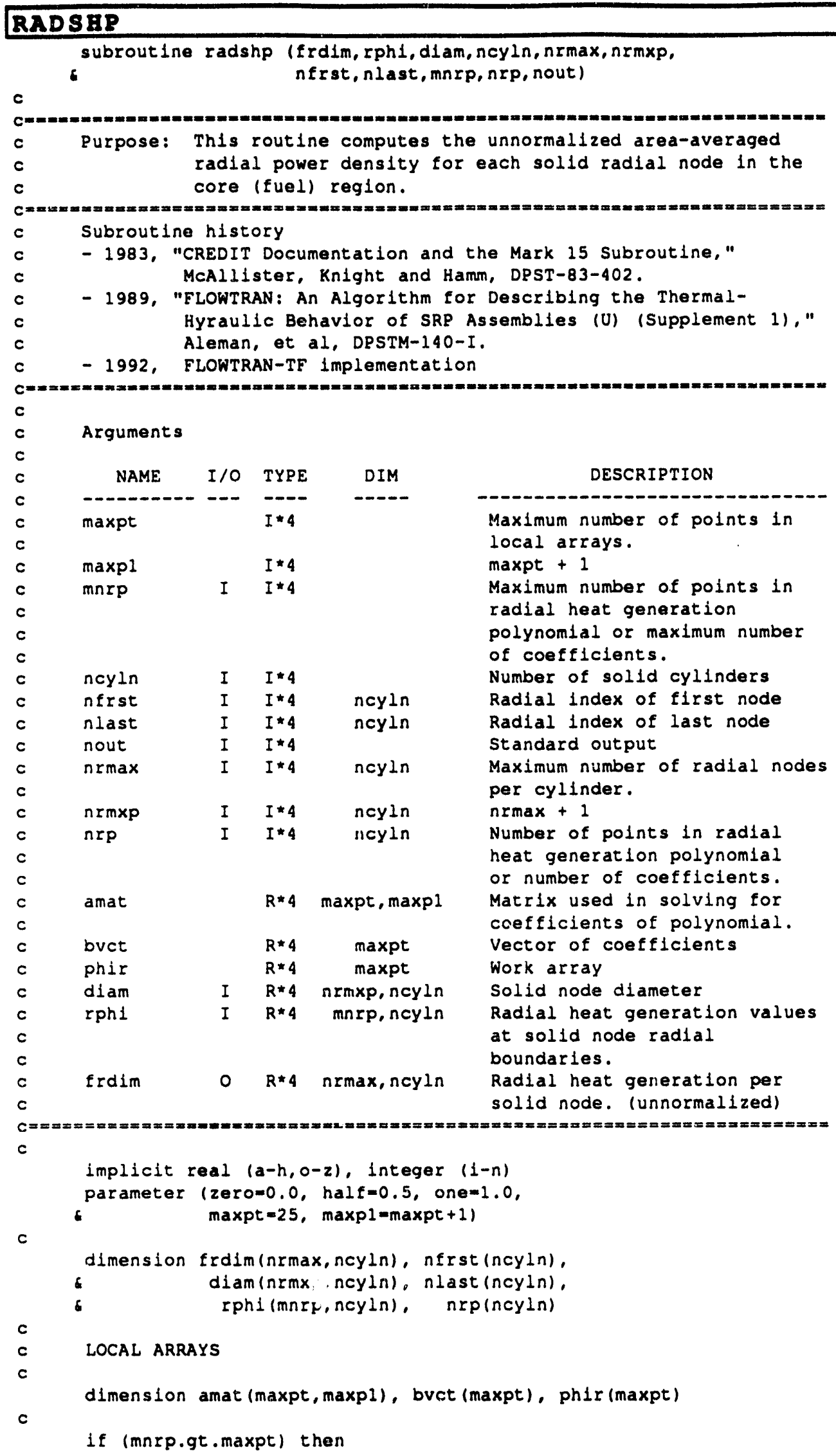




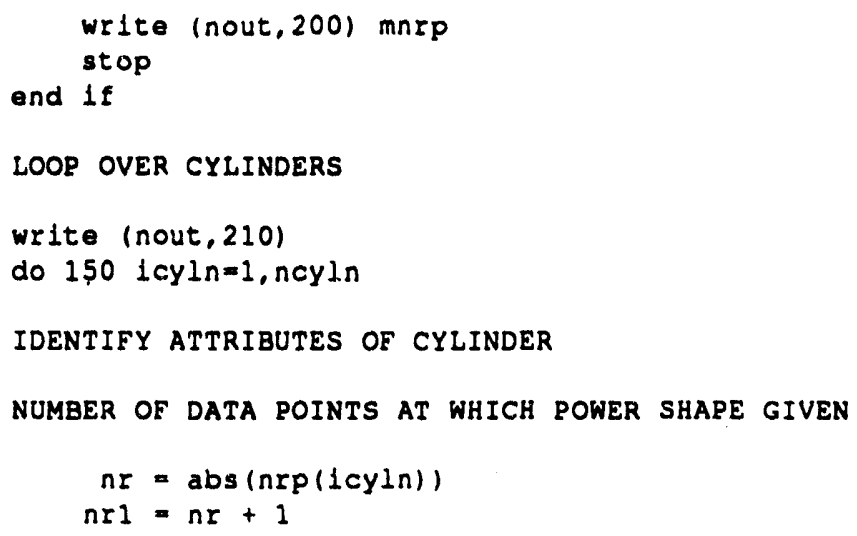


c

c

c

c

c

c

c

50

60

c

c

c

c

c

c

c

c

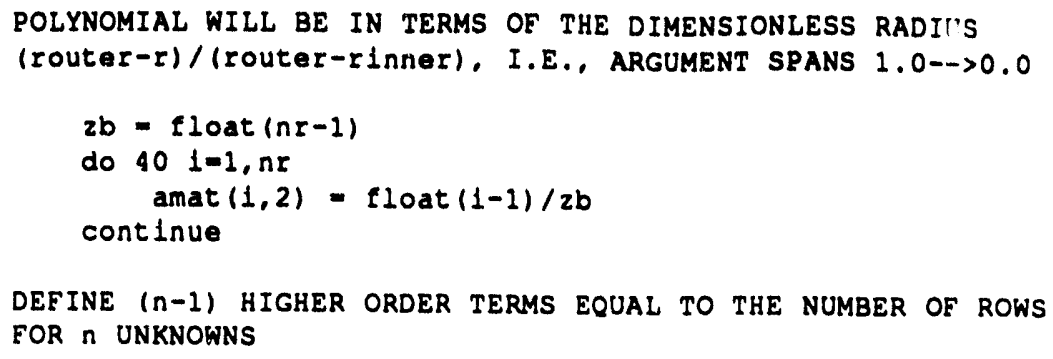

COMPUTE AREA-AVERAGED RADIAL POWER DENSITY FOR EACH SOLID NODE IN CORE REGION.

LOOP OVER NODES IN CYLINDER CORE

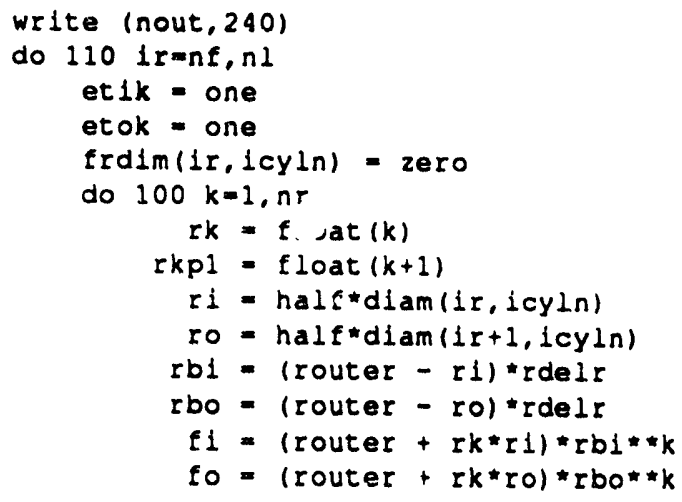




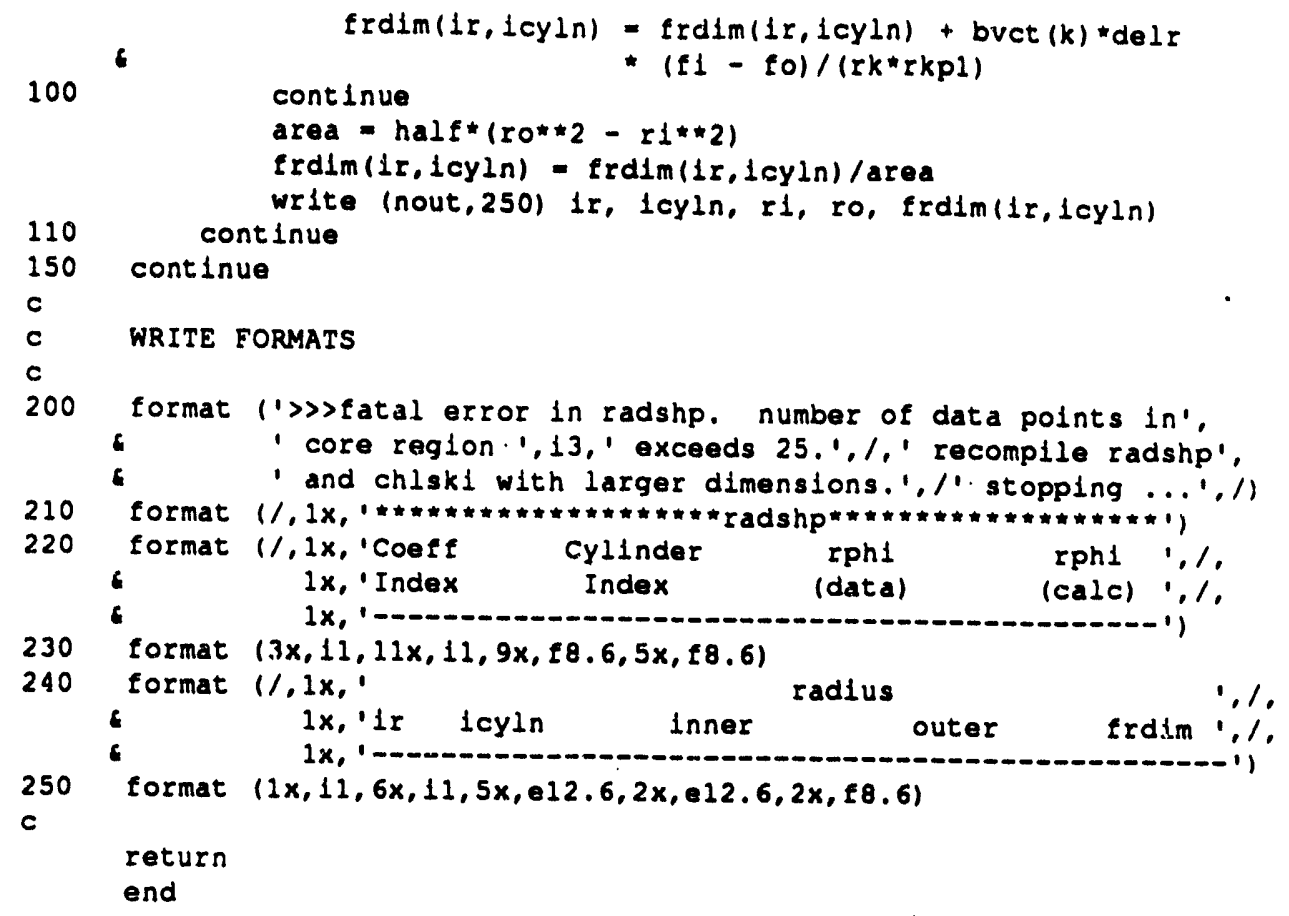

\section{READIN}

subroutine readin$$
\text { c }
$$$$
\text { c }
$$$$
\text { c }
$$$$
\text { c }
$$$$
\text { c }
$$$$
\text { c }
$$$$
\text { c }
$$$$
\text { c }
$$$$
c
$$$$
\text { c }
$$$$
\text { c }
$$$$
\text { c }
$$$$
\text { c }
$$$$
\text { c }
$$$$
\text { c }
$$$$
\text { real }(a-h, 0-z) \text { integer }(1-n)
$$ 


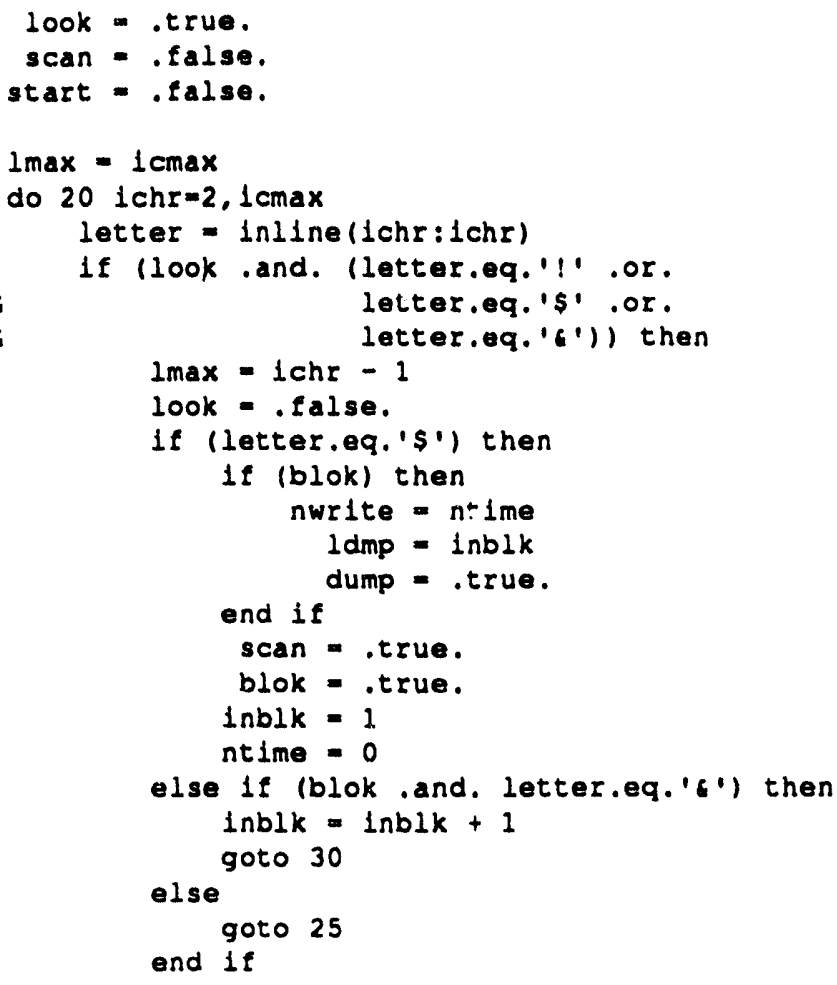


$c$
$c$
5
$c$
$c$
$c$

SAVE DATA BLOCKS OR WRITE SINGLE LINES

If (blok) then

buffer (1nblk) = Inline (1:1en)

length $(1 \mathrm{nb} l k)=$ len

else

write (ninp, 200) inline(1:len)

end if

goto 10

CHECK IF THE FILE ENDED WHILE IN A DATA BLOCK

if (blok) then

do 120 it $=1$, ntime

do 110 iline $=1$, inblk

len = length (iline)

outlin = buffer(iline)

write (ninp, 200) outlin (1:len)

continue

continue

end if

rewind (unit=ninp)

format (a)

return

end

RESET

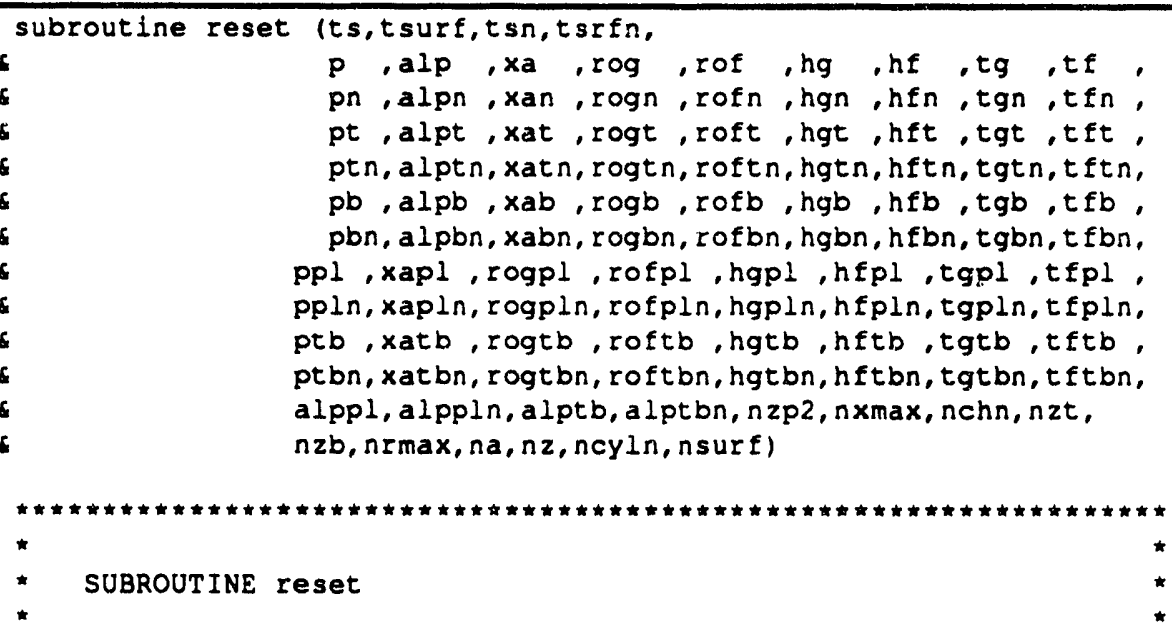




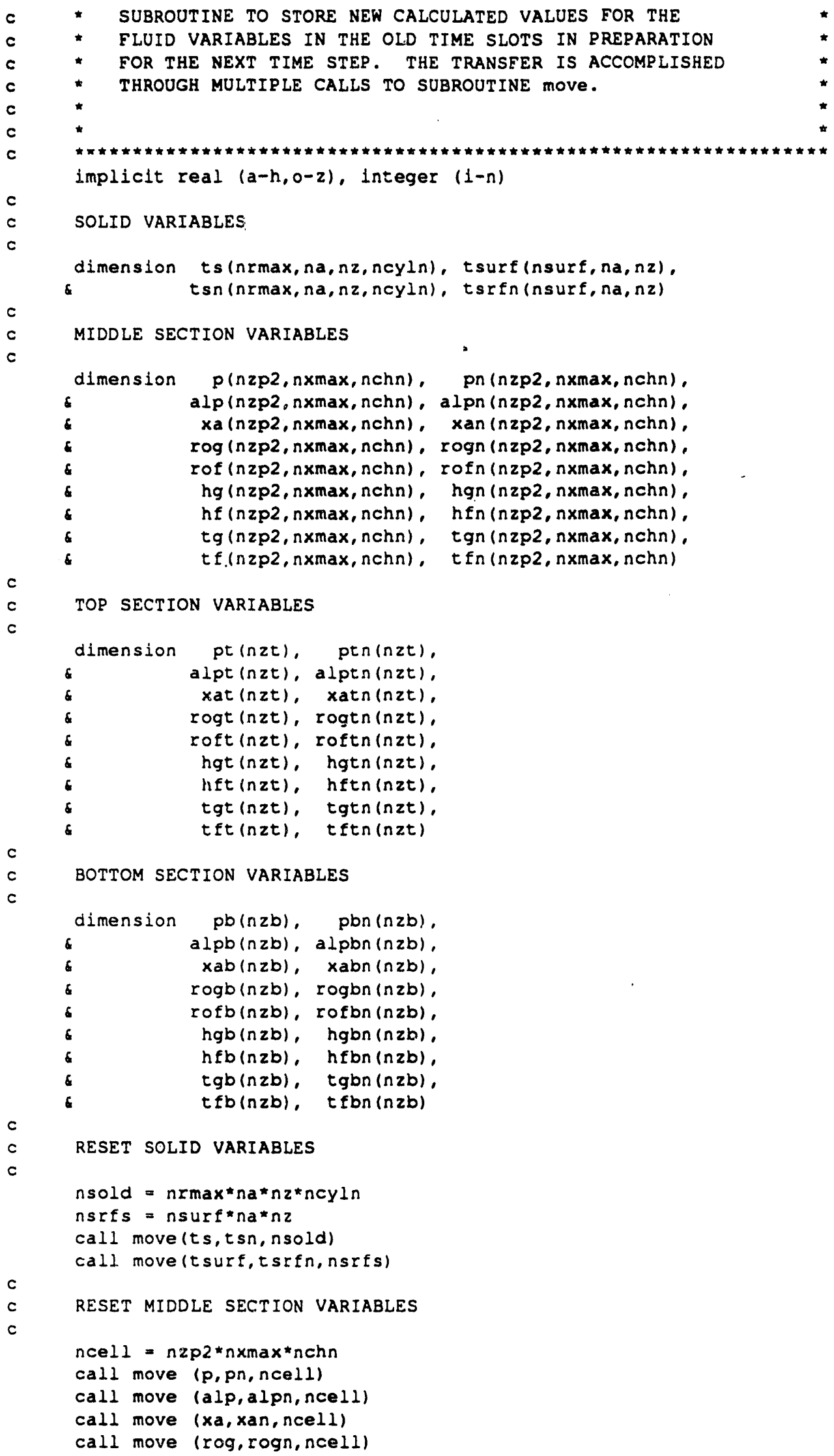


c

c

c

c

c

$\begin{aligned} & \text { ptbn }=p t b \\ & \text { alptbn }=a \text { lptb } \\ & \text { xatbn }=\text { xatb } \\ & \text { tgtbn }=\text { tgtb } \\ & \text { tftbn }=\text { tftb } \\ & \text { rogtbn }=\text { rogtb } \\ & \text { roftbn }=\text { roftb } \\ & \text { hgtbn }=\text { ngtb } \\ & \text { hftbn }=\text { hftb } \\ & \text { return } \\ & \text { end }\end{aligned}$

call move (pt, ptn, nzt)

call move (alpt,alptn, nzt)

call move (xat, xat $n, n z t)$

call move (rogt, rogtn, nzt)

call move (roft, roftn, nzt)

call move (hgt, hgt $n, n z t$ )

call move ( $h f t, h f t n, n z t)$

call move (tgt, tgt $n, n z t$ )

call move (tft, tftn, nzt)

RESET BOTTOM SECTION VARIABLES

call move (pb,pbn, nzb)

call move (alpb, alpbn, $n z b)$

call move ( $x a b, x a b n, n z b)$

call move (rogb, rogbn, nzb)

call move (rofb, rofbn, nzb)

call move (hgb, hgbn, nzb)

call move ( $h f b, h f b n, n z b$ )

call move (tgb,tgbn, nzb)

call move ( $t f b, t f b n, n z b)$

RESET PLENUM VARIABLES

$\mathrm{pp} \perp \mathrm{n}=\mathrm{ppl}$

alppln = alppl

xap.ln $=$ xapl

tgpln $=t g p l$

$t f p l n=t f p l$

rogpln $=$ rogpl

rofpln $=$ rofpl

hgpln $=$ hgpl

hfpln $=\mathrm{hfpl}$

RESET TANK BOTTOM VARIABLES

end

call move (rof, rofn, ncell)

call move (hg, hgn, ncell)

call move ( $h f, h f n, n c e l l$ )

call move (tg,tgn, ncell)

call move (tf,tfn, ncell)

RESET TOP SECTION VARIABLES

RESTRT

subroutine restrt (ts,tsurf,tsn,tsrfn, qwf, qwg, dqfdw, dqgdw,

6

pt, alpt, xat, rogt, roft, hgt, hft, tgt, tft,

pt $n$, alpt $n$, xat $n$, rogt $n$, roft $n$, hgt $n$, hft $n, t g t n, t f t n$,

$p$,alp, xa, rog, rof, hg, hf, tg, tf,

pn, alpn, xan, rogn, rofn, hgn, hfn, tgn, tfn,

$p b, a l p b, x a b, r o g b, r o f b, h g b, h f b, t g b, t f b$, 
c

6

6

pbn, alpbn, xabn, rogbn, rofbn, hgbn, hfbn, tgbn, $t$ fbn, ugt, uft, wgx, wfx, ugz, ufz, ugb, ufb, citn, cizn, cixn, cibn, hfit, hfi, hfib, hgit, hgi, hgib, akgit, akgi, akgib, git, $g 1, g 1 b, e 1 t, e 1, e i b, y s i t, y s 1, y s i b$.

hspl, qonb, $n x, n r, t$ secol

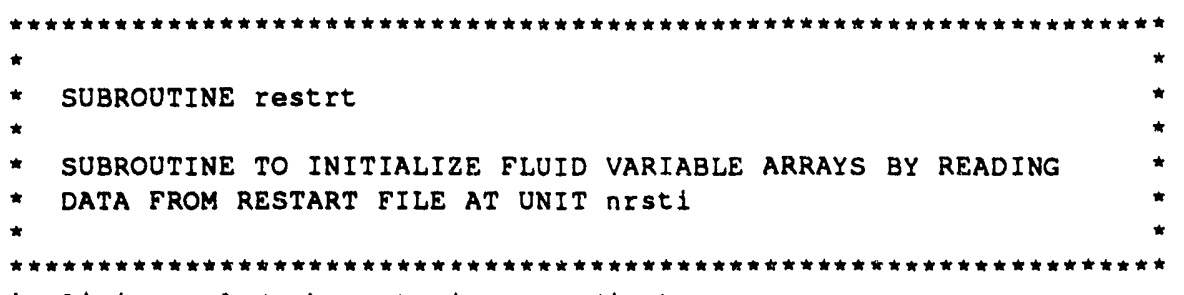

implicit real $(a-h, o-z)$, integer $(i-n)$

common /lounit/ ninp, nout, ndmp, ntmp, nrsti, nrsto

include 'params.inc'

SOLID VARIABLES

dimension $t s$ (nrmax, na, nz, ncyln),

6 $\operatorname{tsn}(n r m a x, n a, n z, n c y l n)$.

* nr(ncyln)

dimension qwf(nsurf, na,nz), dqfdw(nsurf,na,nz),

- qwg (nsurf,na,nz), dqgdw(nsurf,na,nz),

gonb (nsurf, na,nz), hspl (nsurf,na,nz),

tsurf (nsurf, na, nz), tsrfn(nsurf,na,nz)

MIDDLE SECTION PHYSICAL PARAMETERS

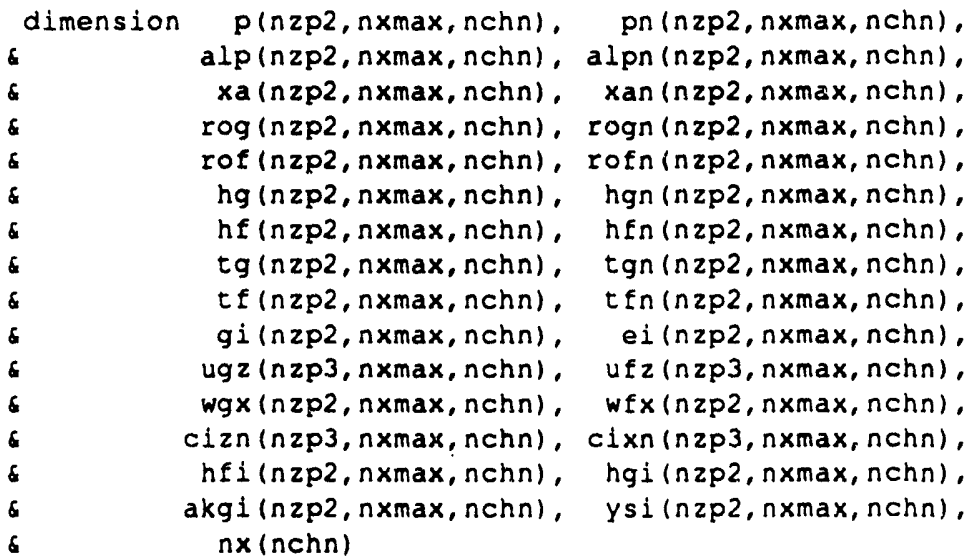

TOP SECTION PHYSICAL PARAMETERS

\begin{tabular}{|c|c|c|}
\hline \multirow[t]{12}{*}{ dimension } & \multirow{2}{*}{ pt (nzt) } & $\operatorname{ptn}(n z t)$ \\
\hline & & $\operatorname{alptn}(n z t)$ \\
\hline & $\operatorname{xat}(n z t)$ & $\operatorname{xatn}(n z t)$ \\
\hline & rogt $(n z t)$. & $\operatorname{rogtn}(n z t)$ \\
\hline & roft $(n z t)$, & roftn $(n z t)$ \\
\hline & $\begin{array}{l}\text { hgt (nzt), } \\
\text { hft (nzt). }\end{array}$ & $\begin{array}{l}\text { hgtn (nzt) } \\
\text { hftn (nzt) }\end{array}$ \\
\hline & $\operatorname{tgt}(n z t)$, & $\operatorname{tgtn}(n z t)$ \\
\hline & $t \in t(n z t)$, & $t \in t n(n z t)$ \\
\hline & git (nzt), & elt (nzt) \\
\hline & ugt (nzt), & $u \in t(n z t)$ \\
\hline & $\operatorname{citn}(n z t)$, & $h \in 1 t(n z t)$ \\
\hline & hgit (nzt), & it $(n z t)$ \\
\hline
\end{tabular}




$c$
$c$
$c$

6 ysit (nzt)

BOTTOM SECTION PHYSICAL PARAMETERS

dimension pb(nzb), pbn(nzb),

- alpb(nzb), alpbn(nzb),

- $\quad x a b(n z b), x a b n(n z b)$.

$\operatorname{rog} b(n z b), \operatorname{rogbn}(n z b)$,

rofb $(n z b), \operatorname{rofbn}(n z b)$,

hgb (nzb), hgbn(nzb),

$h f b(n z b), h f b n(n z b)$,

$\operatorname{tgb}(n z b), \operatorname{tgbn}(n z b)$,

$t f b(n z b), t f b n(n z b)$,

gib (nzb), eib(nzb).

$u g b(n z b), u f b(n z b)$,

$c 1 b n(n z b), h f i b(n z b)$,

ngib(nzb), akgib(nzb),

ysib (nzb)

c

c

c

c

c

c

$c$

c

10

c

c

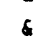

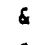

c

c

c

$c$

$c$

c

c

c

c

c

$$
\text { c }
$$

BEGIN FLUID INITIALIZATION:

READ LOCAL PRESSURE, VOID FRACTION, AIR MASS FRACTION, GAS TEMPERATURE, FLUID TEMPERATURE, GAS DENSITY, FLUID DENSITY, GAS ENTHALPY, FLUID ENTHALPY, INTERFACIAL MASS AND ENERGY TRANSFER RATES, AND GAS AND FLUID VELOCITIES

PLENUM

read (nrsti) ppl, alppl, xapl, tgpl, tfpl,

TOP SECTION

read (nrsti) ( pt (Jz).

a alpt $(j z), \operatorname{xat}(j z)$,

a tgt $(j z), \operatorname{tet}(j z)$,

1) $\operatorname{rogt}(j z), \operatorname{roft}(j z)$. 


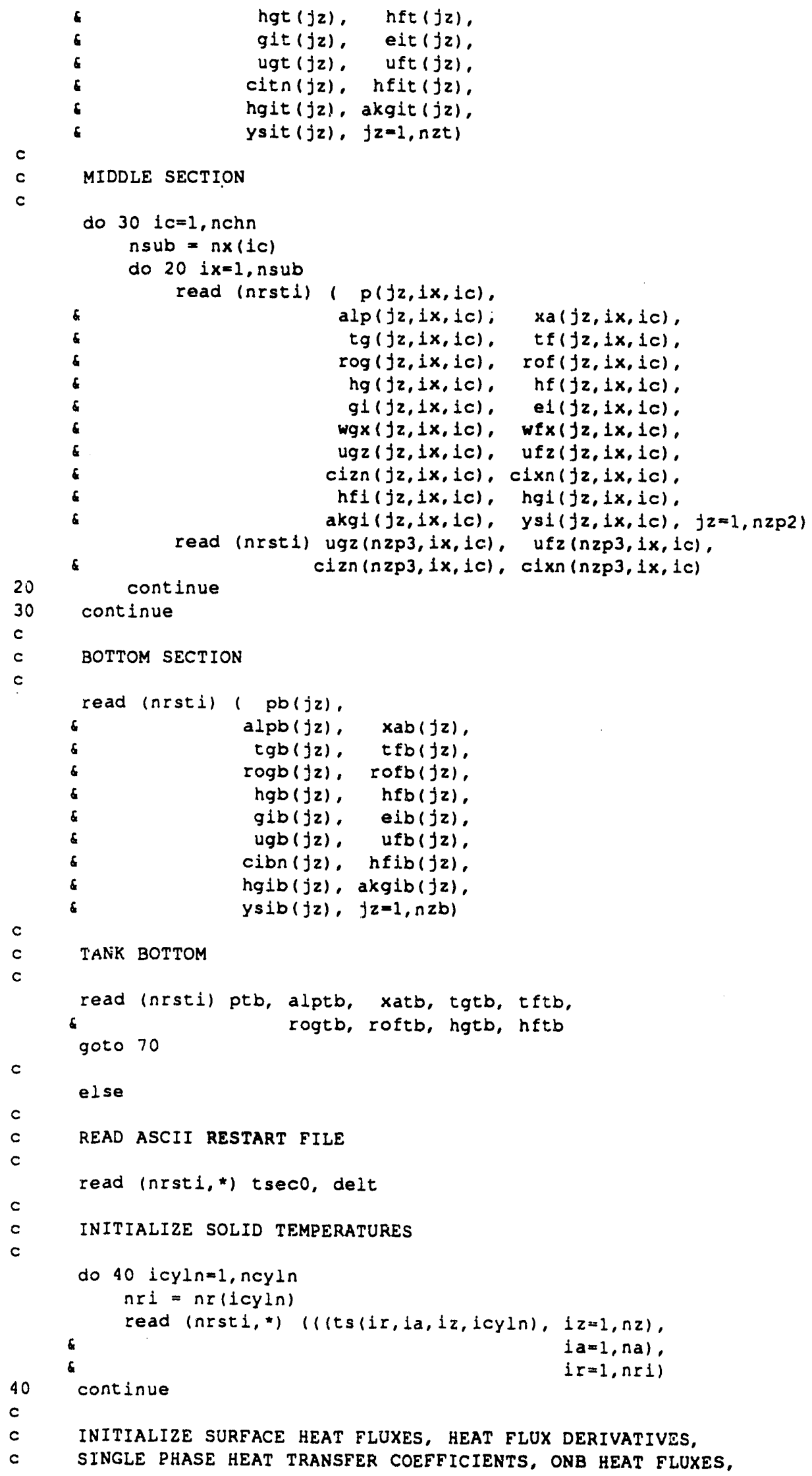


c

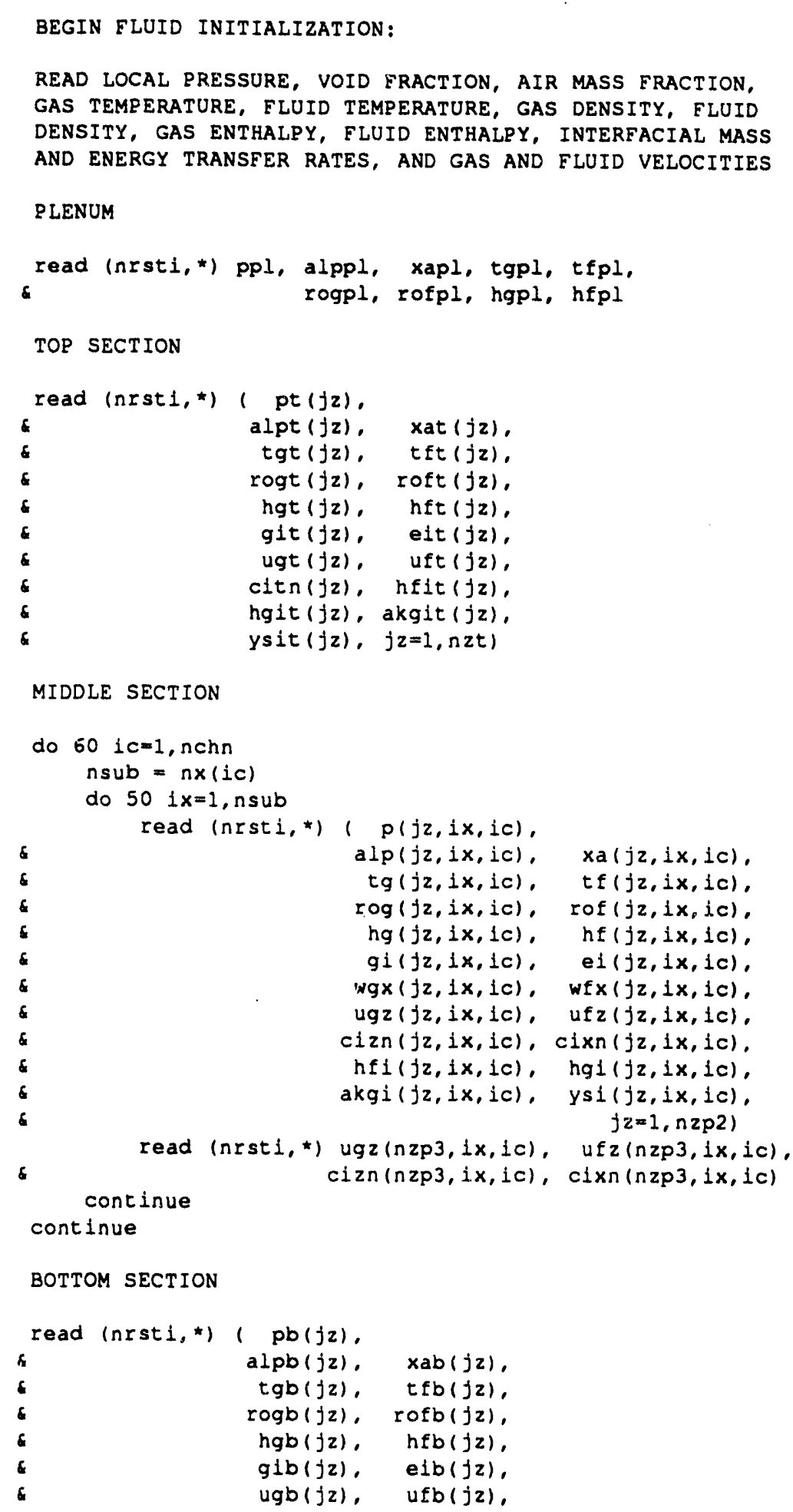




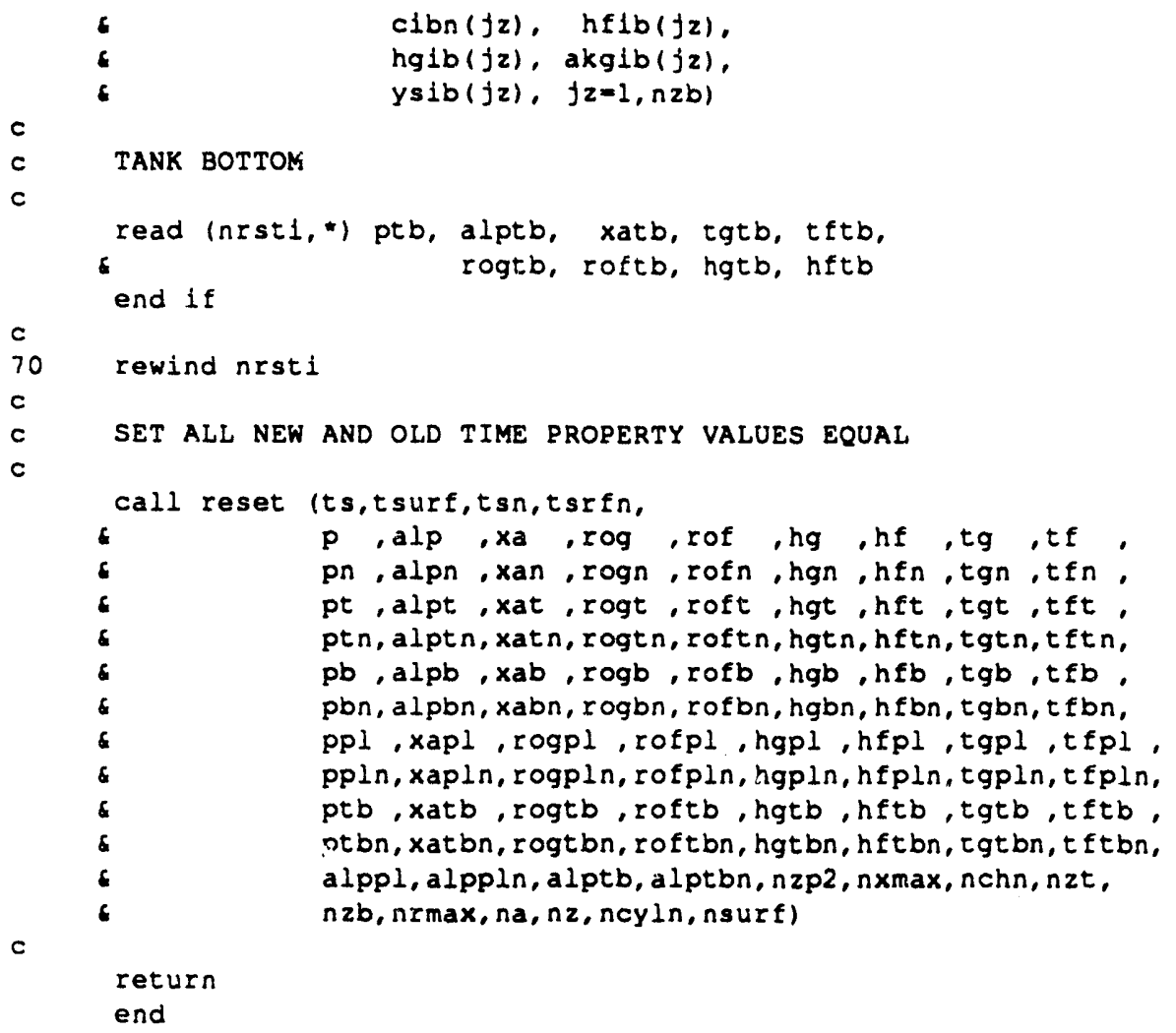

\section{RING}

subroutine ring (ts, tsurf, tsrfn, dr, cond, radc, ar, rhs, diag, cra,

\& crc, ca, trhs, cmag, cmrhs, cma, cmb, cmd, cri, cro,

\& d, rhsi, rhso, rf, ncms, da, dz, nr, nrp, nrmax, na, nz, nsurf, ncyln, icyln, icent, qwe, qwg, dqfdw, dqgdw) 

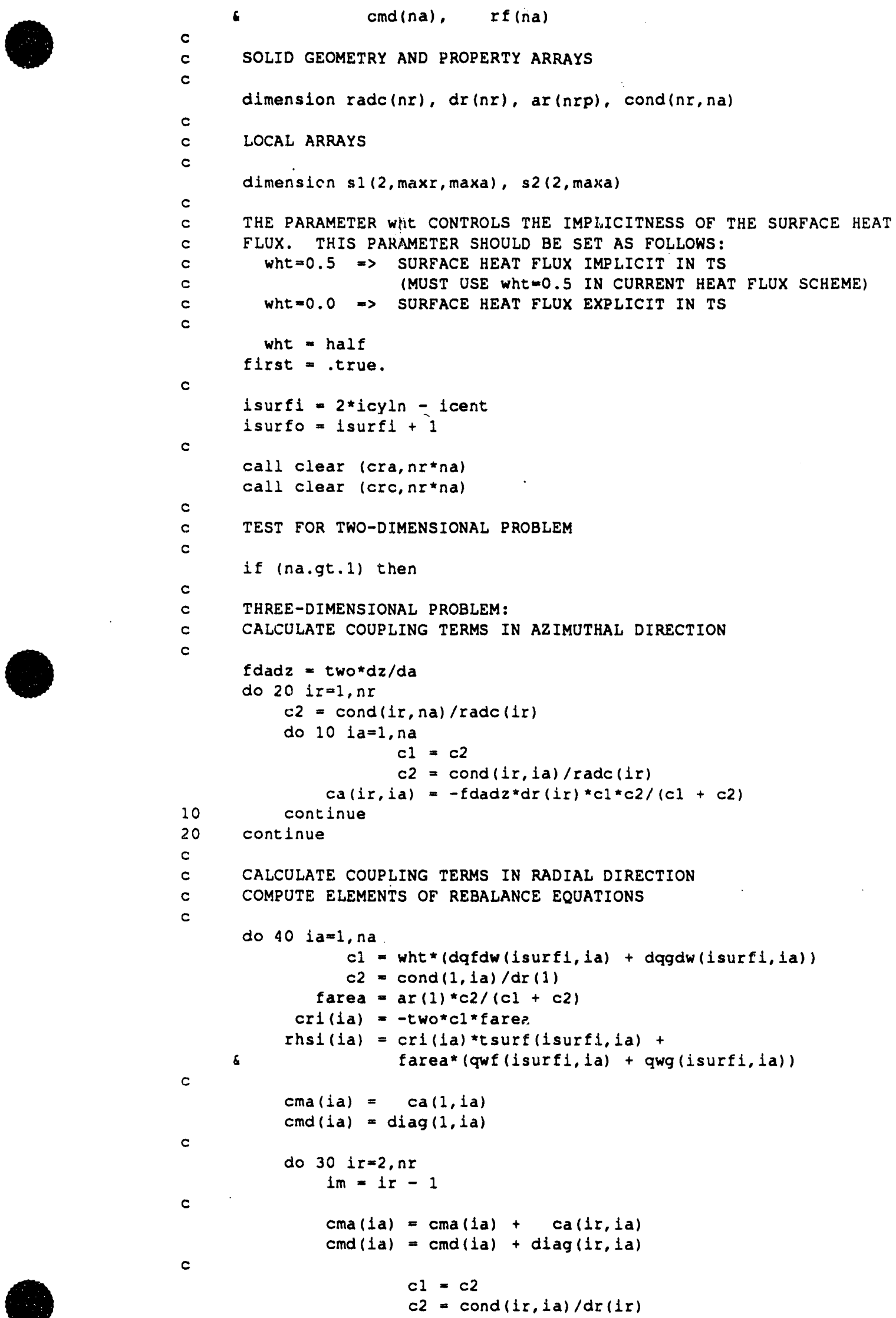
30

c

0

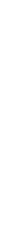

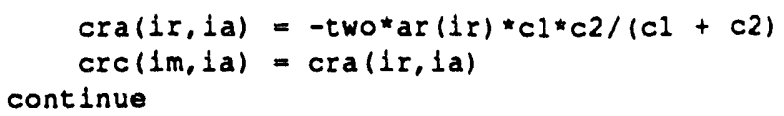


$c$

500 continue

first $=$.false.

$\approx$

$c$

PERFORM COARSE MESH REBALANCE

do 700 la=1, na

$1 \mathrm{am}=1 \mathrm{a}-1$

if (1am.eq.0) $1 \mathrm{am}=\mathrm{na}$

1 ap $=1 a+1$

if (1a.eq.na) lap $=1$

$\operatorname{cmrhs}(1 a)=\operatorname{trhs}(i a)+\operatorname{cri}(1 a) \star t s(1, i a)$

$\sqrt{2}$

$+\operatorname{cro}(1 a) * t \sin r, 1 a)$

do 600 ir $=1, n r$

$\operatorname{cmrhs}(1 a)=\operatorname{cmrhs}(1 a)-\operatorname{ca}(1 r, 1 a) * t s(1 r, 1 a m)$

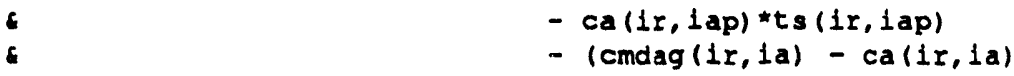

continue

- $\operatorname{ca}(1 r, 1 a p)) * t s(1 r, 1 a)$

continue

c

call trldag (firstc, 1, na, na, cma, cmd, cmb, cmrhs, rf, s2)

firstc $=$.false.

$c$

do 800 ia=1, na

do 800 ir $=1, n r$

$t s(1 r, i a)=t s(1 r, i a)+r f(i a)$

800

continue

1000 continue

c

else

TWO-DIMENSIONAL PROBLEM $(r, z)$ :

CALCULATE COUPLING TERMS IN RADIAL DIRECTION

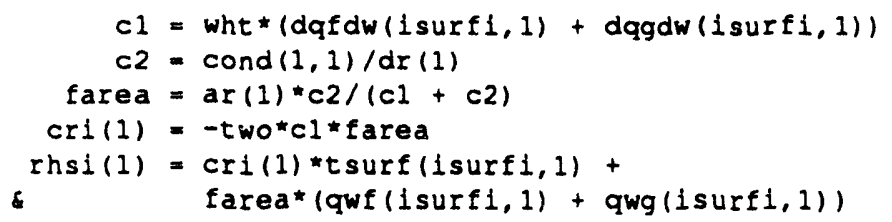

c

do 2100 ir $=2, n r$

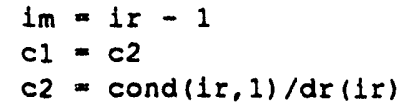


c

c

2200

COMPUTE DIAGONAL ELEMENTS

do $22001 \mathrm{r}=1, \mathrm{nr}$

$\operatorname{diag}(1 r, 1)=\operatorname{diag}(1 r, 1)-\operatorname{cra}(1 x, 1)-\operatorname{crc}(1 r, 1)$

continue

$\operatorname{diag}(1,1)=\operatorname{diag}(1,1)-\operatorname{cri}(1)$

$\operatorname{dlag}(n r, 1)=\operatorname{diarg}(n r, 1)-\operatorname{cro}(1)$

c

c

c

c

c

c

c

3000

continue

SOLVE TRIDIAGONAL SYSTEM FOR TEMPERATURES

call tridag (first, $1, n r, n r, \operatorname{cra}(1,1)$, diag, crc $(1,1)$, rhs, ts, s 1 )

end if

COMPUTE SUREACE TEMPERATURES AND HEAT FLUXES AT NEW TIME

do 3000 1a=1, na

$c 1=\operatorname{cond}(1,1 a) / d r(1)$

$c 2=\operatorname{half*}(\operatorname{dgfdw}(1 \operatorname{surf1}, 1 a)+\operatorname{dggdw}(1 \operatorname{surf1}, 1 a))$

tsurf(1surfi, ia)

- $(c 1 * t s(1,1 a)+c 2 * t \operatorname{surf}(1 \operatorname{surf1} 1,1 a)-$ half*(qwf (1surf1,1a) + qwg(isurfi, 1a))) $(c)+c 2)$

$\operatorname{delt} 1=\operatorname{tsurf}(1 \operatorname{surf} 1, i a)-\operatorname{tscfn}(1 \operatorname{surf} 1,1 a)$

qwf (isurfi,ia) = qwf(isurfi,ia) + dqfdw(1surfi,ia)*delti

$q w g(i s u r f i, i a)=q w g(i \operatorname{surf} i, i a)+d q g d w(i s u r f i, i a) * d e l t i$

return

end

\section{ROESAT}

subroutine rofsat $(t, 1$, rofs,drfsdt)

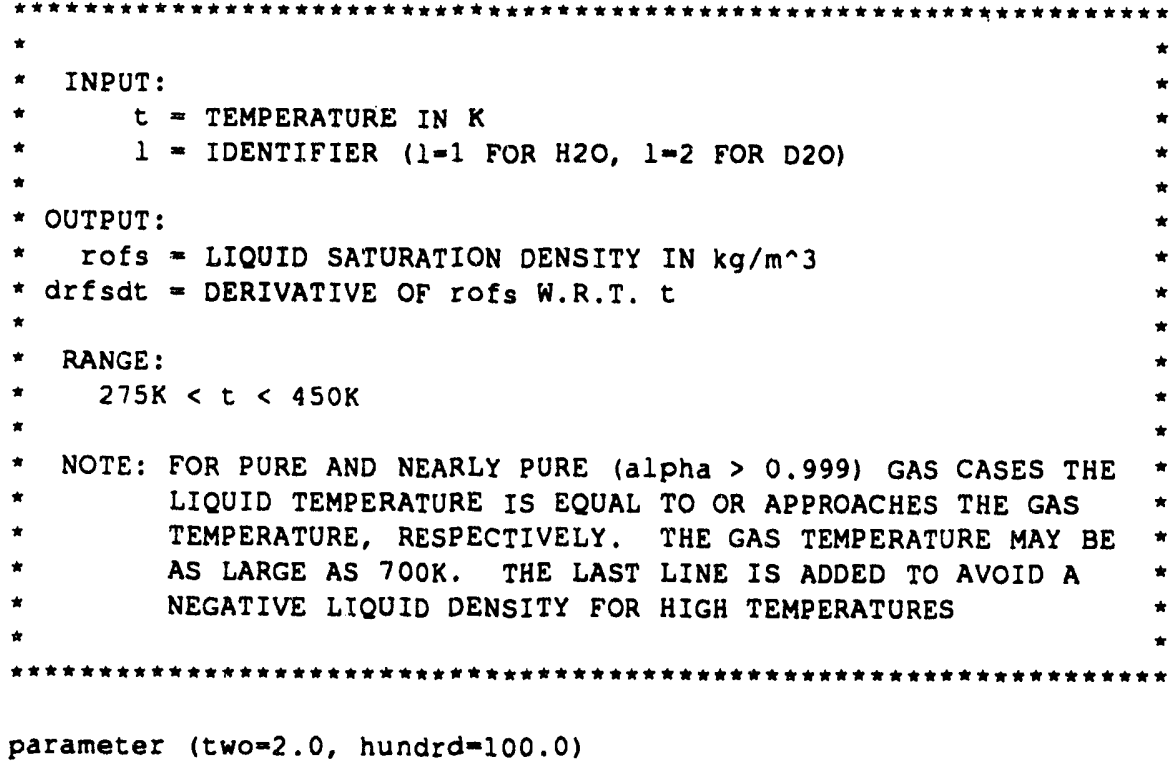




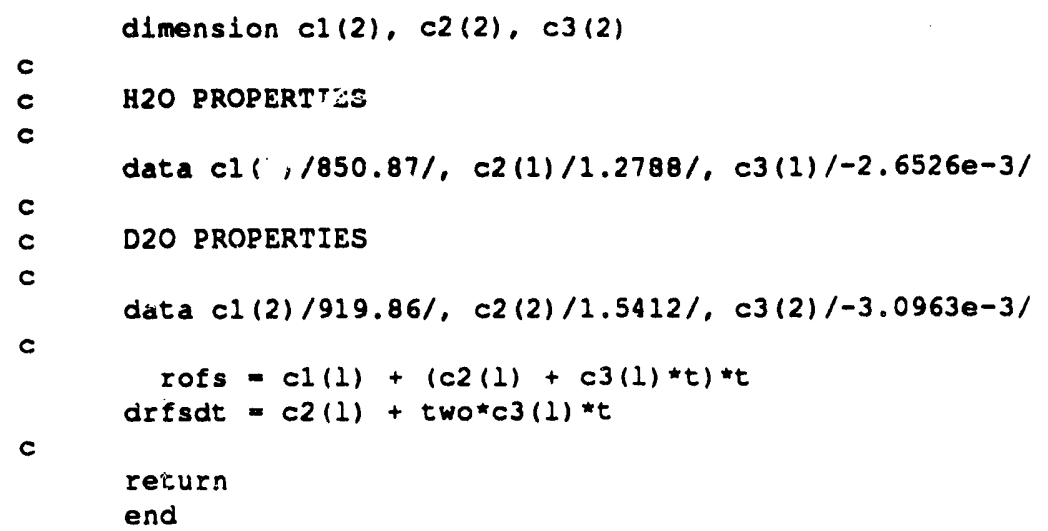

\section{RSTEAM}

subroutine rsteam(p,t, 1, ros, drosdp, drosdt:

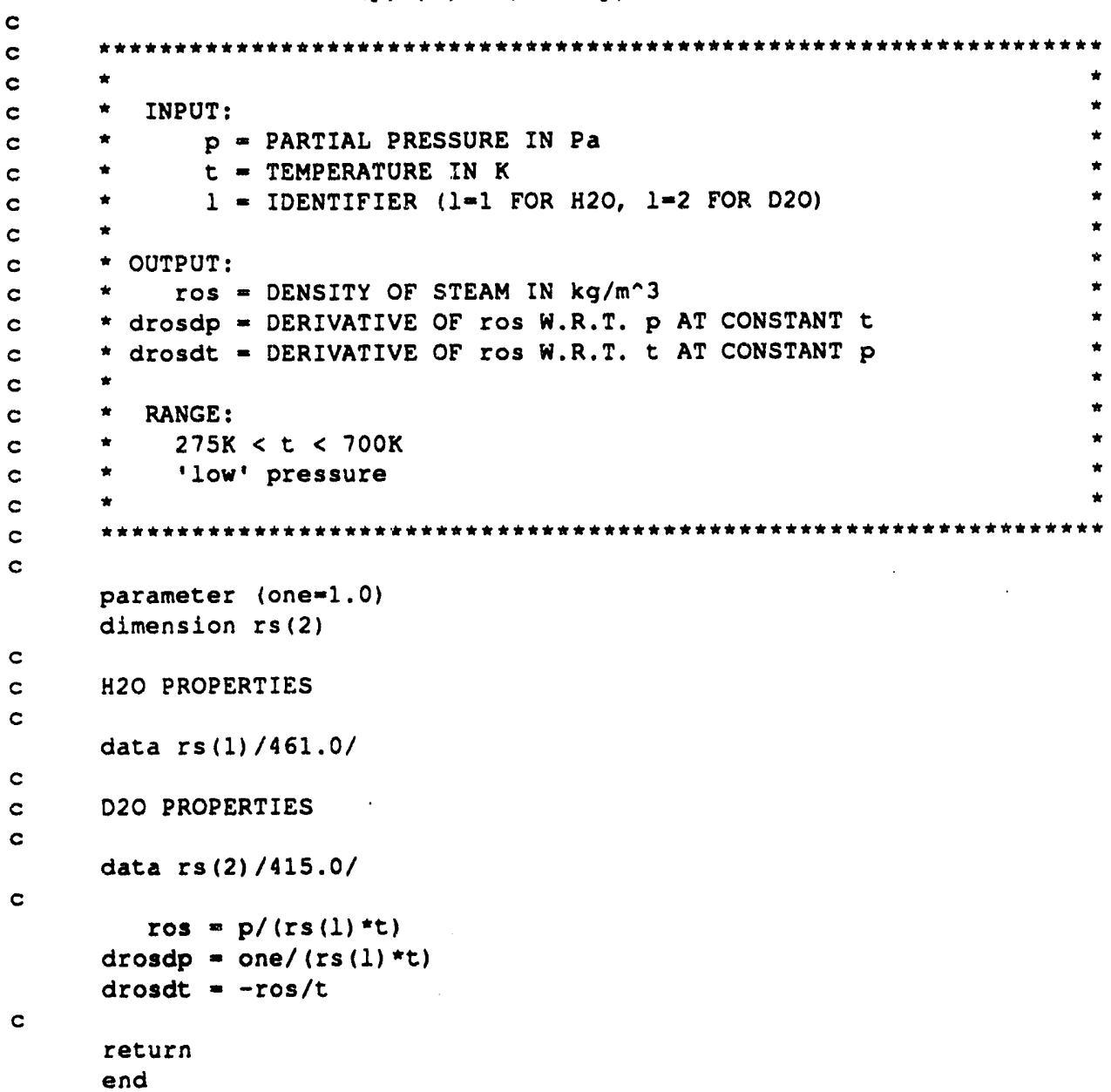

\section{SCMSIB}

subroutine scmslb (in)

c

write (in, 10)

10 format $(1$,
c $9 x$,
c iftat
- $1.9 x$,

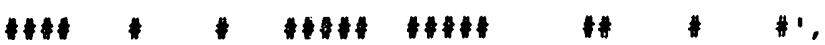




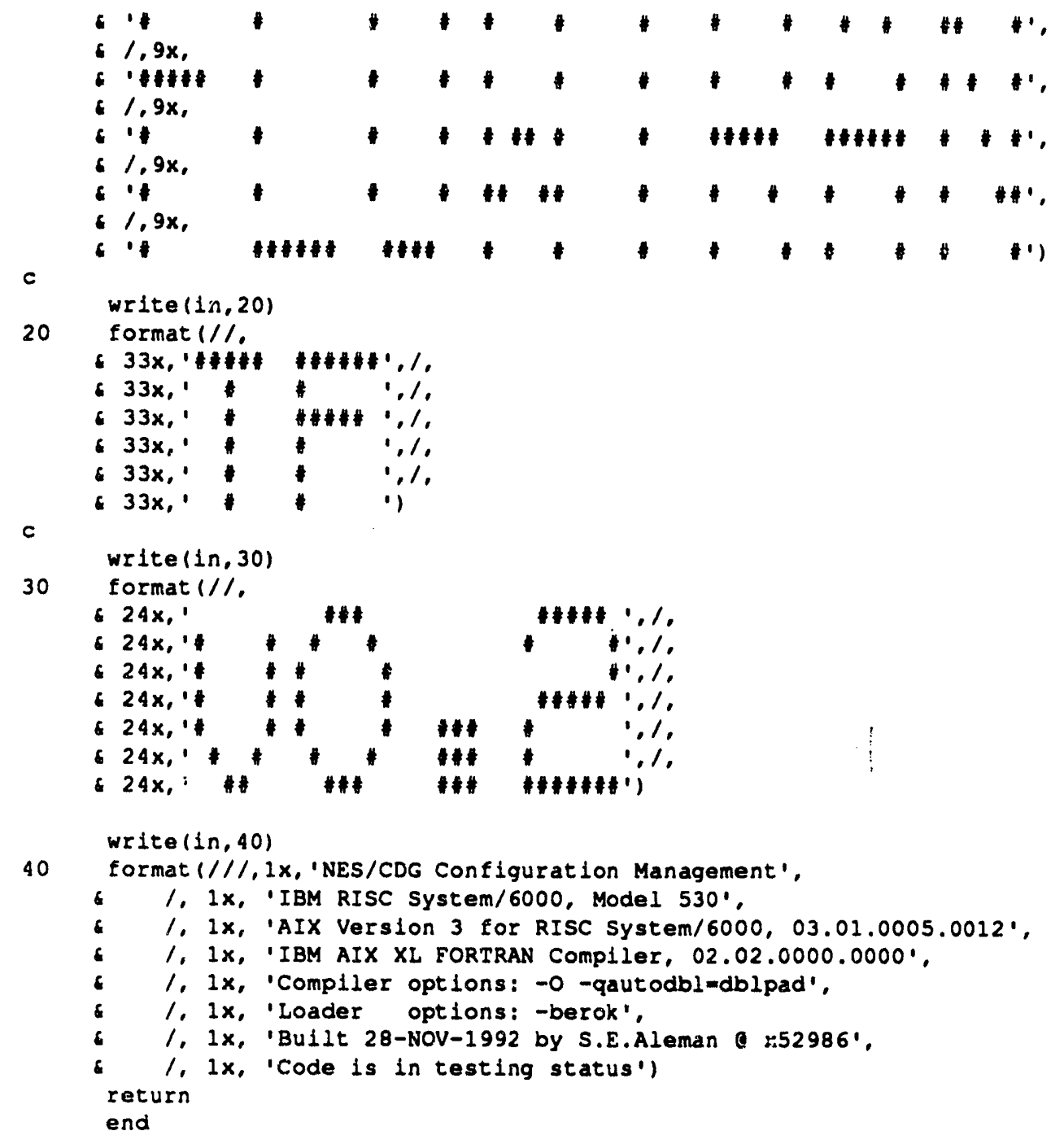

\section{SETSID}

subroutine setsld (radc,ar,dr, vols, zfacm, da, diam,dz, rdz,
hactiv, mat, ioxid, nfrst, nlast, nr, nz, na, nrmax,
nrmxp, ncyln, izht, izhb, icent, iradbc, douts,
emisi, emiso, cradi)

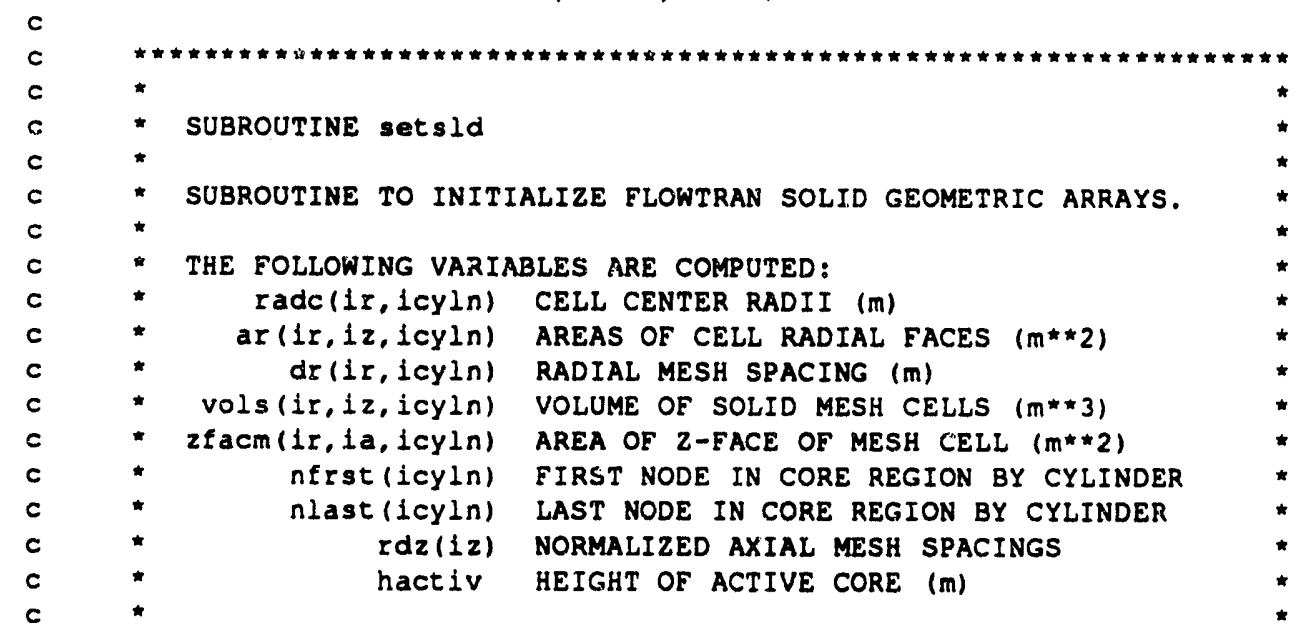


c

$c$

c

$c$

$c$

$c$

c

c

C

c

c

c

c

c

dimension node (10)

COMPUTE SOLID CELL GEOMETRIC PARAMETERS

$p 1=a \cos (-o n e)$

da $=t w o * p i / n a$

do 40 icyln=1, ncyln

$n r i=n r(1 c y l n)$

$n r p=n r i+1$

do 20 ir $=1, n r i$

$d r(i r, i c y l n)=\operatorname{half}(\operatorname{diam}(i r+1, i c y l n)-\operatorname{diam}(i r, i c y l n))$

$\operatorname{radc}(i r, i c y l n)=\operatorname{gart} *(\operatorname{diam}(i r+1, i c y l n)+\operatorname{diam}(i r, i c y l n))$

$z f a c m(i r, i c y l n)=d r(1 r, i c y l n) * \operatorname{radc}(1 r, i c y l n) \star d a$

do $10 \mathrm{iz}=1, \mathrm{nz}$

$\operatorname{ar}(i r, i z, i c y l n)=\operatorname{half} * d i a m(i r, i c y l n) * d a * d z(i z)$

$\operatorname{vols}(i r, i z, i c y l n)=z \mathrm{facm}(i r, i c y l n) * d z(i z)$

continue

continue

COMPUTE RADIAL AREAS AT OUTER FACES

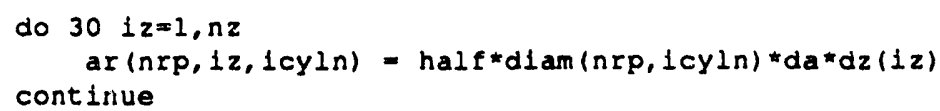

CHECK ASSEMBLY MATERIAI. NODALIZATION, COMPUTE AND STORE FIRST ANU LAST NODES IN CORE REGION

do 150 icy $l n=1$, ncyln

$n r i=n r(i c y l n)$

CHECK MATERIAL ON OUTSIDE OF CYLINDER

mtest $=\operatorname{mat}(n r i, 1,1$ cy $1 n)$

if (mtest.eq.1 .or. mtest.eq.4 .or. mtest.eq.5) then

write $(*, 200)$ icyln

stop

end if

CHECK MATERIAL ON INSIDE OF CYLINDER

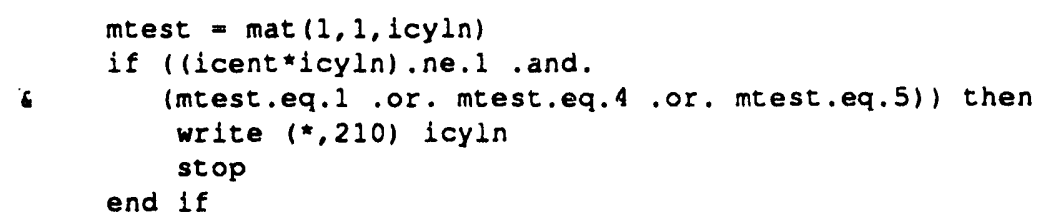


Inode -2

c

c

c

c

110

c

c

c

c $c$

150

c

c

FIND NUMBER OF DIFFERENT MATERIAL SECTIONS IN CYLINDER AND SAVE THE FIRST AND LAST NODES OF EACH SECTION

node $(1)=1$

do 110 ir $r=2, n r i$

if (mat $(1 r, 1,1 c y 1 n)$.ne.mtest) then

node (inode) $=1 r-1$

node $($ Inode +1$)=1 r$

nsect $=$ nsect +1

Inode $=$ inode +2

end if

mtest $=\operatorname{mat}(1 \mathrm{r}, 1,1$ cyln)

continue

node (Inode) $=n r 1$

FIND BOUNDS FOR CYLINDER CORE REGION

if (nsect.eq.1 .or. nsect.eq. 3 .or. nsect.eq.5) then

nfrst $(i c y \mid n)=$ node (nsect)

nlast $($ icyln $n=$ node $($ nsect +1$)$

else if (nsect.eq. 2 .or. nsect.eq.4) then

if $($ ioxid(lcyln).eq.1) then

$n$ frst $($ Icy $1 n)=$ node $(n$ sect -1$)$

nlast $(i c y l n)=$ node (nsect)

else if (ioxid(icyln).eq.2) then

nfrst $(1$ cyln $)=$ node (nsect +1$)$

else

nlast $(1 c y l n)=$ node $($ nsect +2$)$

write $(*, 220)$ icyln
stop

end if

else

write $(*, 230)$ icyln

stop

end if

continue

COMPUTE HEIGHT OF ACTIVE CORE AND NORMALIZED

AXIAL MESH SPACINGS OVER HEATED CORE

hactiv $=$ zero

do 160 iz=izht, izhb

hactiv = nactiv $+d z(i z)$

continue

call clear (rdz,nz)

do 170 izmizht, izhb

$r d z(1 z)=d z(1 z) /$ hactiv

continue

IF A RADIATION BOUNDARY CONDITION IS SPECIFIED, CALCULATE

THE CONSTANT TERM IN THE RADIATION HEAT FLUX EQUATION

if (iradbc.eq.1) then

nrout $=n r(n c y l n)+1$

ratio = diam(nrout,ncyln)/douts

if (ratio.gt.one) then

write $(*, 240)$

stop 


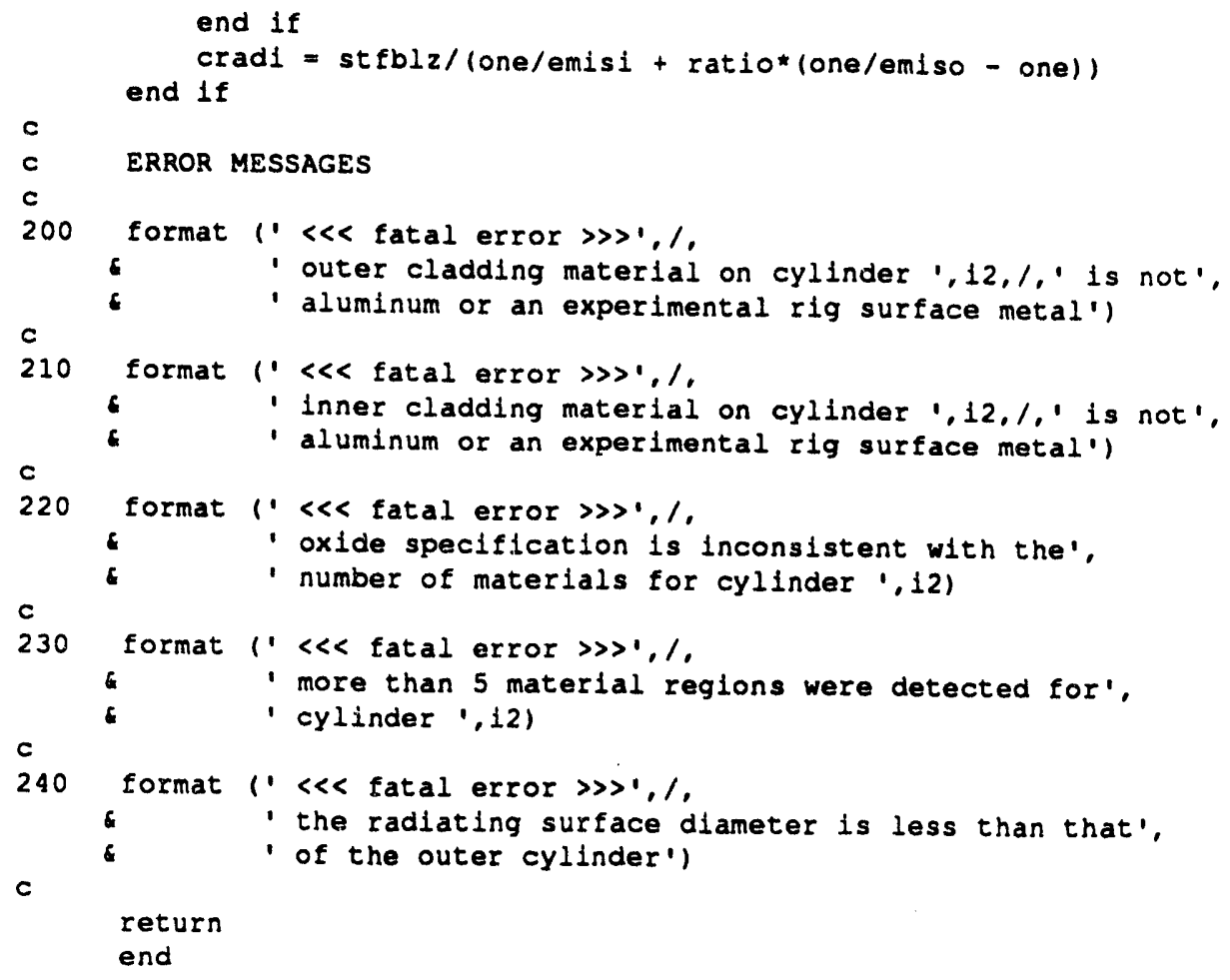

\section{SOLID}

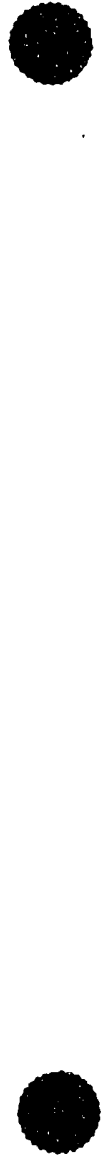

(ts, tsn, tsurf, tsrfn, pows, qwf, qwg, dqfdw, dqgdw, wt, radc, ar, vols, zfacm, mat, rdelt, da, dr, dz, nr, na, nsurf, nz, ncy $1 n$, nrmax, nrmxp, wss, icent, iaxial, noms)

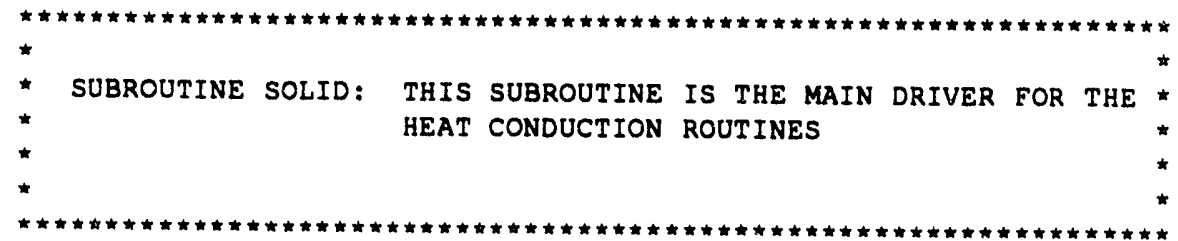

ARGUMENTS

NOTE: ALL VARIABLES LISTED BELOW ARE AT CURRENT TIME LEVEL UNLESS OTHERWISE INDICATED

$0 \quad t s(*)$

I $\operatorname{tsn}(*)$

tsurf (1surf, ia, 1z)

tsren (isurf, $1 a, 12$ )

pows (*)

I qwf(isurf, ia, iz)

I qwg(1surf, $1 a, 1 z)$

I dqfdw(isurf, ia, iz)

I dqgdw(isurf, 1a, 1z)

I $\operatorname{radc}(* \star)$

I $\operatorname{ar}(* \star)$

c I vols $(* \star)$
SOLID TEMPERATURES FOR ALL CYLINDERS AT ALL AXIAL, AZIMUTHAL, AND RADIAL LOCATIONS SOLID TEMPERATURES AT OLD TIME LEVEL SURFACE TEMPERATURES SURFACE TEMPERATURES AT PREVIOUS ITERATION THERMAL ENERGY DEPOSITED IN THE SOLID DURING CURRENT TIME STEP SURFACE HEAT FLUX TO LIQUID SURFACE HEAT FLUX TO GAS DERIVATIVE OF LIQUID SURFACE HEAT FLUX WITH RESPECT TO SURFACE TEMPERATURE DERIVATIVE OF GAS SURFACE HEAT FLUX WITH RESPECT TO SURFACE TEMPERATURE CELL CENTERED RADII FOR EACH CYLINDER AREAS OF THE RADIAL FACES OF THE MESH CELLS VOLUMES OF THE MESH CELLS 


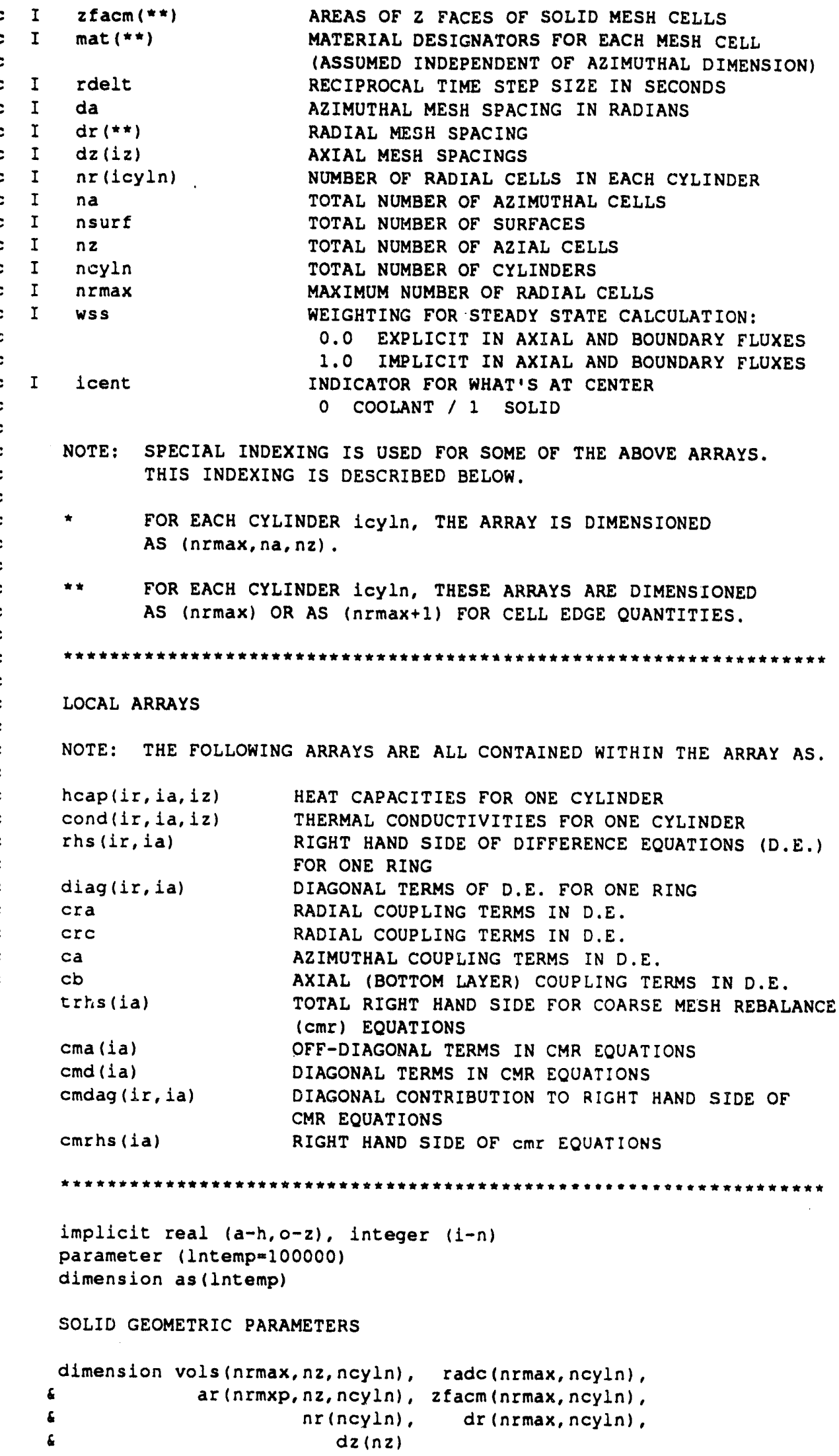

\section{LOCAL ARRAYS}

NOTE: THE FOLLONING ARRAYS ARE ALL CONTAINED WITHIN THE ARRAY AS. 
$c$

c

$c$

c

SOLID PROPERTIES

dimension ts(nrmax, na, nz,ncyln),

c $\quad \sin (n r m a x, n a, n z, n c y \perp n)$,

- pows (nrmax, na, nz, ncyln),

c $\operatorname{mat}(n \max , n z, n c y l n)$,

6 wt (ncyln)

SURFACE PROPERTIES

dimension qwf(nsurf,na,nz), dqfdw(nsurf,na,nz),

- qwg (nsurf, na, nz), dqgdw(nsurf, na, nz),

- tsurf (nsurf,na,nz), tsren(nsurf,na,nz)

logical first

save first, Ihcap, lcond, Irhs, Idiag, lcra, lcrc, 1ca, Icb, It rhs,

- Id, lcri, lcro, lrhsi, I chso, $1 \mathrm{rf}, 1 \mathrm{cma}, 1 \mathrm{cmb}, 1 \mathrm{cmd}, 1 \mathrm{cmdag}$,

c lcmrhs

data first/.true./

c

c

SET UP POINTERS FOR TEMPORARY ARRAYS IF FIRST ENTRY

if (first) then

first $=$.false.

len $=$ nrmax*na

lhcap $=1$

lcond $=1$ hcap + len*nz

lrhs = lcond + len*nz

ldiag $=$ lihs + len

lcra = ldiag + len

lcrc $=1 \mathrm{cra}+1 \mathrm{en}$

$1 c a=1 c r c$ t len

$1 \mathrm{cb}=1 \mathrm{ca} \quad+1 \mathrm{en}$

Itrhs $=1 \mathrm{cb} \quad+1 e n$

$1 d=1$ trhs + na

lcri $=1 d+n \max$

lcro = lcri + na

lrhsi = lcro + na

Irhso $=\operatorname{lrhsi}+n a$

lrf $=$ lrhso + na

lcma $=1 \mathrm{rf}+\mathrm{na}$

$1 \mathrm{cmb}=1 \mathrm{cma}+\mathrm{na}$

$1 \mathrm{cmd}=1 \mathrm{cmb}+\mathrm{na}$

$1 \mathrm{cmdag}=1 \mathrm{cmd}+\mathrm{na}$

lcmrhs = lcmag + len

lend $=1 \mathrm{cmrhs}+\mathrm{na}$

c

10

if (lend.gt. Intemp) then write $(*, 10)$ lend, Intemp

format $(/ " \ll$ fatal error $\gg$ insufficient temporary'.

- 'storage space in solid\% length of as () = :

⿷ i6, ' maximum as () $=1, i 6 /)$

$$
\text { stop }
$$

end if

end if

c

c

LOOP OVER ALL CYLINDERS

do 100 icyln=1, ncyln

$n r i=n r(i c y l n)$

$n r i p=n r i+1$

OBTAIN SOLID PROPERTIES 


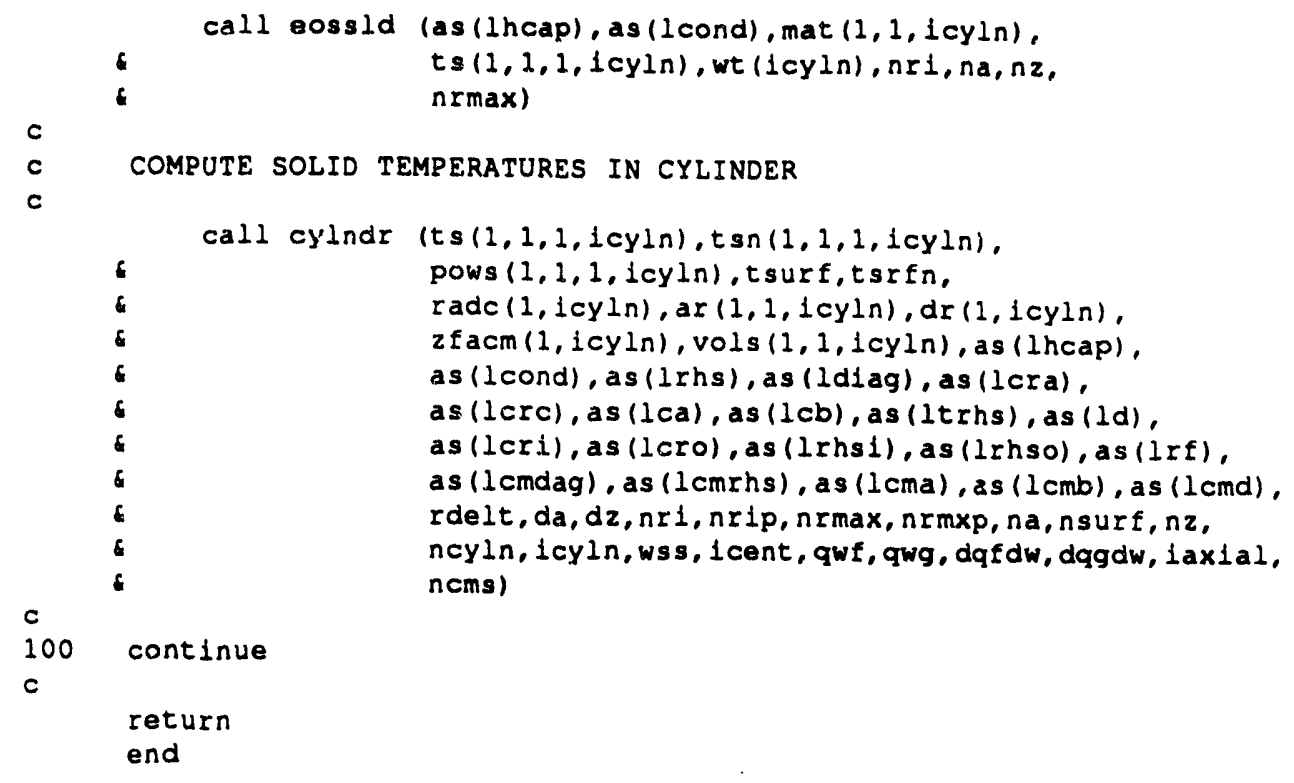

\section{SPLINE}

subroutine spline(1type, $n, t, y, h, b, u, v, z)$

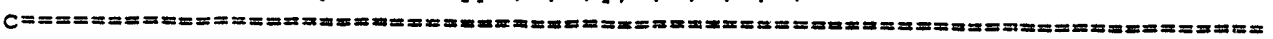

c Purpose: Thls routine computes the slopes of piecewise linear

c splines or the second derivatives of natural cubic

c splines.

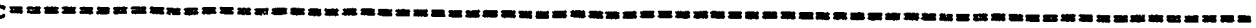

c Subroutine history

c - algorithm given in "Numerical Mathematics and Computing" by

c Cheney and Kincaid, 2nd Edition, 1985.

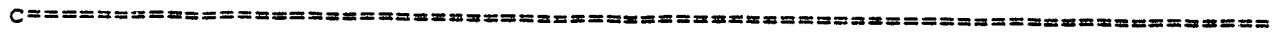

$c$

$c$

$c$

$c$

C

c

C

$c$

c

$c$

c

c

$c$ $c$

Arguments

\begin{tabular}{lcll} 
NAME & I/O & TYPE & DIM \\
\hline Itype & $I$ & I 4 &
\end{tabular}

DESCRIPTION
Identifier of type of spline
$=0$ (Natural cubic spline)
$=1$ (First-degree spline)
Number of points
Abscissa values or knots
Ordinate values
Intermediate working arrays
slopes of first-degree splines
or second derivatives of
natural cubic splines at the
knots

implicit real $(a-h, 0-z)$, integer $(1-n)$

parameter (zero $=0 ., t w o=2, s i x=6$.

dimension $t(n), y(n), h(n), b(n), u(n), v(n), z(n)$

if (itype ne. 0) then

do $10 i=1, n-1$

$$
z(i)=(y(i+1)-y(1)) /(t(i+1)-t(1))
$$

10

continue

return

end if

c

$$
\text { do } \begin{aligned}
20 \quad i & =1, n-1 \\
h(i) & =t(i+1)-t(i)
\end{aligned}
$$




\begin{tabular}{|l|ll|l|}
\hline $2 / 15 / 93$ & WSRC-TR-93-086 Rev. 0 & FLOWTRAN-TF v1.2 Source Code & Pg. 301 of 354 \\
\hline
\end{tabular}

20

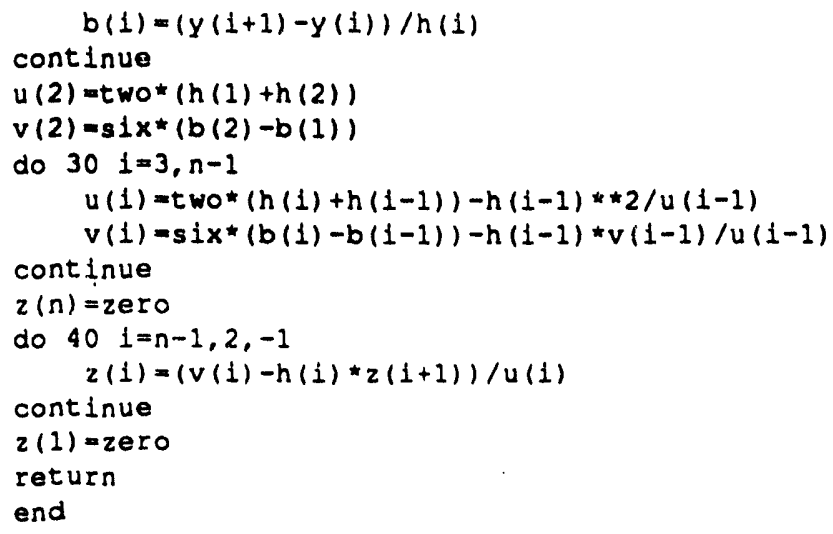

\section{SRIATC}

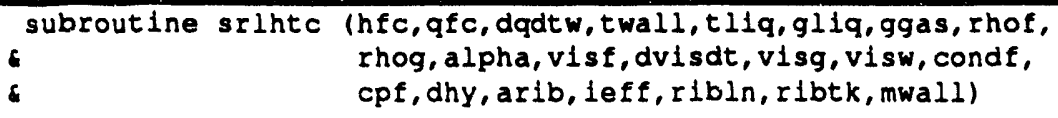


c

c

c

$c$

c

c

$c$

cC

cc

$\mathrm{cC}$

$\mathrm{cc}$

cc

CC

c

c

c

$c$

c

c

c

c

c

c

data rnul/4.0/, delal/0.01/

CALCULATE FLUID CELL PROPERTIES

NOTE: PHASIC LIQUID VELOCITY CORRELATION

re $=g l i q^{*}$ dhy/visf

$\mathrm{pr}=\mathrm{cpf} * \mathrm{visf} /$ condf

CALCULATE INVERSE MARTINELLI FACTOR AND TWO-PHASE

REYNOLDS NUMBER FACTOR (NORMALLY OFE)

$$
f=\text { one }
$$

CALCULATE LIQUID PHASE HEAT TRANSFER COEFFICIENT FOR

NON-BOILING SITUATION, CHECK FOR ANNULAR FLOW REGIME

CHURN-TURBULENT CORRELATION

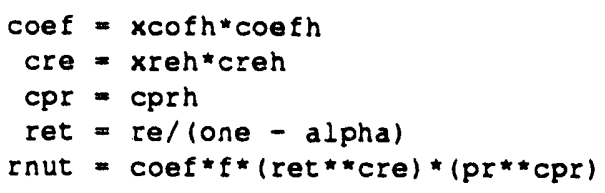

COMBINE CHURN-TURBULENT AND ANNULAR CORRELATIONS

if (delal.le.zero) then else

CHECK FOR LAMINAR FLOW

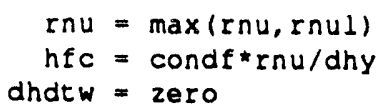


end if

psi $=$ ribln*tanh $(x 1) / x 1$

$h f c=h f c *(o n e+a r i b *(p s i-r i b t k))$

CALCULATE HEAT FLUX AND DERIVATIVE

delt $=$ twall - tliq

qfc $=h f c^{\star}$ delt

$d q d t w=h f c+q f c^{*} d h d t w$

c

return

end

\section{STATE}

subroutine state $(p, t g, t f, x a$, factor, 1, inftz, $n z, n z p 2, n x m a x, n c h n, 1 x$, c

ic, rog, drogdp, drogdt, drgdxa, hg, dhgdp, dhgdt, dhgdxa, rof, drofdp, drofdt, hf, dhfdp, dhfdt)

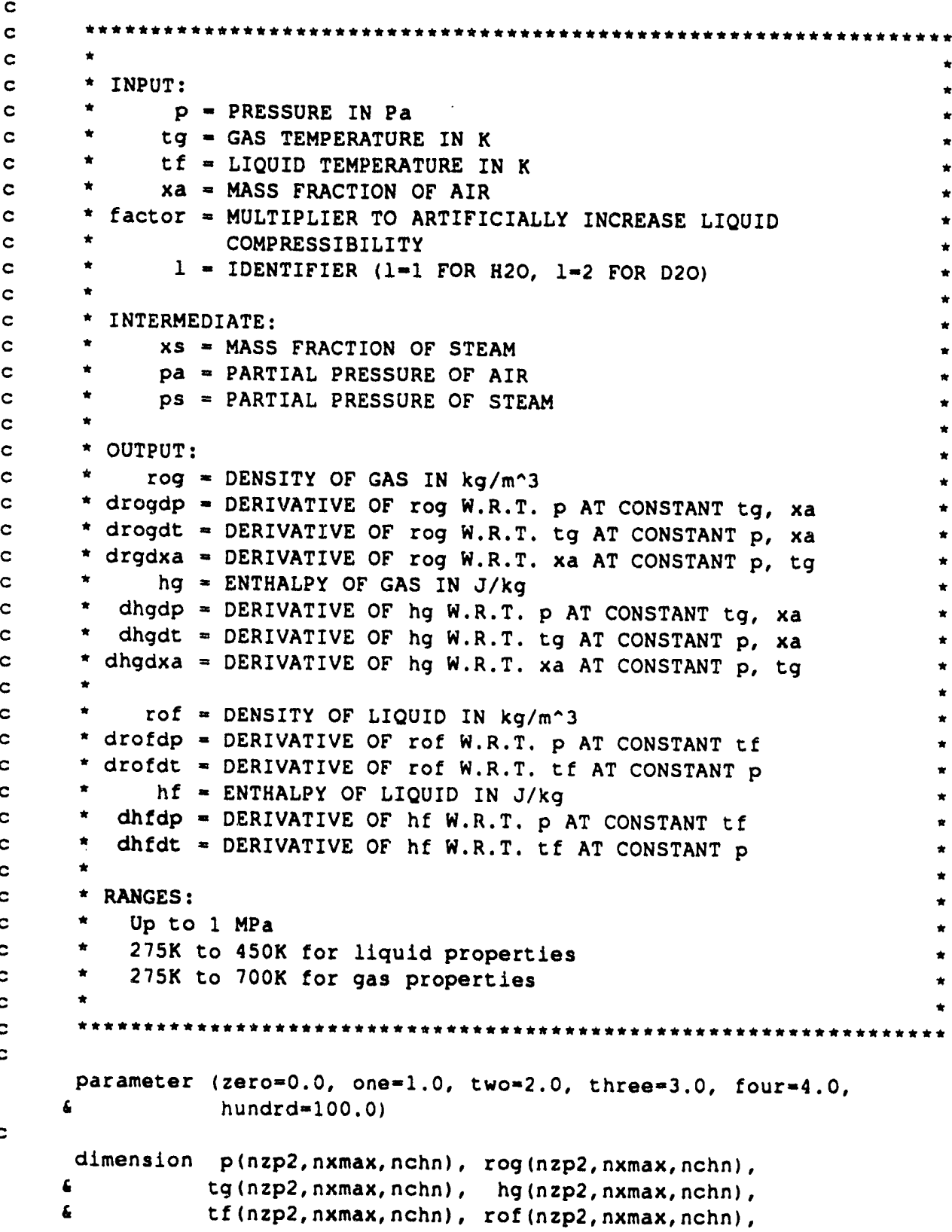


C

c

C

c 
c

c

c

c

c

c

c

c

c

c

c

6

COMPUTE GAS PROPERTIES

$\operatorname{rog}(j z, 1 x, 1 c)=\mathrm{roa}+\mathrm{ros}$

$\operatorname{drogdp}(j z)=\left(p a^{\star} d r o a d p+p s^{*} d r o s d p\right) / p(j z, i x, 1 c)$

$d r o g d t(f z)=d r o a d t+d r o s d t$

$\operatorname{drgdxa}(j z)=(w a * p a+w s(1) * p s) * * 2 /(w a * w s(1) * p(j z, i x, i c))$

$h g(j z, i x, i c)=x a(j z, i x, i c) * h a+x s * h s$

dhgdp $(j z)=x a(j z, i x, i c)$ \#hadp + xs*dhsdp

dhgdt $(j z)=x a(j z, i x, i c) * d h a d t+x s^{\star} d h s d t$

dhgdxa(jz) $=$ ha - hs

200

c

continue

return

end 


\section{STORE}

subroutine store (tsn, tsifn, qwf, qwg, dqfdw, dqgdw, hspl, qonb,

4 ptn, alptn, xatn, rogtn, roftn, hgtn, hetn, tgtn, tetn,

6 pn, alpn, xan, rogn, rofn, hgn, hen, tgn, ten,

- pbn, alpbn, xabn, rogbn, rofbn, hgbn, hfbn, tgbn, $t$ fbn,

6 ugt, uft, wgx, wfx, ugz, ufz, ugb, ufb, g1t, g1, gib,

c elt, el, e1b,c1tn, c1zn, c1xn, cibn, he1t, hel, hfib,

6 hg1t, hg1, hglb, akg1t, akg1, akg1b,ys1t, ys1, ys1b,

6 ppln, alppln, xapln, rogpln, rofpln, hgpln, hfpln,

- tgpln, tepln,ptbn, alptbn, xatbn, rogtbn, roftbn.

chgtbn, hetbn, tgtbn, tftbn, tsec, delt, nx, nr, nzp2,

nzp3, nxmax, nct
nsurf, isave)

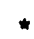

* SUBROUTINE store

- subroutine to save fluid VARIAble arRays by storing

* data for a restart file at unit nisto. data prom the

* LASt CONVERged Iteration (OLd time values) are stored.

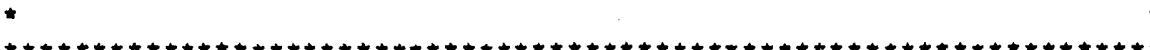

implicit real $(a-h, 0-z)$, integer $(1-n)$

common /iounit/ ninp, nout, ndmp, ntmp, nrsti, nrsto

\section{SOLID VARIABLES}

dimension qwf (nsurf,na,nz), dqfdw(nsurf,na,nz),

dimension tsn (nrmax, na, nz, ncyln) gwg (nsurf, na,nz), dggdw (nsurf, na,nz). tsrfn (nsurf, na,nz). hspl(nsurf, na,nz). qonb (nsurf, na, nz)

MIDDLE SECTION PHYSICAL PARAMETERS

dimension pn (nzp2, nxmax, nchn).

- alpn (nzp2, nxmax, nchn).

$\operatorname{rog} n(n z p 2, n x \max , n c h n), \operatorname{rof} n(n z p 2, n x \max , n c h n)$

hgn (nzp2, nxmax, nchn), hfn (nzp2, nxmax, nchn),

$\operatorname{tgn}(n z p 2, n x \max , n c h n), \quad t f n(n z p 2, n x \max , n c h n)$, gi (nzp2, nxmax, nchn), ei (nzp2, nxmax, nchn). ugz (nzp3, nxmax, nchn), ufz(nzp3, nxmax, nchn), wgx (nzp2, nxmax, nchn), wfx(nzp2, nxmax, nchn), cizn (nzp3, nxmax, nchn), cixn(nzp3, nxmax, nchn), hfi (nzp2, nxmax, nchn), hg1 (nzp2, nxmax, nchn). akgi(nzp2, nxmax, nchn), ysi(nzp2, nxmax, nchn), $n \times(n c h n)$

TOP SECTION PHYSICAL PARAMETERS

dimension ptn(nzt).

c alptn(nzt).

rogtn(nzt), roftn(nzt),

hgtn(nzt), hftn(nzt),

$\operatorname{tgtn}(n z t), t \in t n(n z t)$.

git (nzt), e1t (nzt)

ugt (nzt), uft(nzt),

citn(nzt), helt (nzt).

$n g 1 t(n z t), \operatorname{axg} 1 t(n z t)$.

ysit (nzt) 
c

c

c

c

C

c

c

C

c

c

C

$\pm 0$

c

$c$

c

BOTTOM SECTION PHYSICAL PARAMETERS

dimension pbn (nzb).

- alpbn(nzb), $x a b n(n z b)$.

rogbn (nzb), rofbn (nzb).

hgbn (nzb), hebn(nzb).

tgbn(nzb), tfbn(nzb).

$g i b(n z b)$, eib(nzb).

$u g b(n z b), u f b(n z b)$,

cibn (n>b), $n f i b(n z b)$,

hgib(nzb), akgib(nzb).

ysib(nzb)

WRITE BINARY OR ASCII RESTART FILE

if (1save.gt.0) then

WRITE BINARY RESTART FILE

write (nrsto) tsec, delt

STORE SOLID TEMPERATURES

do 10 lcy $\ln =1$, ncyln

$n r 1=n r(1$ cyln $)$

write (nrsto) ( (tsn(ir, ia, iz, icyln), $1 z=1, n z)$,

$c$

continue

$i a=1, n a)$

$1 r=1, n(1)$

WRITE SURFACE HEAT FLUXES, HEAT FLUX DERIVATIVES, SINGIE PHASE

HEAT TRANSFER COEFFICIENTS, ONB HEAT FLUXES, AND TEMPERATURES

write (nrsto) (( $q w f(i s u r f, i a, 1 z)$, qwg (isurf,ia, iz),

6 dqfdw(isurf, $1 \mathrm{a}, 1 z), \operatorname{dqgdw}(i \operatorname{surf}, 1 \mathrm{a}, 1 z)$,

hspl (1surf, 1a,1z), qonb(1surf,1a,1z),

tsrfn(1surf, $1 a, 1 z), \quad(z=1, n z), 1 a=1, n a)$,

isurf $=1$, nsurf)

STORE LOCAL PRESSURE, VOID FRACTION, AIR MASS FRACTION, GAS TEMPERATURE, FLUID TEMPERATURE, GAS DENSITY, FLUID DENSITY, GAS ENTHALPY, FLUID ENTHALPY, INTERFACIAL MASS AND ENERGY TRANSFER RATES, AND GAS AND FLUID VELOCITIES

PI.INUM

write (nrsto) ppln, alppln, xapln, tgpln, tfpln,

6

rogpln, rofpln, hapin, hfpln

TOP SECTION

write (nrsto) $($ ptn(jz).

c $\operatorname{alptn}(j z), \operatorname{xatn}(j z)$.

$\operatorname{tgtn}(j z), \operatorname{tftn}(j z)$.

$\operatorname{rogtn}(j z), \operatorname{roftn}(j z)$,

hgtn(jz), hftn(jz),

gitijz), eit $(j z)$.

ugt (jz), Let (jz).

$\operatorname{citn}(j z), h f i t(j z)$.

hgit $(j z)$, akgit $(j z)$.

$y \operatorname{sit}(j z), j z=1, n z t)$

MIDDLE SECTION 
C

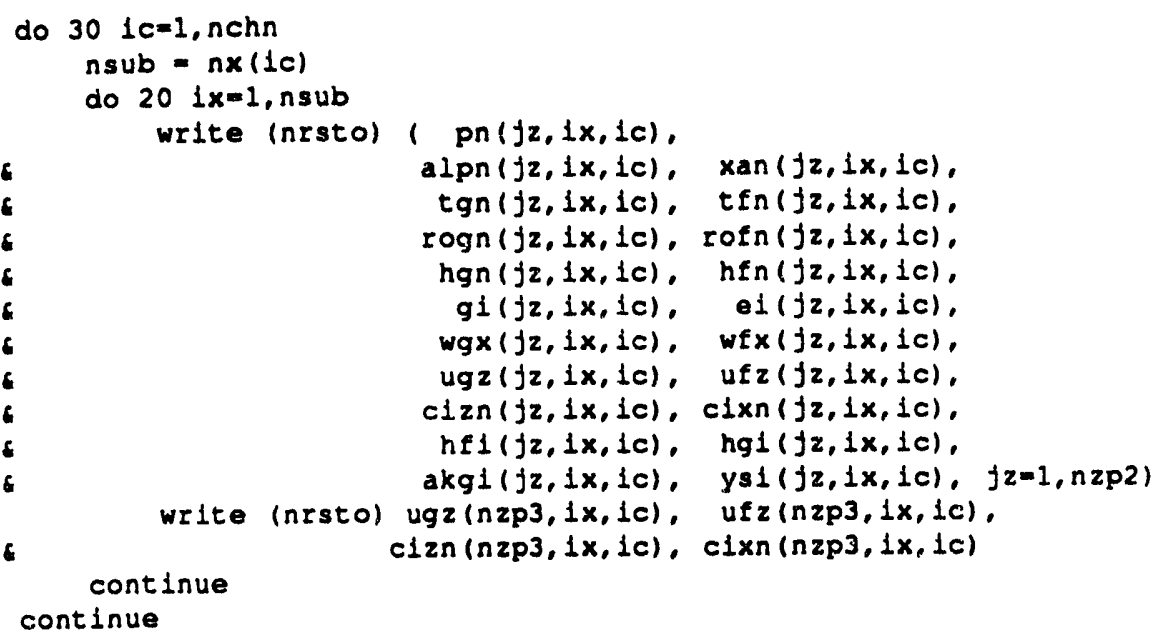

c

c

TANK BOTTOM

write (nrsto) ptbn, alptbn, xatbn, tgtbn, tftbn,

6

rogtbn, roftbn, hgtbn, hftbn

return

else

WRITE ASCII RESTART FILE

write (nrsto, *) tsec, delt

STORE SOLID TEMPERATURES

do 40 lcyln=1, ncyln $n r 1=n r(10 y l n)$

6

write (nrsto,*) ((t)sn(ir,ia,iz,icyln), $i z=1, n z)$,

$i a=1, n a)$.

continue

WRITE SURFACE HEAT FLUXES, HEAT FLUX DERIVATIVES, SINGLE PHASE HEAT TRANSFER COEFFICIENTS, ONB HEAT FLUXES, AND TEMPERATURES

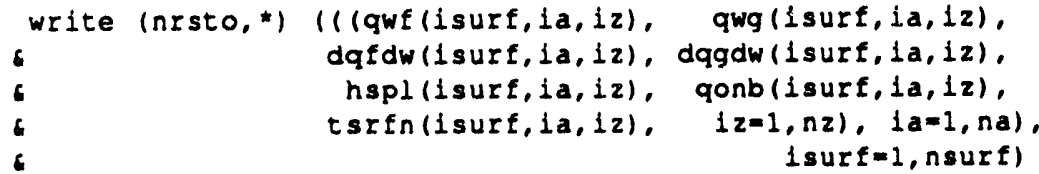

c

STORE LOCAL PRESSURE, VOID FRACTION, AIR MASS FRACTION, 
TANK BOTTOM

write (nrsto,*) ptbn, alptbn, xatbn, tgtbn, tftbn,

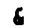

rogtbn, roftbn, hgtbn, hetbn

end $1 f$

return 


\begin{tabular}{|l|ll|r|}
\hline Pg. 310 of 354 & WSRC-TR-93-086 Rev.0 & FLOWTRAN-TF v1.2 Source Code & $2 / 15 / 93$ \\
\hline
\end{tabular}

end
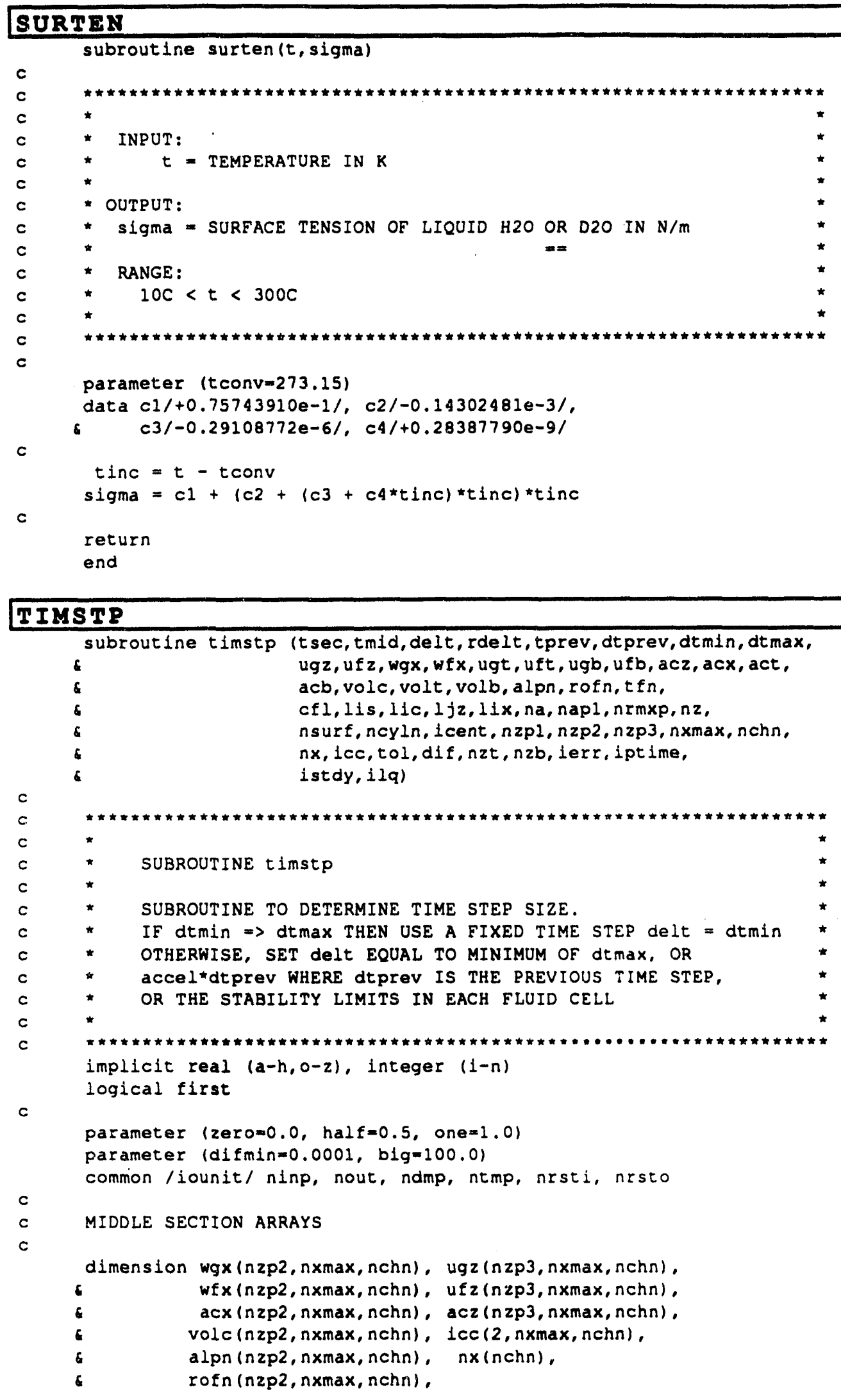
c

c

c

c

c

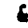

$\operatorname{tn}(n z p 2, n \times \max , n \operatorname{chn})$

TOP AND BOTTOM SECTION ARRAYS

dimension ugt(nzt), uft(nzt), act(nzt), volt (nzt), $u g b(n z b), u f b(n z b), a c b(n z b), \operatorname{volb}(n z b)$

$\operatorname{data} \operatorname{accel} / 0.9 /, \operatorname{xcfl} 1 / 0.9 /$

RESET CFL INDICATORS

cfl $=$-one

1 is $=0$

lic $=0$

$1 j z=0$

$1 i x=0$

c

c

BEGIN TIME STEP CONTROL LOGIC:

ON ERROR, CUT TIME STEP AND REITERATE

reduc $=$ half

if (ierr.eq.3) then

reduc $=\operatorname{accel} *($ tol $/ \mathrm{d} I f)$

ierr $=1$

end if

if ((ierr.eq.1) and. (istdy.1t.2)) then

$t \sec =t \sec -$ delt

delt $=$ reduc*delt

$t$ sec $=t \sec +$ delt

tmid = tsec - half delt

goto 190

elseif ((1err.eq.1) .and. (istdy.ge.2)) then

delt = reduc*delt.

end if

goto 190

c

WITH NO ERROR, SAVE OLD VALUES AND

GO ON TO THE NEXT TIME INTERVAL

tprev $=t \sec$

dtprev $=$ delt

if (dtmin.ge.dtmax) then

delt $=d t m i n$

$t$ sec $=t \sec +$ delt

$t \operatorname{mid}=t \sec -$ half*delt

rdelt $=$ one/delt

return

end if

C

c

c

CHECK STABILITY LIMITS IN TOP SECTION

$c f l=b i g$

$\operatorname{tcf} 1=b i g$

do $120 \quad j z=1, n z t$

$j z p=j z+1$

$(j-1 / 2$ FACE $)$

sumg $=-\operatorname{act}(j z) \star \min (z e r o, \operatorname{ugt}(j z))$

$\operatorname{sumf}=-\operatorname{act}(j z) \star \min (z \operatorname{ero}, u f t(j z))$ 
c

c

100

110

120

c

c

c

c

C

C

$c$

c

c

c

$(j+1 / 2$ FACE $)$

if (jzp .le. nzt) then

sumg $=$ sumg $+\operatorname{act}(j z p) * \max (z e r o, u g t(f z p))$

sumf $=\operatorname{sumf}+\operatorname{act}(j z p) \star_{\max }(z e r o, u f t(j z p))$

else

do $1101 c=1$, nchn

nsub $=n \times(i c)$

do 100 ix $x=1$, nsub

sumg $=\operatorname{sumg}+\operatorname{acz}(2,1 x, 1 c) \star_{\max }(z e r 0, \operatorname{ugz}(2,1 x, 1 c))$

sumf $=\operatorname{sumf}+\operatorname{acz}(2, i x, i c) * \max (z e r o$, ufz $(2,1 x, 1 c))$

continue

continue

end if

If (sumg .gt. zero) tcfl = xcfl*volt (fz)/sumg

if (sumf.gt. zero) tcfl $=\min (t c f l, x \in f l * \operatorname{volt}(j z) / \operatorname{sumf}$ )

if (tcfl. it. cfl) then

cf $1=t c f l$

1 is $=1$

$1 j z=j z$

end if

continue

CHECK STABILITY LIMITS IN MIDDLE SECTION

do 150 ic=1, nchn

$n$ sub $=n \times(1 c)$

do 140 ix=1, nsub

$i x r=1 c c(2, i x, i c)$

do $130 j z=2, n z p 1$

$j z m=j z-1$

$j z p=j z+1$

$(j-1 / 2$ FACE)

sumg $=\quad-\operatorname{acz}(j z, i x, i c) * \min (z e r o, u g z(j z, i x, i c))$

sumf $=\quad-\operatorname{acz}(j z, i x, i c) * \min (z e r o$, ufz $(j z, i x, i c))$

$(j+1 / 2$ FACE $)$

sumg $=$ sumg $+\operatorname{acz}(j z p, i x, i c) * \max (z e r o, u g z(j z p, i x, i c))$

$\operatorname{sumf}=\operatorname{sumf}+\operatorname{acz}(j 2 p, i x, i c) \star_{\max }(z e r o, u f z(j z p, i x, i c))$

$(i-1 / 2$ FACE $)$

sumg $=$ sumg $-\operatorname{acx}(j z, 1 x, 1 c) \star_{m} \ln (z e r o, w g x(j z, 1 x, 1 c))$

sumf $=\operatorname{sumf}-\operatorname{acx}(j z, 1 x, 1 c){ }^{*} \min (z e r o, w f x(j z, 1 x, 1 c))$

$(i+1 / 2$ FACE)

sumg $=\operatorname{sumg}+a c x(j z, i x r, i c) * \max (z e r 0, w g x(j z, i x r, i c))$

sumf $=\operatorname{sumf}+\operatorname{acx}(j z, i x r, i c) * \max (z e r 0, w f x(j z, i \times r, i c))$

if (sumg .gt. zero) tcfl $=x c f l * v o l c(j z, 1 x, i c) /$ sumg

if (sumf .gt. zero) tcfl $=\min (t c f l, x c f l * v o l c(j z, 1 x, i c) / s u m f$ )

If (tcfl. It. cfl) then

$c f l=t c f l$

1 is $=2$

$11 \mathrm{c}=1 \mathrm{c}$

$1 j z=j z m$

$1 i x=i x$

end if 

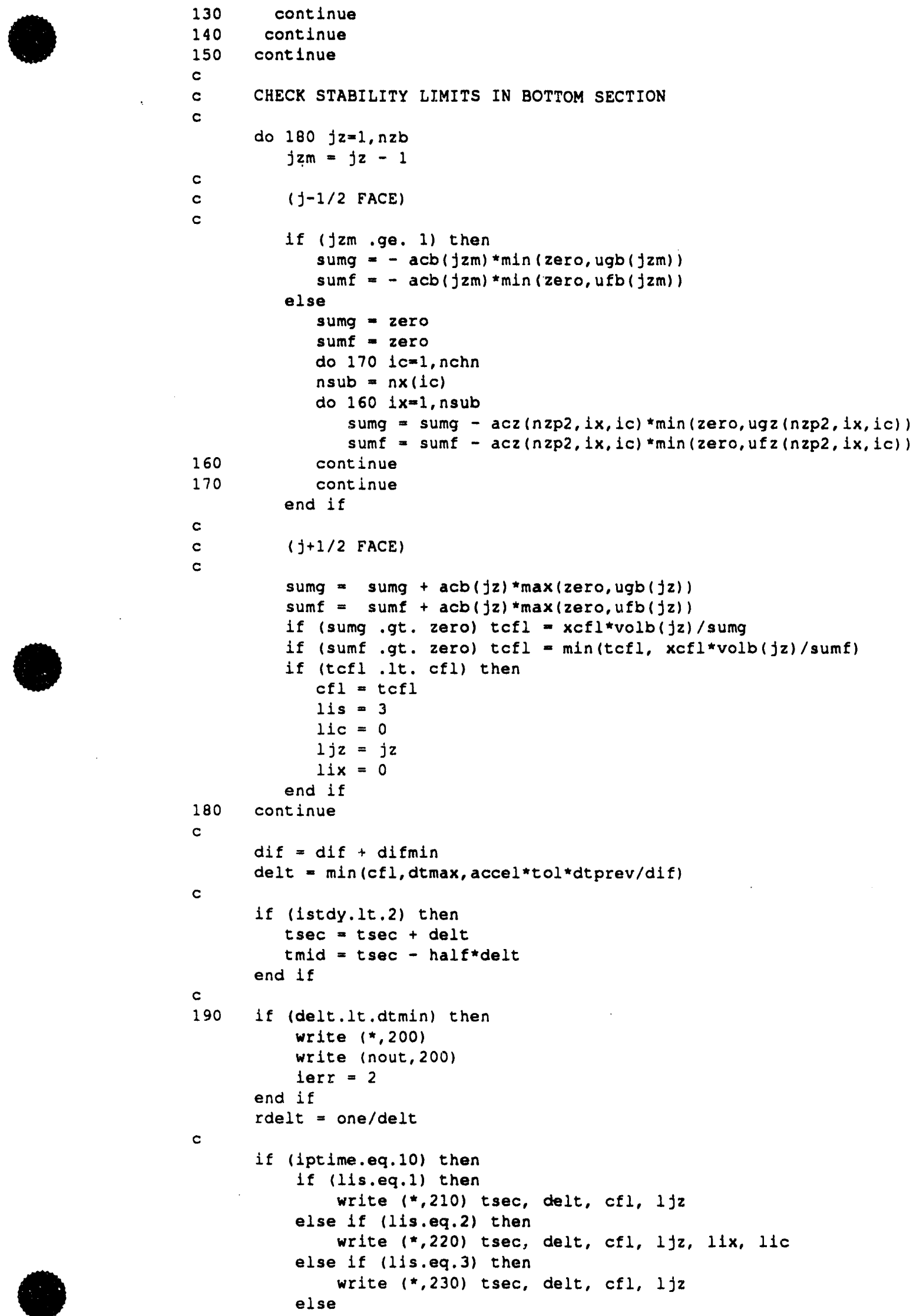


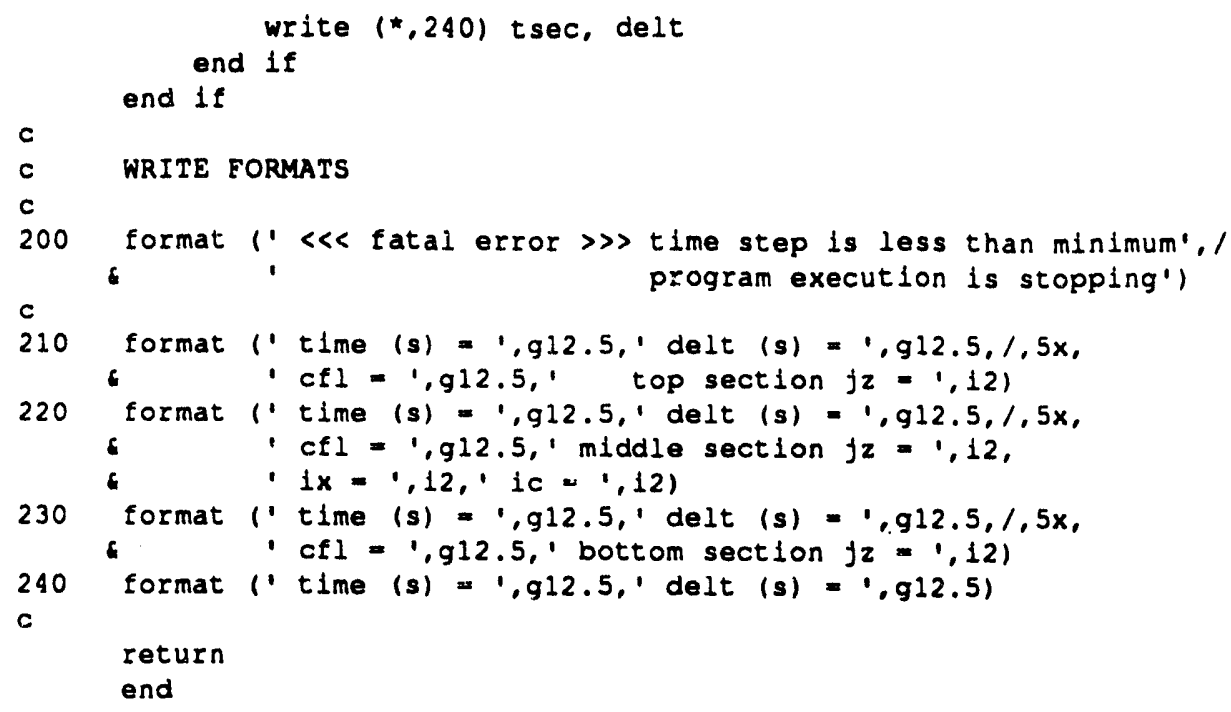

\section{TRANS}

subroutine trans (critr, tcrit, lcrit, iredo)

c

C $\quad$ C

c

c

c

c

INITIALIZE FLAGS AND TIME COUNTERS

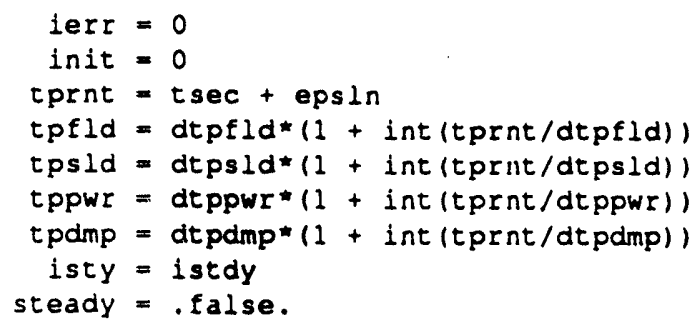




\begin{tabular}{|l|ll|l|}
\hline $2 / 15 / 93$ & WSRC-TR-93-086 Rev. 0 & FLOWTRAN-TF v1.2 Source Code & Pg. 315 of 354 \\
\hline
\end{tabular}

end $1 f$

c

c

100

c

c

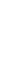

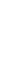

end if

call polst (a(lpows), sumdry,tnklev, a(laxial), a (laxal0), deldry,

- delmod, a(ldz+1), hactiv, 1zht, 1zhb, gap, asypwr, tmid,

- a(lbigm), a (lbigmw), a (lbigmd), a (lxtp), a (lytp),

- a(1ztp), la(lntp), la(11ntp), la(11type), 1fissn,

- 1tnklv, tnklvo, 1modh, 1dryh, 1conh, 1exp, inlt, na, ncyln,

- la(lnr),nz,nrmax, ntpmax, ntptot,nspln, nout)

CALCULATE SURFACE HEAT FLUXES FOR THIS TIME STEP

if (1err.eq.0) then

call qwall (a (lqwf),a(lqwg), a (ldqfdw), a (ldqgdw), a(1theta),

$a($ lgw), a (lhspl), a (lqonb), a (lchfq). $a(1 t s u r f), a(l p), a(l a l p), a(1 \times a), a(1 r \circ g), a(1 r \circ f)$, $a(1 t g), a(1 t f), a(1 \cup g z), a(1 \cup f z), a(1 d h z), a(1 a r)$, tmodo, hmod, hactiv, tnklev, gap, tsec, a $(1 d z+1)$, $a(1 r$ lbl), a (1ribt), 1a(1iorib), la(lmat), 1boil, 1chf, matgas, persat, nsurf, na, napl, nxmax, la (lnr), nrmax, nrmxp, 1a $(\operatorname{lnx}), n z, n z p 2, n z p 3, n c h n, n c y l n, 1$ cent, la (liaz1), 1a (Inazc), ht damp, iradbc, crad1, Itmod, $a(1 x t p), a(1 y t p), a(1 z t p)$, la (lntp), la (lintp), ia (l1type), nspln, ntpmax, ntptot, ilq)

CALCULATE SOLID INTERNAL AND SURFACE TEMPERATURES

if (1stdy.ge.1) then

else

rdelts $=\mathrm{rdelt} / \mathrm{xdelt}$

rdelts $=$ rdelt

end if

call solid (a(lts),a(1tsn), a(ltsurf), a(ltsrfn), a(lpows),

- $a(l q w f), a(l q w g), a(1 d q f d w), a(1 d q g d w), a(l w t)$,

6 a(lradc), a(lar), a(lvols), a(lzfacm), ia(lmat),

- rdelts, da, a (ldr), a (ldz+1), la (lnr), na, nsurf,

d $n z, n c y l n, n r m a x, n r m x p$, wss, icent, iaxial, ncms)

end $1 f$

OBTAIN FLUID DYNAMICS BOUNDARY CONDITIONS AT TOP AND BOTTOM OF ASSEMBLY EOR NEW TIME 


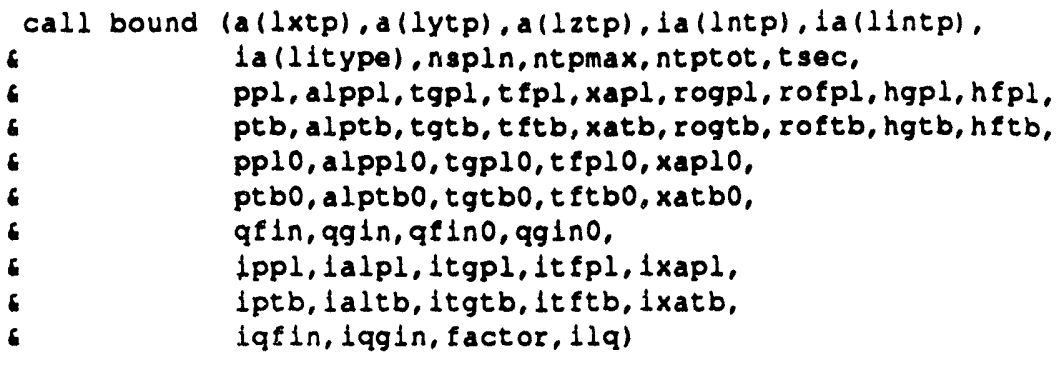

if (1err.eq.0) then

COMPUTE INTERFACIAL HEAT, MASS, AND MOMENTUM TRANSFER COEFEICIENTS

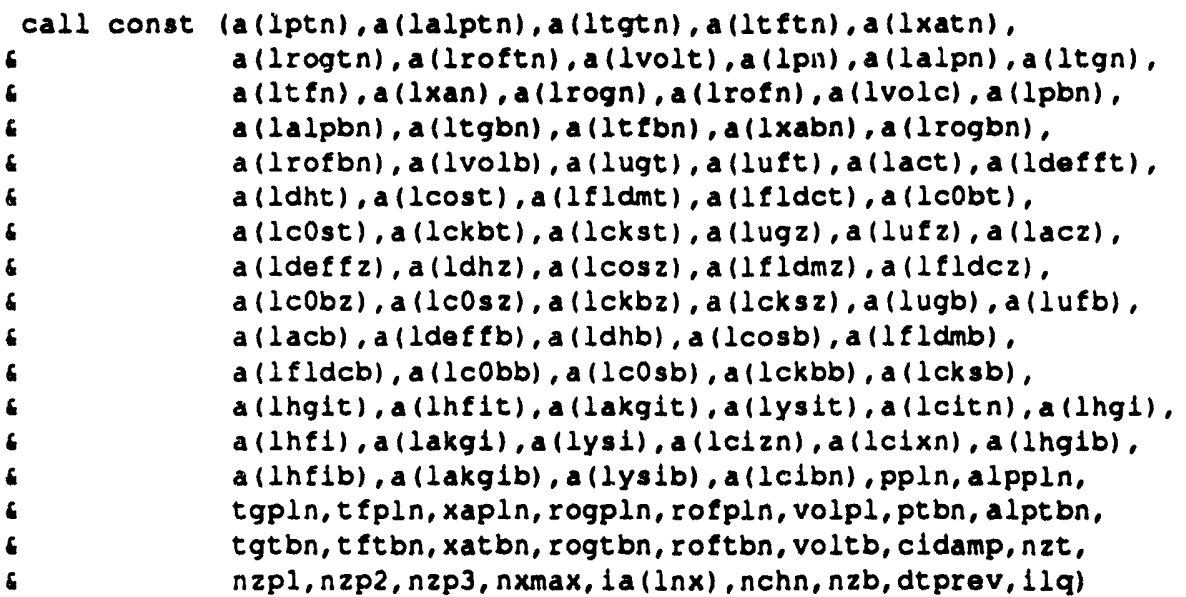

CALCULATE EXPLICIT TURBULENT MASS EXCHANGE RATES

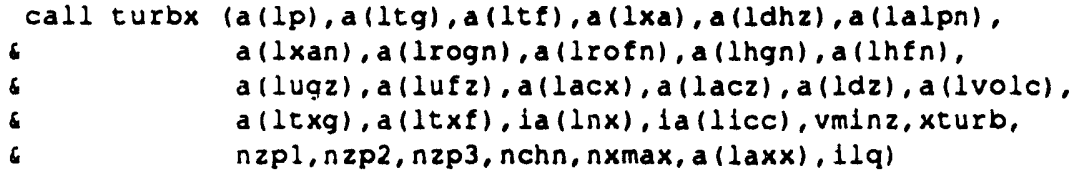
$a(1 \times a n), a(1 \operatorname{rog} n), a(1$ rof $n), a(1 h g n), a(1 h f n)$, $a(1 \cup g z), a(l u f z), a(l a c x), a(l a c z), a(1 d z), a(1 v o l c)$, $a(1 t x g), a(1 t x f), 1 a(\ln x), 1 a(11 c c)$, vminz, xturb, $n z p 1, n z p 2, n z p 3, n c h n, n x \max , a(\operatorname{axx}), 11 q)$ 
c

c

SET UP DONOR ARRAYS USED BY JACOB IN TOP SECTION

call donort (a(lalpt $n), a(1 \times a t n), a($ lrogt $n), a($ lroftn), a(lhgt $n)$, - a(lhftn), alppln, xapln, rogpln, rofpln, hgpln, hfpln, - a (lugt), a(luft), a(lbgt), a (lcgt), a(1bft), a (1cft), - a(lact), a(ladvbt), a(ladvct), vminz,nzt)

\section{SET UP DONOR ARRAYS USED BY JACOB IN MIDDLE SECTION}

call donorm (a(lalpn), a(lxan), a(lrogn), a(1rofn), a(lhgn), a (1hfn), - $a(\operatorname{lug} z), a(\operatorname{lufz}), a(\log z), a(1 \log z), a(1 b f z), a(1 \subset f z)$, 6 $a(\operatorname{lwg} x), a(\operatorname{lwf} x), a(\operatorname{ldg} x), a(\log x), a(1 d f x), a(\operatorname{lef} x)$, c $a(1 a c z), a(1 a c x), a($ ladvbz), $a(1 a d v c z), a(1 a d v d x)$, - a (ladvex), vminz,vminx, nzp1, nzp2,nzp3, nchn, nxmax, - $\operatorname{la}(\ln x), 1 a(11 c c))$

SET UP DONOR ARRAYS USED BY JACOB IN BOTTOM SECTION

call donorb (a (lalpbn), a (1xabn), a (1rogbn), a (1rofbn), a (1hgbn), - a (lhfbn), alptbn, xatbn, rogtbn, roftbn, hgtbn, hftbn, 4 $a(1$ (lugb), $a(1 \cup f b), a(1 b g b), a(1<g b), a(1 b f b), a(1 c f b)$, $a(1 u g b), a(1 u f b), a(1 b g b), a(1 c g b), a(1 b f b)$
$a(1 a c b), a(1 a d v b b), a(1 a d v c b), v m i n z, n z b)$

end if

PEREORM NEWTON ITERATIONS TO SOLVE FLUID CONSERVATION EQUATIONS

call newton (a ( $\operatorname{azm} 1), a(\operatorname{lazm} 2), a(\operatorname{laxm1}), a(\operatorname{laxm} 2), a(1$ rhs), a (1sx), b $a(1 s z), a(1 t), a(1 \mathrm{dpm}), a(1 \mathrm{atml}), a(1 \mathrm{rhst}), a(1 s t), a(1 \mathrm{abml}), a(1 \mathrm{rhsb})$, - $a(1 \mathrm{sb}), a(1 \mathrm{cmesh}), a(1 \mathrm{ar}), a(1 \mathrm{volc}), a(1 \mathrm{volt}), a(1 \mathrm{volb}), a(1 \mathrm{cpat})$.

6 a (lcptgt), a (leptft), a (lcpxat), a (lfat), a (lftgt), a (lftft), a (lfxat),

- a (1dpt), a (lcpa), a (lcptg), a (lcptf), a (lcpxa), a (lfa), a (lftg),

- $a(1 f t f), a(1 f \times a), a(1 d p), a(1 c p a b), a(1 c p t g b), a(1 c p t f b), a(1 c p \times a b)$,

- $a(1 f a b), a(1 f t g b), a(1 f t f b), a(1 f \times a b), a(1 d p b), a(1 p t n), a(1 a l p t n)$,

- a(1xatn), a(1rogtn), a(1hgtn), a(1roftn), a(1hftn),a(ltgtn),a(1tftn),

- $a(1 p t), a($ lalpt), a (lxat), a (lrogt), a (lngt), a (lroft), a (lnft),

\& $a(1 t g t), a(1 t f t), a(1 p n), a(1 a l p n), a(1 \times a n), a(1 r \circ g n), a(1 h g n)$,

- $a(1 \operatorname{rof} n), a(1 h f n), a(1 t g n), a(1 t f n), a(1 p), a(1 a l p), a(1 \times a), a(1 r \circ g)$,

a $a(1 h g), a(1 r o f), a(1 h f), a(1 t g), a(1 t f), a(1 p b n), a(1 a l p b n), a(1 \times a b n)$,

- $a(1 r \circ g b n), a(1 h g b n), a(1 r \circ f b n), a(1 h f b n), a(1 t g b n), a(1 t f b n), a(1 p b)$,

- $a(1 a l p b), a(1 \times a b), a(1<\circ g b), a(1 h g b), a(1<\circ f b), a(1 h f b), a(1 t g b)$,

- $a(1 t f b), a(\lg i t), a(1 e i t), a(1 y s i t), a(\operatorname{lng} i t), a(\operatorname{lnf}(t), a(\operatorname{lakgit})$,

- a(lgi), a(lei), a(lysi), a(lhgi), a(lhfi), a(lakgi), a(lglb), a(leib),

- $a($ lysib), $a($ lngib), $a(\ln f(b), a($ lakgib), a (ladvbz), a (ladvcz),

- $a(1$ advax), a (ladvex), a (ladvbt), a (1advct), a (ladvbb), a (1advcb),

- $a(\operatorname{lbg} t), a(1 b f t), a(\log t), a(1<f t), a(\operatorname{lbg} z), a(\operatorname{lbf} z), a(\log z), a(\operatorname{lcf} z)$,

- $a(\operatorname{ldg} x), a(\operatorname{ldf} x), a(\operatorname{leg} x), a(\operatorname{le} f x), a(\operatorname{lbg} b), a(\operatorname{lof} b), a(\log b), a(\operatorname{lcfb})$,

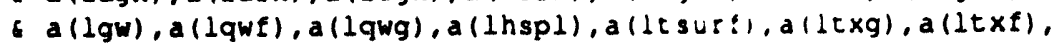

- $1 a(\operatorname{llaz} 1), 1 a(\ln a z c), 1 a(\ln x), 1 a(\ln r), \operatorname{la}(: \operatorname{lec})$

\section{PERFORM ACCURACY CHECK ON FLUID CALCU:AT:ONS}

If (1err.eq.0) then call accchk (a(1pt),a(lalpt),a(1tgt),a(1tft),a(1xat), $a(1 r \circ g t), a(1 r \circ f), a(1 h g t), a(\ln f t), a(1 p)$, $a(1 a \perp p), a(1 t g), a(1 t f), a(1 \times a), a(1 r \circ g)$. $a(1 r o f), a(1 h g), a(1 h f), a(1 p b), a(1 a l p b)$, $a(1 t g b), a(1 t f b), a(1 \times a b), a(1 r \circ g b), a(1 r \circ f b)$, $a($ lhgb), $a($ lhfb), $a(1 p t n), a(1 a \mid p t n), a(1 t g t n)$, $a(1 t f t n), a(1 \times a t n), a(1 \operatorname{rog} t n), a(1 \operatorname{roftn})$, $a(1 h g t n), a(1 h f t n), a(1 p n), a(l a l p n), a(1 t g n)$, $a(1 t f n), a(1 x a n), a(1 \operatorname{rog} n), a(1 \operatorname{rof} n), a(1 h g n)$, 


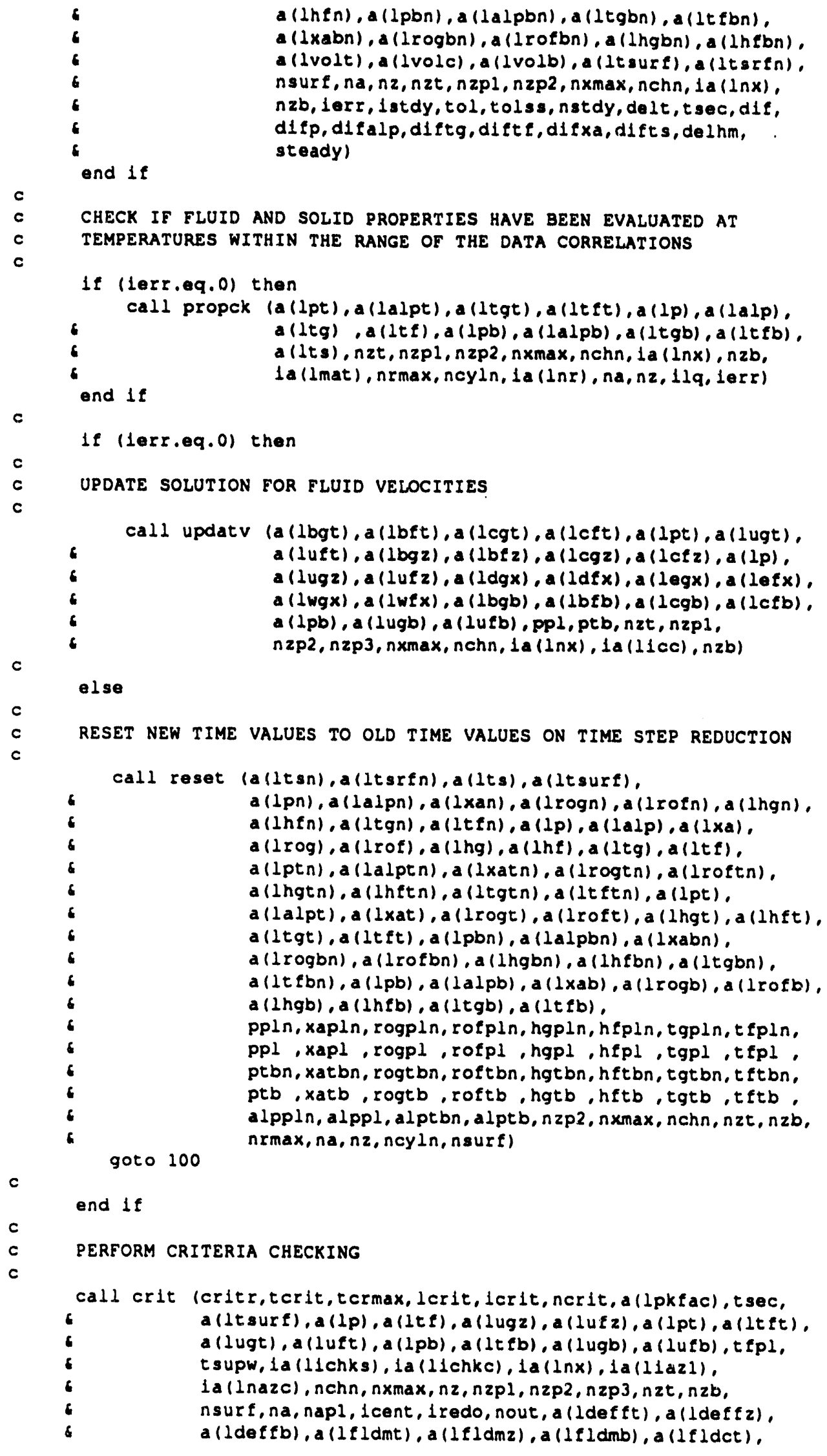




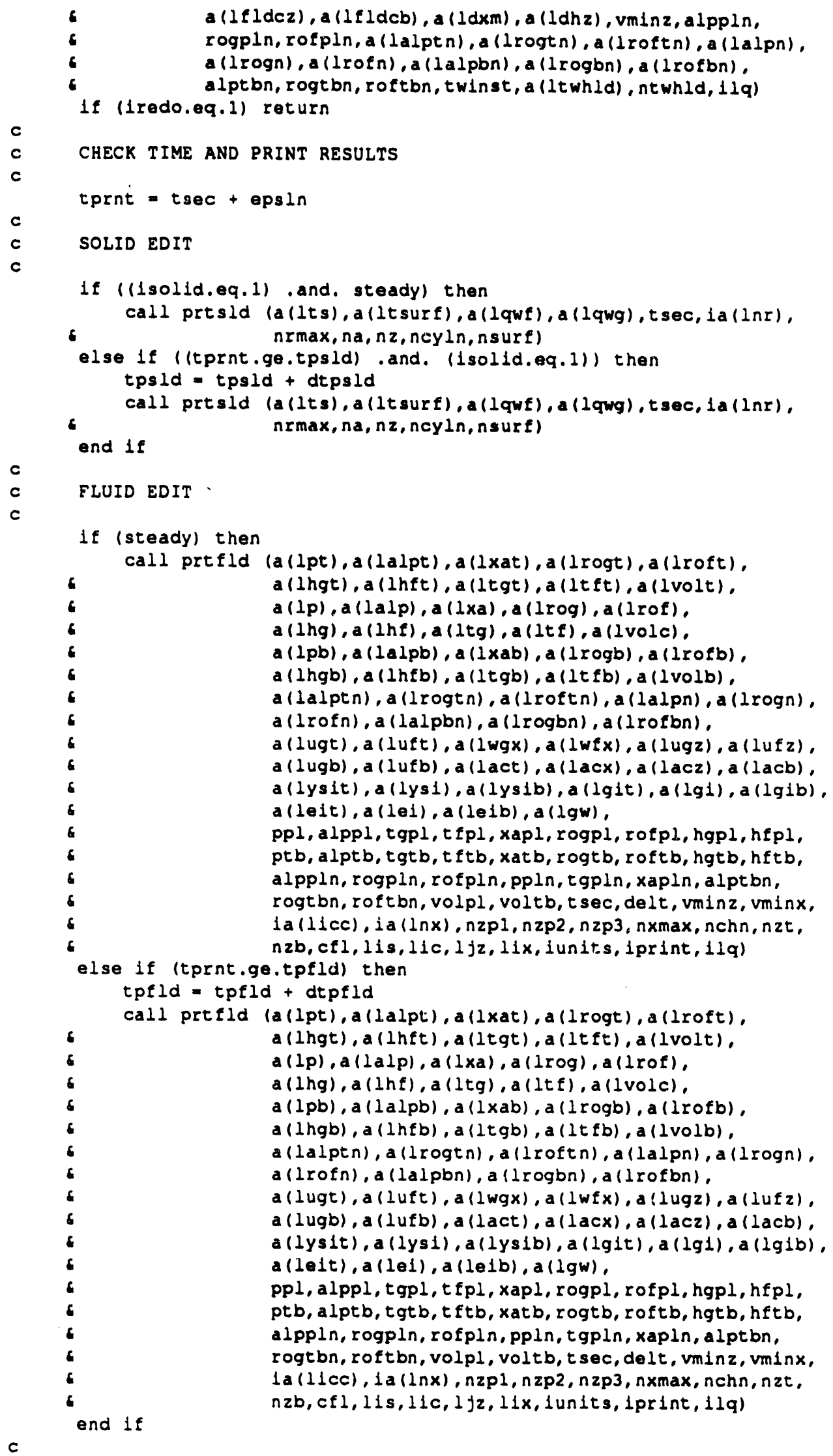


if ((dtpdmp.1e.runsec) and. steady) then call dmpplt (a (1ts),a (ltsurf), a (lqwf), a (lqwg), a (lpt), a (lalpt), a (lxat), a (lrogt), a (1roft), a (lngt), $a(1 h f t), a(1 t g t), a(1 t f t), a(1 p), a(1 a l p), a(1 \times a)$, $a(\operatorname{lrog}), a(1 r o f), a(1 h g), a(1 h f), a(1 t g), a(1 t f)$, $a(1 p b), a(1 a \perp p b), a(1 \times a b), a(1 r \circ g b), a(1 r \circ f b)$, $a(1 h g b), a(1 h f b), a(1 t g b), a(1 t f b), a(1 u g t), a(1 u f t)$, $a(1 w g x), a(1 w f x), a(l u g z), a(l u f z), a(l u g b), a(1 u f b)$, ppl, alppl, tgpl, tfpl, xapl, rogpl, rofpl, hqpl, hfpl, ptb, alptb, tgtb, tftb, xatb, rogtb, roftb, hgtb, hftb, t sec, $1 a(\ln x), 1 a(\ln r), n z p 1, n z p 2, n z p 3, n x m a x, n c h n$, $n s u b c, n z t, n z b, n r m a x, n a, n z, n c y l n, n s u r f)$

else if (tprnt.ge.tpdmp) then $t p d m p=t p d m p+d t p d m p$ call dmpplt (a (1ts), a (ltsurf), a (lqwf), a (lqwg), a (lpt), $a(1 a l p t), a(1 x a t), a(1$ rogt), a (lroft), a (lhgt), $a(1 h f t), a(1 t g t), a(1 t f t), a(1 p), a(1 a l p), a(1 \times a)$, $a(1 r o g), a(1 r o f), a(1 h g), a(1 h f), a(1 t g), a(1 t f)$, $a(1 p b), a(1 a l p b), a(1 \times a b), a(1 r \circ g b), a(1 r \circ f b)$. $a(1 h g b), a(1 h f b), a(1 t g b), a(1 t f b), a(1 u g t), a(1 u f t)$. $a(1 w g x), a(1 w f x), a(\operatorname{lugz}), a(1 \cup f z), a(1 u g b), a(l u f b)$. ppl, alppl, tgpl, tfpl, xapl, rogpl, rofpl, hgpl, hfpl, ptb, alptb, tgtb, tftb, xatb, rogtb, roftb, hgtb, hftb, tsec, ia $(\ln x), i a(\ln r), n z p 1, n z p 2, n z p 3, n x \max , n c h n$, end if nsubc, nzt, nzb, nrmax, na, nz, ncy $1 n, n s u r f)$

PRINT RESULTS TO SCREEN

if (1scrn.gt.0 and. 1scrn.1t.3) then

call prtsin (a(lpt), a(lalpt), a(ltgt), a(1t: :),a(:xat), a (lrogt), a (lroft), a (lhgt), a ( $i n:: 1$, $a(1 p), a(1 a \mid p), a(1 t g), a(1 t f), d(: x a)$, $a(1 r \circ g), a(1 r o f), a(1 h r), a(1 h t)$. $a(1 p b), a(1 a \mid p b), a(1 t g b), a(1 t \leq b), a(; x a b)$, $a(1 r \circ g b), a(1 r \circ f b), a(1 h g b), a(1 n f b)$, ppl, alppl, tgpl, tfpl, xapl, rogpl, rofpl, hgpl, hfpl, ptb, alptb, tgtb, tetb, xatb, rogtb, roftb, hgtb, hftb, $a(1 \cup g t), a(1 u f t), a(1 u g z), a(1 u f z), a(1 w g x), a(1 w f x)$, $a(1 u g b), a(1 u f b), n z t, n z p l, n z p 2, n z p 3, n x \max , n c h n$, la $(\ln x), n z b, t \sec$, delt, dtmin, dtmax, nitno, 11 tot, dif, difp, difdlp, diftg, diftf, difxa, difts, delhm, a (lysit), a(lysi), a (lysib),a(lgit), $a(\lg 1), a(\lg 1 b), c r 1 t r, n c r i t, 1$ scrn, twinst, cfl, vininz, a (lact)) 
end if

RESET OLD TIME $(n)$ VALUES OF VARIABLES FOR NEW TIME STEP

call reset (a(lts), a(ltsurf), a(ltsn), a(ltsrfn),

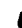

$a(1 p), a(1 a l p), a(1 \times a), a(1<\circ g), a(1 r \circ f), a(1 h g), a(1 h f)$, $a(1 t g), a(1 t f), a(l p n), a(l a l p n), a(l x e n), a(\log n)$, $a(1 \operatorname{lof} n), a(1 h g n), a(\ln f n), a(l t g n), a(l t f n), a(l p t)$, $a($ lalpt), a (lxat), a (lrogt), a (lroft), a (lngt), a (lhft), $a(1 t g t), a(1 t f t), a(1 p t n), a(1 a l p t n), a(1 \times a t n)$, $a(\operatorname{lrogtn}), a(1$ roftn), a (lhgtn), a(lhftn), a (ltgtn), $a\left(1 t^{c_{4}}, n\right), a(1 p b), a(1 a l p b), a(1 \times a b), a(1 r \circ g b), a(1 r \circ f b)$, $a(1 h g b), a(1 h f b), a(1 t g b), a(1 t f b), a(l p b n), a(1 a l p b n)$, $a(1 \times a b n), a(1$ rogbn), a (Irofbn), a (lhglon), a (Ihfbn), a (1tgbn), a (1tfun), ppl, xapl, rogpl, rofpl, hgpl, hfpl ,tgpl, tfpl . ppln, xapln, rogpln, rofpln, rgpln, hfpln, tgpln, tfpln, ptb, xatb , rogtb, roftr, hgtb , hftb ,tgtb ,tftb, ptbn, xatbn, rogtbn, roftbn, hgtbn, hftbn, tgtbn, tftbn, alppl, alppln, alptb, alptbn, nzp2, nxmax, nchn, nzt, nzb, nrmax, na, nz, ncy $1 n$, nsurf)

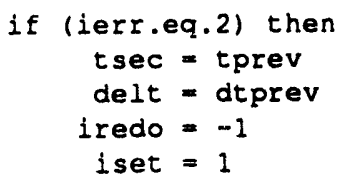




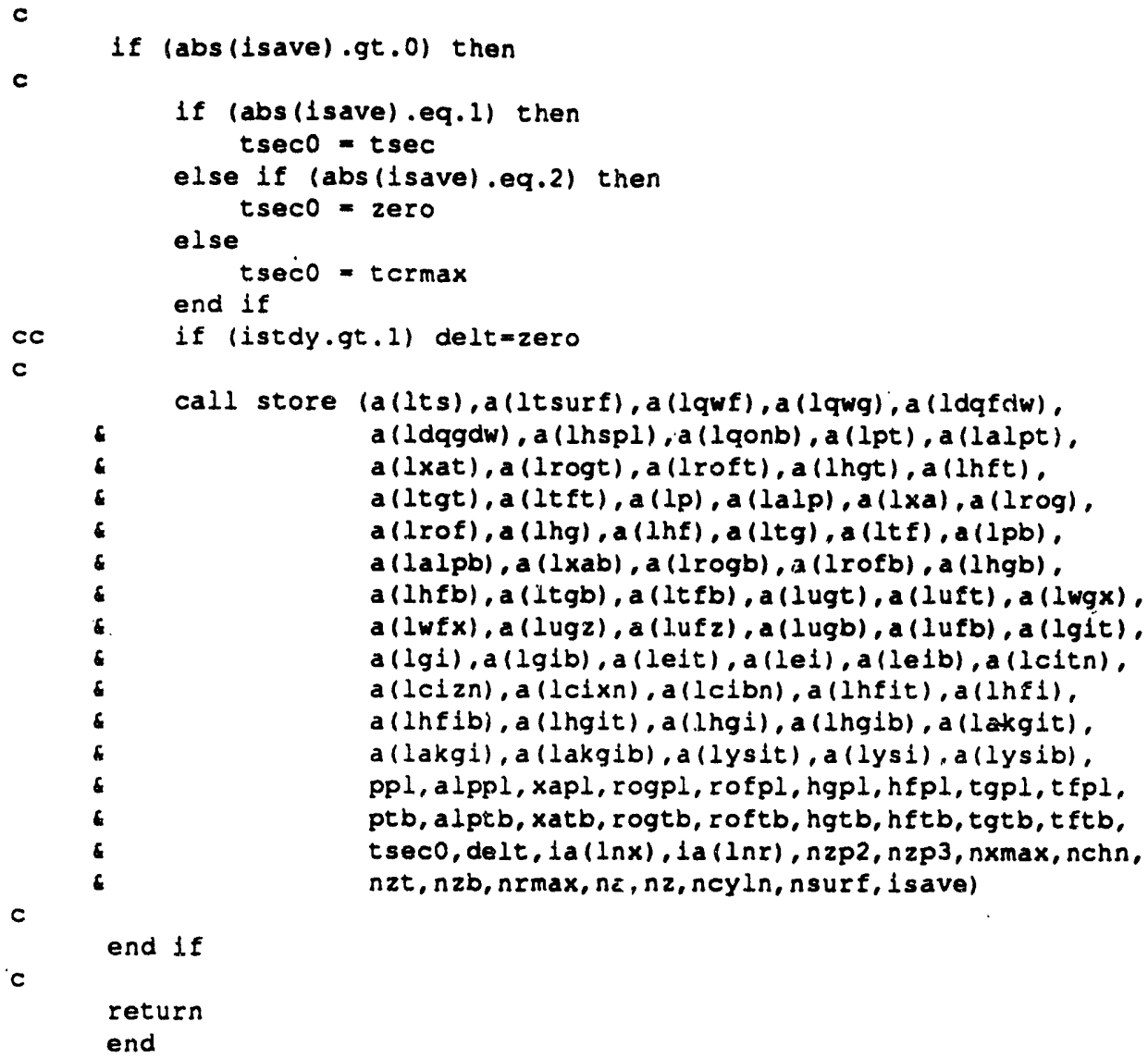

\section{TRIDAG}

subroutine tridag (first, istart, iend, neq, $a, b, c, d, x, s$ )

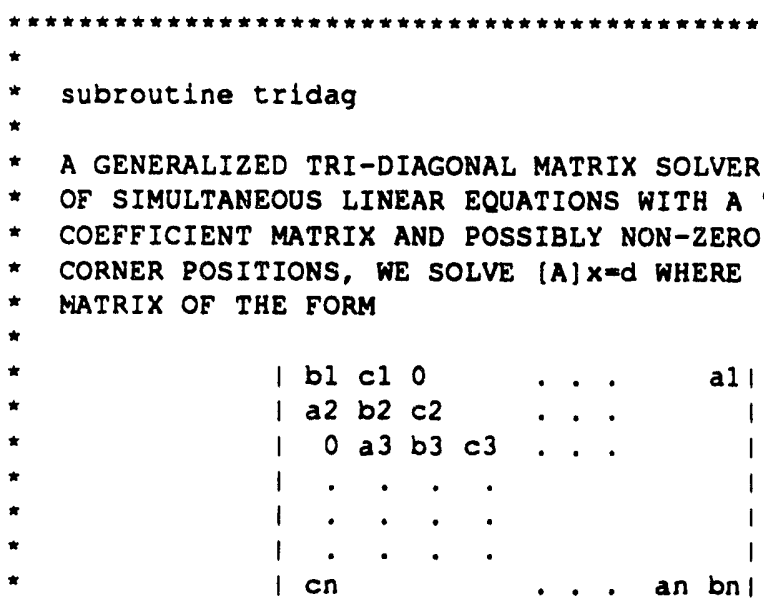

WHERE al AND Cn MAY BE ZERo, THE pOSSIBILITy OF NON-ZERo

- terms is iNCLUDEd for azimUThal COUPLING IN CYLINDRICAL

- geometry

$\star$

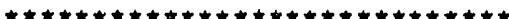

implicit real $(a-h, 0-z)$, integer $(1-n)$

parameter $(1 \max =100)$

parameter (zero=0.0, one=1.0)

loglcal first 
c

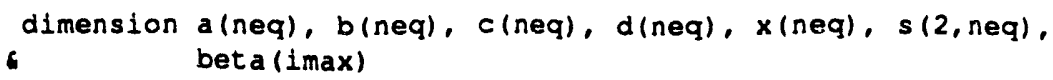

ON THE FIRST ENTRY, DECOMPOSE THE MATRIX BY COMPUTING THE INTERMEDIATE ARRAYS beta AND $S$ AND THE VALUE OF THE COEFEICIENT ThO

THE S VECTORS ARE INTRODUCED TO SAVE INTERMEDIATE RESULTS WHICH DO NOT CHANGE FOR ITERATIVE SOLUTIONS, THESE TERMS ONLY ARISE FROM THE EXTRA CORNER ELEMENTS IN THE MATRIX

NOTE THAT MATRIX VECTORS b AND C ARE MODIFIED SO THE ORIGINAL [A] MATRIX IS LOST

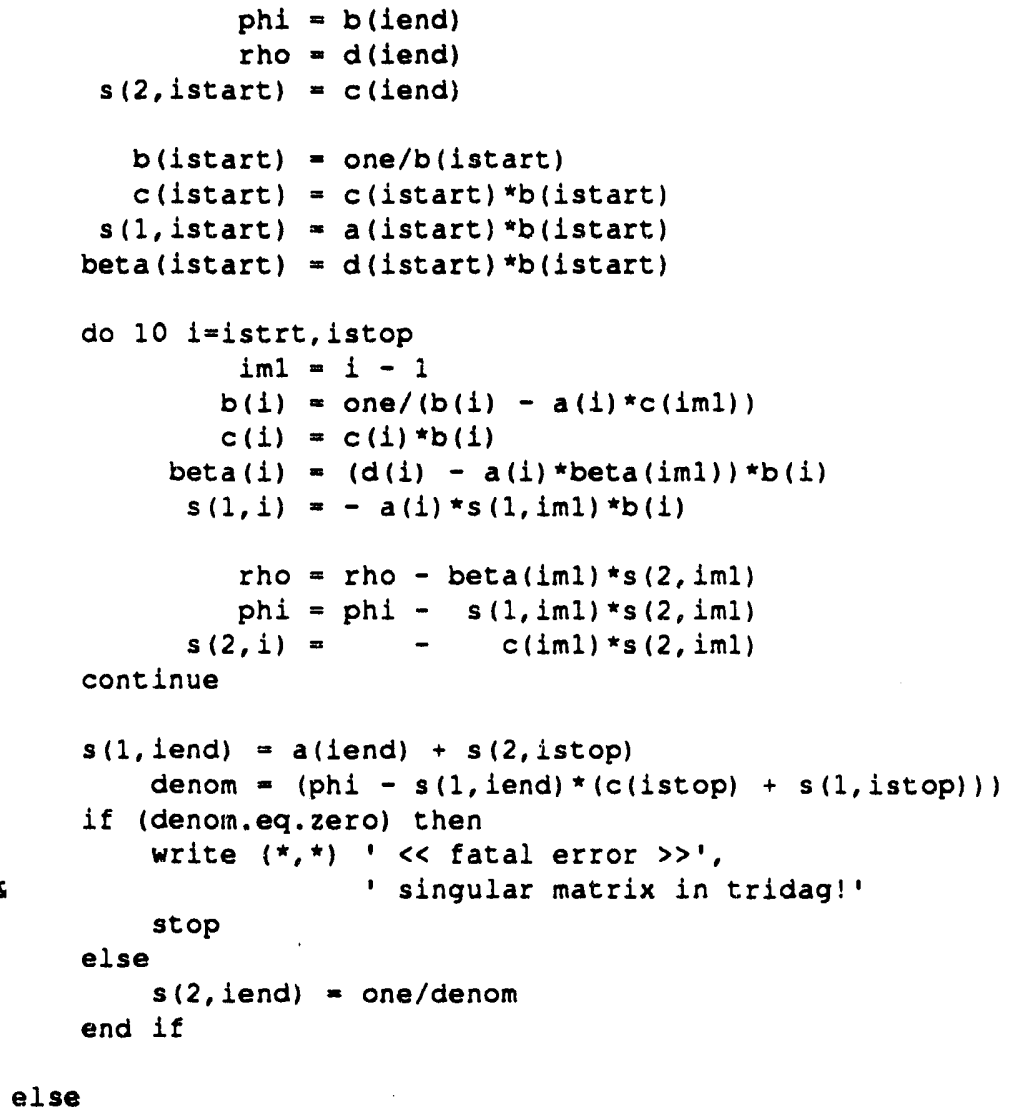

ON SUBSEQUENT ENTRIES, FOR AN ITERATIVE SOLUTION, ONLY RECOMPUTE beta AND tho WHICH CHANGE WHEN THE RIGHT HAND SIDE IS ALTERED

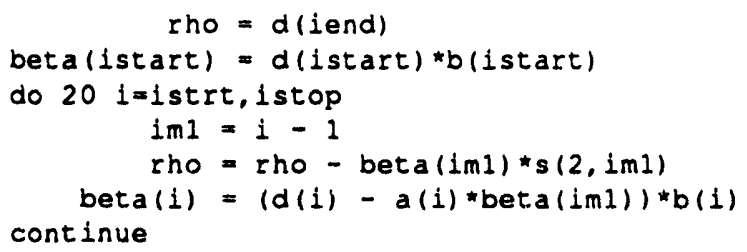


c

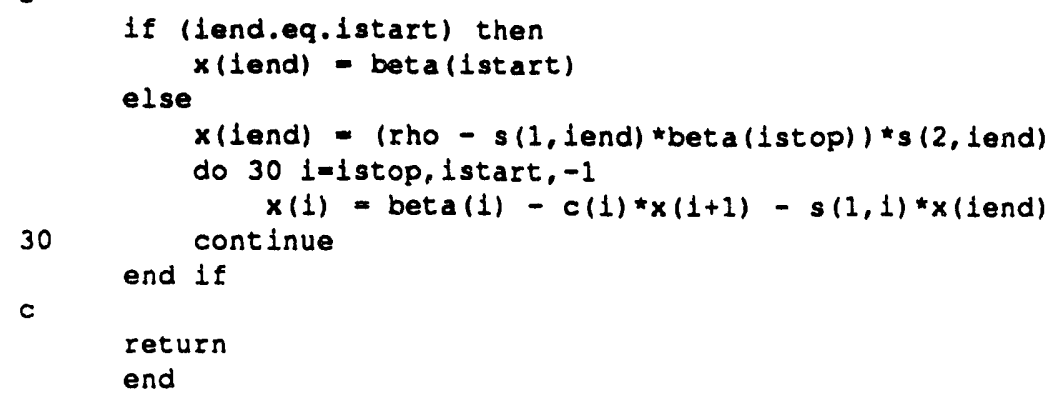

\section{TSAT}

subrout ine tsat $(p, 1, t s, d t s d p)$

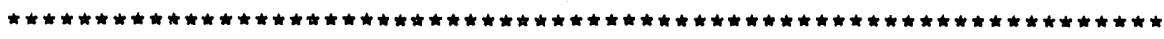

$\star$

* INPUT:

$\mathbf{p}=$ PRESSURE IN Pa

$1=$ IDENTIFIER $(1=1$ FOR H2O, $1=2$ FOR D2O

* output:

- ts = Saturation temperature in k

- desdp = DERIVAtive of ts W.R.T. p

* RANGE:

* $\quad 611.3 \mathrm{~Pa}<\mathrm{p}<1.0 \mathrm{e}+6 \mathrm{~Pa}$

$\star$

\section{TURBX}

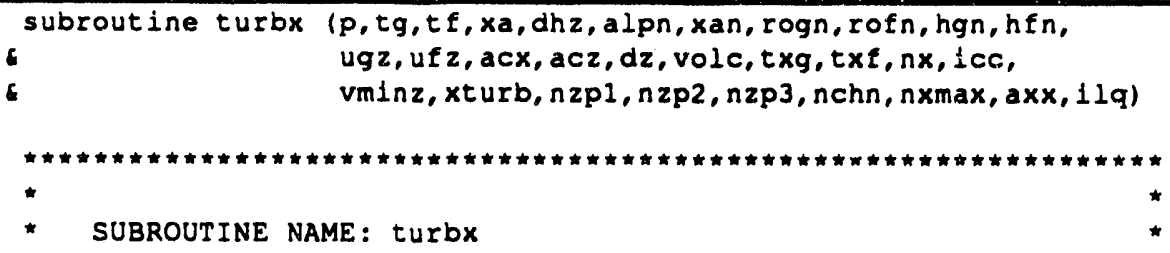




\begin{tabular}{|l|ll|l|}
\hline $2 / 15 / 93$ & WSRC-TR-93-086 Rev. 0 & FLOWTRAN-TF v1.2 Source Code & Pg. 325 of 354 \\
\hline
\end{tabular}

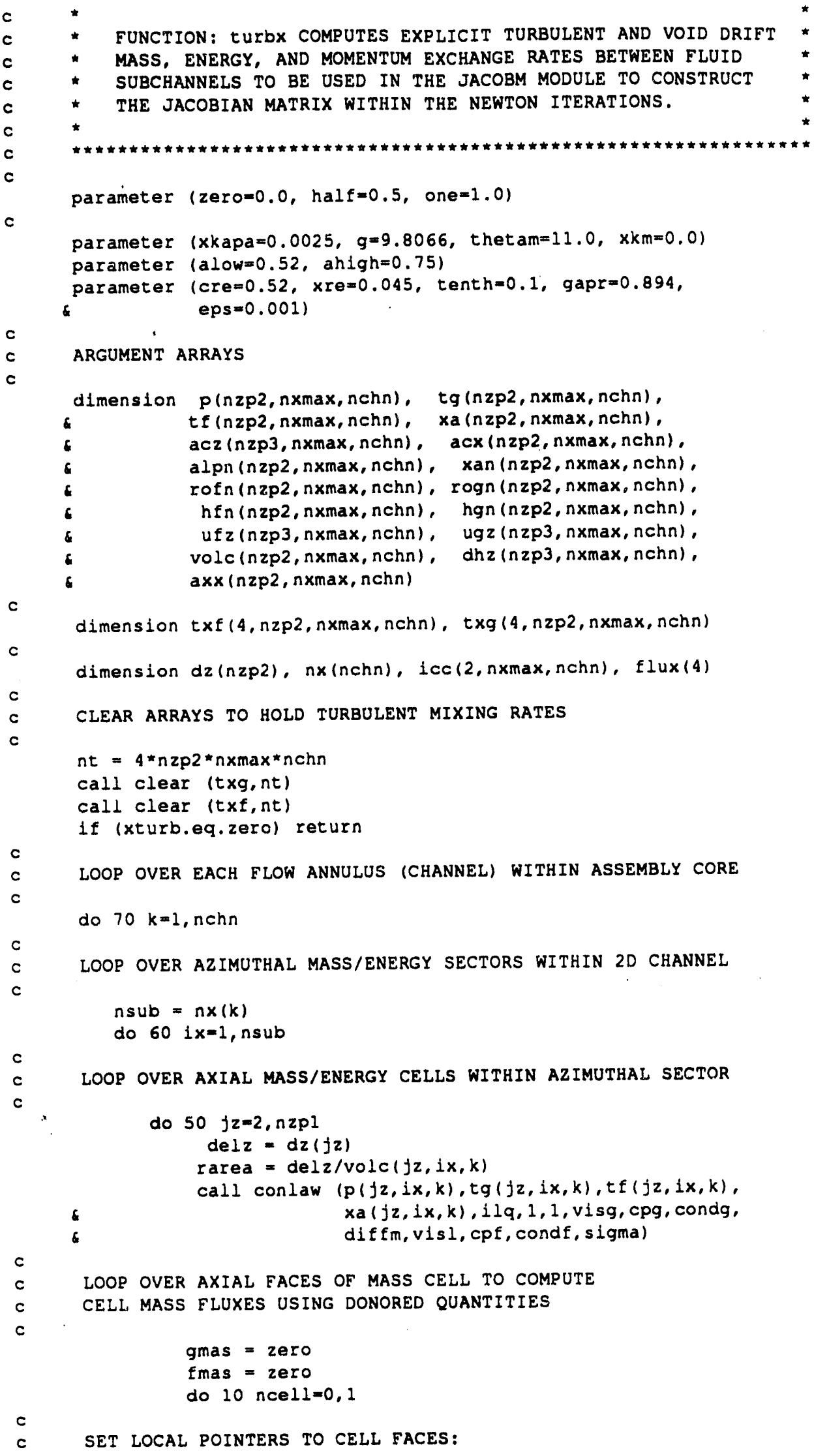


c jCl = LOWER MASS CELL INDEX

c $\quad \mathrm{jCU}$ = UPPER MASS CELL INDEX

c If = INDEX FOR INTERMEDIATE CELL FACE

c

$j c l=j z+n c e l l$

$j c u=j c l-1$

$j f=j e l$

$c$

$u f l d=u f z(j f, 1 x, k)$

ugas $=u g z(j f, i x, k)$

CALCULATE LIQUID DONOR VOID AND DENSITY AT CELL FACE

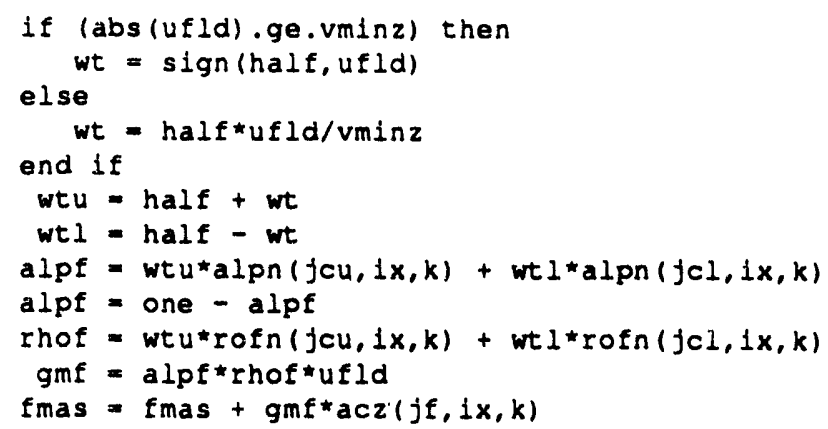

CALCULATE GAS DONOR VOID AND DENSITY AT CELL FACE

if (abs (ugas).ge.vminz) then

FIND REYNOLDS NUMBER BASED ON THE AIR-WATER MIXTURE AND CALCULATE A MIXTURE VELOCITY

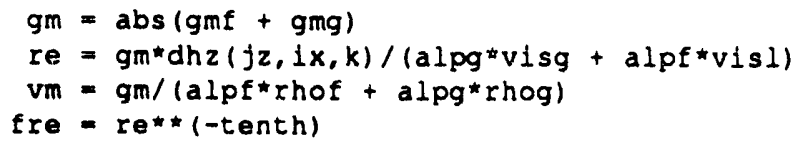

FIND TWO-PHASE MULTIPLIER

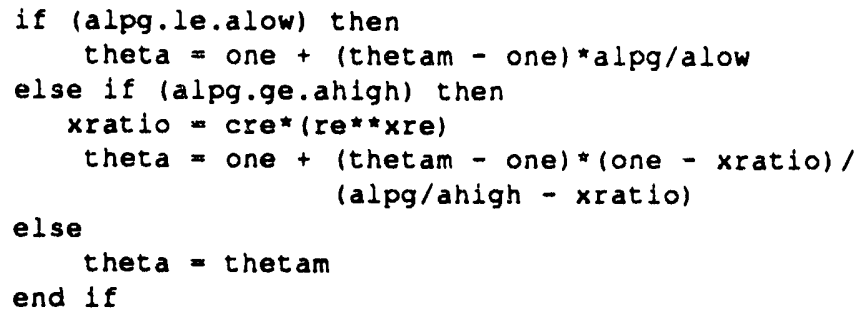


c

$c$

$c$

c

c

c

c

c

c

c

c

c

c

c

c c

FACES AND CALCULATE TURBULENT EXChANGE RATES

do 40 nface=1,2

$1 \times a=1 \operatorname{cc}(\operatorname{nface}, 1 x, k)$

$1 \times f=1 x-(1 x-i x a) *(n f a c e-1)$

nface $=1:$ ixa $=$ MASS CELL TO LEFT OF CURRENT CELL

nface $=2: 1 \times a=$ MASS CELL TO RIGHT OF CURRENT CELL

$1 \times f=$ CELL FACE BETWEEN ADJACENT MASS CELLS

FIND SINGLE PHASE TURBULENT VELOCITY

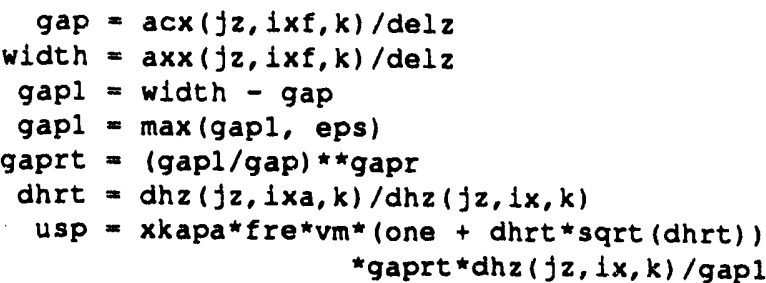

FIND TWO-PHASE TURBULENT VELOCITY

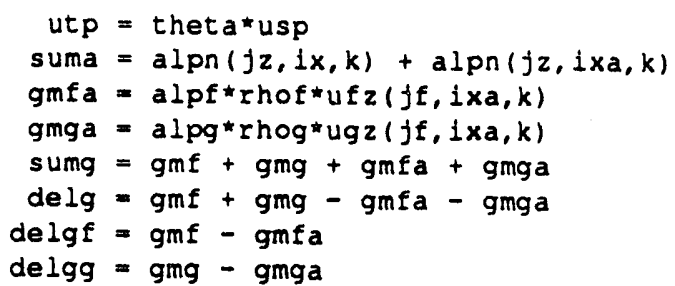

FIND EQUILIBRIUM VOID DIFFERENCE BETWEEN ADJACENT CHANNELS ASSOCIATED WITH VOID DRIFT

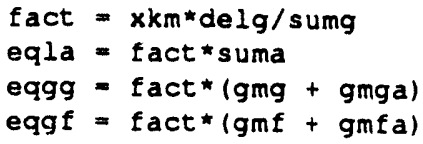

FIND THE VOID FRACTION DIFFERENCE BETWEEN ADJACENT CHANNELS

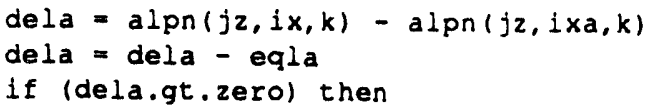

CELL IS DONORING GAS MASS TO ADJACENT CELL

CALCULATE GAS MASS, ENERGY, AND MOMENTUM TRANSFER RATES

INDEX: 1=MASS, 2=ENTHALPY, 3=AXIAL MOMENTUM, $4=A Z$ IMUTHAL MOMENTUM

$x \times t u=x t u r b^{*} r a r e a^{*} u t p^{*} g a p^{*} r$ nog

flux(1) $=x \times t u$ dela

$f \operatorname{lux}(2)=f \operatorname{lux}(1) * \operatorname{hgn}(j z, i x, k)$

flux $(3)=x t u r b * r a r e a * u t p * g a p *($ delgg - eqgg)

flux (4) $=$ zero

ACCUMULATE GAS TURBULENT EXCHANGE TERMS

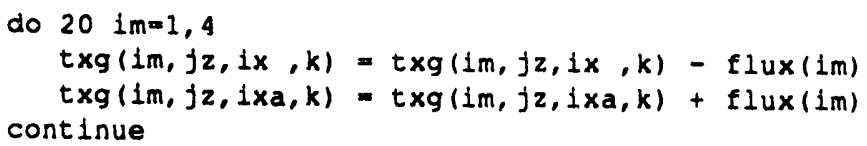


else

CELL IS DONORING LIOUID MASS TO ADJACENT CELL, CALCULATE LIQUID MASS, ENERGY, AND MOMENTUM TRANSFER RATES INDEX: 1-MASS, 2=ENTHALPY, 3=AXIAL MOMENTUM, 4=AZIMUTHAL MOMENTUM

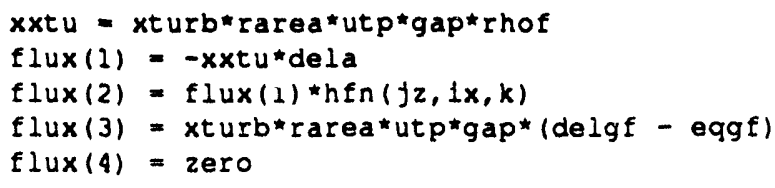
continue

\section{UPDATP}

subroutine updatp (cpat, cptgt, cptft, cpxat, fat, ftgt, ftft, fxat, dpt, cpa , cptg, cptf, cpxa, fa , ftg, ftf , fxa, dp, cpab, cptgb, cpt fb, cpxab, fab, ftgb, ftfb, fxab, dpb, $p t$, alpt, tgt, $t f t$, xat, rogt, hgt, roft, $h f t$, $p, a l p, t g, t f, x a, r o g$, hg , rof, hf , $p b, a l p b, t g b, t f b, x a b, r o g b, h g b, r o f b, h f b$, ppl, alpt n, bgt, cgt, bft, cft, advbt, advet, alpn, bgz, cgz, dgx, egx, bf $z, \operatorname{cf} z, d f x$, ef $x$, $a d v b z, a d v c z, a d v d x, a d v e x$, $p t b, a l p b n, b g b, c g b, b f b, c f b, a d v b b, a d v c b$. factor, nzt, nzp1, nzp2, nzp3, nxmax, nchn, nx, 1cc, $n z b$, ierr, errp, erral, errtg, errtf, errxa, 1lq)

\section{DESCRIPTION :}

Subroutine updatp computes property values over the entire grid for the mth newton 1teration. Subroutine inner supplies the dp corrections. Subroutine jacob supplies the dp coefflclents needed to recover gas volume fraction, gas temperature, liquid temperature, and air mass fraction. Subroutine state is called to compute gas density, gas enthalpy, liquid density, and liquid enthalpy.

jacob output :

$11000001|d p|+\left|a z m^{\prime} s\right||d p 1|=\mid$ rhs $\mid<--$ solvei by inner

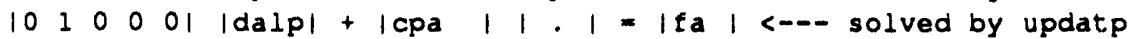

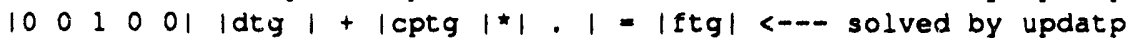

$1000010 \mid$ ldtf $|+|$ cptf $\mid 1$. $|=| f t f \mid$ - - solved by updatp

$10000011|d x a|+|c p x a||d p n|=\mid$ fxa $\mid<--$ solved by updatp

(matrix) surrounding cell pressures 
c 
c O alp (nzp2, nxmax, nchn) = mlddle section alpha

c 0 tg (nzp2, nxmax, nchn) - middle section gas temp.

c O tf (nzp2, nxmax, nchn) = middle section liquld temp.

c $O$ xa (nzp2, nxmax, nchn) = middle section alr mass fract.

c 0 rog (nzp2, nxmax, nchn) = middle section gas density

c 0 hg $(n z p 2, n x m a x, n c h n)=$ middle section gas enthalpy

c $O$ rof $(n z p 2, n \times \max , n c h n)=$ mlddle section liquid density

c O he (nzp2, nxmax, nchn) = middle section gas density

$c$

c ---Bottom Section---

c I $n z b$ number of cells in the bottom section

c I cpab $(2, n z b)=$ coefficients to recover bottom section alpha

c I from surrounding cell pressure corrections

c I cptgb(2,nzb) - coefficlents to recover bottom section gas temp.

c I from surrounding cell pressure corrections

c I cpt $f b(2, n z b)=$ coefficlents to recover bottom section liquid temp.

c I from surrounding cell pressure corrections

c I cpxab $(2, n z b)$ = coefficlents to recover bottom section air mass

c I fract. from surrounding cell pressure

c I corrections

c I fab (nzb) = constants to recover bottom section alpha

c I ftgb $(n z b)$ = constants to recover bottom section gas temp.

c I ft $f b(n z b)$ - constants to recover bottom section liquid temp.

c I fxab (nzb) - constants to recover bottom section alr mass fract.

c I dpb $(n z b)=$ bottom section pressure corrections

c $0 \mathrm{pb} \quad(n z b)=$ bottom section pressure

c $O$ alpb $(n z b)=$ bottom section alpha

c 0 tgb $(n z b)$ - bottom section gas temp.

$c 0$ tfb $(n z b)$ - bottom section liquid temp.

$c 0 \times a b(n z b)=$ bottom section air mass fract.

c 0 rogb $(n z b)=$ bottom section gas density

$c 0$ hgb $(n z b)=$ bottom section gas enthalpy

c O rofb $(n z b)=$ bottom section liquid density

c $O$ hfb $(n z b)=$ bottom section gas density

STORAGE :

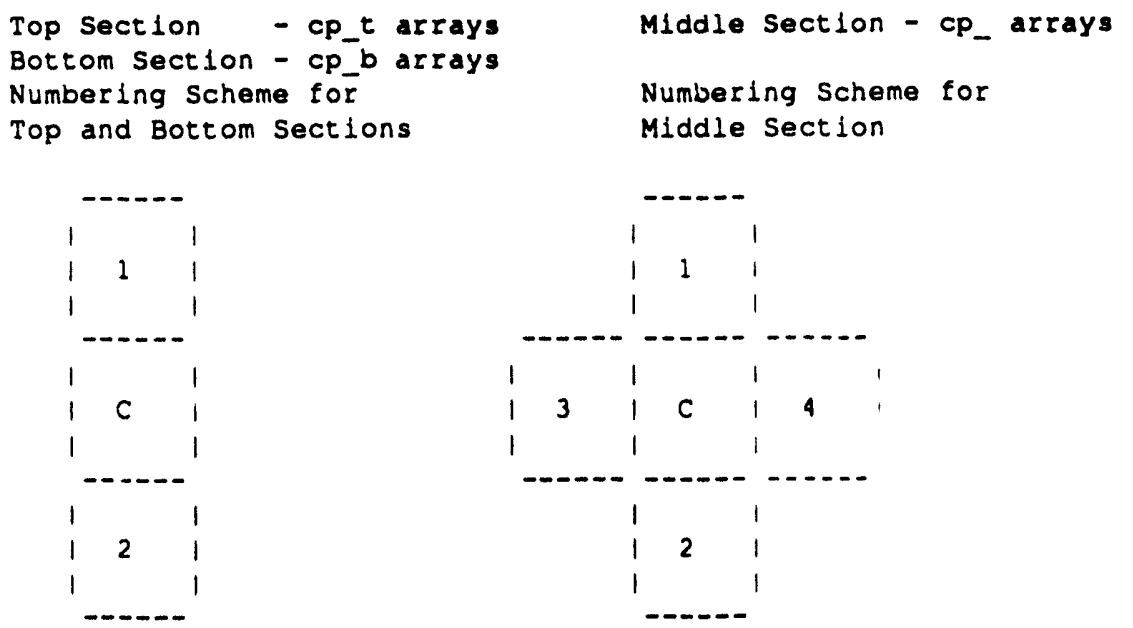

For the bottom cell of the top section and the top cell of the bottom section, the extra storage setup for the middle section fictitious cells is used to store the coupling quantities corresponding to the top and bottom rows of the middle section as follows:

Bottom Cell of Top section - CP_ $(2,1, \ldots)$ locations 


\begin{tabular}{|l|ll|l|}
\hline $2 / 15 / 93$ & WSRC-TR-93-086 Rev. 0 & FLOWTRAN-TF v1.2 Source Code & Pg. 331 of 354 \\
\hline
\end{tabular}

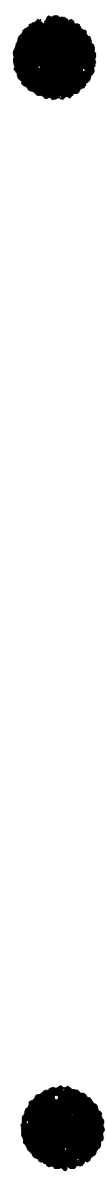

c

c

c

C

c

c

c

c

C

c

c

c

c

c

c

$c$

c

$c$

C

C

$c$

c

$c$

$c$

c

c

c

c

parameter $(z e r 0=0.0$, one=1.0, two=2.0, four $=4.0, \operatorname{ten}=10.0)$

data dtgmax/5.0/, dtfmax/5.0/, dxamax/0.04/

c

include 'knobs.inc'

C

dimension alpch (3)

common /civd/ alpch, delp

dimenston $n x(n c h n), 1 c c(2, n \times \max , n c h n)$

TOP SECTION ARRAYS

dimension cpat $(2, n z t)$, fat (nzt),

c cptgt $(2, n z t)$, ftgt (nzt) cptet $(2, n z t)$, ftet (nzt), cpxat $(2, n z t)$, fxat (nzt) dpt $(n z t)$

c

c

dimension pt (nzt), rogt (nzt),

1) alpt (nzt), hgt (nzt).

tgt (nzt), roft (nzt).

tet (nzt), het (nzt).

xat $(n z t)$

dimension alptn(nzt),

6

6

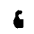

bgt (nzt)

bet $(n z t)$,

$\operatorname{cgt}(n z t)$.

cft (nzt), advbt (nzt, 5), advct (nzt,, 5$)$

MIDDLE SECTION ARRAYS

dimension cpa $(4, n z p 2, n x \max$, nchn), fa (nzp2, nxmax, nchn), 
1. $\operatorname{cptg}(4, n z p 2, n \times \max , n c h n)$, ftg (nzp2, nxmax, nchn) . cpt $f(4, n z p 2, n x m a x, n c h n)$. ft f (nzp2, nxmax, nchn), cpxa (4, nzp2, nxmax, nchn), Exa (nzp2, nxmax, nchn),

dimension $p$ (nzp2, nxmax, nchn), rog(nzp2, nxmax, nchn) c alp (nzp2, nxmax, nchn), hg (nzp2, nxmax, nchn). c $\operatorname{tg}(n 2 p 2, n x \max , n c h n)$, rof (nzp2, nxmax, nchn), tf (nzp2, nxmax, nchn), he (nzp2, nxmax, nchn), $x a$ (nzp2, nxmax, nchn)

dimension alpn (nzp2, nxmax, nchn), $6 \quad \operatorname{dgx}(n z p 2, n \times \max , n c h n)$. dfx (nzp2, nxmax, nchn), egx (nzp2, nxmax, nchn), efx (nzp2, nxmax, nchn), advdx (nzp2, nxmax, nchn, 5), advex (nzp2, nxmax, nchn, 5), bgz (nzp3, nxmax, nchn), $\quad \operatorname{cgz}(n z p 3, n x \max , n c h n)$, bfz (nzp3, nxmax, nchn), cfz (nzp3, nxmax, nchn), advbz (nzp3, nxmax, nchn, 5), advez (nzp3, nxmax, nchn, 5)

c

c

dimension alpun (nzb). $b g b(n z b), \quad \operatorname{cgb}(n z b)$. $b f b(n z b), \quad c f b(n z b)$. $a d v b b(n z b, 5), a d v c b(n z b, 5)$ c

c

c

DERIVATIVE ARRAYS NEEDED BY SUBROUTINE STATE

parameter $(\operatorname{mxnz}=100)$

dimenstion drogdp (mxnz), drogdt (mxnz), drgdxa(mxnz), 6

6

c

c

C

c

c

c

c

C dhgdp (mxnz), dhgdt (mxnz), dhgdxa(mxnz), drofdp $(m \times n z)$, drofdt $(m \times n z)$, dhfdp $(m \times n z)$, dhedt (mxnz)

PARAMETER VALUES

data alplo/-0.0001/, alph1/1.0001/, xa10/-0.005/, xah1/1.001/,

+ tglo/270.0\%, tghi/705.0\%, tflo/270.0/, thil455.0\%,

- epsag/0.0001/, epsaf/0.9999/

data ai/0.218/, am/0.431/, ao/0.351/

G cins/+19.16070/, cin $6 /+0.0 /, \operatorname{cin} 7 /-3.96141 /$

c cmn5/-141.1920/, cmn6/+101.4110/, cmn $7 /-27.3417 /$

c clps/+2.57241/,cip6/+0.0/,cip7/-0.636891/

data $\operatorname{cin} 1 /+1.0 /, \operatorname{cin} 2 /-4.59914 /, \operatorname{cin} 3 /+16.47475 /, \operatorname{cin} 4 /-27.07490 /$,

data cmnl/t1.0/, cmn2/+3.01770/, cmn3/-25.83200/, cmn4/+89.93700/,

data clp1/t1.0/, clp2/-3.685884/, cip3/+8.033505/, c1p4/-6.28314/,

data cmp1/+1.0/, cmp2/-5.72188/, cmp3/+29.67630/, cmp4/-53.35180/, 
4 cmp5/+46.49440/, cmp6/-22.68920/, cmp 7/+5.59218/ c-n-

C

pi - acos (-one)

lerr $=0$

errp = zero

erral $=$ zero

errtg $=$ zero

errt $=$ zero

errxa $=$ zero

c

STUEF FICTITIOUS CELLS ABOVE AND BELOW MIDDLE SECTION

do 11 ic=1, nchn

do $101 \mathrm{x}=1, \mathrm{nx}(1 \mathrm{c})$ $d p(1,1 x, 1 c)=\operatorname{dpt}(n 2 t)$ $d p(n 2 p 2,1 x, 1 c)=d p b(1)$

10 continue

11 continue

c

c--- UPDATE P, alpha, Ig, Tf, Xa

TOP CELL OF TOP SECTION

dalp = fat (1)

$d t g=f t g t(1)$

$d t=f t f t(1)$

$d x a=f x a t(1)$

if (nzt .eq. 1) go to 109

c

dalp = dalp - cpat $(2,1) * d p t(2)$

$d t g=d t g-\operatorname{cptgt}(2,1) * d p t(2)$

$d t f=d t f-\operatorname{cptft}(2,1) \star d p t(2)$

$d x a=d x a-\operatorname{cpxat}(2,1) \star d p t(2)$

pt (1) $=p t$ (1) + dpt (1)

alpt (1) = alpt (1) + dalp

$\operatorname{tgt}(1)=\operatorname{tgt}(1)+\operatorname{sign}(\min (\operatorname{abs}(d t g), d t g m a x), d t g)$

tft (1) $=t f t(1)+\operatorname{sign}(\min (\operatorname{abs}(d t f), d t f \max ), d t f)$

c

xat (1) = xat (1) + sign (min (abs (dxa), dxamax), dxa)

errp = max(errp, abs (dpt (1)))

erral = max(erral, abs(dalp ))

errtg $=\max (e r r t g, a b s(d t g)$ )

ertf $=\max (e r r t f, a b s(d t f)$ )

errxa = $\max (e r r x a, a b s(d x a)$ ))

c

MIDDLE CELLS OF TOP SECTION

do $100 \quad j z=2, n z t-1$

dalp $=f a t(j z)-\operatorname{cpat}(1, j z) * d p t(j z-1)-\operatorname{cpat}(2, f z) * d p t(f z+1)$

$d t g=f t g t(j z)-\operatorname{cptgt}(1, j z) \star d p t(j z-1)-\operatorname{cptgt}(2, j z) \star d p t(j z+1)$

$d t f=\operatorname{ftft}(j z)-\operatorname{cptft}(1, j z) \star d p t(j z-1)-\operatorname{cptft}(2, j z) \star d p t(j z+1)$

c

$d x a=f x a t(j z)-\operatorname{cpxat}(1, j z) \star d p t(j z-1)-\operatorname{cpxat}(2, j z) \star d p t(j z+1)$

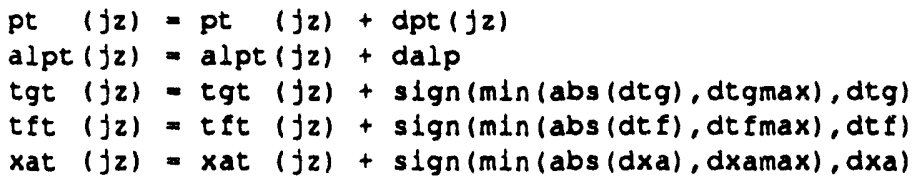

errp $=\max (\operatorname{ercp}, \operatorname{abs}(\operatorname{dpt}(j z)))$ 
100

c

c

c 109

c

c

c

c

c

erral = $\max (\operatorname{erral}$, abs (dalp N)

errtg $=\max (e r r t g$, abs (dtg ))

erte = max(erctf, abs (ttf 1$)$

errxa $=\max (e r r x a$, abs (dxa ) )

continue

BOTTOM CELL OF TOP SECTION

dalp - fat (nzt) - cpat $(1, n z t)$ dpt $(n z t-1)$

$d t g=f t g t(n z t)-\operatorname{cptgt}(1, n z t) \cdot d p t(n z t-1)$

$d t=\operatorname{ftft}(n z t)-\operatorname{cptft}(1, n z t) * d p t(n z t-1)$

$d x a=f x a t(n z t)-\operatorname{cpxat}(1, n z t) * d p t(n z t-1)$

continue

do 111 1c=1, nchn

do $1101 x=1, n \times(1 c)$

dalp = dalp - cpa $(2,1,1 x, 1 c) * d p(2,1 x, 1 c)$

$d t g=d t g-c p t g(2,1,1 x, 1 c) d p(2,1 x, 1 c)$

$d t=d t f-\operatorname{cpt} f(2,1,1 x, 1 c) * d p(2,1 x, 1 c)$

$d x a=d x a-\operatorname{cpxa}(2,1,1 x, 1 c) * d p(2,1 x, 1 c)$

continue

continue

$p t(n z t)=p t(n z t)+d p t(n z t)$

alpt $(n z t)=$ alpt $(n z t)+$ dalp

tgt $(n z t)=t g t(n z t)+\operatorname{gign}(\min (a b s(d t g), d t g m a x), d t g)$

$t f t(n z t)=t f t(n z t)+\operatorname{sign}(\min (a b s(d t f), d t f \max ), d t f)$

xat $(n z t)=x a t(n z t)+\operatorname{sign}(\min (a b s(d x a), d x a m a x), d x a)$

errp $=\max (e r r p, \operatorname{abs}(d p t(n z t)))$

erral = $\max (e r r a l, a b s(d a l p)$ ))

errtg $=\max (e r r t g$, abs (dtg ) )

errtf $=\max (e r r t f, a b s(d t f \quad)$

errxa $=\max (e r r x a$, abs (dxa i)

MIDDLE SECTION

do 202 ic=1, nchn

do $2011 x=1, n x(i c)$

do $200 \quad j z=2, n z p 1$

$i x 1=1 c c(1, i x, i c)$

$1 \times r=1 c c(2,1 x, i c)$

$\operatorname{dalp}=f a(j z, 1 x, 1 c)$

- cpa $(1, j z, 1 x, 1 c) * d p(j z-1,1 x, 1 c)$

- cpa $(2, j z, i x, 1 c)=d p(j z+1,1 x, i c)$

- cpa $(3, j z, i x, i c) d p(j z, i x \mid, i c)$

- cpa $(4, j z, 1 x, 1 c)=d p(j z, 1 x x, 1 c)$

$d t g=f t g(j z, i x, i c)$

$-\operatorname{cptg}(1, f z, 1 x, 1 c) * d p(f z-1,1 x, 1 c)$

- $c p=g(2, f z, 1 x, 1 c) * d p(f z+1, i x, 1 c)$

- $\operatorname{cptg}(3, j z, 1 x, 1 c) \star d p(j z, 1 x 1,1 c)$

- $\operatorname{cptg}(4, j z, 1 x, 1 c) * d p(j z, 1 x I, 1 c)$

$d t f=f t f(j z, 1 x, i c)$

- $\operatorname{cpt} f(1, j z, 1 x, 1 c) * d p(j z-1, i x, i c)$

$-\operatorname{cpt} f(2, j z, i x, 1 c) \approx d p(j z+1, i x, i c)$

$-\operatorname{cptf}(3, j z, 1 x, 1 c) * d p(j z, 1 x 1,1 c)$

$-\operatorname{cpt} f(4, j z, i x, 1 c) \approx d p(j z, i x r, 1 c)$

$d x a=f x a(j z, 1 x, 1 c)$

- $\operatorname{cpxa}(1, j z, 1 x, 1 c) \approx d p(j z-1,1 x, 1 c)$

- $\operatorname{cpxa}(2, j z, 1 x, 1 c) \approx d p(j z+1,1 x, 1 c)$

- $\operatorname{cpxa}(3, j z, i x, i c) \approx d p(j z, i x l, i c)$

$-\operatorname{cpxa}(4, j z, 1 x, i c) * d p(j z, 1 \times r, 1 c)$ 


\begin{tabular}{|l|ll|l|}
\hline $2 / 15 / 93$ & WSRC-TR-93-086 Rev. 0 & FLOWTRAN-TF v1.2 Source Code & Pg. 335 of 354 \\
\hline
\end{tabular}

$c$ $p(j z, i x, i c)=p(j z, i x, i c)+d p(j z, i x, i c)$
$\operatorname{alp}(j z, i x, i c)=\operatorname{alp}(j z, i x, i c)+d a l p$
$t g(j z, i x, i c)=t g(j z, i x, i c)+\operatorname{sign}(\min (a b s(d t g), d t g m a x), d t g)$
$t f(j z, i x, i c)=t f(j z, i x, i c)+\operatorname{sign}(\min (a b s(d t f), d t f \max ), d t f)$
$x a(j z, i x, i c)=x a(j z, i x, i c)+\operatorname{sign}(\min (a b s(d x a), d x a m a x), d x a)$

$c$

errp $=\max (\operatorname{errp}, \operatorname{abs}(d p(j z, i x, i c)))$

erral $=\max ($ erral, abs (dalp )

errtg $=\max (\operatorname{errtg}, \operatorname{abs}(d t g \quad))$

errtf $=\max (\operatorname{errtf}, \operatorname{abs}(d t f \quad)$

errxa $=\max (\operatorname{errxa}$, abs (dxa )

200 continue

201 continue

202 continue

c

c TOP CELL OF BOTTOM SECTION

$c$

dalp $=f a b(1)$

$d t g=f t g b(1)$

$d t f=f t f b(1)$

$\rightarrow x a=f \times a b(1)$

c

do 301 1c=1, nchn

do $300 \mathrm{ix}=1, \mathrm{nx}(\mathrm{ic})$

dalp $=$ dalp - cpa $(1, n z p 2, i x, i c) * d p(n z p 1, i x, i c)$

$d t g=d t g-c p t g(1, n z p 2, i x, 1 c) \star d p(n z p 1, i x, i c)$

$d t f=d t f-\operatorname{cptf}(1, n z p 2, i x, i c) * d p(n z p 1, i x, i c)$

ixa $=d x a-\operatorname{cpxa}(1, n z p 2,1 x, 1 c) \star d p(n z p 1,1 x, 1 c)$

300 continue

301 continue

c

if (nzb .eq. 1) go to 311

c

dalp $=$ dalp $-c p a b(2,1) * d p b(2)$

$d t g=d t g-c p t g b(2,1) \approx d p b(2)$

$d t f=d t f-c p t f b(2,1) * d p b(2)$

$d x a=d x a-c p x a b(2,1) \star d p b(2)$

c

$\mathrm{pb} \quad(1)=\mathrm{pb}$ (1) $+\mathrm{dpb}(1)$

$a l p b(1)=a l p b(1)+d a l p$

$\operatorname{tgb}(1)=\operatorname{tgb}(1)+\operatorname{sign}(\min (a b s(d t g), d t g m a x), d t g)$

$t f b(1)=t f b(1)+\operatorname{sign}(\min (a b s(d t f), d t f \max ), d t f)$

$x a b(1)=x a b(1)+\operatorname{sign}(\min (a b s(d x a), d x a m a x), d x a)$

c

errp $=\max (e r r p, a b s(a p b(1)))$

erral $=\max ($ erral, abs(dalp ))

errtg $=\max (e r r t g, a b s(d t g))$

errtf = $\max (e r r t f, a b s(d t f)$ )

c

errxa $=\max (e r r x a, a b s(d x a))$

MIDDLE CELLS OF BOTTOM SECTION

do $310 \quad j z=2, n z b-1$

dalp = fab $(j z)-\operatorname{cpab}(1, j z) * d p b(j z-1)-\operatorname{cpab}(2, j z) \star d p b(j z+1)$

$d t g=f t g b(j z)-\operatorname{cptgb}(1, j z) \star d p b(f z-1)-\operatorname{cptgb}(2, j z) \approx d p b(j z+1)$

$d t f=f t f b(j z)-c p t f b(1, j z) * d p b(j z-1)-\operatorname{cptfb}(2, j z) * d p b(j z+1)$

$d x a=f \times a b(j z)-\operatorname{cpxab}(1, j z) * d p b(j z-1)-\operatorname{cpxab}(2, j z) \star d p b(j z+1)$

c

$p b \quad(j z)=p b(j z)+d p b(j z)$

$a l p b(j z)=a l p b(j z)+$ dalp

$\operatorname{tgb}(j z)=\operatorname{tgb}(j z)+\operatorname{sign}(\min (a b s(d t g), d t g \max ), d t g)$

$t f b(j z)=t f b(j z)+\operatorname{sign}(\min (a b s(d t f), d t f \max ), d t f)$ 
c

$x a b(f z)=x a b(f z)+\operatorname{sign}(\min (a b s(d x a), d x a m a x), d x a)$

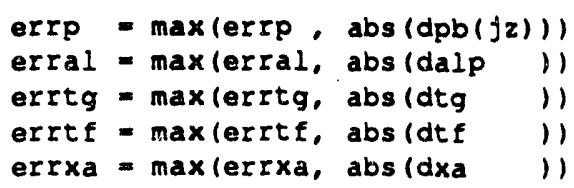


c

6

405

c end if

if (fz eq. $n z t)$ then

do $405 \mathrm{lc}=1, \mathrm{nchn}$

do $405 i x=1, n x(i c)$

$u g p=\operatorname{bg} z(2,1 x, 1 c)$

$+\operatorname{cgz}(2, i x, i c) *(p t(n z t)-p(2, i x, i c))$

if (ugp.gt. zero) then

$\operatorname{advbz}(2,1 x, i c, 1)=z e r o$

$\operatorname{advcz}(2,1 x, 1 c, 1)=z e r o$

$a d v b z(2, i x, 1 c, 3)=z e r o$

$\operatorname{advcz}(2,1 x, 1 c, 3)=z e r o$

$\operatorname{advbz}(2,1 x, 1 c, 5)=z e r o$

$\operatorname{advcz}(2,1 x, i c, 5)=z e r c$

end if

continue

else

$u g p=\operatorname{bgt}(j z+1)+\operatorname{cgt}(j z+1) *(p t(j z)-p t(j z+1))$

if (ugp .gt. zero) then

advbt $(j z+1,1)=$ zero

$\operatorname{advct}(j z+1,1)=$ zero

$\operatorname{advbt}(j z+1,3)=$ zero

$\operatorname{advct}(j z+1,3)=z$ ero

$\operatorname{advbt}(j z+1,5)=z e r o$ $\operatorname{advct}(j z+1,5)=z$ ero

end if

end if

end if

else

if (alpt $(f z)$. It. alphi) then

alpt $(f z)=$ one

else

ierr $=1$

write $(*, 901) \mathrm{jz}$

end if

if (alptn(jz) gt. epsaf) then

if (jz eq. 1) then

$u f m=b f t(j z)+c f t(j z) *(p p l-p t(f z))$

else

$u f m=b f t(j z)+\operatorname{cft}(j z) *(p t(j z-1)-p t(j z))$

end if

if (ufm.1t. zero) then

$\operatorname{advbt}(j z, 2)=$ zero

$\operatorname{advct}(j z, 2)=z e r o$

$\operatorname{advbt}(j z, 4)=$ zero

$\operatorname{advct}(j z, 4)=z e r o$

end if

if $(j z$.eq. $n z t)$ then

do 406 ic $=1$, nchn

do $406 i x=1, n x$ (ic)

$u f p=b f z(2,1 x, 1 c)$

$+\operatorname{cf} z(2, i x, i c) *(p t(n z t)-p(2, i x, i c))$

if (ufp.gt. zero) then

$\operatorname{advbz}(2,1 x, 1 c, 2)=z$ ero

$\operatorname{advcz}(2, i x, i c, 2)=z e r o$

$\operatorname{advbz}(2,1 x, 1 c, 4)=z e r o$

$\operatorname{advcz}(2,1 x, i c, 4)=z e r o$

end $1 f$

continue

else

$u f p=b f t(j z+1)+c f t(j z+1) *(p t(j z)-p t(j z+1))$

if (ufp .gt. zero) then 


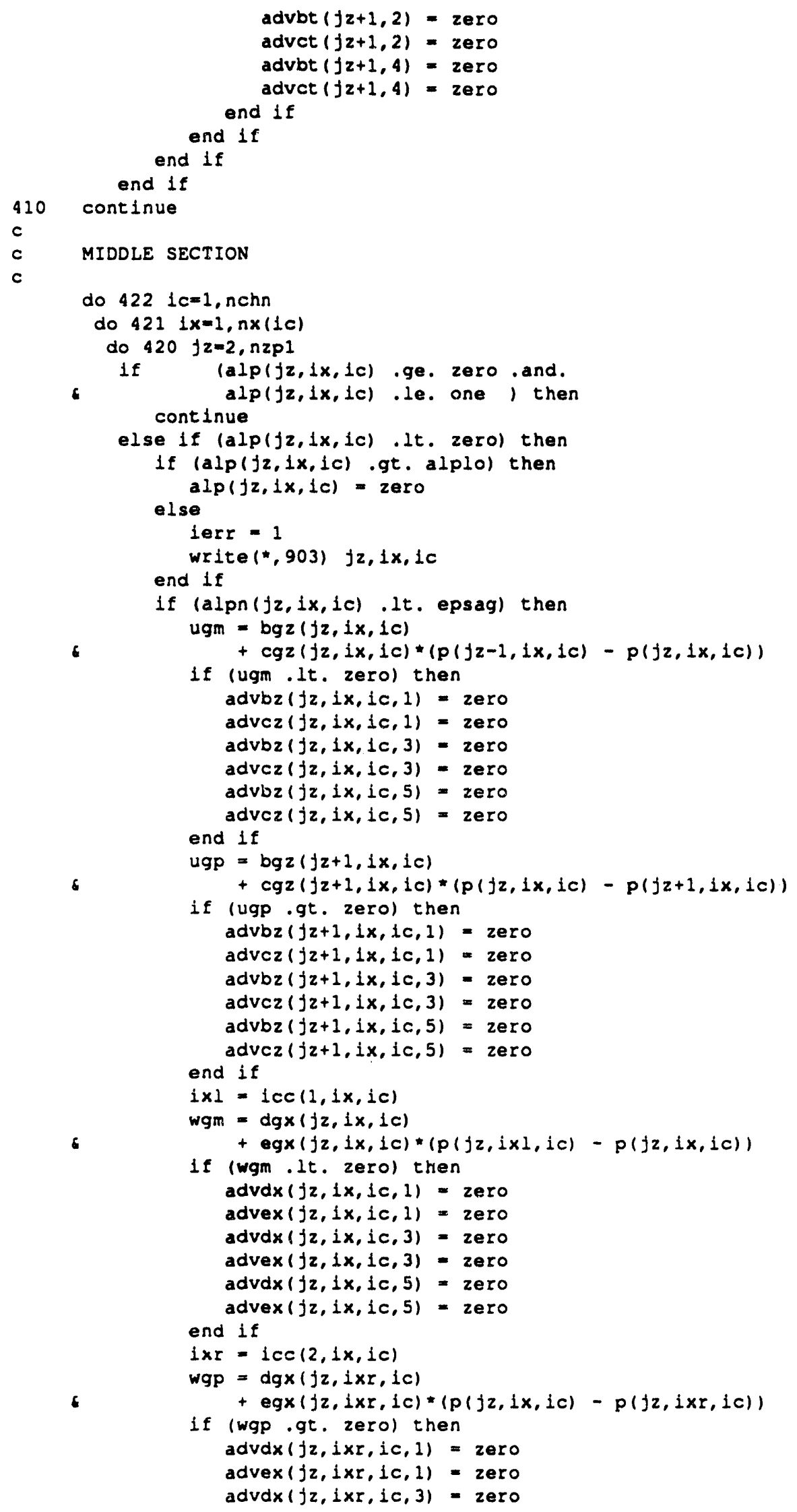




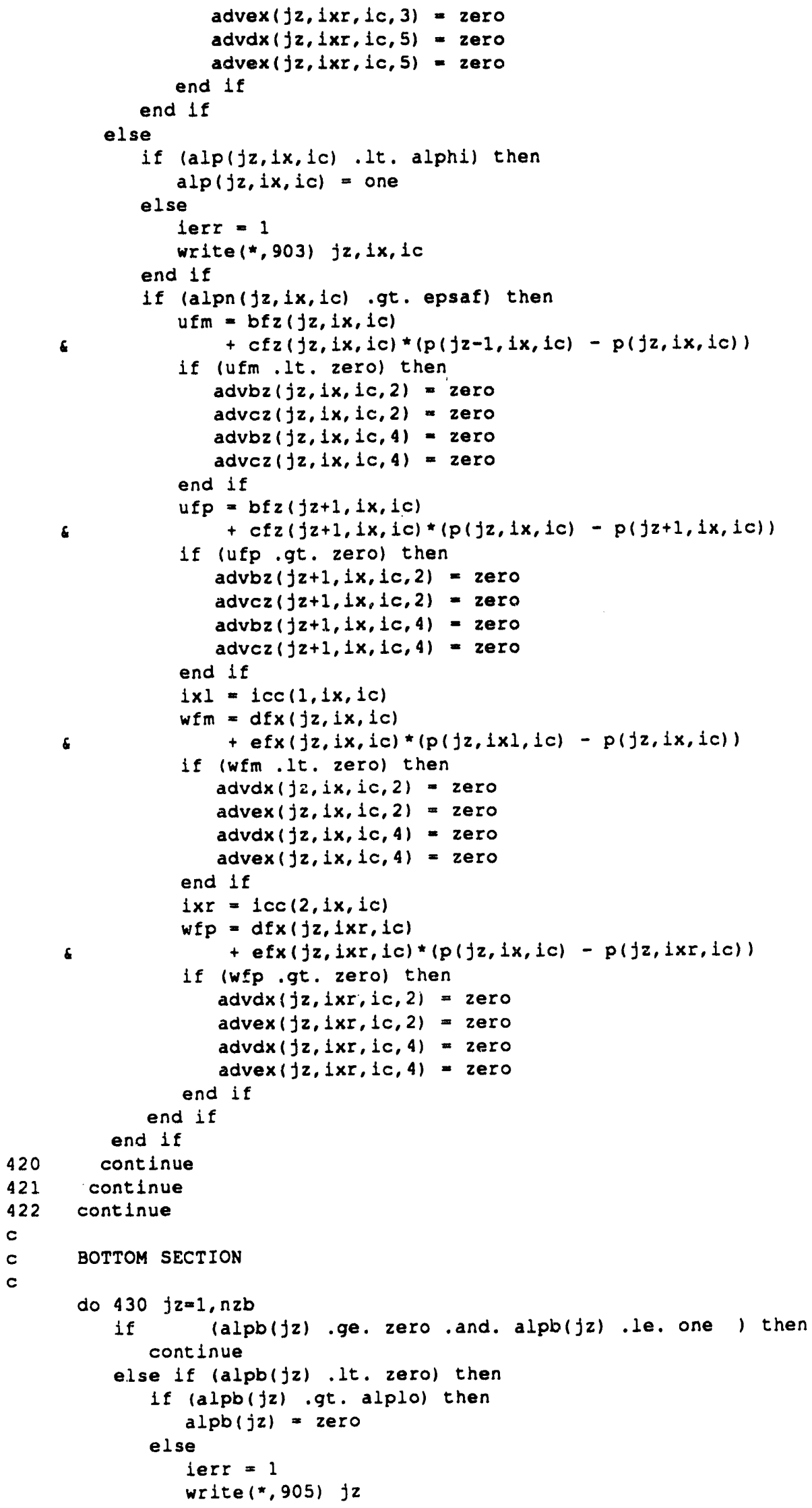


c

$c$

c end if

if (alpbn(jz).1t. epsag) then

if (jz .eq. 1) then

do 425 ic $=1$, nchn

do $4251 x=1, n \times(1 c)$

$u g m=b g z(n z p 2,1 x, i c)$

$+\operatorname{cgz}(n z p 2,1 x, 1 c) *(p(n z p 1,1 x, 1 c)\lfloor p b(1))$

if (ugm.1t. zero) then

$\operatorname{advbz}(n z p 2,1 x, 1 c, 1)=z e r o$

$\operatorname{advcz}(n z p 2,1 x, i c, 1)=z e r o-$

$\operatorname{advbz}(n z p 2,1 x, 1 c, 3)=z e r o$

$\operatorname{advcz}(n z p 2,1 x, 1 c, 3)=z e r o$

$\operatorname{advbz}(n z p 2,1 x, 1 c, 5)=z e r o$

end if

$\operatorname{advcz}(n z p 2,1 x, 1 c, 5)=z e r o$

continue

else

$u g m=b g b(f z-1)+c g b(j z-1) *(p b(j z-1)-p b(f z))$

if (ugm .1t, zero) then advbb $(j z-1,1)=z e r o$ $\operatorname{advcb}(j z-1,1)=$ zero $a d v b b(j z-1,3)=$ zero $\operatorname{advcb}(j z-1,3)=$ zero $a d v b b(j z-1,5)=z e r o$ $\operatorname{advcb}(f z-1,5)=$ zero

end if

end if

if $(y z$.eq. $n z b)$ then

$u g p=b g b(j z)+c g b(j z) *(p b(j z)-p t b \quad$

else

$u g p=b g b(j z)+c g b(j z) *(p b(j z)-p b(j z+1))$

end if

if (ugp .gt. zero) then

$\operatorname{advbb}(f z, 1)=z e r o$

$\operatorname{advcb}(j z, 1)=z e r 0$

$\operatorname{advbb}(j z, 3)=z$ ero

$\operatorname{advcb}(j z, 3)=z$ ero

$\operatorname{advbb}(j z, 5)=z e r 0$

$\operatorname{advcb}(j z, 5)=z e r o$

end if

else

end if

if $(a l p b(j z)$. It. alphi) then

$a l p b(j z)=$ one

else

ierr $=1$

write $(*, 905) \mathrm{jz}$

end $1 f$

if (alpbn(jz) gt. epsaf) then

if $(\mathrm{jz}$.eq. I) then

do 426 ic=1, nchn

do $426 i x=1, n x(i c)$

$u f m=b f z(n z p 2,1 x, 1 c)$

$+c f z(n z p 2, i x, 1 c) *(p(n z p), i x, i c)-p b(1))$

if (ufm.lt. zero) then

$\operatorname{advbz}(n z p 2, i x, 1 c, 2)=z e r o$

$\operatorname{advcz}(n z p 2, i x, i c, 2)=z e r o$

$\operatorname{advbz}(n z p 2,1 x, 1 c, 4)=z e r o$

$\operatorname{advcz}(n z p 2, i x, i c, 4)=z e r o$

end if

continue 


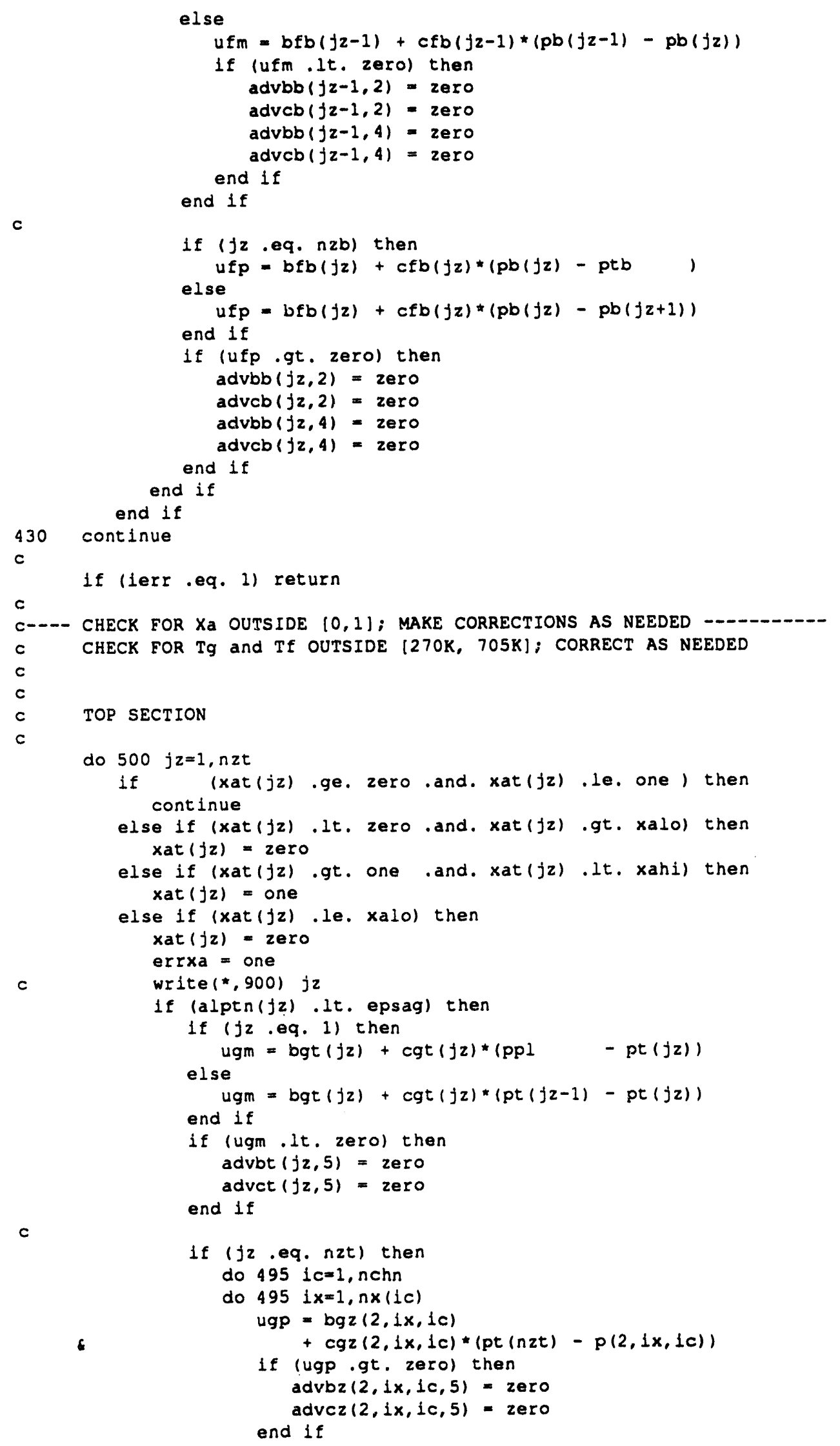


495

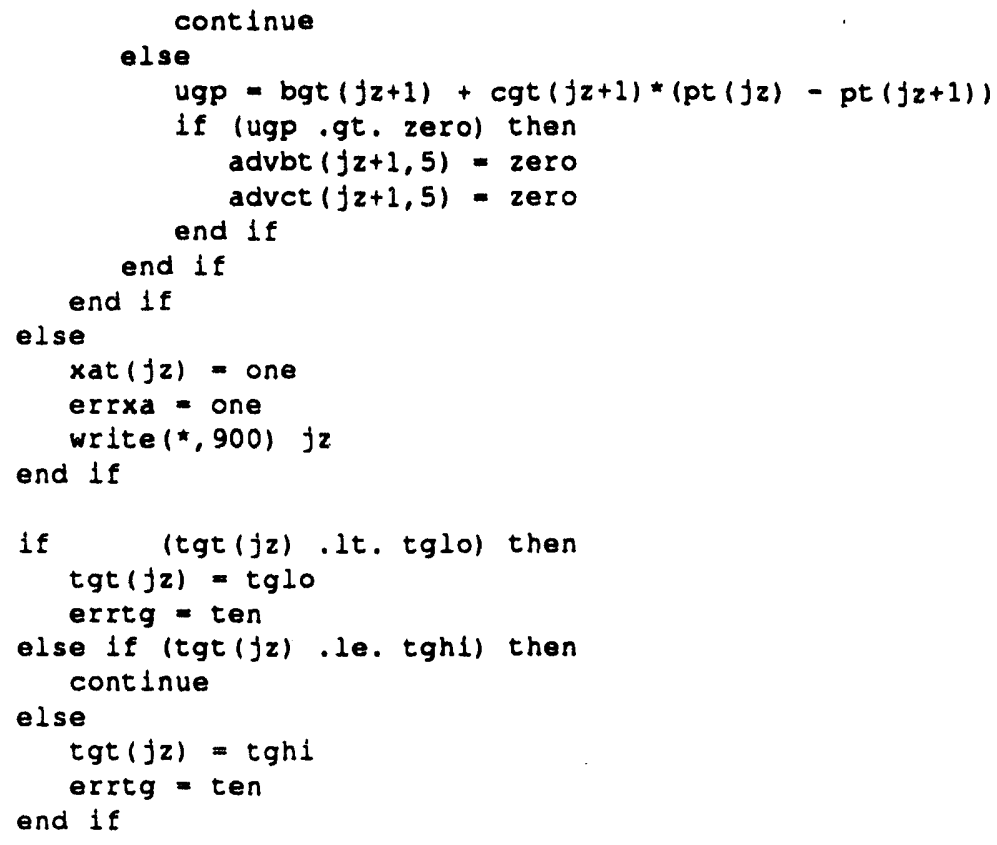

c

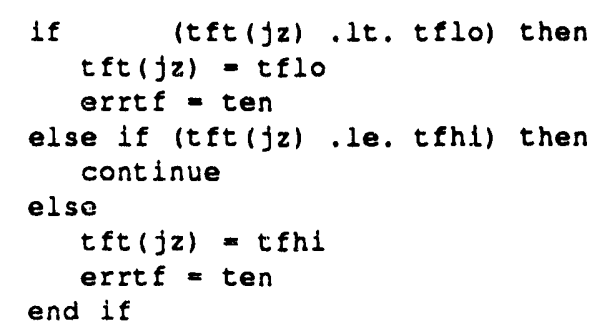



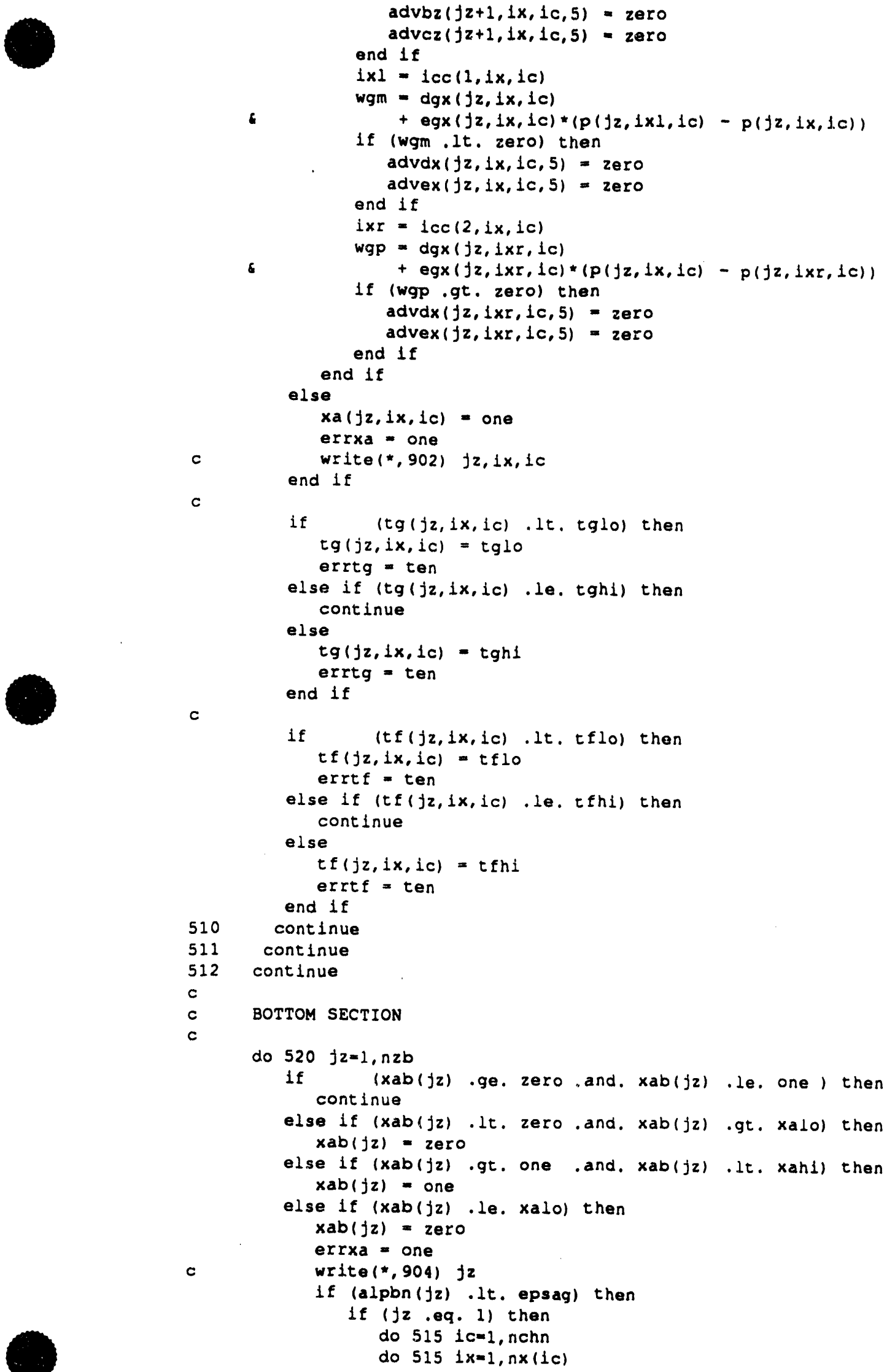


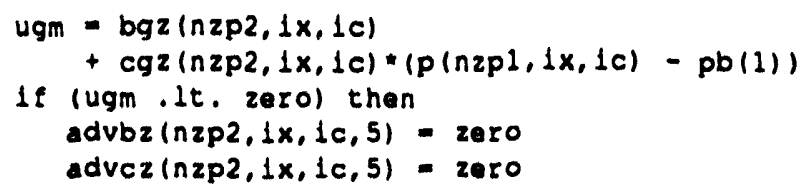

$c$

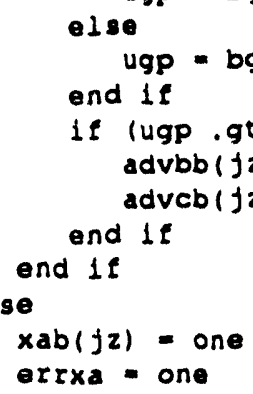


6

het, dhedp, dhedt)

MIDDLE SECTION

do 602 1c $=1$, nchn

do $6011 \mathrm{x}=1,11 \mathrm{x}(1 \mathrm{c})$

call state $(p, t g, t f, x a, f a c t o r, 11 q$,

2, nzp1, nzp2, nxmax, nchn, 1x, 1c,

rog, drogdp, drogdt, drgdxa,

hg , dhgdp, dhgdt, dhgdxa,

rof, drofdp, drofdt,

continue

hf , dhfdp, dhfdt)

602 continue

BOTTOM SECTION

call state (pb,tgb,teb, xab, tactor, 11q,

$6 \quad 1, n z b, n z b, 1,1,1,1$,

rogb, drogdp, drogdt, drgdxa,

hgb , dhgdp, dhgdt, dhgdxa,

rotb, drofdp, drofdt.

heb, dhedp, dhfdt)

c

C---- COMPUTE CHANNEL INLET VOID FRACTIONS

c

c

if (nchn .eq. 3) then

pchn $=$ zero

do $6901 \mathrm{c}=1,3$

do $6901 \times=1, n \times(1 c)$

pchn $=p c h n+p(2,1 x, 1 c)$

690 continue

pchn $=p c h n /(n \times(1)+n \times(2)+n \times(3))$

delp $=p t(n z t)-p e h n$

c

$a v g=a l p t(n z t)$

c

if (delp. le. delpl) then

c

$\operatorname{alp} 1=\operatorname{lcin} 1+(\operatorname{cin} 2+\operatorname{lcin} 3+\cos 4+\cos 5$

$+(\operatorname{cln} 6+\operatorname{cin} 7 * a v g) * a v g) * \operatorname{avg}) * a v g) * a v g) * a v g) * a v g$

+ four*avg* (one - avg)*sigal

$\mathrm{a} l \mathrm{pm}=1 \mathrm{cmn} 1+1 \mathrm{cmn} 2+1 \mathrm{cmn} 3+1 \mathrm{cmn} 4+1 \mathrm{cmn} 5$

$+\left(\mathrm{cmn} 6+\mathrm{cmn} 7\right.$ avg $^{*}$ *avg) *avg)*avg)*avg)*avg)*avg

+ four*avg*(one - avg)*sigam

c

else if (delp. Le. delp2) then

c

$\operatorname{alpln}=(\operatorname{cin} 1+1 \operatorname{cin} 2+(\operatorname{cin} 3+(\operatorname{cin} 4+\operatorname{lcin} 5$

$+(\operatorname{cin} 6+\operatorname{cin} 7 * a v g) * a v g) * a v g) * a v g) * a v g) * a v g) * a v g$

+ four*avg* (one - avg) slgal

alpmn $=1 \mathrm{cmn} 1+1 \mathrm{cmn} 2+1 \mathrm{cmn} 3+1 \mathrm{cmn} 4+1 \mathrm{cmn} 5$

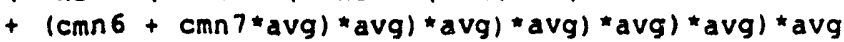

+ four*avg*(one - avg)*sigam

c

$a 1 p i p=1 c 1 p 1+(c 1 p 2+(c 1 p 3+(c 1 p 4+1 c 1 p 5$ $+(c 1 p 6+c 1 p 7 * a v g) * a v g) * a v g) * a v g) * a v g) * a v g) \star a v g$

+ four*avg*(one - avg)*slgf 1

a lpmp $=1 \mathrm{cmpl}+1 \mathrm{cmp} 2+1 \mathrm{cmp} 3+1 \mathrm{cmp} 4+1 \mathrm{cmp} 5$ $+(\mathrm{cmp} 6+\mathrm{cmp} 7$ *avg) *avg)*avg)*avg)*avg)*avg)*avg

+ four*avg*(one - avg)*sigfm

c

$w t=$ one $-(d e l p-d e l p l) /(d e l p 2-d e l p l)$ 


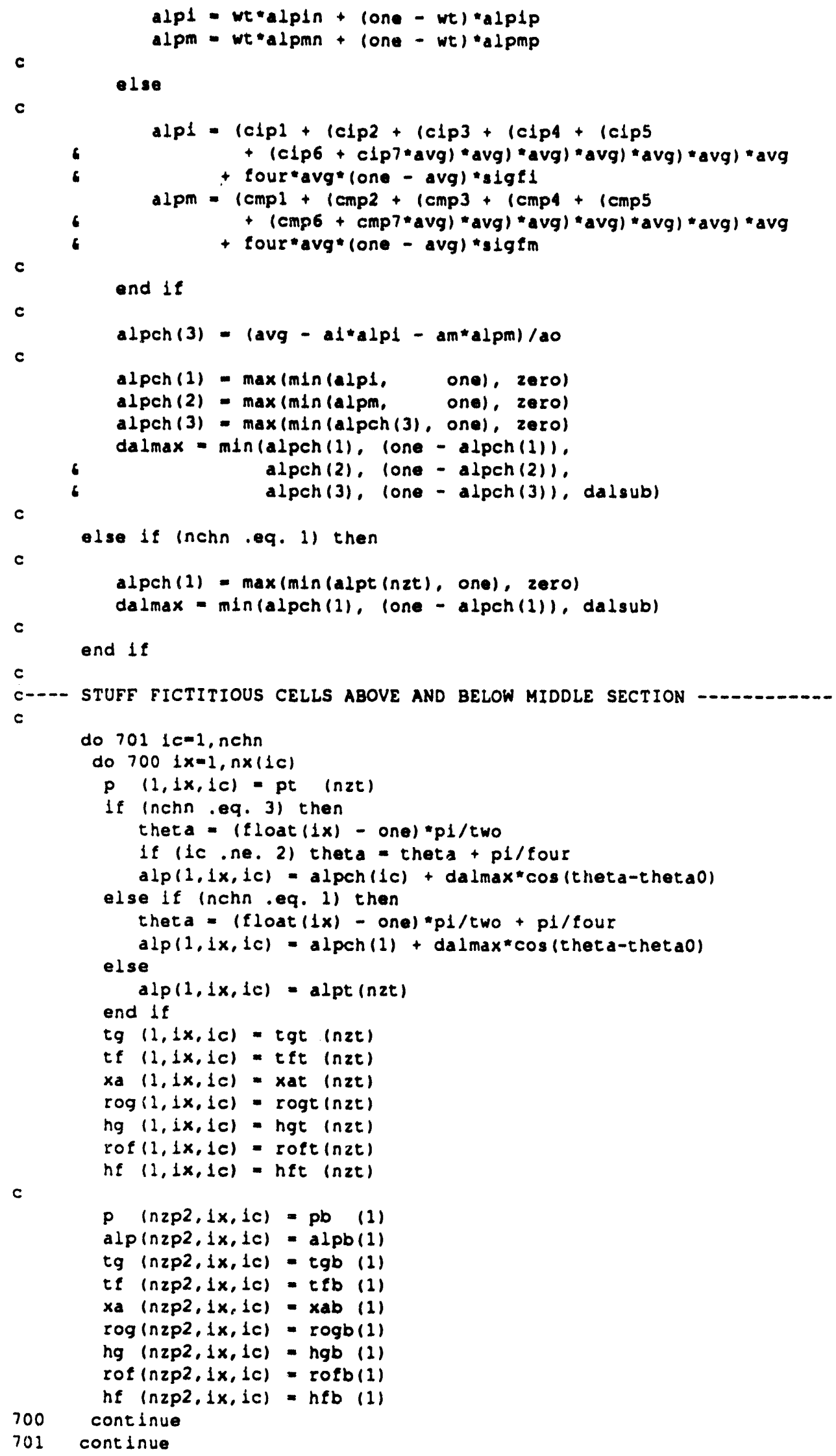




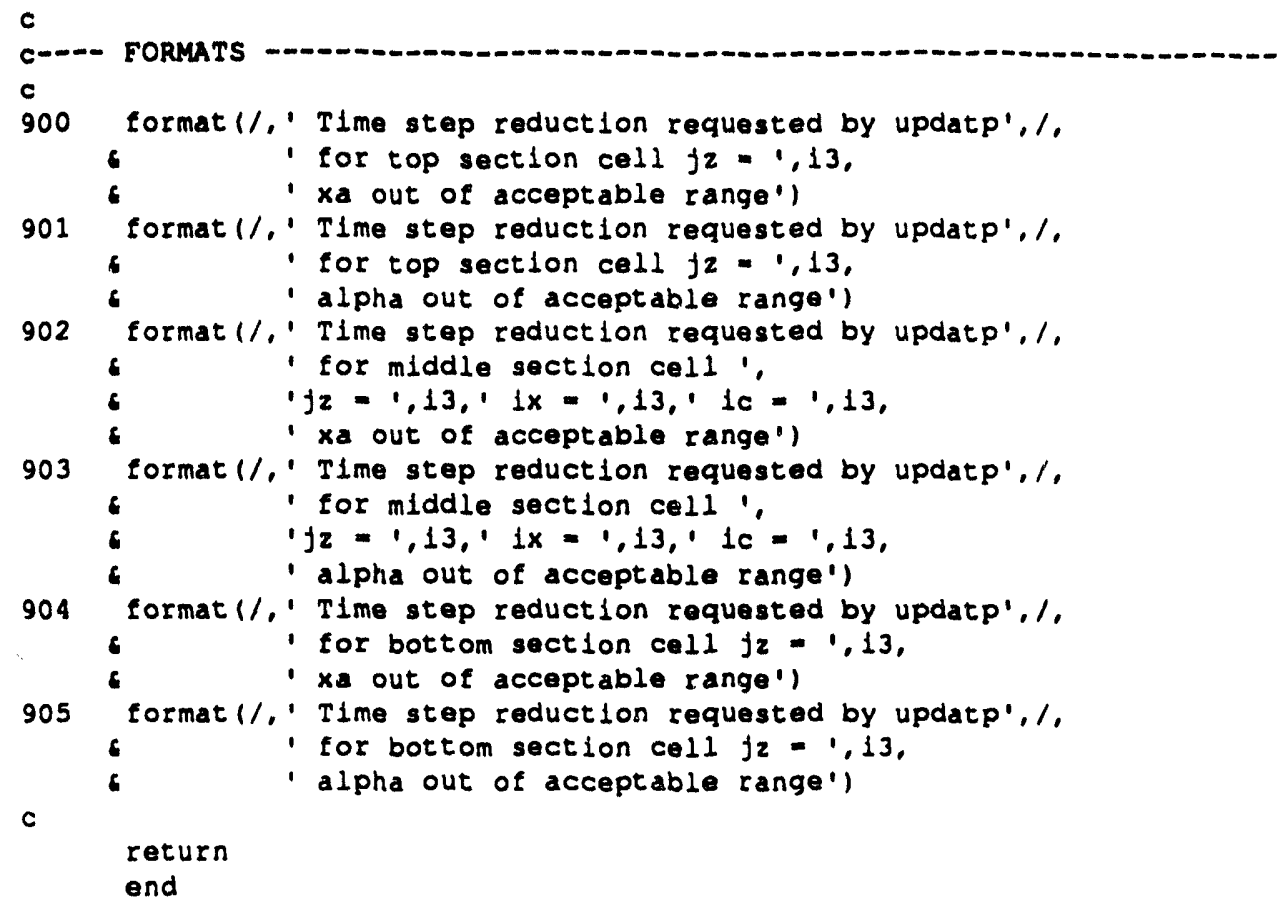

\section{UPDATV}

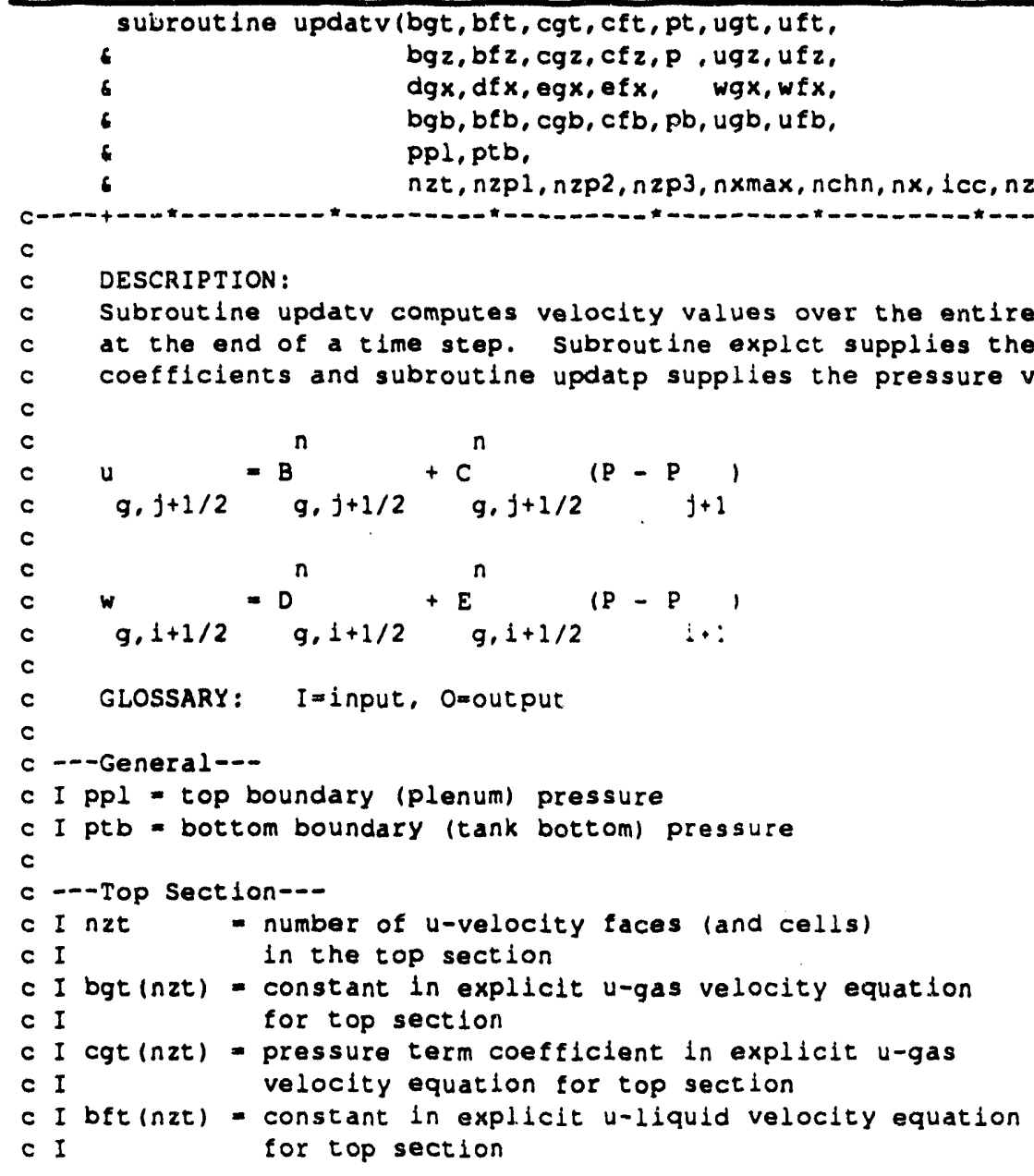




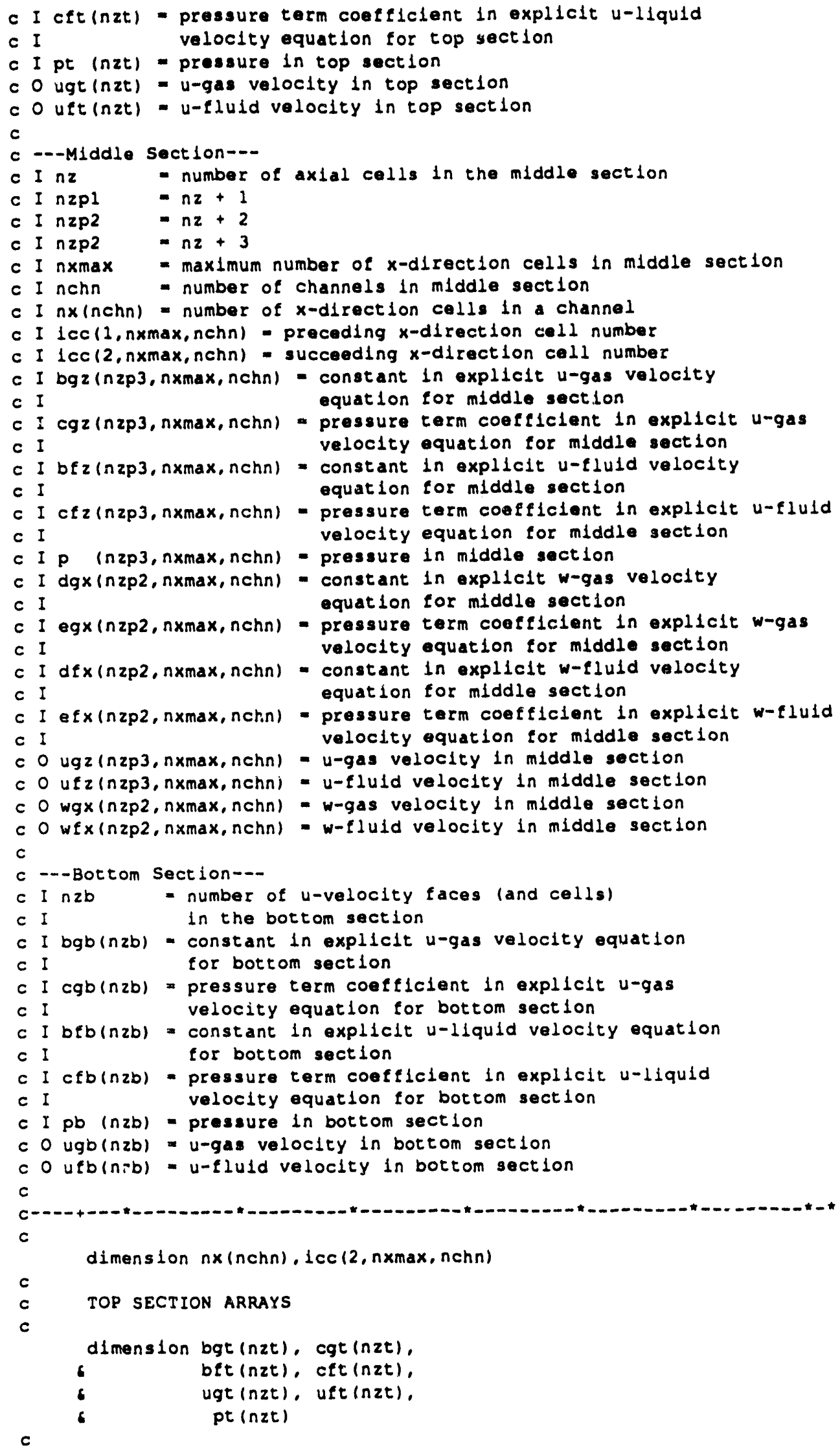




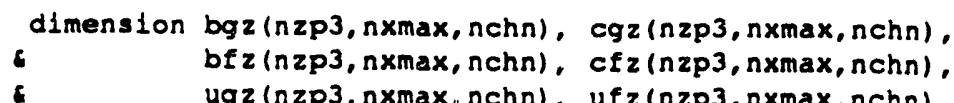


$c$ 300

c

c

c

400

401

c

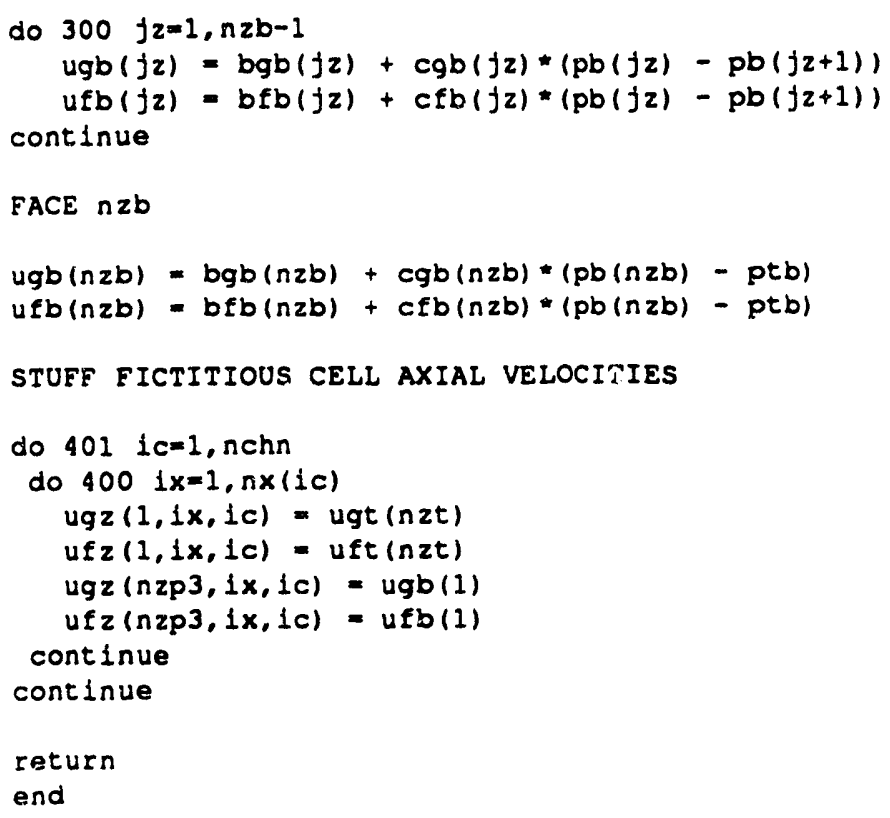

\section{VISGS}

subroutine visgs (p,t, xa, l, visg)

$\star$

- INPUT:

- $\quad p=$ MIXTURE PRESSURE IN Pa

- $t$ = TEMPERATURE IN $K$

- $\quad$ Xa $=$ MASS FRACTION OF AIR

* 1 = IDENTfFier $(1=1$ fOR H20, $1=2$ fOR D2O)

output:

* visg $\approx$ DYNAMIC VISCOSITY OF AIR-STEAM GAS MIXTURE IN Pa-s

- RANGE:

- $275 K<t<700 K$

- $0<x a<1$

* $p<1 M P a$

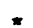

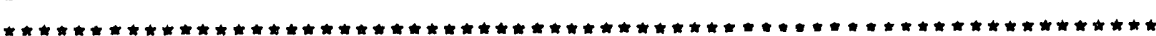

parameter (one=1.0, tconv=273.15)

dimension rs(2), csl(2), cs2(2), cs? (2), cs412)

C

c

$c$

AIR PROPERTIES

data cal/145.8e-8/, ca2/110.4/

H2O PROPERTIES

data rs (1)/461.0/

data $\operatorname{cs} 1(1) / 8.04 \mathrm{e}-6 /, \operatorname{cs} 2(1) / 4.07 \mathrm{e}-8 /$

6 $\operatorname{cs} 3(1) / 1.858 e-7 /, \operatorname{cs} 4(1) / 5.9 e-10 /$

D2O PROPERTIES

data Is(2)/415.0/

data $\operatorname{csl}(2) / 8.3224 \mathrm{e}-6 /, \operatorname{cs} 2(2) / 4.1550 \mathrm{e}-8 /$,

c $\operatorname{cs} 3(2) / 0.01$ $\operatorname{cs} 4(2) / 0.0 /$ 


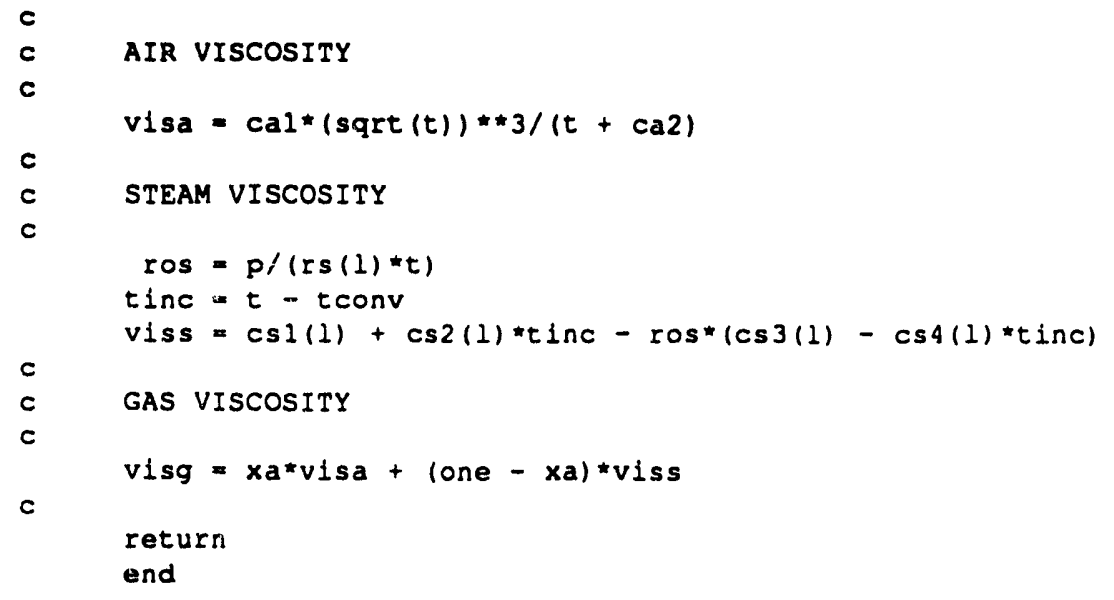

\section{VISIO}

subroutine vislg(t, $1, v i s l, d v d t)$

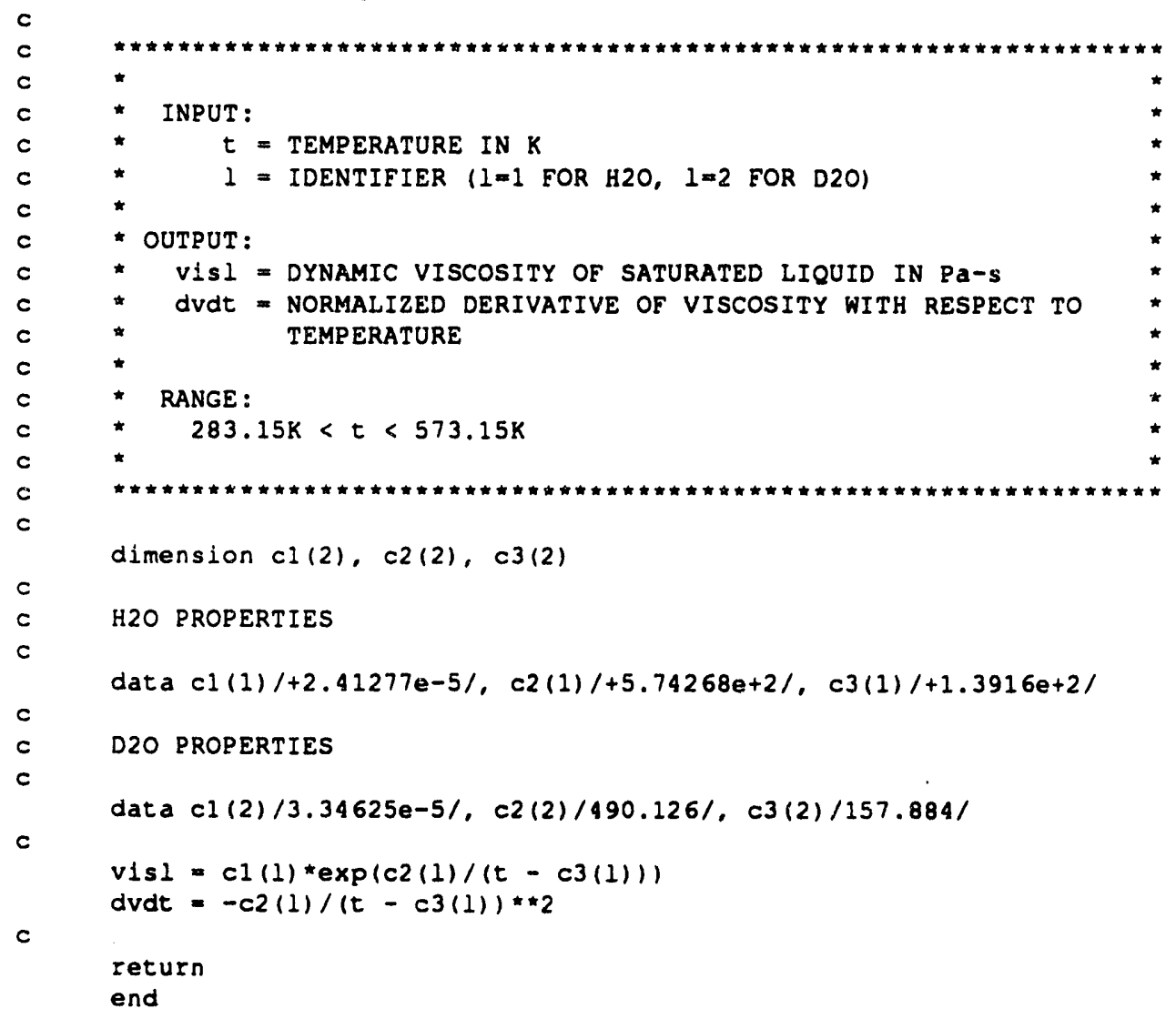

\section{VRIB}

function vrib (alpha)

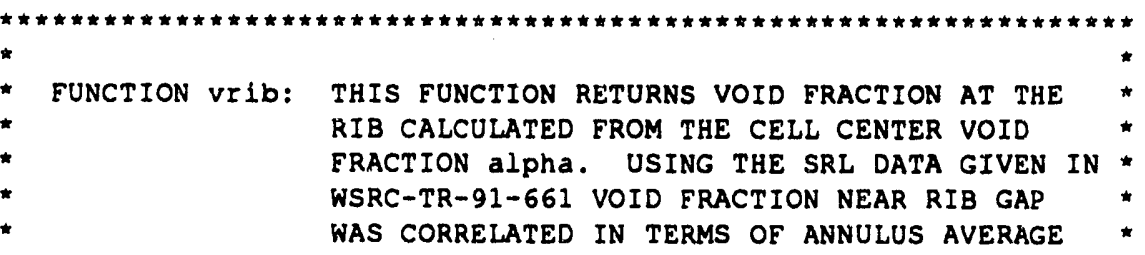




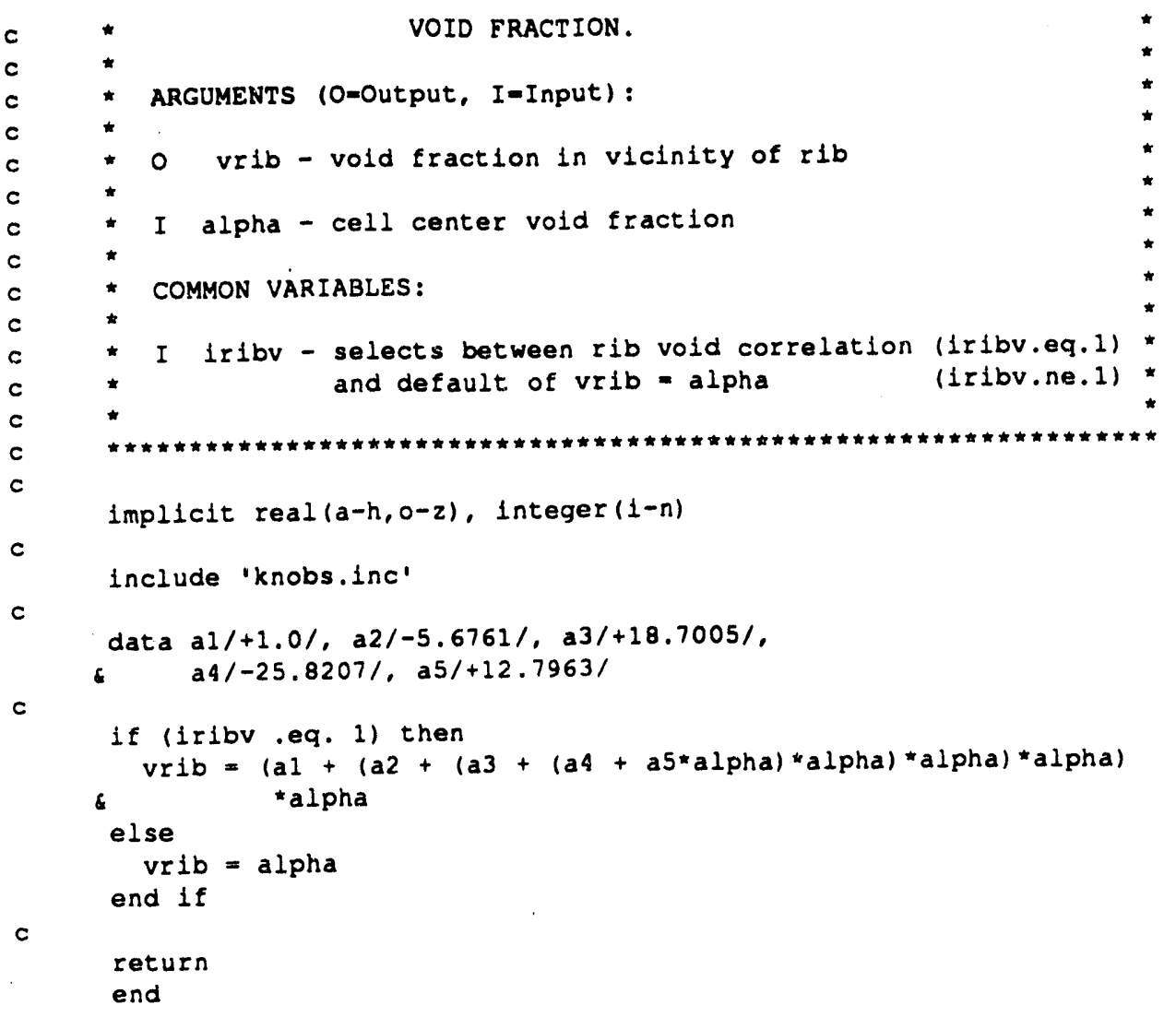

\section{YVALU}

real function yvalu (itype, $n, t, y, z, x$ )

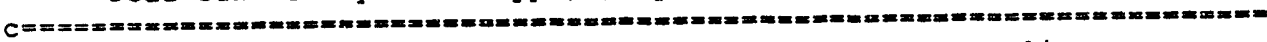

c Purpose: This routine evaluates the first-degree spline or

c natural cubic spline for a given value of $x$.

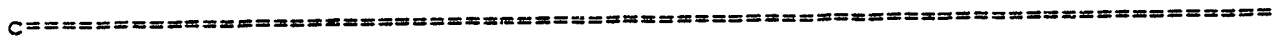

c Subroutine history

- algorithm given in "Numerical Mathematics and Computing" by Cheney and Kincald, 2nd Edition, 1985.

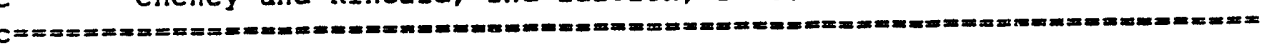

c Arguments

\begin{tabular}{cccc} 
NAME & I/O & TYPE & DIM \\
\hdashline & -- & --0
\end{tabular}

itype I I*4 Identifier of type of spline

$=0$ (Natural cublc spline)

$=1$ (First-degree spline)

Number of points

Abscissa values or knots

Ordinate values

point of interpolation

slopes of first-degree splines

or second derivatives of natural cublc splines at the knots

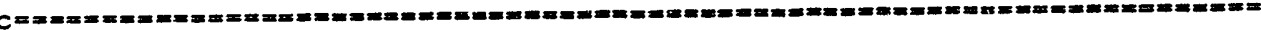

implicit real $(a-h, 0-z)$, integer $(i-n)$

parameter (zero $=0 ., p 5=0.5, t w o=2, . s i x=6$.

dimension $t(n), y(n), z(n)$

c

if $(x . g e . t(n))$ then 


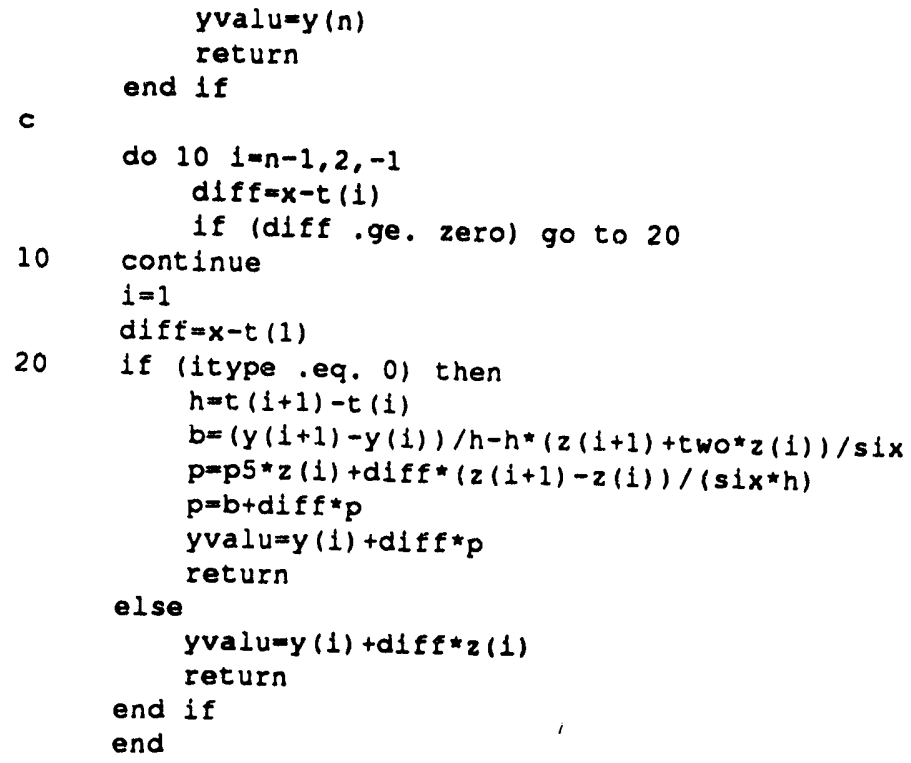

RNOBS . INC

$\begin{array}{ll}\text { common /sense/ } & x \text { cofh, xcofl, xreh, xrel, xkmet, xcvmet, } \\ & x h g 1, x h f 1, x k g 1, x p h 1, \text { cizfac, cixfac, } \\ & x \in \text { ic, plnht, cipln, formhs, alphs, ippu, } \\ & \text { alb2,als2, ala2, expbs, expsa, } \\ & \text { delp1, delp2, sigai, sigam, sigfi, sigfm, } \\ & \text { dalsub, thetao, iribv }\end{array}$

PARAMS . INC

common /rparam/ runsec,tsec, delt, rdelt, dtmin, dtmax, dtpfld,dtpsid,

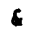

c dt ppwr, dt pdmp, pref, epsin, factor, vminz, vminx, da, alppl, alppln, alptb, alptbn, acpl, actb. volpl, voltb, ppl, xapl, tgpl, tfpl, rogpl, rofpl, hgpl, hfpl, ppln, xapln, tgpln, tfpln, rogpln, rofpln, hgpln, hfpln, ptb, xatb, tgtb, tftb, rogtb, roftb, hgtb, hftb. ptbn, xatbn, tgtbn, tftbn, rogtbn, roftbn, hgtbn, hftbn, topln, subln, bot In, hactiv, gap, tsolid, tsrfac, tol, asypwr, solidv, delmod, deldry, hmod, persat, epsp, epsalp, epstg, epstf, epsxa, tolpow, tcrmax,tmid, hpref, hexpf, tsupw, htdamp, cidamp, xdelt, tolss, wss,

c xturb, douts, emisi, emiso, cradi, xa0, epsy, epsf

c common/tzeros/ pplo,alpplo,tgplo,tfplo, xaplo, qfino, qfin,
ptbo,alptbo, tgtbo,tftbo, xatbo, qgino, qgin, 6 tnklvo, sumdry, tmodo

\footnotetext{
common /iparam/ maxia, maxa, nz, nzpl,nzp2, nzp3,nzt, nzb, izht, izhb, \& nxmax, nchn, nsubc, nlarg, nspln, ntptot, matgas, iboil, c ischem, irebal, ncmr, initmx, nitmax, iltot, nitno, istop, ierr, ibond, iuntes, istart, iptime, isave, ippl,ialpl,itgpl, itfpl, ixapl,iqgin, iprint, - iptb, ialtb, itgtb, itftb, ixatb, iqfin, itnklv, - ifissn, imodh, idryh, iconh, itmod, nreg, mnrp, itime, - lexp, icent, laxial, ncms, iscrn, ncyln, nrmax, nrmxp, - napl, nsurf, igami, igamw, icrit, ncrit, maxpi, isolid,

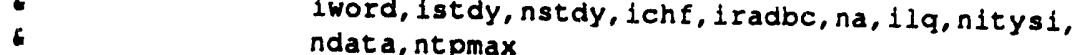


c

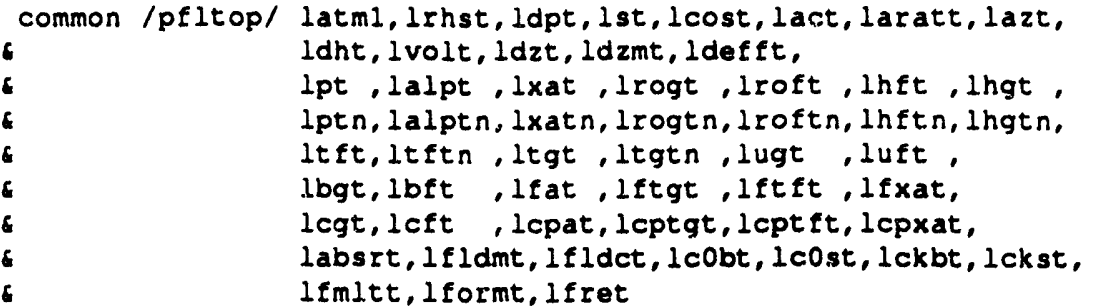

common /pflbot/ labml, Irhsb, ldpb, 1sb, 1cosb, 1acb, laratb, 1azb, 4 ldhb, lvolb, ldzb, ldzmb, ldeffb,

- lpb, lalpb, lxab, lrogb, lrofb, lhfb, lhgb .

- lpbn, lalpbn, lxabn, lrogbn, 1rufbn, lhebn, lhgbn,

- ltfb, ltfbn, ltgb, ltgbn, lugb , lufb .

6 lbgb, lbfb , lfab, lftgb, Iftfb, lfxab,

lcgb, lcfb , lcpab, lcptgb, lcptfb, lcpxab,

c labsrb, If $1 \mathrm{dmb}, 1 f 1 \mathrm{dcb}, 1 \mathrm{cObb}, 1 \mathrm{c0}$ sb, 1 ckbb, $1 \mathrm{cksb}$,

lfmleb, lformb, lfreb

c

common /ajacob/ ladvbz, ladvcz, ladvdx, ladvex,

ladvbt, ladvct, ladvbb, ladvcb

c

common /inlext/ lacin, lrogin,lrofin, lalpin, lugin, lufin,

6 lacex, Irogex, lrofex, lalpex, lugex, lufex

c

common / Intfac/ lcitn, lcixn, lcizn, lcibn,

4 lngi, lnfi, lakgi, lysi, lei, lgi,

- lngit, lhfit, lakgit, lysit, leit, lgit,

\& lngib, lnfib, lakgib, lysib, leib, lgib

C

common /psolid/ Inr, Infrst, Inlast, lloxid, liorib, Imat, Iwt

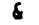

6

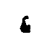

c

common /ppower/ Inrp, Inrgtm, llaxlp, lpfrct, lpfrdd, Ipfrdw, Irphl, - Irphdd, Irphdw, Irdz, laxial, laxa10, 1t imet,

4 1 azmm, lfrdim, lblgm, lbigmw, lbigmd

c

common /splin/ lwb, Iwh, lwu, Iwv, Ixtp, lytp, Iztp, Iintp, litype,

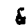

lnep

C

common /critrs/ 1ichks, lichkc, lpkfac

common / le/ ia $(10000)$

common / rc/ a (200000) 

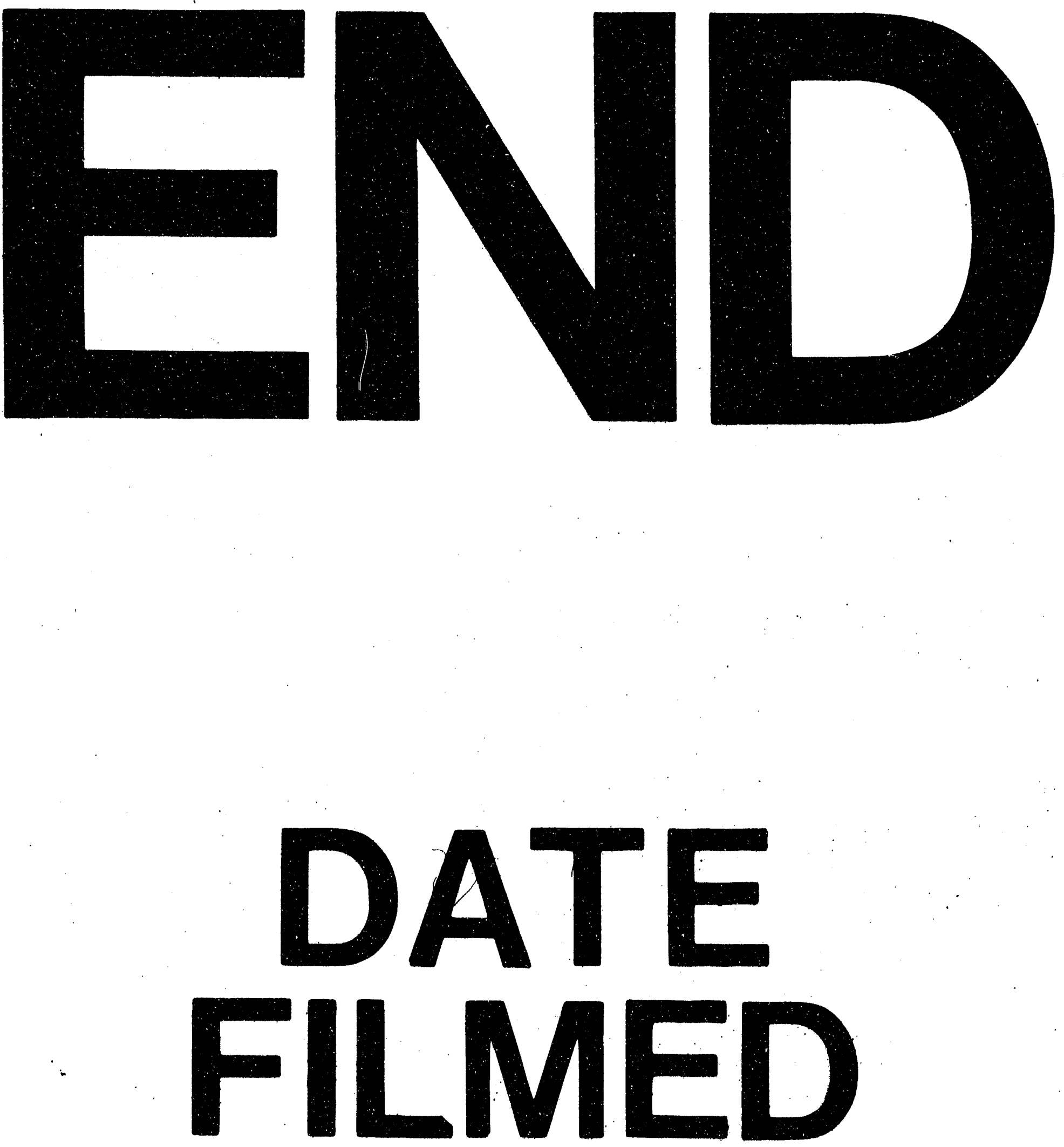

1

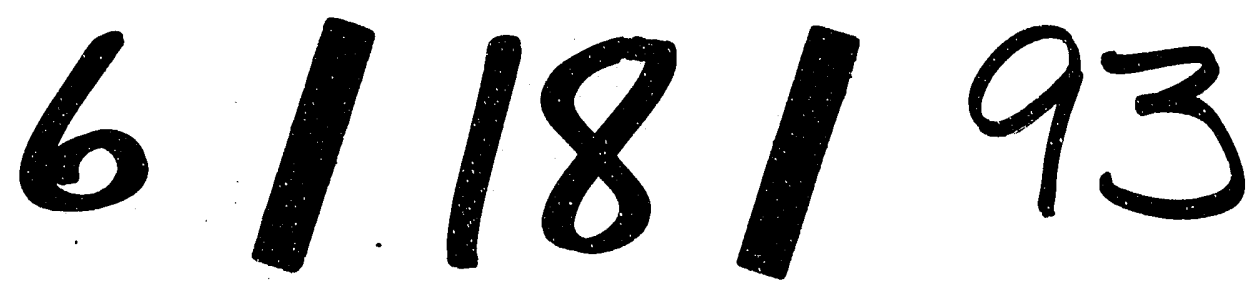


